\title{
Jet energy measurement with the ATLAS detector in proton-proton collisions at $\sqrt{\mathrm{s}}=7 \mathrm{TeV}$
}

\author{
The ATLAS Collaboration ${ }^{\star}$ \\ CERN, 1211 Geneva 23, Switzerland
}

Received: 29 December 2011 / Revised: 20 September 2012 / Published online: 2 March 2013

(C) CERN for the benefit of the ATLAS collaboration 2013. This article is published with open access at Springerlink.com

\begin{abstract}
The jet energy scale and its systematic uncertainty are determined for jets measured with the ATLAS detector at the LHC in proton-proton collision data at a centre-of-mass energy of $\sqrt{s}=7 \mathrm{TeV}$ corresponding to an integrated luminosity of $38 \mathrm{pb}^{-1}$. Jets are reconstructed with the anti- $k_{t}$ algorithm with distance parameters $R=0.4$ or $R=0.6$. Jet energy and angle corrections are determined from Monte Carlo simulations to calibrate jets with transverse momenta $p_{\mathrm{T}} \geq 20 \mathrm{GeV}$ and pseudorapidities $|\eta|<4.5$. The jet energy systematic uncertainty is estimated using the single isolated hadron response measured in situ and in test-beams, exploiting the transverse momentum balance between central and forward jets in events with dijet topologies and studying systematic variations in Monte Carlo simulations. The jet energy uncertainty is less than $2.5 \%$ in the central calorimeter region $(|\eta|<0.8)$ for jets with $60 \leq p_{\mathrm{T}}<800 \mathrm{GeV}$, and is maximally $14 \%$ for $p_{\mathrm{T}}<30 \mathrm{GeV}$ in the most forward region $3.2 \leq|\eta|<4.5$. The jet energy is validated for jet transverse momenta up to $1 \mathrm{TeV}$ to the level of a few percent using several in situ techniques by comparing a well-known reference such as the recoiling photon $p_{\mathrm{T}}$, the sum of the transverse momenta of tracks associated to the jet, or a system of low- $p_{\mathrm{T}}$ jets recoiling against a high- $p_{\mathrm{T}}$ jet. More sophisticated jet calibration schemes are presented based on calorimeter cell energy density weighting or hadronic properties of jets, aiming for an improved jet energy resolution and a reduced flavour dependence of the jet response. The systematic uncertainty of the jet energy determined from a combination of in situ techniques is consistent with the one derived from single hadron response measurements over a wide kinematic range. The nominal corrections and uncertainties are derived for isolated jets in an inclusive sample of high- $p_{\mathrm{T}}$ jets. Special cases such as event topologies with close-by jets, or selections of samples with an enhanced content of jets originating
\end{abstract}

^e-mail: atlas.publications@cern.ch from light quarks, heavy quarks or gluons are also discussed and the corresponding uncertainties are determined.

\section{Contents}

1 Introduction . . . . . . . . . . . . 2

2 The ATLAS detector . . . . . . . . . . . . . . 4

3 Introduction to jet energy calibration methods . . 5

4 Monte Carlo simulation . . . . . . . . . . . . 7

4.1 Event generators . . . . . . . . . . . . 7

4.2 Simulation of the ATLAS detector . . . . . 7

4.3 Nominal Monte Carlo simulation samples . 8

4.4 Simulated pile-up samples _ . . . . . . . . 8

5 Data sample and event selection . . . . . . 8

5.1 Data taking period and LHC conditions . . 8

5.2 Event selection . . . . . . . . . . . . 8

5.3 Data quality assessment . . . . . . . . 9 9

6 Jet reconstruction . . . . . . . . . . . . . . . . . 9

6.1 Reconstructed calorimeter jets . . . . . . 9

6.2 Reconstructed track jets . . . . . . . . . 10

6.3 Monte Carlo truth jets and flavour

association ............ 10

7 Jet quality . . . . . . . . . . . . . . . . . 10

7.1 Criteria to remove non-collision background 10

7.2 Evaluation of the jet quality selection efficiency . . . . . . . . . . 11

7.3 Summary of the jet quality selection . . . . 11

8 Jet energy calibration in the EM+JES scheme . . 13

8.1 Pile-up correction . . . . . . . . . . 13

8.2 Jet origin correction . . . . . . . . . . . 14

8.3 Jet energy correction . . . . . . . . . . . 14

8.4 Jet pseudorapidity correction . . . . . . 16

9 Jet energy scale uncertainties for the EM+JES scheme . . . . . . . . . . . . . 17

9.1 Jet response definition . . . . . . . . . . 18

9.2 Uncertainty in the calibration method . . . 18

9.3 Uncertainty on the jet calorimeter response 18

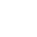

(1)

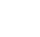

7

8
\section{8} 8 8

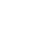

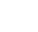

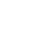
(1) (1) 0 10 (1)

0
1 (1)

13
4 16 7 8

8
18 
9.4 Uncertainties due to the detector simulation

9.5 Uncertainties due to the event modelling in Monte Carlo generators . . . . . . . . . .

9.6 In situ intercalibration using events with dijet topologies . . . . . . . . . .

9.7 Uncertainties due to multiple proton-proton collisions . . . . . . . . . .

9.8 Summary of jet energy scale systematic uncertainties . . . . . . . . . .

9.9 Discussion of special cases . . . . . . . .

10 Jet energy scale uncertainties validation

with in situ techniques for the EM+JES scheme .

10.1 Comparison of transverse momentum balance of jets from calorimeter and tracking

10.2 Photon-jet transverse momentum balance .

10.3 Multijet transverse momentum balance . .

10.4 Summary of JES validation using in situ techniques ............

10.5 JES uncertainty from combination of in situ techniques . . . . . . . . . . . .

11 Jet energy calibration based on global jet properties . . . . . . . . . . . 11.1 Global sequential technique . . . . . . . .

11.2 Properties derived from the internal jet structure .............

11.3 Derivation of the global sequential correction

12 Jet energy scale uncertainties for jet calibrations based on global jet properties . . . . . . . . .

12.1 Validation of the global sequential calibration using dijet events . . . . . . .

12.2 Sensitivity of the global sequential calibration to pile-up . . . . . . . . .

12.3 Summary on the JES uncertainty for the global sequential calibration . . . .

13 Jet calibration schemes based on cell energy weighting $\ldots \ldots \ldots \ldots \ldots$ 13.1 Global cell energy density weighting calibration . . . . . . . . . .

13.2 Local cluster weighting calibration . . . . .

13.3 Jet energy calibration for jets with calibrated constituents

14 Jet energy scale uncertainties for jet calibrations based on cell weighting . . . . . . . . .

14.1 Energy density as input to the global cell weighting calibration $\ldots \ldots \ldots \ldots$

14.2 Cluster properties inside jets as input to the local cluster weighting calibration . .

14.3 Jet energy scale uncertainty from in situ techniques for jets based on cell weighting

15 Summary of jet energy scale uncertainties of various calibration schemes . . . . . . .

16 Jet reconstruction efficiency . . . . . . . . . . 16.1 Efficiency in the Monte Carlo simulation

16.2 Efficiency in situ validation . . . . . . . . 81

16.3 Summary of jet reconstruction efficiency . 82

17 Response uncertainty of non-isolated jets . . . 82

17.1 Evaluation of close-by jet effects . . . . . . 82

17.2 Non-isolated jet response . . . . . . . . . 83

17.3 Non-isolated jet energy scale uncertainty . 84

17.4 Summary of close-by jet uncertainty . . . . 85

18 Light quark and gluon jet response and sample characterisation . . . . . . . . . . 85

18.1 Data samples for flavour dependence studies 85

18.2 Flavour dependence of the calorimeter response ........... 86

18.3 Systematic uncertainties due to flavour dependence . . . . . . . . . . 86

18.4 Average jet flavour determination . . . . . 87

18.5 Systematic uncertainties of average flavour composition ........... 88

18.6 Flavour composition in a photon-jet sample $\quad 90$

18.7 Flavour composition in a multijet sample . 91

18.8 Summary of jet response flavour dependence 92

19 Global sequential calibrated jet response

for a quark sample . . . . . . . . . . . .

20 JES uncertainties for jets with identified heavy quark components . . . . . . . . . . . . . 94

20.1 Selection of identified heavy quark jets . . 94

20.2 Calorimeter response uncertainty . . . . . . 94

20.3 Uncertainties due to Monte Carlo modelling 94

20.4 Final bottom quark JES uncertainty . . . . 95

20.5 Validation of the heavy quark energy scale using tracks . . . . . . . . . . . . 95

21 Study of jet punch-through . . . . . . . . . . 99

21.1 Event selection for punch-through analysis $\quad 99$

21.2 Energy depositions in the hadronic calorimeter . . . . . . . . . . . . 99

21.3 Dijet balance as an indication of punch-through . . . . . . . . . . 100

21.4 Summary of the jet punch-through study . . 102 22 Summary . . . . . . . . . . . . . . . . . 102 Acknowledgements . . . . . . . . . . . . 103 References . . . . . . . . . . . . . . . . . 103 The ATLAS Collaboration . . . . . . . . . . . . . . 106

\section{Introduction}

Collimated sprays of energetic hadrons, called jets, are the dominant feature of high energy hard proton-proton interactions at the Large Hadron Collider (LHC) at CERN. In Quantum Chromodynamics (QCD) jets are produced via the fragmentation of quarks and gluons. They are key ingredients for many physics measurements and for searches for new phenomena.

During the year 2010 the ATLAS detector collected proton-proton collision data at a centre-of-mass energy of 
$\sqrt{s}=7 \mathrm{TeV}$ corresponding to an integrated luminosity of $38 \mathrm{pb}^{-1}$. The uncertainty in the jet energy measurement is the dominant experimental uncertainty for numerous physics results, for example the cross-section measurement of inclusive jets, dijets or multijets [1-5], as well as of vector bosons accompanied by jets [6,7], and new physics searches with jets in the final state [8-13]. The energy measurement of jets produced in proton-proton and electron-proton collisions was also discussed by previous experiments [14-24].

Jets are observed as groups of topologically related energy deposits in the ATLAS calorimeters. The anti- $k_{t}$ jet algorithm [25] is adopted as the standard way to reconstruct jets.

Using a Monte Carlo (MC) simulation the observed jets are calibrated such that, on average, the jet energy corresponds to that of the associated stable particles in the ATLAS detector. The calibration of the jet energy scale (JES) should ensure the correct measurement of the average energy across the whole detector and needs to be independent of proton-proton collision events produced in addition to the event of interest.

In this document, the jet calibration strategies adopted by the ATLAS experiment are outlined and studies to evaluate the uncertainties in the jet energy measurement are presented. A first estimate of the JES uncertainty, described in Ref. [1], was based on information available before the first LHC collisions. It also exploited transverse momentum balance in events with only two jets at high transverse momenta $\left(p_{\mathrm{T}}\right)$. A reduced uncertainty with respect to Ref. [1] is presented that is based on the increased knowledge of the detector performance obtained during the analysis of the first year of ATLAS data taking.

ATLAS has developed several jet calibration schemes [26] with different levels of complexity and different sensitivity to systematic effects, which are complementary in their contribution to the jet energy measurement. Each calibration scheme starts from the measured calorimeter energy at the electromagnetic (EM) energy scale, which correctly measures the energy deposited by electromagnetic showers. In the simplest case, called EM+JES calibration scheme, the jet energy is measured on EM scale and the jet calibration is derived as a simple correction relating the calorimeter's response to the true jet energy. More sophisticated schemes exploit the topology of the calorimeter energy depositions to correct for calorimeter non-compensation (nuclear energy losses, etc.) and other jet reconstruction effects. For the EM+JES calibration scheme the JES uncertainty can be determined from the single hadron response measurements in small data sets collected in situ or in test-beams. With a large data set available the JES uncertainty can also be determined using the ratio of the jet transverse momentum to the momentum of a well measured reference object and by a comparison of the data to the Monte Carlo simulation.
Several techniques have been developed to directly determine the uncertainty on the jet energy measurement in situ. The JES uncertainty can be obtained by comparing the jet energy to a well calibrated reference object. A standard technique to probe the absolute jet energy scale, used also in earlier hadron collider experiments, is to measure the $p_{\mathrm{T}}$ balance between the jet and a well-measured object: a photon or a $Z$ boson. However, the currently limited data statistics imposes a limit on the $p_{\mathrm{T}}$ range that can be tested with this technique. The JES uncertainty on higher jet transverse momenta up to the $\mathrm{TeV}$-scale can be assessed using the multijet balance technique where a recoil system of well-calibrated jets at lower $p_{\mathrm{T}}$ is balanced against a single jet at higher $p_{\mathrm{T}}$. A complementary technique uses the total momentum of the tracks associated to the jets as reference objects. While the resolution of the jet energy measurement using tracks in jets is rather poor, the mean jet energy can be determined to the precision of a few percent.

The standard jet calibration and the corresponding uncertainty on the energy measurement are determined for isolated jets in an inclusive jet data sample. Additional uncertainties are evaluated for the dependence of the calorimeter response to details of jet fragmentation like differences between jets induced by quarks or gluons. Also special event topologies with close-by jets are investigated.

The outline of the paper is as follows. First the ATLAS detector (Sect. 2) is described. An overview of the jet calibration procedures and the various calibration schemes is given in Sect. 3. The Monte Carlo simulation framework is introduced in Sect. 4. The data samples, data quality assessment and event selection are described in Sect. 5. Then, the reconstruction (Sect. 6), and the selection (Sect. 7) of jets are discussed. The jet calibration method is outlined in Sect. 8 which includes a prescription to correct for the extra energy due to multiple proton-proton interactions (pile-up).

Section 9 describes the sources of systematic uncertainties for the jet energy measurement and their estimation using Monte Carlo simulations and collision data. Section 10 describes several in situ techniques used to validate these systematic uncertainties. Section 11 presents a technique to improve the resolution of the energy measurements and to reduce the flavour response differences by exploiting the topology of the jets. The systematic uncertainties associated with this technique are described in Sect. 12. The jet calibration schemes based on calorimeter cell energy weighting in jets are introduced in Sect. 13, and the associated JES uncertainties are estimated from the in situ techniques as described in Sect. 14. Section 15 summarises the systematic uncertainties for all studied jet calibration schemes.

The jet reconstruction efficiency and its uncertainty is discussed in Sect. 16. The response uncertainty of nonisolated jets is investigated in Sect. 17, while Sects. 18 and 19 discuss response difference for jets originating from 
light quarks or gluons and presents a method to determine, on average, the jet flavour content in a given data sample. In Sect. 20 JES uncertainties for jets where a heavy quark is identified are investigated. Finally, possible effects from lack of full calorimeter containment of jets with high transverse momentum are studied in Sect. 21. The overall conclusion is given in Sect. 22.

The present paper discusses the precision of the mean jet energy measurement. The jet energy resolution [27] and calorimeter response uncertainty from single hadron response measurements [28] are discussed elsewhere.

\section{The ATLAS detector}

The ATLAS detector is a multi-purpose detector designed to observe particles produced in proton-proton and heavy ion collisions. A detailed description can be found in Ref. [29]. The detector consists of an inner detector, sampling electromagnetic and hadronic calorimeters and muon chambers. Figure 1 shows a sketch of the detector outline together with an event with two jets at high transverse momenta.

The inner detector (ID) is a tracking system immersed in a magnetic field of $2 \mathrm{~T}$ provided by a solenoid and covers a pseudorapidity ${ }^{1}|\eta| \lesssim 2.5$. The ID barrel region $|\eta| \lesssim 2$ consists of three layers of pixel detectors (Pixel) close to the beam-pipe, four layers of double-sided silicon micro-strip detectors (SCT) providing eight hits per track at intermediate radii, and a transition radiation tracker (TRT) composed of straw tubes in the outer part providing 35 hits per track. At $|\eta|>1$ the ID endcap regions each provide three Pixel discs and nine SCT discs perpendicular to the beam direction.

The liquid argon (LAr) calorimeter is composed of sampling detectors with full azimuthal symmetry, housed in one barrel and two endcap cryostats. A highly granular electromagnetic (EM) calorimeter with accordion-shaped electrodes and lead absorbers in liquid argon covers the pseudorapidity range $|\eta|<3.2$. It contains a barrel part (EMB, $|\eta|<1.475)$ and an endcap part (EMEC, $1.375 \leq|\eta|<3.2$ ) each with three layers in depth (from innermost to outermost EMB1, EMB2, EMB3 and EMEC1, EMEC 2, EMEC3). The middle layer has a $0.025 \times 0.025$ granularity in $\eta \times \phi$ space. In total, the EM calorimeter has a thickness of $X_{0}=22$

\footnotetext{
${ }^{1}$ The ATLAS coordinate system is a right-handed system with the $x$ axis pointing to the centre of the LHC ring and the $y$-axis pointing upwards. The polar angle $\theta$ is measured with respect to the LHC beamline. The azimuthal angle $\phi$ is measured with respect to the $x$-axis. The pseudorapidity $\eta$ is an approximation for rapidity $y$ in the high energy limit, and it is related to the polar angle $\theta$ as $\eta=-\ln \tan \frac{\theta}{2}$. The rapidity is defined as $y=0.5 \times \ln \left[\left(E+p_{z}\right) /\left(E-p_{z}\right)\right]$, where $E$ denotes the energy and $p_{z}$ is the component of the momentum along the beam direction. Transverse momentum and energy are defined as $p_{\mathrm{T}}=p \times \sin \theta$ and $E_{\mathrm{T}}=E \times \sin \theta$, respectively.
}

$\left(X_{0}=24\right)$ radiation lengths. The innermost layer (strips) consists of cells with eight times finer granularity in the $\eta$ direction and with 3-times coarser granularity in the $\phi$ direction.

For $|\eta|<1.8$, a presampler (PS), consisting of an active LAr layer is installed directly in front of the EM calorimeters, and provides a measurement of the energy lost before the calorimeter.

A copper-liquid argon hadronic endcap calorimeter (HEC, $1.5 \leq|\eta|<3.2$ ) is located behind the EMEC. A copper/ tungsten-liquid argon forward calorimeter (FCal) covers the region closest to the beam at $3.1 \leq|\eta|<4.9$. The HEC has four layers and the FCAL has three layers. From innermost to outermost these are: HEC0, HEC $1, \mathrm{HEC} 2, \mathrm{HEC} 3$ and FCa10, FCal1, FCal2. Altogether, the LAr calorimeters correspond to a total of 182,468 readout cells, i.e. $97.2 \%$ of the full ATLAS calorimeter readout.

The hadronic Tile calorimeter $(|\eta|<1.7)$ surrounding the LAr cryostats completes the ATLAS calorimetry. It consists of plastic scintillator tiles and steel absorbers covering $|\eta|<0.8$ for the barrel and $0.8 \leq|\eta|<1.7$ for the extended barrel. Radially, the hadronic Tile calorimeter is segmented into three layers, approximately 1.4, 3.9 and 1.8 interaction lengths thick at $\eta=0$; the $\Delta \eta \times \Delta \phi$ segmentation is $0.1 \times 0.1(0.2 \times 0.1$ in the last radial layer $)$. The last layer is used to catch the tails of the longitudinal shower development. The three radial layers of the Tile calorimeter will be referred to (from innermost to outermost) as Tile0, Tile1, Tile2. ${ }^{2}$

The ATLAS calorimeter covers a total thickness of $\lambda=$ 11 interaction lengths at $\eta=0$.

Between the barrel and the extended barrels there is a gap of about $60 \mathrm{~cm}$, which is needed for the ID and the LAr services. Gap scintillators (Gap) covering the region $1.0 \leq|\eta|<1.2$ are installed on the inner radial surface of the extended barrel modules in the region between the Tile barrel and the extended barrel. Crack scintillators (Scint) are located on the front of the LAr endcap and cover the region $1.2 \leq|\eta|<1.6$.

The muon spectrometer surrounds the ATLAS calorimeter. A system of three large air-core toroids, a barrel and two endcaps, generates a magnetic field in the pseudorapidity range of $|\eta|<2.7$. The muon spectrometer measures muon tracks with three layers of precision tracking chambers and is instrumented with separate trigger chambers.

The trigger system for the ATLAS detector consists of a hardware-based Level 1 (L1) and a software-based higher level trigger (HLT) [30]. Jets are first identified at L1 using a sliding window algorithm from coarse granularity calorimeter towers. This is refined using jets reconstructed from

\footnotetext{
${ }^{2}$ In the barrel, the Tile layers will be called TileBar0, TileBar1, TileBar 2 and in the extended barrel TileExt0, TileExt 1 and TileExt2.
} 


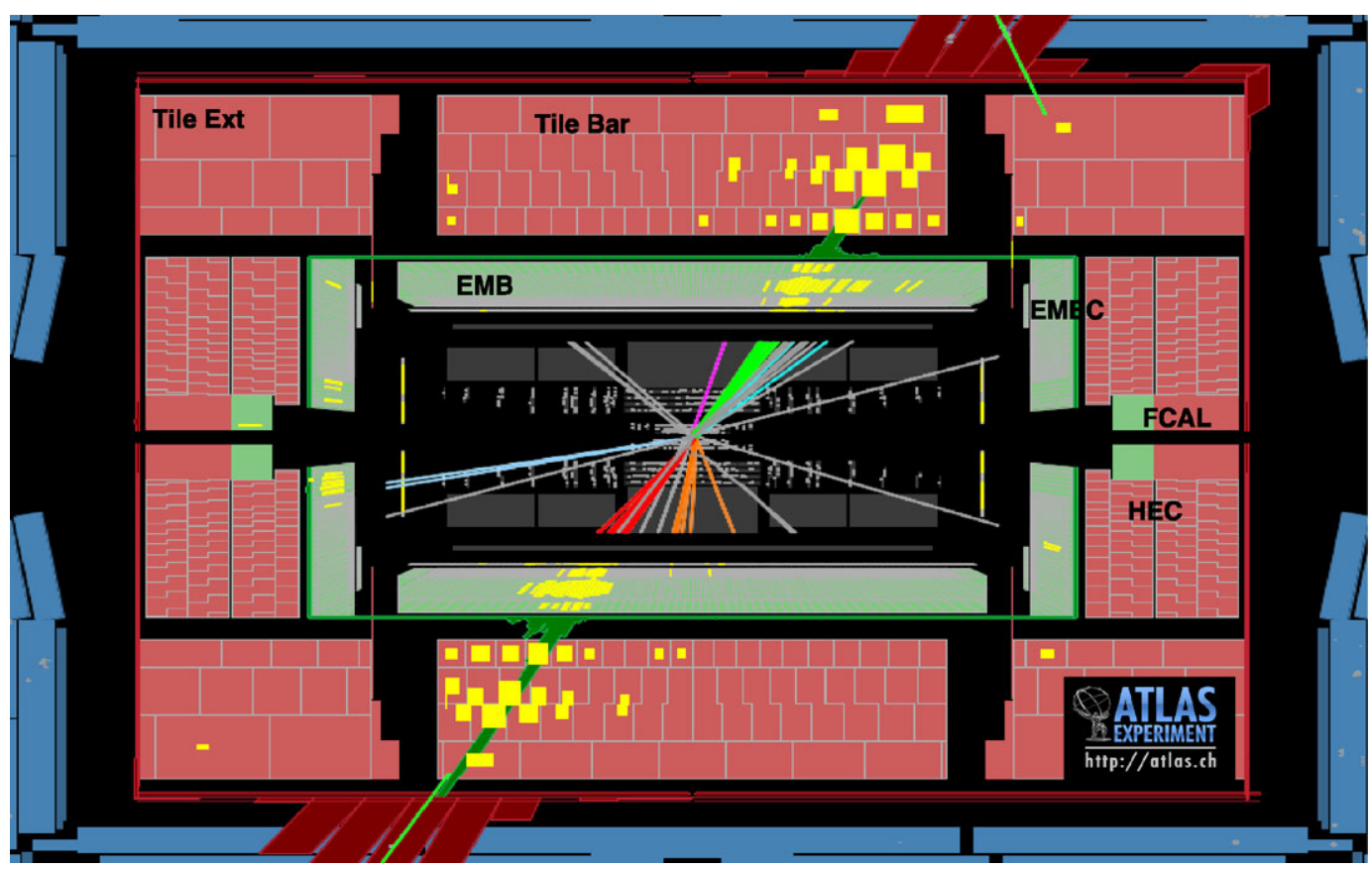

Fig. 1 Display of the central part of the ATLAS detector in the $x-z$ view showing the highest mass central dijet event collected during the 2010 data taking period. The two leading jets have $p_{\mathrm{T}}^{\text {jet }}=1.3 \mathrm{TeV}$ with $y=-0.68$ and $p_{\mathrm{T}}^{\text {jet }}=1.2 \mathrm{TeV}$ with $y=0.64$, respectively. The two leading jets have an invariant mass of approximately $3.1 \mathrm{TeV}$. The missing transverse energy in the event is $46 \mathrm{GeV}$. The lines in the in- ner detector indicate the reconstructed particle trajectories. The energy deposition in the calorimeter cells are displayed as light rectangles. The area of the rectangles is proportional to the energy deposits. The histograms attached to the LAr and the Tile calorimeter illustrate the amount of deposited energy

energy of the corresponding truth jet entering the ATLAS detector.

The jet calibration corrects for the following detector effects that affect the jet energy measurement:

1. Calorimeter non-compensation: partial measurement of the energy deposited by hadrons.

2. Dead material: energy losses in inactive regions of the detector.

3. Leakage: energy of particles reaching outside the calorimeters.

4. Out of calorimeter jet cone: energy deposits of particles inside the truth jet entering the detector that are not included in the reconstructed jet.

5. Noise thresholds and particle reconstruction efficiency: signal losses in the calorimeter clustering and jet reconstruction.

Jets reconstructed in the calorimeter system are formed from calorimeter energy depositions reconstructed at the electromagnetic energy scale (EM) or from energy depositions that are corrected for the lower detector response to hadrons. The EM scale correctly reconstructs the energy deposited by particles in an electromagnetic shower in the calorimeter. This energy scale is established using test-beam measurements for electrons in the barrel [31-35], the endcap [36, 37] and the FCAL [38, 39] calorimeters. The abjet energy measured with the ATLAS calorimeter to the true 


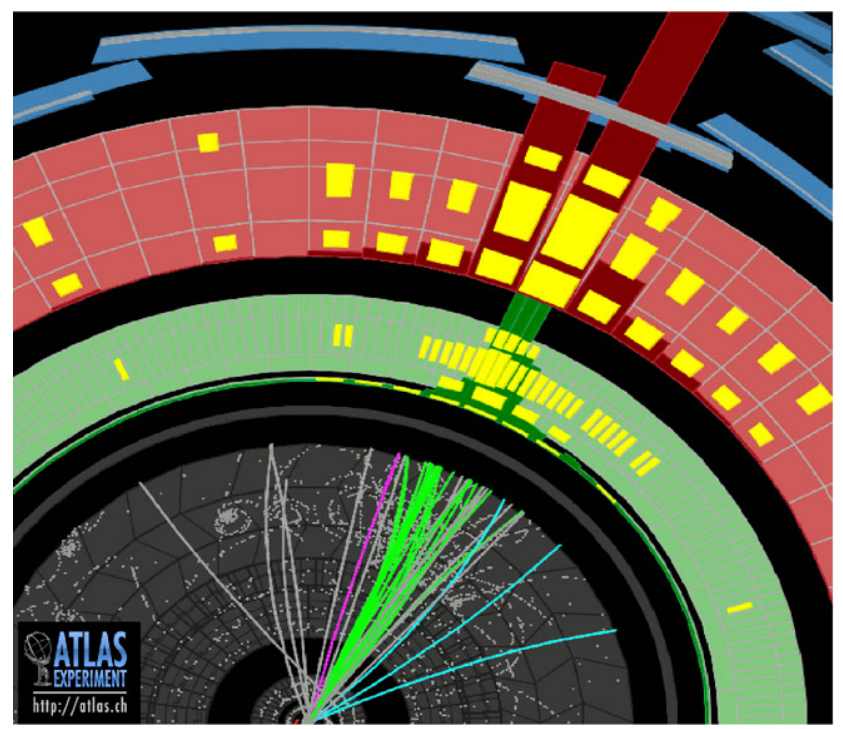

Fig. 2 Zoom of the $x-y$ view of the ATLAS detector showing one of the high- $p_{\mathrm{T}}$ jets of the event shown in Fig. 1. The energy depositions in the calorimeter cells are displayed as light rectangles. The area of the rectangles is proportional to the energy deposits. The dark histograms attached to the LAr ( $\mathrm{Tile}$ ) calorimeter illustrates the amount of deposited energy. The lines in the ID display the reconstructed tracks originating from the interaction vertex

solute calorimeter response to energy deposited via electromagnetic processes was validated in the hadronic calorimeters using muons, both from test-beams $[35,40]$ and produced in situ by cosmic rays [41]. The energy scale of the electromagnetic calorimeters is corrected using the invariant mass of $Z$ bosons produced in proton-proton collisions ( $Z \rightarrow e^{+} e^{-}$events) [42]. The correction for the lower response to hadrons is solely based on the topology of the energy depositions observed in the calorimeter.

In the simplest case, called EM+JES calibration scheme, the jet energy is measured on EM scale and the jet calibration is derived as a simple correction relating the calorimeter response to the true jet energy, as follows:

$$
\begin{aligned}
& E_{\text {calib }}^{\text {jet }}=E_{\text {meas }}^{\text {jet }} / \mathcal{F}_{\text {calib }}\left(E_{\text {meas }}^{\text {jet }}\right), \\
& \quad \text { with } E_{\text {meas }}^{\text {jet }}=E_{\mathrm{EM}}^{\text {jet }}-\mathcal{O}\left(N_{\mathrm{PV}}\right) .
\end{aligned}
$$

The variable $E_{\mathrm{EM}}^{\mathrm{jet}}$ is the calorimeter energy measured at the electromagnetic scale, $E_{\text {calib }}^{\text {jet }}$ is the calibrated jet energy and $\mathcal{F}_{\text {calib }}$ is the calibration function that depends on the measured jet energy and is evaluated in small jet pseudorapidity regions. The variable $\mathcal{O}\left(N_{\mathrm{PV}}\right)$ denotes the correction for additional energy from multiple proton-proton interactions depending on the number of primary vertices $\left(N_{\mathrm{PV}}\right)$.

The simplest calibration scheme applies the JES corrections to jets reconstructed at the electromagnetic scale. This calibration scheme allows a simple evaluation of the systematic uncertainty from single hadron response measurements and systematic Monte Carlo variations. This can be achieved with small data sets and is therefore suitable for early physics analyses.

Other calibration schemes use additional cluster-bycluster and/or jet-by-jet information to reduce some of the sources of fluctuations in the jet energy response, thereby improving the jet energy resolution [26, 27]. For these calibration schemes the same jet calibration procedure is applied as for the EM+JES calibration scheme, but the energy corrections are numerically smaller.

The global calorimeter cell weighting (GCW) calibration exploits the observation that electromagnetic showers in the calorimeter leave more compact energy depositions than hadronic showers with the same energy. Energy corrections are derived for each calorimeter cell within a jet, with the constraint that the jet energy resolution is minimised. These cell corrections account for all energy losses of a jet in the ATLAS detector. Since these corrections are only applicable to jets and not to energy depositions in general, they are called "global" corrections.

The local cluster weighting (LCW) calibration method first clusters together topologically connected calorimeter cells and classifies these clusters as either electromagnetic or hadronic. Based on this classification energy corrections are derived from single pion Monte Carlo simulations. Dedicated corrections are derived for the effects of noncompensation, signal losses due to noise threshold effects, and energy lost in non-instrumented regions. They are applied to calorimeter clusters and are defined without reference to a jet definition. They are therefore called "local" corrections. Jets are then built from these calibrated clusters using a jet algorithm.

The final jet energy calibration (see Eq. (1)) can be applied to EM scale jets, with the resulting calibrated jets referred to as EM+JES, or to GCW and LCW calibrated jets, with the resulting jets referred to as GCW+JES and LCW+JES jets. The jet energy scale (JES) is different for each calibration scheme.

A further jet calibration scheme, called global sequential (GS) calibration, starts from jets calibrated with the EM+JES calibration and exploits the topology of the energy deposits in the calorimeter to characterise fluctuations in the jet particle content of the hadronic shower development. Correcting for such fluctuations can improve the jet energy resolution [27]. The corrections are applied such that the mean jet energy in the inclusive case is left unchanged. The correction uses several jet properties and each correction is applied sequentially. In particular, the longitudinal and transverse structure of the hadronic shower in the calorimeter is exploited.

The simple EM+JES jet calibration scheme does not provide the best performance, but allows in the central detector region the most direct evaluation of the systematic uncertainties from the calorimeter response to single isolated 
hadron measured in situ and in test-beams and from systematic variations of the Monte Carlo simulation. For the GS calibration scheme the systematic uncertainty is obtained by studying the response after applying the GS calibration with respect to the EM+JES calibration. For the GCW+JES and LCW+JES calibration schemes the JES uncertainty is determined from in situ techniques.

For all calibration schemes the JES uncertainty in the forward detector regions is derived from the uncertainty in the central region using the transverse momentum balance in events where only two jets are produced.

In the following, the calibrated calorimeter jet transverse momentum will be denoted as $p_{\mathrm{T}}^{\text {jet }}$, and the jet pseudorapidity as $\eta$.

\section{Monte Carlo simulation}

\subsection{Event generators}

The energy and direction of particles produced in protonproton collisions are simulated using various event generators. An overview of Monte Carlo event generators for LHC physics can be found in Ref. [43]. The samples using different event generators and theoretical models used are described below:

1. Pythia with the MC10 or AMBT1 tune: The event generator PYTHIA [44] simulates non-diffractive protonproton collisions using a $2 \rightarrow 2$ matrix element at leading order in the strong coupling to model the hard subprocess, and uses $p_{\mathrm{T}}$-ordered parton showers to model additional radiation in the leading-logarithmic approximation [45]. Multiple parton interactions [46], as well as fragmentation and hadronisation based on the Lund string model [47] are also simulated. The proton parton distribution function (PDF) set used is the modified leadingorder PDF set MRST LO* [48]. The parameters used for tuning multiple parton interactions include charged particle spectra measured by ATLAS in minimum bias collisions [49], and are denoted as the ATLAS MC10 tune [50].

2. The Perugia2010 tune is an independent tune of PYTHIA with increased final state radiation to better reproduce the jet shapes and hadronic event shapes using LEP and TEVATRON data [51]. In addition, parameters sensitive to the production of particles with strangeness and related to jet fragmentation have been adjusted.

3. HERWIG+JimmY uses a leading order $2 \rightarrow 2$ matrix element supplemented with angular-ordered parton showers in the leading-logarithm approximation [52-54]. The cluster model is used for the hadronisation [55]. Multiple parton interactions are modelled using JIMMY [56]. The model parameters of HERWIG/JIMMY have been tuned to
ATLAS data (AUET1 tune) [57]. The MRST LO* PDF set [48] is used.

4. HeRwig++ [58] is based on the event generator HeRWIG, but redesigned in the $\mathrm{C}++$ programming language. The generator contains a few modelling improvements. It also uses angular-ordered parton showers, but with an updated evolution variable and a better phase space treatment. Hadronisation is performed using the cluster model. The underlying event and soft inclusive interactions are described using a hard and soft multiple partonic interactions model [59]. The MRST LO* PDF set [48] is used.

5. ALPGEN is a tree level matrix-element generator for hard multi-parton processes $(2 \rightarrow n)$ in hadronic collisions [60]. It is interfaced to HERWIG to produce parton showers in the leading-logarithmic approximation. Parton showers are matched to the matrix element with the MLM matching scheme [61]. For the hadronisation, HERWIG is used and soft multiple parton interactions are modelled using JIMMY [56] (with the ATLAS MC09 tune [62]). The PDF set used is CTEQ6L1 [63].

\subsection{Simulation of the ATLAS detector}

The GEANT4 software toolkit [64] within the ATLAS simulation framework [65] propagates the generated particles through the ATLAS detector and simulates their interactions with the detector material. The energy deposited by particles in the active detector material is converted into detector signals with the same format as the ATLAS detector read-out. The simulated detector signals are in turn reconstructed with the same reconstruction software as used for the data.

In GEANT4 the model for the interaction of hadrons with the detector material can be specified for various particle types and for various energy ranges. For the simulation of hadronic interactions in the detector, the GEANT4 set of processes called QGSP_BERT is chosen [66]. In this set of processes, the Quark Gluon String model [67-71] is used for the fragmentation of the nucleus, and the Bertini cascade model [72-75] for the description of the interactions of hadrons in the nuclear medium.

The GEANT4 simulation and in particular the hadronic interaction model for pions and protons, has been validated with test-beam measurements for the barrel [35, 76-79] and endcap [36, 37, 80] calorimeters. Agreement within a few percent is found between simulation and data of the average calorimeter response to pions with momenta between $2 \mathrm{GeV}$ and $350 \mathrm{GeV}$.

Further tests have been carried out in situ comparing the single hadron response, measured using isolated tracks and identified single particles. Agreement within a few percent is found for the inclusive measurement and for identified pions and protons from the decay products of kaon and lambda 
particles produced in proton-proton collisions at $7 \mathrm{TeV}$ [28]. With this method particle momenta of pions and protons in the range from a few hundred $\mathrm{MeV}$ to $6 \mathrm{GeV}$ can be reached. Good agreement between Monte Carlo simulation and data is found.

\subsection{Nominal Monte Carlo simulation samples}

The baseline (nominal) Monte Carlo sample used to derive the jet energy scale and to estimate the sources of its systematic uncertainty is a sample containing high- $p_{\mathrm{T}}$ jets produced via strong interactions. It is generated with the PYTHIA event generator with the MC10 tune (see Sect. 4.1), passed through the full ATLAS detector simulation and is reconstructed as the data.

The ATLAS detector geometry used in the simulation of the nominal sample reflects the geometry of the detector as best known at the time of these studies. Studies of the material of the inner detector in front of the calorimeters have been performed using secondary hadronic interactions [81]. Additional information is obtained from studying photon conversions [82] and the energy flow in minimum bias events [83].

\subsection{Simulated pile-up samples}

For the study of multiple proton-proton interactions, two samples have been used, one for in-time and one for outof-time pile-up. The first simulates additional proton-proton interactions per bunch crossing, while the second one also contains pile-up arising from bunches before or after the bunch where the event of interest was triggered (for more details see Sect. 5 and Sect. 8.1). The bunch configuration of LHC (organised in bunch trains) is also simulated. The additional number of primary vertices in the in-time (bunchtrain) pile-up sample is 1.7 (1.9) on average.

\section{Data sample and event selection}

\subsection{Data taking period and LHC conditions}

Proton-proton collisions at a centre-of-mass energy of $\sqrt{s}=$ $7 \mathrm{TeV}$, recorded from March to October 2010 are analysed. Only data with a fully functioning calorimeter and inner detector are used. The data set corresponds to an integrated luminosity of $38 \mathrm{pb}^{-1}$. Due to different data quality requirements the integrated luminosity can differ for the various selections used in the in situ technique analyses.

Several distinct periods of machine configuration and detector operation were present during the 2010 data taking. As the LHC commissioning progressed, changes in the beam optics and proton bunch parameters resulted in

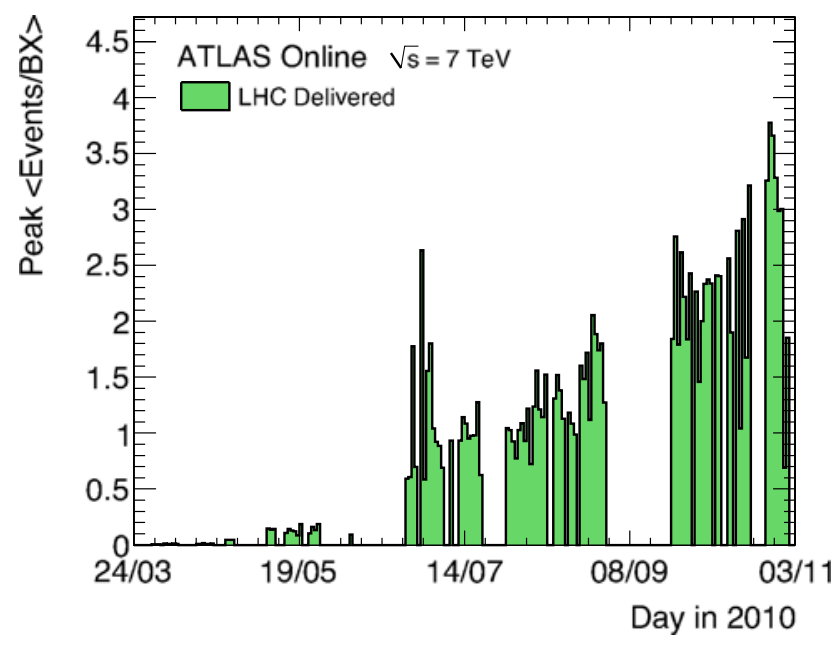

Fig. 3 The peak number of interactions per bunch crossing ("BX") as measured online by the ATLAS luminosity detectors [84]

changes in the number of pile-up interactions per bunch crossing. The spacing between the bunches was no less than $150 \mathrm{~ns}$.

Figure 3 shows the evolution of the maximum of the distribution of the number of interactions (peak) derived from the online luminosity measurement and assuming an inelastic proton-proton scattering cross section of $71.5 \mathrm{mb}$ [84].

The very first data were essentially devoid of multiple proton-proton interactions until the optics of the accelerator beam (specifically $\beta^{*}$ ) were changed in order to decrease the transverse size of the beam and increase the luminosity. ${ }^{3}$ This change alone raised the fraction of events with at least two observed interactions from less than $2 \%$ to between $8 \%$ and $10 \%$ (May-June 2010).

A further increase in the number of interactions occurred when the number of protons per bunch (ppb) was increased from approximately $5-9 \cdot 10^{10}$ to $1.15 \cdot 10^{11} \mathrm{ppb}$. Since the number of proton-proton collisions per bunch crossing is proportional to the square of the bunch intensity, the fraction of events with pile-up increased to more than $50 \%$ for runs between June and September 2010.

Finally, further increasing the beam intensity slowly raised the average number of interactions per bunch crossing to more than three by the end of the proton-proton run in November 2010.

\subsection{Event selection}

Different triggers are used to select the data samples, in order to be maximally efficient over the entire jet $p_{\mathrm{T}}$-range

\footnotetext{
${ }^{3}$ The parameter $\beta^{*}$ is the value of the $\beta$-function (the envelope of all trajectories of the beam particles) at the collision point and smaller values of $\beta^{*}$ imply a smaller physical size of the beams and thus a higher instantaneous luminosity.
} 
of interest. The dijet sample is selected using the hardwarebased calorimeter jet triggers [30,85], which are fully efficient for jets with $p_{\mathrm{T}}^{\text {jet }}>60 \mathrm{GeV}$. For lower $p_{\mathrm{T}}^{\text {jet }}$ a trigger based on the minimum bias trigger scintillators is used.

The multijet sample uses either the inclusive jet trigger or a trigger that requires at least two, three or more jets with $p_{\mathrm{T}}>10 \mathrm{GeV}$ at the EM scale. These triggers are fully efficient for jets with $p_{\mathrm{T}}^{\text {jet }}>80 \mathrm{GeV}$.

Each event is required to have a primary hard scattering vertex. A primary vertex is required to have at least five tracks $\left(N_{\mathrm{pp}}^{\text {tracks }}\right)$ with a transverse momentum of $p_{\mathrm{T}}^{\text {track }}>$ $150 \mathrm{MeV}$. The primary vertex associated to the event of interest (hard scattering vertex) is the one with the highest associated transverse track momentum squared, $\sum\left(p_{\mathrm{T}}^{\text {track }}\right)^{2}$, used in the vertex fit where the sum runs over all tracks used in the vertex fit. This renders the contribution from fake vertices due to beam backgrounds to be negligible.

The $\gamma$-jet sample is selected using a photon trigger [30] that is fully efficient for photons passing offline selections. The higher threshold for the photon $p_{\mathrm{T}}$ is $40 \mathrm{GeV}$ and this trigger was not pre-scaled; the lower threshold is $20 \mathrm{GeV}$ and this trigger was pre-scaled at high luminosity.

\subsection{Data quality assessment}

The ATLAS data quality (DQ) selection is based upon inspection of a standard set of distributions that leads to a data quality assessment for each subdetector, usually segmented into barrel, forward and endcap regions, as well as for the trigger and for each type of reconstructed physics object (jets, electrons, muons, etc.). Each subsystem sets its own DQ flags, which are recorded in a conditions database. Each analysis applies DQ selection criteria, and defines a set of luminosity blocks (each corresponds to approximately two minutes of data taking). The good luminosity blocks used are those not flagged for having issues affecting a relevant subdetector.

Events with minimum bias and calorimeter triggers were required to belong to specific runs and run periods in which the detector, trigger and reconstructed physics objects have passed a data quality assessment and are deemed suitable for physics analysis.

The primary systems of interest for this study are the electromagnetic and hadronic calorimeters, and the inner tracking detector for studies of the properties of tracks associated with jets.

\section{Jet reconstruction}

In data and Monte Carlo simulation jets are reconstructed using the anti- $k_{t}$ algorithm [25] with distance parameters $R=0.4$ or $R=0.6$ using the FASTJET software [86, 87].
The four-momentum recombination scheme is used. For jet finding rapidity $y$ is used, while jet corrections and performance studies use often pseudorapidity $\eta$. The jet $p_{\mathrm{T}}$ reconstruction threshold is $p_{\mathrm{T}}^{\text {jet }}>7 \mathrm{GeV}$.

In the following, only anti- $k_{t}$ jets with distance parameter $R=0.6$ are discussed in detail. The results for jets with $R=$ 0.4 are similar, if not stated otherwise.

\subsection{Reconstructed calorimeter jets}

The input to calorimeter jets can be topological calorimeter clusters (topo-clusters) [37, 88] or calorimeter towers. Only topo-clusters or towers with a positive energy are considered as input to jet finding.

\subsubsection{Topological calorimeter clusters}

Topological clusters are groups of calorimeter cells that are designed to follow the shower development taking advantage of the fine segmentation of the ATLAS calorimeters. The topo-cluster formation algorithm starts from a seed cell, whose signal-to-noise $(S / N)$ ratio (estimated as the absolute value of the energy deposited in the calorimeter cell over the RMS of the energy distribution measured in randomly triggered events without proton-proton collisions) is above a threshold of $S / N=4$. Cells neighbouring the seed (or the cluster being formed) that have a signal-to-noise ratio of at least $S / N=2$ are included iteratively. Finally, all calorimeter cells neighbouring the formed topo-cluster are added. The topo-cluster algorithm efficiently suppresses the calorimeter noise.

The topo-cluster algorithm also includes a splitting step in order to optimise the separation of showers from different close-by particles: All cells in a topo-cluster are searched for local maxima in terms of energy content with a threshold of $500 \mathrm{MeV}$. This means that the selected calorimeter cell has to be more energetic than any of its neighbours. The local maxima are then used as seeds for a new iteration of topological clustering, which splits the original cluster into more topo-clusters.

A topo-cluster is defined to have an energy equal to the energy sum of all the included calorimeter cells, zero mass and a reconstructed direction calculated from the weighted averages of the pseudorapidities and azimuthal angles of the constituent cells. The weight used is the absolute cell energy and the positions of the cells are relative to the nominal ATLAS coordinate system.

\subsubsection{Calorimeter towers}

Calorimeter towers are static, $\Delta \eta \times \Delta \phi=0.1 \times 0.1$, grid elements built directly from calorimeter cells. ${ }^{4}$

${ }^{4}$ For the few calorimeter cells that are larger than the $\Delta \eta \times \Delta \phi=0.1 \times$ 0.1 (like in the last Tile calorimeter layer and the HEC inner wheel) 
ATLAS uses two types of calorimeter towers: with and without noise suppression. Calorimeter towers based on all calorimeter cells are called non-noise-suppressed calorimeter towers in the following. Noise-suppressed towers make use of the topo-clusters algorithm, i.e. only calorimeter cells that are included in topo-clusters are used. Therefore, in a fixed geometrical area the same calorimeter cells are used for noise-suppressed towers and topo-clusters.

Both types of calorimeter towers have an energy equal to the energy sum of all included calorimeter cells. The formed Lorentz four-momentum has zero mass.

\subsection{Reconstructed track jets}

Jets built from charged particle tracks originating from the primary hard scattering vertex (track jets) are used to define jets that are insensitive to the effects of pile-up and provide a stable reference to study close-by jet effects.

Tracks with $p_{\mathrm{T}}^{\text {track }}>0.5 \mathrm{GeV}$ and $|\eta|<2.5$ are selected. They are required to have at least one (six) hit(s) in the Pixel (SCT) detector. The transverse $\left(d_{0}\right)$ and longitudinal $\left(z_{0}\right)$ impact parameters of the tracks measured with respect to the primary vertex are also required to be $\left|d_{0}\right|<1.5 \mathrm{~mm}$ and $\left|z_{0} \sin \theta\right|<1.5 \mathrm{~mm}$, respectively.

The track jets must have at least two constituent tracks and a total transverse momentum of $p_{\mathrm{T}}^{\text {track jet }}>3 \mathrm{GeV}$. Since the tracking system has a coverage up to $|\eta|=2.5$, the performance studies of calorimeter jets is carried out in the range $|\eta|<1.9$ for $R=0.6$ and $|\eta|<2.1$ for $R=0.4$.

\subsection{Monte Carlo truth jets and flavour association}

In the Monte Carlo simulation truth jets are defined from stable particles defined to have proper lifetimes longer than 10 ps excluding muons and neutrinos.

For certain studies, jets in the Monte Carlo simulation are additionally identified as jets initiated by light or heavy quarks or by gluons based on the generator event record. The highest energy parton that points to the truth jet ${ }^{5}$ determines the flavour of the jet. Using this method, only a small fraction of the jets $\left(<1 \%\right.$ at low $p_{\mathrm{T}}$ and less at high $\left.p_{\mathrm{T}}\right)$ could not be assigned a partonic flavour. ${ }^{6}$ This definition is sufficient to study the flavour dependence of the jet response. Any theoretical ambiguities of jet flavour assignment do not need to be addressed in the context of a performance study.

or have a special geometry (like in the FCAL), projective tower grid geometrical weights are defined that specify the fraction of calorimeter cell energy to be attributed to a particular calorimeter tower.

${ }^{5}$ With $\Delta R<0.6$ for jets with $R=0.6$ and $\Delta R<0.4$ for jets with $R=0.4$, where $\Delta R=\sqrt{(\Delta \eta)^{2}+(\Delta \phi)^{2}}$.

${ }^{6}$ This happens when there is no parton at a distance smaller than $\Delta R$ to the jet axis.

\section{Jet quality}

Jets at high transverse momenta produced in proton-proton collisions must be distinguished from background jets not originating from hard scattering events. The main backgrounds are the following:

1. Beam-gas events, where one proton of the beam collided with the residual gas within the beam pipe.

2. Beam-halo events, for example caused by interactions in the tertiary collimators in the beam-line far away from the ATLAS detector.

3. Cosmic ray muons overlapping in-time with collision events.

4. Large calorimeter noise.

The criteria to efficiently reject jets arising from background are only applied to data. They are discussed in the following sections.

\subsection{Criteria to remove non-collision background}

\subsubsection{Noise in the calorimeters}

Two types of calorimeter noise are addressed:

1. Sporadic noise bursts in the hadronic endcap calorimeter $(\mathrm{HEC})$, where a single noisy calorimeter cell contributes almost all of the jet energy. Jets reconstructed from these problematic cells are characterised by a large energy fraction in the HEC calorimeter $\left(f_{\mathrm{HEC}}\right)$ as well as a large fraction of the energy in calorimeter cells with poor signal shape quality ${ }^{7}\left(f_{\mathrm{HEC} \text { quality }}\right)$. Due to the capacitive coupling between channels, the neighbouring calorimeter cells with little genuine energy will have an apparent negative energy $\left(E_{\text {neg }}\right)$.

2. Rare coherent noise in the electromagnetic calorimeter. Similarly, fake jets arising from this source are characterised by a large electromagnetic energy fraction $\left(f_{\mathrm{EM}}\right),{ }^{8}$ and a large fraction of energy in EM calorimeter cells with poor signal shape quality $\left(f_{\text {quality }}\right)$.

\subsubsection{Cosmic rays or non-collision background}

Cosmic rays or non-collision backgrounds can induce events where the jet candidates are not in-time with the beam collision. A cut on the jet time $\left(t_{\text {jet }}\right)$ is applied to reject these backgrounds. The jet time is reconstructed from the energy deposition in the calorimeter by weighting the reconstructed

\footnotetext{
${ }^{7}$ The signal shape quality is obtained by comparing the measured pulse from the calorimeter cell to the expected pulse shape. See Ref. [38] for more details.

${ }^{8}$ The EM fraction is defined as the ratio of the energy deposited in the EM calorimeter to the total energy.
} 
Table 1 Selection criteria used to reject fake jets and non-collision background

\begin{tabular}{lll}
\hline & Loose & Medium \\
\hline $\begin{array}{lll}\text { HEC spikes } \\
\begin{array}{l}\text { Coherent EM } \\
\text { noise }\end{array}\end{array}$ & $f_{\mathrm{EMC}}>0.95$ and $f_{\text {quality }}>0.8$ and $|\eta|<2.8$ & Loose or $f_{\mathrm{HEC}}>1-\left|f_{\mathrm{HECquality}}\right|$ \\
$\begin{array}{l}\text { Non-collision } \\
\text { background }\end{array}$ & $\begin{array}{l}\text { or }\left(t_{\mathrm{EM}} \mid>25 \mathrm{~ns} \text { or }\left(f_{\mathrm{EM}}<0.05 \text { and } f_{\mathrm{ch}}<0.05 \text { and }|\eta|<2\right)\right. \\
\text { Loose or } f_{\mathrm{EM}}>0.9 \text { and } f_{\text {quality }}>0.8 \text { and }|\eta|<2.8\end{array}$ & $\begin{array}{l}\text { Loose or }\left|t_{\text {jet }}\right|>10 \text { ns or }\left(f_{\mathrm{EM}}<0.05 \text { and } f_{\text {ch }}<0.1 \text { and }|\eta|<2\right) \\
\text { or }\left(f_{\mathrm{EM}}>0.95 \text { and } f_{\text {ch }}<0.05 \text { and }|\eta|<2\right)\end{array}$ \\
\hline
\end{tabular}

time of calorimeter cells forming the jet with the square of the cell energy. The calorimeter time is defined with respect to the event time recorded by the trigger.

A cut on the $f_{\mathrm{EM}}$ is applied to make sure that the jet has some energy deposited in the calorimeter layer closest to the interaction region as expected for a jet originating from the nominal interaction point.

Since a real jet is expected to have tracks, the $f_{\mathrm{EM}}$ cut is applied together with a cut on the minimal jet charged fraction $\left(f_{\text {ch }}\right)$, defined as the ratio of the scalar sum of the $p_{\mathrm{T}}$ of the tracks associated to the jet divided by the jet $p_{\mathrm{T}}$, for jets within the tracking acceptance.

A cut on the maximum energy fraction in any single calorimeter layer $\left(f_{\max }\right)$ is applied to further reject noncollision background.

\subsubsection{Jet quality selections}

Two quality selections are provided:

1. A loose selection is designed with an efficiency above $99 \%$, that can be used in most of the ATLAS physics analyses.

2. A medium selection is designed for analyses that select jets at high transverse momentum, such as for jet crosssection measurements [1].

A tight quality selection has been developed for the measurement of the jet quality selection efficiency described in Sect. 7.2, but is not used in physics analyses, since the medium jet quality selection is sufficient for removing fake jets. The quality selection criteria used to identify and reject fake jets are listed in Table 1.

\subsection{Evaluation of the jet quality selection efficiency}

The criteria for the jet quality selection are optimised by studying samples with good and fake jets classified by their amount of missing transverse momentum significance: ${ }^{9}$

\footnotetext{
${ }^{9}$ The missing transverse momentum $\left(E_{\mathrm{T}}^{\mathrm{miss}}\right)$ significance is defined as $E_{\mathrm{T}}^{\text {miss }} / \sqrt{\sum E_{\mathrm{T}}}$, where $\sum E_{\mathrm{T}}$ is the scalar sum of the transverse energies of all energy deposits in the calorimeter.
}

1. Good jets belong to events where the two leading jets have $p_{\mathrm{T}}^{\text {jet }}>20 \mathrm{GeV}$, and are back-to-back $\left(\Delta \phi_{\mathrm{j}-\mathrm{j}}>\right.$ 2.6 radian) in the plane transverse to the beam, and with a small missing transverse momentum significance $E_{\mathrm{T}}^{\text {miss }} / \sqrt{\sum E_{\mathrm{T}}}<1$.

2. Fake jets belong to events with a high transverse momentum significance $E_{\mathrm{T}}^{\mathrm{miss}} / \sqrt{\sum E_{\mathrm{T}}}>3$ and with a reconstructed jet back-to-back to the missing transverse momentum direction $\left(\Delta \phi_{E_{\mathrm{T}}^{\text {miss }}-\mathrm{j}}>2.6\right.$ radian $)$.

The good jets sample is used to study the jet selection inefficiency and the bad jet sample is used to study the rejection power.

As the jet quality selection criteria are only applied to data an efficiency correction for data is determined. This efficiency is measured using a tag-and-probe method in events with two jets at high transverse momentum. The reference jet $\left(p_{\mathrm{T}}^{\text {ref }}\right)$ is required to pass the tightened version of the jet quality selections, and to be back-to-back $\left(\Delta \phi_{E_{\mathrm{T}}^{\text {miss }}-\mathrm{j}}>\right.$ 2.6 radian) and well-balanced with the probe jet $\left(p_{\mathrm{T}}^{\text {probe }}\right)$ :

$$
\begin{gathered}
\left(\left|p_{\mathrm{T}}^{\text {probe }}-p_{\mathrm{T}}^{\text {ref }}\right| / p_{\mathrm{T}}^{\text {avg }}<0.4\right), \\
\text { with } p_{\mathrm{T}}^{\text {avg }}=\left(p_{\mathrm{T}}^{\text {probe }}+p_{\mathrm{T}}^{\text {ref }}\right) / 2 .
\end{gathered}
$$

The jet quality selection criteria were then applied to the probe jets, measuring the fraction of jets passing as a function of $\eta$ and $p_{\mathrm{T}}^{\text {jet }}$.

The resulting efficiencies for jets with $R=0.6$ for loose and medium selections applied to the probe jets are shown in Fig. 4. The tight selection of the reference jet was varied to study the systematic uncertainty. The loose selection criteria are close to $100 \%$ efficient. In the forward region the medium selection criteria are also close to fully efficient. In the central region they have an efficiency of $99 \%$ for $p_{\mathrm{T}}^{\text {jet }}>50 \mathrm{GeV}$. For lower $p_{\mathrm{T}}$ jets of about $25 \mathrm{GeV}$ an inefficiency of up to $3-4 \%$ is observed.

\subsection{Summary of the jet quality selection}

Quality selections used to reject fake jets with the ATLAS detector have been developed. Simple variables allow the removal of fake jets due to sporadic noise in the calorimeter 


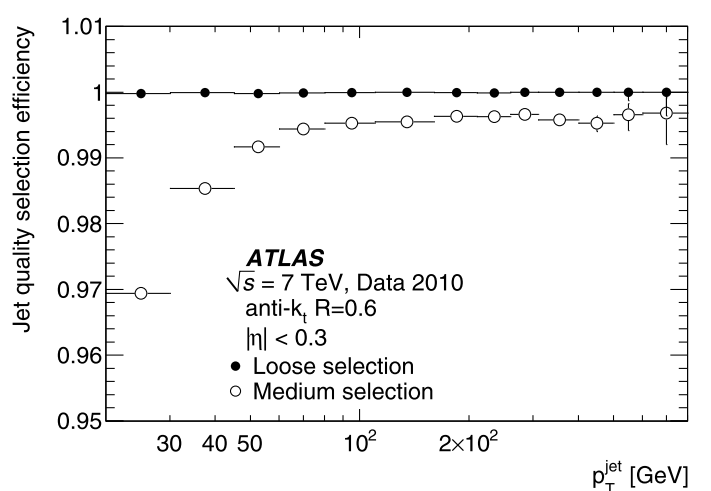

(a) $|\eta|<0.3$

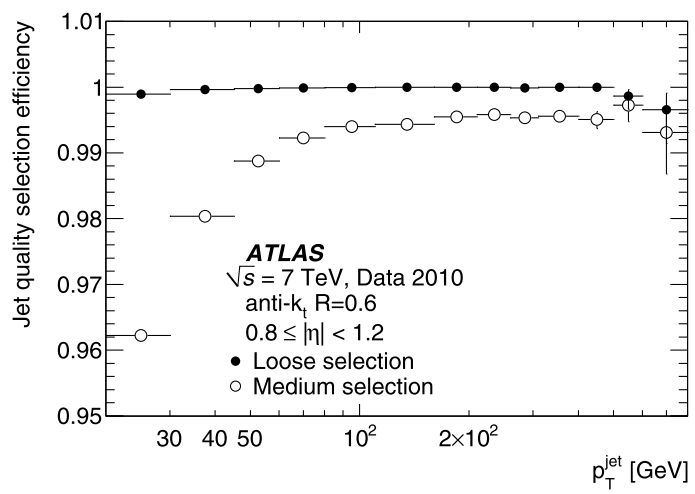

(c) $0.8 \leq|\eta|<1.2$

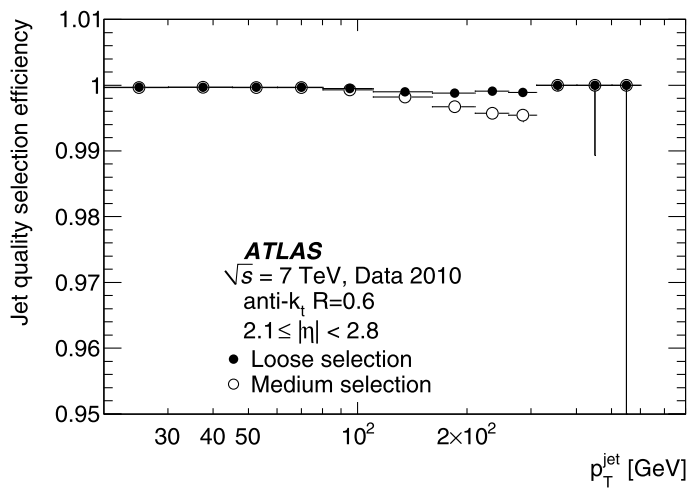

(e) $2.1 \leq|\eta|<2.8$

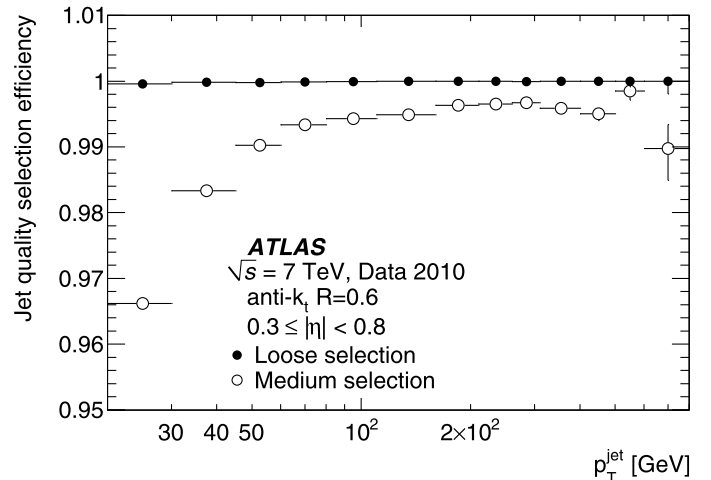

(b) $0.3 \leq|\eta|<0.8$

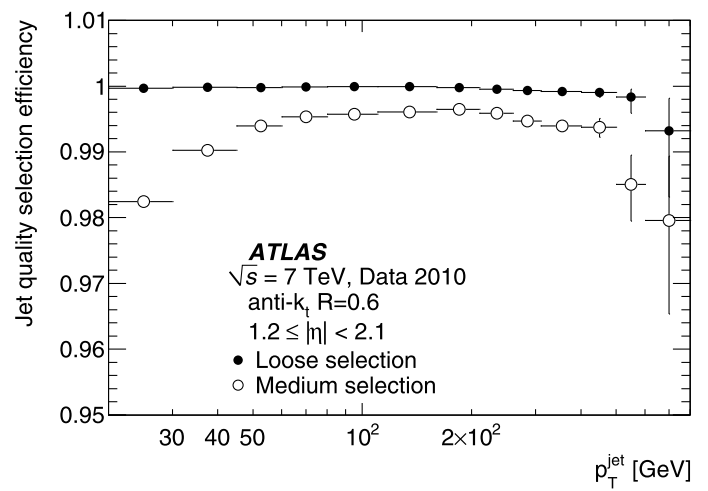

(d) $1.2 \leq|\eta|<2.1$

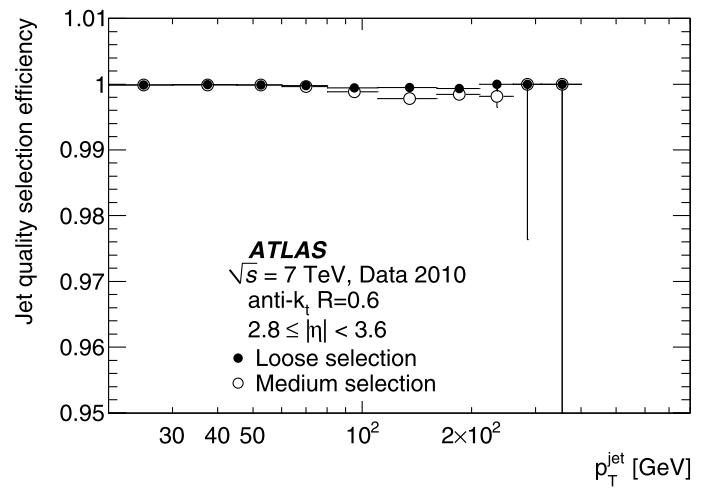

(f) $2.8 \leq|\eta|<3.6$

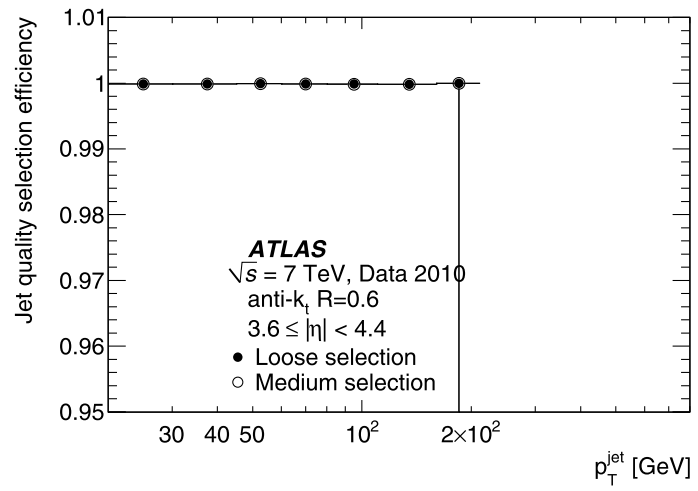

(g) $3.6 \leq|\eta|<4.4$

Fig. 4 Jet quality selection efficiency for anti- $k_{t}$ jets with $R=0.6$ measured with a tag-and-probe technique as a function of $p_{\mathrm{T}}^{\text {jet }}$ in bins of $\eta$, for loose and medium selection criteria (see Table 1). Only sta- tistical uncertainties are shown. In (e), (f), (g) the loose and medium results overlap 
or non-collision background at the analysis level, with an efficiency greater than $99 \%$ over a wide kinematic range.

\section{Jet energy calibration in the EM+JES scheme}

The simple EM+JES calibration scheme applies corrections as a function of the jet energy and pseudorapidity to jets reconstructed at the electromagnetic scale.

The additional energy due to multiple proton-proton collisions within the same bunch crossing (pile-up) is corrected before the hadronic energy scale is restored, such that the derivation of the jet energy scale calibration is factorised and does not depend on the number of additional interactions measured.

The EM+JES calibration scheme consists of three subsequent steps as outlined below and detailed in the following subsections:

1. Pile-up correction: The average additional energy due to additional proton-proton interactions is subtracted from the energy measured in the calorimeters using correction constants obtained from in situ measurements.

2. Vertex correction: The direction of the jet is corrected such that the jet originates from the primary vertex of the interaction instead of the geometrical centre of the detector.

3. Jet energy and direction correction: The jet energy and direction as reconstructed in the calorimeters are corrected using constants derived from the comparison of the kinematic observables of reconstructed jets and those from truth jets in Monte Carlo simulation.

\subsection{Pile-up correction}

\subsubsection{Correction strategy}

The measured energy of reconstructed jets can be affected by contributions that do not originate from the hard scattering event of interest, but are instead produced by additional proton-proton collisions. An offset correction for pile-up is derived from minimum bias data as a function of the number of reconstructed primary vertices, $N_{\mathrm{PV}}$, the jet pseudorapidity, $\eta$, and the bunch spacing.

This offset correction applied to the jet transverse energy $\left(E_{\mathrm{T}}\right)$ at the EM scale as the first step of jet calibration can be written generically as:

$E_{\mathrm{T}}^{\text {corrected }}=E_{\mathrm{T}}^{\text {uncorrected }}-\mathcal{O}\left(\eta, N_{\mathrm{PV}}, \tau_{\text {bunch }}\right)$,

where $\mathcal{O}\left(\eta, N_{\mathrm{PV}}, \tau_{\text {bunch }}\right)$ corrects for the jet offset due to pile-up.

Due to the varying underlying particle spectrum and the variation in the calorimeter geometry the jet offset is derived as a function of the jet pseudorapidity. The amount of intime pile-up is parametrised by $N_{\mathrm{PV}}$. The spacing between consecutive bunches, $\tau_{\text {bunch }}$, is considered, because it can impact the amount by which collisions in previous bunch crossings affect the jet energy measurement. ${ }^{10}$

The jet offset correction is proportional to the number of constituent towers in a jet as a measure of the jet area. For jets built directly from dynamically-sized topological clusters, for which no clear geometric definition is available, a model is used that describes the average area of a jet in terms of the equivalent number of constituent towers.

\subsubsection{Constituent tower multiplicity of jets}

The multiplicity of calorimeter towers in jets depends on the internal jet composition and on the presence of pile-up. The average tower multiplicity can be measured in situ.

Figure 5 depicts the distribution of the constituent tower multiplicity for jets based on towers with $p_{\mathrm{T}}^{\text {jet }}>7 \mathrm{GeV}$ as a function of the jet pseudorapidity. The average number of constituent towers is also indicated. This distribution is governed by the change in physical size of calorimeter towers for a constant interval in pseudorapidity, as well as by differences in the noise spectrum for the various calorimeters and sampling regions.

\subsubsection{Pile-up offset for towers and jets}

The calorimeter tower offset at the EM scale is derived by measuring the average tower transverse energy for all towers in events with $N_{\mathrm{PV}}=1,2, \ldots, N$ and comparing directly to events with $N_{\mathrm{PV}}=N_{\mathrm{PV}}^{\mathrm{ref}}=1$ :

$\mathcal{O}_{\text {tower }}\left(\eta, N_{\mathrm{PV}}\right)=\left\langle E_{\mathrm{T}}^{\text {tower }}\left(\eta, N_{\mathrm{PV}}\right)\right\rangle-\left\langle E_{\mathrm{T}}^{\text {tower }}\left(\eta, N_{\mathrm{PV}}^{\text {ref }}\right)\right\rangle$,

where the angled brackets denote a statistical average over all events. The average is computed for events at each primary vertex multiplicity. For this measurement non-noisesuppressed calorimeter towers are used (see Sect. 6.1.2) in order to remain sensitive to low energy depositions that may not rise above noise threshold except inside of a jet. The calorimeter tower offset is shown in Fig. 6a for $1 \leq N_{\mathrm{PV}} \leq 5$.

The tower offset can be extrapolated to an EM scale jet offset using:

$\mathcal{O}_{\text {jet } \mid \text { tower }}\left(\eta, N_{\mathrm{PV}}\right)=\mathcal{O}_{\text {tower }}\left(\eta, N_{\mathrm{PV}}\right) \cdot A^{\text {jet }}$

\footnotetext{
${ }^{10}$ The dependence on $\tau_{\text {bunch }}$ is explicitly allowed for due to the possibility of pile-up contributions from previous proton-proton bunch crossings for closely spaced bunches. This will be an important consideration for the 2011-2012 LHC run as the number of bunches is increased and the spacing between consecutive bunches is reduced.
} 


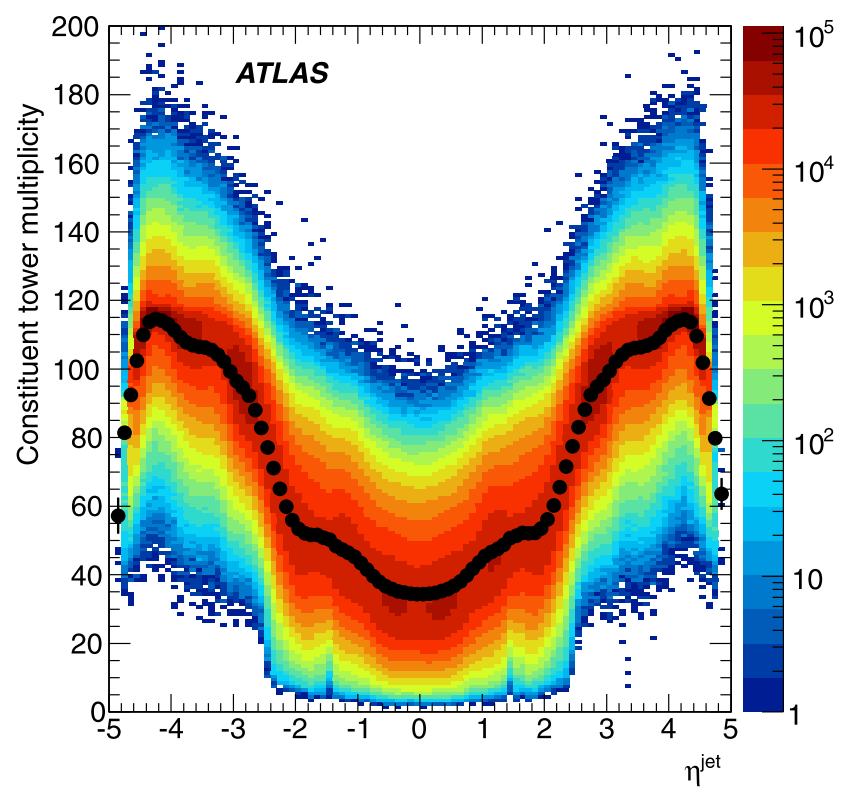

Fig. 5 Distribution of the number constituent calorimeter towers as a function of the jet pseudorapidity for anti- $k_{t}$ jets with $R=0.6$ and $p_{\mathrm{T}}^{\mathrm{jet}}>7 \mathrm{GeV}$. The black dots indicate the average number of tower constituents

where $A^{\text {jet }}$ is the jet area that, for jets built from calorimeter towers, can be estimated from the constituent tower multiplicity, $A^{\text {jet }}=N_{\text {towers }}^{\text {jet }}$. For jets built from topo-clusters, the mean equivalent constituent tower multiplicity $\left(A^{\text {jet }}=\right.$ $\left.\left\langle N_{\text {towers }}^{\text {jet }}\right\rangle\right)$ is used. ${ }^{11}$ The small dependencies of the constituent multiplicity on $p_{\mathrm{T}}^{\text {jet }}$ and $N_{\mathrm{PV}}$ are neglected in the correction, but incorporated as systematic uncertainties (see Sect. 9.7).

The jet offset for jets with $R=0.6$ is shown in Fig. $6 \mathrm{~b}$.

\subsubsection{Track jet based validation and offset correction}

Track jets constructed from charged particles originating from the primary hard-scattering vertex matched to the calorimeter jets provide a stable reference that can be used to measure the variation of the calorimeter $E_{\mathrm{T}}^{\mathrm{jet}}$ as a function of $N_{\mathrm{PV}}$. It is therefore possible to validate the tower-based offset correction and also to directly estimate the pile-up energy contribution to jets.

As this method is only applicable to jets within the inner detector acceptance, it serves primarily as a cross-check for the tower-based method discussed above. It can also be used, however, to derive a dedicated offset correction that can be applied to jets at energy scales other than the electro-

\footnotetext{
${ }^{11}$ The equivalent constituent tower multiplicity for jets based on topoclusters is calculated from the location of the calorimeter cells of the constituent topo-clusters in the jet.
}

magnetic energy scale. Studying the variation of the offset correction as a function of $p_{\mathrm{T}}^{\text {track jet }}$ can establish the systematic uncertainty of the pile-up correction.

The criterion to match a track jet to a calorimeter jet with $R=0.6$ is

$\Delta R($ jet, track jet $)<0.4$,

where $\Delta R=\sqrt{(\Delta \eta)^{2}+(\Delta \phi)^{2}}$. The offset is calculated by measuring the average calorimeter jet $E_{\mathrm{T}}^{\mathrm{jet}}$ as a function of $N_{\mathrm{PV}}$ and the transverse momentum of the matched track jet, $p_{\mathrm{T}}^{\text {track jet }}$ :

$\mathcal{O}_{\text {track jet }}=\left\langle E_{\mathrm{T}}^{\text {jet }}\left(N_{\mathrm{PV}} \mid p_{\mathrm{T}}^{\text {track jet }}\right)\right\rangle-\left\langle E_{\mathrm{T}}^{\mathrm{jet}}\left(N_{\mathrm{PV}}^{\text {ref }} \mid p_{\mathrm{T}}^{\text {track jet }}\right)\right\rangle$.

The reference $N_{\mathrm{PV}}^{\text {ref }}=1$ is used.

Both tower and topo-cluster jets at the EM-scale are used. The most probable value of the calorimeter jet $E_{\mathrm{T}}$ is determined from a fit using a Landau distribution convolved with a Gaussian for each range of $p_{\mathrm{T}}^{\text {track jet }}$. A consistent offset of nearly $\mathcal{O}=0.5 \mathrm{GeV}$ per vertex is found for $|\eta|<1.9$. No systematic trend of the offset as a function of $p_{\mathrm{T}}^{\text {track jet }}$ is observed.

Figure 7 presents the jet-based offset correction as a function of $N_{\mathrm{PV}}$ derived with respect to $N_{\mathrm{PV}}^{\mathrm{ref}}=1$ for tower and topo-cluster based jets using the EM and the EM+JES scale. The magnitude of the offset is higher after EM+JES calibration (see Figs. $7 \mathrm{c}$ and $7 \mathrm{~d}$ ), and the increase corresponds to the average jet energy correction (see Sect. 8.3).

\subsection{Jet origin correction}

Calorimeter jets are reconstructed using the geometrical centre of the ATLAS detector as reference to calculate the direction of jets and their constituents (see Sect. 6). The jet four-momentum is corrected for each event such that the direction of each topo-cluster points back to the primary hard-scattering vertex. The kinematic observables of each topo-cluster are recalculated using the vector from the primary hard-scattering vertex to the topo-cluster centroid as its direction. The raw jet four-momentum is thereafter redefined as the vector sum of the topo-cluster four-momenta. The origin-corrected pseudorapidity is called $\eta_{\text {origin }}$. This correction improves the angular resolution and results in a small improvement $(<1 \%)$ in the jet $p_{\mathrm{T}}$ response. The jet energy is unaffected.

\subsection{Jet energy correction}

The final step of the EM+JES jet calibration restores the reconstructed jet energy to the energy of the Monte Carlo truth jet. Since pile-up effects have already been corrected for, the 


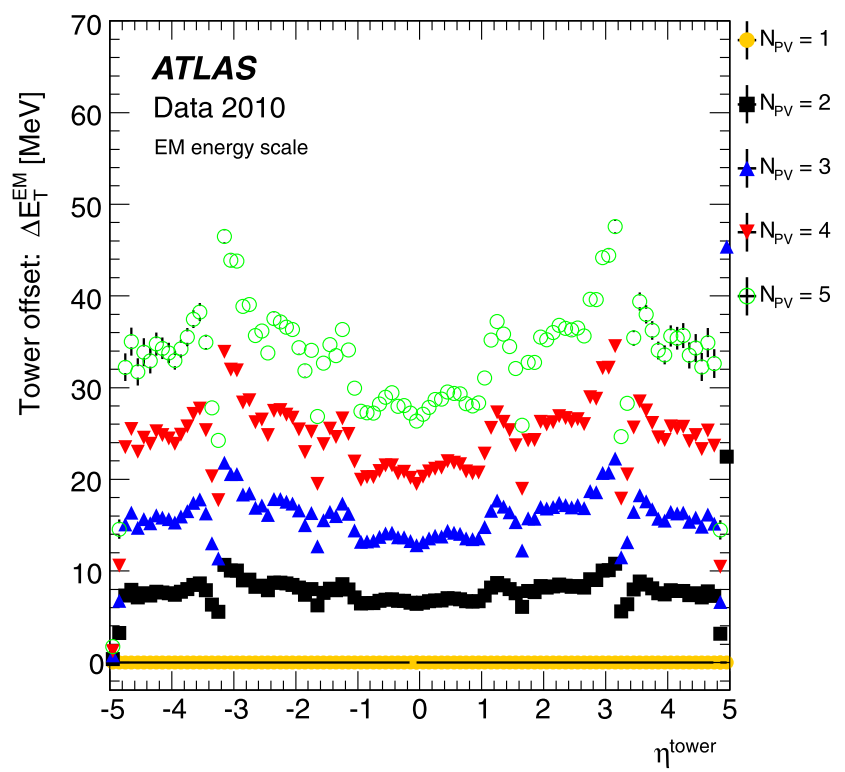

(a) Tower offset

Fig. 6 Tower offset (a) and jet offset (b) at the EM scale as a function of the tower or jet pseudorapidity in bins of the number of reconstructed primary vertices. The jet offset is shown for anti- $k_{t}$ jets with

Monte Carlo samples used to derive the calibration do not include multiple proton-proton interactions.

The calibration is derived using all isolated calorimeter jets that have a matching isolated truth jet within $\Delta R=0.3$. Here, an isolated jet is defined as a jet having no other jet with $p_{\mathrm{T}}^{\text {jet }}>7 \mathrm{GeV}$ within $\Delta R=2.5 R$, where $R$ is the distance parameter of the jet algorithm. A jet is defined to be isolated, if it is isolated with respect to the same jet type, i.e. either a calorimeter or a truth jet.

The final jet energy scale calibration is first parametrised as a function of uncalibrated jet energy and $\eta$. Here the detector pseudorapidity is used rather than the origin-corrected $\eta$ (used by default in physics analyses), since it more directly corresponds to a region of the calorimeter. Energy is used rather than $p_{\mathrm{T}}$, since the calorimeter responds to energy, and as a consequence, the response curves when shown as a function of energy for various $\eta$ regions can be directly compared. The method to derive this calibration is detailed below.

The EM-scale jet energy response

$\mathcal{R}_{\mathrm{EM}}^{\mathrm{jet}}=E_{\mathrm{EM}}^{\mathrm{jet}} / E_{\mathrm{truth}}^{\mathrm{jet}}$

for each pair of calorimeter and truth jets is measured in bins of the truth jet energy $E_{\text {truth }}^{\text {jet }}$ and the calorimeter jet detector pseudorapidity $\eta_{\text {det. }}{ }^{12}$ For each $\left(E_{\text {truth }}^{\text {jet }}, \eta_{\text {det }}\right)$-bin,

\footnotetext{
${ }^{12}$ Here, pseudorapidity refers to the original reconstructed jet before the origin correction.
}

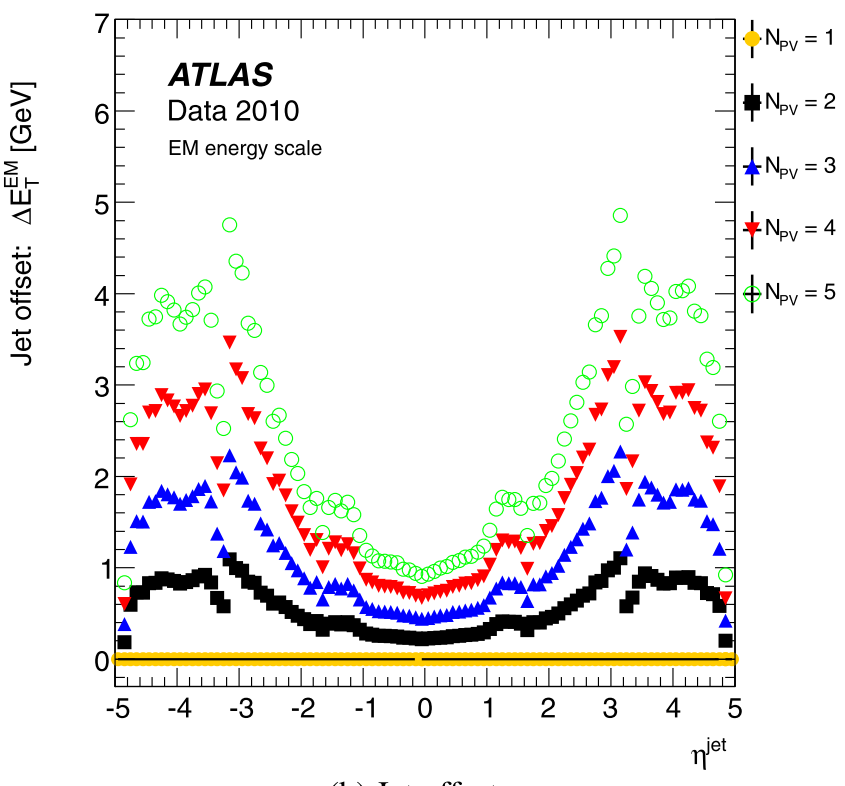

(b) Jet offset

$R=0.6$. Only statistical uncertainties are shown. They are typically smaller than the marker size

the averaged jet response $\left\langle\mathcal{R}_{\mathrm{EM}}^{\text {jet }}\right\rangle$ is defined as the peak position of a Gaussian fit to the $E_{\mathrm{EM}}^{\mathrm{jet}} / E_{\mathrm{truth}}^{\mathrm{jet}}$ distribution. In the same $\left(E_{\text {truth }}^{\text {jet }}, \eta_{\text {det }}\right)$-bin, in addition, the average jet energy $\left(\left\langle E_{\mathrm{EM}}^{\mathrm{jet}}\right\rangle\right)$ is derived from the mean of the $E_{\mathrm{EM}}^{\mathrm{jet}}$ distribution. For a given $\eta_{\text {det }}$-bin $k$, the jet response calibration function $\mathcal{F}_{\text {calib }, k}\left(E_{\mathrm{EM}}^{\mathrm{jet}}\right)$ is obtained using a fit of the $\left(\left\langle E_{\mathrm{EM}}^{\mathrm{jet}}\right\rangle_{j},\left\langle\mathcal{R}_{\mathrm{EM}}^{\mathrm{jet}}\right\rangle_{j}\right)$ values for each $E_{\text {truth }}^{\text {jet }}$-bin $j$.

The fitting function is parameterised as:

$\mathcal{F}_{\text {calib }, k}\left(E_{\text {EM }}^{\text {jet }}\right)=\sum_{i=0}^{N_{\max }} a_{i}\left(\ln E_{\mathrm{EM}}^{\mathrm{jet}}\right)^{i}$,

where $a_{i}$ are free parameters, and $N_{\max }$ is chosen between 1 and 6 depending on the goodness of the fit.

The final jet energy scale correction that relates the measured calorimeter jet energy to the true energy is then defined as $1 / \mathcal{F}_{\text {calib }}\left(E_{\mathrm{EM}}^{\text {calo }}\right)$ in the following:

$E_{\mathrm{EM}+\mathrm{JES}}^{\mathrm{jet}}=\frac{E_{\mathrm{EM}}^{\mathrm{jet}}}{\left.\mathcal{F}_{\text {calib }}\left(E_{\mathrm{EM}}^{\mathrm{jet}}\right)\right|_{\eta_{\text {det }}}}$,

where $\left.\mathcal{F}_{\text {calib }}\left(E_{\mathrm{EM}}^{\mathrm{jet}}\right)\right|_{\eta_{\text {det }}}$ is the jet response calibration function for the relevant $\eta_{\text {det }}$-bin $k$.

The average jet energy scale correction $\left\langle 1 / \mathcal{F}_{\text {calib }, k}\left(E_{\text {calo }}^{\mathrm{EM}}\right)\right\rangle$ is shown as a function of calibrated jet transverse momentum for three jet $\eta$-intervals in Fig. 8. In this and the following figures the correction is only shown over the accessible 


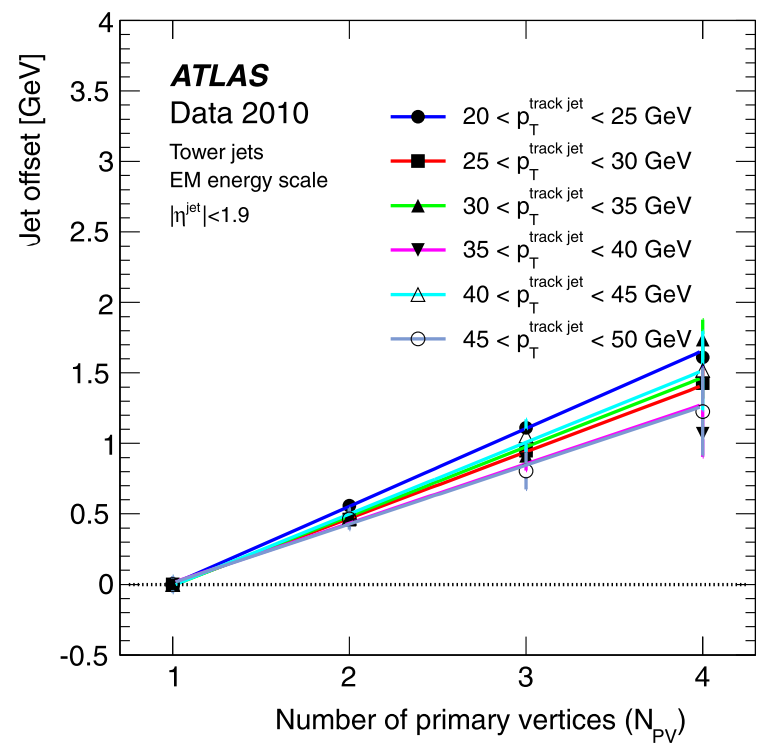

(a) Tower jet offset (EM scale)

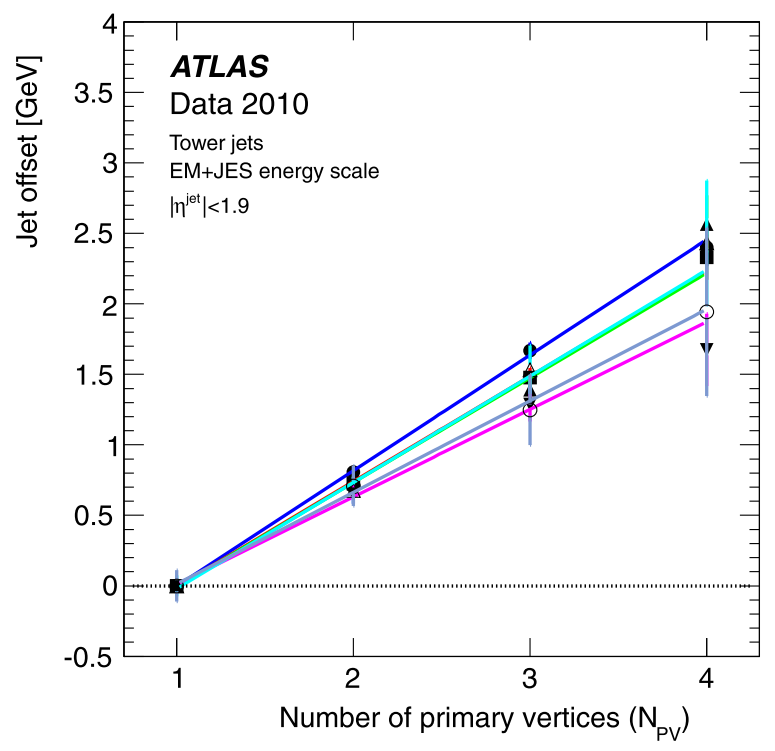

(c) Tower jet offset (EM+JES scale)

Fig. 7 Jet offset as a function of the number of primary vertices for several ranges of $p_{\mathrm{T}}^{\text {track jet }}$ values in bins of track jet $p_{\mathrm{T}}$. The track jet offset is derived for calorimeter tower jets at the EM scale (a), topocluster jets at the EM scale (b), calorimeter tower jets at the EM+JES

kinematic range, i.e. values for jets above the kinematic limit are not shown.

The calorimeter jet response $\mathcal{R}_{\mathrm{EM}}^{\text {jet }}$ is shown for various energy- and $\eta_{\text {det }}$-bins in Fig. 9. The values of the jet energy correction factors range from about 2.1 at low jet energies in the central region to less than 1.2 for high energy jets in the most forward region.

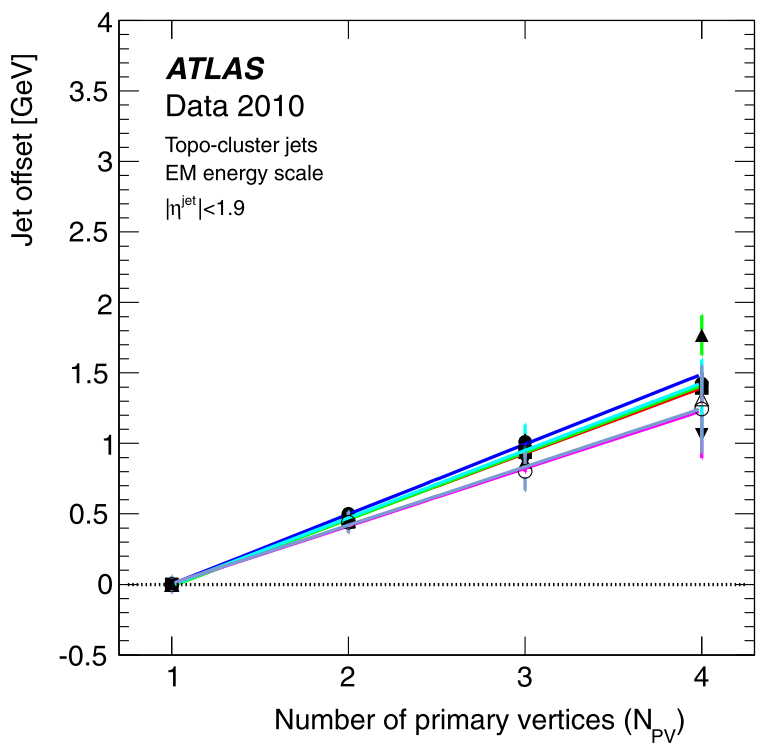

(b) Topo-cluster jet offset (EM scale)

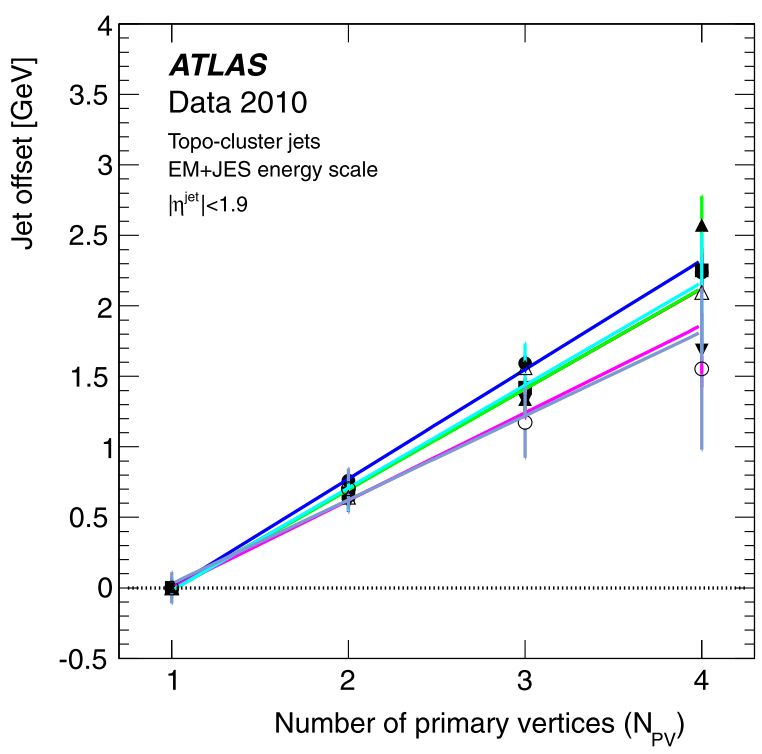

(d) Topo-cluster jet offset (EM+JES scale)

scale (c), and topo-cluster jets at the EM+JES scale (d). Only statistical uncertainties from the fit results are shown. The lines are fits using a linear function

\subsection{Jet pseudorapidity correction}

After the jet origin and energy corrections the origin-corrected jet $\eta$ is further corrected for a bias due to poorly instrumented regions of the calorimeter. In these regions topoclusters are reconstructed with a lower energy with respect to better instrumented regions (see Fig. 9). This causes the 


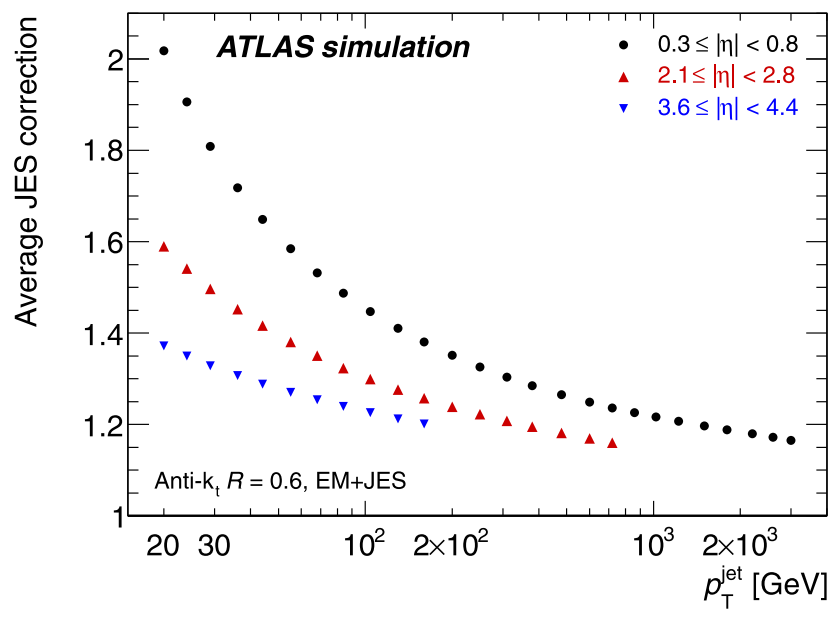

Fig. 8 Average jet energy scale correction as a function of the calibrated jet transverse momentum for three representative $\eta$-intervals obtained from the nominal Monte Carlo simulation sample. This correction corresponds to $\mathcal{F}_{\text {calib }}$ in the text. It is only shown over the accessible kinematic range

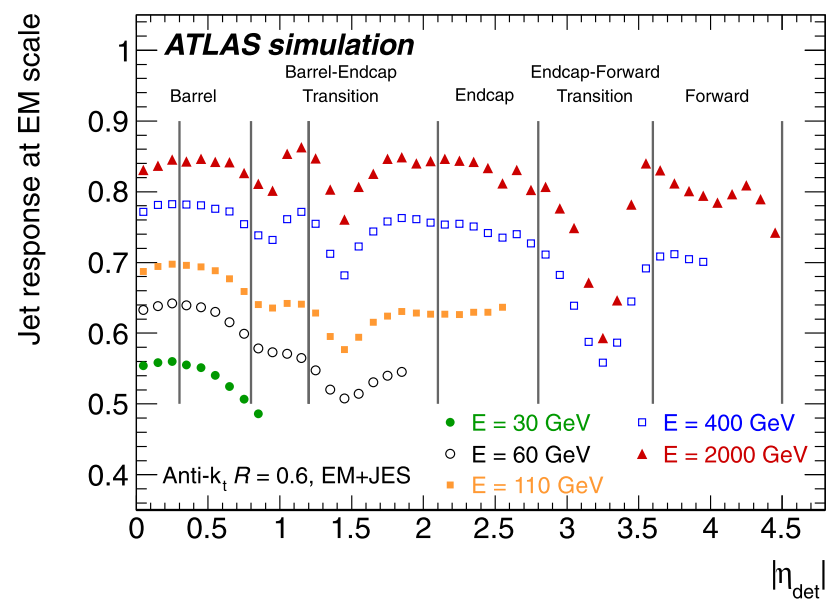

Fig. 9 Average simulated jet response $\left(\mathcal{R}_{\mathrm{EM}}^{\mathrm{jet}}\right)$ at the electromagnetic scale in bins of EM+JES calibrated jet energy and as a function of the detector pseudorapidity $\eta_{\text {det }}$. Also shown are the $\eta$-intervals used to evaluate the JES uncertainty (see Table 2). The inverse of the response shown in each bin is equal to the average jet energy scale correction $\left(\mathcal{F}_{\text {calib }}\right)$

jet direction to be biased towards the better instrumented calorimeter regions.

The $\eta$-correction is derived as the average difference $\Delta \eta=\eta_{\text {truth }}-\eta_{\text {origin }}$ in $\left(E^{\text {truth }}, \eta_{\text {det }}\right)$-bins, and is parameterised as a function of the calibrated jet energy $E_{\mathrm{EM}+\mathrm{JES}}^{\text {calo }}$ and the uncorrected $\eta_{\text {det }}$. The correction is very small ( $\Delta \eta<$ 0.01 ) for most regions of the calorimeter but larger in the transition regions. The size of the bias is illustrated as a function of the detector pseudorapidity $\left|\eta_{\text {det }}\right|$ and EM+JES calibrated jet energy in Fig. 10.

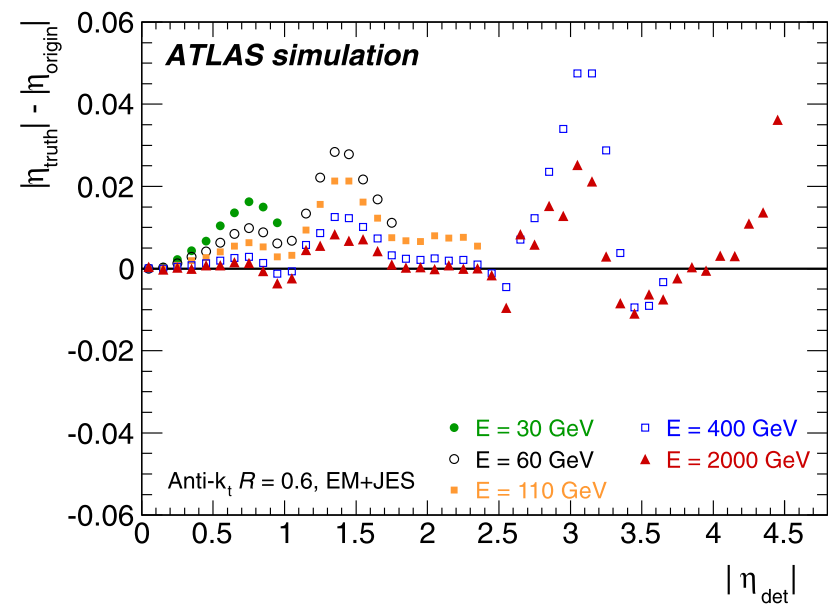

Fig. 10 Difference between the jet pseudorapidity calculated using an origin correction and the true jet pseudorapidity in bins of the calorimeter jet energy calibrated with the EM+JES scheme as a function of the detector pseudorapidity $\left|\eta_{\text {det }}\right|$

\section{Jet energy scale uncertainties for the EM+JES scheme}

The JES systematic uncertainty is derived combining information from the single hadron response measured in situ and single pion test-beam measurements, uncertainties on the amount of material of the ATLAS detector, the description of the electronic noise, and the Monte Carlo modelling used in the event generation. Dedicated Monte Carlo simulation test samples are generated with different conditions with respect to the nominal Monte Carlo sample described in Sect. 4.3. These variations are expected to provide an estimate of the systematic effects contributing to the JES uncertainty.

The pseudorapidity bins used for the estimate of the JES uncertainty divide the ATLAS detector in the eight $\eta$-regions specified in Table 2 and Fig. 9.

The JES systematic uncertainty for all jets with pseudorapidity $|\eta|>0.8$ is determined using the JES uncertainty for the central barrel region $(0.3 \leq|\eta|<0.8)$ as a baseline, with a contribution from the relative calibration of the jets with respect to the central barrel region. This choice is motivated by the good knowledge of the detector geometry in the central region, and by the use of pion response measurements in the ATLAS combined test-beam, which used a full slice of the ATLAS barrel detector, for the estimate of the calorimeter response uncertainties. The region $0.3 \leq|\eta|<0.8$ is the largest fully instrumented $|\eta|$ region considered where combined test-beam results, used to estimate the calorimeter uncertainty, are available for the entire pseudorapidity range.

This section describes the sources of systematic uncertainties and their effect on the response of EM+JES calibrated jets. In Sect. 9.1, the selection of jets used to derive Monte Carlo based components of the JES systematic uncer- 
Table 2 Detector regions used for the JES uncertainty estimate

\begin{tabular}{ll}
\hline$\eta$ region & ATLAS detector regions \\
\hline$|\eta|<0.3$ & Central Barrel \\
$0.3 \leq|\eta|<0.8$ & \\
$0.8 \leq|\eta|<1.2$ & Barrel-Endcap Transition \\
$1.2 \leq|\eta|<2.1$ & \\
$2.1 \leq|\eta|<2.8$ & Endcap \\
$2.8 \leq|\eta|<3.2$ & Endcap-Forward Transition \\
$3.2 \leq|\eta|<3.6$ & \\
$3.6 \leq|\eta|<4.5$ & Forward \\
\hline
\end{tabular}

tainty is discussed. The contributions to the JES systematics due to the following effects are then described:

1. JES calibration method (Sect. 9.2).

2. Calorimeter response (Sect. 9.3).

3. Detector simulation (Sect. 9.4).

4. Physics model and parameters employed in the Monte Carlo event generator (Sect. 9.5).

5. Relative calibration for jets with $|\eta|>0.8$ (Sect. 9.6).

6. Additional proton-proton collisions (pile-up) (Sect. 9.7).

Section 9.8 discusses how the final uncertainties are calculated. Additional uncertainties such as those for close-by jets are mentioned in Sect. 9.9 and discussed in more detail in Sect. 17.

\subsection{Jet response definition}

The average energy or $p_{\mathrm{T}}$ response, defined as

$\left\langle\mathcal{R}^{\text {jet }}\right\rangle=\left\langle E^{\text {jet }} / E^{\text {truth }}\right\rangle \quad$ or $\left\langle\mathcal{R}\left(p_{\mathrm{T}}^{\text {jet }}\right)\right\rangle=\left\langle p_{\mathrm{T}}^{\text {jet }} / p_{\mathrm{T}}^{\text {truth }}\right\rangle$,

is obtained as the peak position from a Gaussian fit to the distribution of the ratio of reconstructed energy, respectively $p_{\mathrm{T}}$, for reconstructed and truth jets by matching isolated calorimeter jets to Monte Carlo truth jets as described in Sect. 8.3, but without the isolation cut for truth jets. ${ }^{13}$ This is done separately for the nominal and each of the alternative Monte Carlo samples. Only MC truth jets with $p_{\mathrm{T}}^{\text {truth }}>$ $15 \mathrm{GeV}$, and calorimeter jets with $p_{\mathrm{T}}^{\text {jet }}>7 \mathrm{GeV}$ after calibration, are considered. The calibrated response $\left\langle\mathcal{R}^{\text {jet }}\right\rangle$ is studied in bins of the truth jet transverse momentum $p_{\mathrm{T}}^{\text {truth }}$.

The uncertainties are estimated in bins of $p_{\mathrm{T}}^{\text {truth }}$, and the same bins are used to assign an uncertainty to reconstructed jets based on its calibrated jet $p_{\mathrm{T}}$. It was verified that most reconstructed jets stay in the $p_{\mathrm{T}}$ bin of the associated truth jet bin.

\footnotetext{
${ }^{13}$ The isolation cut for truth jets on the average jet response has a negligible impact on the average jet response given that truth jets are matched to isolated reconstructed jets.
}

9.2 Uncertainty in the calibration method

After the jets in the nominal jet Monte Carlo simulation sample are calibrated (see Sect. 8), the jet energy and $p_{\mathrm{T}}$ response still show slight deviations from unity at low $p_{\mathrm{T}}$. This so-called "non-closure" refers to a failed consistency test when the calibration is applied to the same sample from which it is derived. This can be seen in Fig. 11, showing the jet response for $p_{\mathrm{T}}$ and energy as a function of $p_{\mathrm{T}}^{\text {jet }}$ for the nominal Monte Carlo sample in the barrel (a) and endcap (b) and the most forward (c) regions for anti- $k_{t}$ jets with $R=0.6$.

Any deviation from unity in the jet energy or $p_{\mathrm{T}}$ response after the application of the JES to the nominal Monte Carlo sample implies that the kinematic observables of the calibrated calorimeter jet are not restored to that of the corresponding truth jet. Besides approximations made when deriving the calibration (fit quality, parametrisation of calibration curve), the non-closure is due to the application of the same correction factor for energy and transverse momentum. Closure can therefore only be achieved if the reconstructed jet mass is close to the true jet mass. If this is not the case, such as for low $p_{\mathrm{T}}$ jets, restoring only the jet energy and pseudorapidity will lead to a bias in the $p_{\mathrm{T}}$ calibration. The non-closure is also affected by jet resolution and by details how the Monte Carlo samples are produced in order to cover the large kinematic range in jet transverse momentum.

The systematic uncertainty due to the non-closure of the nominal JES calibration is taken as the larger deviation of the response in either energy or $p_{\mathrm{T}}$ from unity. In the barrel region $(0.3 \leq|\eta|<0.8)$ this contribution amounts to about $2 \%$ at low $p_{\mathrm{T}}^{\text {jet }}$ and less than $1 \%$ for $p_{\mathrm{T}}^{\text {jet }}>30 \mathrm{GeV}$. In the endcap and forward regions, the closure is less than $1 \%$ for $p_{\mathrm{T}}^{\mathrm{jet}}>20 \mathrm{GeV}$, and the energy response is within $1 \%$ for jets with transverse momentum above $30 \mathrm{GeV}$. The deviation of the jet response from unity after calibration is taken as a source of systematic uncertainty.

For physics analysis the non-closure uncertainty only needs to be considered when an absolute jet energy or transverse momentum is needed. For analyses where only the description of the data by the Monte Carlo simulation is important, this uncertainty does not need to be considered.

\subsection{Uncertainty on the jet calorimeter response}

The response and corresponding uncertainties for single particles interacting in the ATLAS calorimeters can be used to derive the jet energy scale uncertainty in the central calorimeter region as detailed in Ref. [28].

In the ATLAS simulation infrastructure the true calorimeter energy deposits in each calorimeter cell can be traced to the particles generated in the collision. The uncertainty in the calorimeter response to jets can then be obtained from 


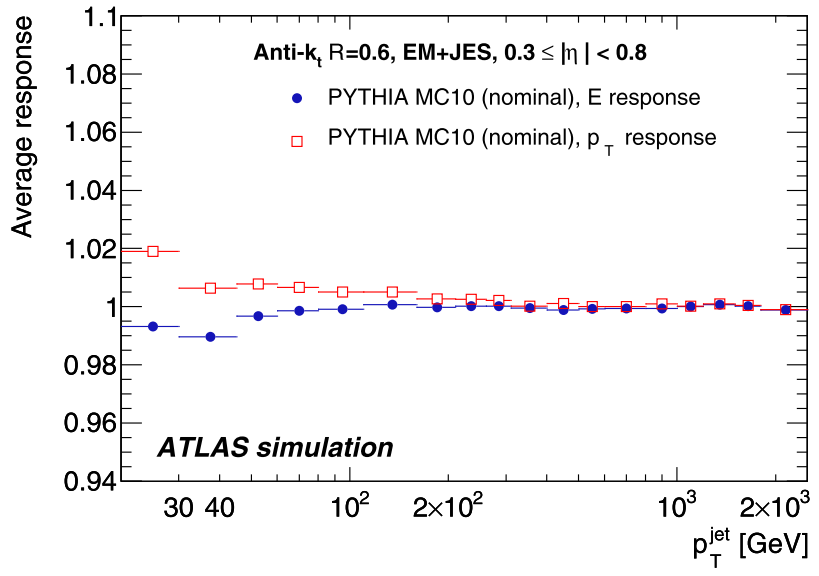

(a) $0.3 \leq|\eta|<0.8$

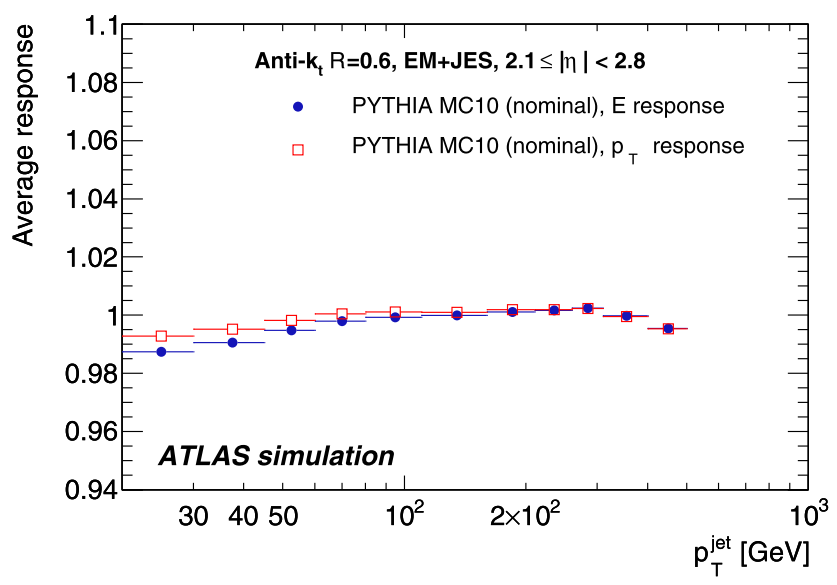

(b) $2.1 \leq|\eta|<2.8$

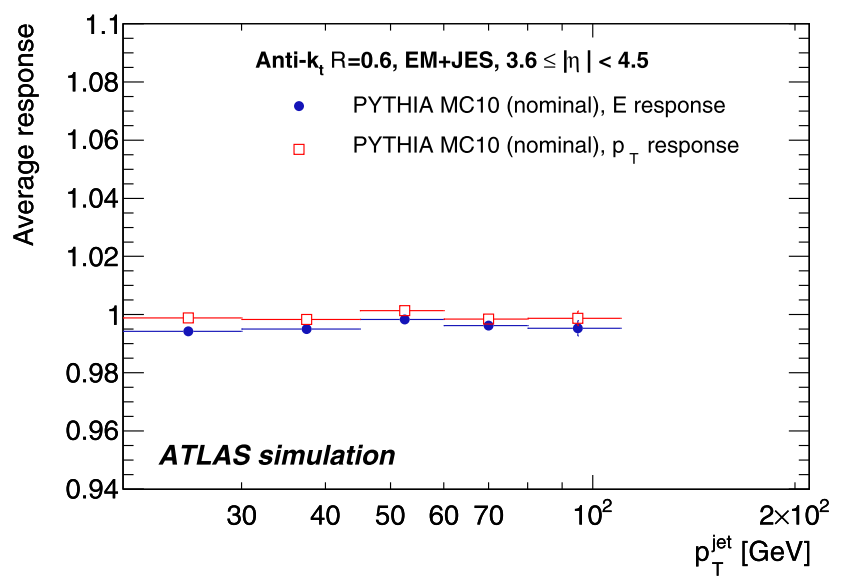

(c) $3.6 \leq|\eta|<4.5$

Fig. 11 Average simulated jet $p_{\mathrm{T}}$ response (open squares) after the $\mathrm{EM}+\mathrm{JES}$ calibration and jet energy response (full circles) as a function of $p_{\mathrm{T}}^{\text {jet }}$ for the nominal Monte Carlo sample for jets in the central (a), endcap (b) and most forward (c) calorimeter regions. Systematic uncertainties are not shown. Statistical uncertainties are smaller than the marker size the response uncertainty in the individual particles constituting the jet. The in situ measurement of the single particle response detailed in Ref. [28] significantly reduces the uncertainty due to the limited knowledge of the exact detector geometry, in particular that due to the presence of additional dead material, and the modelling of the exact way particles interact in the detector.

The following single particle response measurements are used:

1. The single hadron energy measured in a cone around an isolated track with respect to the track momentum $(E / p)$ in the momentum range from $0.5 \leq p^{\text {track }}<20 \mathrm{GeV}$.

2. The pion response measurements performed in the 2004 combined test-beam, where a full slice of the ATLAS detector was exposed to pion beams with momenta between $20 \mathrm{GeV}$ and $350 \mathrm{GeV}$ [78].

Uncertainties for charged hadrons are estimated from these measurements as detailed in Ref. [28]. Additional uncertainties are related to:

1. The calorimeter acceptance for low $p_{\mathrm{T}}$ particles that do not reach the calorimeter or are not reconstructed in a topo-cluster due to the noise thresholds.

2. Calorimeter response to particles with $p>400 \mathrm{GeV}$ for which the uncertainty is conservatively estimated as $10 \%$, to account for possible calorimeter non-linearities and longitudinal leakage.

3. The baseline absolute electromagnetic scale for the hadronic and electromagnetic calorimeters for particles in the kinematic range not measured in situ.

4. The calorimeter response to neutral hadrons is estimated by comparing various models in GEANT4. An uncertainty of $10 \%$ for particles with an energy $E<3 \mathrm{GeV}$ and $5 \%$ for higher energies is obtained.

At high transverse momentum, the dominant contribution to the calorimeter response uncertainties is due to particles with momenta covered by the test-beam. In the pseudorapidity range $0 \leq|\eta|<0.8$ the shift of the relative jet energy scale expected from the single hadron response measurements in the test-beam is up to $\approx 1 \%$, and the uncertainty on the shift is from $1 \%$ to $3 \%$. The total envelope (the shift added linearly to the uncertainty) of about $1.5-4 \%$, depending on the jet transverse momentum, is taken as the relative JES calorimeter uncertainty. The calorimeter uncertainty is shown in Sect. 9.8.

\subsection{Uncertainties due to the detector simulation}

\subsubsection{Calorimeter cell noise thresholds}

As described in Sect. 6.1.1, topo-clusters are constructed based on the signal-to-noise ratio of calorimeter cells, where 
the noise is defined as the RMS of the measured cell energy distribution in a data taking period without proton-proton collisions. Discrepancies between the simulated noise and the real noise in data can lead to differences in the cluster shapes and to the presence of fake topo-clusters. For data, the noise can change over time, ${ }^{14}$ while the noise RMS used in the simulation is fixed at the time of the production of the simulated data sets. These effects can lead to biases in the jet reconstruction and calibration, if the electronic noise injected in the Monte Carlo simulation does not reflect that data. Additionally in the MC simulation the noise is generated from the RMS measured in data assuming a Gaussian distribution.

The effect of the calorimeter cell noise mis-modelling on the jet response is estimated by reconstructing topo-clusters, and thereafter jets, in Monte Carlo using the noise RMS measured from data. The actual energy and noise simulated in the Monte Carlo are left unchanged, but the values of the thresholds used to include a given calorimeter cell in a topocluster are shifted according to the cell noise RMS measured in data at one particular time.

The response for jets reconstructed with the modified noise thresholds are compared with the response for jets reconstructed in exactly the same sample using the default Monte Carlo noise thresholds.

To further understand the effect of the noise thresholds on the jet response, the noise thresholds were shifted. An increase of each calorimeter cell threshold by $7 \%$ in the Monte Carlo simulation is found to give a similar shift in the jet response as using the noise RMS from data. Raising and lowering the cell thresholds by $7 \%$ shows that the effect on the jet response from varying the cell noise thresholds is symmetric. This allows the use of the calorimeter cell noise thresholds derived from data as a representative sample to determine the jet energy scale uncertainty and covers the cases when the data have either more or less noise than the simulation.

The maximal observed change in jet response is used to estimate the uncertainty on the jet energy measurement due to the calorimeter cell noise modelling. It is found to be below $2 \%$ for the whole pseudorapidity range, and negligible for jets with transverse momenta above $45 \mathrm{GeV}$. The uncertainties assigned to jets with transverse momenta below $45 \mathrm{GeV}$ are:

1. $1 \%$ and $2 \%$ for $20 \leq p_{\mathrm{T}}^{\text {jet }}<30 \mathrm{GeV}$ for anti- $k_{t}$ jets with $R=0.4$ and $R=0.6$ jets, respectively,

2. $1 \%$ for $30 \leq p_{\mathrm{T}}^{\text {jet }}<45 \mathrm{GeV}$ for both $R$ values.

\footnotetext{
${ }^{14}$ Time-dependent noise changes for single cells in data are accounted for using regular measurements.
}

\subsubsection{Additional detector material}

The jet energy scale is affected by possible deviations in the material description as the jet energy scale calibration has been derived to restore the energy lost assuming a geometry as simulated in the nominal Monte Carlo sample. Simulated detector geometries that include systematic variations of the amount of material have been designed using test-beam measurements [32], in addition to $900 \mathrm{GeV}$ and $7 \mathrm{TeV}$ data $[82,83,89,90]$. The possible additional material amount is estimated from these in situ measurements and the a priori knowledge of the detector construction. Specific Monte Carlo simulation samples have been produced using these distorted geometries.

In the case of uncertainties derived with in situ techniques, such as those coming from the single hadron response measurements detailed in Sect. 9.3, most of the effects on the jet response due to additional dead material do not apply, because in situ measurements do not rely on simulation where the material could be misrepresented. However, the quality criteria of the track selection for the single hadron response measurement, effectively only allow particles that have not interacted in the Pixel and SCT layers of the inner detector to be included in the measurement.

Therefore the effect of possible additional dead material in these inner detector layers on the calorimeter response to jets needs to be taken into account for particles in the momentum range of the in situ single hadron response measurement. This is achieved using a specific Monte Carlo sample where the amount of material is systematically varied by adding $5 \%$ of material to the existing inner detector services [42]. The jet response in the two cases is shown in Fig. 12.

Electrons, photons, and hadrons with momenta $p>$ $20 \mathrm{GeV}$ are not included in the single hadron response measurements and therefore there is no estimate based on in situ techniques for the effect of any additional material in front of the calorimeters. This uncertainty is estimated using a dedicated Monte Carlo simulation sample where the overall detector material is systematically varied within the current uncertainties [42] on the detector geometry. The overall changes in the detector geometry include:

1. The increase in the inner detector material mentioned above.

2. An extra 0.1 radiation length $\left(X_{0}\right)$ in the cryostat in front of the barrel of the electromagnetic calorimeter $(|\eta|<$ $1.5)$.

3. An extra $0.05 X_{0}$ between the presampler and the first layer of the electromagnetic calorimeter.

4. An extra $0.1 X_{0}$ in the cryostat after the barrel of the electromagnetic calorimeter. 


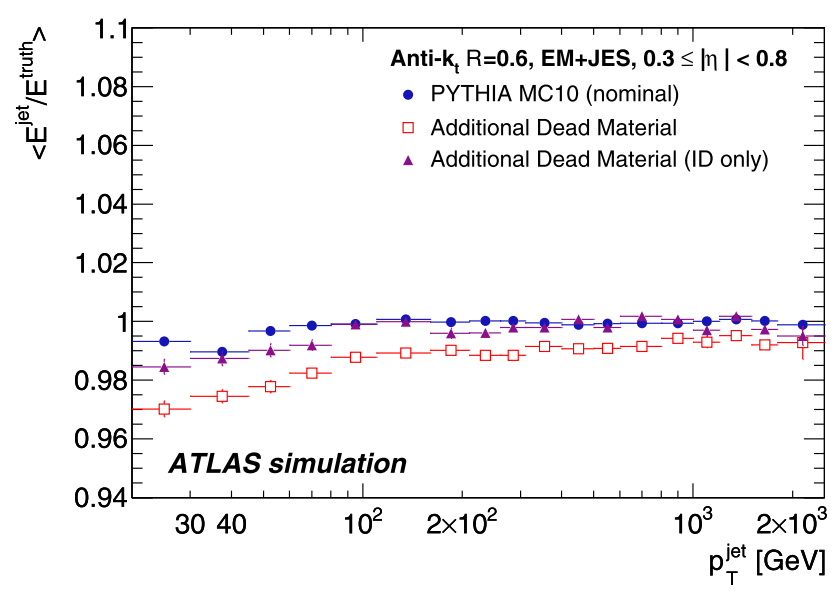

(a) Energy response

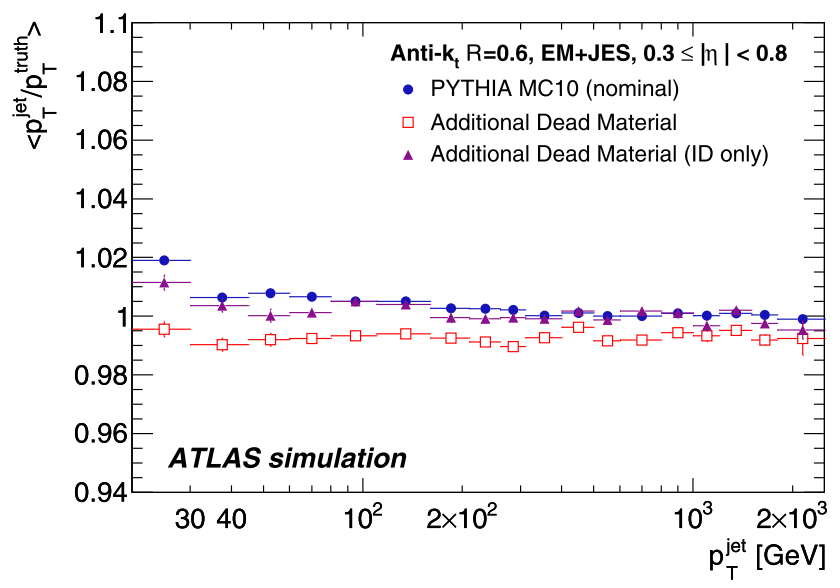

(b) Transverse momentum response

Fig. 12 Average simulated jet response in energy (a) and in $p_{\mathrm{T}}(\mathbf{b})$ as a function of $p_{\mathrm{T}}^{\text {jet }}$ in the central region $(0.3 \leq|\eta|<0.8)$ in the case of additional dead material in the inner detector (full triangles) and in both the inner detector and the calorimeters (open squares). The amount of additional dead material is specified in the text. The response within the nominal Monte Carlo sample is shown for comparison (full circles). Only statistical uncertainties are shown

5. Extra material in the barrel-endcap transition region in the electromagnetic calorimeter $(1.37<|\eta|<1.52)$. An increase of 1.5 times the nominal simulated material is adopted.

The uncertainty contribution due to the overall additional detector material is estimated by comparing the EM+JES jet response in the nominal Monte Carlo simulation sample with the jet response in a Monte Carlo simulation sample with a distorted geometry (see Fig. 12). This uncertainty is then scaled by the average energy fraction of electrons, photons and high transverse momentum hadrons within a jet as a function of $p_{\mathrm{T}}$.
9.5 Uncertainties due to the event modelling in Monte Carlo generators

The contributions to the JES uncertainty from the modelling of the fragmentation, the underlying event and other choices in the event modelling of the Monte Carlo event generator are obtained from samples based on ALPGEN+HERWIG+ Jimmy and the Pythia Perugia2010 tune discussed in Sect. 4.

By comparing the baseline PythiA Monte Carlo sample to the Pythia Perugia2010 tune, the effects of soft physics modelling are tested. The PerUgia2010 tune provides, in particular, a better description of the internal jet structure recently measured with ATLAS [3]. The ALPGEN Monte Carlo uses different theoretical models for all steps of the event generation and therefore gives a reasonable estimate of the systematic variations. However, the possible compensation of modelling effects that shift the jet response in opposite directions cannot be excluded.

Figure 13 shows the calibrated jet kinematic response for the two Monte Carlo generators and tunes used to estimate the effect of the Monte Carlo theoretical model on the jet energy scale uncertainty. The kinematic response for the nominal sample is shown for comparison. The ratio of the nominal response to that for each of the two samples is used to estimate the systematic uncertainty to the jet energy scale, and the procedure is further detailed in Sect. 9.8.

\subsection{In situ intercalibration using events} with dijet topologies

The response of the ATLAS calorimeters to jets depends on the jet direction, due to the different calorimeter technology and to the varying amounts of dead material in front of the calorimeters. A calibration is therefore needed to ensure a uniform calorimeter response to jets. This can be achieved by applying correction factors derived from Monte Carlo simulations. Such corrections need to be validated in situ given the non-compensating nature of the calorimeters in conjunction with the complex calorimeter geometry and material distribution.

The relative jet calorimeter response and its uncertainty is studied by comparing the transverse momenta of a wellcalibrated central jet and a jet in the forward region in events with only two jets at high transverse momenta (dijets). Such techniques have been applied in previous hadron collider experiments $[14,15]$.

\subsubsection{Intercalibration method using a fixed central reference region}

The traditional approach for $\eta$-intercalibration with dijet events is to use a fixed central region of the calorimeters 


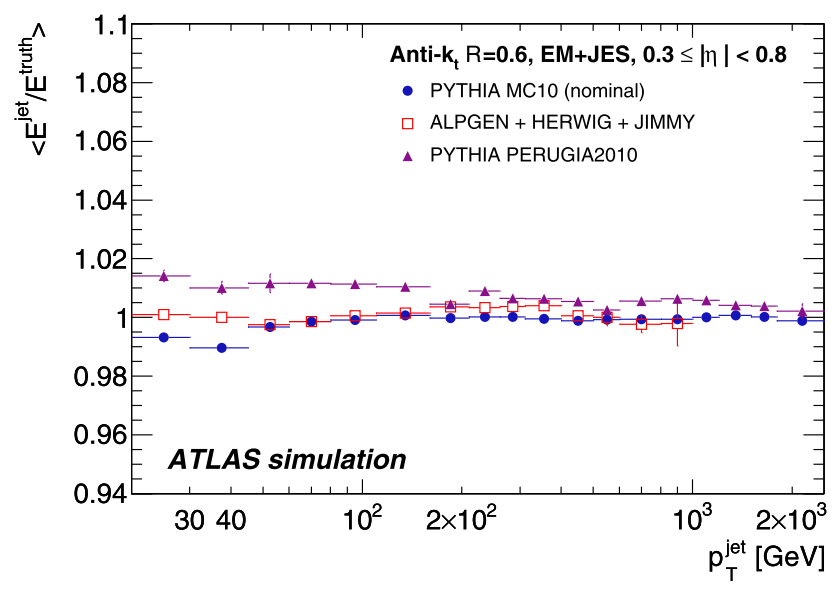

(a) Energy response

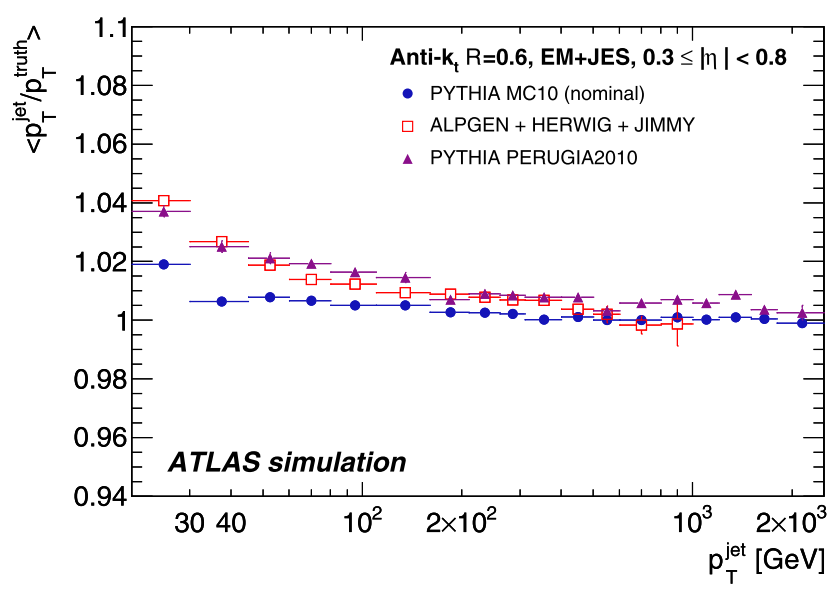

(b) Transverse momentum response

Fig. 13 Average simulated response in energy (a) and in $p_{\mathrm{T}}$ (b) as a function of $p_{\mathrm{T}}^{\text {jet }}$ in the central region $(0.3 \leq|\eta|<0.8)$ for ALPGEN+HERWIG+JIMMY (open squares) and PYTHIA with the PERUGIA2010 tune (full triangles). The response of the nominal Monte Carlo simulation sample is shown for comparison (full circles). Only statistical uncertainties are shown

as the reference region. The relative calorimeter response to jets in other calorimeter regions is then quantified by the $p_{\mathrm{T}}$ balance between the reference jet and the probe jet, exploiting the fact that these jets are expected to have equal $p_{\mathrm{T}}$ due to transverse momentum conservation. The $p_{\mathrm{T}}$ balance can be characterised by the asymmetry $\mathcal{A}$, defined as

$\mathcal{A}=\frac{p_{\mathrm{T}}^{\text {probe }}-p_{\mathrm{T}}^{\text {ref }}}{p_{\mathrm{T}}^{\text {avg }}}$

with $p_{\mathrm{T}}^{\text {avg }}=\left(p_{\mathrm{T}}^{\text {probe }}+p_{\mathrm{T}}^{\text {ref }}\right) / 2$. The reference region is chosen as the central region of the barrel: $|\eta|<0.8$. If both jets fall into the reference region, each jet is used, in turn, as the reference jet. As a consequence, the average asymmetry in the reference region will be zero by construction.
The asymmetry is then used to measure an $\eta$-intercalibration factor $c$ for the probe jet, or its response relative to the reference jet $1 / c$, using the relation

$\frac{p_{\mathrm{T}}^{\text {probe }}}{p_{\mathrm{T}}^{\text {ref }}}=\frac{2+\mathcal{A}}{2-\mathcal{A}}=1 / c$.

The asymmetry distribution is calculated in bins of jet $\eta_{\text {det }}$ and $p_{\mathrm{T}}^{\text {avg }}$ : The bins are labeled $i$ for each probe jet $\eta_{\text {det }}$ and $k$ for each $p_{\mathrm{T}}^{\text {avg }}$-bin. Intercalibration factors are calculated for each bin according to Eq. (13):

$c_{i k}=\frac{2-\left\langle\mathcal{A}_{i k}\right\rangle}{2+\left\langle\mathcal{A}_{i k}\right\rangle}$,

where the $\left\langle\mathcal{A}_{i k}\right\rangle$ is the mean value of the asymmetry distribution in each bin. The uncertainty on $\left\langle\mathcal{A}_{i k}\right\rangle$ is taken to be the RMS $/ \sqrt{N}$ of each distribution, where $N$ is the number of events per bin.

\subsubsection{Intercalibration using the matrix method}

A disadvantage with the method outlined above is that all events are required to have a jet in the central reference region. This results in a significant loss of event statistics, especially in the forward region, where the dijet cross section drops steeply as the rapidity interval between the jets increases. In order to use the full event statistics, the default method can be extended by replacing the "probe" and "reference" jets by "left" and "right" jets defined as $\eta^{\text {left }}<\eta^{\text {right }}$. Equations (12) and (13) then become:

$\mathcal{A}=\frac{p_{\mathrm{T}}^{\text {left }}-p_{\mathrm{T}}^{\text {right }}}{p_{\mathrm{T}}^{\text {avg }}}$ and

$\mathcal{R}_{l r}=\frac{p_{\mathrm{T}}^{\text {left }}}{p_{\mathrm{T}}^{\text {right }}}=\frac{c^{\text {right }}}{c^{\text {left }}}=\frac{2+\mathcal{A}}{2-\mathcal{A}}$,

where the term $\mathcal{R}$ denotes the ratio of the responses, and $c^{\text {left }}$ and $c^{\text {right }}$ are the $\eta$-intercalibration factors for the left and right jets, respectively.

In this approach there is a response ratio distribution, $\mathcal{R}_{i j k}$, whose average value $\left\langle\mathcal{R}_{i j k}\right\rangle$ is evaluated for each $\eta^{\text {left }}$ bin $i, \eta^{\text {right }}$-bin $j$ and $p_{\mathrm{T}}^{\text {avg }}$-bin $k$. The relative correction factor $c_{\alpha k}$ for a given jet $\eta$-bin $\alpha$ and for a fixed $p_{\mathrm{T}}^{\text {avg }}$-bin $k$, is obtained by minimising a matrix of linear equations:

$$
\begin{aligned}
& S\left(c_{1 k}, \ldots, c_{N k}\right) \\
& \quad=\sum_{j=1}^{N} \sum_{i=1}^{j-1}\left(\frac{1}{\Delta\left\langle\mathcal{R}_{i j k}\right\rangle}\left(c_{i k}\left\langle\mathcal{R}_{i j k}\right\rangle-c_{j k}\right)\right)^{2}+X\left(c_{i k}\right),
\end{aligned}
$$

where $N$ denotes the number of $\eta$-bins, $\Delta\left\langle\mathcal{R}_{i j k}\right\rangle$ is the statistical uncertainty of $\left\langle\mathcal{R}_{i j k}\right\rangle$ and the function $X\left(c_{i k}\right)$ is used 
to quadratically suppress deviations from unity of the average corrections. ${ }^{15}$ The $\eta$-bins are assumed to be ordered such that $\eta_{i}<\eta_{i+1}$. Note that if the jet response does not vary with $\eta$, then the relative response will be unity for each $\left(\eta_{\text {left }}, \eta_{\text {right }}\right)$-bin combination (see Eq. (15)). A perfect minimization $S=0$ is achieved when all correction factors equal unity.

The minimisation of Eq. (16) is done separately for each $p_{\mathrm{T}}^{\text {avg }}$-bin $k$, and the resulting calibration factors $c_{i k}$ (for each jet $\eta$-bin $i$ ) are scaled such that the average calibration factor in the reference region $|\eta|<0.8$ equals unity.

\subsubsection{Selection of dijet events}

Events are retained if there were at least two jets above the jet reconstruction threshold of $p_{\mathrm{T}}^{\text {jet }}>7 \mathrm{GeV}$. The event is rejected if either of the two leading jets did not satisfy the standard jet selection criteria (see Sect. 7).

Events are required to satisfy a specific logic using a central or a forward jet trigger, which select events based on jet activity in either the central $(|\eta|<3.2)$ or the forward $(|\eta|>3.2)$ trigger regions, respectively [30]. The use of a logical OR allows the selection to capture dijet events where the jet is in either of the two jet trigger regions: central-central, central-forward and forward-forward configurations. The requirements are chosen such that the trigger efficiency, for a specific region of $p_{\mathrm{T}}^{\text {avg }}$, was greater than $99 \%$ and approximately flat as a function of the pseudorapidity of the probe jet.

To cover the region $p_{\mathrm{T}}^{\text {avg }}<45 \mathrm{GeV}$, events triggered by the minimum bias trigger scintillators were used. To enhance events which have only two jets at high $p_{\mathrm{T}}$, the following selection criteria are applied;

$p_{\mathrm{T}}^{\mathrm{avg}}>20 \mathrm{GeV}, \quad \Delta \phi\left(\mathrm{j}_{1}, \mathrm{j}_{2}\right)>2.6 \mathrm{rad}$,

$p_{\mathrm{T}}\left(\mathrm{j}_{3}\right)<\max \left(0.15 p_{\mathrm{T}}^{\mathrm{avg}}, 7 \mathrm{GeV}\right)$,

where $\mathrm{j}_{i}$ denotes the $i$ th highest $p_{\mathrm{T}}$ jet in the event and $\Delta \phi\left(\mathrm{j}_{1}, \mathrm{j}_{2}\right)$ is the azimuthal angle between the two leading jets.

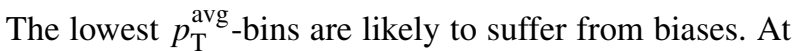
very low $p_{\mathrm{T}}^{\text {avg }}$, it is expected that this technique may not measure accurately the relative response to jets, because the assumption of dijet balance at hadron level may start to fail. First, there are residual low- $p_{\mathrm{T}}$ jet effects since the selection criterion on the third jet, which is used to suppress the unbalancing effects of soft QCD radiation, is not as efficient due to the jet reconstruction threshold of $7 \mathrm{GeV}$. Second, the jet reconstruction efficiency is worse for low- $p_{\mathrm{T}}$ jets.

\footnotetext{
${ }^{15} X\left(c_{i k}\right)=K\left(N_{\text {bins }}^{-1} \sum_{i=1}^{N_{\text {bins }}} c_{i k}-1\right)^{2}$ is defined with $K$ being a constant and $N_{\text {bins }}$ being the number of $\eta$-bins (number of indices $i$ ). This term prevents the minimisation from choosing the trivial solution: all $c_{i k}$ equal to zero. The value of the constant $K$ does not impact the solution as long as it is sufficiently large $\left(K \approx 10^{6}\right)$.
}

\subsubsection{Comparison of intercalibration methods}

The relative jet response obtained with the matrix method is compared to the relative jet response obtained using the method with a fixed reference region. Figure 14 shows the jet response relative to central jets $(1 / c)$ for two $p_{\mathrm{T}}^{\text {avg }}$-bins, $30 \leq p_{\mathrm{T}}^{\text {avg }}<40 \mathrm{GeV}$ and $60 \leq p_{\mathrm{T}}^{\text {avg }}<80 \mathrm{GeV}$. These results are obtained for a reference region $0.1 \leq|\eta|<0.6$ and therefore not directly comparable to the results discussed below where $0.1 \leq|\eta|<0.8$ is used. The matrix method has a higher statistical precision relative to the fixed reference method for low $p_{\mathrm{T}}$, since in this region the dijet cross section is particularly large for large pseudorapidity separations.

The response observed using the fixed reference region method is compatible with those obtained using the matrix method. ${ }^{16}$ These results are representative of all the phase space regions studied in this analysis and the matrix method is therefore used to give the final uncertainty on the in situ $\eta$-intercalibration due to its higher statistical precision.

\subsubsection{Comparison of data with Monte Carlo simulation}

Figure 15 shows the relative response obtained with the matrix method as a function of the jet pseudorapidity for data and Monte Carlo simulations in four $p_{\mathrm{T}}^{\text {avg }}$ regions. The results are normalized such that average relative response in $|\eta|<0.8$ equals unity, both for data and Monte Carlo simulation.

The response in data is reasonably well reproduced by the Monte Carlo simulations for $p_{\mathrm{T}}^{\text {jet }}>60 \mathrm{GeV}$, with the Monte Carlo simulation and data agreeing typically better than $2 \%$ in the central region $(|\eta|<2.8)$ and 5-10\% (depending on $\left.p_{\mathrm{T}}^{\text {avg }}\right)$ in the forward region $(|\eta|>2.8)$. At lower values of $p_{\mathrm{T}}$, the data do not agree as well with the Monte Carlo simulations and the Monte Carlo simulations themselves show a large spread around the data. For $20 \leq p_{\mathrm{T}}^{\text {avg }}<30 \mathrm{GeV}$, the Monte Carlo simulation deviates from the data by about $10 \%$ for $|\eta|>2.8$, with the different Monte Carlo simulations predicting both higher and lower relative responses than that observed in the data.

The main differences, due to residual low- $p_{\mathrm{T}}$ jet effects (see Sect. 9.6.3), occur between PYTHIA with the MC10 or the PERUgia tune on one side and ALPGEN/HERwig++ on the other. The differences therefore apparently reflect a difference in physics modelling between the event generators.

Figure 16 shows the relative response as a function of $p_{\mathrm{T}}^{\mathrm{avg}}$. The distributions are shown for jets in the region $1.2 \leq$ $|\eta|<2.1$ and also for those in the region $3.6 \leq|\eta|<4.5$. Again, the response is reasonably well described by the Monte Carlo simulation for all calorimeter regions at high $p_{\mathrm{T}}$ and the more central region at low $p_{\mathrm{T}}$.

\footnotetext{
${ }^{16}$ As discussed in Sect. 9.6.3, even for an ideal detector the asymmetry, and hence the relative response, is not expected to be exactly flat due to the effects of soft QCD radiation and other soft particle activities.
} 


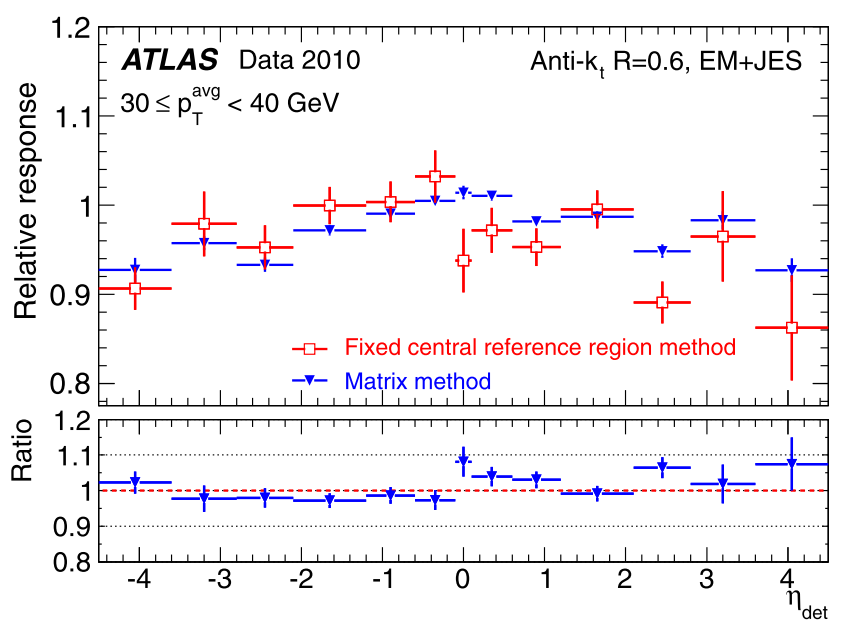

(a) $30 \leq p_{\mathrm{T}}^{\text {avg }}<40 \mathrm{GeV}$

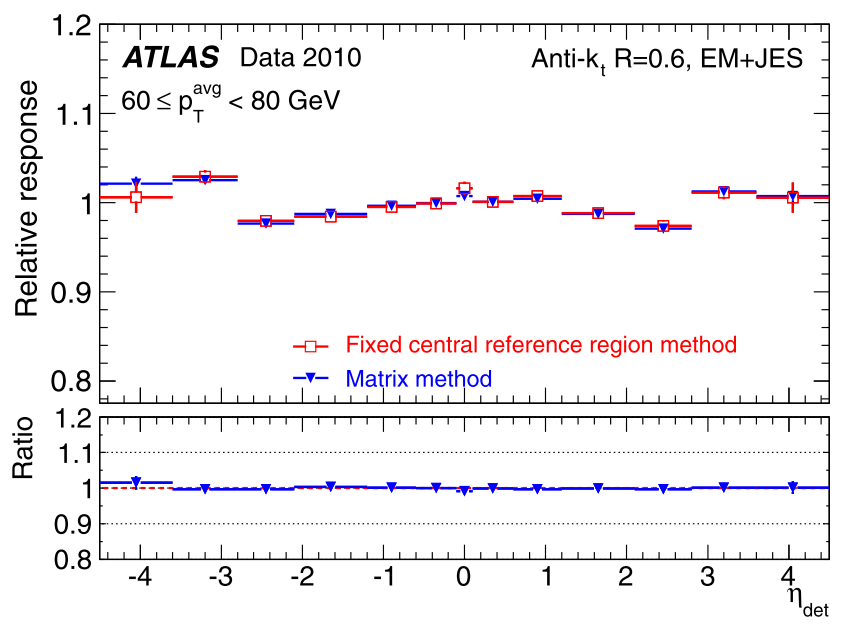

(b) $60 \leq p_{\mathrm{T}}^{\text {avg }}<80 \mathrm{GeV}$

Fig. 14 Relative response of anti- $k_{t}$ jets with $R=0.6$ calibrated with the EM+JES scheme, $1 / c$, as a function of the pseudorapidity measured using the matrix and fixed central reference region $\eta$-intercalibration methods. Results are presented for two bins of $p_{\mathrm{T}}^{\text {avg }}: 30 \leq p_{\mathrm{T}}^{\text {avg }}<40 \mathrm{GeV}$ measured in minimum bias data (a), and $60 \leq p_{\mathrm{T}}^{\text {avg }}<80 \mathrm{GeV}$ measured in data collected using jet triggers (b). The lower part of the figures shows the ratio of the two methods. The central reference region is $0.1 \leq|\eta|<0.6$. Only statistical uncertainties are shown

\subsubsection{Total uncertainties in the forward region}

The Monte Carlo simulation predictions for the relative jet response diverge at low values of $p_{\mathrm{T}}^{\text {avg }}$ (see Fig. 15). The data themselves lie between the different predictions. The uncertainty on the relative jet response must reflect this disagreement because there is no a priori reason to believe one theoretical prediction over another. The differences in the jet $p_{\mathrm{T}}$ balance are already visible when comparing the two simulations using partons only (switching of the hadronisation process and using no detector simulation) and are therefore most likely due to the different parton shower modelling. This is also confirmed by the observation that differences of the calorimeter response to jets evaluated as $p_{\mathrm{T}}^{\text {jet }} / p_{\mathrm{T}}^{\text {truth }}$ between PYTHIA and HERWIG are rather small. The observed differences are therefore due to the modelling of the additional parton radiation and not due to the modelling of the jet fragmentation.

The uncertainty on the relative response is taken to be the RMS deviation of the Monte Carlo predictions from the data. At high $p_{\mathrm{T}}$, where the spread of Monte Carlo simulation predictions is small, the uncertainty mainly reflects the true difference between the response in data and simulation. At low $p_{\mathrm{T}}$ and large $|\eta|$, the uncertainty mainly reflects the physics modelling uncertainty, although the detector-based differences between data and simulation are also accounted for. Other uncertainty sources, such as trigger selection or the $\mathrm{QCD}$ radiation suppression using the third jet, are either negligible, or included in the total uncertainty assigned from the spread of Monte Carlo predictions around the data.

Figure 17 shows the uncertainty in the jet response, relative to jets in the central region $|\eta|<0.8$, as a function of the jet $p_{\mathrm{T}}$ and $|\eta|$. The JES uncertainty, determined in the central detector region using the single particle response and systematic variations of the Monte Carlo simulations, is transferred to the forward regions using the results from the dijet balance. These uncertainties are included in the final uncertainty as follows:

1. The total JES uncertainty in the central region $0.3 \leq$ $|\eta|<0.8$ is kept as a baseline.

2. The uncertainty from the relative intercalibration is taken as the RMS deviation of the MC predictions from the data and is added in quadrature to the baseline uncertainty.

The measurements are performed for transverse momenta in the range $20 \leq p_{\mathrm{T}}^{\mathrm{avg}}<110 \mathrm{GeV}$. The uncertainty for jets with $p_{\mathrm{T}}>100 \mathrm{GeV}$ is taken as the uncertainty of the last available $p_{\mathrm{T}}$-bin. ${ }^{17}$ The uncertainties are evaluated separately for jets reconstructed with distance parameters $R=0.4$ and $R=0.6$, and are in general found to be slightly larger for $R=0.4$ (not shown).

Figure 18 shows a summary of the $\eta$-intercalibration results and the associated intercalibration uncertainty calculated as detailed above, as a function of jet $|\eta|$ for two representative $p_{\mathrm{T}}^{\text {avg }}$-bins.

\subsection{Uncertainties due to multiple proton-proton collisions}

The offset to the jet transverse energy due to pile-up interactions can be measured at the EM scale from the average en-

\footnotetext{
${ }^{17}$ This is justified by the decrease of the intercalibration uncertainty with $p_{\mathrm{T}}$, but cannot completely exclude the presence of calorimeter non-linearities for jet energies above those used for the intercalibration.
} 


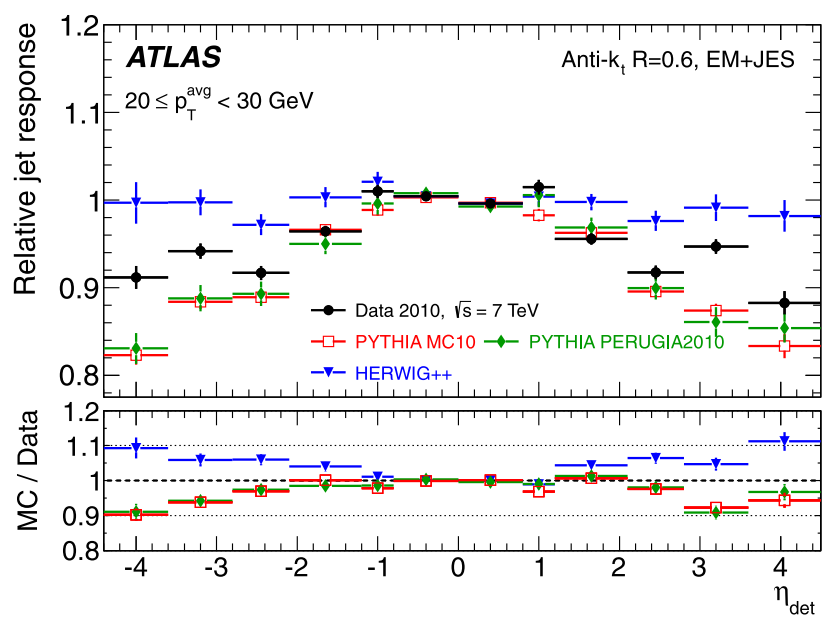

(a) $20 \leq p_{\mathrm{T}}^{\text {avg }}<30 \mathrm{GeV}$

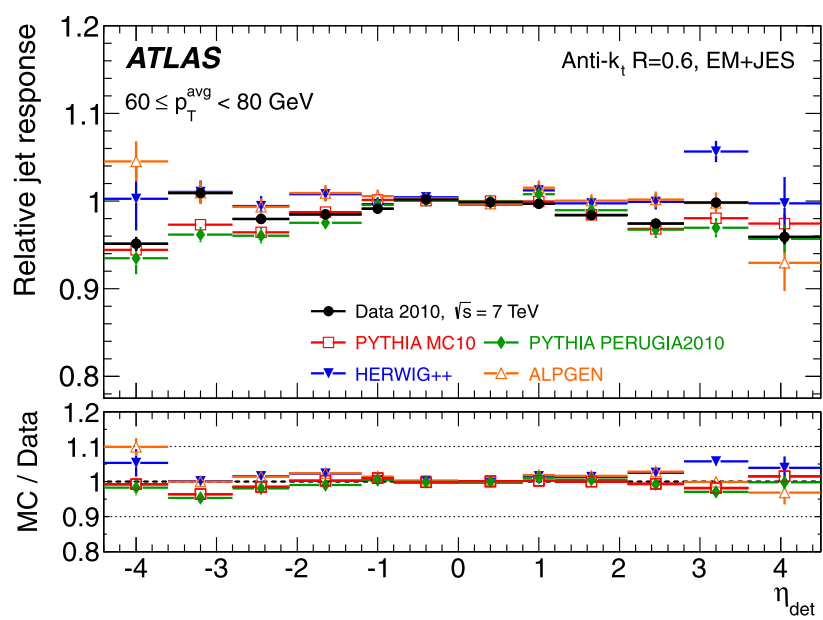

(c) $60 \leq p_{\mathrm{T}}^{\text {avg }}<80 \mathrm{GeV}$

Fig. 15 Relative jet response, $1 / c$, of anti- $k_{t}$ jets with $R=0.6$ as a function of the jet pseudorapidity measured using the matrix $\eta$-intercalibration method in bins of the average $p_{\mathrm{T}}$ of the two leading jets (a) $20 \leq p_{\mathrm{T}}^{\mathrm{avg}}<30 \mathrm{GeV}$, (b) $30 \leq p_{\mathrm{T}}^{\text {avg }}<45 \mathrm{GeV}$, (c) $60 \leq p_{\mathrm{T}}^{\text {avg }}<80 \mathrm{GeV}$ and $80 \leq p_{\mathrm{T}}^{\text {avg }}<110 \mathrm{GeV}$. The lower part

ergy in calorimeter towers in minimum bias events. The uncertainty in the pile-up corrections can be obtained by varying certain analysis choices and by studying the jet response with respect to the transverse momentum of track jets as a function of the number of primary vertices.

\subsubsection{Jet offset correction uncertainty from track jets}

The systematic uncertainty in the jet offset correction can be evaluated using track jets. Figure 7 shows the variation of the offset among the various ranges of $p_{\mathrm{T}}^{\text {track jet }}$. From the offset spread an uncertainty on the correction can be derived. It is approximately $\delta\left(\mathcal{O}_{\text {track jet }}^{\text {EM }}\right)<100 \mathrm{MeV}$ per additional vertex at the EM scale and $\delta\left(\mathcal{O}_{\text {track jet }}^{\mathrm{EM}+\mathrm{JES}}\right)<200 \mathrm{MeV}$ per ad-

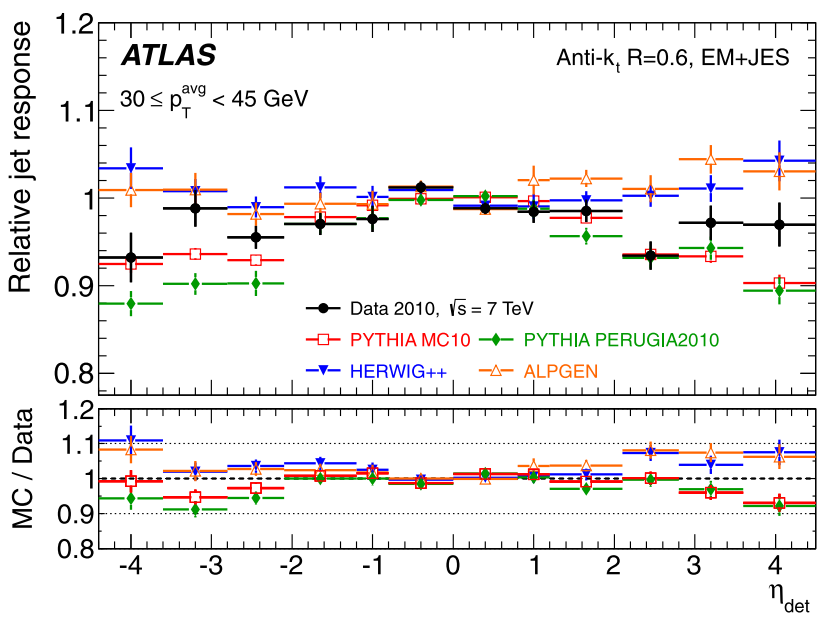

(b) $30 \leq p_{\mathrm{T}}^{\text {avg }}<45 \mathrm{GeV}$

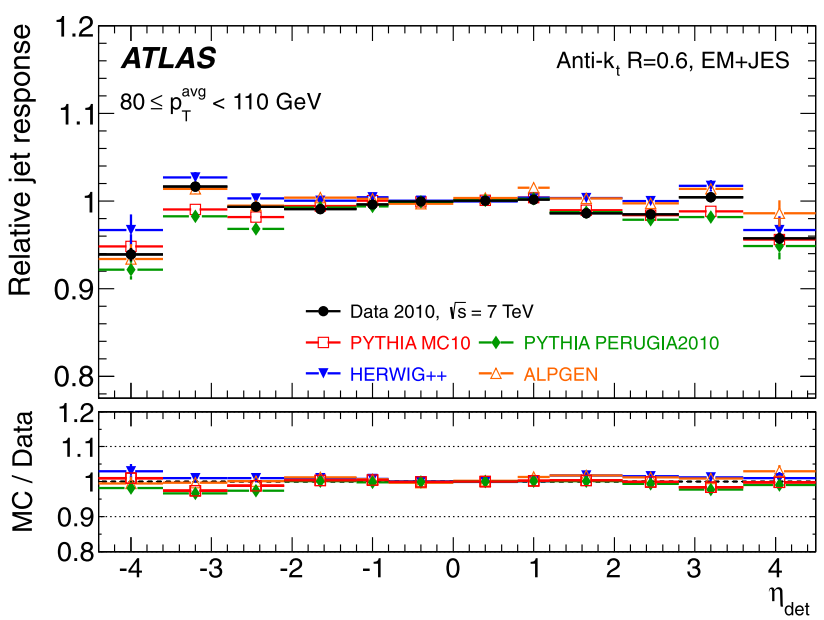

(d) $80 \leq p_{\mathrm{T}}^{\text {avg }}<110 \mathrm{GeV}$

of each figure shows the ratio of Monte Carlo simulation to data. The results are normalized such that average relative response in $|\eta|<0.8$ equals unity, both for data and Monte Carlo simulation. Only statistical uncertainties are shown

ditional vertex at the EM+JES scale. Since the jet pile-up offset was about $500 \mathrm{MeV}$ before correction, even with this conservative estimate the application of the offset correction represents an improvement of a factor of five obtained over the systematic bias associated with pile-up effects on the calorimeter jet $p_{\mathrm{T}}$ without pile-up correction.

The full offset correction shows reasonable closure, i.e. little dependence on $N_{\mathrm{PV}}$ after pile-up offset correction, when using the actual constituent tower multiplicity directly (tower-based) and a slight under-correction using the average constituent multiplicity in the jet (jet-based). ${ }^{18}$ Fig-

\footnotetext{
${ }^{18}$ See Sect. 8.1.3 for definition of tower-based and jet-based pile-up offset corrections.
} 


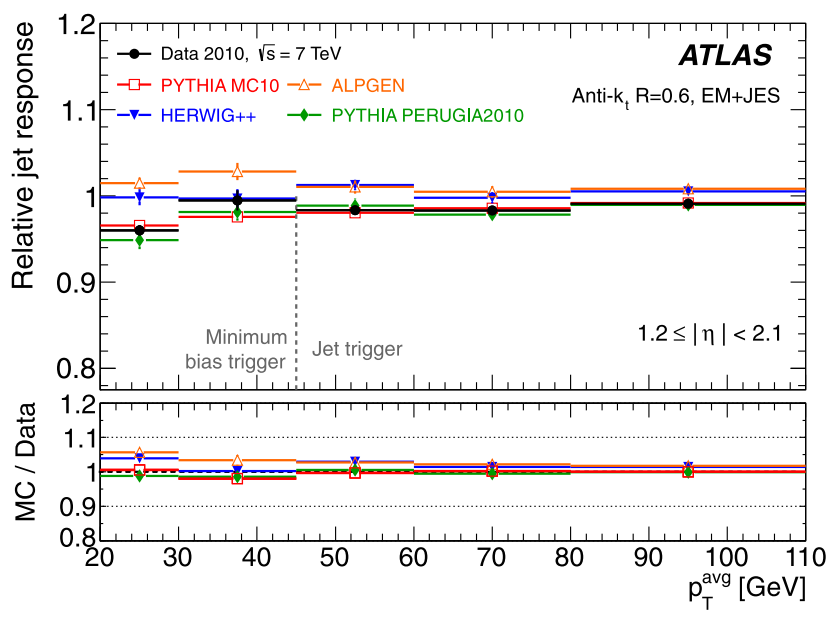

(a) $1.2 \leq|\eta|<2.1$

Fig. 16 Relative jet response, $1 / c$, of anti- $k_{t}$ jets with $R=0.6$ as a function of $p_{\mathrm{T}}^{\text {avg }}$ found using the matrix $\eta$-intercalibration method for (a) $1.2 \leq|\eta|<2.1$ and (b) $3.6 \leq|\eta|<4$.5. For $p_{\mathrm{T}}^{\text {avg }}<45 \mathrm{GeV}$, the data are collected using the minimum bias trigger stream. For

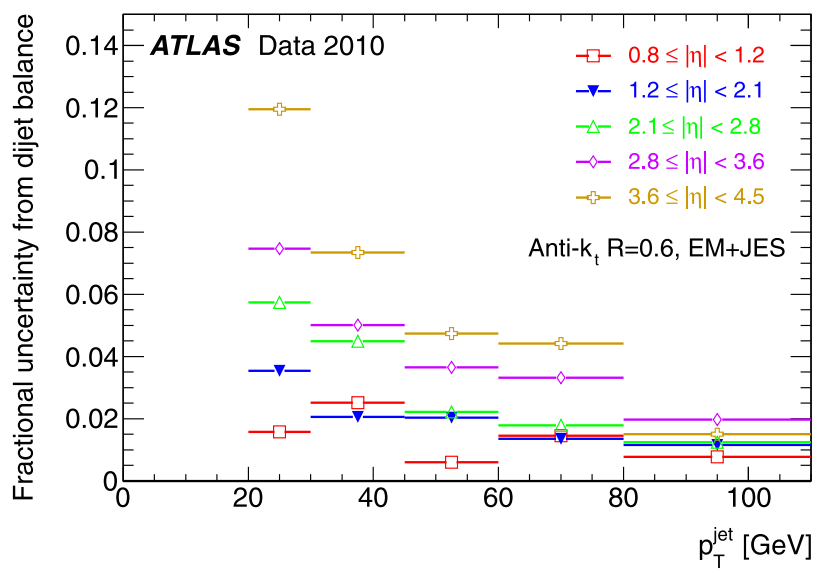

(a) Transverse momentum

Fig. 17 Fractional response uncertainty for anti- $k_{t}$ jets with $R=0.6$ calibrated with the EM+JES scheme as obtained from the dijet balance

ure 19a shows the tower-based correction applied to tower jets at the EM scale as a function of the reconstructed vertex multiplicity. The tower-based correction exhibits a closure consistent with zero slope in $E_{\mathrm{T}}^{\mathrm{jet}}$ as a function of $N_{\mathrm{PV}}$. Figures $19 \mathrm{~b}$ and $19 \mathrm{c}$ show the jet-based correction applied to both tower jets and topo-cluster jets, respectively. The use of the jet-based offset correction slightly under-corrects for the effect of pile-up for jets constructed from both towers and topo-clusters.

The implication of this observation is two-fold:

1. There is no significant difference in the sensitivity of topo-cluster jets to pile-up as compared to tower jets.

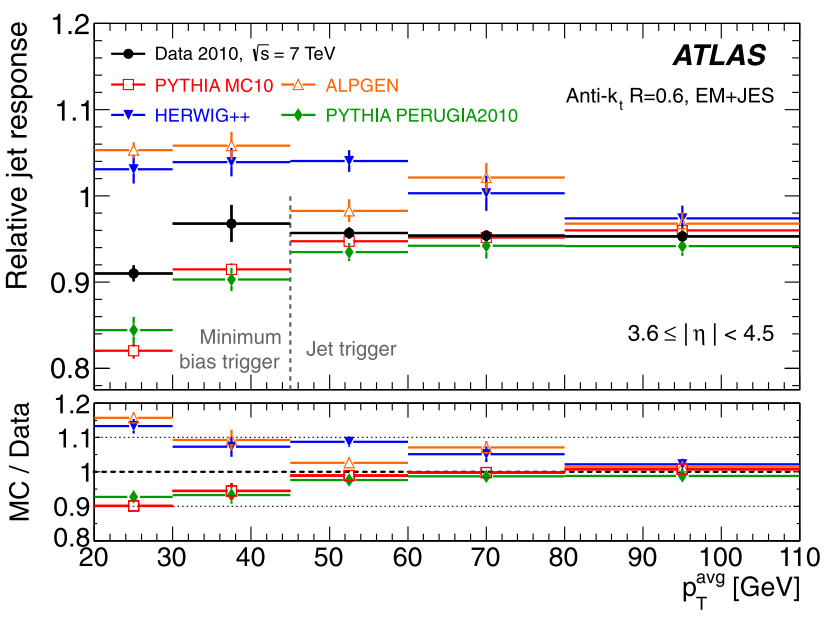

(b) $3.6 \leq|\eta|<4.5$

$p_{\mathrm{T}}^{\mathrm{avg}}>45 \mathrm{GeV}$, the data are collected using the calorimeter trigger stream. The lower part of each figure shows the ratio of Monte Carlo simulation to data. Only statistical uncertainties are shown

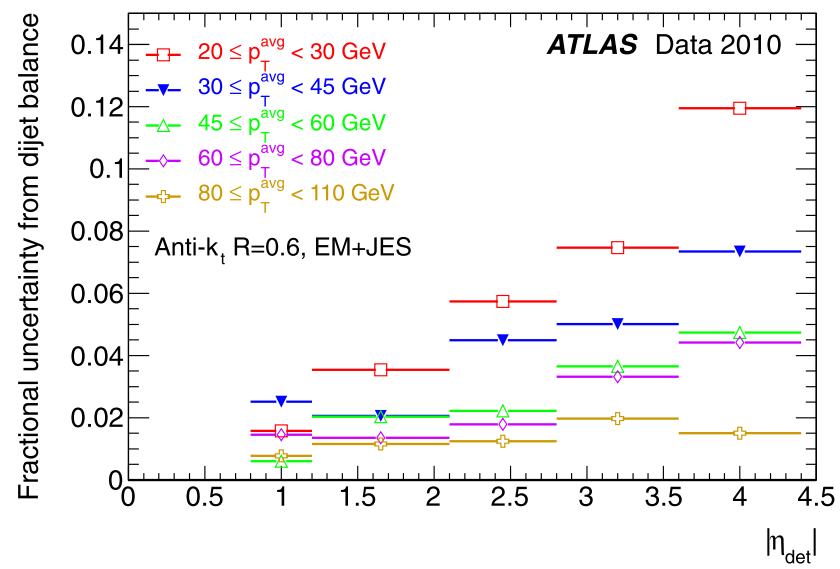

(b) Pseudorapidity

in situ technique as a function of $p_{\mathrm{T}}^{\text {jet }}$ for various $|\eta|$-regions of the calorimeter (a) and as a function of $|\eta|$ in various $p_{\mathrm{T}}^{\text {jet }}$ bins (b)

2. There is a systematic underestimation of the average tower multiplicity in jets due to the effect of pile-up or due to differences in the jet transverse energy distribution in the derivation and the validation of the pile-up correction.

\subsubsection{Jet offset correction uncertainties}

The contributions to the jet offset correction uncertainty are estimated from studies that account for:

1. The effect of variations of the trigger selection on the measured non-noise-suppressed tower energy distribution that is input to the offset correction. 


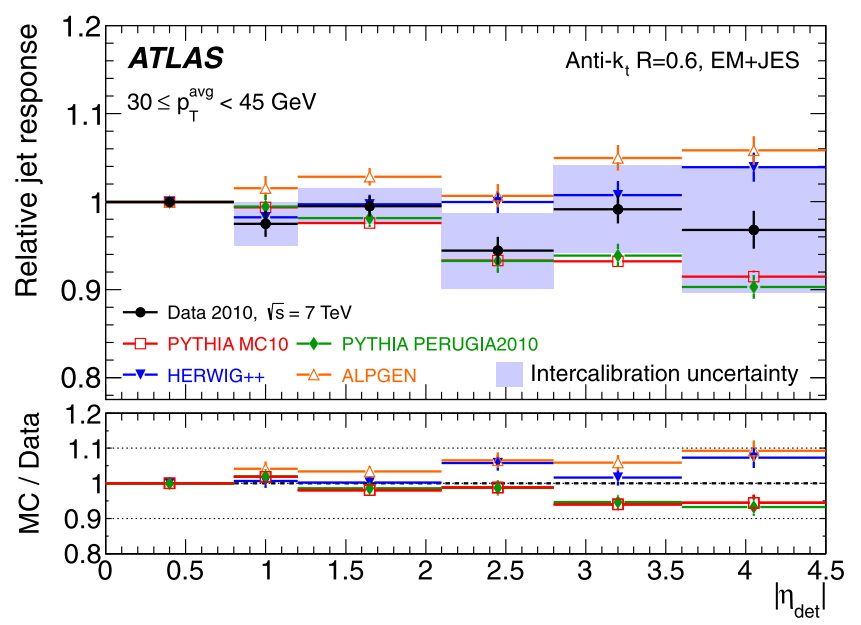

(a) $30 \leq p_{\mathrm{T}}^{\text {avg }}<45 \mathrm{GeV}$

Fig. 18 Average jet response for anti- $k_{t}$ jets with $R=0.6$ calibrated with the EM+JES scheme measured relative to a central reference jet within $|\eta|<0.8$ using the matrix method in data and various Monte Carlo generator samples as a function of $|\eta|$ for $p_{\mathrm{T}}^{\text {avg }}$ in the ranges 30-

2. The variation with $p_{\mathrm{T}}^{\text {jet }}$ and $N_{\mathrm{PV}}$ of the tower multiplicity in jets based on topo-clusters. ${ }^{19}$

3. The variation of the offset correction derived from track jets as a function of the number of primary vertices for various values of track jet $p_{\mathrm{T}}$.

4. The residual dependence of the corrected calorimeter jet energy for calorimeter jets matched to track jets as a function of the number of primary vertices.

The JES uncertainty is estimated by adding all uncertainties in quadrature, including the one from the non-closure of the correction. The track jet method can be used only up to $|\eta|=1.9$, since a full coverage of the jet area by the tracking acceptance is needed. Beyond $|\eta|=1.9$, the dijet balance method detailed in Sect. 9.6 is used. This approach compares the relative jet response in events with only one reconstructed vertex with the response measured in events with several reconstructed vertices. The dijet balance method yields uncertainties similar to those intrinsic to the method also in the case of $|\eta|<1.9$.

Table 3 gives the contribution of each source of systematic uncertainty in percent to the average offset correction which is given in Table 4.

For jets based on towers the total systematic uncertainty is significantly larger than the validation of the correction using track jets indicate ( $2 \%$ in Table 3 ). The larger of the two individual uncertainties (21\% and $16 \%$ in Table 3 ) is therefore adopted. This yields for the correction of the aver-

\footnotetext{
${ }^{19}$ This is determined from the variation in tower multiplicity for $N_{\mathrm{PV}}=1$ in jets matched to track jets with $25 \leq p_{\mathrm{T}}<30 \mathrm{GeV}$ as compared to $N_{\mathrm{PV}}=4$ in track jets with $35 \leq p_{\mathrm{T}}<40 \mathrm{GeV}$.
}

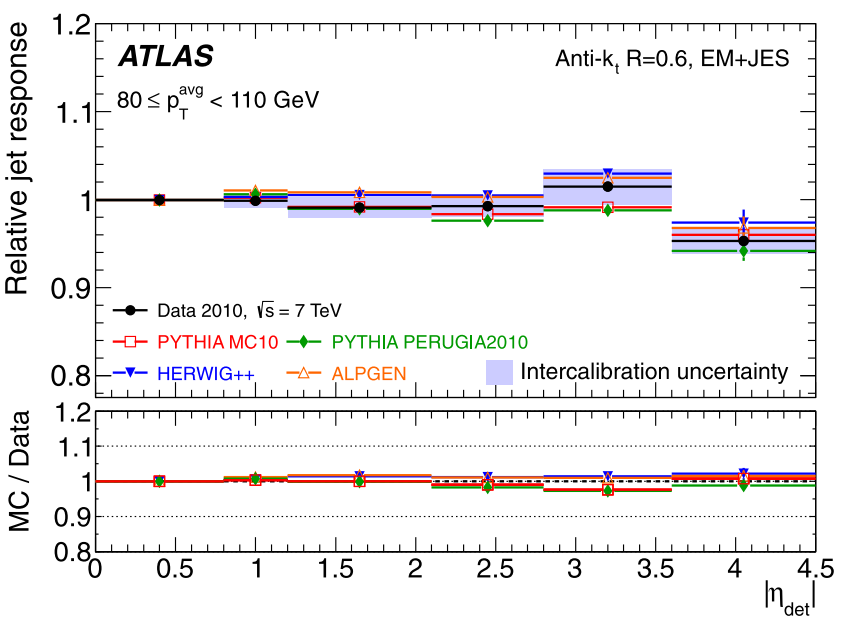

(b) $80 \leq p_{\mathrm{T}}^{\text {avg }}<110 \mathrm{GeV}$

$45 \mathrm{GeV}$ (a) and 80-110 GeV (b). The resulting systematic uncertainty component is shown as a shaded band around the data points. The errors bars on the data points only show the statistical uncertainties

age offset of $0.48 \mathrm{GeV} /$ vertex (see Table 4 ) the uncertainty $\delta\left(\mathcal{O}^{\text {tower-based }}\right)=100 \mathrm{MeV}$ per vertex..$^{20}$ The resulting total uncertainty is a factor of five smaller than the bias attributable to pile-up $(\approx 500 \mathrm{MeV}$ per vertex) even with this conservative systematic uncertainty estimation.

The offset correction for jets based on topo-clusters receives an additional uncertainty due to the average tower multiplicity approximation. This contribution is estimated to introduce a $20 \%$ uncertainty in the constituent tower multiplicity by comparing jets in events with $N_{\mathrm{PV}}=1-3$ and for the five highest $p_{\mathrm{T}}^{\text {track jet }}$-bins. This estimation translates directly into a $20 \%$ uncertainty on the jet-based offset. The resulting systematic uncertainty on jets corrected by the offset correction is estimated to be $\delta\left(\mathcal{O}^{\text {jet-based }}\right) \approx 160 \mathrm{MeV}$ per vertex; a factor of three smaller than the bias due to pile-up.

Figure 20 shows the relative uncertainty due to pile-up in the case of two measured primary vertices. In this case, the uncertainty due to pile-up for central jets with $p_{\mathrm{T}}=20 \mathrm{GeV}$ and pseudorapidity $|\eta| \leq 0.8$ is about $1 \%$, while it amounts to about $2 \%$ for jets with pseudorapidity $2.1 \leq|\eta|<2.8$ and to less than $2.5 \%$ for all jets with $|\eta| \leq 4.5$. In the case of three primary vertices, $N_{\mathrm{PV}}=3$, the pile-up uncertainty is approximately twice that of $N_{\mathrm{PV}}=2$, and with four primary vertices the uncertainty for central, endcap and forward jets is less than $3 \%, 6 \%$ and $8 \%$, respectively. The relative uncertainty due to pile-up for events with up to five

\footnotetext{
${ }^{20}$ Using twice the RMS of the variation in the closure test yields a similar value.
} 


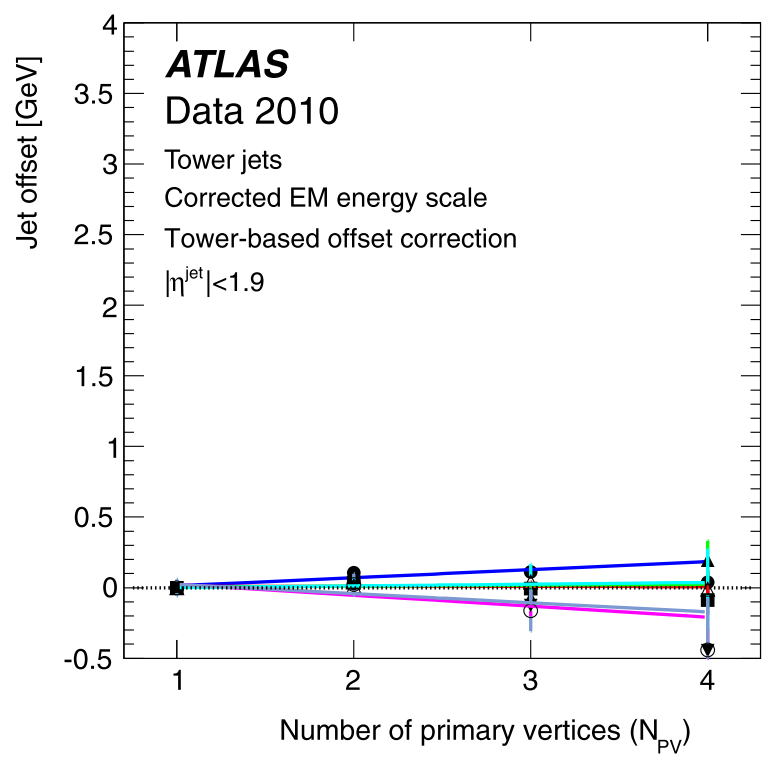

(a) Tower jets (tower-based correction)

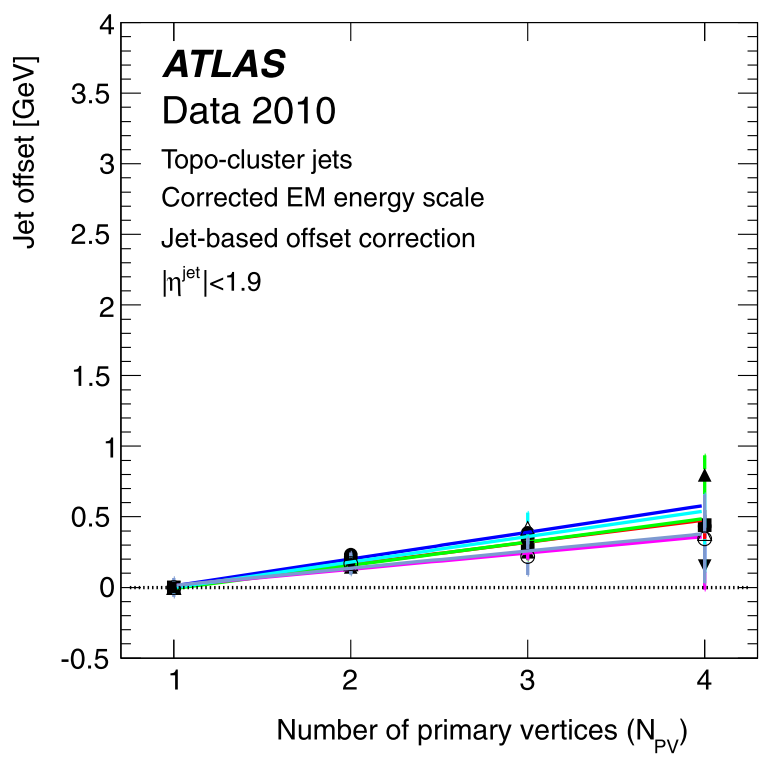

(c) Topo-cluster jets (jet-based correction)

Fig. 19 Jet residual offset measured at the EM scale after pile-up correction using the most probable value $E_{\mathrm{T}}^{\text {jet }}$ obtained from a fit to a Landau+Gauss distribution for various bins in track jet transverse momentum $\left(p_{\mathrm{T}}^{\text {track jet }}\right)$ as a function of the primary vertex multiplicity: tower jets corrected with tower-based offset correction (using the actual number constituent towers) (a), tower jets corrected with the jet-based offset correction (using the average number of constituent

additional collisions becomes less than $1 \%$ for all jets with $p_{\mathrm{T}}^{\text {jet }}>200 \mathrm{GeV}$. The pile-up uncertainty needs to be added separately to the estimate of the total jet energy scale uncertainty detailed in Sect. 9.8.

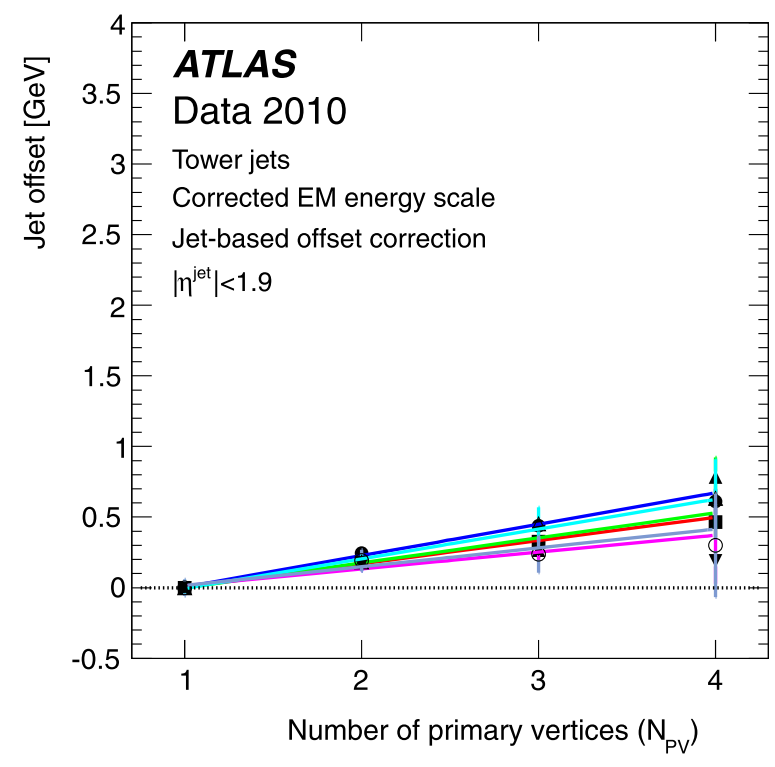

(b) Tower jets (jet-based correction)

$$
\begin{aligned}
& \longrightarrow 20<p_{T}^{\text {track jet }}<25 \mathrm{GeV} \\
& \longrightarrow-25<p_{T}^{\text {track jet }}<30 \mathrm{GeV} \\
& \square 30<p_{T}^{\text {track jet }}<35 \mathrm{GeV} \\
& \longrightarrow \quad 35<p_{T}^{\text {track jet }}<40 \mathrm{GeV} \\
& \triangle 40<p_{T}^{\text {track jet }}<45 \mathrm{GeV} \\
& \square 45<p_{T}^{\text {track jet }}<50 \mathrm{GeV}
\end{aligned}
$$

towers) (b) and topo-cluster jets corrected with the jet-based offset correction (using the average number of equivalent constituent towers) (c). The axis ranges are identical to Fig. 7 for ease of comparison. The jet offset is given for anti- $k_{t}$ jets at the EM scale with $R=0.6$. Only the statistical uncertainties of the fit results are shown. The lines are fits using a linear function

\subsubsection{Out-of-time pile-up}

The effect of additional proton-proton collisions from previous bunch crossings within trains of consecutive bunches 
Table 3 Summary of systematic uncertainties associated with the offset correction for both the tower-based offset applied jet-by-jet to tower jets and the jet-level offset applied to topo-cluster jets. The uncertainty is expressed as a percentage of the average offset correction, shown in Table 4. The tower-based correction uses the actual number of towers for each jet, while the jet-based offset uses an average tower multiplicity (see Sect. 8.1.1)

\begin{tabular}{llll}
\hline Systematic & Tower-based offset & Jet-based offset & Comments \\
\hline Trigger selection & $16 \%$ & $16 \%$ & MBTS vs. Jet triggers \\
Tower multiplicity variation & - & $20 \%$ & $\left\langle N_{\text {towers }}^{\text {jet }}\right\rangle$ vs. $p_{\mathrm{T}}^{\text {track jet }}$ and $N_{\mathrm{PV}}$ \\
$p_{\mathrm{T}}^{\text {track jet }}$ variation & $21 \%$ & $22 \%$ & Variation of $100 \mathrm{MeV} / \mathrm{vertex}$ \\
Total (quadrature sum) & $26 \%$ & $34 \%$ & Assumes uncorrelated errors \\
Closure of tack jet validation & $2 \%$ & $35 \%$ & Determined from average \\
\hline
\end{tabular}

Table 4 Variation of the calorimeter $E_{\mathrm{T}}^{\text {jet }}$ with pile-up for several bins in track jet $p_{\mathrm{T}}$. Slopes are given in GeV/vertex at the electromagnetic scale for each primary vertex from additional proton-proton collisions in the event, and represent the slope of the jet offset before and after the tower-based offset correction. Tower-based corrections are applied to tower jets and jet-based corrections are applied to topo-cluster jets. The reported uncertainties are purely statistical

\begin{tabular}{|c|c|c|c|c|}
\hline \multirow[t]{2}{*}{ Track jet $p_{\mathrm{T}}$} & \multicolumn{2}{|c|}{ Tower jets $[\mathrm{GeV} / \mathrm{vertex}]$} & \multicolumn{2}{|c|}{ Topo-cluster jets [GeV/vertex] } \\
\hline & Before & After & Before & After \\
\hline $20-25 \mathrm{GeV}$ & $0.55 \pm 0.02$ & $0.06 \pm 0.02$ & $0.50 \pm 0.02$ & $0.19 \pm 0.02$ \\
\hline 25-30 GeV & $0.47 \pm 0.02$ & $0.00 \pm 0.02$ & $0.47 \pm 0.02$ & $0.16 \pm 0.02$ \\
\hline 30-35 GeV & $0.49 \pm 0.03$ & $0.01 \pm 0.03$ & $0.47 \pm 0.03$ & $0.17 \pm 0.03$ \\
\hline $35-40 \mathrm{GeV}$ & $0.42 \pm 0.03$ & $-0.08 \pm 0.03$ & $0.41 \pm 0.03$ & $0.12 \pm 0.03$ \\
\hline $40-45 \mathrm{GeV}$ & $0.51 \pm 0.05$ & $0.01 \pm 0.05$ & $0.48 \pm 0.05$ & $0.18 \pm 0.05$ \\
\hline $45-50 \mathrm{GeV}$ & $0.42 \pm 0.06$ & $-0.07 \pm 0.06$ & $0.41 \pm 0.06$ & $0.12 \pm 0.06$ \\
\hline Average & $0.48 \pm 0.02$ & $-0.01 \pm 0.02$ & $0.46 \pm 0.02$ & $0.16 \pm 0.02$ \\
\hline
\end{tabular}

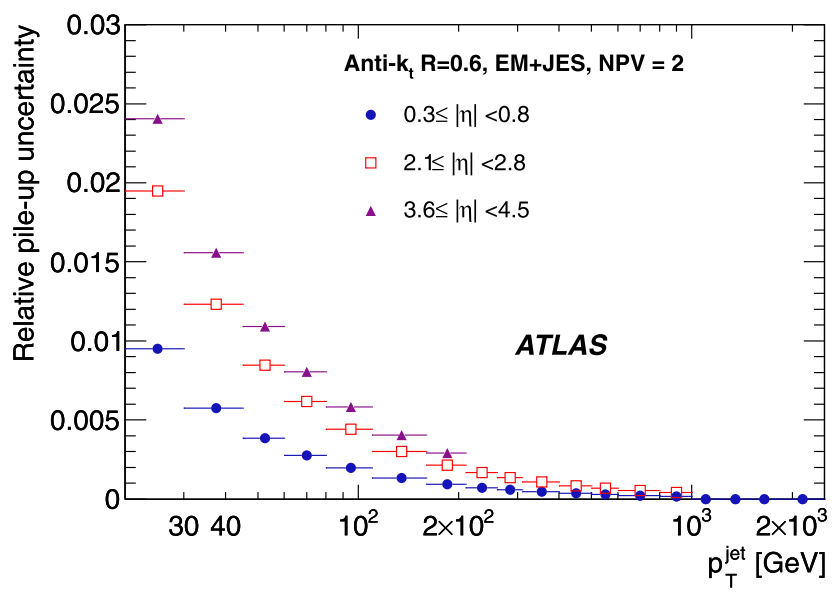

Fig. 20 Relative JES uncertainty from pile-up for anti- $k_{t}$ jets with $R=0.6$ in the case of two measured primary vertices, $N_{\mathrm{PV}}=2$, for central $(0.3 \leq|\eta|<0.8$, full circles $)$, endcap $(2.1 \leq|\eta|<2.8$, open squares $)$ and forward $(3.6 \leq|\eta|<4.5$, full triangles $)$ jets as a function of jet $p_{\mathrm{T}}^{\text {jet }}$

(out-of-time pile-up) has been studied separately. The effect is found to be negligible in the 2010 data.

\subsubsection{Pile-up corrections applied to jet shape measurements}

The measurement of internal jet properties like the energy flow inside jets can be made considerably more difficult in the presence of additional proton-proton collisions. The applicability of the tower-based offset presented in Sect. 9.7.1 to correct the mean jet energy can also be tested on the internal jet shape measurements.

The offset correction is applied to the measurement of the differential jet shape for $R=0.6$ tower jets, as described in Ref. [3].

The jet shape variable used, $\rho^{a}(r)$, is defined as:

$$
\begin{aligned}
\rho^{a}(r)= & \frac{1}{\pi\left[(r+\delta r / 2)^{2}-(r-\delta r / 2)^{2}\right]} \\
& \cdot\left\langle\frac{p_{\mathrm{T}}\left(r-\frac{\delta r}{2}, r+\frac{\delta r}{2}\right)}{p_{\mathrm{T}}(0,0.7)}\right\rangle,
\end{aligned}
$$

where $r=\sqrt{(d \eta)^{2}+(d \phi)^{2}}$ is the distance of the jet constituents to the jet four-momentum vector and the angled brackets denote an average over all jets, $p_{\mathrm{T}}(b, c)$ is the sum of the $p_{\mathrm{T}}$ of all towers with an opening angle $b \leq \Delta R<c$ with respect to the jet axis, and $\delta r=0.1$. 
This definition differs from the canonical jet shape variable $\rho(r)$ [3] in two important ways. First, by normalising to area, the variable measures an energy density. Therefore, $\rho^{a}(r)$ will approach an asymptotic value far from the jet axis. The level of the asymptote is related to the energy density in the calorimeter and is measurably higher in events with pile-up. Second, all towers are included in the definition. This allows an examination of energy outside of the jet cone, in some sense measuring "energy flow" around the jet axis.

Figure 21 depicts $\rho^{a}(r)$ with and without a correction of the tower constituent energy for the mean energy induced by pile-up interactions as representative examples. In events with two (three) reconstructed vertices, differences in this particular jet shape variable of up to $35 \%$ (70 \%) just outside the jet $(r>0.6)$ and $20 \%(40 \%)$ near the nominal jet radius $(r=0.6)$ are observed. The bulk of the shape $(0.1 \leq r<0.6)$ is restored to that observed in events with only a single interaction, in both the core $(r<0.1)$ and the periphery $(r>0.6)$ of the jet.

The results demonstrate that the tower-based offset correction can be applied on a fine scale granularity and is valid both inside and near jets.

\subsection{Summary of jet energy scale systematic uncertainties}

The total jet energy scale uncertainty is derived by considering all the individual contributions described in the previous sections. In the central region $(|\eta|<0.8)$, the estimate proceeds as follows:

1. For each $p_{\mathrm{T}}^{\text {jet }}$ and $\eta$ bin, the uncertainty due to the calibration procedure is calculated as described in Sect. 9.2 for both jet energy and $p_{\mathrm{T}}$ response. For each bin, the maximum deviation from unity between the energy and $p_{\mathrm{T}}$ response is taken as the final non-closure uncertainty.

2. The calorimeter response uncertainty is estimated as a function of jet $\eta$ and $p_{\mathrm{T}}$ from the propagation of single particle uncertainties to the jets, as detailed in Sect. 9.3.

3. Sources of uncertainties estimated using Monte Carlo samples with a systematic variation are accounted as follows:

(a) the response in the test sample $\mathcal{R}_{\text {var }}$ and the response in the nominal sample $\mathcal{R}_{\text {nom }}$ is considered as a starting point for the estimate of the JES uncertainty. The deviation of this ratio from unity is defined as:

$$
\Delta_{\mathrm{JES}}\left(p_{\mathrm{T}}^{\mathrm{jet}}, \eta\right)=\left|1-\frac{\mathcal{R}_{\mathrm{var}}\left(p_{\mathrm{T}}^{\mathrm{jet}}, \eta\right)}{\mathcal{R}_{\mathrm{nom}}\left(p_{\mathrm{T}}^{\mathrm{jet}}, \eta\right)}\right| .
$$

This deviation is calculated from both the energy and $p_{\mathrm{T}}$ response, leading to $\Delta_{\mathrm{JES}}^{\mathrm{E}}\left(p_{\mathrm{T}}^{\mathrm{jet}}, \eta\right)$ for the deviation in the energy response, and to $\Delta_{\mathrm{JES}}^{p_{\mathrm{T}}}\left(p_{\mathrm{T}}^{\text {jet }}, \eta\right)$ for the deviation in the transverse momentum response.

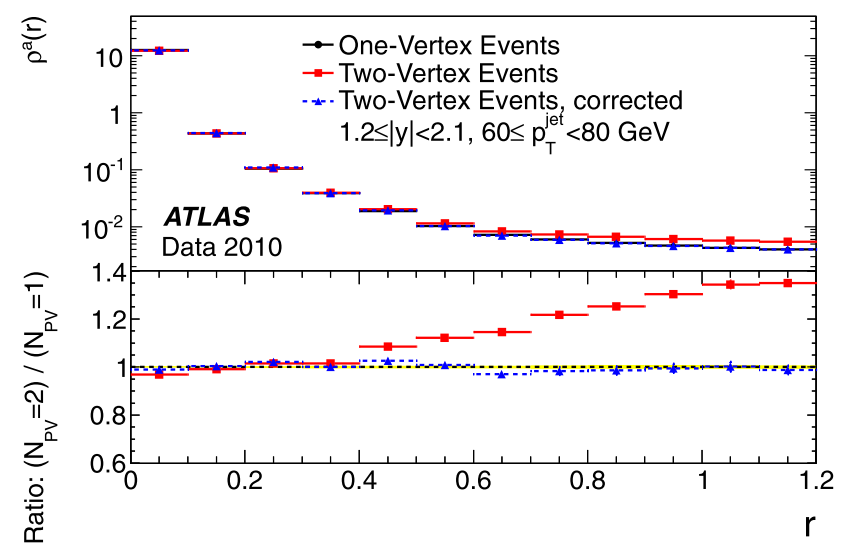

(a) Comparison of $N_{\mathrm{PV}}=1$ and $N_{\mathrm{PV}}=2$

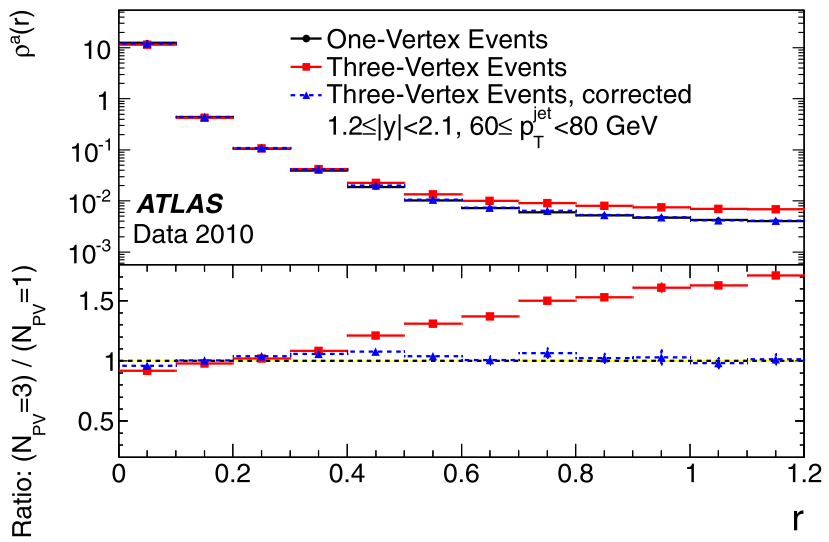

(b) Comparison of $N_{\mathrm{PV}}=1$ and $N_{\mathrm{PV}}=3$

Fig. 21 Measured sum $p_{\mathrm{T}}$ in annuli around the jet axis, divided by the total $p_{\mathrm{T}}$ around the jet within $\Delta R=0.7$ of the jet axis and normalised by the area of each annulus as a function of the distance of the jet constituent to the jet axis. The shapes of jets in the rapidity range $1.2 \leq|y|<2.1$ are compared, before and after the offset corrections, in events with one and two reconstructed vertices (a), and one and three reconstructed vertices (b). The corrected distribution is also shown (full triangles). Note that the single vertex data (full circles) are partially hidden behind the corrected multi-vertex data. Anti- $k_{t}$ jets with $R=0.6$ reconstructed from calorimeter towers are used and calibrated with the EM+JES scheme

(b) The larger $\Delta_{\text {JES }}$ in each bin derived from the jet energy or transverse momentum response is considered as the contribution to the final JES systematic uncertainty due to the specific systematic effect:

$$
\Delta_{\mathrm{JES}}\left(p_{\mathrm{T}}^{\mathrm{jet}},|\eta|\right)=\max \left(\Delta_{\mathrm{JES}}^{\mathrm{E}}\left(p_{\mathrm{T}}^{\mathrm{jet}}, \eta\right), \Delta_{\mathrm{JES}}^{p_{\mathrm{T}}}\left(p_{\mathrm{T}}^{\mathrm{jet}}, \eta\right)\right) .
$$

4. The estimate of the uncertainty contributions due to additional material in the inner detector and overall additional dead material are estimated as described in the previous step. These uncertainties are then scaled by the average fraction of particles forming the jet that have $p<20 \mathrm{GeV}$ 
(for the inner detector distorted geometry) and by the average fraction of particles outside the kinematic range of the single hadron response in situ measurements (for the overall distorted geometry).

For each $\left(p_{\mathrm{T}}^{\mathrm{jet}}, \eta\right)$-bin, the uncertainty contributions from the calorimeter, the jet calibration non-closure, and systematic Monte Carlo simulation variations are added in quadrature.

For pseudorapidities beyond $|\eta|>0.8$, the $\eta$-intercalibration contribution is estimated for each pseudorapidity bin in the endcap region as detailed in Sect. 9.6.6. The pseudorapidity intercalibration contribution is added in quadrature to the total JES uncertainty determined in the $0.3 \leq|\eta|<$ 0.8 region to estimate the JES uncertainty for jets with $|\eta|>$ 0.8 , with the exception of the non-closure term that is taken from the specific $\eta$-region. For low $p_{\mathrm{T}}^{\text {jet }}$, this choice leads to partially double counting the contribution from the dead material uncertainty, but it leads to a conservative estimate in a region where it is difficult to estimate the accuracy of the material description.

The contribution to the uncertainty due to additional proton-proton interactions described in Sect. 9.7 is added separately, depending on the number of primary vertices in the event. In the remainder of the section only the uncertainty for a single proton-proton interaction is shown in detail.

Figure 22 shows the final fractional jet energy scale systematic uncertainty and its individual contributions as a function of $p_{\mathrm{T}}^{\text {jet }}$ for three selected $\eta$ regions. The fractional JES uncertainty in the central region amounts to $2 \%$ to $4 \%$ for $p_{\mathrm{T}}^{\text {jet }}<60 \mathrm{GeV}$, and it is between $2 \%$ and $2.5 \%$ for $60 \leq$ $p_{\mathrm{T}}^{\text {jet }}<800 \mathrm{GeV}$. For jets with $p_{\mathrm{T}}^{\text {jet }}>800 \mathrm{GeV}$, the uncertainty ranges from $2.5 \%$ to $4 \%$. The uncertainty amounts to up to $7 \%$ and $3 \%$, respectively, for $p_{\mathrm{T}}^{\text {jet }}<60 \mathrm{GeV}$ and $p_{\mathrm{T}}^{\mathrm{jet}}>60 \mathrm{GeV}$ in the endcap region, where the central uncertainty is taken as a baseline and the uncertainty due to the intercalibration is added. In the forward region, a $13 \%$ uncertainty is assigned for $p_{\mathrm{T}}^{\mathrm{jet}}=20 \mathrm{GeV}$. The increase in the uncertainty is dominated by the modelling of the soft physics in the forward region that is accounted for in the $\eta$ intercalibration contribution. This uncertainty contribution is estimated conservatively.

Table 5 presents a summary of the maximum uncertainties in the different $\eta$ regions for anti- $k_{t}$ jets with $R=0.6$ and with $p_{\mathrm{T}}^{\text {jet }}$ of $20 \mathrm{GeV}, 200 \mathrm{GeV}$ and $1.5 \mathrm{TeV}$ as examples.

The same study has been repeated for anti- $k_{t}$ jets with distance parameter $R=0.4$, and the estimate of the JES uncertainty is comparable to that obtained for anti- $k_{t}$ jets with $R=0.6$. The JES uncertainty for anti- $k_{t}$ jets with $R=0.4$ is between $\approx 4 \%(8 \%, 14 \%)$ at low $p_{\mathrm{T}}^{\text {jet }}$ and $\approx 2.5 \%-3 \%$

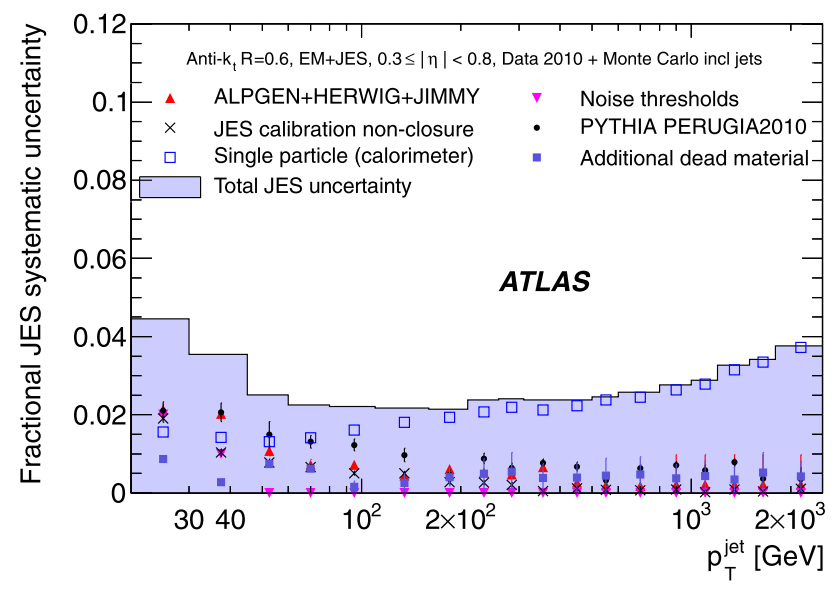

(a) $0.3 \leq|\eta|<0.8$

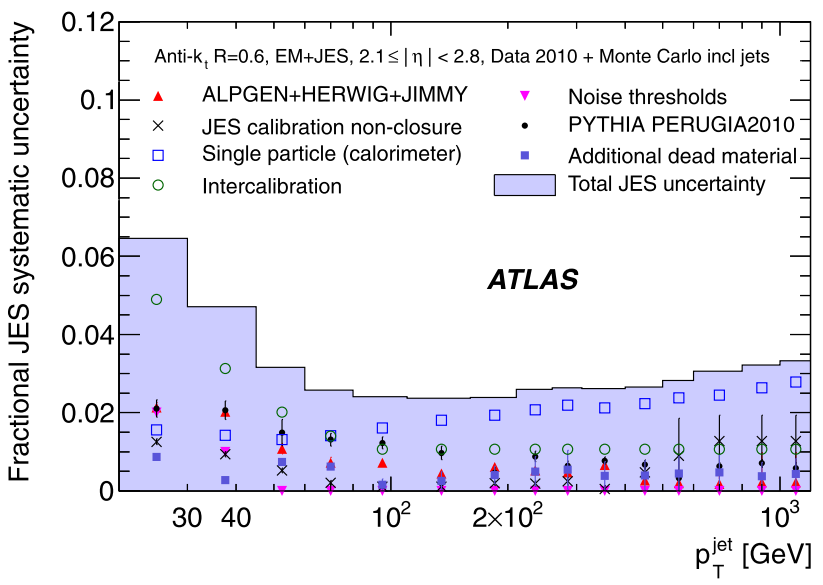

(b) $2.1 \leq|\eta|<2.8$

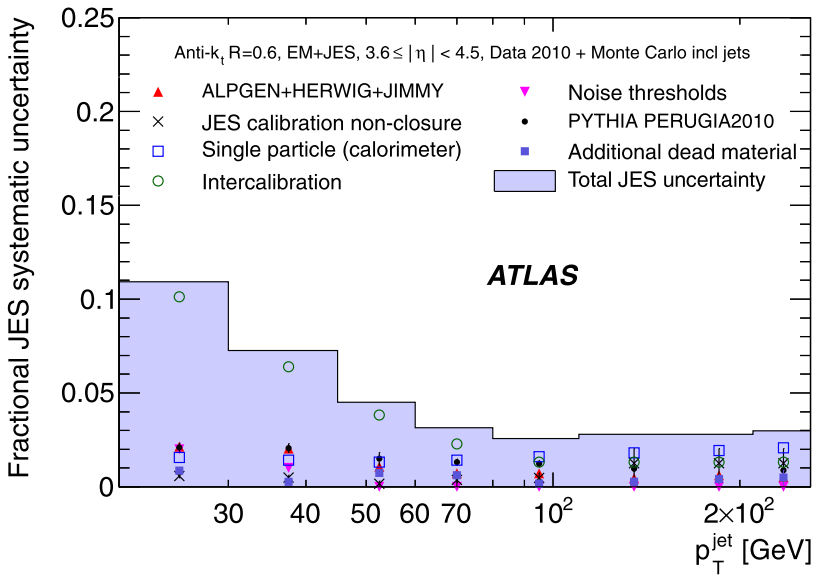

(c) $3.6 \leq|\eta|<4.5$

Fig. 22 Fractional jet energy scale systematic uncertainty as a function of $p_{\mathrm{T}}^{\text {jet }}$ for jets in the pseudorapidity region $0.3 \leq|\eta|<0.8$ in the calorimeter barrel (a), $2.1 \leq|\eta|<2.8$ in the calorimeter endcap (b), and in the forward pseudorapidity region $3.6 \leq|\eta|<4.5$ (c). The total uncertainty is shown as the solid light shaded area. The individual sources are also shown together with uncertainties from the fitting procedure if applicable 
Table 5 Summary of the maximum EM+JES jet energy scale systematic uncertainties for different $p_{\mathrm{T}}^{\text {jet }}$ and $\eta$ regions from Monte Carlo simulation based study for anti- $k_{t}$ jets with $R=0.6$

\begin{tabular}{lccc}
\hline$\eta$ region & \multicolumn{3}{c}{ Maximum fractional JES Uncertainty } \\
\cline { 2 - 4 } & $p_{\mathrm{T}}^{\text {jet }}=20 \mathrm{GeV}$ & $200 \mathrm{GeV}$ & $1.5 \mathrm{TeV}$ \\
\hline $0 \leq|\eta|<0.3$ & $4.6 \%$ & $2.3 \%$ & $3.1 \%$ \\
$0.3 \leq|\eta|<0.8$ & $4.5 \%$ & $2.2 \%$ & $3.3 \%$ \\
$0.8 \leq|\eta|<1.2$ & $4.4 \%$ & $2.3 \%$ & $3.3 \%$ \\
$1.2 \leq|\eta|<2.1$ & $5.4 \%$ & $2.4 \%$ & $3.4 \%$ \\
$2.1 \leq|\eta|<2.8$ & $6.5 \%$ & $2.5 \%$ & \\
$2.8 \leq|\eta|<3.2$ & $7.9 \%$ & $3.0 \%$ & \\
$3.2 \leq|\eta|<3.6$ & $8.1 \%$ & $3.0 \%$ & \\
$3.6 \leq|\eta|<4.5$ & $10.9 \%$ & $2.9 \%$ & \\
\hline
\end{tabular}

Table 6 Summary of the maximum EM+JES jet energy scale systematic uncertainties for different $p_{\mathrm{T}}^{\text {jet }}$ and $\eta$ regions from Monte Carlo simulation based study for anti- $k_{t}$ jets with $R=0.4$

\begin{tabular}{llll}
\hline$\eta$ region & \multicolumn{3}{c}{ Maximum fractional JES Uncertainty } \\
\cline { 2 - 4 } & $p_{\mathrm{T}}^{\text {jet }}=20 \mathrm{GeV}$ & $200 \mathrm{GeV}$ & $1.5 \mathrm{TeV}$ \\
\hline $0 \leq|\eta|<0.3$ & $4.1 \%$ & $2.3 \%$ & $3.1 \%$ \\
$0.3 \leq|\eta|<0.8$ & $4.3 \%$ & $2.4 \%$ & $3.3 \%$ \\
$0.8 \leq|\eta|<1.2$ & $4.4 \%$ & $2.5 \%$ & $3.4 \%$ \\
$1.2 \leq|\eta|<2.1$ & $5.3 \%$ & $2.6 \%$ & $3.5 \%$ \\
$2.1 \leq|\eta|<2.8$ & $7.4 \%$ & $2.7 \%$ & \\
$2.8 \leq|\eta|<3.2$ & $9.0 \%$ & $3.3 \%$ & \\
$3.2 \leq|\eta|<3.6$ & $9.3 \%$ & $3.5 \%$ & \\
$3.6 \leq|\eta|<4.5$ & $13.4 \%$ & $4.9 \%$ & \\
\hline
\end{tabular}

$(2.5 \%-3.5 \%, 5 \%)$ for jets with $p_{\mathrm{T}}>60 \mathrm{GeV}$ in the central (endcap, forward) region, and is summarised in Table 6.

\subsection{Discussion of special cases}

The jet energy scale is derived using the simulated sample of inclusive jets described in Sect. 4.3, with a particular mixture of quark and gluon initiated jets and with a particular selection of isolated jets. The differences in fragmentation between quark and gluon initiated jets and the effect of closeby jets give rise to a topology and flavour dependence of the energy scale. Since the event topology and flavour composition (quark and gluon fractions) may be different in final states other than the considered inclusive jet sample, the dependence of the jet energy response on jet flavour and topology has to be accounted for in physics analyses. The flavour dependence is discussed in more detail in Sect. 18 and an additional uncertainty specific to jets with heavy quark components is discussed in Sect. 20.
The JES systematic uncertainty is derived for isolated jets. ${ }^{21}$ The response of jets as a function of the distance to the closest reconstructed jet needs to be studied and corrected for separately if the measurement relies on the absolute jet energy scale. The contribution to the JES uncertainty from close-by jets also needs to be estimated separately, since the jet response depends on the angular distance to the closest jet. This additional uncertainty can be estimated from the Monte Carlo simulation to data comparison of the $p_{\mathrm{T}}$-ratio between calorimeter jets and matched track jets in inclusive jet events as a function of the isolation radius. This is discussed in more detail in Sect. 17.

\section{Jet energy scale uncertainties validation with in situ techniques for the EM+JES scheme}

The jet energy calibration can be tested in situ using a wellcalibrated object as reference and comparing data to the nominal Pythia Monte Carlo simulation. The following in situ techniques have been used by ATLAS:

\section{Comparison to the momentum carried by tracks asso-} ciated to a jet: The mean transverse momentum sum of tracks that are within a cone with size $R$ provides an independent test of the calorimeter energy scale over the entire measured $p_{\mathrm{T}}^{\text {jet }}$ range within the tracking acceptance. The comparison is done in the jet $\eta$ range $0 \leq|\eta|<2.1$.

2. Direct $p_{\mathrm{T}}$ balance between a photon and a jet: Events with a photon and one jet at high transverse momentum are used to compare the transverse momentum of the jet to that of the photon. To account for effects like soft QCD radiation and energy migrating out of the jet area the data are compared to the Monte Carlo simulation. The comparison is done in the jet $\eta$ range $|\eta|<1.2$ and for photon transverse momenta $25 \leq p_{\mathrm{T}}^{\gamma}<250 \mathrm{GeV}$.

3. Photon $p_{\mathrm{T}}$ balance to hadronic recoil: The photon transverse momentum is balanced against the full hadronic recoil using the projection of the missing transverse momentum onto the photon direction. This method, called missing transverse momentum fraction (MPF) technique, does not explicitly involve a jet algorithm. The comparison is done in the same kinematic region as the direct photon balance method.

4. Balance between a high- $p_{\mathrm{T}}$ jet and low- $p_{\mathrm{T}}$ jet system: If jets at low transverse momentum are wellcalibrated, jets at high transverse momentum can be balanced against a recoil system of low transverse momentum jets. This method can probe the jet energy scale up

\footnotetext{
${ }^{21}$ This choice is motivated by the minor differences observed in the average kinematic jet response of isolated and non-isolated jets in the nominal inclusive jet Monte Carlo sample and by the need to factorise the topology dependence of the close-by jet energy scale uncertainty for final states other than the inclusive jets considered.
} 
to the TeV-regime. The $\eta$ range used for the comparison is $|\eta|<2.8$.

All methods are applied to data and Monte Carlo simulation.

The in situ techniques usually rely on assumptions that are only approximately fulfilled. An example is the assumption that the jet to be calibrated and the reference object are balanced in transverse momentum. This balance can be altered by the presence of additional high- $p_{\text {T }}$ particles. For the determination of the JES uncertainties the modelling of physics effects has to be disentangled from detector effects. This can be studied by systematically varying the event selection criteria. The ability of the Monte Carlo simulation to describe extreme variations of the selection criteria determines the systematic uncertainty in the in situ methods, since physics effects can be suppressed or amplified by these variations.

So far the in situ techniques are used to validate the systematic uncertainty in the jet energy measurement. However, they can also be used to obtain jet energy corrections. This is an interesting possibility when the statistical and systematic uncertainties in the samples studied become smaller than the standard JES uncertainty from the single hadron response. The results of the in situ techniques are discussed in the following sections.

\subsection{Comparison of transverse momentum balance of jets from calorimeter and tracking}

The transverse momentum of each jet can be compared with the total transverse momentum of tracks associated with the jet by means of a geometrical selection. The charged-tototal-momentum ratio defined as

$r_{\text {trk }}=\frac{\left|\sum \vec{p}_{\mathrm{T}}{ }^{\text {track }}\right|}{p_{\mathrm{T}}^{\text {jet }}}$

can be used to test the jet calibration. If all produced particles were pions, the symmetry of QCD under isospin transformation would require that this ratio be $2 / 3$ once the energy is high enough so that the total particle multiplicity is large and the initial isospin of the proton-proton system can be ignored. Production of other particles such as kaons, $\eta$ mesons, and baryons gives different fractions, but their contributions can be calculated using a properly tuned event generator.

Since the tracking system provides a measurement that is independent of the calorimeter, the ratio $r_{\text {trk }}$ can be used to determine the calorimeter jet energy scale. The $r_{\text {trk }}$ distribution is broad but a meaningful calibration does not require very many events, since the statistical uncertainty on the mean scales as $1 / \sqrt{N}$. This calibration can be used for jets confined within the tracking detector coverage. Dominant systematic uncertainties result from the knowledge of the tracking efficiency, variations in the predicted value of $r_{\mathrm{trk}}$ for various generator tunes and loss of tracking efficiency in the dense core of high- $p_{\mathrm{T}}$ jets.

To test the $p_{\mathrm{T}}^{\text {jet }}$ dependence of the jet energy measurement, the double ratio of charged-to-total momentum observed in data to that obtained in Monte Carlo simulation is studied:

$R_{r_{\text {trk }}} \equiv \frac{\left[\left\langle r_{\text {trk }}\right\rangle\right]_{\text {Data }}}{\left[\left\langle r_{\text {trk }}\right\rangle\right]_{\mathrm{MC}}}$

\subsubsection{Jet and track selection}

To ensure that the majority of tracks associated with the jets found in the calorimeter are within the inner detector fiducial volume, jets are required to have $|\eta|<2.1^{22}$ and $p_{\mathrm{T}}^{\text {jet }}>20 \mathrm{GeV}$. To reduce the influence of nearby jets on the measurement, if two jets are separated by a distance $\Delta R<2 R$ then the softer of these two jets is rejected from the analysis.

Tracks with $p_{\mathrm{T}}^{\text {track }}>1 \mathrm{GeV}$ are selected using the criteria detailed in Sect. 6.2. The $p_{\mathrm{T}}^{\text {track }}>1 \mathrm{GeV}$ requirement is intended to select mainly tracks from fragmentation rather than those arising from soft interactions.

Tracks are associated with jets using a geometric algorithm. If the distance $\Delta R_{\text {track,jet between the track and the }}$ jet is less than the distance parameter used in the jet reconstruction $(R=0.4$ or $R=0.6)$, the track is associated to the jet. Track parameters are evaluated at the distance of closest approach to the primary hard-scattering vertex and are not extrapolated to the calorimeter. This simple association algorithm facilitates comparison with charged particles from truth jets whose parameters correspond to those measured at the origin.

\subsubsection{Comparison of data and Monte Carlo simulation}

The jet response validation using the total momentum measured in tracks depends on a comparison of the mean value of $r_{\text {trk }}$ observed in the data to that predicted in the Monte Carlo simulation. It is therefore important to demonstrate that the baseline Monte Carlo generator and simulation provide a reasonable description of the data.

ATLAS has measured the charged particle fragmentation function for jets with $25 \leq p_{\mathrm{T}}^{\text {jet }}<500 \mathrm{GeV}$ and $|\eta|<1.2$

\footnotetext{
${ }^{22}$ Sect. 9.7 discusses "track jets" obtained by running the anti- $k_{t}$ jet algorithm using tracks as input. Those studies are restricted to $|\eta|<1.9$ to avoid bias in the position of the centre of the jet due to tracking inefficiencies. Since the jets in this section are found using calorimeter information, no such bias is present and it is therefore possible to extend the pseudorapidity coverage to $|\eta|<2.1$.
} 
and has compared the measurement with the predictions of several Monte Carlo generators and generator tunes [91].

The jet fragmentation function and the transverse jet profile are compared to various Monte Carlo event generators and tunes. The jet fragmentation function is measured using charged particles with momentum fraction $z$ with respect to the jet momentum $F\left(z, p_{\mathrm{T}}^{\text {jet }}\right)=1 / N_{\text {jet }} d N_{\mathrm{ch}} / d z$.

The growth of the mean charged particle multiplicity with $p_{\mathrm{T}}^{\mathrm{jet}}$ is well modelled by the Monte Carlo simulation. The measured jet fragmentation function agrees well with the Pythia MC10 and the Perugia2010 tunes within the measurement uncertainties. The jet fragmentation function is described by the Pythia tunes. The HeRWIG++ Monte Carlo generator is not consistent with the data.

For observables related to jet properties in the direction transverse to the jet axis the Monte Carlo generators (Herwig and the various PythiA tunes) show reasonable agreement with data, but none of the generators agrees within the experimental uncertainties over the full kinematic range. For instance, the PYTHIA MC10 tune shows an excess of about $10 \%$ in the transverse charged particle distributions close to the jet axis.

These measurements [91] indicate that the PYTHIA MC10 and PERUGIA2010 tunes span the range of fragmentation functions that are consistent with the data. The studies presented here use the MC10 tune to obtain the central values of the Monte Carlo predictions. Systematic uncertainties are assessed from the difference between the MC10 and PERUGIA2010 PYTHIA tunes.

The $r_{\text {trk }}$ distributions used to validate the JES are shown for data and simulation for two typical bins of jet $p_{\mathrm{T}}$ in Figs. 23a and 23b. Agreement between data and simulation is good, although the data distribution is somewhat wider than the Monte Carlo simulation. Figures $23 \mathrm{c}$ and $23 \mathrm{~d}$ show $\left\langle r_{\text {trk }}\right\rangle$ for data and simulation and the average double ratio $R_{r_{\text {trk }}}$, respectively, as a function of $p_{\mathrm{T}}^{\mathrm{jet}}$. Figure $23 \mathrm{~d}$ demonstrates that the measured JES calibration agrees with that predicted by the Monte Carlo simulation to better than $2 \%$ for $p_{\mathrm{T}}^{\text {jet }}>25 \mathrm{GeV}$. Measurements using the minimum bias and jet triggers are consistent for those $p_{\mathrm{T}}^{\text {jet }}$ bins where both triggers are accessible.

\subsubsection{Systematic uncertainties}

The systematic uncertainties associated with the method using the total track momentum to test the JES are discussed below.

Generator model dependence While basic isospin arguments constrain the mean fraction of the jet momentum observed in charged tracks, the prediction for $r_{\text {trk }}$ does depend on details of the physics model used in the Monte Carlo generator. Systematic uncertainties arise from:
1. The parametrisation of the fragmentation function and of the underlying event (which mainly affect the fraction of the momentum carried by particles below the $p_{\mathrm{T}}=1 \mathrm{GeV}$ cut used for this analysis).

2. The model of colour reconnection (which can change the distribution of particles with low momenta).

3. The probability of producing strange quarks and baryons (which are iso-doublets rather than iso-triplets like the pion) and of producing iso-scalars such as the $\eta$.

The size of these uncertainties has been estimated by studying a wide range of PythiA tunes. ${ }^{23} \mathrm{~A}$ list of the PYTHIA tunes studied is given in Table 7.

These studies have been done at the generator level and have been cross-checked using simulated samples when the appropriate tunes were available with full simulation.

The data have also been compared to default tunes of Herwig++ and Herwig+Jimmy. Pythia tune 117, and the default HERWIG++ and HERWIG+JIMMY tunes are not consistent with the measured $f(z)$ distributions. Since these generators do not describe the fragmentation functions measured by ATLAS [91] they are excluded from consideration when determining the systematic uncertainty on the JES measurement.

At low $p_{\mathrm{T}}^{\text {jet }}$, the variations between tunes arise mainly from differences in the hardness of the jet fragmentation, which affects the fraction of charged particles falling below the $1 \mathrm{GeV}$ cut on $p_{\mathrm{T}}^{\text {track }}$. In general, PYTHIA tunes that include the "colour annealing" model of colour reconnection exhibit harder fragmentation than similar tunes without colour annealing. At high $p_{\mathrm{T}}^{\text {jet }}$, differences among tunes are primarily associated with the strangeness and baryon content of the truth jets. Versions of PYTHIA tuned to LEP data (including flavour-dependent fragmentation measurements) using the tuning software PROFESSOR [92] in general show a charged fraction about $1 \%$ higher than the other tunes considered here. Using a conservative approach, the value of systematic uncertainty has been symmetrised around the PYTHIA MC10 baseline tune using the absolute value of the largest deviation of the tunes considered from the baseline.

Inner detector material description The dominant systematic uncertainty on the reconstruction efficiency for isolated tracks is derived from the uncertainty on the description of inner detector material in the simulation. The systematic uncertainty on the efficiency is independent of $p_{\mathrm{T}}^{\text {track }}$ for tracks with $p_{\mathrm{T}}^{\text {track }}>500 \mathrm{MeV}$ but is $\eta$-dependent, ranging from $2 \%$ for $\left|\eta^{\text {track }}\right|<1.3$ to $7 \%$ for $2.3 \leq\left|\eta^{\text {track }}\right|<2.5$ [93]. Convolving these uncertainties with the appropriate $\eta^{\text {track }}$ distributions results in systematic uncertainties on $r_{\text {trk }}$ that

\footnotetext{
${ }^{23}$ Additional information about the PYTHIA tunes can be found in Ref. [51].
} 


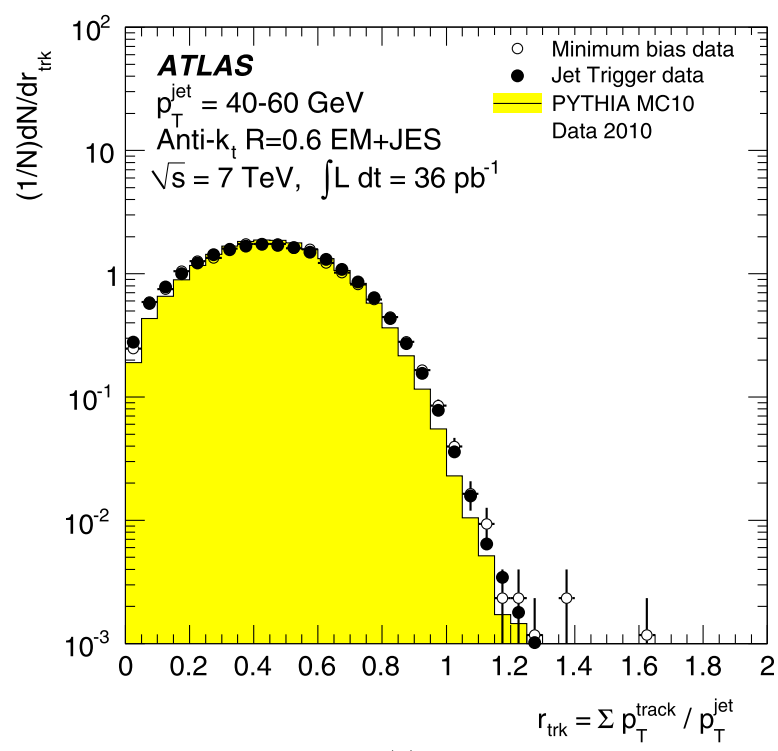

(a)

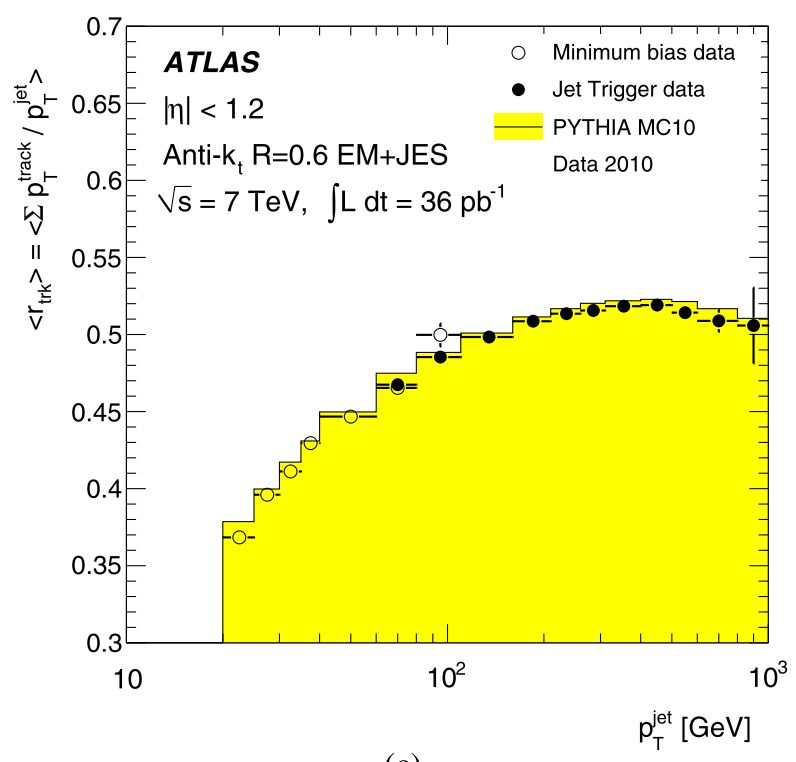

(c)

Fig. 23 The distribution of the charged-to-total momentum ratio $r_{\text {trk }}$ for $40 \leq p_{\mathrm{T}}^{\text {jet }}<60 \mathrm{GeV}$ (a) and for $600 \leq p_{\mathrm{T}}^{\text {jet }}<800 \mathrm{GeV}(\mathbf{b})$, the average charged-to-total momentum ratio $\left\langle r_{\text {trk }}\right\rangle$ for data and Monte Carlo simulation as a function of $p_{\mathrm{T}}^{\mathrm{jet}}$ (c) and the ratio of $\left\langle r_{\text {trk }}\right\rangle$ for data and Monte Carlo simulation $\left(R_{r_{\text {trk }}}\right)$ as a function of $p_{\mathrm{T}}^{\text {jet }}$ for the pseu-

range from $2 \%$ for jet pseudorapidities $|\eta|<1.2$ to $3.5 \%$ for pseudorapidities $1.7 \leq|\eta|<2.1$.

Uncertainties in the material distributions also affect the probability that photon conversions produce charged particles that can be included in the $r_{\text {trk }}$ measurement. The track selection used here requires at least one Pixel hit and most of the material in the ID is at a larger radius than the Pixel detector, resulting in a small systematic uncertainties associated with rate of conversions.

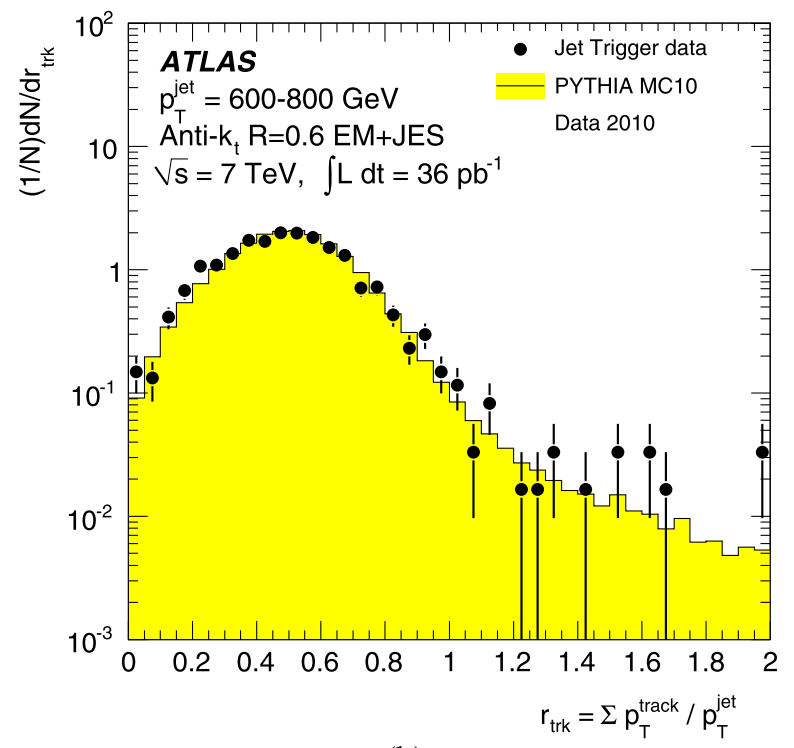

(b)

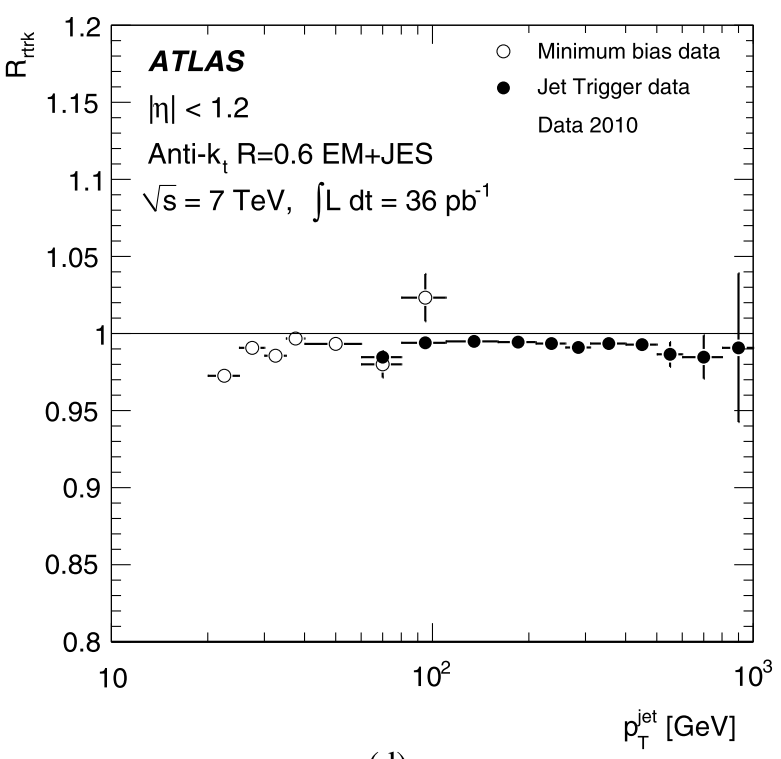

(d)

dorapidity range $|\eta|<1.2$ (d) for anti- $k_{t}$ jets with $R=0.6$ calibrated using the EM+JES scheme. The data measured with the jet (minimum bias) trigger are shown as closed (open) circles. Only statistical uncertainties are shown

Tracking efficiency in the jet core There are several effects that change the tracking efficiency and resolution inside a jet compared to those for isolated tracks:

1. When two tracks are close together, their hits may overlap. While the pattern recognition software allows tracks to share hits, the resolution is degraded since the calculated position of the hit is affected by the presence of the 
Table 7 Pythia generator tunes used to study the systematic uncertainty on the prediction for $r_{\text {trk }}$. Tunes specified by number (e.g. 100) refer to the value of the PYTUNE parameter [51]. A dash in the table indicates that the particular tune has no PYTUNE value

\begin{tabular}{lll}
\hline Tune Name & PYTUNE Value & Comments \\
\hline MC10 & - & ATLAS default $\left(p_{\mathrm{T}}\right.$ ordered showering) \\
MC09 & - & ATLAS default for Summer 2010 $\left(p_{\mathrm{T}}\right.$ ordered showering) \\
RFTA & 100 & Rick Field Tune A $Q^{2}$ ordered showering \\
& 107 & Tune A with "colour annealing" colour reconnection \\
& 110 & Tune A with LEP tune from Professor \\
& 117 & Tune 110 with "colour annealing" colour reconnection \\
& 129 & Tune of $Q^{2}$ ordered showering and UE with Professor \\
PERUGIA2010 & 320 & PERUGIA0 ( $p_{\mathrm{T}}$ ordered showering) \\
\hline
\end{tabular}

other track. The probability of not assigning hits to tracks increases.

2. When the hit density becomes high in the core of the jet, failures in the pattern recognition may result in the creation of tracks by combining hits that in fact came from several particles. Such tracks are called fake tracks.

3. When two high- $p_{\mathrm{T}}$ tracks are close together in space, they will share hits over many layers. In this case, one of the two tracks may be lost. This effect, referred to as loss of efficiency, becomes more important as the $p_{\mathrm{T}}^{\text {jet }}$ increases.

The reliability of the simulation to predict the size of these effects depends on whether the software properly models merging of ID hits. Detailed comparisons of the data and Monte Carlo simulation indicate that the simulation accurately reproduces the degradation of response in the jet core and models the degradation in resolution well. Furthermore, the fraction of tracks with $z>1$ constrain the size of possible tails. The non-Gaussian tails in the track resolution are small and the data are described by the Monte Carlo simulation.

Any residual difference in resolution between data and simulation is absorbed in the quoted uncertainty due to ID alignment.

Fake tracks and loss of efficiency are studied in the simulation using a hit-based matching algorithm using truth jets. These studies indicate that the rate for reconstructing fake tracks remains at $0.1 \%$ for the full $p_{\mathrm{T}}^{\text {jet }}$ range considered here, but that there is loss of tracking efficiency near the core of high- $p_{\mathrm{T}}$ jets. This effect has a negligible effect on $r_{\text {trk }}$ for jets with $p_{\mathrm{T}}^{\text {jet }}<500 \mathrm{GeV}$, but increases with $p_{\mathrm{T}}^{\text {jet }}$ such that on average $\sim 7.5 \%$ of the charged track momentum is lost for jets in the range $800 \leq p_{\mathrm{T}}^{\text {jet }}<1000 \mathrm{GeV}$. A relative uncertainty of $50 \%$ is assigned to the value of the inefficiency that is caused by merged hits. While this effect gives the largest systematic uncertainty on the JES for $p_{\mathrm{T}}^{\text {jet }} \gtrsim 600 \mathrm{GeV}\left(1.9 \%\right.$ for $600 \leq p_{\mathrm{T}}^{\text {jet }}<800 \mathrm{GeV}$ and
$3.7 \%$ for $800 \leq p_{\mathrm{T}}^{\text {jet }}<1000 \mathrm{GeV}$ ), it is still smaller than the present statistical uncertainty of the jet response measurements using the track-based method at these values of $p_{\mathrm{T}}^{\mathrm{jet}}$.

Inner detector alignment For high $p_{\mathrm{T}}$ tracks, the momentum resolution achieved in the ID is worse than that of the simulation. This degradation in resolution is attributed to an imperfect alignment of the ID. The systematic uncertainty on $r_{\text {trk }}$ is obtained by degrading the tracking resolution in the simulation. The size of this additional resolution smearing is determined by studying the width of the measured mass distribution for $Z$-decays $Z \rightarrow \mu^{+} \mu^{-}$. This procedure results in a systematic uncertainty of less than $0.2 \%$ for all $p_{\mathrm{T}}^{\text {jet }}$ and $\eta$.

Calorimeter jet $p_{\mathrm{T}}$ resolution The systematic uncertainty due to jet transverse momentum resolution uncertainties [27] is determined by smearing the jet four-momentum (without changing $\eta$ or $\phi$ ) in Monte Carlo simulation. The relative uncertainty on the $p_{\mathrm{T}}^{\text {jet }}$ resolution is $5 \%$ for $0 \leq$ $|\eta|<0.8$ and $10 \%$ for $0.8 \leq|\eta|<2.1$. The effect of this variation is largest for low values of $p_{\mathrm{T}}^{\text {jet }}$ and high values of $\eta$; for $p_{\mathrm{T}}^{\text {jet }}<40 \mathrm{GeV}$ and $0.8<|\eta|<2.1$ the uncertainty on $R_{r_{\text {trk }}}$ is $\sim 2 \%$.

Combined systematic uncertainty The above uncertainties are assumed to be uncorrelated and are combined in quadrature. The resulting total uncertainties are shown in Fig. 24 as a function of $p_{\mathrm{T}}^{\text {jet }}$ for several regions of $\eta$.

\subsubsection{Summary of JES uncertainty from tracks}

Final results for anti- $k_{t}$ jets with $R=0.6$ and EM+JES corrections are shown in Fig. 25 for five bins in $\eta$ with the derived systematic uncertainties. To facilitate comparisons at high $p_{\mathrm{T}}^{\mathrm{jet}}$, where the statistical uncertainties are large, the combined data from the three bins with $|\eta|<1.2$ are 


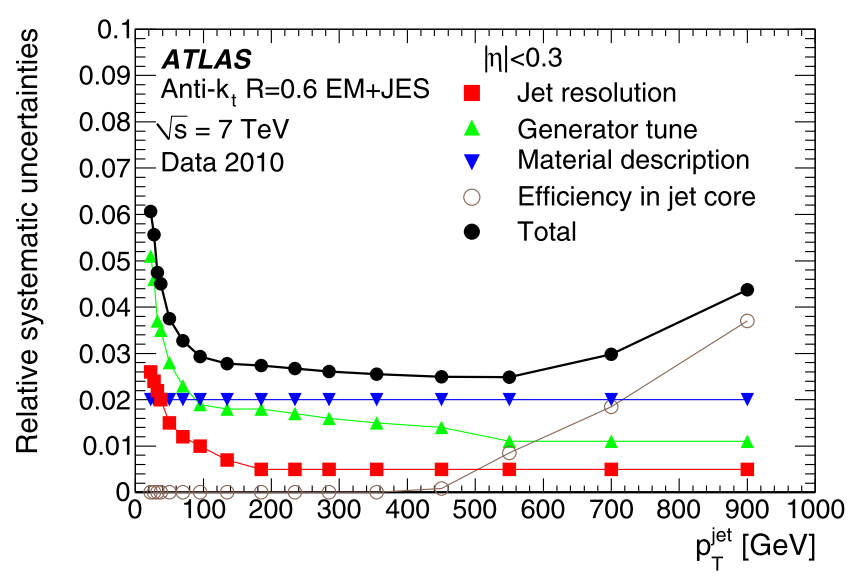

(a) $|\eta|<0.3$

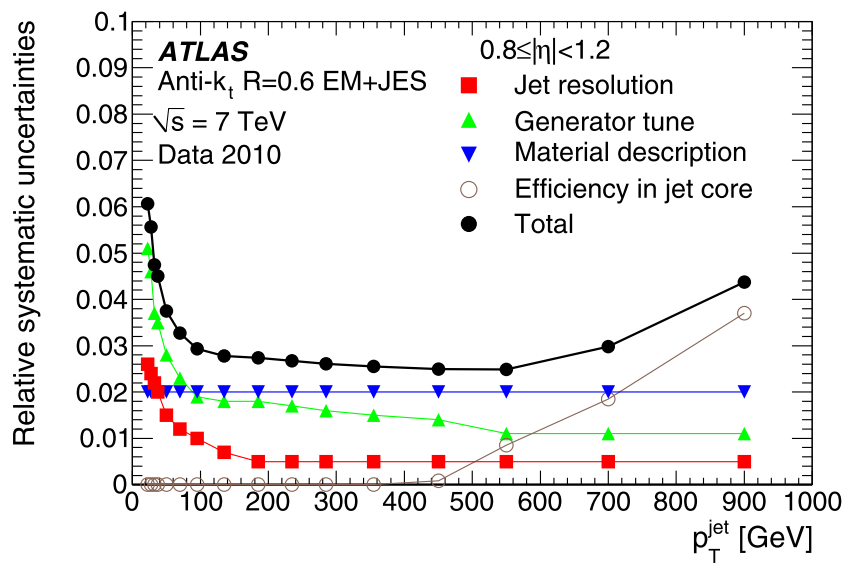

(c) $0.8 \leq|\eta|<1.2$

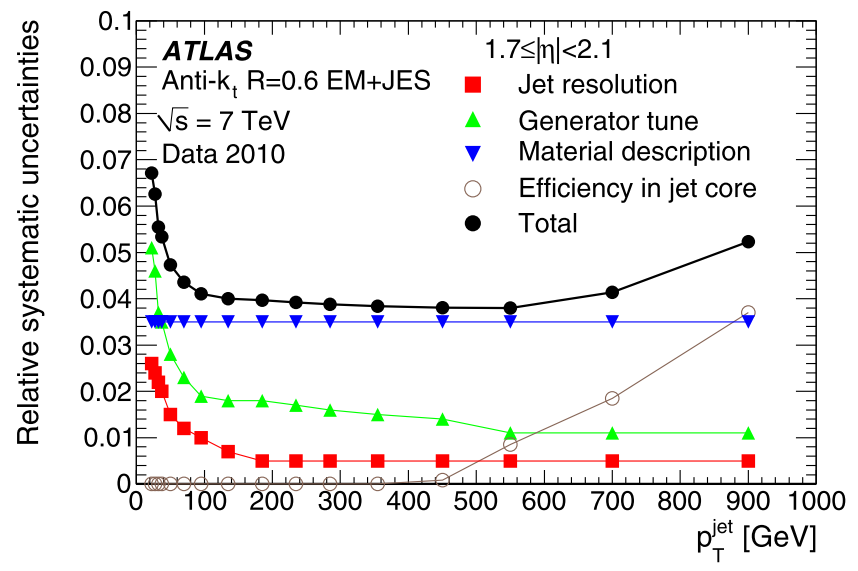

(e) $1.7 \leq|\eta|<2.1$

Fig. 24 Relative systematic uncertainty on the JES obtained by comparing the total momentum of tracks associated to jets to the calorimeter measurements for different $\eta$ regions for anti- $k_{t}$ jets with $R=0.6$
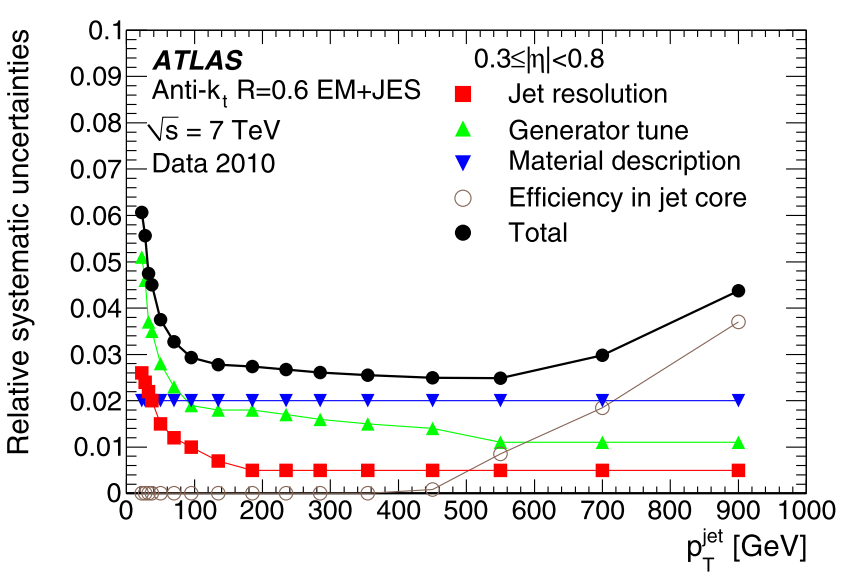

(b) $0.3 \leq|\eta|<0.8$

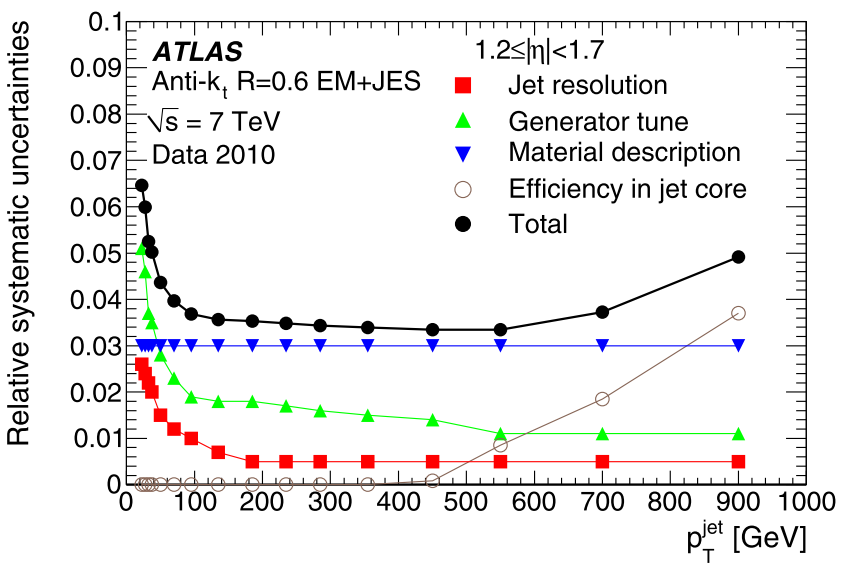

(d) $1.2 \leq|\eta|<1.7$

calibrated with the EM+JES scheme as a function of $p_{\mathrm{T}}^{\text {jet }}$. The total and the individual systematic uncertainties, as evaluated from the inclusive jet Monte Carlo simulation, are shown 


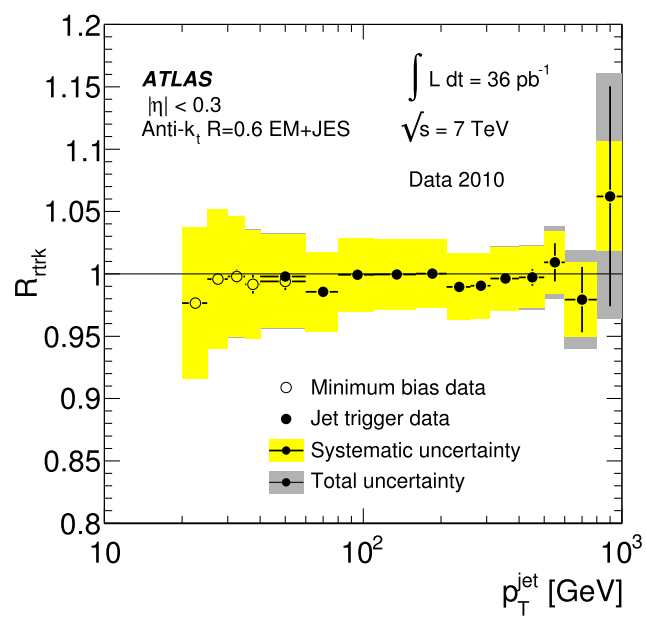

(a) $|\eta|<0.3$

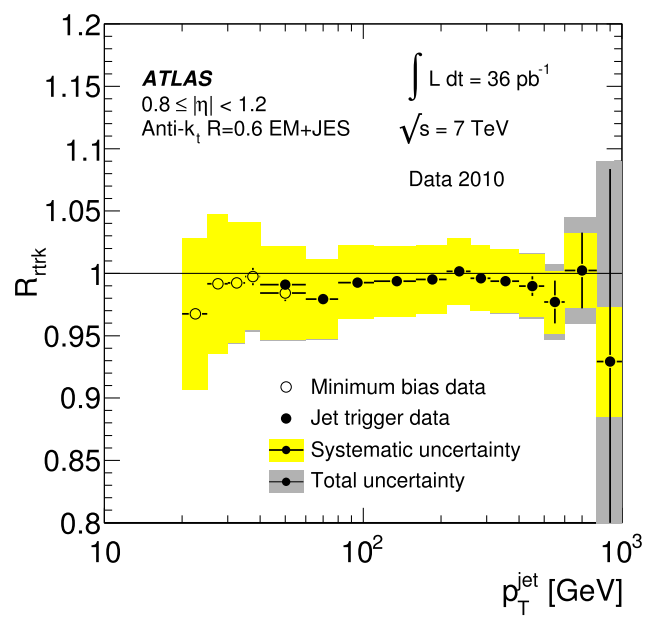

(c) $0.8 \leq|\eta|<1.2$

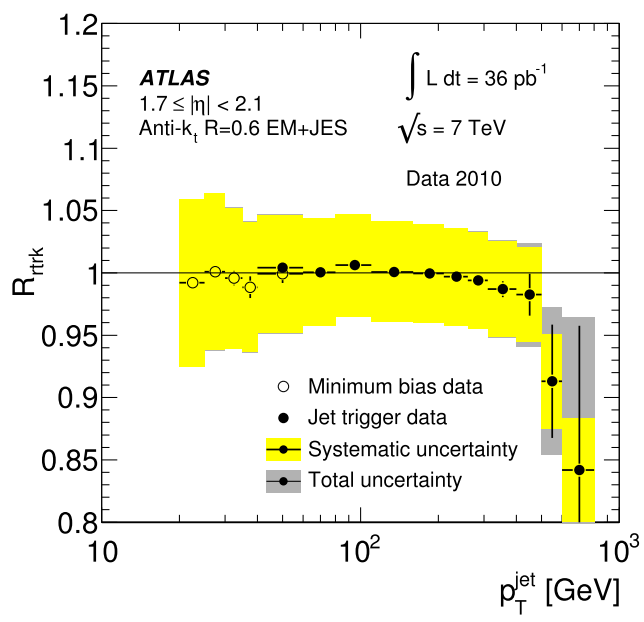

(e) $1.7 \leq|\eta|<2.1$

Fig. 25 Double ratio of the mean track to calorimeter response ratio in data and Monte Carlo simulation $R_{r_{\text {trk }}}=\left[r_{\text {trk }}\right]_{\text {Data }} /\left[r_{\text {trk }}\right]_{\text {MC }}$ for anti- $k_{t}$ jets with $R=0.6$ calibrated with the EM+JES scheme as a function of

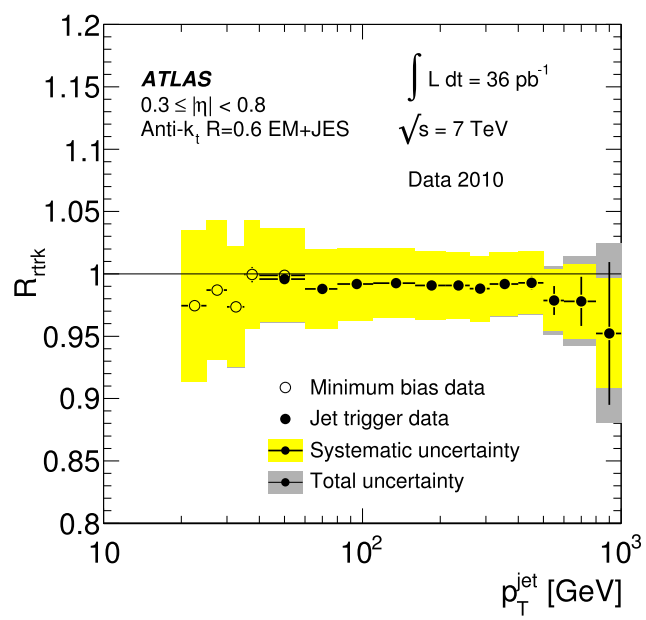

(b) $0.3 \leq|\eta|<0.8$

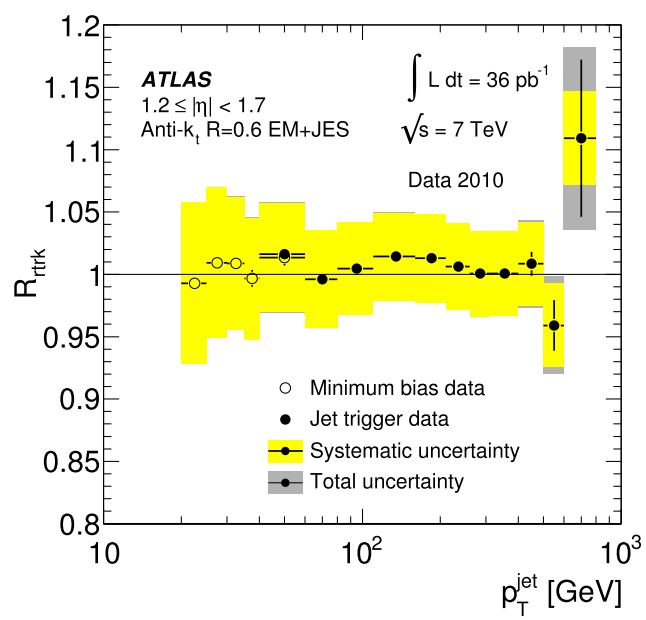

(d) $1.2 \leq|\eta|<1.7$

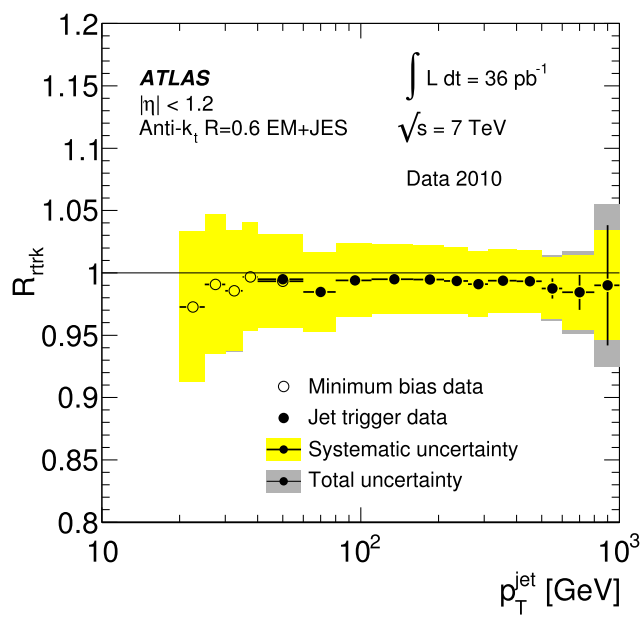

(f) $|\eta|<1.2$

$p_{\mathrm{T}}^{\text {jet }}$ for various $\eta$ bins. Systematic (total) uncertainties are shown as a light (dark) band 
also displayed. Averaging all data with $p_{\mathrm{T}}^{\text {jet }}>25 \mathrm{GeV}$ and $|\eta|<1.2$ yields a value of $r_{\text {trk }}$ that agrees with the simulation to better than $1 \%$. This small discrepancy is well within the quoted systematic uncertainty, which is highly correlated between bins in $p_{\mathrm{T}}^{\text {jet }}$. No significant variation of $R_{r_{\text {trk }}}$ with $p_{\mathrm{T}}^{\text {jet }}$ is observed. For $|\eta|>1.2$, the statistical uncertainties are large for $p_{\mathrm{T}}^{\text {jet }}>500 \mathrm{GeV}$. For $p_{\mathrm{T}}^{\text {jet }}<500 \mathrm{GeV}$, the level of agreement between data and simulation is similar to that obtained at low $\eta$.

In summary, $r_{\text {trk }}$, the ratio of track to calorimeter transverse momentum, is used to validate the JES for anti- $k_{t}$ jets with $R=0.4$ and $R=0.6$ calibrated with the EM+JES calibration scheme. Systematic uncertainties associated with jet modelling and track reconstruction are assessed and the method is shown to provide a JES uncertainty evaluation independent of the modelling of the calorimeter response. Systematic uncertainties are below $3 \%$ for $0 \leq|\eta|<0.8$ and rise to $\sim 4 \%$ for $1.7 \leq|\eta|<2.1$ for $40 \leq p_{\mathrm{T}}^{\text {jet }}<800 \mathrm{GeV}$. The results agree within systematic uncertainties with those predicted using the ATLAS calorimeter simulation and provide an independent estimate of the overall jet energy scale and its uncertainty.

\subsection{Photon-jet transverse momentum balance}

In $\gamma$-jet events, a jet recoils against a photon at high transverse momentum. The photon energy, being accurately measured in the electromagnetic calorimeter, is used as a reference. Such a topology can be used to validate the jet energy measurement. Any discrepancy between data and simulation may be taken as an uncertainty on the jet energy calibration.

Two methods of balancing the photon and the recoiling jet transverse momentum with different sensitivities and systematic uncertainties are used: the direct $p_{\mathrm{T}}$ balance technique and the missing transverse momentum projection fraction technique.

\subsubsection{Direct transverse jet momentum balance technique}

The direct $p_{\mathrm{T}}$ balance technique exploits the approximate transverse momentum balance in events with only one photon and one jet with high $p_{\mathrm{T}}$. The ratio of the jet $p_{\mathrm{T}}$ to the photon $p_{\mathrm{T}}\left(p_{\mathrm{T}}^{\mathrm{jet}} / p_{\mathrm{T}}^{\gamma}\right)$ is used to estimate the jet response. Since the photon $p_{\mathrm{T}}$ is well-measured and well-described by the simulation, the quality of the jet $p_{\mathrm{T}}$ calibration can be assessed by comparing data and Monte Carlo simulation using the ratio $p_{\mathrm{T}}^{\mathrm{jet}} / p_{\mathrm{T}}^{\gamma}$. This technique was used at the $\mathrm{CDF}$ experiment [14].

\subsubsection{Missing transverse momentum projection fraction technique}

The missing transverse momentum projection fraction (MPF) technique exploits the momentum balance, in the transverse plane, of the photon and the hadronic recoil to derive the detector response to jets. This technique has been used in the past for the D0 experiment [15].

The missing transverse momentum vector $\left(\vec{E}_{\mathrm{T}}^{\text {miss }}\right)$ is defined as the opposite of the vector sum of the transverse projections of calorimeter energy deposits. The missing transverse momentum is calculated from the energy deposits in the calorimeter cells that are included in topo-clusters. The calorimeter cell energy is computed using the same calibration as the one used in the jet calibration scheme to be tested. The missing transverse momentum is corrected for the photon four-momentum. The reconstructed jet four-momentum is not directly used in the missing transverse momentum calculation.

The MPF technique is based on the assumption that the only missing transverse momentum in a $\gamma$-jet event arises from calorimeter non-compensation, signal losses due to noise suppression and energy losses in the non-active regions of the detector by the hadronic jet. The transverse momentum balance can be written as:

$\vec{p}_{\mathrm{T}}^{\gamma}+\vec{p}_{\mathrm{T}}^{\mathrm{jet}}=\overrightarrow{0}$,

where $\vec{p}_{\mathrm{T}}^{\gamma}$ and $\vec{p}_{\mathrm{T}}^{\mathrm{jet}}$ is the photon and jet transverse momentum vector. The particles produced by the hard scatter and their interaction in the calorimeter can be expressed in terms of the observables:

$\mathcal{R}^{\gamma} \vec{p}_{\mathrm{T}}^{\gamma}+\mathcal{R}^{\text {jet }} \vec{p}_{\mathrm{T}}^{\mathrm{jet}}=-\vec{E}_{\mathrm{T}}^{\mathrm{miss}}$,

where $\mathcal{R}_{\gamma}$ is the calorimeter response to photons. Since the calorimeter is well calibrated for photons, $\mathcal{R}_{\gamma}=1$. The variable $\mathcal{R}^{\text {jet }}$ denotes the calorimeter response to jets. By using the above two equations and projecting the $E_{\mathrm{T}}^{\text {miss }}$ in the direction of the photon the response can be written as:

$\mathcal{R}_{\mathrm{MPF}}=1+\frac{\vec{p}_{\mathrm{T}}^{\gamma} \cdot \vec{E}_{\mathrm{T}}^{\mathrm{miss}}}{\left|p_{\mathrm{T}}^{\gamma}\right|^{2}}$,

where $\mathcal{R}_{\mathrm{MPF}}$ is the calorimeter response to all the particle recoiling against the photon.

Note that the MPF technique measures the calorimeter response relying only on the photon and $E_{\mathrm{T}}^{\text {miss }}$ quantities and does not test the jet calibration directly. Therefore the MPF response is independent of the jet algorithm.

\subsubsection{Photon-jet Monte Carlo simulation sample}

The $\gamma$-jet sample is simulated with the event generator PYTHIA using the ATLAS MC10 tune [50].

The systematic uncertainty from jets which are identified as photons (fakes) are studied with an inclusive PYTHIA jet sample using the MC09 tune [62]. ${ }^{24}$ To efficiently produce

\footnotetext{
${ }^{24}$ Since a large event statistics is needed for this sample, only a sample with an older tune was available.
} 
this sample a generator level event filter is applied to select events with jets that are more likely to satisfy photon identification criteria. A cone based jet algorithm is applied to the four-momenta of all stable generated particles within a $0.18 \times 0.18$ region in $\eta \times \phi$. The size of this jet is chosen to better model the narrow energy deposition pattern of photons. A generated event is only fully simulated if it contains at least one particle jet with $p_{\mathrm{T}}>17 \mathrm{GeV}$ passing the generator-level filter described above. Events in the dijet sample with prompt photons, e.g. that are produced by radiation are removed.

\subsubsection{Selection of the photon-jet data sample}

The leading photon in each event must have $p_{\mathrm{T}}^{\gamma}>25 \mathrm{GeV}$ and lie in the pseudorapidity range $\left|\eta^{\gamma}\right|<1.37$. In this range the photon is fully contained within the electromagnetic barrel calorimeter. Furthermore, events in which the leading photon is in a calorimeter region where an accurate energy measurement is not possible are rejected. In each event only the leading photon is considered.

The leading photon candidate must also satisfy strict photon identification criteria [94], meaning that the pattern of energy deposition in the calorimeter is consistent with the expected photon showering behaviour. The photon candidate must be isolated from other activity in the calorimeter ( $E_{\mathrm{T}}^{\gamma \text { Isolation }}$ ) with an isolation cone of size $R=0.4$. If the leading photon does not meet all of these criteria, the event is rejected.

Only events are retained that fired an online trigger requiring a photon candidate with $p_{\mathrm{T}}^{\gamma}>20 \mathrm{GeV}$ or $p_{\mathrm{T}}^{\gamma}>$ $40 \mathrm{GeV}$. At the trigger level the photon identification requirements are less strict than those of the off-line selection.

The $p_{\mathrm{T}}$ distribution of photons in events selected with the above criteria is shown in Fig. 26. The small discrepancies between the $p_{\mathrm{T}}^{\gamma}$ spectrum in data and Monte Carlo simulation do not affect the comparison of the jet response in data and Monte Carlo simulation.

The leading jet must be in the fiducial region $|\eta|<1.2$. Jets that have a distance within $\Delta R>0.2$ to the selected photons are rejected.

Soft QCD radiation can affect the $p_{\mathrm{T}}$ balance between the jet and photon. The following two selection cuts are applied to suppress this effect. To select events in which the photon and the leading jet are back-to-back, $\Delta \phi_{\text {jet- } \gamma}>\pi-0.2$ radians is required. The presence of sub-leading jets is suppressed by requiring that the transverse momentum of the sub-leading jet $p_{\mathrm{T}}^{\text {jet2 }}$ is less than $10 \%$ of the $p_{\mathrm{T}}$ of the leading photon. ${ }^{25} \mathrm{~A}$ summary of the event selection criteria can be found in Table 8 .

\footnotetext{
${ }^{25}$ This cut is not applied, if it would be below the jet $p_{\mathrm{T}}$ reconstruction threshold of $p_{\mathrm{T}}^{\text {jet }}=7 \mathrm{GeV}$. If in this case a sub-leading jet with $p_{\mathrm{T}}^{\text {jet }} \geq$ $7 \mathrm{GeV}$ is present, the event is rejected.
}

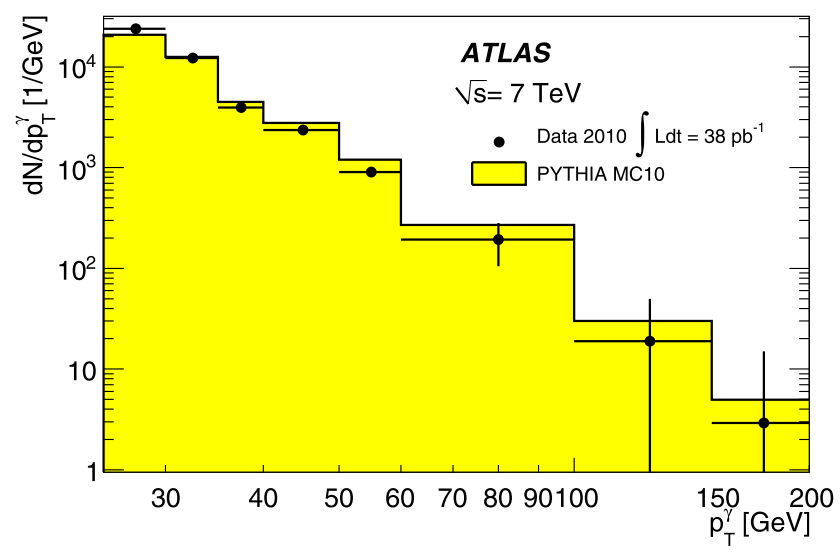

Fig. 26 Distribution of the photon transverse momentum for events passing the photon selection criteria described in Sect. 10.2.4. A correction is made in the first $p_{\mathrm{T}}^{\gamma}$ bin for the pre-scale applied to the trigger in this $p_{\mathrm{T}}^{\gamma}$ range. The Monte Carlo simulation is normalised to the observed number of events observed in data and corrected for the trigger pre-scale. Uncertainties are statistical only

Table 8 Criteria used to select events with a photon and a jet with high transverse momentum

\begin{tabular}{ll}
\hline Variable & Threshold \\
\hline$|\eta|$ & $<1.2$ \\
$p_{\mathrm{T}}^{\gamma}$ & $>25 \mathrm{GeV}$ \\
$\left|\eta^{\gamma}\right|$ & $<1.37$ \\
$E_{\mathrm{T}}^{\gamma \text { Isolation }}$ & $<3 \mathrm{GeV}$ \\
$\Delta \phi_{\mathrm{jet}-\gamma}$ & $>\pi-0.2 \mathrm{rad}$ \\
$p_{\mathrm{T}}^{\text {jet2 }} / p_{\mathrm{T}}^{\gamma}$ & $<10 \%$ \\
\hline
\end{tabular}

\subsubsection{Systematic uncertainties of the photon-jet in situ validation technique}

Uncertainties due to background from jets identified as photons (fakes), soft QCD radiation, in-time pile-up, nonfunctional calorimeter read-out regions and the photon energy scale are studied.

Background in the photon-jet sample The systematic uncertainty from jets which are identified as photons (fakes) are studied with the inclusive jet Monte Carlo simulation sample described in Sect. 10.2.3. Dijet events in which one of the jets is misidentified as a photon contribute to the data sample but not to Monte Carlo simulation signal sample. The rate of dijet events faking photons is sensitive to the detailed modelling of the jet fragmentation and the detector simulation, and is therefore subject to large uncertainties.

The systematic uncertainty from this background is determined in two steps. First the detector response of the $\gamma$-jet sample $\left(\mathcal{R}_{\gamma \text {-jet }}\right)$ and the filtered dijet sample $\left(\mathcal{R}_{\text {dijet }}\right)$ is determined in the Monte Carlo simulation as seen in Fig. 27. Also shown is the response difference relative to the response of 


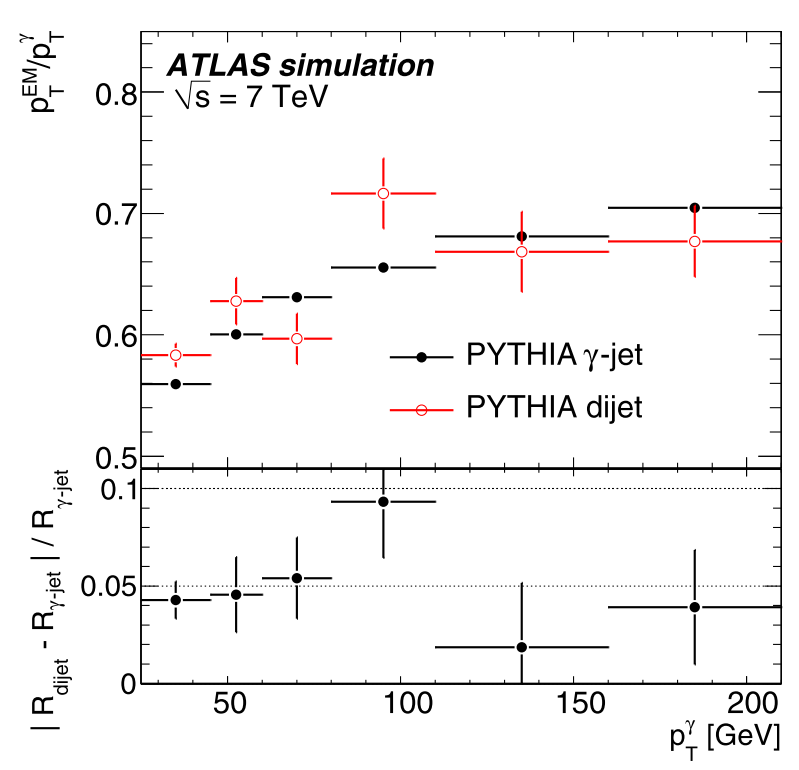

(a) Direct $p_{\mathrm{T}}$ balance technique

Fig. 27 Average jet response measured at the EM scale as a function of $p_{\mathrm{T}}^{\gamma}$ as determined by the direct $p_{\mathrm{T}}$ balance technique for anti- $k_{t}$ jets with $R=0.6$ within $|\eta|<1.2$ (a) and by the MPF technique (b) for $\gamma$-jet events and inclusive jet events where one jet has been recon-

the $\gamma$-jet sample $\left(\left|\mathcal{R}_{\text {dijet }}-\mathcal{R}_{\gamma \text {-jet }}\right| / \mathcal{R}_{\gamma \text {-jet }}\right)$. A response difference of 3-5\% is estimated. It is larger for the direct balance technique than for the MPF technique.

The contribution from background in the signal region is estimated with the sideband technique described in the prompt photon analysis [94]. Using the photon isolation and the photon identification criteria to define signal and control samples, the signal purity is measured. The purity $P$ is about 0.6 at $p_{\mathrm{T}}=25 \mathrm{GeV}$ and rises to about 0.95 at higher $p_{\mathrm{T}}{ }^{26}$

The systematic uncertainty on the response due to background in the $\gamma$-jet sample is given by:

$\varepsilon=\left(\frac{\mathcal{R}_{\text {dijet }}-\mathcal{R}_{\gamma \text {-jet }}}{\mathcal{R}_{\gamma \text {-jet }}}\right) \cdot(1-P)$.

The systematic uncertainty is calculated in bins of $p_{\mathrm{T}}^{\gamma}$. For $p_{\mathrm{T}}=45 \mathrm{GeV}$ the systematic uncertainty is below $1 \%$ for the direct balance technique and below $0.6 \%$ for the MPF technique.

The effect of background contamination in the $\gamma$-jet sample has been further validated by relaxing the photon identification criteria. Both data and Monte Carlo simulation show a $3 \%$ variation in response for the direct $p_{\mathrm{T}}$ balance technique, mostly at low $p_{\mathrm{T}}$. This is consistent with the systematic uncertainty computed with the purity method using

\footnotetext{
${ }^{26}$ This is similar to the purity measured in Ref. [94] and small differences are due to the different data samples.
}

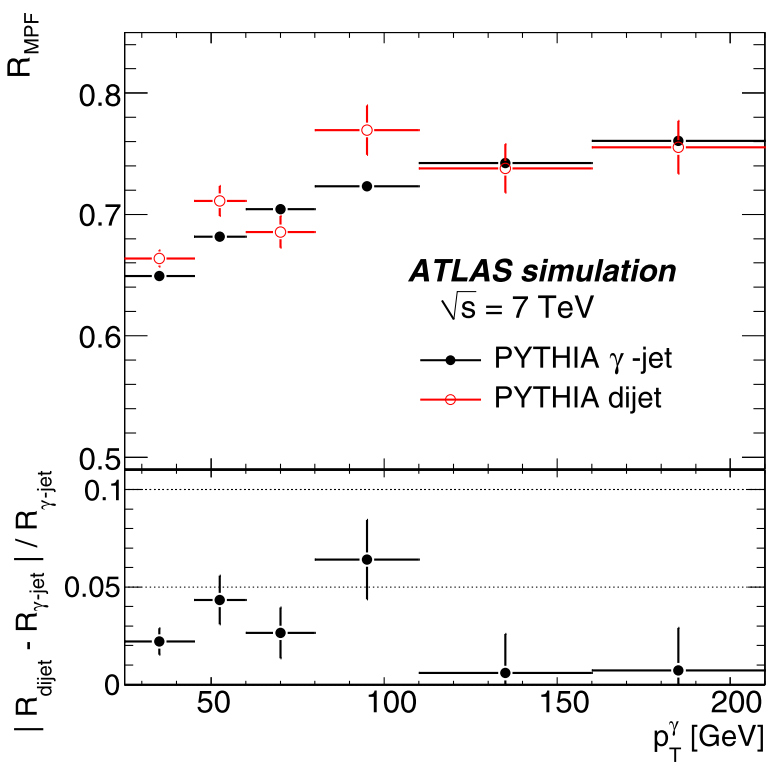

(b) MPF technique

structed as a photon, as derived in the Monte Carlo simulation. The lower part of the figures shows the absolute response difference between the dijet and $\gamma$-jet events with respect to the response of $\gamma$-jet events. Only statistical uncertainties are shown

Eq. (27), e.g. for the lowest $p_{\mathrm{T}}$ bin $40 \%$ of the events are expected to be dijet background giving a response that is $5 \%$ higher than the response of $\gamma$-jet events.

Soft QCD radiation suppression cuts The stability of the jet response ratio of the data to the Monte Carlo simulation is explored by varying the radiation suppression cuts. Figure 28a shows the thresholds for the $p_{\mathrm{T}}^{\text {jet2 }} / p_{\mathrm{T}}^{\gamma}$ and $\Delta \phi_{\text {jet }-\gamma}$ cuts for 13 sets of cuts. Figure 28b illustrates the change in the ratio of the data to the Monte Carlo simulation of the MPF response for each of these 13 sets of cuts, for one typical $p_{\mathrm{T}}^{\gamma}$ bin. The result demonstrates that the ratio of the data response to the Monte Carlo response is not sensitive to the exact values of the radiation cuts, within the $1 \%$ level. The systematic uncertainty is taken as the difference in the data to Monte Carlo ratio between the nominal cuts defining the signal sample, and the loosest cuts in all $p_{\mathrm{T}}$-bins, labelled as "Point 13" in Fig. 28a.

The MPF-determined response changes slightly between the data and the Monte Carlo simulation, the systematic uncertainty is $0.7 \%$ at $p_{\mathrm{T}}^{\gamma}=50 \mathrm{GeV}$ and falls to $0.4 \%$ at $p_{\mathrm{T}}^{\gamma}=135 \mathrm{GeV}$. The quoted values are determined from linear fits to the points analogous to those shown in Fig. 28b.

The stability of the ratio of the data to the Monte Carlo simulation for the response measured with the direct $p_{\mathrm{T}}$ balance technique is shown in Fig. 29. The response measured in either data or in Monte Carlo simulation varies by up to $10 \%$ due to differing radiation suppression cuts. However, 


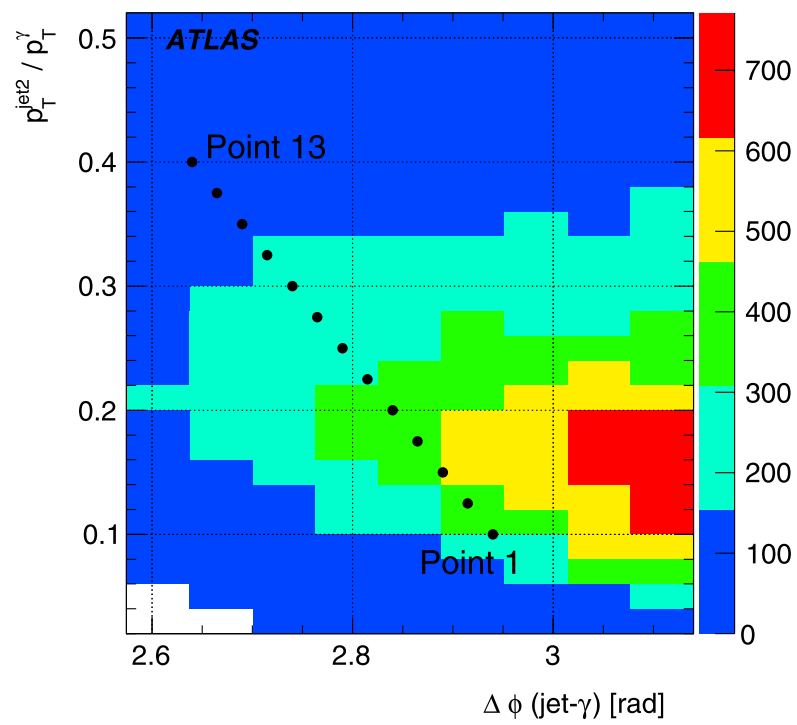

(a)

Fig. 28 The values of radiation-suppressing cut thresholds (points) used to probe the soft QCD radiation systematic uncertainty, as a function of $\Delta \phi_{\text {jet }-\gamma}$ and $p_{\mathrm{T}}^{\text {jet} 2} / p_{\mathrm{T}}^{\gamma}$ overlaid with the number of events observed in data (a). The nominal selection is the bottomrightmost point labelled "Point 1". Relative change in the MPF re-

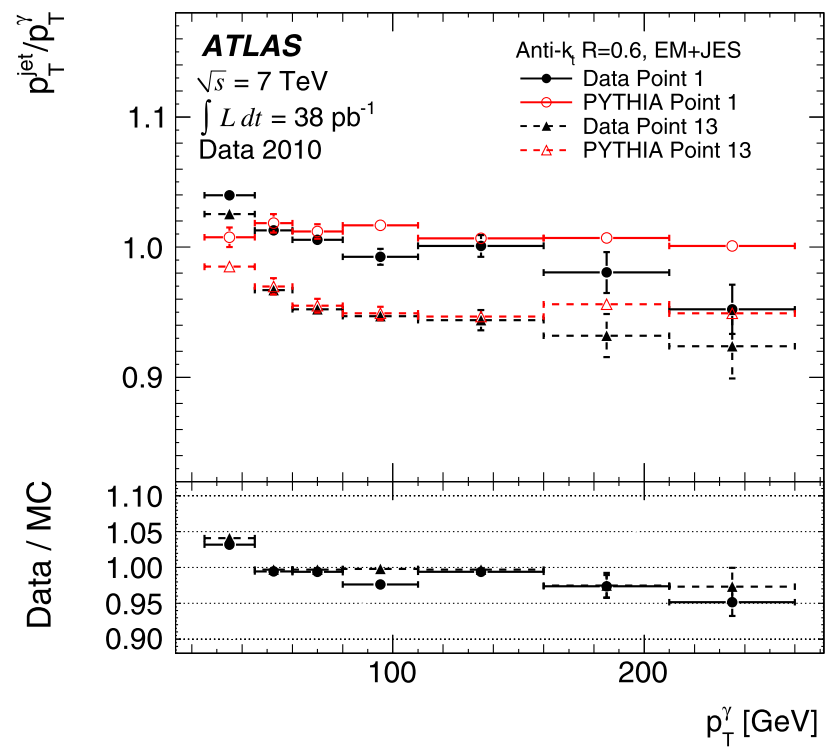

Fig. 29 Average jet response as determined by the direct $p_{\mathrm{T}}$ balance technique with the nominal selection (Point 1 ) and with a set of relaxed radiation suppression cuts (Point 13), for anti- $k_{t}$ jets with $R=0.6$ calibrated with the EM+JES scheme within $|\eta|<1.2$ as a function of the photon transverse momentum for data and Monte Carlo simulation. Only statistical uncertainties are shown

the data to Monte Carlo ratio with and without the radiation suppression cuts is stable within $\sim 1 \%$.

In-time pile-up The average number of proton-proton collisions in each bunch crossing grew significantly during the

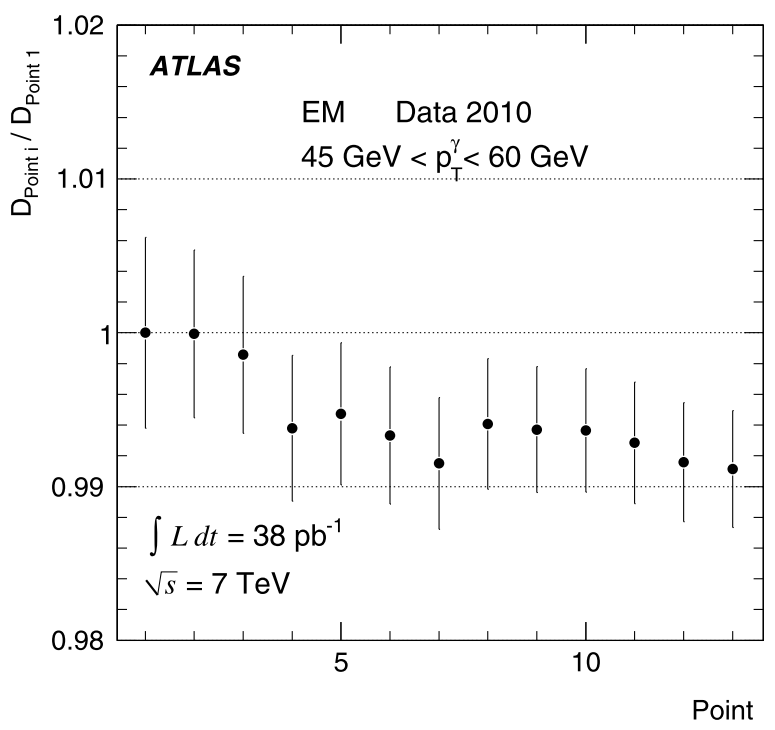

(b)

sponse between data and Monte Carlo simulation (b), defined as $D=$ $\left[\mathcal{R}_{\mathrm{MPF}}\right]_{\text {Data }} /\left[\mathcal{R}_{\mathrm{MPF}}\right]_{\mathrm{MC}}$ from the point given on the $x$-axis to point 1 , when relaxing the soft QCD radiation suppression as indicated in (a). Only statistical uncertainties are shown

data-taking period. Thus, there is a non-negligible fraction of events containing in-time pile-up (see Sect. 8.1). The additional collisions produce extra particles which can overlap with the hard interaction of interest in the ATLAS detector. The increased energy is about $0.5 \mathrm{GeV}$ per additional reconstructed primary vertex (see Sect. 8.1.4).

The MPF technique is expected to be insensitive to intime pile-up events. Because in-time pile-up is random and symmetric in $\phi$, the mean of the quantity $\vec{p}_{\mathrm{T}}^{\gamma} \times \vec{E}_{\mathrm{T}}^{\text {miss }}$ should be robust against in-time pile-up. The missing transverse fraction (MTF) is defined as:

$\mathrm{MTF}=\frac{\left(\vec{p}_{\mathrm{T}}^{\gamma} \times \vec{E}_{\mathrm{T}}^{\mathrm{miss}}\right)_{z}}{\left|\vec{p}_{\mathrm{T}}^{\gamma}\right|^{2}}=\frac{\left|\vec{E}_{\mathrm{T}}^{\text {miss }}\right|}{\left|\vec{p}_{\mathrm{T}}^{\gamma}\right|} \sin \left(\phi_{\vec{E}_{\mathrm{T}}^{\text {miss }}}-\phi_{\vec{p}_{\mathrm{T}}^{\gamma}}\right)$,

where $\left(\vec{p}_{\mathrm{T}}^{\gamma} \times \vec{E}_{\mathrm{T}}^{\mathrm{miss}}\right)_{z}$ is the $z$-component of the vector resulting from the cross product. The MTF measures the activity in the plane perpendicular to the photon $\vec{p}_{\mathrm{T}}$. The mean of the MTF is zero, if there is no bias due to in-time pile-up.

Figure 30 shows the MTF distribution for data with and without in-time pile-up. For both these distributions the means are compatible with zero.

From the study of the MTF distribution and other checks, such as the dependence of the MPF on $N_{\mathrm{PV}}$, it can be justified that in-time pile-up can be neglected and no systematic uncertainty is attributed to the MPF method. In the case of the direct $p_{\mathrm{T}}$ balance technique the impact of in-time pileup is explored by comparing the $p_{\mathrm{T}}$ balance between events with exactly one identified primary vertex and events with 


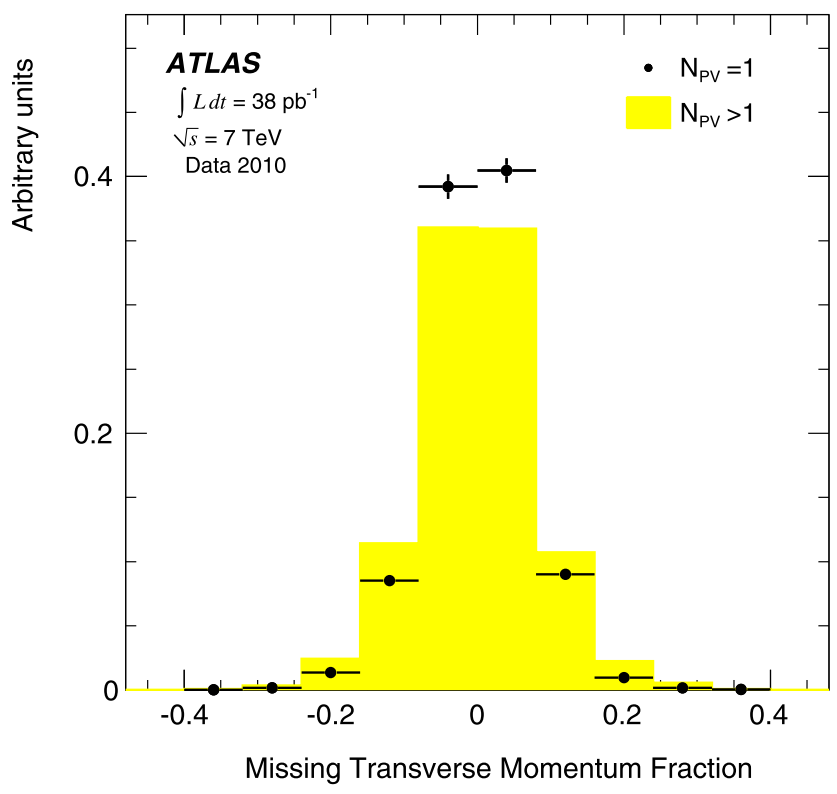

Fig. 30 The missing transverse momentum fraction (MTF) distribution for data with exactly one reconstructed primary vertex $N_{\mathrm{PV}}$, and with more than one reconstructed primary vertex. Only statistical uncertainties are shown

any number of vertices. As seen in Fig. 31 the ratio of the response in data to the response in Monte Carlo simulation for events with exactly one vertex and for events with more than one vertex is consistent with a variation of $0.8 \%$. This is taken as a systematic uncertainty.

No effect due to the offset correction for in-time pileup is seen (see Sect. 8.1), and no systematic uncertainty is attributed to the offset correction for in-time pile-up.

Impact of missing calorimeter read-out regions For a small subset of the calorimeter channels the calorimeter readout is not functioning properly. The energy of these calorimeter cells is evaluated using the trigger tower information, which has larger granularity and less accurate resolution. While photons reconstructed in or near such a region are not considered in the analysis, there is no such rejection applied to jets. A sub-sample of events with no jet containing such a cell has been used to evaluate a possible systematic uncertainty between data and simulation. Within the statistical uncertainty, no bias is observed for the MPF $\gamma$-jet technique or the direct $p_{\mathrm{T}}$ balance technique, therefore no systematic uncertainty is assigned.

Photon energy scale Both the direct $p_{\mathrm{T}}$ balance and the MPF techniques are sensitive to the photon energy scale. The absolute electron energy scale has been measured in situ using the invariant mass constraint in $Z \rightarrow e^{+} e^{-}$for electrons. The uncertainty on the photon energy scale results in a systematic uncertainty smaller than $1 \%$, depending on $p_{\mathrm{T}}^{\mathrm{jet}}$ and $\eta$.

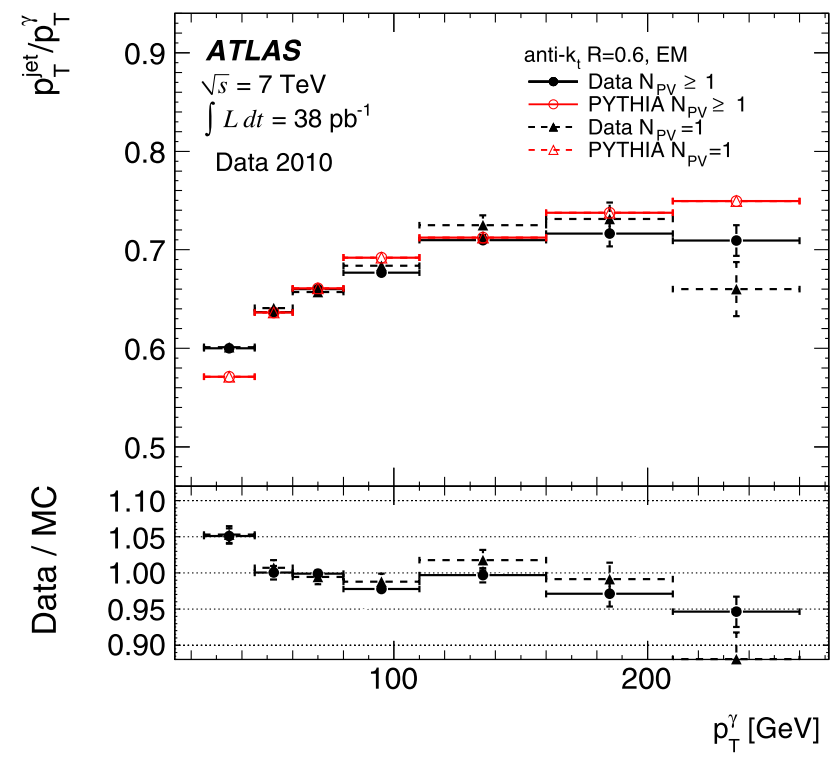

Fig. 31 Average jet response for anti- $k_{t}$ jets with $R=0.6$ at the EM scale within $|\eta|<1.2$ as determined by the direct $p_{\mathrm{T}}$ balance technique in events with any number of reconstructed primary vertices and in events with exactly one reconstructed vertex as a function of the photon transverse momentum for both data and Monte Carlo simulation. The lower part of the figure shows the data to Monte Carlo simulation ratio. Only statistical uncertainties are shown

The direct $p_{\mathrm{T}}$ balance technique and the MPF technique find a systematic uncertainty which is approximately opposite in sign. This sign difference is caused by the upwards shift in photon energy leading to an equivalent downwards shift in $\vec{E}_{\mathrm{T}}^{\text {miss }}$, and vice versa.

The response measured with both the MPF and the direct $p_{\mathrm{T}}$ balance techniques has been studied for converted and non-converted photons. The results of both samples agree within the statistical uncertainties. No additional systematic uncertainty has been considered for this effect, which is already accounted for in the photon energy scale and the photon background systematic uncertainty.

Total systematic uncertainty Table 9 shows a summary of the systematic uncertainties studied for the direct $p_{\mathrm{T}}$ balance and MPF techniques. The total systematic uncertainties for each method are similar, although each method is sensitive to different effects. Total systematic uncertainties are found on the data to Monte Carlo simulation jet response ratio of smaller than $1 \%$ for the MPF method and of smaller than $1.6 \%$ for direct balance method.

\subsubsection{Results from the photon-jet balance}

The direct $p_{\mathrm{T}}$ balance and MPF techniques are used to validate the jet response in situ by comparing data and Monte Carlo simulation. The response in data and Monte Carlo simulation for the EM scale energy is shown in Fig. 32. 


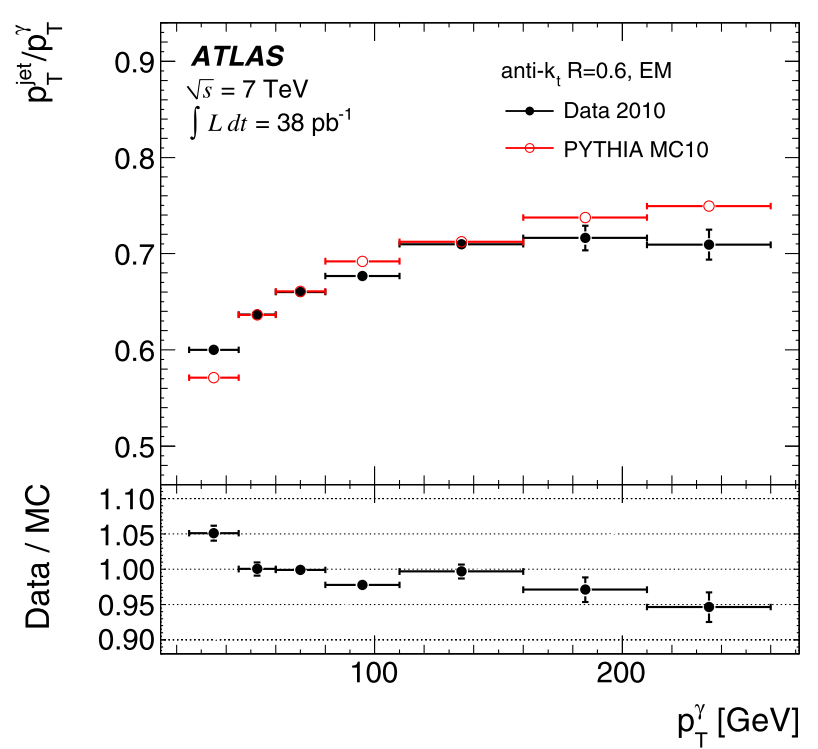

(a) Direct $p_{\mathrm{T}}$ balance technique

Fig. 32 Average jet response as determined by the direct $p_{\mathrm{T}}$ balance for anti- $k_{t}$ jets with $R=0.6$ (a) and the MPF technique (b) using the EM scale for both data and Monte Carlo simulation as a function of

Table 9 Individual systematic uncertainties in the jet energy scale from both the direct $p_{\mathrm{T}}$ balance and the MPF techniques at two values of $p_{\mathrm{T}}^{\gamma}$

\begin{tabular}{llllll}
\hline$p_{\mathrm{T}}^{\gamma}$ range [GeV] & \multicolumn{2}{c}{ Direct $p_{\mathrm{T}}$ balance [\%] } & & \multicolumn{2}{c}{ MPF [\%] } \\
\cline { 5 - 6 } & $45-60$ & $110-160$ & & $45-60$ & $110-160$ \\
\hline Background & \pm 1.0 & \pm 0.4 & & \pm 0.6 & \pm 0.1 \\
Soft QCD radiation & \pm 0.8 & \pm 0.9 & & \pm 0.7 & \pm 0.4 \\
In-time pile-up & \pm 0.8 & \pm 0.8 & & \pm 0 & \pm 0 \\
Photon scale & ${ }_{-0.3}^{0.5}$ & ${ }_{-0.3}^{+0.5}$ & & ${ }_{-0.5}^{+0.2}$ & ${ }_{-0.5}^{+0.3}$ \\
Total systematics & +1.6 & +1.4 & & +0.9 & +0.5 \\
\hline
\end{tabular}

The jet response in data and Monte Carlo simulation agrees within uncertainties in the range $p_{\mathrm{T}}^{\gamma}>45 \mathrm{GeV}$. In the range $25 \leq p_{\mathrm{T}}^{\gamma}<45 \mathrm{GeV}$ there is a shift in the data to Monte Carlo ratio of $5 \%$ for the direct $p_{\mathrm{T}}$ balance technique and $3 \%$ for the MPF technique.

Figure 33 shows the jet response measured in both data and Monte Carlo simulation using the direct $p_{\mathrm{T}}$ balance technique with the anti- $k_{t}$ jet algorithm with $R=0.6$ for the EM+JES calibration scheme. The data to Monte Carlo simulation agreement is within $\pm 5 \%$.

Figure 34 shows the ratio of $p_{\mathrm{T}}^{\mathrm{jet}} / p_{\mathrm{T}}^{\gamma}$ between data and Monte Carlo simulation together with the total uncertainty on the determination of the data to Monte Carlo simulation ratio, for anti- $k_{t}$ jets with $R=0.6$ on EM scale (Fig. 34a) and calibrated with the EM+JES scheme (Fig. 34b). The results are the same for jets on EM scale and calibrated

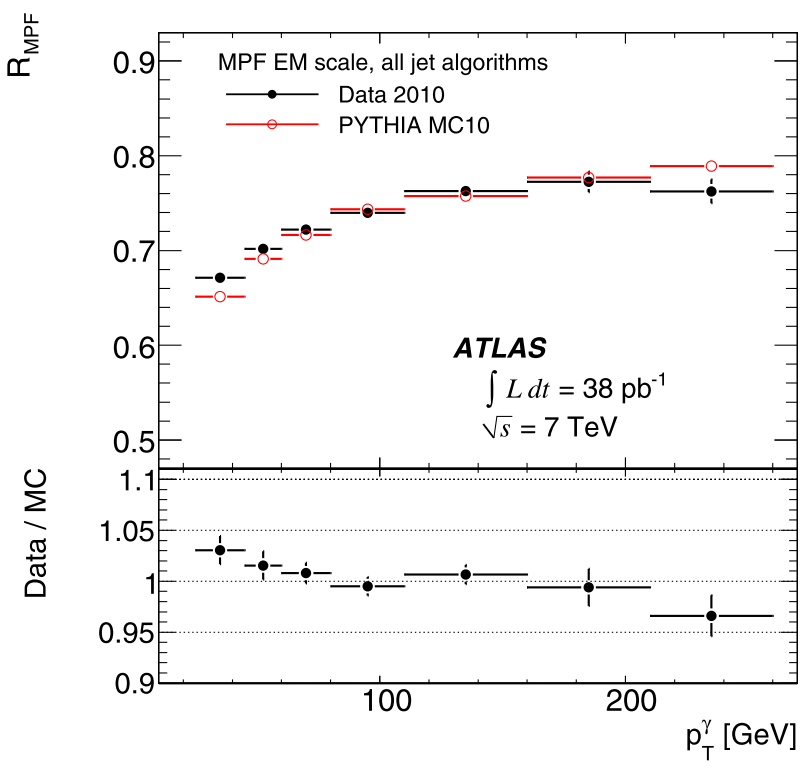

(b) MPF technique

the photon transverse momentum. Only jets within $|\eta|<1.2$ are used. The lower part of the figure shows the data to Monte Carlo simulation ratio. Only statistical uncertainties are shown

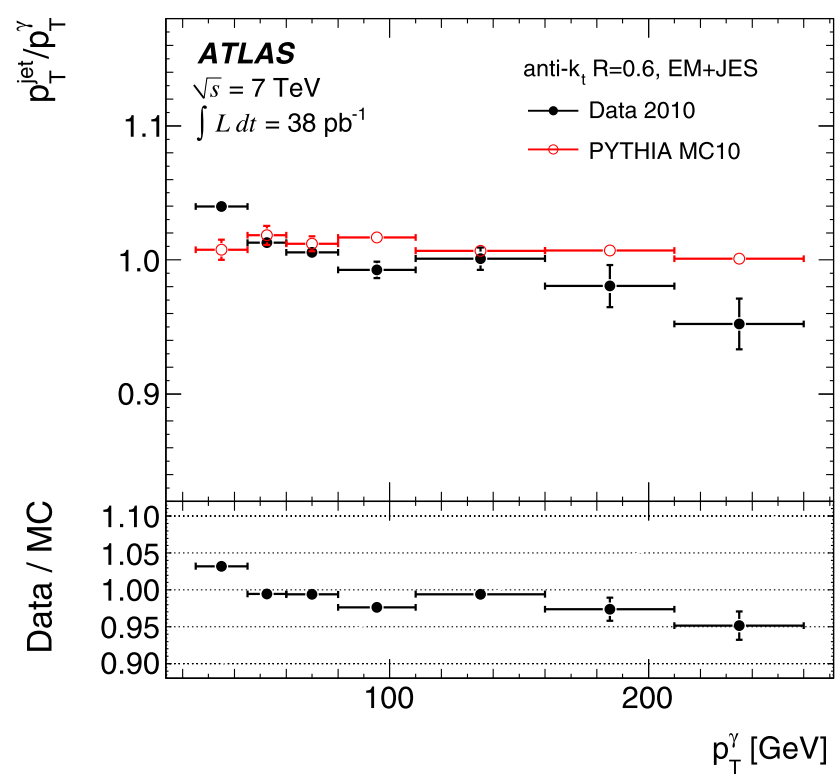

Fig. 33 Average jet response as determined by the direct $p_{\mathrm{T}}$ balance technique for anti- $k_{t}$ jets with $R=0.6$ calibrated with the EM+JES scheme within $|\eta|<1.2$ as a function of the photon transverse momentum for both data and Monte Carlo simulation. The lower part of the figure shows the data to Monte Carlo simulation ratio. Only statistical uncertainties are shown

with the EM+JES scheme, since the EM+JES calibration depends only on the $p_{\mathrm{T}}$ and $\eta$ of the jet and it is applied to data and Monte Carlo simulation. Similarly, Fig. 35 shows the response ratio of data to Monte Carlo simulation, as 


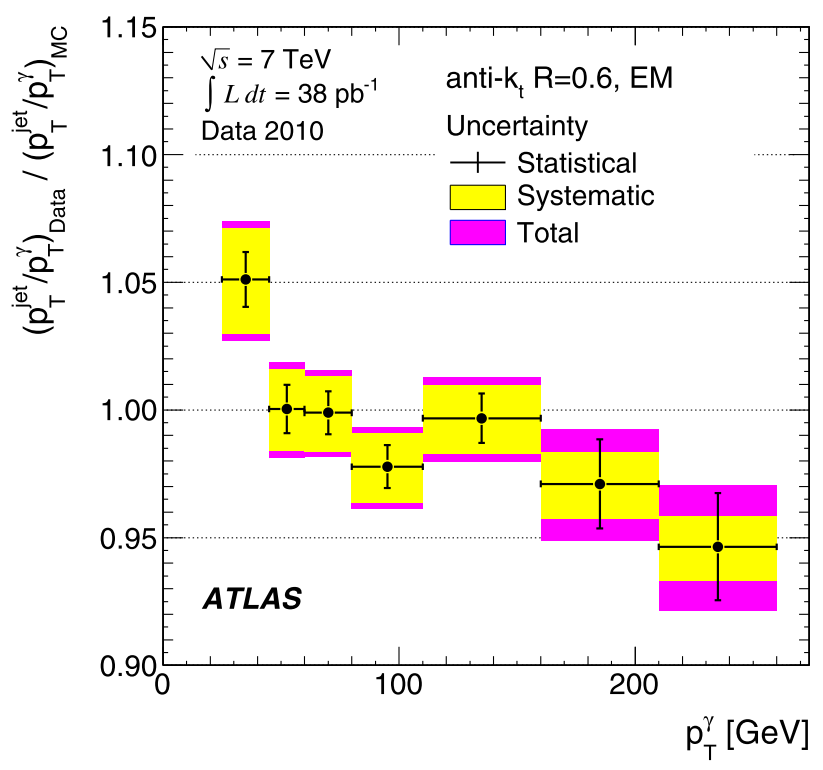

(a) EM

Fig. 34 Average jet response ratio of data to Monte Carlo simulation using the direct $p_{\mathrm{T}}$ balance technique for each input energy scale, EM (a) and EM+JES (b), as a function of the photon transverse momen-

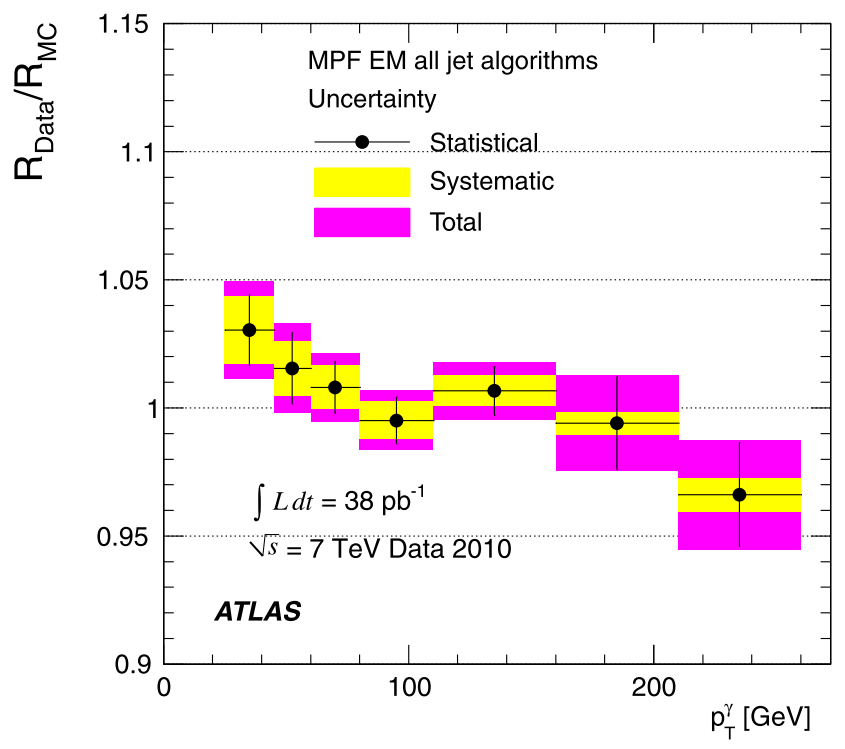

Fig. 35 Average jet response ratio of data to Monte Carlo simulation using the MPF method at the EM scale as a function of the photon transverse momentum. Statistical and systematic uncertainties (light band) are included. The total uncertainty is shown as the dark band. Jets within $|\eta|<1.2$ are used

determined using the MPF technique together with the total uncertainty on the determination of the data to Monte Carlo simulation ratio for the $\gamma$-jet in situ technique as outlined in Sect. 10.2.5. For $p_{\mathrm{T}}^{\gamma}>45 \mathrm{GeV}$, the response in data and Monte Carlo simulation agrees to within $3 \%$ for both

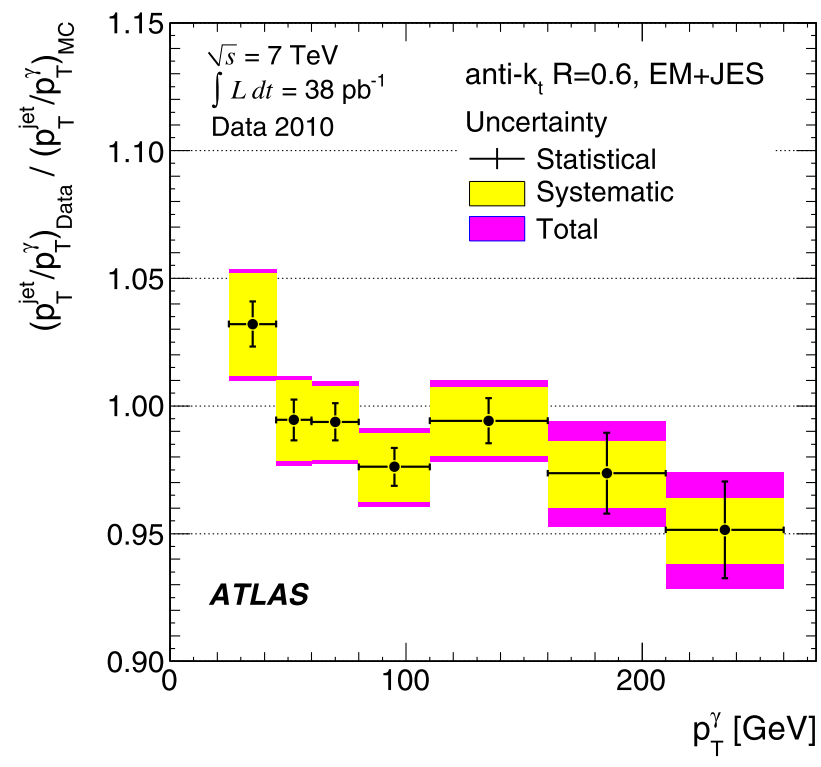

(b) $\mathrm{EM}+\mathrm{JES}$

tum. Statistical and systematic uncertainties (light band) are included with the total uncertainty shown as the dark band. Jets within $|\eta|<1.2$ are used

MPF and direct balance techniques up to about $180 \mathrm{GeV}$. For $p_{\mathrm{T}}>180 \mathrm{GeV}$ the data are $5 \%$ lower than the MC. However, this is not statistically significant as discussed in Sect. 10.5.2. In the range $25 \leq p_{\mathrm{T}}^{\gamma}<45 \mathrm{GeV}$ there is an observed shift of $5 \%$ for the direct $p_{\mathrm{T}}$ balance technique and $3 \%$ for the MPF technique.

The size of these shifts is consistent with the systematic uncertainty on the EM+JES jet energy calibration (see Sect. 9). At high $p_{\mathrm{T}}^{\gamma}$ the dominant uncertainty is statistical while the systematic uncertainty dominates at low $p_{\mathrm{T}}^{\gamma}$.

\subsubsection{Summary of the photon-jet balance}

The validation of the EM+JES calibration scheme for jets with the anti- $k_{t}$ jet algorithm reconstructed from topo-clusters using in situ methods is presented. Agreement between the response in data and Monte Carlo simulation is found to be within statistical uncertainties for $45 \leq p_{\mathrm{T}}^{\gamma}<210 \mathrm{GeV}$. Both techniques observe a shift in the data to Monte Carlo simulation ratio for $25 \leq p_{\mathrm{T}}^{\gamma}<45 \mathrm{GeV}$. The total systematic uncertainties of the $\gamma$-jet in situ technique is estimated to be less than $1.6 \%$ for $45 \leq p_{\mathrm{T}}^{\gamma}<240 \mathrm{GeV}$.

\subsection{Multijet transverse momentum balance}

The $p_{\mathrm{T}}$ reach in the $\gamma$-jet transverse momentum balance technique is limited by the available event statistics. The multijet balance technique where a recoil system consisting of several low- $p_{\mathrm{T}}$ jets balances against a high- $p_{\mathrm{T}}$ jet can be 
used to assess the jet calibration of jet transverse momenta up to the $\mathrm{TeV}$ region. The same method can also be used to obtain correction factors for possible non-linearities at very high $p_{\mathrm{T}}^{\text {jet }}$. Here, the method is only used to assess the JES uncertainty.

\subsubsection{The multijet balance technique}

The method exploits the $p_{\mathrm{T}}$ balance in events where the highest $p_{\mathrm{T}}$ jet (leading jet) is produced back-to-back in $\phi$ to a multijet system. The leading jet is required to have significantly larger transverse momentum than other jets in the event. In this way the leading jet is at a higher $p_{\mathrm{T}}^{\text {jet }}$ scale compared to other reconstructed jets, called non-leading jets. The ensemble of the non-leading jets passing the selection cuts is referred to as the recoil system.

The event topology used in this analysis is sketched in Fig. 36. The vectorial sum of the transverse momenta of all non-leading jets defines the transverse momentum of the recoil system ( $p_{\mathrm{T}}^{\text {Recoil }}$ ), which is expected to approximately balance the transverse momentum of the leading jet $\left(p_{\mathrm{T}}^{\text {Leading }}\right)$. Thus a correlation between the momentum scale of the leading jet and the scale of the non-leading jets can be established. If the absolute JES is well-known for all nonleading jets, the JES of the leading jet can be verified by studying the multijet balance (MJB) that is defined as the ratio:

$\mathrm{MJB}=\frac{\left|\vec{p}_{\mathrm{T}}^{\text {Leading }}\right|}{\left|\vec{p}_{\mathrm{T}}^{\text {Recoil }}\right|}$.

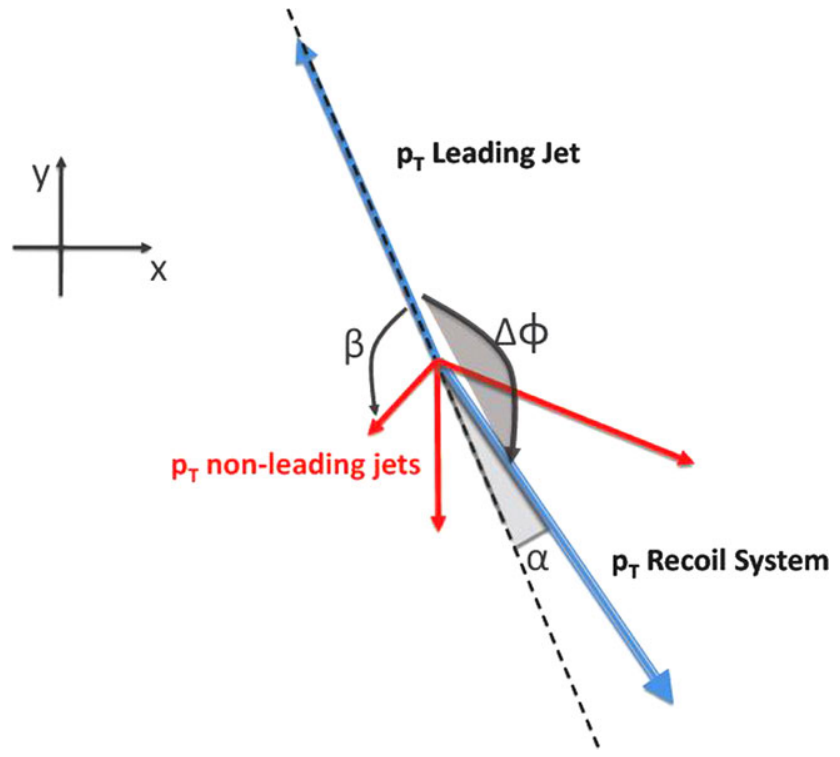

Fig. 36 Sketch of the event topology used for the multijet balance technique in the $x-y$-plane
Moreover, the $p_{\mathrm{T}}^{\text {Recoil }}$ is a good estimator of the true leading jet $p_{\mathrm{T}}$, and it is therefore interesting to study MJB as a function of $p_{\mathrm{T}}^{\text {Recoil }}$. In the ideal case MJB should be equal to one; however, various effects such as the presence of closeby jets, soft gluon emission, pile-up or the selection criteria themselves may introduce a bias.

The comparison between the balance measured in the simulation $\left([\mathrm{MJB}]_{\mathrm{MC}}\right)$ and the data $\left([\mathrm{MJB}]_{\text {Data }}\right)$ can be interpreted as a source of systematic uncertainty and therefore the ratio

$r=[\mathrm{MJB}]_{\text {Data }} /[\mathrm{MJB}]_{\mathrm{MC}}$

can be used to assess the high $p_{\mathrm{T}}$ JES uncertainty.

The jets belonging to the recoil system must be confined to a lower jet energy scale with respect to the leading jet in order to ensure that the multijet balance is testing the absolute high $p_{\mathrm{T}}$ scale and not only the intercalibration between jets. There are various analysis methods to constrain the leading jet to a higher $p_{\mathrm{T}}$ scale with respect to the jets in the recoil system. In this analysis it is done by setting an upper limit on the ratio between the transverse momentum of the second highest $p_{\mathrm{T}}$ jet $\left(p_{\mathrm{T}}^{\mathrm{Jet} 2}\right)$ and the $p_{\mathrm{T}}^{\text {Recoil }}$. This cut is very efficient in selecting multijet events while minimising the bias on the transverse momentum of the leading jet.

\subsubsection{Selection of multijet events}

Two jet trigger selections have been used to cover a wide $p_{\mathrm{T}}$ range with large enough statistics. The first trigger selection requires at least one jet with $p_{\mathrm{T}}>15 \mathrm{GeV}$ at the EM scale in the level-1 calorimeter trigger. The data collected with this trigger are used to cover the region of $p_{\mathrm{T}}^{\text {Recoil }}<260 \mathrm{GeV}$. The second trigger selection, which requires at least one jet with $p_{\mathrm{T}}>95 \mathrm{GeV}$ for the level-1 trigger, is used to populate the region of $p_{\mathrm{T}}^{\text {jet }} \geq 260 \mathrm{GeV}$. The two triggers are fully efficient for jets with $p_{\mathrm{T}}^{\text {Recoil }}>80 \mathrm{GeV}$ and $p_{\mathrm{T}}^{\text {jet }}>250 \mathrm{GeV}$, respectively. To avoid a trigger bias, the multijet balance is studied in events containing a recoil system with transverse momentum larger than $80 \mathrm{GeV}$.

At least three jets in the event with $p_{\mathrm{T}}<25 \mathrm{GeV}$ and $|y|<2.8$ are required. In order to select events with one jet being produced against a well-defined recoil system, a selection is applied using two angular variables ( $\alpha$ and $\beta$ as depicted in Fig. 36):

1. $\alpha=|\Delta \phi-\pi|$, where $\Delta \phi$ is the azimuthal opening angle between the highest $p_{\mathrm{T}}$ jet and the recoil system.

2. $\beta$ is the azimuthal opening angle of the non-leading jet that is closest to the leading jet in $\phi$, measured with respect to the leading jet.

Events are selected by requiring:

1. $\alpha=|\Delta \phi-\pi|<0.3$ radian. 
2. $\beta>1$ radian, i.e. no jets within $|\Delta \phi|=1$ radian around the leading jet.

The cuts applied to $\alpha$ and $\beta$ retain the bulk of the events.

A further selection is applied to ensure that the leading jet is at a higher scale with respect to the jets composing the recoil system. This is done by requiring that the asymmetry ratio $A$ of $p_{\mathrm{T}}^{\mathrm{Jet} 2}$ to the transverse momentum of the recoil system satisfies the following inequality:

$A=\frac{p_{\mathrm{T}}^{\mathrm{Jet} 2}}{p_{\mathrm{T}}^{\text {Recoil }}}<0.6$.

This cut enables the efficient suppression of events with topologies very close to those of dijet events. This can be seen from the distributions of the ratio of the $p_{\mathrm{T}}^{\mathrm{Jet} 2}$ to the leading jet $p_{\mathrm{T}}$ shown in Fig. 37 before and after the cut is applied. Events are weighted according to the pre-scale values applied at the trigger level.

This selection therefore ensures that the leading jet is at a higher scale with respect to the jets forming the recoil system. At the same time this cut does not bias either the leading jet $p_{\mathrm{T}}$ or the recoil system $p_{\mathrm{T}}$. This has been confirmed using Monte Carlo simulation by checking that the average response of the leading jet and recoil system $p_{\mathrm{T}}$ is not significantly shifted from one after the asymmetry cut is applied. A summary of the selection criteria used in the analysis is given in Table 10.

\subsubsection{Measurement of the multijet balance}

The multijet balance is studied as a function of the transverse momentum of the recoil system, $p_{\mathrm{T}}^{\text {Recoil }}$, which is a good

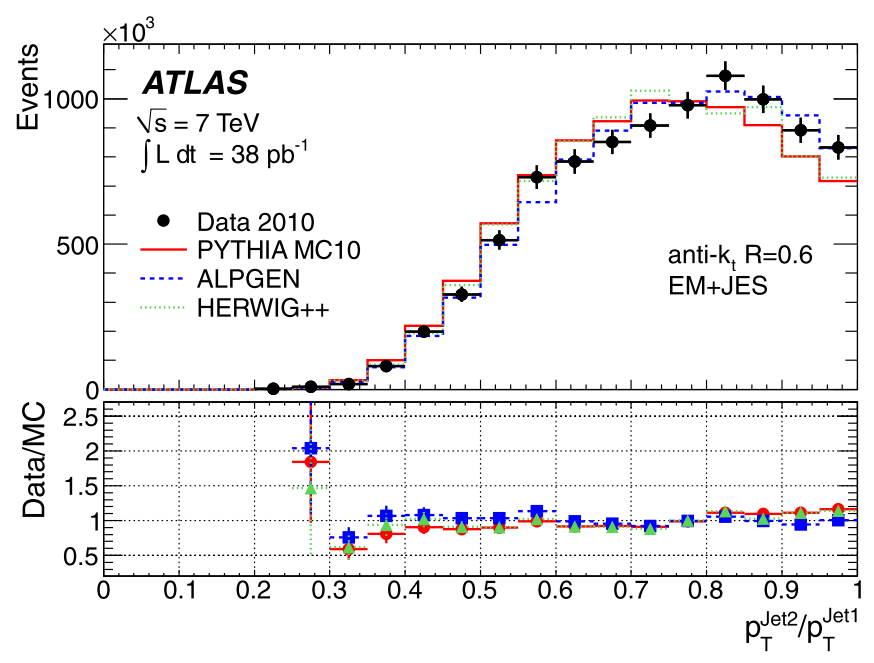

(a) Before asymmetry cut

Fig. 37 Distribution of the ratio of the sub-leading to the leading jet $p_{\mathrm{T}}$ for anti- $k_{t}$ jets with $R=0.6$ before (a) and after (b) the asymmetry cut, see Eq. (31), has been applied for data (full circles) and for simulation (lines). All the distributions in the simulation are normalised to estimator of the true leading jet $p_{\mathrm{T}}$ as shown in Fig. 38 for various Monte Carlo simulations. The ratio of reconstructed $p_{\mathrm{T}}^{\text {Recoil }}$ to the true leading jet $p_{\mathrm{T}}$ as a function of the true leading jet $p_{\mathrm{T}}$ is, on average, consistent with unity to better than $1 \%$.

The multijet balance obtained from the selected events for the anti- $k_{t}$ jet algorithm with $R=0.6$ is shown in Fig. 39 for data and Monte Carlo simulation. The transverse momentum of the recoil system ranges from $80 \mathrm{GeV}$ up to $1.0 \mathrm{TeV}$ for the anti- $k_{t}$ jets with $R=0.6$.

The multijet balance at low $p_{\mathrm{T}}^{\text {Recoil }}$ values shows a bias towards values lower than one. This is due to effects which broaden the leading jet and the $p_{\mathrm{T}}^{\text {Recoil }}$, and is a direct consequence of binning in $p_{\mathrm{T}}^{\text {Recoil }}$. This effect is observed already for truth jets and is, after reconstruction, correctly reproduced by the Monte Carlo simulation.

The data to Monte Carlo simulation ratio obtained from the multijet balance distributions are shown in the lower part of Fig. 39. The average value of the data to Monte Carlo

Table 10 Selection criteria to define the event sample for the multijet balance analysis

\begin{tabular}{ll}
\hline Variable & Cut value \\
\hline Jet $p_{\mathrm{T}}$ & $>20 \mathrm{GeV}$ \\
Jet rapidity & $|y|<2.8$ \\
Number of good jets & $\geq 3$ \\
$p_{\mathrm{T}}^{\text {Recoil }}$ & $>80 \mathrm{GeV}$ \\
$\alpha$ & $<0.3$ radian \\
$\beta$ & $>1$ radian \\
$p_{\mathrm{T}}^{\text {Jet2 }} / p_{\mathrm{T}}^{\text {Recoil }}$ & $<0.6$ \\
\hline
\end{tabular}

(b) After asymmetry cut

the number of data events. Events selected by pre-scaled triggers have entered the histogram weighted by the pre-scale value. Only statistical uncertainties are shown 


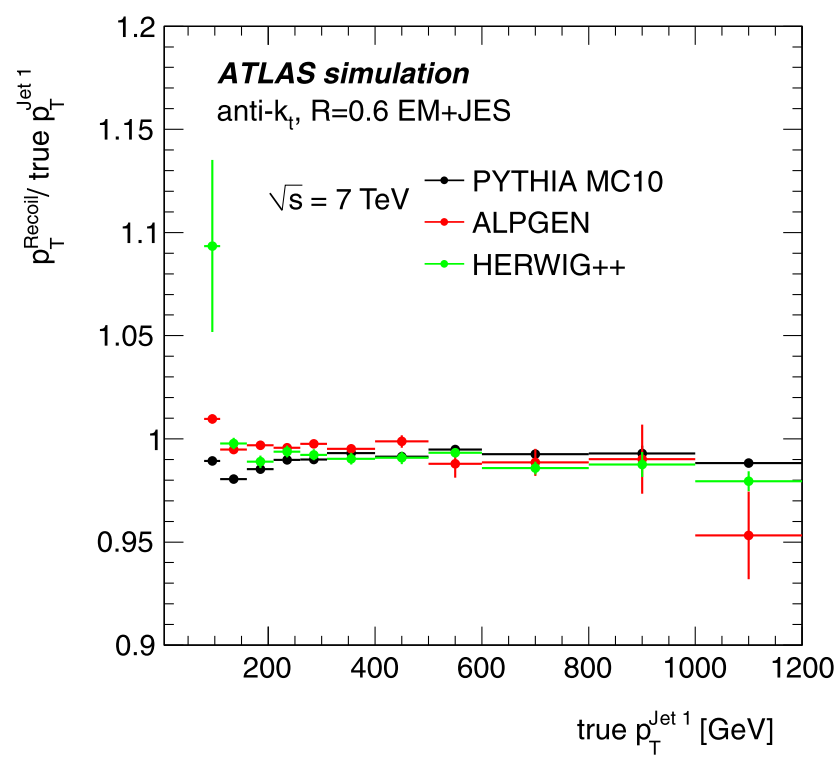

Fig. 38 Ratio of the reconstructed $p_{\mathrm{T}}$ of the recoil system to the true leading jet $p_{\mathrm{T}}$ for anti- $k_{t}$ jets with $R=0.6$ as a function of the true leading jet $p_{\mathrm{T}}$ for three samples of Monte Carlo simulations. Only statistical uncertainties are shown

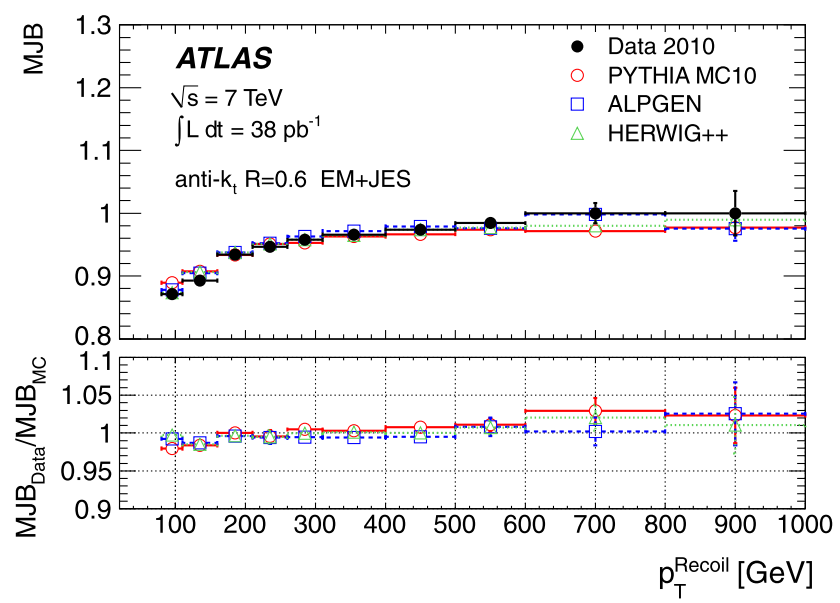

Fig. 39 Multijet balance MJB as a function of the recoil system $p_{\mathrm{T}}$ for data and Monte Carlo simulation for anti- $k_{t}$ jets with $R=0.6$. Only statistical uncertainties are shown

simulation ratio is within $3 \%$ for transverse jet momenta up to the $\mathrm{TeV}$-region. The data to Monte Carlo simulation ratio provides an estimate of the uncertainty on the leading jet $p_{\mathrm{T}}$ scale.

\subsubsection{Estimate of the systematic uncertainty on the multijet balance}

Two main categories of systematic uncertainty have been considered:

1. The reference $p_{\mathrm{T}}^{\text {jet }}$ of the recoil system.
2. The MJB used to probe the leading jet $p_{\mathrm{T}}$, due to selection criteria or an imperfect Monte Carlo simulation modelling of the event.

The standard JES uncertainty has been obtained for isolated jets. In the case of multijet events the additional uncertainty due to close-by jets (see Sect. 17) and the different flavour composition (see Sect. 18) should be taken into account.

The systematic uncertainty on the recoil system has been calculated taking into account the following effects:

1. JES uncertainty: The JES uncertainty described in Sect. 9 is applied to each jet composing the recoil system.

2. Close-by jet: Jets belonging to the recoil system are often produced with another jet nearby in the multijet environment, and the jet response is dependent on the angular distance to the closest jet. The close-by jet uncertainty has been estimated by studying the $p_{\mathrm{T}}$ ratio between the calorimeter jets and matched track jets as a function of the jet transverse momentum for different jet isolation cuts. This uncertainty is discussed in more detail in Sect. 17.

3. Flavour composition of the recoil system: The JES uncertainty is estimated for the average jet composition of the inclusive jet sample. A discrepancy in the specific flavour composition between data and Monte Carlo simulation may result in an additional JES uncertainty. The procedure described in Sect. 18 is used to estimate this uncertainty. It requires as input the average jet response and the flavour composition uncertainty as a function of the jet $p_{\mathrm{T}}$. In the samples used, the uncertainty on the jet $p_{\mathrm{T}}$ due to flavour composition is about $1 \%$.

The systematic uncertainty on MJB due to the uncertainty on $p_{\mathrm{T}}^{\text {Recoil }}$ is estimated by calculating the multijet balance after shifting the $p_{\mathrm{T}}$ of all jets in the recoil system up and down by the systematic uncertainties. The total systematic uncertainty is obtained by summing in quadrature the contribution of each source and is shown in Fig. 40 for anti- $k_{t}$ jets with $R=0.6$. The contributions of each single source are also shown separately. The standard JES uncertainty is the dominant source of uncertainty over the entire $p_{\mathrm{T}}^{\text {jet }}$ range.

The second category of systematic uncertainties includes sources that affect MJB used to probe the jet energy scale at high $p_{\mathrm{T}}^{\text {jet }}$. These are discussed below.

In the following the various sources considered are discussed:

1. Selection criteria: The imperfect description given by the Monte Carlo simulation for the variables used to select the events might induce a systematic uncertainty on the multijet balance. In order to evaluate this systematic uncertainty, all relevant selection criteria are varied 


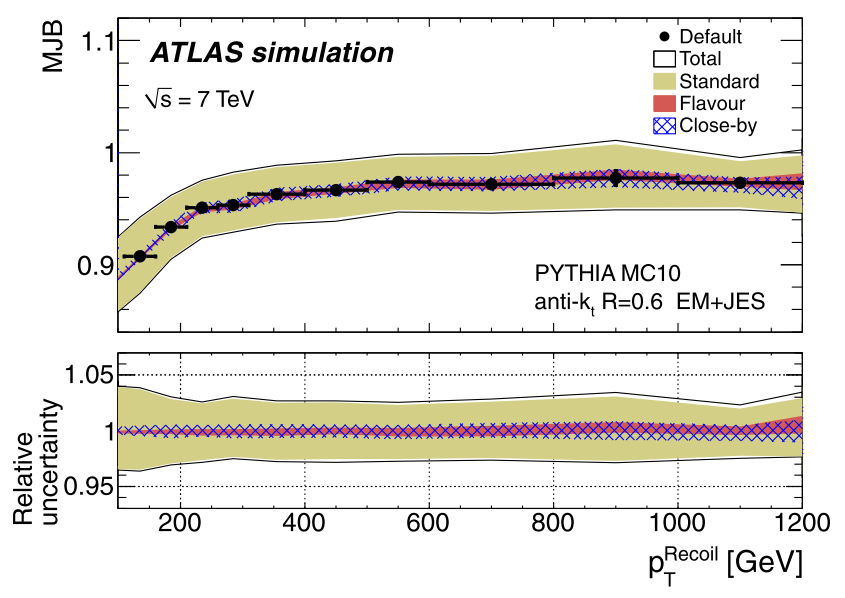

Fig. 40 The multijet balance MJB as a function of $p_{\mathrm{T}}^{\text {Recoil }}$ (full dots) with statistical uncertainties for anti- $k_{t}$ jets with $R=0.6$. The three bands are defined by the maximum shift of MJB when the jets that compose the recoil system are shifted up and down by the standard JES uncertainty, close-by jet and flavour uncertainties. The black lines show the total uncertainty obtained by adding in quadrature the individual uncertainties. The lower part of the figure shows the relative uncertainty due to the scale uncertainty of the jets that compose the recoil system, defined as the maximum relative shift with respect to the nominal value, as a function of $p_{\mathrm{T}}^{\text {Recoil }}$

in a range where the corresponding kinematic variables are not strongly biased and can be examined with small enough statistical fluctuations. The nominal values and the range of variations of the selection criteria are listed in Table 11.

The systematic uncertainty on MJB originating from these sources is evaluated by calculating the multijet balance after varying the cut for each variable in the range mentioned above. For each value of the selection criteria the ratio $(r)$ between the MJB values calculated from data and Monte Carlo simulation is evaluated as a function of the recoil system $p_{\mathrm{T}}$. The maximum deviation of the $r$ with varied cuts $\left(r_{\text {varied }}\right)$ with respect to the nominal ratio $\left(r_{\text {nominal }}\right)$, being expressed in the double ratio

$r_{\text {varied }} / r_{\text {nominal }}$

is assumed to represent the systematic uncertainty for the source. A quadratic sum of the systematic uncertainties for all sources is taken as the total systematic uncertainty.

2. Jet rapidity acceptance: The analysis uses only jets with $|y|<2.8$ to have a smaller jet energy scale uncertainty on the recoil system. This selection, however, could cause an additional systematic uncertainty, if the fraction of jets produced outside the rapidity range differs in the data and Monte Carlo simulation. This effect is evaluated by studying MJB (calculated as usual from only jets with $|y|<2.8$ ) for events with $p_{\mathrm{T}}^{\text {Recoil }}>$ $80 \mathrm{GeV}$, as a function of the total transverse energy $\left(\sum E_{\mathrm{T}}\right)$ summed over all jets with $2.8 \leq|y|<4.5$, in
Table 11 Nominal cut values and the range of variation used to evaluate the systematic uncertainty on the selection criteria for the multijet balance technique. Events below the values are rejected

\begin{tabular}{lll}
\hline Variable & Nominal & Range \\
\hline Jet $p_{\mathrm{T}}$ & $20 \mathrm{GeV}$ & $15-35 \mathrm{GeV}$ \\
$\alpha$ & 0.3 radian & $0.1-0.4$ radian \\
$\beta$ & 1.0 radian & $0.5-1.5$ radian \\
$p_{\mathrm{T}}$ asymmetry cut & 0.6 & $0.4-0.7$ \\
\hline
\end{tabular}

the data and Monte Carlo simulation. The agreement between the data and Monte Carlo simulation is satisfactory, and MJB is stable over the entire $\sum E_{\mathrm{T}}$ range with the largest deviations up to $3 \%$ at relatively high $\sum E_{\mathrm{T}}$. Since the majority of events have a very small $\sum E_{\mathrm{T}}$, this effect is considered to be negligible.

3. Soft physics modelling: Imperfect modelling of multiple parton interactions (underlying event), of fragmentation and of parton shower radiation may affect the multijet balance in two ways. Firstly the selection criteria may act differently on samples with different modelling of the event topology. Secondly MJB itself can be directly affected, since the modelling variation acts differently on the leading jet and the recoil system.

The systematic uncertainty is estimated by evaluating the ratio between the MJB measured using the nominal Monte Carlo simulation and an alternative Monte Carlo simulation sample where the particular source of uncertainty is varied. As alternative Monte Carlo simulation samples Herwig++ and Pythia with the Perugia tune are used.

In addition, the parameter controlling the centre-ofmass energy dependence of the cut-off parameter determining whether an event is produced via a matrix element or by the underlying event model $(\operatorname{PARP}(90))$ is lowered from PYTHIA PARP $(90)=0.25$ to PYTHIA $\operatorname{PARP}(90)=0.16$. This change increases the energy in the forward region. The systematic uncertainty introduced by these variations is at most $2 \%$.

4. Pile-up: Imperfect description of the pile-up may introduce a systematic uncertainty. This effect is estimated by evaluating the ratio

$\mathrm{MJB}^{\text {pile-up }} / \mathrm{MJB}^{\text {nominal }}$,

where the nominal sample is simulated without pile-up collisions. The systematic uncertainty due to pile-up is smaller than $1 \%$ for the whole $p_{\mathrm{T}}^{\text {jet }}$ range considered.

All systematic uncertainties due to the selection criteria, event modelling and pile-up, and the total uncertainty obtained by summing them in quadrature are shown as a function of $p_{\mathrm{T}}^{\text {Recoil }}$ in Fig. 41 for anti- $k_{t}$ jets with $R=0.6$. 


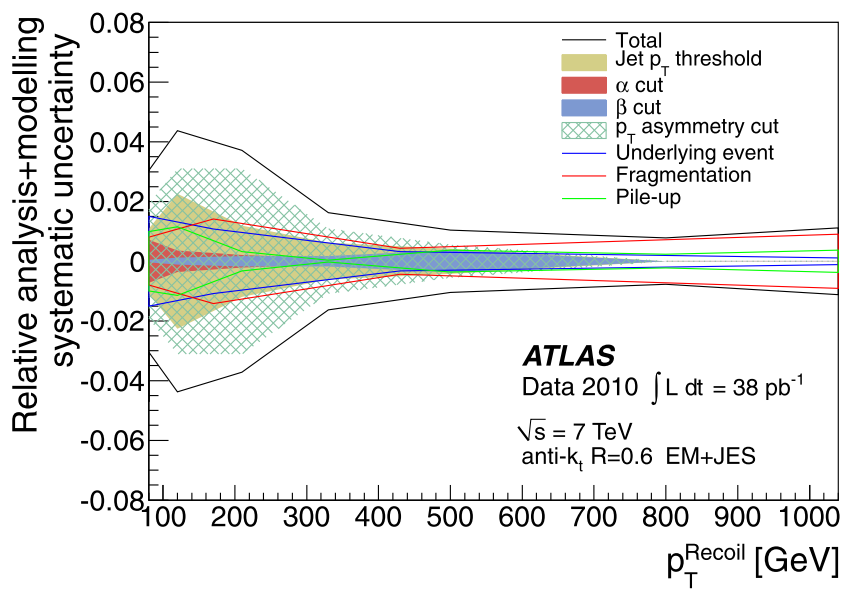

(a)

Fig. 41 (a) Single contributions as a function of $p_{\mathrm{T}}^{\text {Recoil }}$ to the relative uncertainty on MJB due to the sources considered in the selection criteria and event modelling for anti- $k_{t}$ jets with $R=0.6$ (various lines) and the total uncertainty (full line) obtained as the squared sum of all uncertainties. (b) Ratio of data to Monte Carlo simulation for the multijet balance (MJB) as a function of the recoil system $p_{\mathrm{T}}$ for anti- $k_{t}$ jets

The final systematic uncertainty resulting from the uncertainties of the recoil reference system and from the multijet balance variable added in quadrature is presented in Fig. 41b for anti- $k_{t}$ jets with $R=0.6$. The total systematic uncertainty amounts to about $4 \%$ for jets of $p_{\mathrm{T}}^{\text {jet }}=1 \mathrm{TeV}$. At high transverse momentum the main contribution to the systematic uncertainty is due to the standard JES uncertainty of the EM+JES scheme. The relatively large uncertainty due to the asymmetry cut is mainly due to topology dependence of the multijet balance. The maximum values of the uncertainties in the $p_{\mathrm{T}}^{\text {jet }}$ range considered for each source are summarised in Table 12.

\subsubsection{Summary of the multijet balance results}

The data sample collected in 2010 allows the validation of the high- $p_{\mathrm{T}}$ jet energy scale to within $5 \%$ up to $1 \mathrm{TeV}$ for anti- $k_{t}$ jets with $R=0.6$ calibrated with the EM+JES scheme. In this range the statistical uncertainty is roughly equivalent to, or smaller than, the systematic uncertainty.

\subsection{Summary of JES validation using in situ techniques}

The jet energy calibration can be tested in situ using a wellcalibrated object as reference and comparing data to the Pythia Monte Carlo simulation tuned to ATLAS data [49]. The in situ techniques have been discussed in the previous sections, i.e. the comparison of jet calorimeter energy to the momentum carried by tracks associated to a jet (Sect. 10.1),

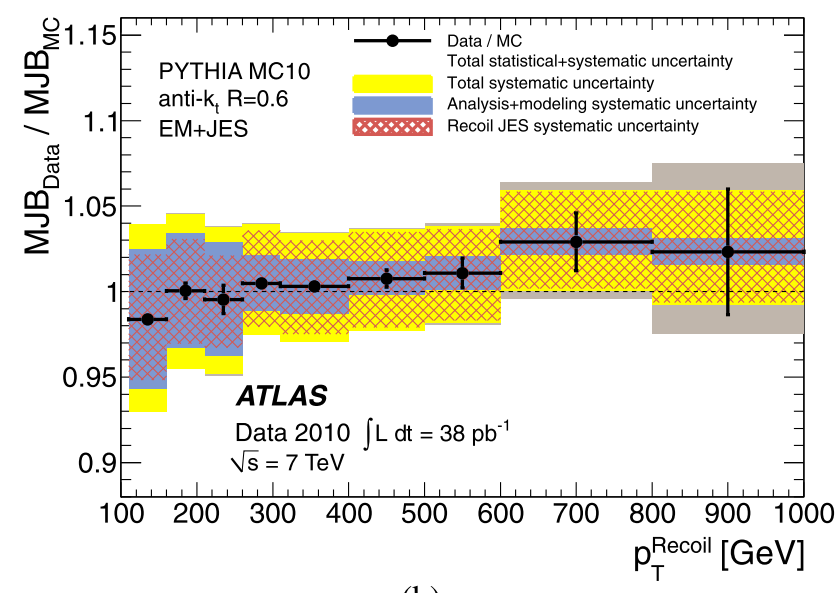

(b)

with $R=0.6$. The various shaded regions show the total uncertainty obtained as the squared sum of all total systematic uncertainties and of the statistical uncertainty. Also displayed are the contributions to the systematic uncertainty due to multijet analysis cuts and event modelling (darkest band) and to the jet energy scale for jets in the recoil system (hatched band)

Table 12 Maximum values of the systematic uncertainties in the whole $p_{\mathrm{T}}^{\text {Recoil }}$ range for anti- $k_{t}$ jets with $R=0.4$ or $R=0.6$, for all effects considered in the multijet balance analysis

\begin{tabular}{ll}
\hline Source & Uncertainty \\
\hline Jet energy scale of the recoil system & $4 \%$ \\
Flavour composition & $\simeq 1 \%$ \\
Close-by jets & $2 \%$ \\
Jet $p_{\mathrm{T}}$ threshold & $<2 \%$ \\
$\alpha$ cut & $<1 \%$ \\
$\beta$ cut & $<1 \%$ \\
$p_{\mathrm{T}}^{\text {Jet} 2 / p_{\mathrm{T}}^{\text {Recoil }} \text { cut }}$ & $3 \%$ \\
Underlying event modelling & $2 \%$ \\
Fragmentation modelling & $1.5 \%$ \\
Pile-up & $<1 \%$ \\
\hline
\end{tabular}

the direct transverse momentum balance between a jet and a photon and the photon balance using the missing transverse momentum projection technique (Sect. 10.2) as well as $p_{\mathrm{T}}$ balance between a high- $p_{\mathrm{T}}$ jet recoiling against a system of lower $p_{\mathrm{T}}$ jets (Sect. 10.3)

The comparison of data to Monte Carlo simulation for all in situ techniques for the pseudorapidity range $|\eta|<1.2$ is shown in Fig. 42 together with the JES uncertainty region as estimated from the single hadron response measurements and systematic variations of the Monte Carlo simulations. The results of the in situ techniques support the estimate of the JES uncertainty obtained using the independent method described in Sect. 9. 


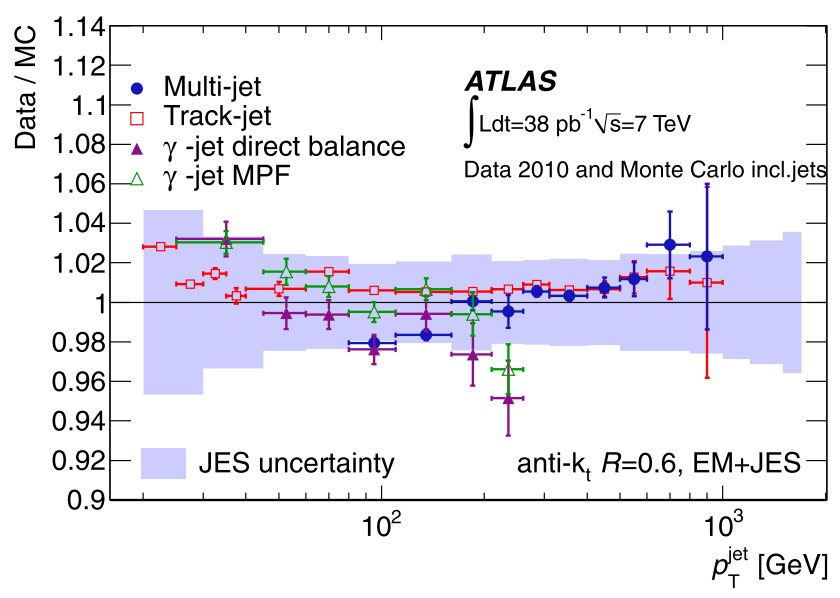

Fig. 42 Ratio of $p_{\mathrm{T}}^{\text {jet }}$ over reference $p_{\mathrm{T}}$ in data and Monte Carlo simulation based on PYTHIA with the MC10 tune for several in situ techniques for $|\eta|<1.2$. Only statistical uncertainties are shown. Superimposed is the jet energy scale uncertainty obtained from single hadron response measurements and systematic Monte Carlo simulation variations as a function of $p_{\mathrm{T}}^{\mathrm{jet}}$ (light band) averaged over the range $|\eta|<1.2$

\subsection{JES uncertainty from combination of in situ techniques}

The JES uncertainty for jets in the range $|\eta|<1.2$ as obtained from in situ techniques is described in the previous sections. In the following section the data to Monte Carlo simulation response ratio for the track jet, the direct balance $\gamma$-jet and the multijet balance method will be combined. ${ }^{27}$

In this combination the ability of the Monte Carlo simulation to describe the data, the individual uncertainties of the in situ techniques and their compatibility are considered.

\subsubsection{Combination technique}

The requirements for combining the uncertainties from the individual in situ techniques are:

1. Propagate all uncertainties of the individual in situ techniques to the final uncertainty.

2. Minimise biases on the shapes of the measured distributions, i.e. on the $p_{\mathrm{T}}$ dependence of the data to Monte Carlo simulation ratio.

3. Optimise the variance of the known uncertainties on the average of the in situ methods while respecting the two previous requirements.

The combination proceeds in the following steps:

1. Toy Monte Carlo method: Monte Carlo pseudo-experiments are created that represent the ensemble of measurements and contain the full data treatment chain including interpolation and averaging (as described in the

\footnotetext{
${ }^{27}$ For the purpose of the combination the multijet balance method is applied for jets within $|\eta|<1.2$.
}

following steps). These pseudo-experiments are used to consistently propagate all uncertainties into the evaluation of the average. The pseudo-experiments represent the full list of available measurements and take into account all known correlations.

2. Interpolation method: A linear interpolation is used to obtain the nominal values of the data to Monte Carlo simulation response ratio for each in situ technique. ${ }^{28}$ The interpolation function for each in situ method is discretized into small $(1 \mathrm{GeV})$ bins. This fine binning is common to all in situ methods for the purpose of averaging.

3. Averaging: The response ratios of the in situ methods are then averaged in the fine bins taking into all known correlations to minimise the spread in the average measured from the Monte Carlo pseudo-experiments. This is equivalent to minimise the $\chi^{2}$ between the average and the individual measurements.

The combination of the in situ calibration data is performed using the software package HVPTools [95]. The systematic uncertainties are introduced in HVPTools for each component as an algebraic function of $p_{\mathrm{T}}$ or as a numerical value for each data point. The systematic uncertainties belonging to the same source are taken to be fully correlated throughout all measurements affected.

The HVPTools package transforms the in situ data and associated statistical and systematic covariance matrices into fine-grained $p_{\mathrm{T}}$ bins, taking into account the best knowledge of the correlations between the points within each in situ measurement. Statistical and systematic correlations between the measurements could also be included, but as the different measurements use independent events, these correlations are neglected. ${ }^{29}$ The covariance matrices are obtained by assuming systematic uncertainties corresponding to the same source are fully correlated. Statistical uncertainties, taken as independent between the data points, are added in quadrature to these matrices.

The interpolated measurements from different in situ methods contributing to a given momentum bin are averaged taking correlations between measurement points into account. The measurements are performed at different $p_{\mathrm{T}}^{\mathrm{jet}}$ values and use different binning (point densities). ${ }^{30}$

\footnotetext{
${ }^{28} \mathrm{~A}$ second order polynomial interpolation provides in principle a better shape description. However, due to the smooth variations in the results of each in situ measurement, the differences between the results obtained with the two interpolation procedures are found to be negligible.

${ }^{29}$ Care was taken to avoid an overlap of the multijet balance and $\gamma$-jet result. Allowing for an overlap would have required taking into account the (strong) correlations, without a potential gain in precision.

${ }^{30}$ The method avoids replacing missing information in case of a lower point density (wider binning) by extrapolating information from the polynomial interpolation.
} 
To derive proper averaging weights for each in situ method, wider averaging regions ${ }^{31}$ are defined. These regions are constructed such that all in situ method covering the corresponding $p_{\mathrm{T}}$ range have at least one measurement inside. The averaging regions are used to compute weights for the in situ methods, which are later applied in the binwise average in fine $1 \mathrm{GeV}$ bins.

The averaging weights for each in situ method are computed as follows:

1. The generation of pseudo-experiments fluctuates the data points around the original measurements taking into account all known correlations. The polynomial interpolation is redone for each pseudo-experiment for each in situ method.

2. For each in situ measurement and each Monte Carlo pseudo-experiment the new bin content for each wider region is calculated from the integral of the interpolating polynomials.

3. The contents of the wide bins are treated as new measurements and are again interpolated with polynomials. The interpolation function is used to obtain new measurements in small $(1 \mathrm{GeV})$ bins for each in situ method in the $p_{\mathrm{T}}^{\text {jet }}$ range covered by it.

4. In each small bin a covariance matrix (diagonal here) between the measurements of each in situ method is computed. Using this matrix the averaging weights are obtained by $\chi^{2}$ minimisation.

For the averaging weights the procedure using the large averaging regions as an intermediate step is important in order to perform a meaningful comparison of the precision of the different in situ methods. The average is computed avoiding shape biases which would come from the use of large bins. Therefore at this next step the fine $1 \mathrm{GeV}$ bins are obtained directly from the interpolation of the original bins.

The bin-wise average between measurements is computed as follows:

1. The generation of Monte Carlo pseudo-experiments fluctuates the data points around the original measurements taking into account all known correlations. The polynomial interpolation is redone for each generated Monte Carlo pseudo-experiment for each in situ method.

2. For each generated pseudo-experiment, small $(1 \mathrm{GeV})$ bins are filled for each measurement in the momentum intervals covered by that in situ method, using the polynomial interpolation.

\footnotetext{
${ }^{31}$ For example, when averaging two measurements with unequal point spacing, a useful averaging region would be defined by the measurement of the in situ method with the larger point spacing, and the points of the other measurement would be statistically merged before computing the averaging weights.
}

3. The average and its uncertainty are computed in each small bin using the weights previously obtained. This will be displayed as a band with the central value given by the average while the total uncertainty on the average is represented by the band width.

4. The covariance matrix among the measurements is computed in each small bin.

5. $\chi^{2}$ rescaling corrections are computed for each bin as follows: if the $\chi^{2}$ value of a bin-wise average exceeds the number of degrees of freedom $\left(n_{\text {dof }}\right)$, the uncertainty on the average is rescaled by $\sqrt{\chi^{2} / n_{\text {dof }}}$ to account for inconsistencies. ${ }^{32}$

The final systematic uncertainty for a given jet momentum is (conservatively) estimated by the maximum deviation between the data to Monte Carlo simulation response ratio together with its total uncertainty (average band) and unity. The central value (measured bias) and the uncertainty on the average measurement are hence taken into account. If a correction for the measured bias were performed, only the relative uncertainty on the average would affect the final JES calibration.

A smoothing procedure using a sliding interval with a Gaussian kernel is applied to the systematic uncertainty on the average. The width of the Gaussian increases as a function of $p_{\mathrm{T}}$. It removes spikes due to statistical fluctuations in the measurements, as well as discontinuities at the first and/or last point in a given measurement.

\subsubsection{Combination results}

Following the method described in the previous section the JES uncertainty for jets with $|\eta|<1.2$ can be obtained. For the purpose of combination the multijet balance analysis is repeated $^{33}$ for jets with $|\eta|<1.2$ and the uncertainty for low- $p_{\mathrm{T}}$ jets forming the recoil system is taken from the $\gamma$-jet analysis.

Figure 43 shows the ratio of the jet response in data and Monte Carlo simulation as a function of the transverse jet momentum for the three in situ techniques using as reference objects photons ( $\gamma$-jet), a system of low-energetic jets (multijet) or the transverse momentum of all tracks associated to jets (track jet). The errors shown for each in situ technique are the statistical and systematic uncertainties added in quadrature.

The results from the track jets cover the widest $p_{\mathrm{T}}^{\text {jet }}$ range from the lowest to the highest $p_{\mathrm{T}}^{\text {jet }}$ values. Compared to

\footnotetext{
${ }^{32}$ Such (small) inconsistencies are seen in the comparison of the $\gamma$-jet and track jet results in one $p_{\mathrm{T}}^{\text {jet }}$ bin.

${ }^{33}$ The restriction in the $\eta$ range applies to all jets. The resulting uncertainty of the multijet method is therefore larger than the one in Sect. 10.3.
} 
the $\gamma$-jet results they have a relatively large systematic uncertainty. The $\gamma$-jet results cover a $p_{\mathrm{T}}^{\text {jet }}$ range up to about $300 \mathrm{GeV}$. From this point onwards the multijet balance method helps to constrain the JES uncertainty.

Figure 44 shows the contribution of each in situ technique to the total JES uncertainty in form of their weight. In the region $30 \lesssim p_{\mathrm{T}}^{\text {jet }} \lesssim 300 \mathrm{GeV}$ the $\gamma$-jet results make the highest contribution to the overall JES uncertainty determination. The contribution is about $80 \%$ at $p_{\mathrm{T}}^{\text {jet }}=30 \mathrm{GeV}$ and decreases to about $60 \%$ at $p_{\mathrm{T}}^{\text {jet }}=300 \mathrm{GeV}$. At the lowest $p_{\mathrm{T}}^{\text {jet }}$ the method based on tracks determines the JES uncertainty. At about $p_{\mathrm{T}}^{\text {jet }}=300 \mathrm{GeV}$ the $\gamma$-jet results and the ones based on tracks have an about equal contribution. Above $p_{\mathrm{T}}^{\text {jet }}=300 \mathrm{GeV}$ the results based on tracks have the highest contribution to the JES uncertainty. In this region the multijet balance contributes to the JES uncertainty to about $20 \%$. For the highest $p_{\mathrm{T}}^{\text {jet }}$ only the multijet balance is used to determine the JES uncertainty.

The final JES uncertainty obtained from the combination of the in situ techniques is shown in Fig. 45. The JES uncertainty is about $9 \%$ at $p_{\mathrm{T}}^{\text {jet }}=20 \mathrm{GeV}$ and decreases to about $2-3 \%$ for $50 \leq p_{\mathrm{T}}^{\text {jet }}<200 \mathrm{GeV}$. At the lowest $p_{\mathrm{T}}^{\text {jet }}$ the systematic uncertainty is determined by the in situ method based on tracks. At $250 \mathrm{GeV}$, the uncertainty increases because the $\gamma$-jet results are $5 \%$ below unity and therefore pull the central value of the average down as shown in Fig. 43. Moreover, the $\gamma$-jet and the track methods give different results. While for all other $p_{\mathrm{T}}^{\text {jet }}$ values the $\chi^{2} / n_{\text {dof }}$ is within $0.2 \leq \chi^{2} / n_{\text {dof }}<0.8$, it rises to $\chi^{2} / n_{\text {dof }}=2$ at $250 \mathrm{GeV}$.

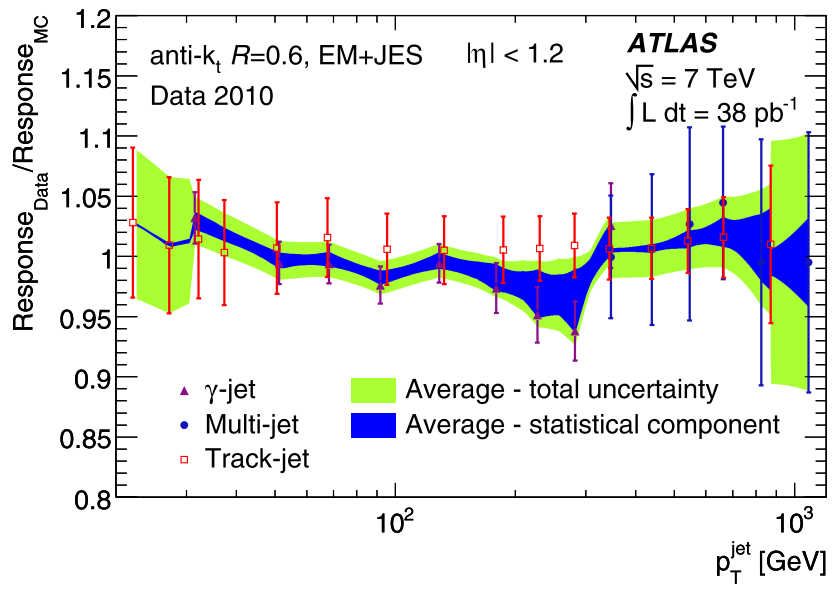

Fig. 43 Average jet response ratio of the data to the Monte Carlo simulation (based on PYTHIA with the MC10 tune) for jets with $|\eta|<1.2$ as a function of the transverse jet momentum $p_{\mathrm{T}}$ for three in situ techniques. The error displays the statistical and systematic uncertainties added in quadrature. Shown are the results for anti- $k_{t}$ jets with $R=0.6$ calibrated with the EM+JES scheme. The light band indicates the total uncertainty from the combination of the in situ techniques. The inner dark band indicates the statistical component
For $p_{\mathrm{T}}^{\text {jet }}>350 \mathrm{GeV}$ the multijet balance contributes to the uncertainty and the resulting uncertainty is about $4-5 \%$ up to $700 \mathrm{GeV}$. At the highest reachable $p_{\mathrm{T}}^{\text {jet }}$ the JES uncertainty increases to $10 \%$.

Figure 45 also compares the JES uncertainty obtained from a combination of in situ techniques to the one derived from the single hadron response measurements and the systematic Monte Carlo simulation variations (see Sect. 9). The in situ JES uncertainty is larger than the standard JES uncertainty in most $p_{\mathrm{T}}^{\mathrm{jet}}$ regions. It is similar in the region $30 \lesssim p_{\mathrm{T}}^{\text {jet }} \lesssim 150 \mathrm{GeV}$. Figure 45 also shows the JES uncertainty, that could have been achieved, if the in situ techniques had been used to correct the jet energy scale in data.

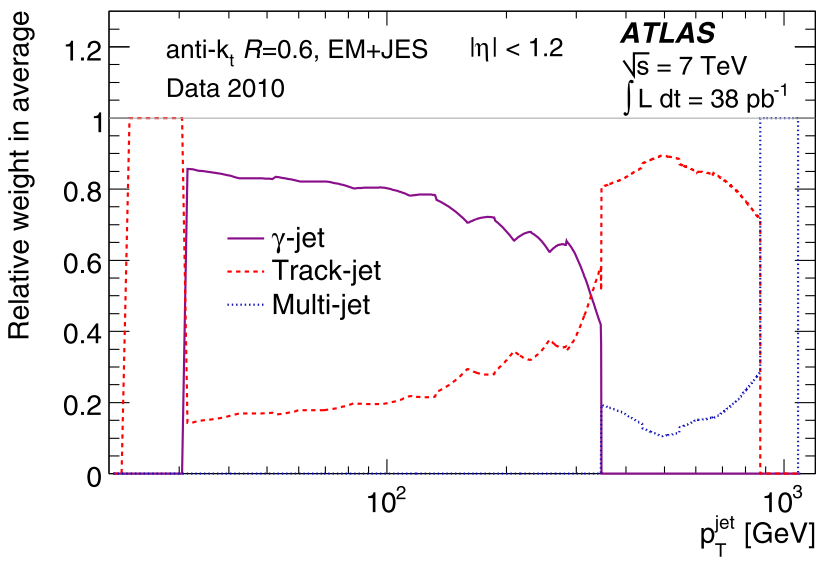

Fig. 44 Weight carried by each in situ technique in the combination to derive the jet energy scale uncertainty as a function of the jet transverse momentum $p_{\mathrm{T}}^{\text {jet }}$ for anti- $k_{t}$ jets with $R=0.6$ calibrated with the EM+JES scheme

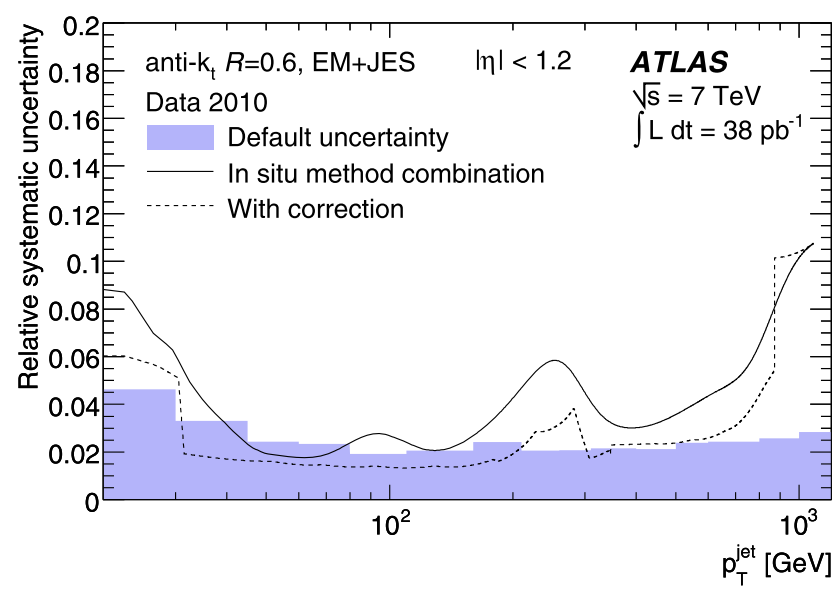

Fig. 45 Jet energy scale uncertainty from the combination of in situ techniques (solid line) as a function of the jet transverse momentum $p_{\mathrm{T}}$ for anti- $k_{t}$ jets with $R=0.6$ calibrated with the EM+JES scheme for $|\eta|<1.2$. The dashed line shows the JES uncertainty that could have been achieved, if in situ techniques had been used to recalibrate the jets. For comparison, the shaded band indicates the JES uncertainties as derived from the single hadron response measurements and systematic Monte Carlo variations for $|\eta|<0.3$ 
In this case the JES uncertainty obtained from a combination of in situ techniques would be slightly smaller than the standard JES uncertainty over a wide $p_{\mathrm{T}}^{\text {jet }}$ range of $30-700 \mathrm{GeV}$.

\section{Jet energy calibration based on global jet properties}

\subsection{Global sequential technique}

The global sequential calibration (GS) technique is a multivariate extension of the EM+JES calibration. After the EM+JES calibration, the dependence of the calorimeter response to the jet on the transverse momentum and pseudo rapidity is removed. However, the response can also depend on other variables that can be removed by the GS technique. Its main effect is to reduce the spread of the reconstructed energy and, thus, improve the jet energy resolution [27].

Any variable $x$ that is correlated with the calorimeter response to the jet can be used. The average response $\mathcal{R}$ as a function of $x$ is defined as

$$
\langle\mathcal{R}(x)\rangle=\left\langle p_{\mathrm{T}}^{\text {jet }}(x) / p_{\mathrm{T}}^{\text {truth }}\right\rangle,
$$

where $p_{\mathrm{T}}^{\mathrm{jet}}$ is the transverse momentum of the calorimeter jet calibrated with EM+JES scheme and $p_{\mathrm{T}}^{\text {truth }}$ that of the truth jet. As for the EM+JES calibration, only isolated calorimeter jets that have a matched isolated truth jet are used (see Sect. 8.3). The average in Eq. (34) is defined as the peak position from a Gaussian fit in a given $p_{\mathrm{T}}^{\text {truth }}, \eta$ and $x$ bin.

The response dependence on $x$ is removed when multiplying $p_{\mathrm{T}}^{\text {jet }}$ by:

$C(x)=1 /\langle\mathcal{R}(x)\rangle$.

Several variables can be used sequentially to achieve the optimal resolution. This procedure requires that the correction for a given variable $x_{i}\left(C^{i}\right)$ is calculated using jets to which the correction for the previous variable $x_{i-1}\left(C^{i-1}\right)$ has already been applied. The jet transverse momentum after correction number $i$ is given by:

$p_{\mathrm{T}}^{i}=C^{i}\left(x_{i}\right) \times p_{\mathrm{T}}^{i-1}=C^{i}\left(x_{i}\right) \times C^{i-1}\left(x_{i-1}\right) \times p_{\mathrm{T}}^{i-2}=\cdots$

In this sequential procedure, care must be taken that corrections for a variable $x_{i}$ are not spoiled by the corrections for a variable $x_{k}(k>i)$. It has been thoroughly checked in this study that the response remains unchanged as a function of all the variables until the last correction.

\subsection{Properties derived from the internal jet structure}

The variables (denoted by $x$ in Eq. (35)) used in the GS calibration are properties characterising the longitudinal and transverse topology of the energy deposited by the jet. A large energy deposit in the calorimeter layers close to the interaction point indicates that the shower started to develop before the calorimeter, leading to a lower detector response, since a part of the jet energy could not be detected. A large energy deposit in the hadronic layers indicates, for example, a larger hadronic component of the jet implying on average a lower calorimeter response. The transverse extent of the jet can be affected by out-of-cluster energy deposits that lead to a lower average response. In the barrel region the energy in the last electromagnetic calorimeter layer and in the first hadronic calorimeter layer will be correlated to energy losses in the poorly instrumented region in between the electromagnetic and hadronic calorimeters. The average detector response to jets and the transverse extent of the jet is correlated to how many particles of the jet hit the poorly instrumented transition region between the barrel and the endcap calorimeters.

Each of these jet properties may be sensitive to several effects: energy deposited in the dead material, noncompensation of the calorimeter, or unmeasured energy due to the noise suppression. In the GS calibration, no attempt is made to separate these effects. The jet properties help to significantly improve the jet energy resolution, and implicitly correct on average for these effects.

The longitudinal structure of the jet ${ }^{34}$ is characterised by the fractional energy deposited in the different layers of the calorimeters before any jet calibration is applied ("layer fractions"):

$$
f_{\text {layer }}=\frac{E_{\mathrm{EM}}^{\text {layer }}}{E_{\mathrm{EM}}^{\mathrm{jet}}},
$$

where $E_{\mathrm{EM}}^{\mathrm{jet}}$ is the jet energy at the EM scale and $E_{\mathrm{EM}}^{\text {layer }}$ the energy deposited in the layer of interest, also defined at the EM scale. The transverse jet structure can be characterised by the jet width defined as:

width $=\frac{\sum_{i} p_{\mathrm{T}}^{i} \Delta R_{i, \text { jet }}}{\sum_{i} p_{\mathrm{T}}^{i}}$,

where the sums are over the jet constituents $(i)$ and $p_{\mathrm{T}}$ is the transverse constituent momentum. $\Delta R_{i, \text { jet }}$ is the distance in $\eta \times \phi$-space between the jet constituents and the jet axis. In the following study topo-clusters are used as jet constituents.

\subsection{Derivation of the global sequential correction}

The GS corrections are determined in jet $|\eta|$ bins of width 0.1 from $|\eta|=0$ to $|\eta|=4.5$. In each bin, the jet properties that provide the largest improvement in jet energy resolution have been selected in an empirical way. The chosen

\footnotetext{
${ }^{34}$ Here, longitudinal refers to the direction along the jet axis.
} 


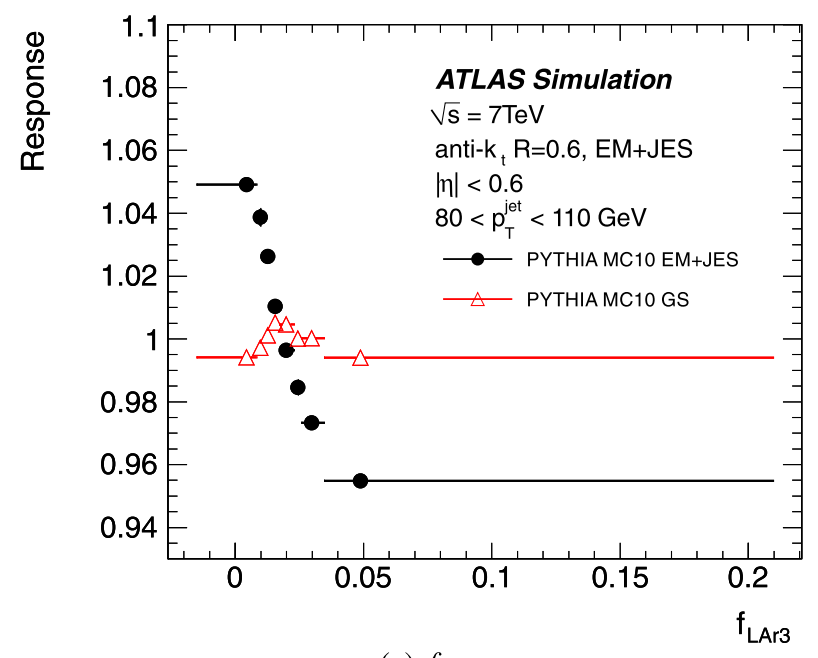

(a) $f_{\mathrm{LAr} 3}$

Fig. 46 Average jet response calculated using truth jets before (circles) and after (triangles) GS calibration as a function of $f_{\mathrm{LAr} 3}$ (a) and the lateral jet width (b) in the Pythia MC10 sample. Anti-

Table 13 Sequence of corrections in the GS calibration scheme in each $|\eta|$ region

\begin{tabular}{lllll}
\hline$|\eta|$ region & Corr 1 & Corr 2 & Corr 3 & Corr 4 \\
\hline$|\eta|<1.2$ & $f_{\mathrm{Tile} 0}$ & $f_{\mathrm{LAr} 3}$ & $f_{\mathrm{PS}}$ & width \\
$1.2 \leq|\eta|<1.4$ & $f_{\mathrm{Tile} 0}$ & & & width \\
$1.4 \leq|\eta|<1.7$ & $f_{\mathrm{Tile} 0}$ & $f_{\mathrm{HEC} 0}$ & & width \\
$1.7 \leq|\eta|<3.0$ & & $f_{\mathrm{HEC} 0}$ & & width \\
$3.0 \leq|\eta|<3.2$ & & $f_{\mathrm{LAr} 3}$ & & width \\
$3.2 \leq|\eta|<3.4$ & & $f_{\mathrm{LAr} 3}$ & & \\
$3.4 \leq|\eta|<3.5$ & & $f_{\mathrm{LAr} 3}$ & & width \\
$3.5 \leq|\eta|<3.8$ & $f_{\mathrm{FCal1}}$ & & width \\
$3.8 \leq|\eta|<4.5$ & $f_{\mathrm{FCal1}}$ & & & \\
\hline
\end{tabular}

jet properties and the order in which they are applied are summarised in Table 13. The improvement in resolution obtained is found to be independent of which property is used first to derive a correction.

In the following section, "GSL" refers to the calibration applied up to the third correction (containing only the calorimeter layer fraction corrections) and "GS" to the calibration applied up to the last correction (including the width correction).

The effect of the GS calibration is shown in Fig. 46 for the case of the $f_{\mathrm{LAr} 3}$ and the lateral jet width properties. In both cases, the jet response is shown before and after GS calibration. Before GS, the response decreases as $f_{\mathrm{LAr} 3}$ and the jet width increase. After GS, the dependence of the calorimeter response to jets on the $f_{\mathrm{LAr} 3}$ and the width variables is reduced to less than $1 \%$. The average response remains unchanged. A similar behaviour is observed as a function of $f_{\mathrm{PS}}$ and $f_{\mathrm{Ti}} \mathrm{e} 0$.

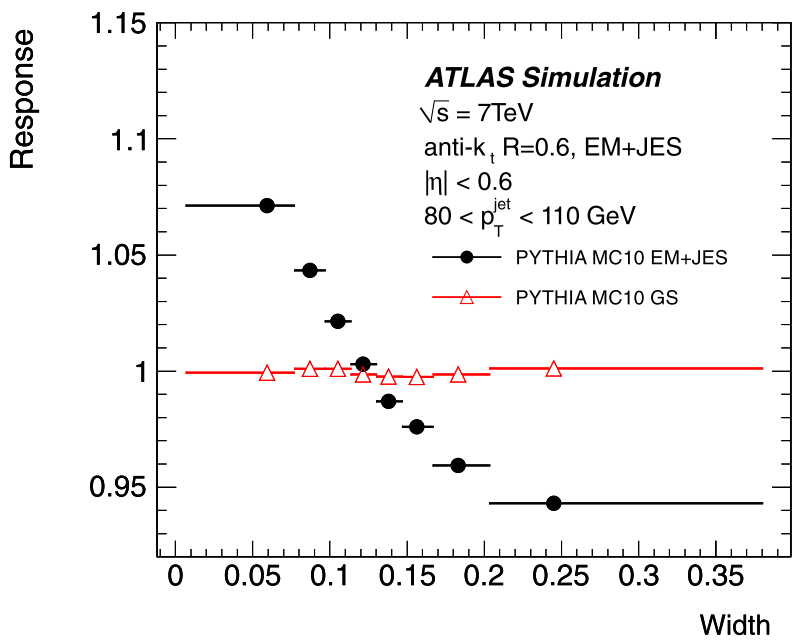

(b) jet width

$k_{t}$ jets with $R=0.6$ calibrated with the EM+JES scheme having $80 \leq p_{\mathrm{T}}<110 \mathrm{GeV}$ and $|\eta|<0.6$ are used

The GS calibration acts in the same way on all the variables shown in Table 13. As explained in Sect. 11.1, the GS results in an improved jet energy resolution [27].The jet energy scale systematic uncertainty and response sensitivity to jet flavor for this calibration are described in Sects. 12, 18 and 19.

\section{Jet energy scale uncertainties for jet calibrations based on global jet properties}

The JES uncertainties in the global sequential jet calibration scheme are evaluated using the transverse momentum balance in events with only two jets at high transverse momentum.

By construction the GS calibration scheme preserves the energy scale of the EM+JES calibration scheme for the event sample from which the corrections have been derived. Possible changes of the JES in event samples with different topologies or jet flavours are studied in Sect. 19.

\subsection{Validation of the global sequential calibration using dijet events}

\subsubsection{Dijet balance method}

The GS corrections can be derived from dijet events using the dijet balance method. This method is a tag-and-probe technique exploiting the $p_{\mathrm{T}}$ imbalance between two backto-back jets. In contrast to the method presented in Sect. 9.6 a correction for the truth jet imbalance is applied. This correction will be described in more detail at the end of this section. 
Dijet events are selected by requiring that the two highest $p_{\text {T }}$ jets are back-to-back ( $\Delta \phi>2.8$ radian). The two jets are required to be in the same pseudorapidity region.

The jet whose response dependence on the layer fractions or width is studied, is referred to as the probe jet, while the other is referred to as the reference jet. The average transverse momentum of the probe and the reference jet is defined as

$p_{\mathrm{T}}^{\text {avg }}=\left(p_{\mathrm{T}}^{\text {probe }}+p_{\mathrm{T}}^{\text {ref }}\right) / 2$.

Since the choice of the reference jet and the probe jet is arbitrary, events are always used twice, inverting the roles of reference and probe.

The GS corrections are measured through the asymmetry variable defined as:

$A(x)=\frac{p_{\mathrm{T}}^{\text {probe }}(x)-p_{\mathrm{T}}^{\text {ref }}}{p_{\mathrm{T}}^{\text {avg }}(x)}$,

where $x$ is any of the properties used in the GS calibration (see Table 13). Both $p_{\mathrm{T}}^{\text {probe }}$ and $p_{\mathrm{T}}^{\text {ref }}$ depend on $x$, but in a given event the value of $x$ of the probe jet is different from that of the reference jet. For this reason the dependence on $x$ is explicitly written in Eq. (40) only for the probe jet.

The probe and the reference jet transverse momenta are defined with the same calibration. When computing the correction factor $C_{i}$, they are both corrected up to the $(i-1)$ th correction (see Sect. 11.1). The mean response as a function of $x$ is given by:

$\langle R(x)\rangle=\left\langle\frac{p_{\mathrm{T}}^{\text {probe }}}{p_{\mathrm{T}}^{\text {ref }}}\right\rangle \simeq \frac{2+\langle A(x)\rangle}{2-\langle A(x)\rangle}$.

For more details see also discussion in Sect. 9.6.1.

The measurement of the response through the asymmetry defined in Eq. (40) assumes that the asymmetry for truth jets is zero. This is true on average (inclusive in $x$ ), but not when computed in bins of $x$. The measured asymmetry $A(x)$ is therefore a mixture of detector effects and imbalance at the level of the generated particles. In order to remove the effect of imbalance at the level of generated particles, a new asymmetry is defined:

$A^{\prime}(x)=A(x)-A_{\text {true }}(x)$,

where $A(x)$ is given by Eq. (40) and $A_{\text {true }}(x)$ is:

$A_{\text {true }}(x)=\frac{p_{\mathrm{T}, \text { truth }}^{\text {probe }}(x)-p_{\mathrm{T}, \text { truth }}^{\text {ref }}}{p_{\mathrm{T}, \text { truth }}^{\text {avg }}(x)}$,

where $p_{\mathrm{T}, \text { truth }}^{\text {avg }}(x)=\left(p_{\mathrm{T}, \text { truth }}^{\text {probe }}(x)+p_{\mathrm{T}, \text { truth }}^{\text {ref }}\right) / 2$ with $p_{\mathrm{T} \text {,truth }}^{\text {probe }}(x)$ and $p_{\mathrm{T}, \text { truth }}^{\text {ref }}$ being the probe and reference jets transverse momenta, respectively. The variable $A_{\text {true }}(x)$ denotes the true asymmetry. The variable $x$ in Eq. (42) is that of the reconstructed jet matched to the truth jet. Matching is done using the same criterion as for the EM+JES calibration (see Sect. 8.3). When using $A^{\prime}(x)$ instead of $A(x)$ in Eq. (41), the effects of imbalance at the level of generated particles are removed and the resulting response depends only on detector effects. Accounting for the truth jet imbalance is particularly important for the corrections that depend on the energy in the presampler and the jet width.

\subsubsection{Validation of the dijet balance method in the Monte Carlo simulation}

The dijet balance method can be checked in two different ways.

The first uses the default Pythia event sample with the MC10 tune and compares the average response calculated using Eq. (41) to the average response calculated using the truth jets (see Eq. (34)). Figure 47 shows this comparison for jets after the EM+JES calibration for $80 \leq p_{\mathrm{T}}^{\text {jet }}<110 \mathrm{GeV}$ and $|\eta|<0.6$. The results obtained using the asymmetry defined as in Eq. (40) and when incorporating the true asymmetry are shown. If the true asymmetry were ignored, the calculated response would be different from the true jet response by up to $4 \%$ for high values of the jet width and the presampler fraction in this particular $p_{\mathrm{T}}^{\text {jet }}$ bin. This difference increases with decreasing $p_{\mathrm{T}}^{\text {jet }}$ reaching $8 \%$ for jets of $p_{\mathrm{T}}^{\text {jet }} \approx 20 \mathrm{GeV}$. These differences are reduced to less than $2 \%$ when a correction for $A_{\text {true }}$ is used. Similar results are found in the other $p_{\mathrm{T}}^{\text {jet }}$ and $|\eta|$ bins.

The second test compares the true asymmetry between different simulated samples. Figure 48 shows the true asymmetry as a function of $f_{\mathrm{PS}}, f_{\mathrm{LAr} 3}, f_{\mathrm{Tile} 0}$ and the jet width in the central region for $40 \leq p_{\mathrm{T}}^{\mathrm{jet}}<60 \mathrm{GeV}$ for various event samples: the reference PYTHIA sample with the MC10 tune, the Pythia sample with the Perugia2010 tune and the HERWIG++ sample. The distributions of the jet properties are also shown. The last two samples test the sensitivity to the description of soft physics or the specifics of the hadronisation process that could cause differences in the truth jet imbalance. The true asymmetry differs by no more than $5 \%$ in this particular $p_{\mathrm{T}}^{\text {jet }}$ and $\eta$ bin. For $p_{\mathrm{T}}^{\text {jet }}>60 \mathrm{GeV}$ and other $|\eta|$ bins, the true asymmetries differ by less than $2 \%$. At low $p_{\mathrm{T}}^{\text {jet }}$ (below $40 \mathrm{GeV}$ in the barrel), the $\Delta \phi$ cut, in particular combined with the small PERUGIA2010 and HERWIG++ samples yield statistical uncertainties of the order of $5 \%$.

In summary, the dijet balance method allows the determination of the response as a function of the layer fractions and the jet width over the entire transverse jet momentum and pseudorapidity ranges. This method can therefore be applied to data to validate the corrections derived in the Monte Carlo simulation. 


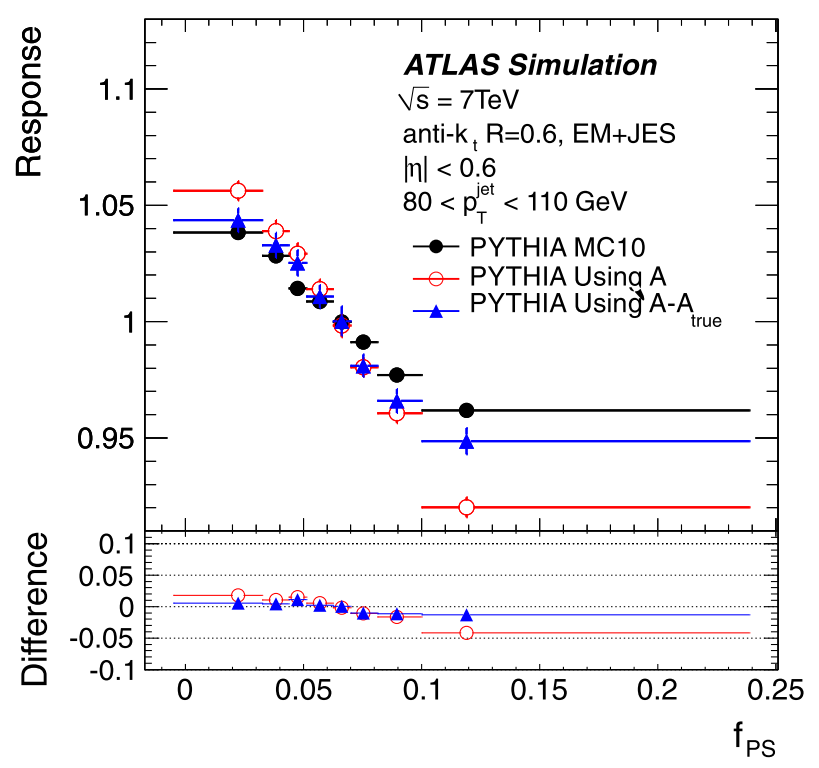

(a) $f_{\mathrm{PS}}$

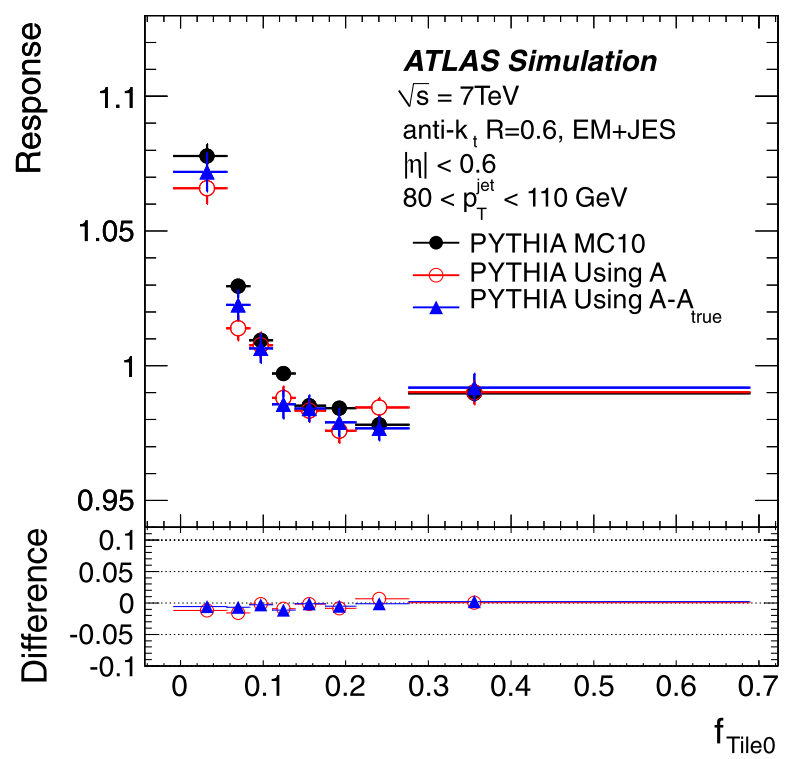

(c) $f_{\text {Tile } 0}$

Fig. 47 Average jet response calculated using truth jets (full circles), using the reconstructed asymmetry $A$ (open circles), and using $A-A_{\text {true }}$ (triangles) as a function of the calorimeter layer energy fraction $f_{\mathrm{PS}}(\mathbf{a}), f_{\mathrm{LAr} 3}(\mathbf{b}), f_{\mathrm{Tile} 0}(\mathbf{c})$ and the lateral jet width $(\mathbf{d})$ in the PYTHIA MC10 sample before applying GS calibration. The lower part

\subsubsection{Differences between data based and Monte Carlo based corrections}

Figure 49 shows the difference between the reconstructed asymmetry and the true asymmetry for the PYTHIA MC10 sample as a function of $f_{\mathrm{PS}}, f_{\mathrm{LAr} 3}, f_{\mathrm{Tile} 0}$ and width for jets with $80 \leq p_{\mathrm{T}}^{\text {jet }}<110 \mathrm{GeV}$ and $|\eta|<0.6$. The reconstructed

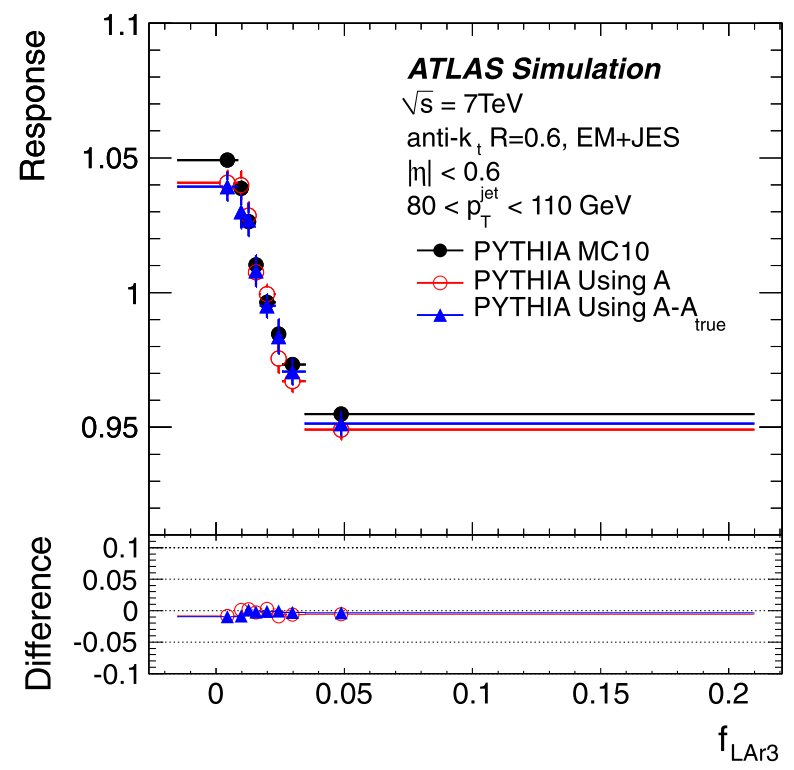

(b) $f_{\text {LAr3 }}$

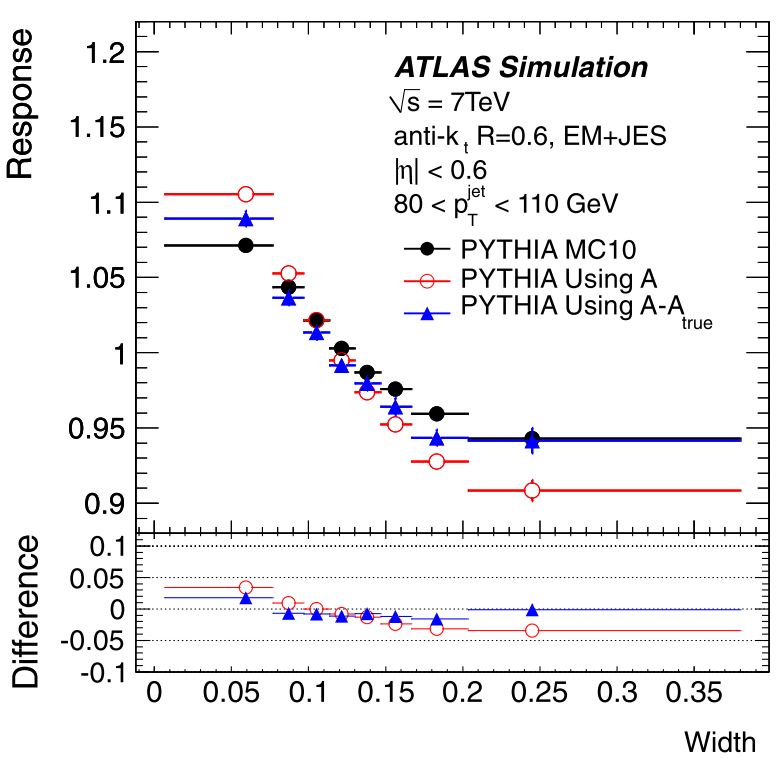

(d) jet width

of each figure shows the differences between the response calculated using the truth jet and the one calculated with the dijet balance method without $A_{\text {true }}$ (full triangles) and with $A_{\text {true }}$ (open circles). Anti- $k_{t}$ jets with $R=0.6$ calibrated with the EM+JES scheme are used and have $80 \leq p_{\mathrm{T}}^{\text {jet }}<110 \mathrm{GeV}$ and $|\eta|<0.6$

asymmetries in data and the PYTHIA MC10 sample are compatible within statistical uncertainties. Similar agreement is found in the other $\eta$ and $p_{\mathrm{T}}^{\text {jet }}$ regions.

The asymmetries as shown in Fig. 49 are used to derive data based corrections. The difference between data and Monte Carlo simulation provides a quantitative measure of the additional jet energy scale uncertainty intro- 


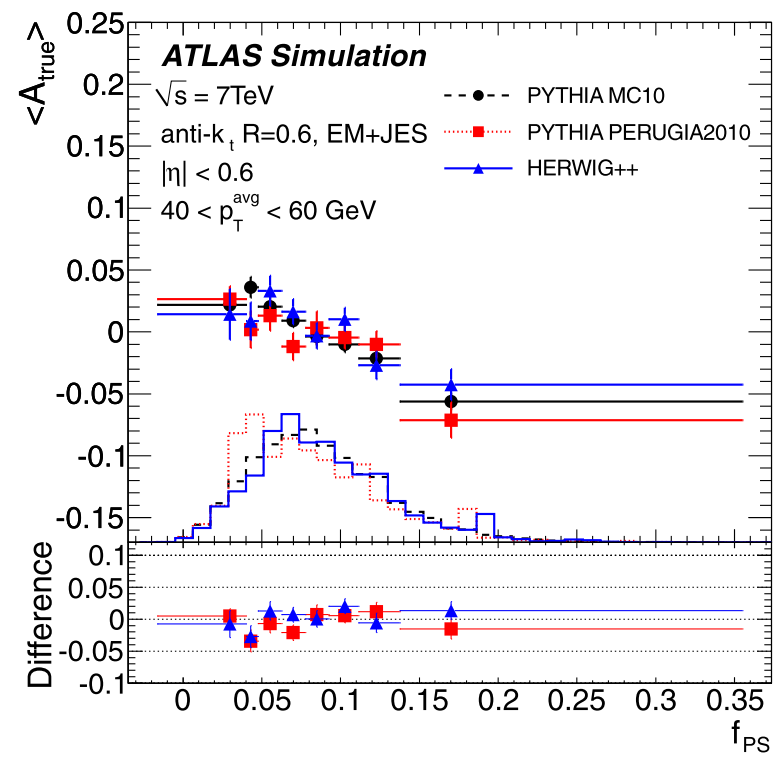

(a) $f_{\mathrm{PS}}$

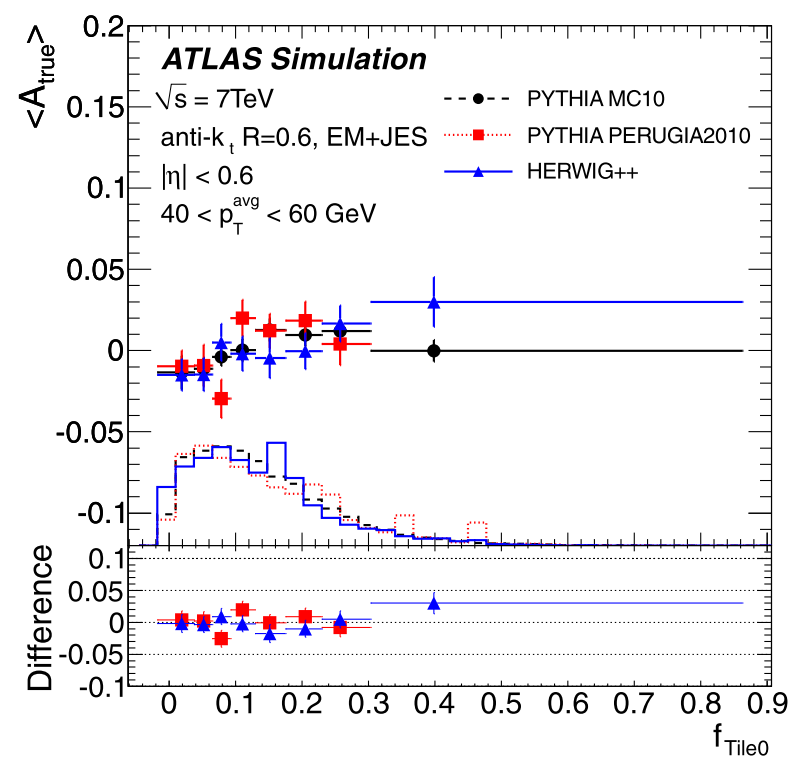

(c) $f_{\text {Tile } 0}$

Fig. 48 Average asymmetry for truth jets obtained from various Monte Carlo event generators and tunes (PYTHIA with the MC10 and the PERUgia2010 tune and Herwig++) as a function of the calorimeter layer fraction $f_{\mathrm{PS}}(\mathbf{a}), f_{\mathrm{LAr} 3}(\mathbf{b}), f_{\mathrm{Tile} 0}(\mathbf{c})$ and the lateral jet width (d) of the probe jet. Anti- $k_{t}$ jets with $R=0.6$ calibrated with the

duced by the GS calibration. After the first two corrections in Table 13 the response changes by less than $1 \%$ for data based and Monte Carlo based corrections. The response changes by an additional $1 \%$ to $2 \%$ after the third (PS) and the fourth (width) corrections are applied in the barrel. The agreement in the endcap is within $2 \%(4 \%)$ for $p_{\mathrm{T}}^{\text {truth }}>60 \mathrm{GeV}(<60 \mathrm{GeV})$.

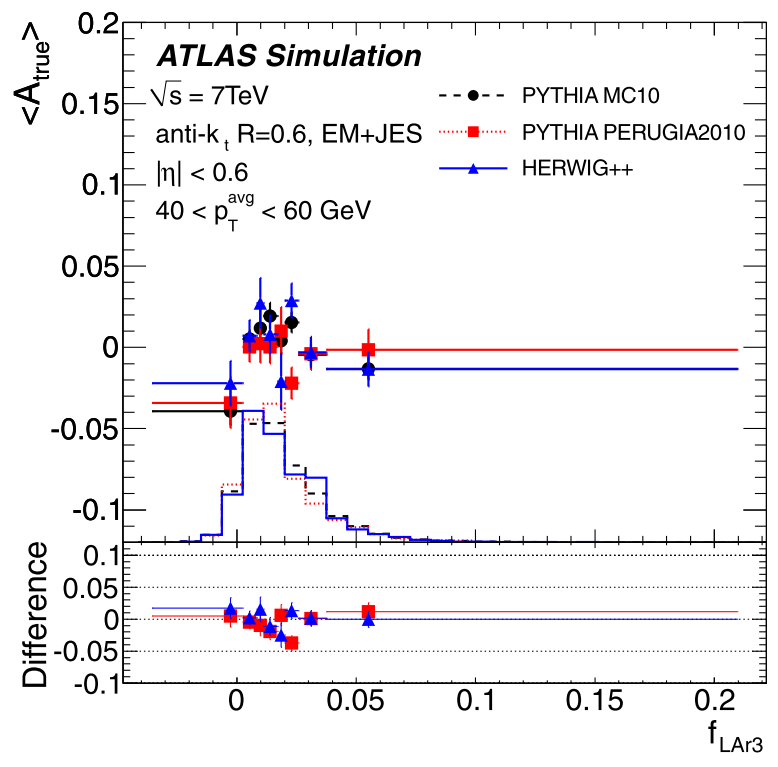

(b) $f_{\mathrm{LAr} 3}$

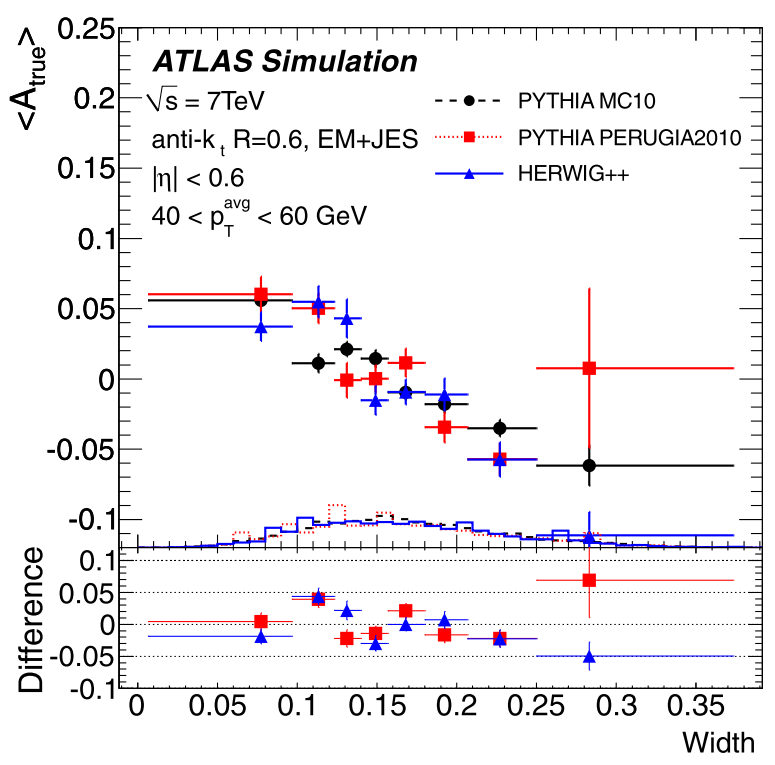

(d) Jet width

EM+JES scheme are used and have $40 \leq p_{\mathrm{T}}^{\text {avg }}<60 \mathrm{GeV}$ and $|\eta|<0.6$. The distributions of the jet properties are superimposed on each figure. The lower part of each figure shows the differences between PYTHIA with the Perugia2010 tune or Herwig++ and Pythia with the MC10 tune

Data based corrections are also derived with true asymmetries coming from the Perugia2010 and HerwiG++ samples. These corrections are then applied to the reference PyTHIA MC10 sample and the response yielded is compared to the response obtained after applying the reference data based corrections using the true asymmetry from the reference PYTHIA MC10 sample. The difference in response 


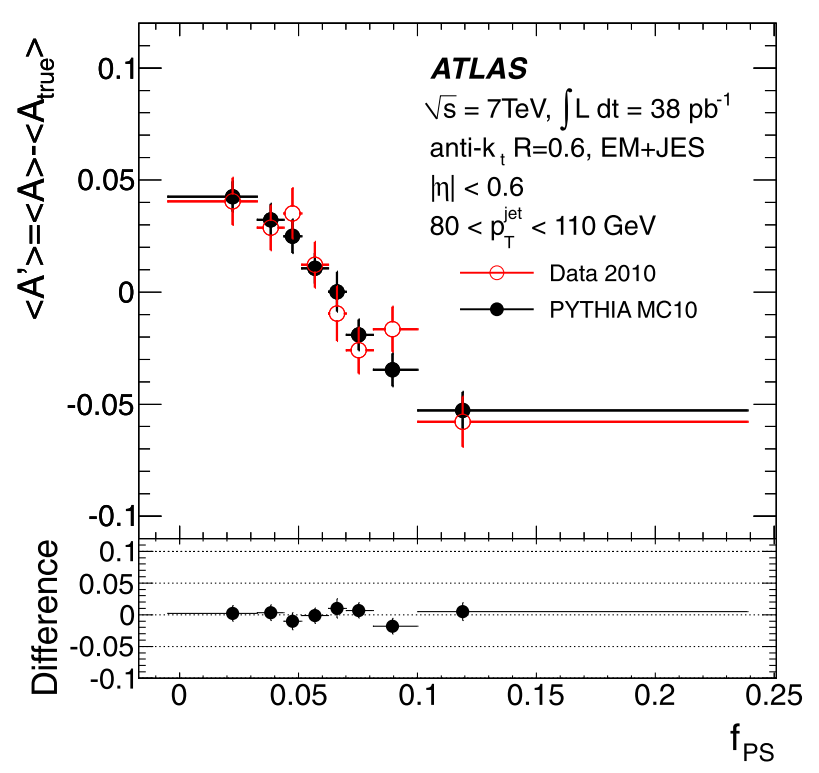

(a) $f_{\mathrm{PS}}$

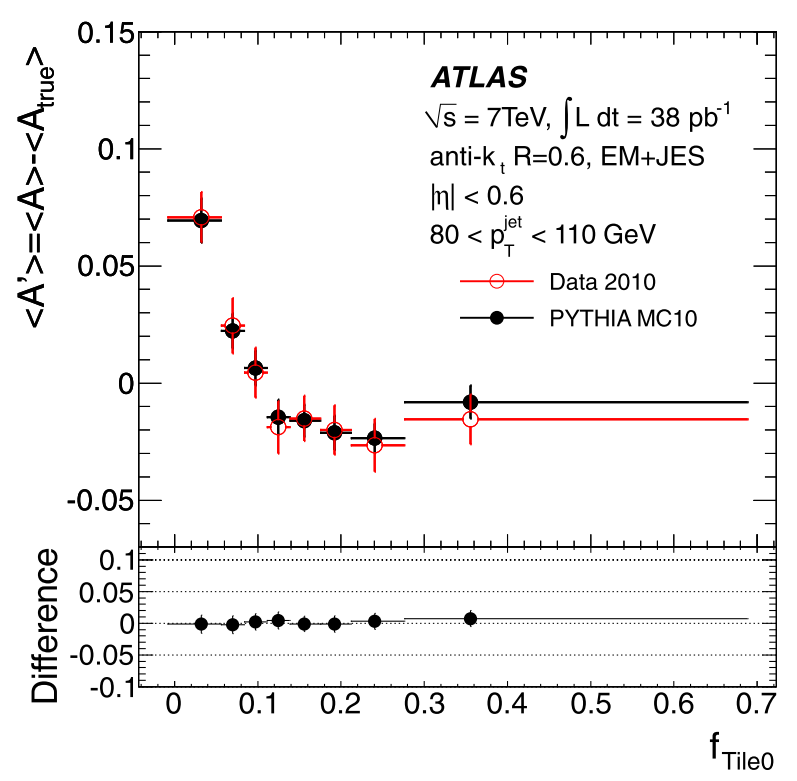

(c) $f_{\text {Tile } 0}$

Fig. 49 Difference between the average reconstructed asymmetry and the average true asymmetry in data (open circles) and in the reference PyTHIA MC10 sample (full circles) as a function of the calorimeter layer fractions $f_{\mathrm{PS}}(\mathbf{a}), f_{\mathrm{LAr} 3}(\mathbf{b}), f_{\mathrm{Tile} 0}(\mathbf{c})$ and the lateral jet

is found to be lower than $0.5 \%$ in all the $p_{\mathrm{T}}^{\text {jet }}$ and $|\eta|$ bins where the statistical uncertainty is small enough.

As a further cross-check the same GS corrections (here the Monte Carlo based ones) are applied to both data and Monte Carlo simulation samples. The difference between data and simulation reflects differences in the jet properties used as input to the GS calibration in the inclusive samples.

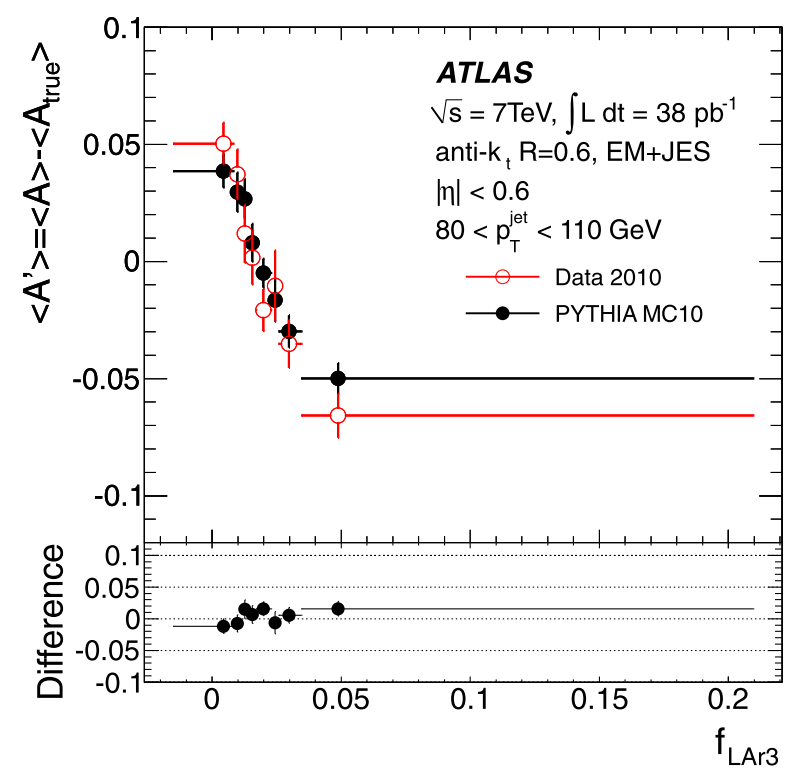

(b) $f_{\mathrm{LAr} 3}$

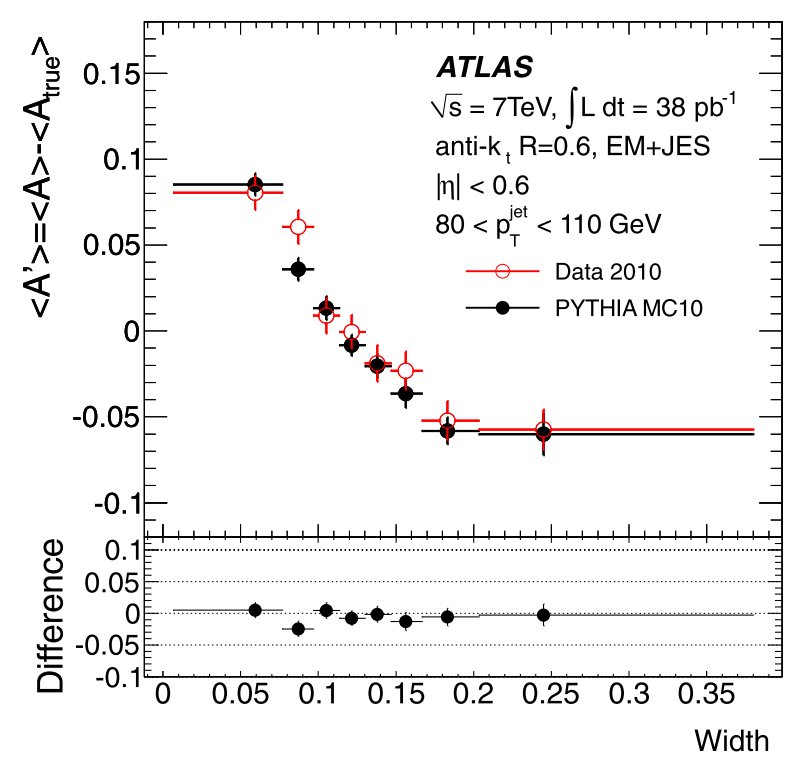

(d) Jet width

width (d). The lower part of each figure shows the differences between Monte Carlo simulation and data. Anti- $k_{t}$ jets with $R=0.6$ calibrated with the EM+JES scheme are used and have $80 \leq p_{\mathrm{T}}^{\text {jet }}<110 \mathrm{GeV}$ and $|\eta|<0.6$

Figure 50 shows the mean value of $f_{\mathrm{PS}}, f_{\mathrm{LAr} 3}, f_{\mathrm{Til}} 0$ and width as a function of $p_{\mathrm{T}}^{\text {jet }}$ in the barrel for data and various Monte Carlo simulation samples: the nominal PYTHIA MC10, Pythia Perugia2010 and Herwig++. The agreement for $f_{\mathrm{Tile} 0}$ and $f_{\mathrm{PS}}$ between data and PYTHIA with the $\mathrm{MC} 10$ tune is within $5 \%$ over the entire $p_{\mathrm{T}}^{\text {jet }}$ range. For $f_{\mathrm{LAr} 3}$, this agreement is also within $5 \%$ except for 


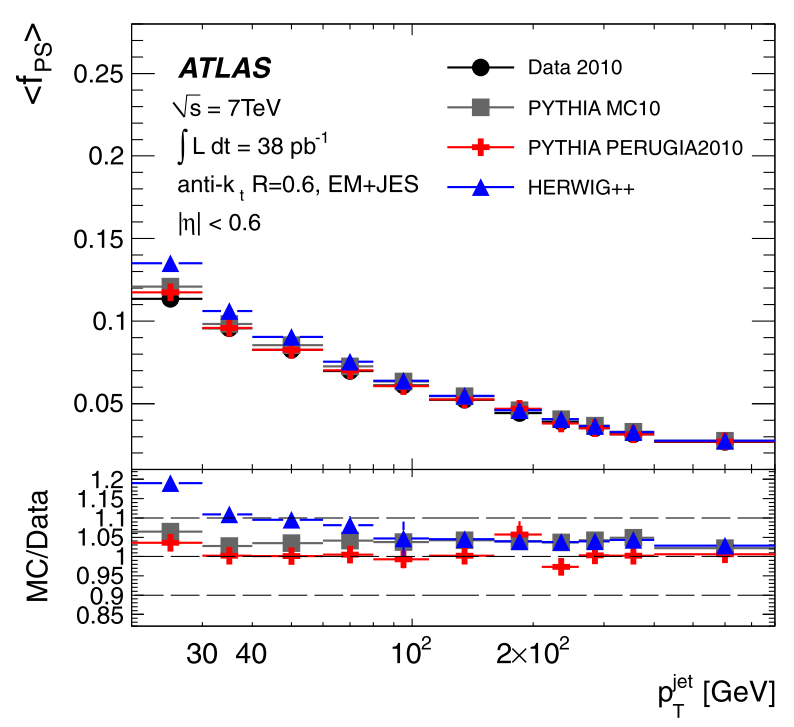

(a) $f_{\mathrm{PS}}$

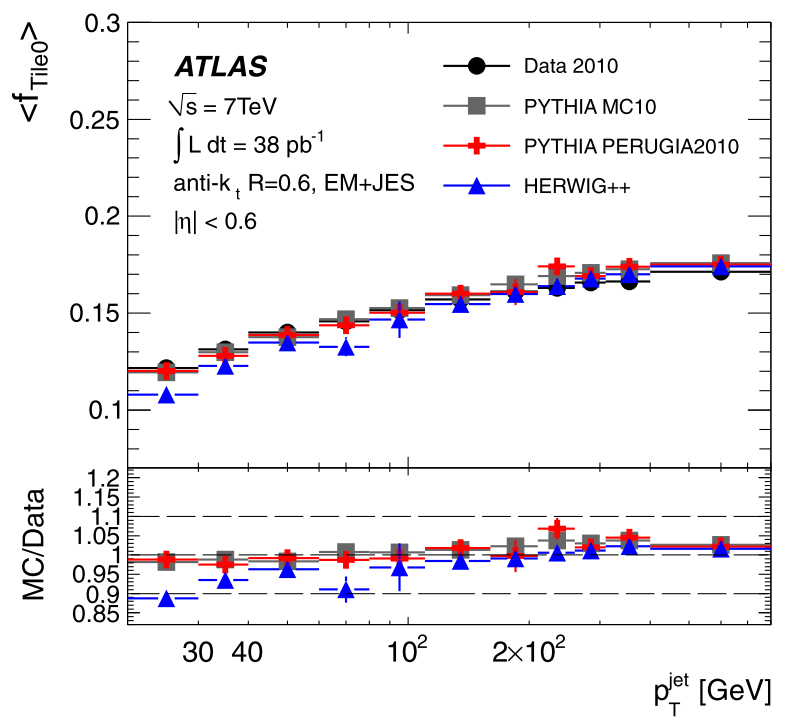

(c) $f_{\mathrm{Tile}}$

Fig. 50 Mean value of the jet calorimeter layer fractions $f_{\mathrm{PS}}(\mathbf{a})$, $f_{\mathrm{LAr} 3}(\mathbf{b}), f_{\mathrm{Tile} 0}(\mathbf{c})$ and the jet width $(\mathbf{d})$ as a function of $p_{\mathrm{T}}^{\text {jet }}$ for $|\eta|<0.6$ for data and various Monte Carlo simulations. Anti- $k_{t}$ jets

$20 \leq p_{\mathrm{T}}^{\text {jet }}<30 \mathrm{GeV}$ where a disagreement of $7.5 \%$ is observed. A larger disagreement is found for the jet width. Jets are $5 \%(10 \%)$ wider in data than in Monte Carlo simulation at $200 \mathrm{GeV}(600 \mathrm{GeV})$ and more narrow at $p_{\mathrm{T}}<30 \mathrm{GeV}$.

The standard deviations of the $f_{\mathrm{LAr} 3}$ and the $f_{\mathrm{PS}}$ distributions show also agreement within $5 \%$ between data and PYTHIA MC10 simulation over the entire $p_{\mathrm{T}}^{\text {jet }}$ range (not shown). For $f_{\text {Tile } 0}$ and width, disagreements of $10 \%$ are observed in some $p_{\mathrm{T}}^{\text {jet }}$ bins. Similar results are found in the

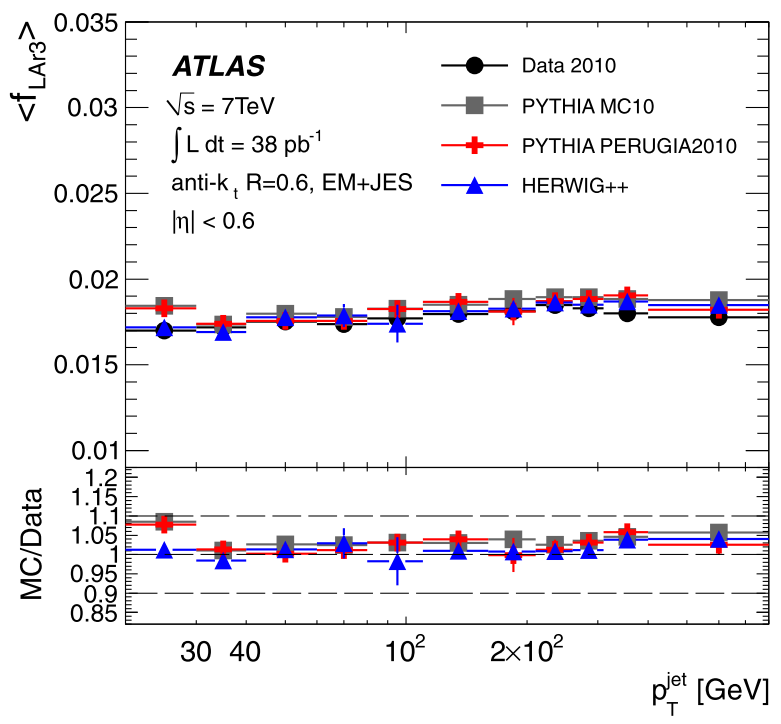

(b) $f_{\mathrm{LAr} 3}$

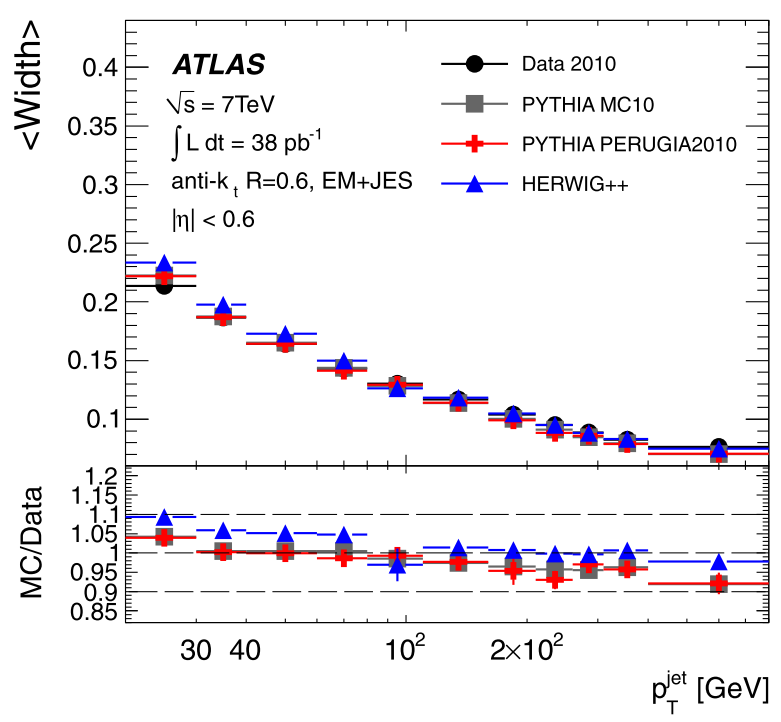

(d) Jet width

with $R=0.6$ calibrated with the EM+JES scheme are used. The ratio of Monte Carlo simulation to data is shown in the lower part of each figure

other $|\eta|$ bins for the calorimeter layer fractions and the jet width, except for $2.1 \leq|\eta|<2.8$, where the agreement for the width is slightly worse than in the other eta ranges.

Figure 50 shows that PythiA with the MC10 and the Pythia PeRUgia2010 tunes agree to within a few per cent. The agreement of the HERWIG sample with data is as good as for the other samples for $f_{\mathrm{LAr} 3}$ and $f_{\mathrm{Tile} 0}$, except for $20 \leq p_{\mathrm{T}}^{\text {jet }}<30 \mathrm{GeV}$. For $f_{\mathrm{PS}}$ and the width, disagreements of $5-10 \%$ are observed between HERWIG++ and the other 
samples for $p_{\mathrm{T}}^{\text {jet }}<60 \mathrm{GeV}$. For $p_{\mathrm{T}}^{\text {jet }}>160 \mathrm{GeV}$, HeRWIG++ is found to describe the width observed in data better than the other samples.

The systematic uncertainty can be quantitatively estimated by comparing how the correction coefficients $E_{\mathrm{GS}}^{\mathrm{jet}} /$ $E_{\mathrm{EM}+\mathrm{JES}}^{\mathrm{jet}}$ differ between data and Monte Carlo simulation. The correction coefficient as a function of $p_{\mathrm{T}}^{\text {jet }}$ in the barrel calorimeter in data and in the PYTHIA MC10 sample after

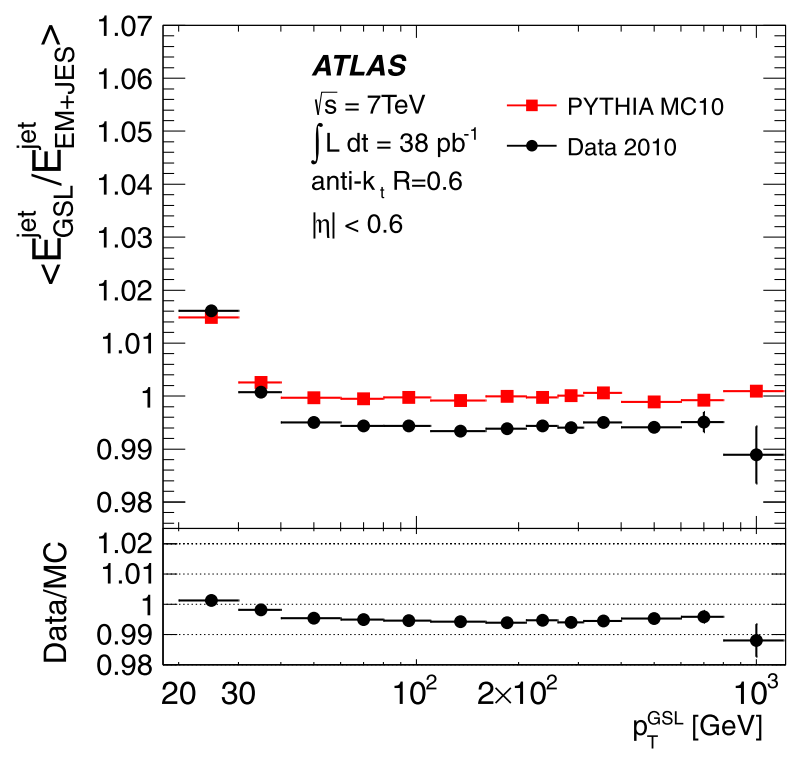

(a) GSL jet $p_{\mathrm{T}}$ comparison

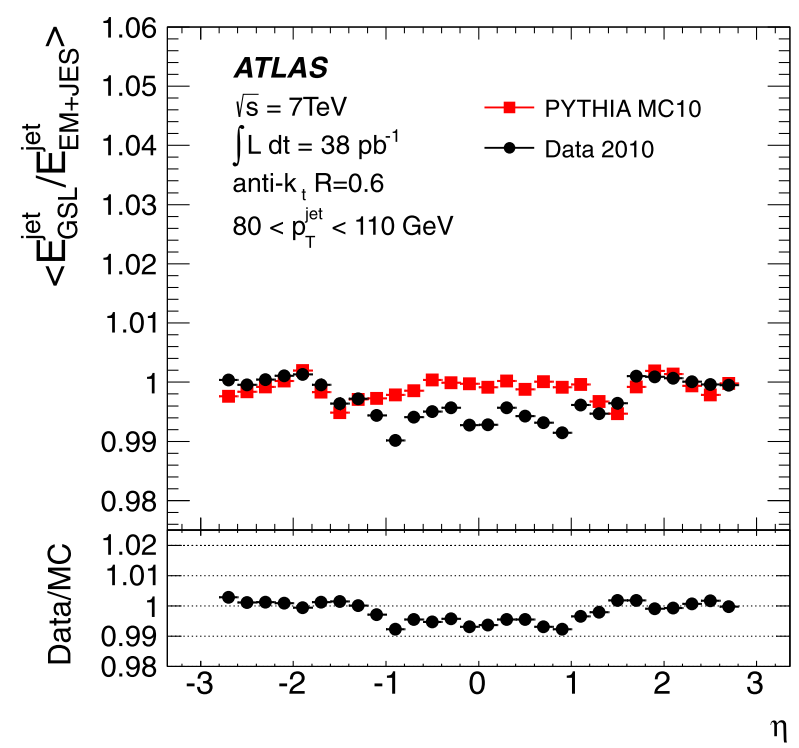

(c) GSL jet $\eta$ comparison

Fig. 51 Average jet energy after GSL $(\mathbf{a}, \mathbf{c})$ and GS (b, d) corrections divided by the average jet energy after the EM+JES calibration as a function of $p_{\mathrm{T}}^{\text {jet }}(\mathbf{a}, \mathbf{b})$ in the calorimeter barrel and as a function of $\eta$ for $80 \leq p_{\mathrm{T}}^{\text {jet }}<100 \mathrm{GeV}(\mathbf{c}, \mathbf{d})$ in data and the Monte
GSL and GS corrections are shown in Figs. 51a and 51b. The ratios of data to Monte Carlo simulation are shown in the lower part of each figure. Figures $51 \mathrm{c}$ and $51 \mathrm{~d}$ show the same quantity, but as a function of $\eta$ for $80 \leq p_{\mathrm{T}}^{\text {jet }}<110 \mathrm{GeV}$.

Deviations from unity in the ratios between data and Monte Carlo simulation as shown in Fig. 51 represent the systematic uncertainty associated to the GS corrections. This uncertainty is added in quadrature to the EM+JES un-

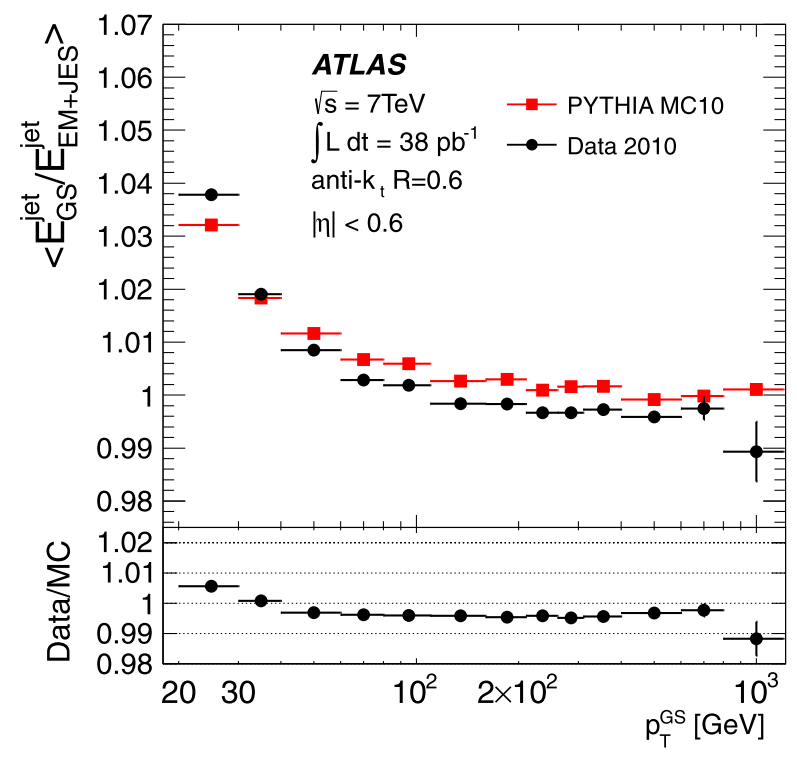

(b) GS jet $p_{\mathrm{T}}$ comparison

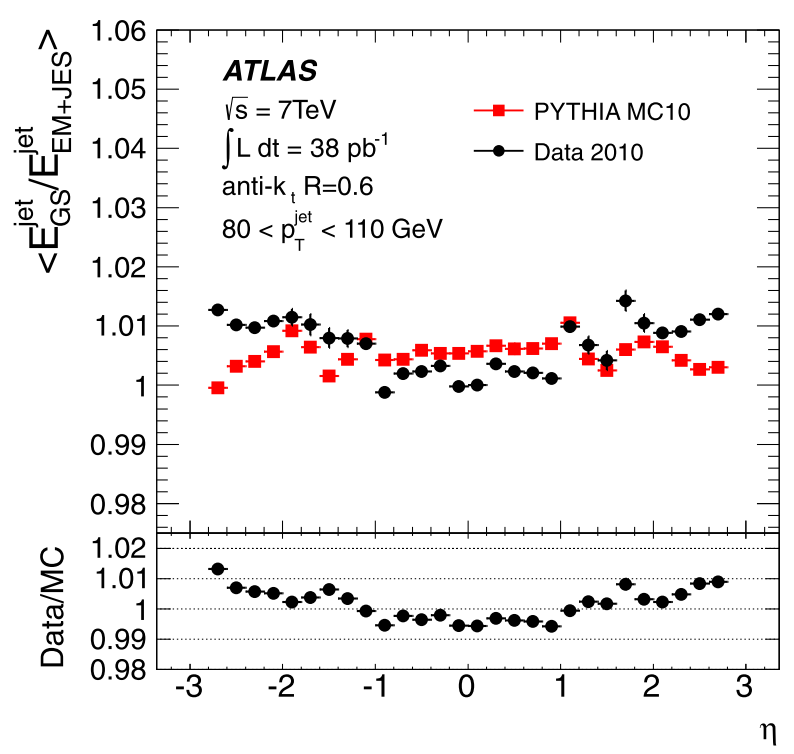

(d) GS jet $\eta$ comparison

Carlo simulation. Anti- $k_{t}$ jets with $R=0.6$ are used. The double ratio $\left[E_{\mathrm{GS}(\mathrm{GSL})} / E_{\mathrm{EM}+\mathrm{JES}}\right]_{\text {Data }} /\left[E_{\mathrm{GS}(\mathrm{GSL})} / E_{\mathrm{EM}+\mathrm{JES}}\right]_{\mathrm{MC}}$ is shown in the lower part of each figure 
certainty. The results for all the $p_{\mathrm{T}}^{\mathrm{jet}}$ and $\eta$ ranges are the following:

For $20 \leq p_{\mathrm{T}}^{\text {jet }}<30 \mathrm{GeV}$ and $0 \leq|\eta|<2.1$, the data to Monte Carlo ratio varies from $0.5 \%$ to $0.7 \%$ depending on the $|\eta|$ region. For $p_{\mathrm{T}}^{\text {jet }}>30 \mathrm{GeV}$ and $0 \leq|\eta|<2.1$, the uncertainty is lower than $0.5 \%$. For $2.1 \leq|\eta|<2.8$, the data to Monte Carlo ratio varies from $0.4 \%$ to $1 \%$ depending on the $p_{\mathrm{T}}^{\text {jet }}$ bin. For a given $p_{\mathrm{T}}^{\text {jet }}$, the uncertainty is higher for $2.1 \leq|\eta|<2.8$ than for $0 \leq|\eta|<2.1$, because of the poorer description of the jet width. For $2.1 \leq|\eta|<2.8$ the GSL scheme shows slightly larger difference than the GS scheme. In general, the uncertainty on the data to Monte Carlo ratio is lower than $1 \%$ for $20 \leq p_{\mathrm{T}}^{\text {jet }}<800 \mathrm{GeV}$ and $0 \leq|\eta|<2.8$.

The uncertainty coming from the imperfect description of the jet properties and the differences between data based and Monte Carlo simulation based corrections presented in Sect. 12.1.3 are not independent. The average jet response after the GS calibration in each $p_{\mathrm{T}}^{\mathrm{jet}}$ and $\eta$ bin, which depends on both the distribution of the properties and the GS corrections, is close to the response after the EM+JES calibration.

A change in the distribution of a jet property therefore translates into a change in the GS correction as a function of this property such that the average jet response stays the same in the sample used to derive the correction. The differences observed after applying data based and Monte Carlo simulation based GS corrections described in Sect. 12.1.3 are therefore partly caused by differences in the jet properties and not only by differences in the GS corrections themselves.

\subsection{Sensitivity of the global sequential calibration} to pile-up

An important feature of the GS calibration is its robustness when applied in the presence of pile-up interactions, which translates into small variations in the size of each of the corrections and the distributions of the jet properties. The corrections derived in the sample without pile-up are directly applicable to the sample with pile-up with only a small additional effect on the jet energy scale.

The quantitative estimate of the effect of pile-up is obtained applying the GS corrections derived in the PYTHIA MC10 sample without pile-up to simulated samples with pile-up. The resulting response is then compared to the response after the EM+JES calibration, which includes the offset correction as described in Sect. 8.1. It is found that the response after the various GS corrections remains unchanged to better than $1 \%(2 \%)$ for $p_{\mathrm{T}}>30 \mathrm{GeV}\left(p_{\mathrm{T}}<\right.$ $30 \mathrm{GeV}$ ). These variations are smaller than the uncertainty on the jet energy in the absence of pile-up over the entire $p_{\mathrm{T}}$ range, thus demonstrating the robustness of the additional corrections with respect to pile-up.

\subsection{Summary on the JES uncertainty}

for the global sequential calibration

The systematic uncertainty on the global sequential calibration in the inclusive jet sample has been evaluated. It is found to be lower than $1 \%$ for $0 \leq|\eta|<2.8$ and $20 \leq p_{\mathrm{T}}^{\text {jet }}<$ $800 \mathrm{GeV}$. This uncertainty is added in quadrature to the JES based on the EM+JES calibration scheme.

\section{Jet calibration schemes based on cell energy weighting}

Besides the simple EM+JES calibration scheme, ATLAS has developed several calibration schemes [26] with different levels of complexity and different sensitivity to systematic effects. The EM+JES calibration facilitates the evaluation of systematic uncertainties for the early analyses, but the energy resolution is rather poor and it exhibits a rather high sensitivity of the jet response to the flavour of the parton inducing the jet. These aspects can be improved using more sophisticated calibrations.

The ATLAS calorimeters are non-compensating and give a lower response to hadrons than to electrons or photons. Furthermore reconstruction inefficiencies and energy deposits outside the calorimeters lower the response to both electromagnetic and hadronic particles, but in different ways. The main motivation for calibration schemes based on cell energy density is to improve the jet energy resolution by weighting differently energy deposits from electromagnetic and hadronic showers [27]. The calorimeter cell energy density is a good indicator, since the radiation length $X_{0}$ is much smaller than the hadronic interaction length $\lambda_{\mathrm{I}}$.

Methods based on cell energy weighting were pioneered by the CDHS experiments [96, 97] and later extensively used by the $\mathrm{H} 1$ experiment $[98,99]$. A further development was presented in Ref. [100].

Two calibration schemes implementing this idea have been developed by ATLAS:

1. For the global calorimeter cell energy density calibration (GCW) the weights depend on the cell energy density and are obtained from Monte Carlo simulation by optimising the reconstructed jet energy resolution with respect to the true jet energy. This calibration is called "global" because the jet is calibrated as a whole and, furthermore, the weights that depend on the calorimeter cell energy density are derived such that fluctuations in the measurement of the jet energy are minimised and this minimisation corrects for all effects at once.

2. For the local cluster calibration (LCW) multiple variables at the calorimeter cell and the topo-cluster levels 
are considered in a modular approach treating the various effects of non-compensation, dead material deposits and out-of-cluster deposits independently. The corrections are obtained from simulations of charged and neutral particles. The topo-clusters in the calorimeter are calibrated "locally", without considering the jet context, and jets are then reconstructed directly from calibrated topoclusters.

Final jet energy scale corrections also need to be applied to the GCW and LCW calibrated jets, but they are numerically smaller than the ones for the EM+JES calibration scheme. These corrections are derived with the same procedure as described in Sect. 8. The resulting jets are referred to as calibrated with GCW+JES and LCW+JES schemes.

\subsection{Global cell energy density weighting calibration}

This calibration scheme (GCW) attempts to assign a larger cell-level weight to hadronic energy depositions in order to compensate for the different calorimeter response to hadronic and electromagnetic energy depositions. The weights also compensate for energy losses in the dead material.

In this scheme, jets are first found from topo-clusters or calorimeter towers at the EM scale. Secondly the energies of the calorimeter cells forming jets are weighted according to their energy density. Finally, a JES correction is derived from the sum of the weighted energy in the calorimeter cells associated to the jet as a function of the jet $p_{\mathrm{T}}$ and pseudorapidity.

The weights are derived using Monte Carlo simulation information. A reconstructed jet is first matched to the nearest truth jet requiring $R_{\min }<0.3$. No second truth jet should be within a distance of $\Delta R=1$. The nearest truth jet should have a transverse energy $E_{\mathrm{T}}>20 \mathrm{GeV}$. The transverse energy of the reconstructed jet should be $E_{\mathrm{T}}^{\mathrm{EM}}>5 \mathrm{GeV}$, where $E_{\mathrm{T}}^{\mathrm{EM}}$ is the transverse energy of the reconstructed jet measured at the electromagnetic scale.

For each jet, calorimeter cells are identified with an integer number $i$ denoting a calorimeter layer or a group of layers in the ATLAS calorimeters. Afterwards, each cell is classified according to its energy density which is defined as the calorimeter cell energy measured at the electromagnetic scale divided by the geometrical cell volume $(E / V)$.

A weight $w_{i j}$ is introduced for each calorimeter cell within a layer $i$ at a certain energy density bin $j$. The cells are classified in up to $16 \mathrm{E} / \mathrm{V}$ bins according to the following formula:

$j=\frac{\ln \frac{E / \mathrm{GeV}}{V / \mathrm{mm}^{3}}}{\ln 2}+26$,

where $j$ is an integer number between 0 and 15. Calorimeter cells in the presampler, the first layer of the electromagnetic calorimeter, the gap and crack scintillators (Gap, Scint) are excluded from this classification. A constant weight is applied to these cells independent of their $E / V$. The cryostat (Cryo) term is computed as the geometrical average of the energy deposited in the last layer of the electromagnetic barrel LAr calorimeter and the first layer of the Tile calorimeter. This gives a good estimate of the energy loss in the material between the LAr and the Tile calorimeters.

Table 14 shows the number of energy density bins for each calorimeter layer. In the case of the seven layers without energy density segmentation the weights are denoted by $v_{i}$.

The jet energy is then calculated as:

$E_{\mathrm{GCW}}^{\mathrm{jet}}=\sum_{i=1}^{10} \sum_{j=1}^{16} w_{i j} E_{i j}+\sum_{i=1}^{7} v_{i} E_{i}$

where $w_{i j}\left(v_{i}\right)$ are the GCW calibration constants and $E_{i j}$ are the calorimeter cell energies in layer $j$ and energy density bin $j$. In order to reduce the number of degrees of freedom, for a given layer $i$, the dependence on the energy density bin of each element $w_{i j}$ is parameterised by a common polynomial function of third and fourth degree depending on the layer (see Table 14). In this way the number of free parameters used to calibrate any jet is reduced from 167 to 45 .

Table 14 Number of energy density bins per calorimeter layer used in the GCW jet calibration scheme and the degree of the polynomial function used in the weight parametrisation

\begin{tabular}{lll}
\hline Calorimeter Layer & $\begin{array}{l}\text { Nb. E/V } \\
\text { bins }\end{array}$ & $\begin{array}{l}\text { Poly. Degree } \\
\text { on E/V }\end{array}$ \\
\hline PSB & 1 & 1 \\
PSE & 1 & 1 \\
EMB1 & 1 & 1 \\
EME1 & 1 & 1 \\
EMB2 and EMB3 with $|\eta|<0.8$ & 16 & 4 \\
EMB2 and EMB3 with $|\eta| \geq 0.8$ & 16 & 4 \\
EME2 and EME3 with $|\eta|<2.5$ & 16 & 4 \\
EME2 and EME3 with $|\eta| \geq 2.5$ & 16 & 4 \\
TileBar0, TileBar1 and TileBar2 & 16 & 4 \\
TileExt0, TileExt1 and TileExt2 & 16 & 4 \\
HEC0-3 with $|\eta|<2.5$ & 16 & 4 \\
HEC0-3 with $|\eta| \geq 2.5$ & 16 & 4 \\
FCAL0 & 16 & 3 \\
FCAL1 and FCAL2 & 16 & 3 \\
Cryo term & 1 & 1 \\
Gap & 1 & 1 \\
Scint & 1 & 1 \\
\hline
\end{tabular}


The weights are computed by minimising the following function:

$\chi^{2}=\frac{1}{N_{\text {jet }}} \sum_{\text {jet }=1}^{N_{\text {jet }}}\left(\frac{E_{\mathrm{GCW}}^{\mathrm{jet}}}{E_{\text {truth }}^{\mathrm{jet}}}-1\right)^{2}$,

where $N_{\text {jet }}$ is the total number of jets in the Monte Carlo sample used. This procedure provides weights that minimise the jet energy resolution. The mathematical bias on the mean jet energy that is introduced in particular at low jet energies (see Ref. [101]) is corrected by an additional jet energy calibration following the method described in Sect. 8 and discussed in Sect. 13.3.

\subsection{Local cluster weighting calibration}

This calibration scheme $[37,102]$ corrects locally the topoclusters in the calorimeters independent of any jet context. The calibration starts by classifying topo-clusters as mainly electromagnetic or hadronic depending on cluster shape variables [88]. The cluster shape variables characterise the topology of the energy deposits of electromagnetic or hadronic showers and are defined as observables derived from calorimeter cells with positive energy in the cluster and from the cluster energy. All weights depend on this classification and both hadronic and electromagnetic weights are applied to each cluster.

\subsubsection{Barycentre of the longitudinal cluster depth}

The barycentre of the longitudinal depth of the topo-cluster $\left(\lambda_{\text {centre }}\right)$ is defined as the distance along the shower axis from the front of the calorimeter to the shower centre. The shower centre has coordinates:

$\langle i\rangle=\frac{\sum_{k \mid E_{k}>0} E_{k} i_{k}}{\sum_{k \mid E_{k}>0} E_{k}}$,

with $i$ taking values of the spatial coordinates $x, y, z$ and $E_{k}$ denoting the energy in the calorimeter cell $k$. Only calorimeter cells with positive energy are used.

The shower axis is determined from the spatial correlation matrix of all cells in the topo-cluster with positive energies:

$C_{i j}=\frac{\sum_{k \mid E_{k}>0} E_{k}^{2}\left(i_{k}-\langle i\rangle\right)\left(j_{k}-\langle j\rangle\right)}{\sum_{k \mid E_{k>0}} E_{k}^{2}}$,

with $i, j=x, y, z$. The shower axis is the eigenvector of this matrix closest to the direction joining the interaction point and the shower centre.

\subsubsection{Cluster isolation}

The cluster isolation is defined as the ratio of the number of unclustered calorimeter cells ${ }^{35}$ that are neighbours of a given topo-cluster to the number of all neighbouring cells. The neighbourhood relation is defined in two dimensions, i.e. within the individual calorimeter layer. ${ }^{36}$

After calculating the cluster isolation for each individual calorimeter layer, the final cluster isolation variable is obtained by weighting the individual layer cell ratios by the energy fractions of the topo-cluster in these layers. This assures that the isolation is evaluated where the topo-cluster has most of its energy.

The cluster isolation is zero for topo-clusters where all neighbouring calorimeter cells in each layer are inside other topo-clusters and one for topo-clusters with no neighbouring cell inside any other topo-cluster.

\subsubsection{Cluster energy correction}

All corrections are derived from the Monte Carlo simulations for single charged and neutral pions. The hadronic shower simulation model used is QGSP_BERT. The detector geometry and topo-cluster reconstruction is the same as in the nominal Monte Carlo simulation sample. A flat distribution in the logarithm of pion energies from $200 \mathrm{MeV}$ to $2 \mathrm{TeV}$ is used.

The corrections are derived with respect to the true deposited energy in the active and inactive detector region ("calibration hits").

True energy depositions are classified in three types by the ATLAS software:

1. The visible energy, like the energy deposited by ionisation.

2. The invisible energy, like energy absorbed in nuclear reactions.

3. The escaped energy, like the energy carried away by neutrinos. 37

The local cluster calibration proceeds in the following steps:

1. Cluster classification: The expected number of pions in logarithmic bins of the topo-cluster energy, the cluster depth in the calorimeter, and the average cell energy density are used to calculate classification weights. The

\footnotetext{
${ }^{35}$ Unclustered calorimeter cells that are not contained in any topocluster.

${ }^{36}$ In general, topo-clusters are formed in a three dimensional space defined by $\eta, \phi$ and the calorimeter depth.

${ }^{37}$ The escaped energy is recorded at the place where the particle that escapes the detector volume ("world volume" in GEANT4 terminology) is produced.
} 
weights are calculated for small $|\eta|$ regions by mixing neutral and charged pions with a ratio of 1:2. This assumes that $2 / 3$ of the pions should be charged. Clusters are classified as mostly electromagnetic or mostly hadronic. The calculated weight denotes the probability $p$ for a cluster to stem from a hadronic interaction.

2. Hadronic weighting: Topo-clusters receive calorimeter cell correction weights derived from detailed Monte Carlo simulations of charged pions. Calorimeter cells in topo-clusters are weighted according to the topo-cluster energy and the calorimeter cell energy density. The hadronic energy correction weights are calculated from the true energy deposits as given by the Monte Carlo simulation $\left(w_{\mathrm{HAD}}\right)$ multiplied by a weight to take into account the different nature of hadronic and electromagnetic showers.

The applied weight is

$w_{\mathrm{HAD}} \cdot p+w_{\mathrm{EM}} \cdot(1-p)$,

where $w_{\mathrm{EM}}=1$ and $p$ is the probability of the topo-cluster to be hadronic as determined by the classification step. Dedicated correction weight tables for each calorimeter layer in 0.2-wide $|\eta|$-bins are used. The correction weight tables are binned logarithmically in topo-cluster energy and cell energy density $(E / V)$.

3. Out-of-cluster (OOC) corrections: A correction for isolated energy deposits inside the calorimeter, but outside topo-clusters is applied. These are energy depositions not passing the noise thresholds applied during the clustering. These corrections depend on $|\eta|$, the energy measured around the topo-cluster and the cluster barycentre $\lambda_{\text {centre }}$. There are two sets of constants for hadronic and electromagnetic showers and both are used for each cluster with the respective weights of $p$ and $1-p$. The OOC correction is finally multiplied with the cluster isolation value discussed in Sect. 13.2.2 in order to avoid double counting.

4. Dead material (DM) corrections: The topo-cluster cluster energy is corrected for energy deposits in materials outside the calorimeters. Energy deposits in upstream material like the inner wall of the cryostat are highly correlated to the presampler signals. The corrections are derived from the sum of true energy depositions in the material in front and behind the calorimeter and from the presampler signal. The correction for energy deposited in the outer cryostat wall between the electromagnetic and the hadronic barrel calorimeters is based on the geometrical mean of the energies in the layers just before and just beyond the cryostat wall. Corrections for other energy deposits without clear correlations to topo-cluster observables are obtained from look-up tables binned in topo-cluster energy, the pseudorapidity $|\eta|$, and the shower depth. Two sets of DM weights for hadronic and electromagnetic showers are used. The weights are applied according to the classification probability $p$ defined above.

All corrections are defined with respect to the electromagnetic scale energy of the topo-cluster. Since only calorimetric information is used, the LCW calibration does not account for low-energy particles which do not create a topocluster in the calorimeter. This is, for instance, the case when the energy is absorbed entirely in inactive detector material or particles are bent outside of the calorimeter acceptance.

\subsection{Jet energy calibration for jets with calibrated constituents}

The simulated response to jets at the GCW and LCW energy scales, i.e. after applying weights to the calorimeter cells in jets or after the energy corrections to the topo-clusters, are shown in Fig. 52 as a function of $\eta_{\text {det }}$ for various jet energy bins. For both jet calibration schemes the jet response is about $0.6-0.8$ for jet energies of $30 \mathrm{GeV}$, but is bigger than 0.9 for high energetic jets. The inverse of the jet response calculated on the LCW or GCW scale is used in each bin as jet energy scale correction as described in Sect. 8.3 for the EM+JES calibration scheme.

The final jet energy correction needed to restore the reconstructed jet energy to the true jet energy is much smaller than in the case of the EM+JES calibration shown in Fig. 9.

\section{Jet energy scale uncertainties for jet calibrations based on cell weighting}

The jet energy scale uncertainty for jets based on cell weighting is obtained using the same in situ techniques as described in Sect. 10. The results for each in situ technique together with the combination of all in situ techniques are discussed in Sect. 14.3.

In order to build up confidence in the Monte Carlo simulation the description of the variables used as inputs to the cell weighting by the Monte Carlo simulation is discussed in Sect. 14.1 for the global cell weighting scheme and in Sect. 14.2 for the local cluster weighting scheme.

\subsection{Energy density as input}

to the global cell weighting calibration

The global cell energy density weighting calibration scheme (see Sect. 13.1) applies weights to the energy deposited in each calorimeter cell according to the calorimeter cell energy density ( $E / V$, where $V$ is the calorimeter cell volume and the energy is defined on EM-scale). This attempts to compensate for the different calorimeter response to 


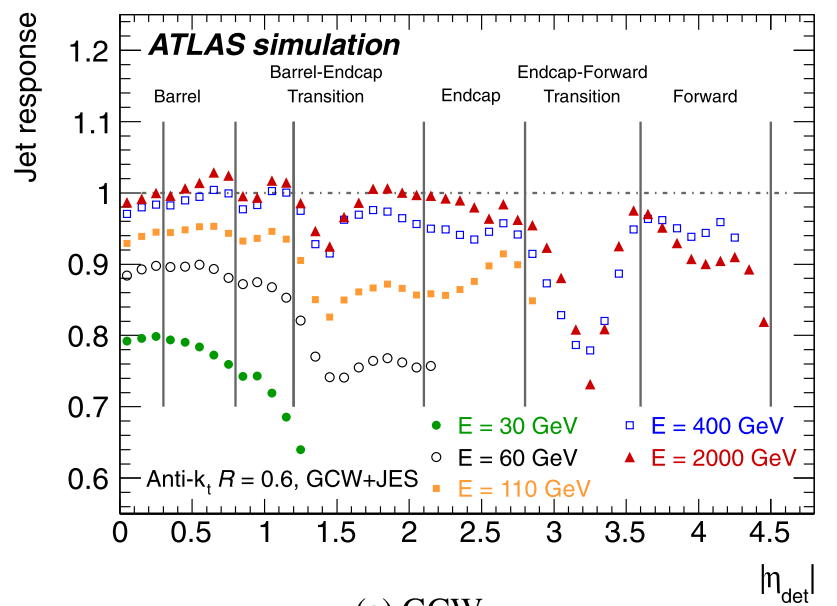

(a) GCW

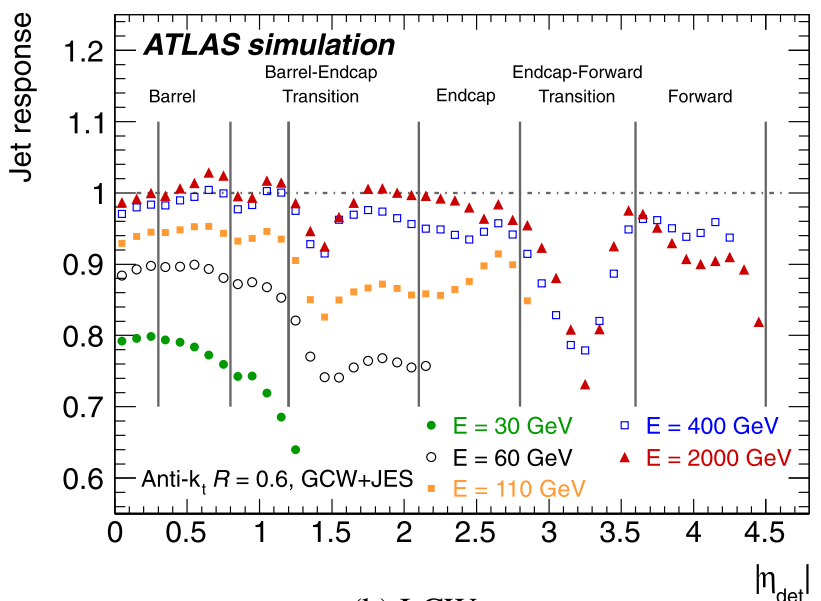

(b) LCW

Fig. 52 Average simulated jet energy response at the GCW (a) and the LCW (b) scale in bins of the GCW+JES and LCW+JES calibrated jet energy and as a function of the detector pseudorapidity $\left|\eta_{\text {det }}\right|$

hadronic and electromagnetic showers, but it also compensates for energy losses in the dead material. The description of the calorimeter cell energy density in the Monte Carlo simulation is therefore studied to validate this calibration scheme.

Only calorimeter cells inside jets with $p_{\mathrm{T}}^{\text {jet }}>20 \mathrm{GeV}$ and $|y|<2.8$ built of topo-clusters and with a cell energy of at least two standard deviations above the noise thresholds are considered for this comparison. Similar results have been obtained using cells inside jets built from calorimeter towers. The Monte Carlo simulation reproduces the generic features of the data over many orders of magnitude. However, the following paragraphs discusses those differences, all of which are on the order of a few percent.

Figure 53 shows the calorimeter cell energy density distributions in data and Monte Carlo simulation for cells in representative longitudinal segments of the barrel and forward calorimeters. Fewer cells with high energy density are observed in data than predicted by Monte Carlo simulation in the barrel presampler (a) and in the second layer of the barrel electromagnetic calorimeter (b). This behaviour is observed for other segments of the barrel electromagnetic calorimeter, but not for the second layer of the Tile barrel calorimeter (c). Here, a good agreement between data and Monte Carlo simulation is found over the full energy density spectrum. Only for the lowest energy densities are slight differences found. Good agreement is also present in the first layer of the Tile extended barrel calorimeter, while the energy density is on average smaller for the second and third layer in the data than in the Monte Carlo simulation. Such a deficit of high energy density cells in data is also observed for the second and third layer of the scintillators placed in the gap between the Tile barrel and extended barrel modules. Better agreement is found between data and Monte Carlo simulation for the first layer of the scintillators.
The second layer of the endcap electromagnetic calorimeter (d) shows a similar behaviour to that observed in the barrel: fewer cells are found at high energy density in the data than in the Monte Carlo simulation. This effect is present in all three layers of the endcap electromagnetic calorimeter, yet it becomes more pronounced with increasing calorimeter depth. A similar effect, but of even larger magnitude has been observed for cells belonging to the endcap presampler. The first layer of the endcap hadronic calorimeter (e) shows a better agreement between data and Monte Carlo simulation. This agreement is also present for other layers of the HEC. In the first layer of the forward calorimeter more cells with energy densities in the middle part of the spectrum are found in data than in Monte Carlo simulation (f). This effect has been observed in other FCAL layers, and it becomes slightly more pronounced with increasing FCAL depth.

\subsection{Cluster properties inside jets as input} to the local cluster weighting calibration

The LCW weights are defined with respect to the electromagnetic scale energy of the topo-clusters and can therefore be applied in any arbitrary order. This allows systematic checks of the order in which the corrections are applied. There are four cluster properties used in the LCW calibration scheme:

1. The energy density in cells in topo-clusters.

2. The cluster energy fraction deposited in different calorimeter layers.

3. The isolation variable characterising the energy around the cluster.

4. The depth of the cluster barycentre in the calorimeter.

In addition, the cluster energy after each correction step and the cluster location can be compared in data and Monte Carlo simulation. 


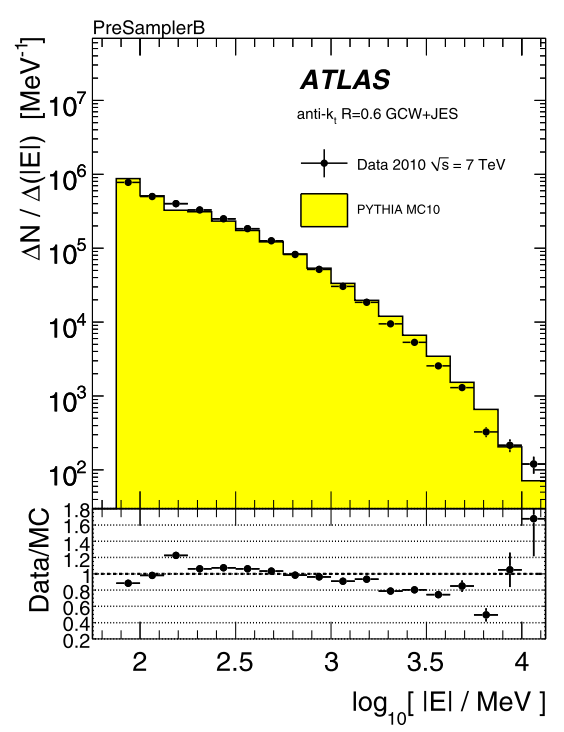

(a) Barrel PS

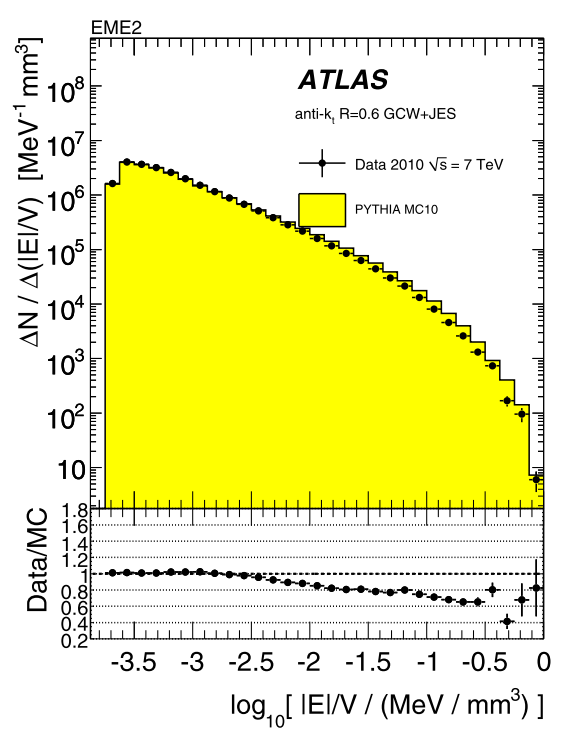

(d) Second layer of EMEC

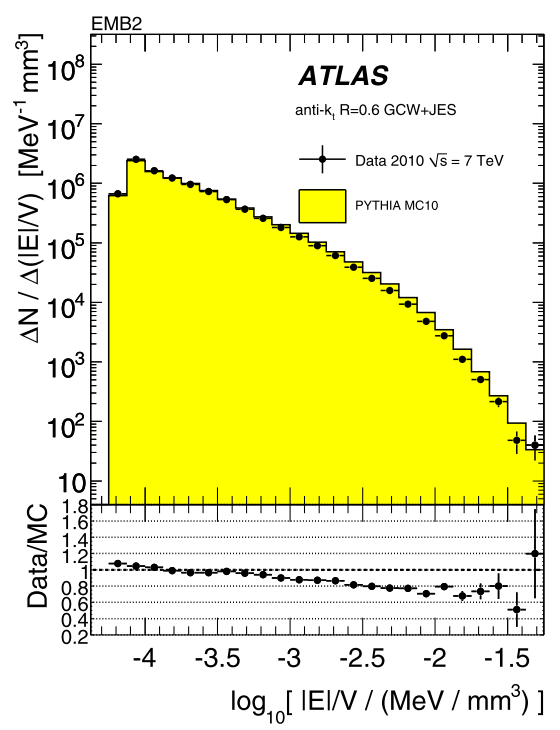

(b) Second layer EMB

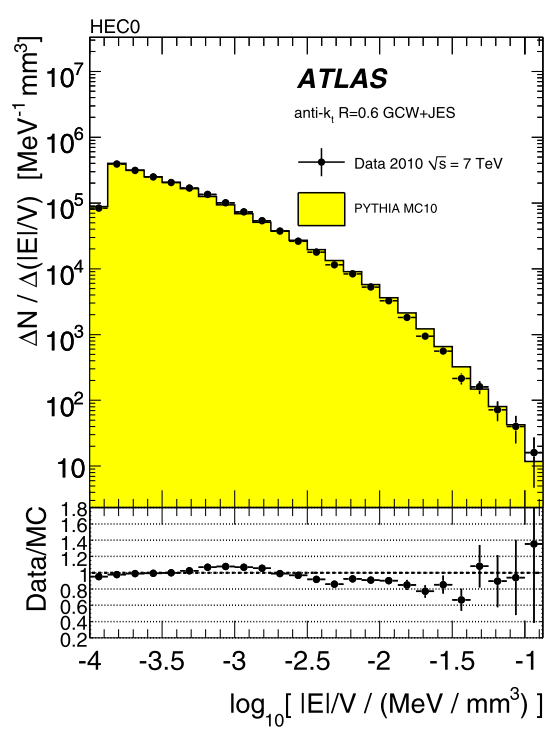

(e) First layer of HEC

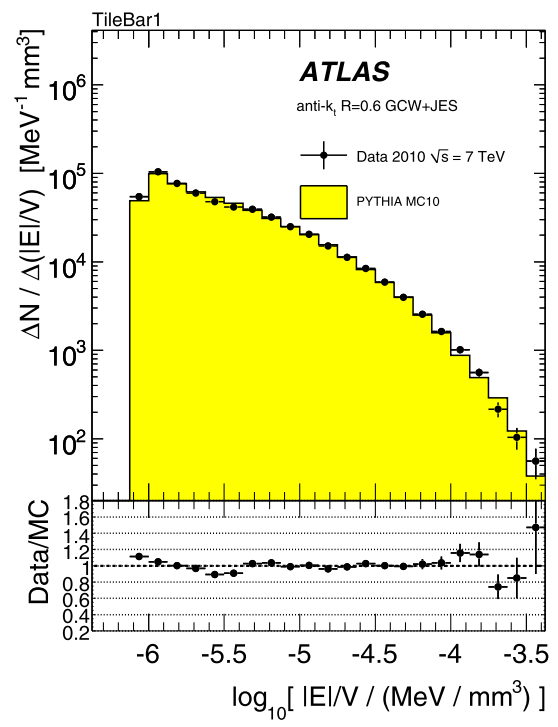

(c) Second layer Tile in barrel

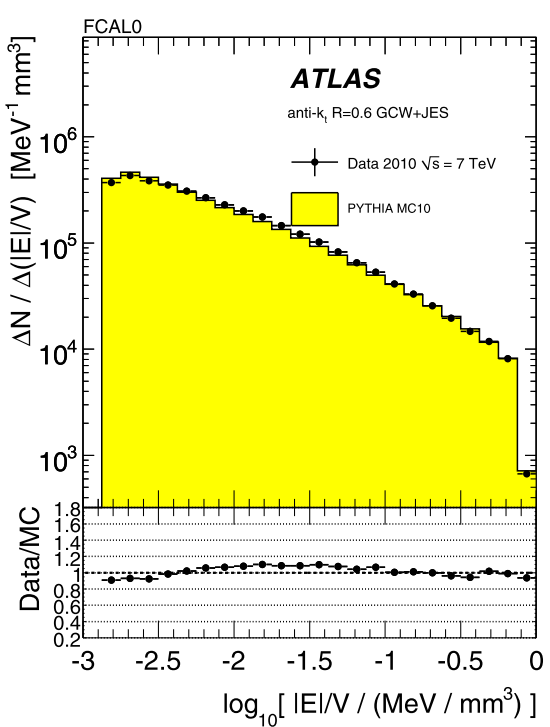

(f) First layer of FCAL
Fig. 53 Calorimeter cell energy density distributions measured at EM scale used in the GCW jet calibration scheme in data (points) and Monte Carlo simulation (shaded area) for calorimeter cells in the barrel presampler (a), the second layer of the barrel electromagnetic calorimeter (b), the second layer of the barrel hadronic Tile calorimeter (c), the second layer of the endcap electromagnetic calorimeter (d), the first layer of the endcap hadronic calorimeter (e) and the first layer

\subsubsection{Cluster isolation}

Figure 54 shows the distributions of the cluster isolation variable for all topo-clusters in calibrated jets with $p_{\mathrm{T}}^{\text {jet }}>$ $20 \mathrm{GeV}$ and $|y|<2.8$ for topo-clusters classified as electromagnetic (a) and hadronic (b).

The cluster isolation variable is bounded between 0 and 1 , with higher values corresponding to higher isola- of the forward calorimeter (f). Anti- $k_{t}$ jets with $R=0.6$ requiring $p_{\mathrm{T}}^{\text {jet }}>20 \mathrm{GeV}$ and $|y|<2.8$ calibrated with the GCW+JES scheme are used. Monte Carlo simulation distributions are normalised to the number of cells in data distributions. The ratio of data to Monte Carlo simulation is shown in the lower part of each figure. Only statistical uncertainties are shown

tion (see Sect. 13.2.2). Most of the topo-clusters in lower energetic jets have a high degree of isolation. The peaks at $0.25,0.5$ and 0.75 are due to the topo-clusters in boundary regions which are geometrically difficult to model or regions with a small number of calorimeter cells. Such topo-clusters contain predominantly gap scintillator cells or are located at the boundary of the HEC and the FCAL calorimeters. 


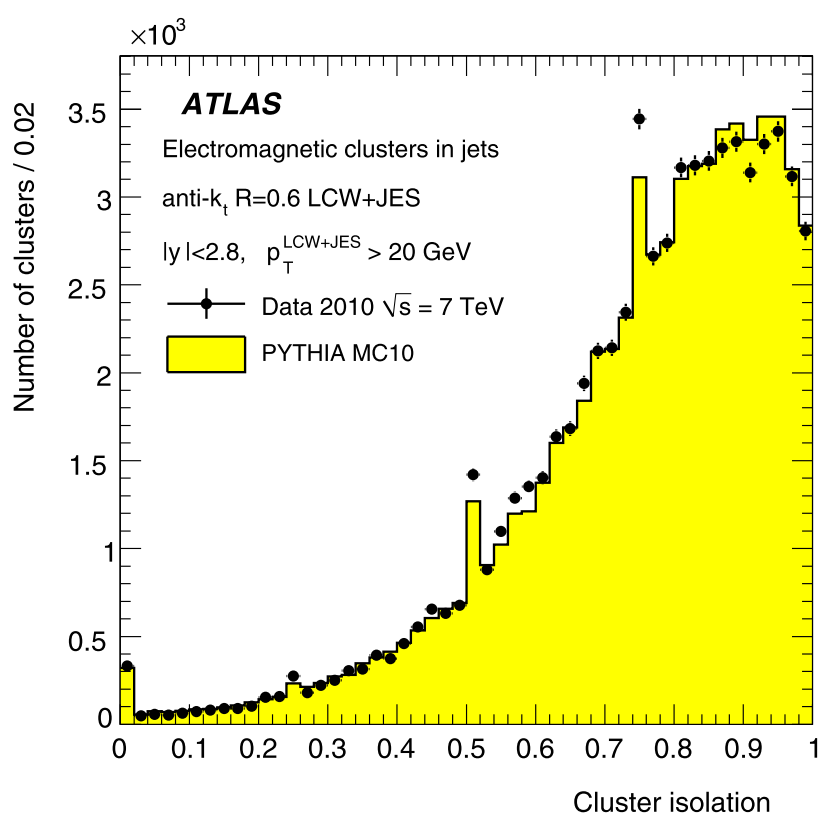

(a) Electromagnetic topo-clusters

Fig. 54 Distributions of the isolation variable for topo-clusters classified as electromagnetic (a) and as hadronic (b) in data (points) and Monte Carlo simulation (shaded area). Topo-clusters associated to

The features observed are similar for topo-clusters classified as mostly electromagnetic and those classified as mostly hadronic. A reasonable agreement between data and Monte Carlo simulation (see Fig. 54) is found. The agreement in the peaks corresponding to the transition region between calorimeters is not as good as in the rest of the distribution.

Figure 55 shows the mean value of the topo-cluster isolation variable as a function of the topo-cluster energy for all topo-clusters in jets with $p_{\mathrm{T}}^{\text {jet }}>20 \mathrm{GeV}$ and $|y|<$ 2.8 for topo-clusters classified as electromagnetic (a) or as hadronic (b). The Monte Carlo simulation consistently predicts more isolated topo-clusters than observed in the data, particularly at topo-cluster energies $E<2 \mathrm{GeV}$ and for both hadronic and electromagnetic cluster classifications. This feature is present in all rapidity regions, except for very low energy topo-clusters classified as mostly electromagnetic in very central jets.

\subsubsection{Longitudinal cluster barycentre}

Figure 56 shows the cluster barycentre $\lambda_{\text {centre }}$ (see Sect. 13.2.1) distributions for all topo-clusters in LCW calibrated jets with $p_{\mathrm{T}}^{\text {jet }}>20 \mathrm{GeV}$ and $|y|<2.8$ and for both cluster classifications. Most topo-clusters classified as electromagnetic have their centre in the electromagnetic calorimeter, as

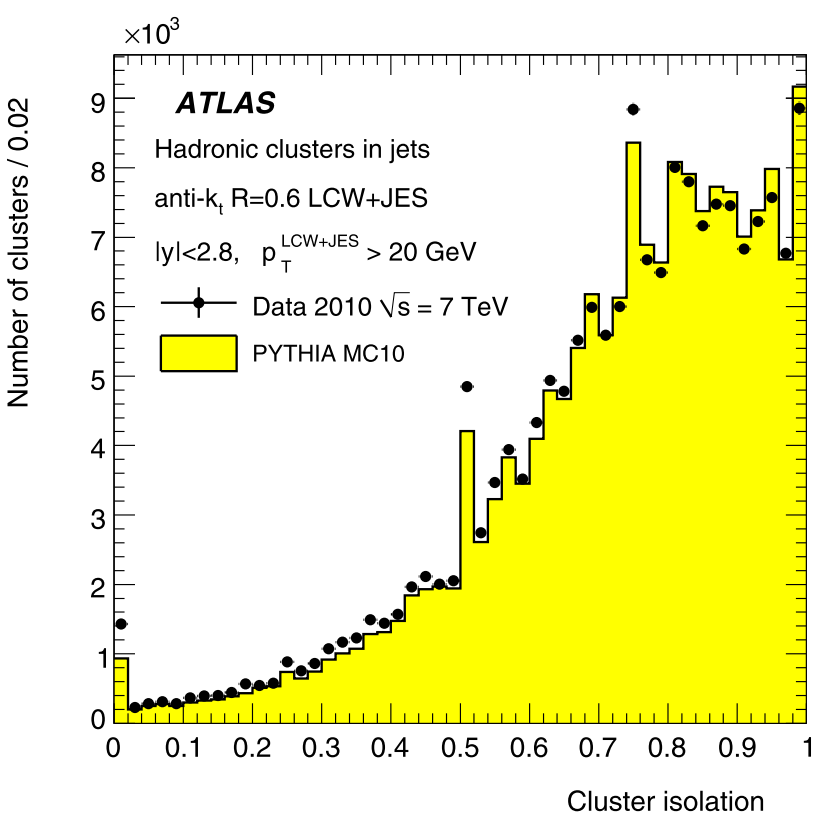

(b) Hadronic topo-clusters

anti- $k_{t}$ jets with $R=0.6$ with $p_{\mathrm{T}}^{\text {jet }}>20 \mathrm{GeV}$ and $|y|<2.8$ calibrated with the LCW+JES scheme are used

expected. Those topo-clusters classified as mostly hadronic are very often in the electromagnetic calorimeter, since these low $p_{\mathrm{T}}$ jets do not penetrate far into the hadronic calorimeter. However, a structure is observed, related to the position of the different longitudinal layers in the hadronic calorimeter. This structure is more prominent when looking at individual rapidity regions, being smeared where the geometry is not changing in this inclusive distribution. Good agreement is observed between data and Monte Carlo simulation.

Figure 57 shows the mean value of distributions of $\lambda_{\text {centre }}$ as a function of the cluster energy for all topo-clusters in jets with $p_{\mathrm{T}}^{\mathrm{jet}}>20 \mathrm{GeV}$ and $|y|<2.8$, again for both types of topo-clusters. In this case, topo-clusters classified as mostly electromagnetic have their barycentre deeper in the calorimeter on average as the cluster energy increases. A different behaviour is observed for clusters tagged as hadronic, for which the mean depth in the calorimeter increases until approximately $2 \mathrm{GeV}$, at which point the mean depth decreases again. The shape of the mean depth as a function of energy is different for different jet rapidities due to the changing calorimeter geometry. However, the qualitative features are similar, with a monotonic increase up to some topo-cluster energy, and a decrease thereafter. This is likely due to an increased probability of a hadronic shower to be split into two or more clusters with increased shower energy. A good agreement is observed between data and Monte Carlo simulation. 


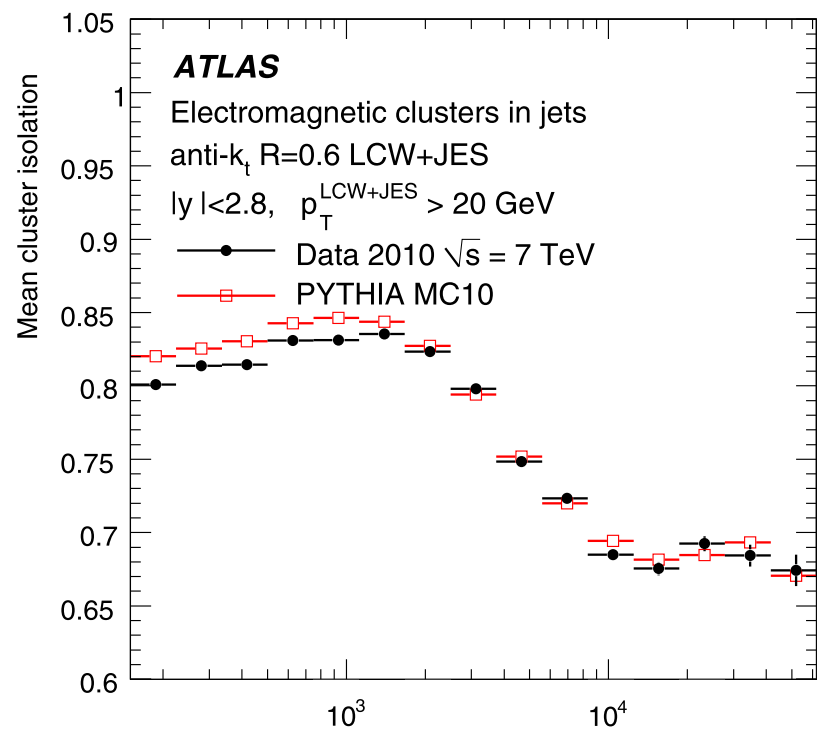

Cluster energy at EM-scale [MeV]

(a) Electromagnetic topo-clusters

Fig. 55 Mean value of the cluster isolation variable for topo-clusters classified as electromagnetic (a) and as hadronic (b) as a function of the topo-cluster energy measured at the EM scale, in data (closed cir-

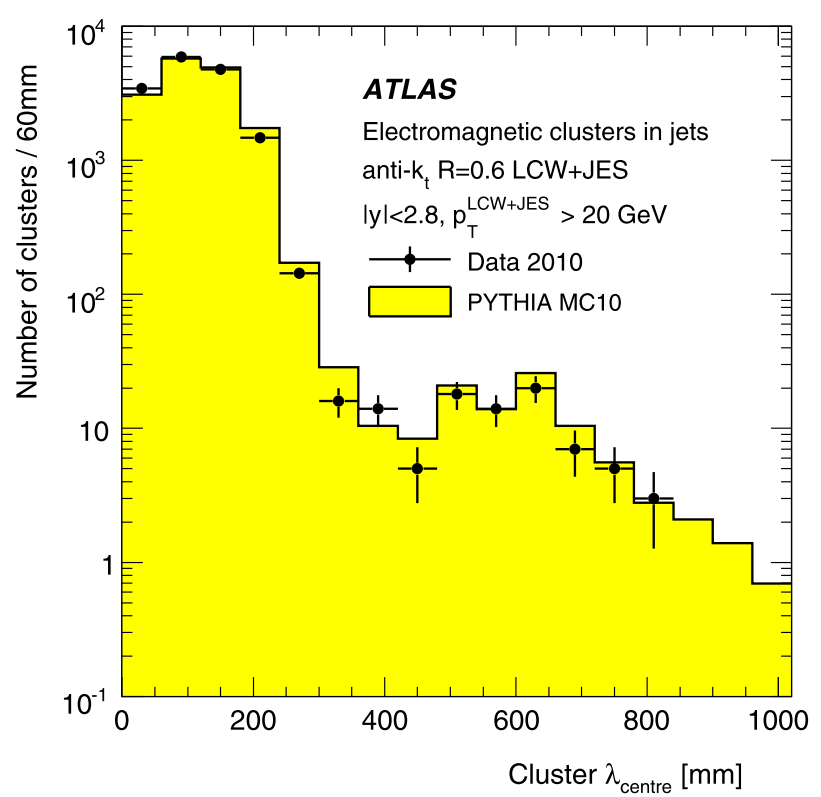

(a) Electromagnetic topo-clusters

Fig. 56 Distributions of the longitudinal cluster barycentre $\lambda_{\text {centre }}$ for topo-clusters classified as electromagnetic (a) and as hadronic (b) in data (points) and Monte Carlo simulation (shaded area). Topo-clust-

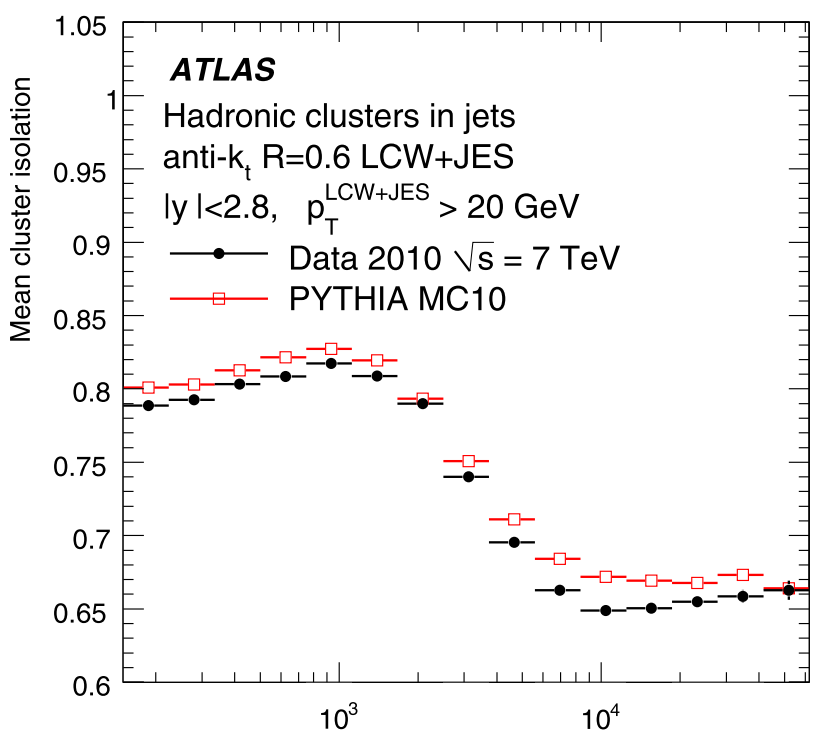

Cluster energy at EM-scale [MeV]

(b) Hadronic topo-clusters

cles) and Monte Carlo simulation (open squares). Topo-clusters associated to anti- $k_{t}$ jets with $R=0.6$ with $p_{\mathrm{T}}^{\text {jet }}>20 \mathrm{GeV}$ and $|y|<2.8$ calibrated with the LCW+JES scheme are used

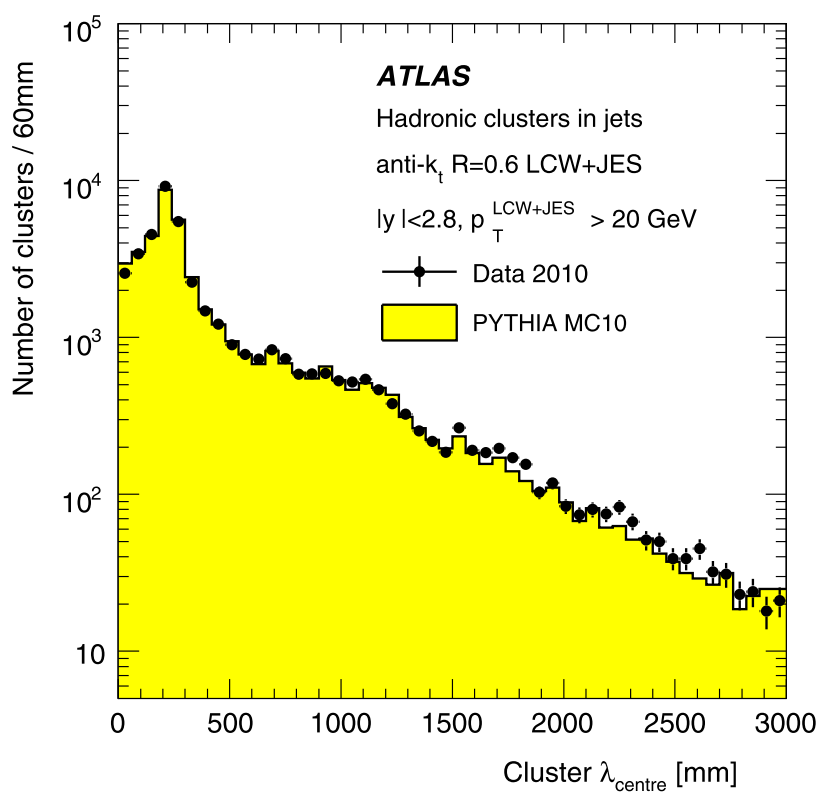

(b) Hadronic topo-clusters

ers associated to anti- $k_{t}$ jets with $R=0.6$ with $p_{\mathrm{T}}^{\text {jet }}>20 \mathrm{GeV}$ and $|y|<2.8$ calibrated with the LCW+JES scheme are used 


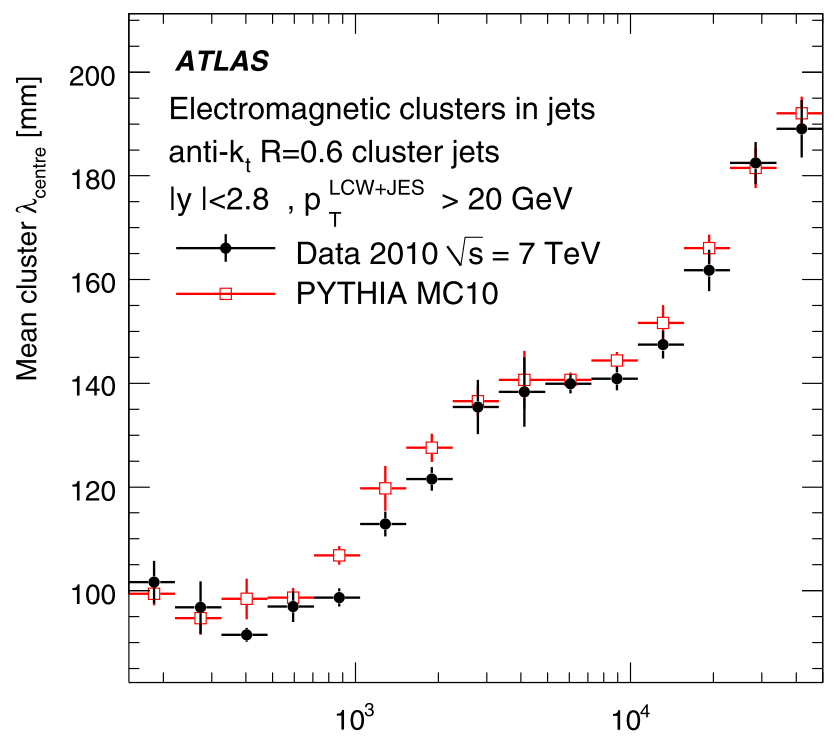

Cluster energy at EM-scale [MeV]

(a) Electromagnetic topo-clusters

Fig. 57 Mean value of the longitudinal cluster barycentre $\lambda_{\text {centre }}$ as a function of the topo-cluster energy measured at the EM scale for topoclusters classified as electromagnetic (a) and as hadronic in data (b) in

\subsubsection{Cluster energy after LCW corrections}

In this section the size of each of the three corrections of the topo-cluster calibration is studied in data and Monte Carlo simulation. This provides a good measure of how the differences between data and Monte Carlo simulation observed in previous sections impact the size of the corrections applied.

Figure 58 shows the mean value of the ratio of the calibrated topo-cluster energy to the uncalibrated topo-cluster energy after each calibration step as a function the topocluster energy and pseudorapidity. Only topo-clusters in LCW calibrated jets with $p_{\mathrm{T}}^{\text {jet }}>20 \mathrm{GeV}$ are considered. For the results shown as a function of topo-cluster energy the rapidity of the jets is, in addition, restricted to $|y|<0.3$.

The agreement between data and Monte Carlo simulation is within 5-10\% for the full topo-cluster pseudorapidity range $\eta_{\text {topo-cluster }}$ and is generally better for lower topo-cluster energies where the correction for the out-of-cluster energy dominates. As the topo-cluster energy increases the largest corrections become the hadronic response and the dead material corrections.

An agreement to about $1 \%$ is observed in a wide region in most of the barrel region after each correction. The agreement between data and Monte Carlo simulation is within $2 \%$ for all topo-cluster pseudorapidities after the hadronic and the out-of-cluster corrections. Larger differences are observed between data and Monte Carlo simulation in the tran-

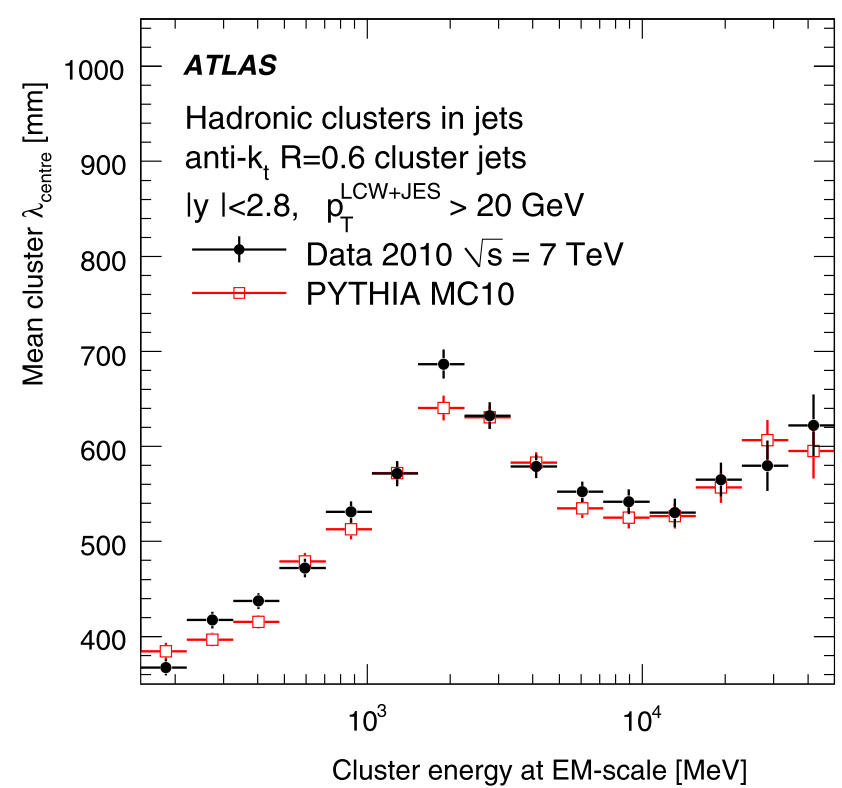

(b) Hadronic topo-clusters

data (closed circles) and Monte Carlo simulation (open squares). Topoclusters associated to anti- $k_{t}$ jets with $R=0.6$ with $p_{\mathrm{T}}^{\text {jet }}>20 \mathrm{GeV}$ and $|y|<2.8$ calibrated with the LCW+JES scheme are used

sition region between the barrel and the endcap and in the forward region once the dead material correction is applied.

14.3 Jet energy scale uncertainty from in situ techniques for jets based on cell weighting

For the jet calibration schemes based on cell weighting the JES uncertainty is evaluated using in situ techniques. The same techniques as described in Sect. 10 are employed. The final JES uncertainty is obtained from a combination of all in situ techniques following the prescription in Sect. 10.5.

\subsubsection{Comparison of transverse momentum balance from calorimeter and tracking}

The result of the JES validation using the total transverse momentum of the tracks associated to jets (see Sect. 10.1) is shown in Fig. 59 for jets in data and Monte Carlo simulation calibrated with the GCW+JES scheme and in Fig. 60 for jets calibrated with the LCW+JES scheme in various jet pseudorapidity regions within $|\eta|<2.1$. The bin $|\eta|<1.2$ is obtained by combining the $|\eta|<0.3,0.3 \leq|\eta|<0.8$ and $0.8 \leq|\eta|<1.2$ bins.

Similar results as for the EM+JES scheme are obtained. In both cases, the agreement between data and simulation is excellent and within the uncertainties of the in situ method. The calibration schemes agree to within a few per cent, except for the bins with very low numbers of events. 

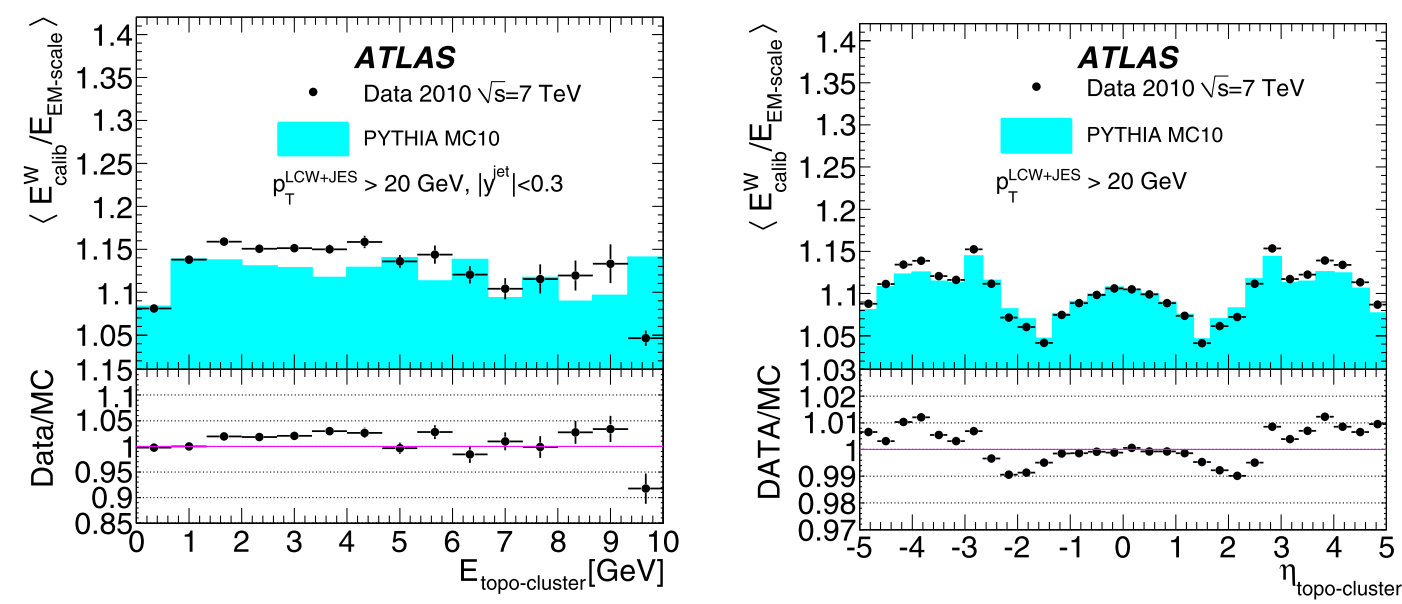

(a) Hadronic response weights
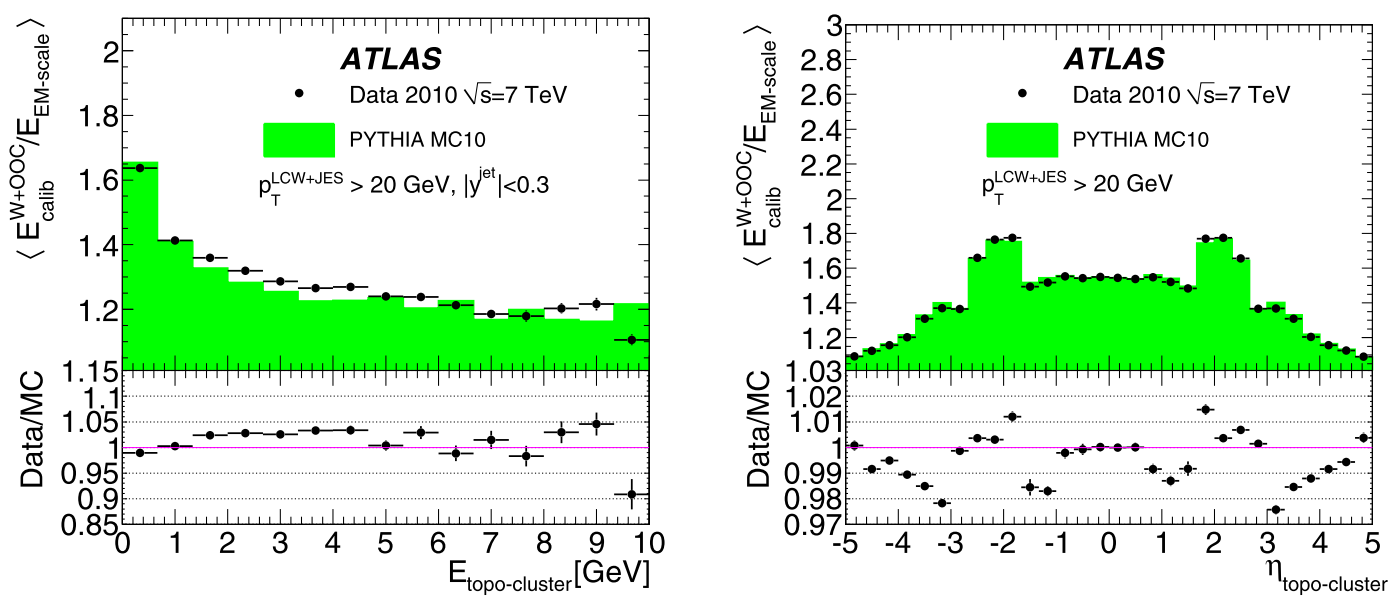

(b) Hadronic response and out-of-cluster weights
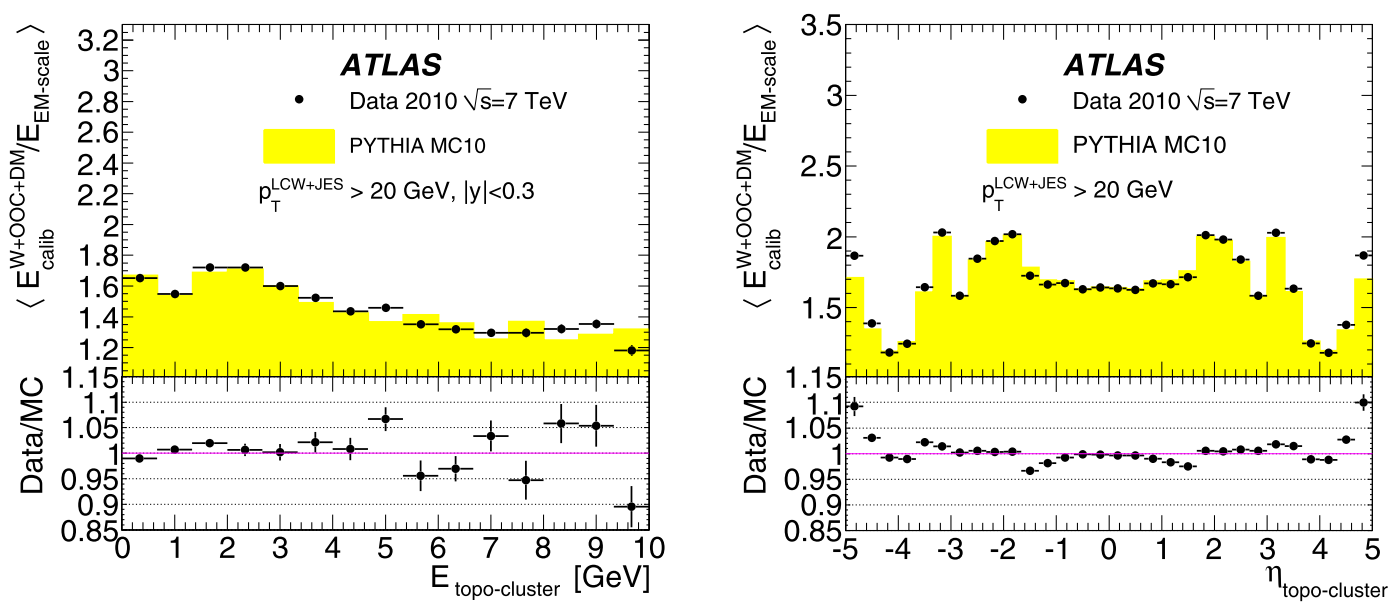

(c) Hadronic response, out-of-cluster and dead material weights

Fig. 58 Mean calibrated topo-cluster energy divided by the uncalibrated topo-cluster energy in data (points) and Monte Carlo simulation (shaded area) as a function of the uncalibrated topo-cluster energy (left) and topo-cluster pseudorapidity (right) after hadronic response weighting (a), adding out-of-cluster corrections (b), and adding dead material corrections (c) applied to topo-clusters in jets. The corrections are sequentially applied. Anti- $k_{t}$ jets with $R=0.6$ in the LCW+JES scheme are required to have $p_{\mathrm{T}}^{\text {jet }}>20 \mathrm{GeV}$. In addition, for the results as a function of the topo-cluster energy (left) the rapidity of the jets is restricted to $|y|<0.3$ 


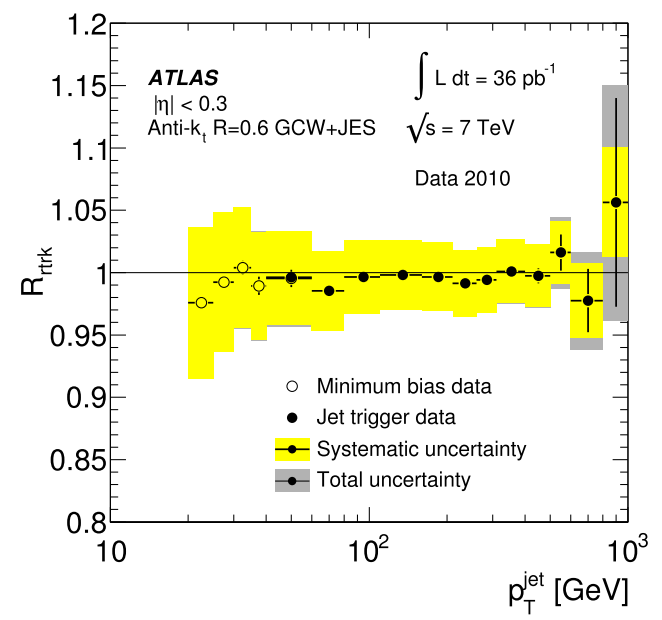

(a) $|\eta|<0.3$

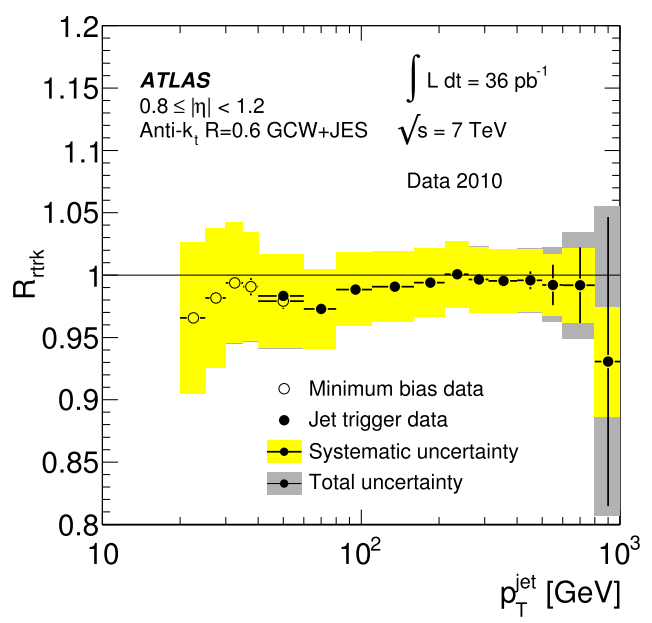

(c) $0.8 \leq|\eta|<1.2$

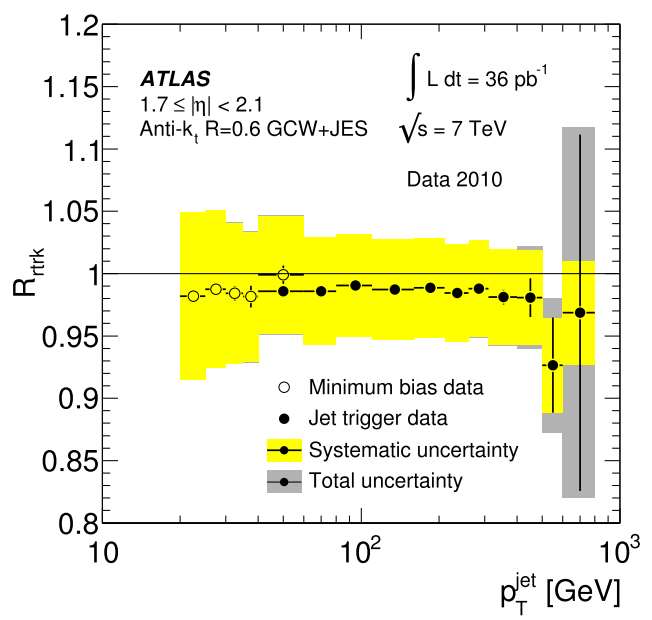

(e) $1.7 \leq|\eta|<2.1$

Fig. 59 Double ratio of the track to calorimeter response in data and Monte Carlo simulation, $R_{r_{\text {trk }}}=\left[\left\langle r_{\text {trk }}\right\rangle\right]_{\text {Data }} /\left[\left\langle r_{\text {trk }}\right\rangle\right]_{\mathrm{MC}}$, for anti- $k_{t}$ jets with $R=0.6$ using the GCW+JES calibration scheme as a function

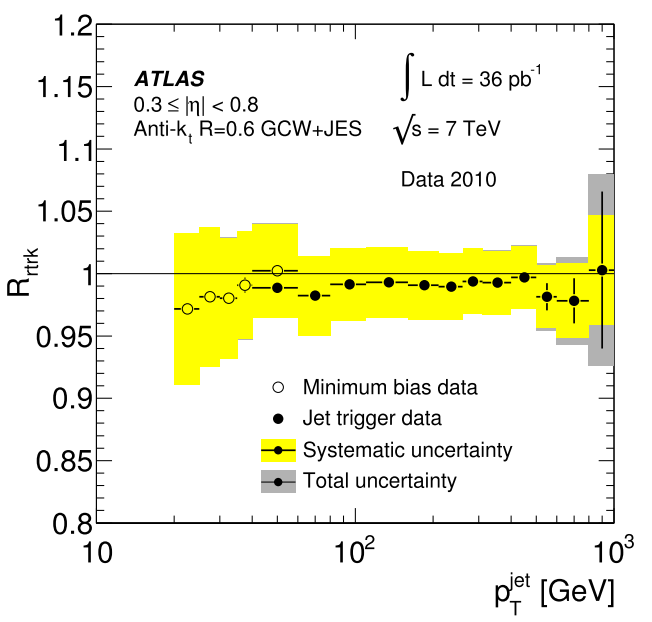

(b) $0.3 \leq|\eta|<0.8$

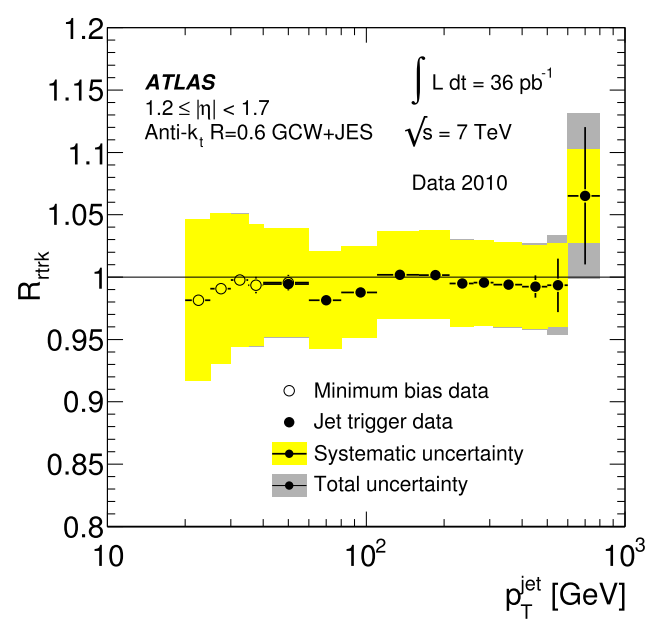

(d) $1.2 \leq|\eta|<1.7$

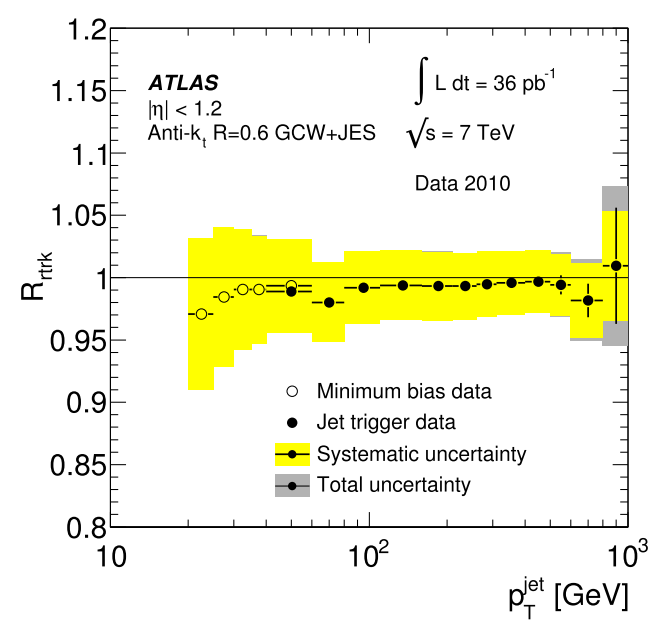

(f) $|\eta|<1.2$

of $p_{\mathrm{T}}^{\mathrm{GCW}+\mathrm{JES}}$ for various $\eta$ bins. Systematic (total) uncertainties are shown as a light (dark) band 


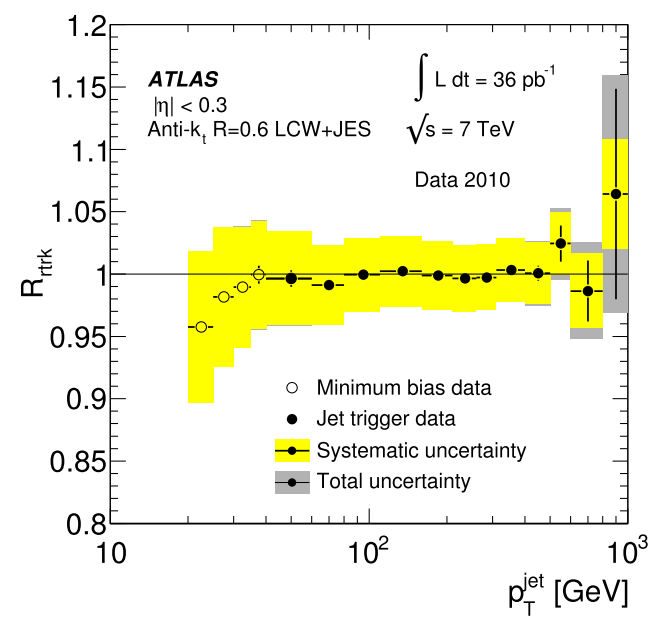

(a) $|\eta|<0.3$

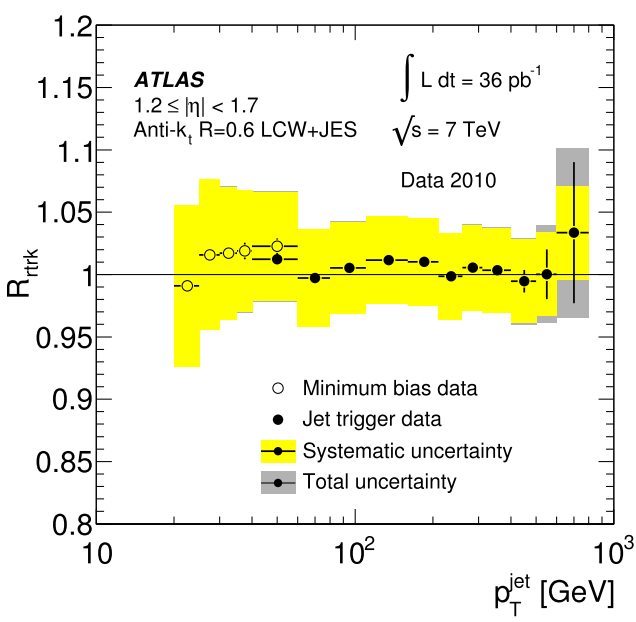

(c) $0.8 \leq|\eta|<1.2$

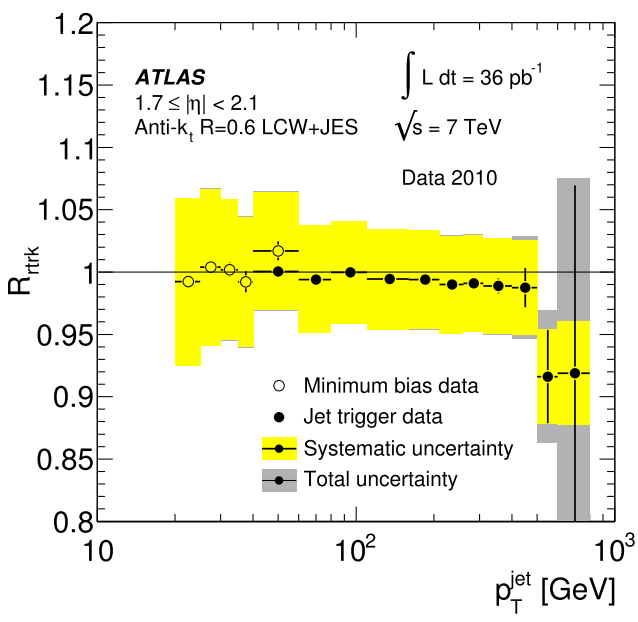

(e) $1.7 \leq|\eta|<2.1$

Fig. 60 Double ratio of the track to calorimeter response comparison in data and Monte Carlo simulation, $R_{r_{\text {trk }}}=\left[\left\langle r_{\text {trk }}\right\rangle\right]_{\text {Data }} /\left[\left\langle r_{\text {trk }}\right\rangle\right]_{\mathrm{MC}}$, for anti- $k_{t}$ jets with $R=0.6$ using the LCW $+\mathrm{JES}$ calibration scheme as a

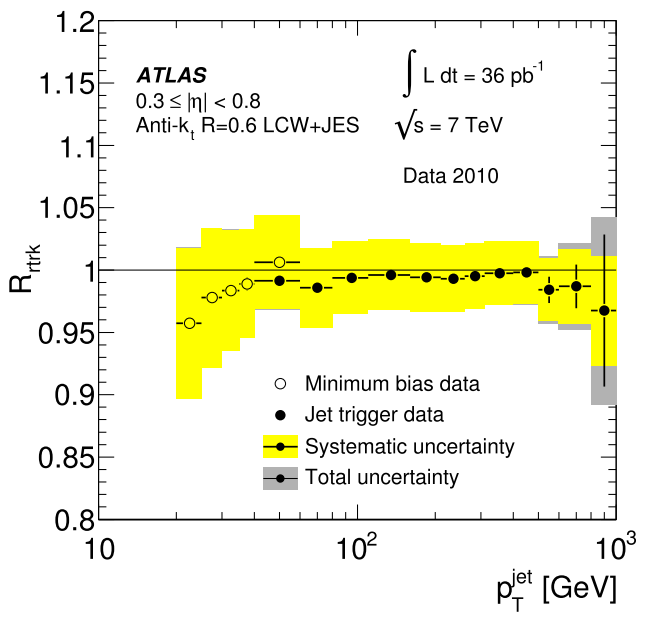

(b) $0.3 \leq|\eta|<0.8$

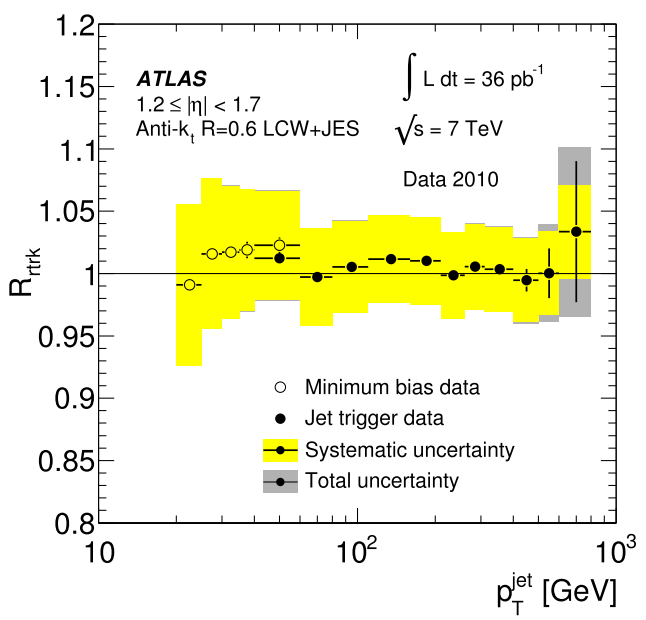

(d) $1.2 \leq|\eta|<1.7$

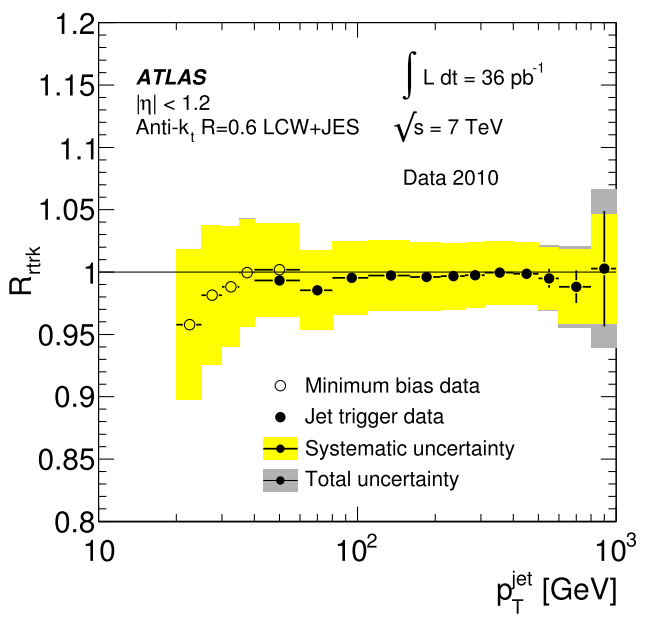

(f) $|\eta|<1.2$

function of $p_{\mathrm{T}}^{\mathrm{LCW}+\mathrm{JES}}$ for various $\eta$ bins. Systematic (total) uncertainties are shown as a light (dark) band 


\subsubsection{Photon-jet transverse momentum balance}

The response measured by the direct $p_{\mathrm{T}}$ balance technique (see Sect. 10.2.1) for the GCW+JES and LCW+JES calibrations is shown in Fig. 61. The agreement of the Monte Carlo

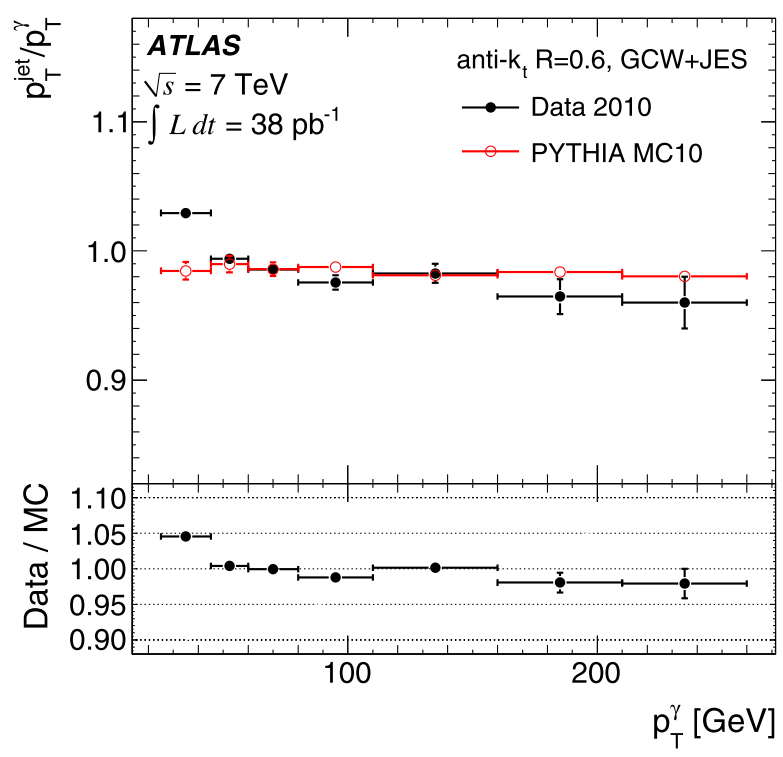

(a) GCW+JES

Fig. 61 Average jet response as determined by the direct $p_{\mathrm{T}}$ balance technique for anti- $k_{t}$ jets with $R=0.6$ calibrated with the GCW+JES (a) and LCW+JES (b) scheme as a function of photon transverse mo-

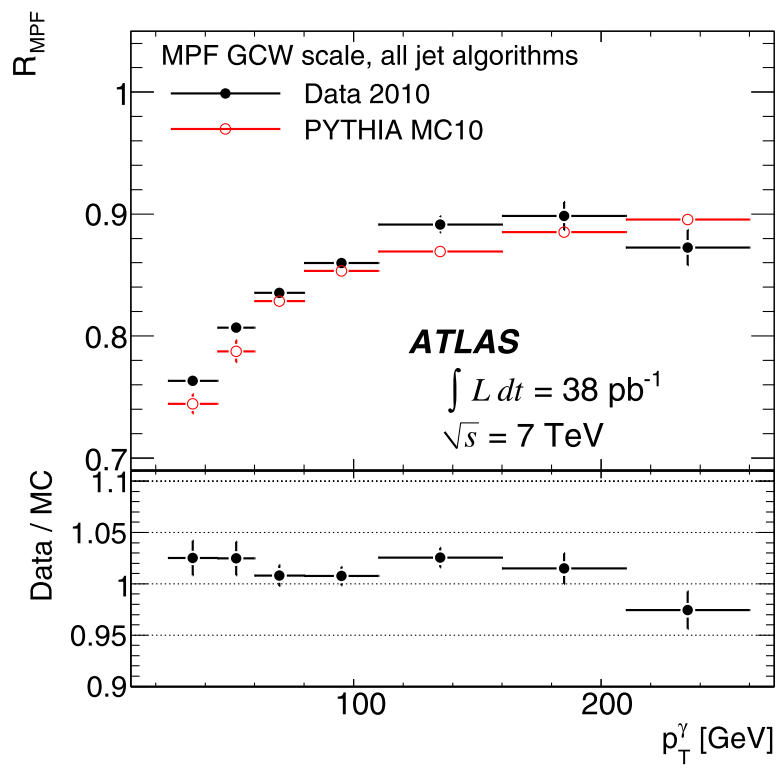

(a) GCW

Fig. 62 Average calorimeter response as determined by the MPF technique for the GCW (a) and LCW (b) calibration scheme as a function of photon transverse momentum for both data and Monte Carlo sim- simulation with data is similar for both calibration schemes. The data to Monte Carlo agreement is 3 to $5 \%$.

Figure 62 shows the comparison of the response determined by the MPF technique (see Sect. 10.2.2), measured in data and Monte Carlo simulation at the GCW and LCW jet energy scales (without a JES correction). To calculate

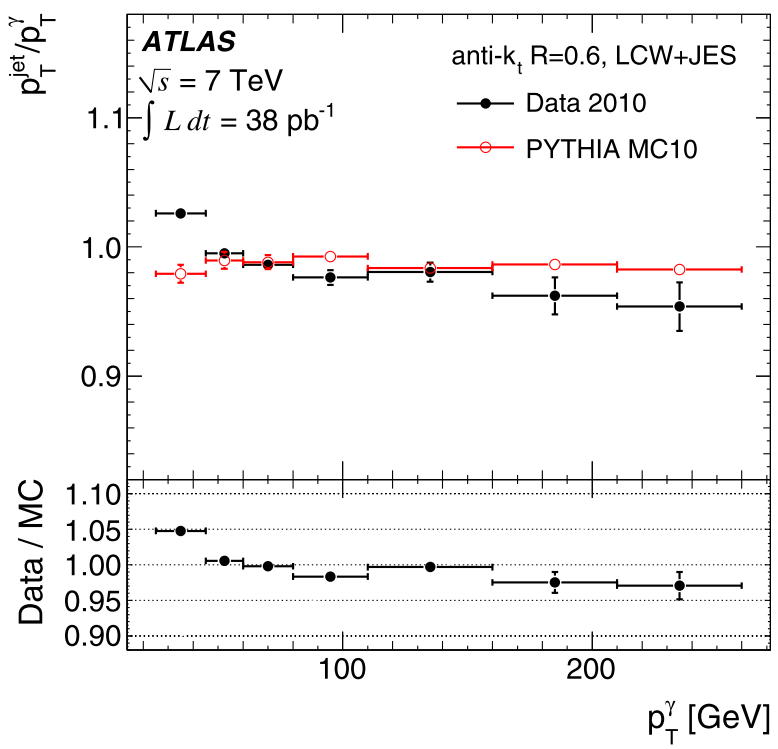

(b) $\mathrm{LCW}+\mathrm{JES}$

mentum for both data and Monte Carlo simulation. The lower part of each figure shows the data to Monte Carlo simulation ratio. Only statistical uncertainties are shown

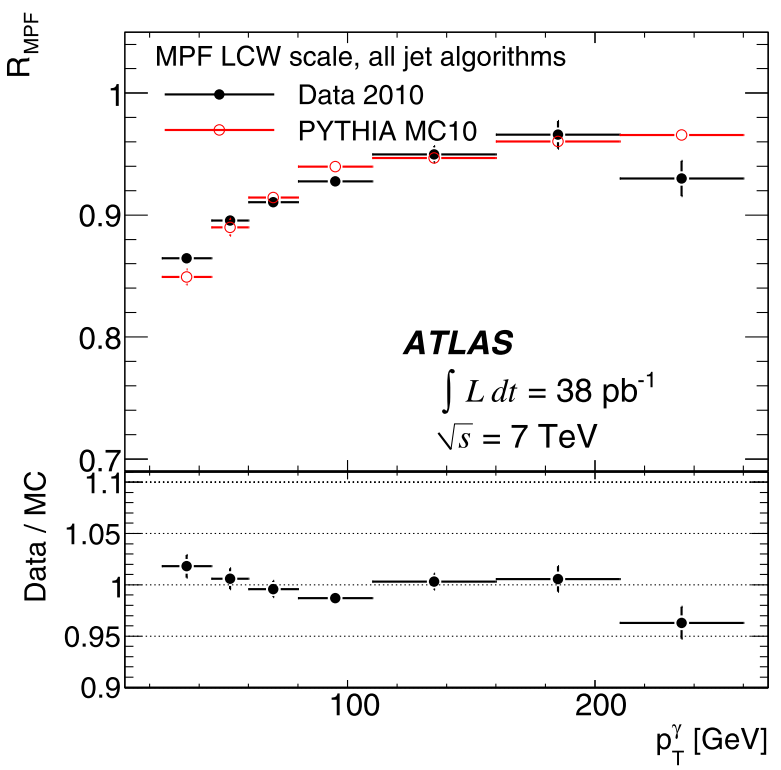

(b) LCW

ulation. The lower part of each figure shows the data to Monte Carlo simulation ratio. Only statistical uncertainties are shown 


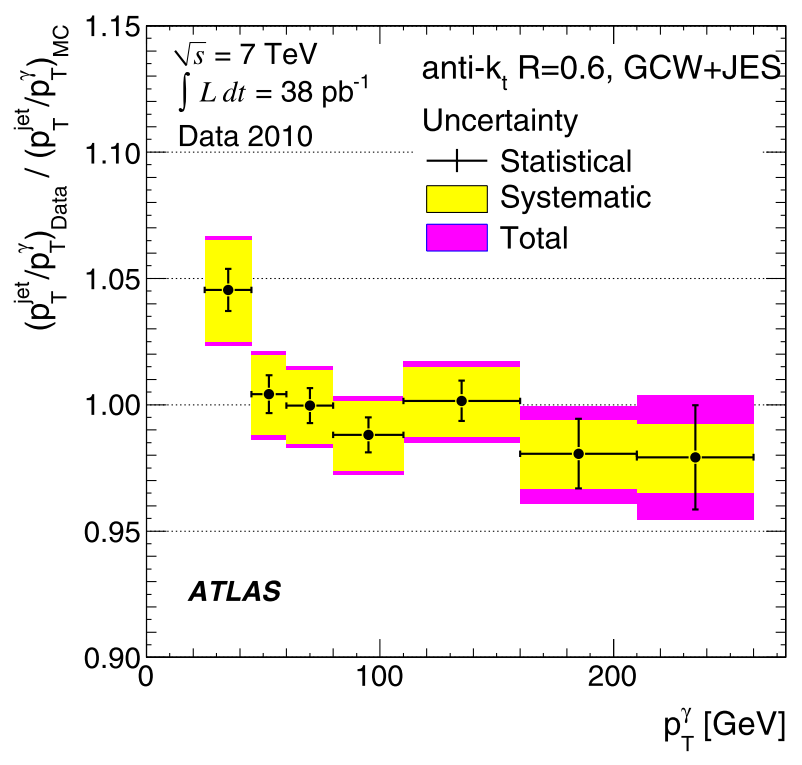

(a) GCW+JES

Fig. 63 Average jet response in data to the response in Monte Carlo simulation using the direct $p_{\mathrm{T}}$ balance technique of anti- $k_{t}$ jets with $R=0.6$ calibrated with the GCW+JES (a) and LCW+JES (b) scheme

the response using the MPF technique at these energy scales the $E_{\mathrm{T}}^{\text {miss }}$ is calculated using GCW or LCW calibrated topoclusters as an input. ${ }^{38}$ All the calibrations schemes are found to be consistent between data and Monte Carlo simulation to within 3 to $4 \%$.

The ratios of jet response in data to the response in Monte Carlo simulation using the direct $p_{\mathrm{T}}$ balance technique for the GCW+JES and LCW+JES jet calibration schemes as a function of the photon transverse momentum are shown in Fig. 63. The agreement of data and Monte Carlo simulation is within $5 \%$ and is compatible with unity within the statistical and systematic uncertainties. A similar result for the MPF technique is shown in Fig. 64 for GCW and LCW calibration schemes (without a JES correction). Good agreement between data and Monte Carlo simulation is found.

\subsubsection{Intercalibration of forward jets using events with dijet topologies}

The transverse momentum balance in events with only two jets at high transverse energy can be used to determine the JES uncertainty for jets in the forward detector region. The matrix method, described in Sect. 9.6, is used in order to test the performance of the GCW+JES and LCW+JES calibrations for jets with $|\eta|>1.2$ and to determine the JES uncertainty in the forward region based on the well calibrated jet in the central reference region.

\footnotetext{
${ }^{38}$ For the GCW calibration scheme the cell energies in the topo-clusters are multiplied by the cell energy weights described in Sect. 13.1.
}

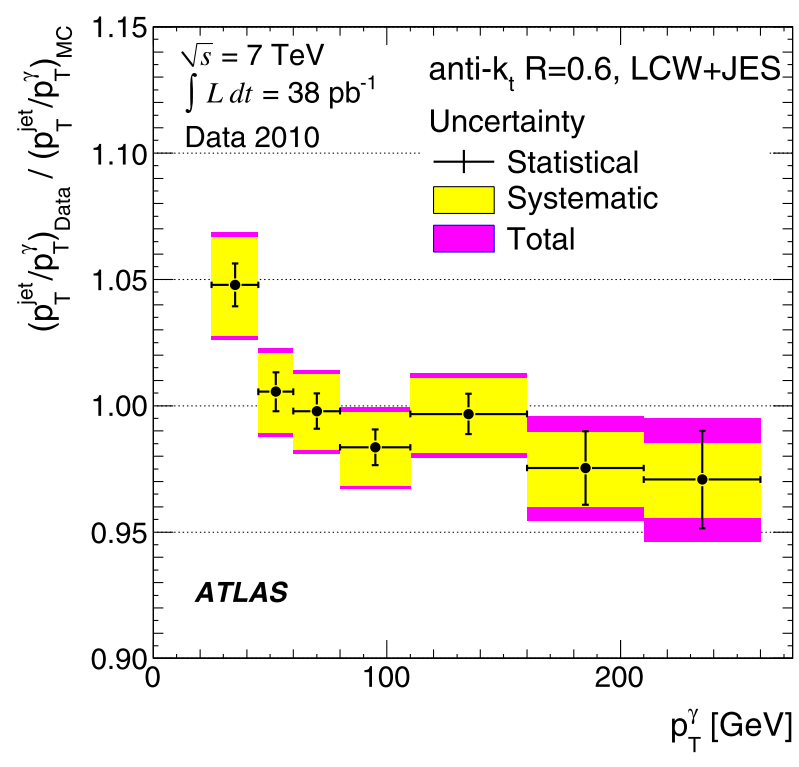

(b) LCW+JES

as a function of photon transverse momentum. Statistical and systematic uncertainties (light band) are included with the total uncertainty shown as the dark band

The same selection and method as for the test of the EM+JES calibration is applied, with two exceptions: the reference region is defined by $\left|\eta_{\text {det }}\right|<1.2$ instead of $\left|\eta_{\text {det }}\right|<$ 0.8 , and a fit is applied to smooth out statistical fluctuations that has essentially no impact for the low- $p_{\mathrm{T}}$ bins, but stabilize the result at high $p_{\mathrm{T}}$ where some bins suffer from a low number of events.

The JES uncertainty in the reference regions is obtained from the $\gamma$-jet results (see Sect. 14.3.2) and using the sum of track momenta (see Sect. 14.3.1).

Figure 65 shows the resulting uncertainties as a function of $p_{\mathrm{T}}^{\text {jet }}$ for various $\eta$-bins. The uncertainty is taken as the RMS spread of the relative response from the Monte Carlo predictions around the relative response measured in data (see Sect. 9.6.6). The JES uncertainty introduced by the dijet balance is largest at lower $p_{\mathrm{T}}^{\text {jet }}$ and smallest at higher $p_{\mathrm{T}}^{\text {jet }}$. For $p_{\mathrm{T}}^{\text {jet }}>100 \mathrm{GeV}$ the JES uncertainty for the GCW+JES scheme is less than $1 \%$ for $1.2 \leq|\eta|<2.1$ and about $2.5 \%$ for $2.8 \leq|\eta|<3.2$. For $p_{\mathrm{T}}^{\text {jet }}=20 \mathrm{GeV}$ the JES uncertainty is about $2 \%$ for $1.2 \leq|\eta|<2.1$ and about $9.5 \%$ for $3.6 \leq$ $|\eta|<4.5$.

The JES uncertainties for the LCW+JES calibration scheme are slightly larger than those for GCW+JES scheme.

\subsubsection{Multijet transverse momentum balance}

The multijet balance (MJB) technique, described in Sect. 10.3, is used to evaluate the JES uncertainty in the 


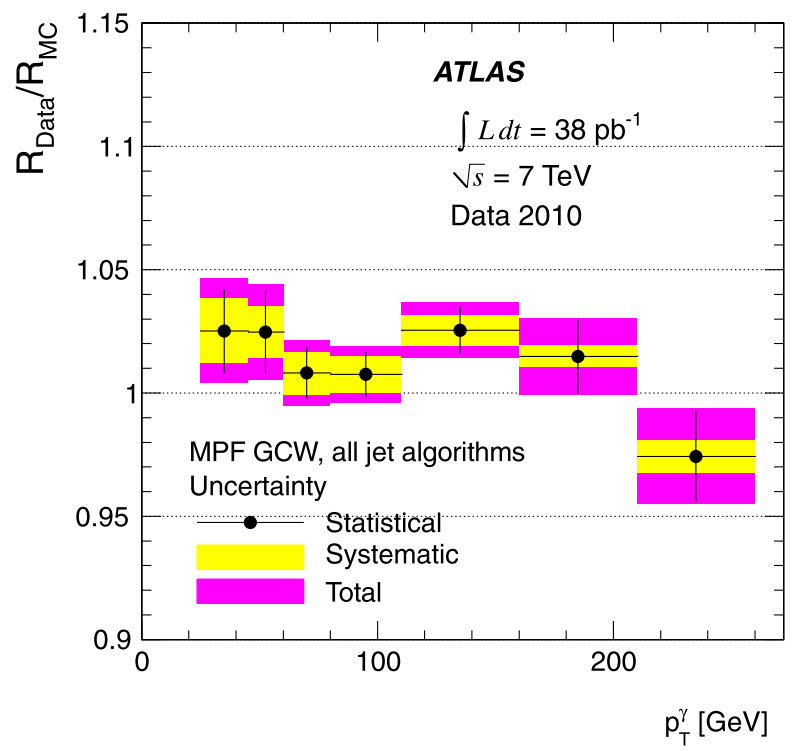

(a) GCW

Fig. 64 The ratios of the MPF calorimeter response in data to the response in Monte Carlo simulation using the MPF method for each input energy scale GCW (a), and LCW (b) as a function of the photon

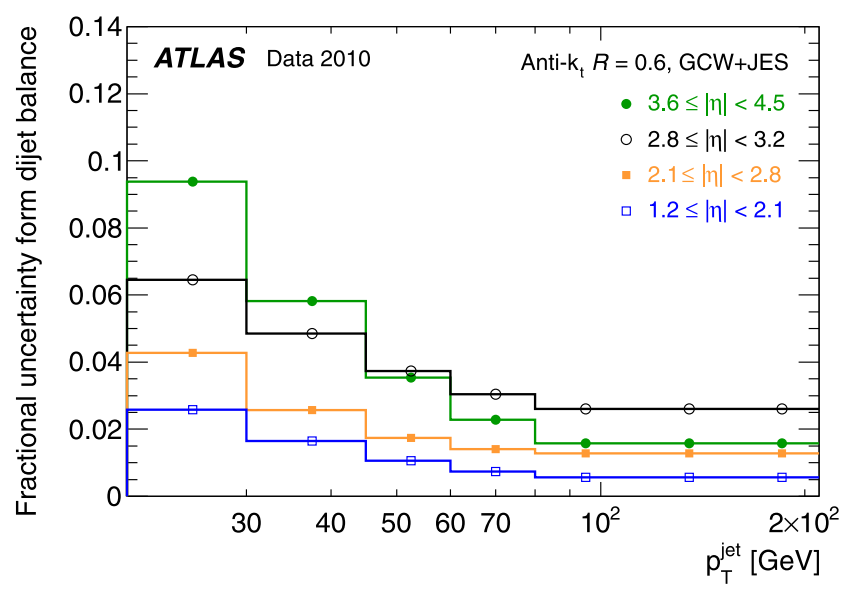

(a) GCW+JES

Fig. 65 Uncertainty in the jet response obtained from the dijet $\eta$ intercalibration technique for anti- $k_{t}$ jets with $R=0.6$ as a function of the jet $p_{\mathrm{T}}$ for various $|\eta|$-regions of the calorimeter. The jets are

high transverse momentum region for the GCW+JES and LCW+JES calibration schemes. The method and selection cuts used are the same as those for the EM+JES calibrated jets.

Figure 66 shows the MJB for anti- $k_{t}$ jets with $R=0.6$ obtained using the GCW+JES and LCW+JES calibrations in the data and Monte Carlo simulation as a function of the recoil $p_{\mathrm{T}}$. The agreement between the data and MC simulations, evaluated as the data to Monte Carlo simulation ratio, are very similar to those for the EM+JES calibration as shown in Fig. 39.

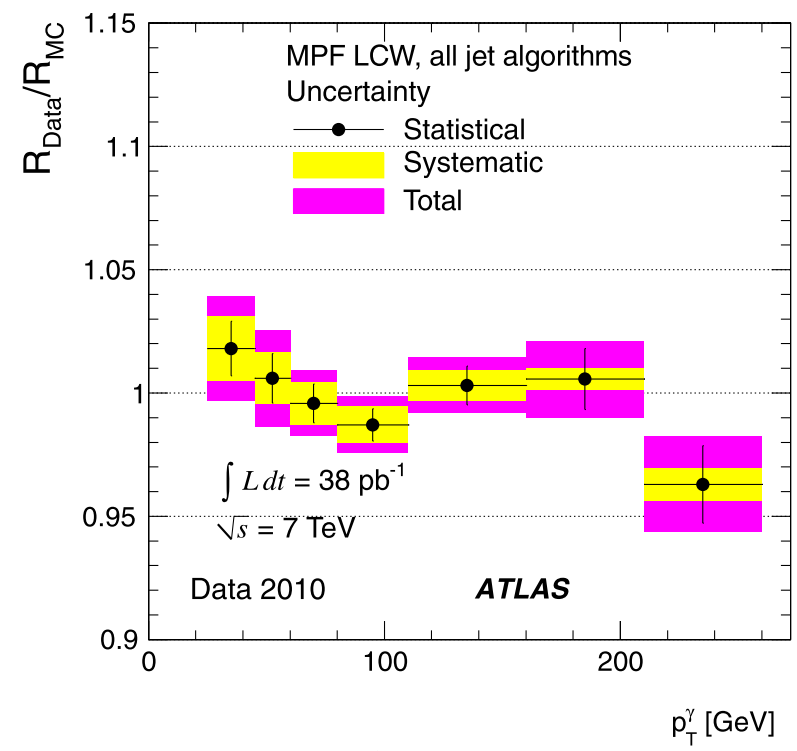

(b) LCW

transverse momentum. Statistical and systematic uncertainties (light band) are included. The total uncertainty is shown as the dark band

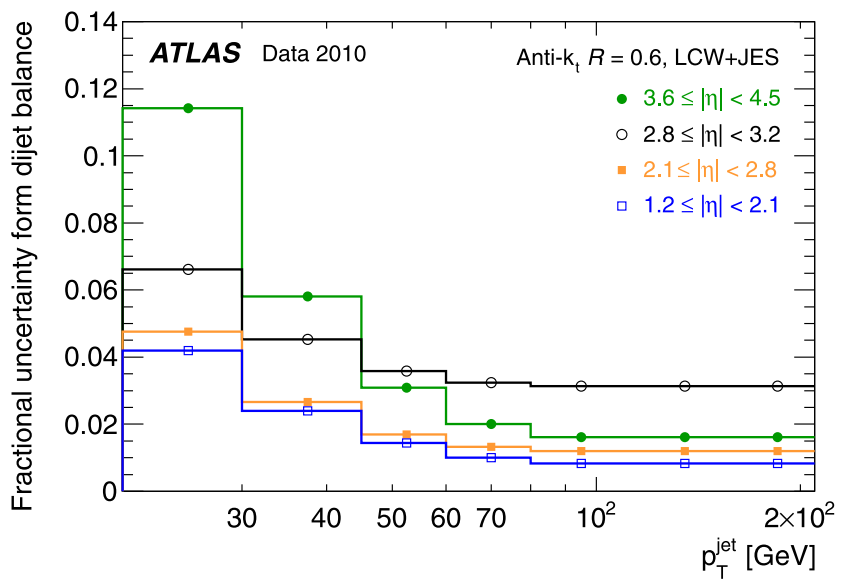

(b) LCW+JES

calibrated with the GCW+JES (a) and the LCW+JES (b) calibration schemes. Only statistical uncertainties are shown

The systematic uncertainties on the MJB for these cell energy weighting calibration schemes are evaluated in the same way as the EM+JES calibration, described in Sect. 10.3.4, except for the component of the standard JES uncertainty on the recoil system. The JES uncertainty for jets in the recoil system is obtained from the in situ $\gamma$-jet balance discussed in Sect. 14.3.2. In this case, the systematic uncertainty on the MJB due to the recoil system JES uncertainty is then calculated by shifting the $p_{\mathrm{T}}$ of recoil jets up and down by the $\gamma$-jet JES uncertainty. In order to apply the $\gamma$-jet JES uncertainty to the recoil system, the 
MJB analysis is performed with jets selected within the range $|\eta|<1.2$, where the JES uncertainty based on $\gamma$-jet events has been derived. The close-by jet and flavour composition systematic uncertainties are also re-evaluated for the GCW+JES and LCW+JES jets using the same method (see Sect. 17).

Figure 67 shows the total and individual JES systematic uncertainties on the recoil system for anti- $k_{t}$ jets with $R=0.6$ calibrated by the GCW+JES and LCW+JES schemes. The total uncertainty is dominated by the uncertainty from the $\gamma$-jet uncertainty. The increase of the JES uncertainty at high $p_{\mathrm{T}}^{\text {jet }}$ above $800 \mathrm{GeV}$ is caused by a large JES systematic uncertainty due to limited $\gamma$-jet event statistics at high $p_{\mathrm{T}}$.

The systematic uncertainties associated with the analysis method and event modelling are re-evaluated in the same way as for the EM+JES calibration scheme and then added to the recoil system JES systematic uncertainties. The summary of all systematic uncertainties and the total uncertainty obtained by adding the statistical and systematic uncertainties in quadrature is shown in Fig. 68 for anti- $k_{t}$ jets with $R=0.6$.

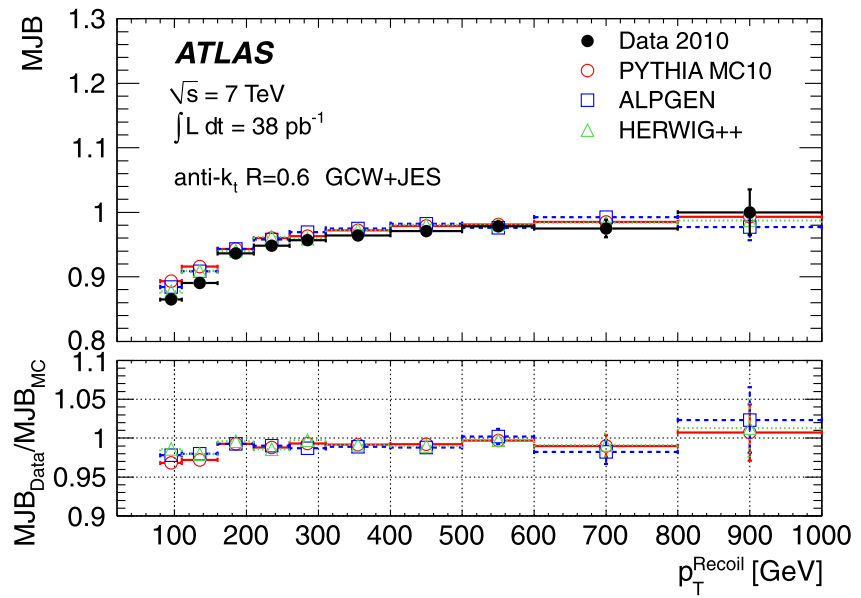

(a) GCW+JES

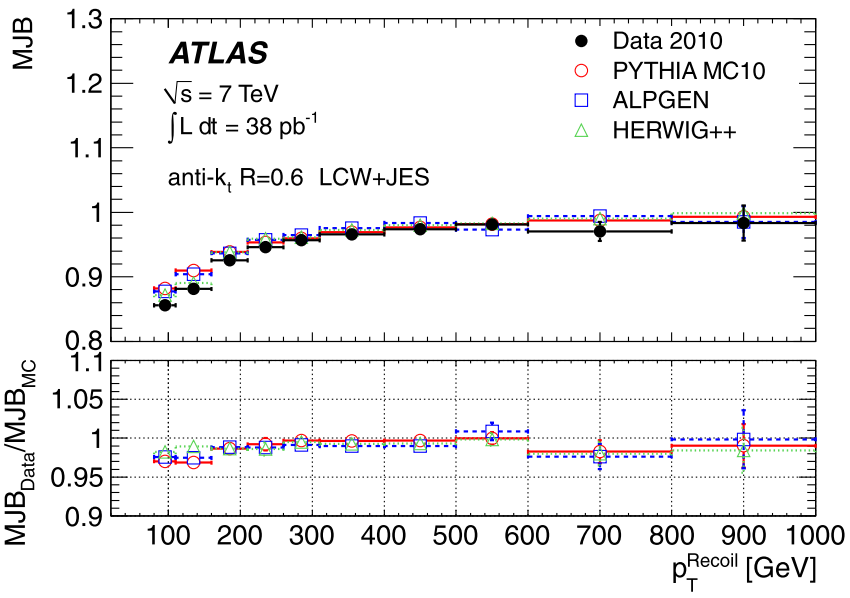

(b) LCW+JES

Fig. 66 Multijet balance MJB as a function of the recoil system $p_{\mathrm{T}}$ for data and Monte Carlo simulation for the anti- $k_{t}$ algorithm with $R=0.6$ using the GCW+JES (a) and LCW+JES (b) calibration scheme. Only statistical uncertainties are shown

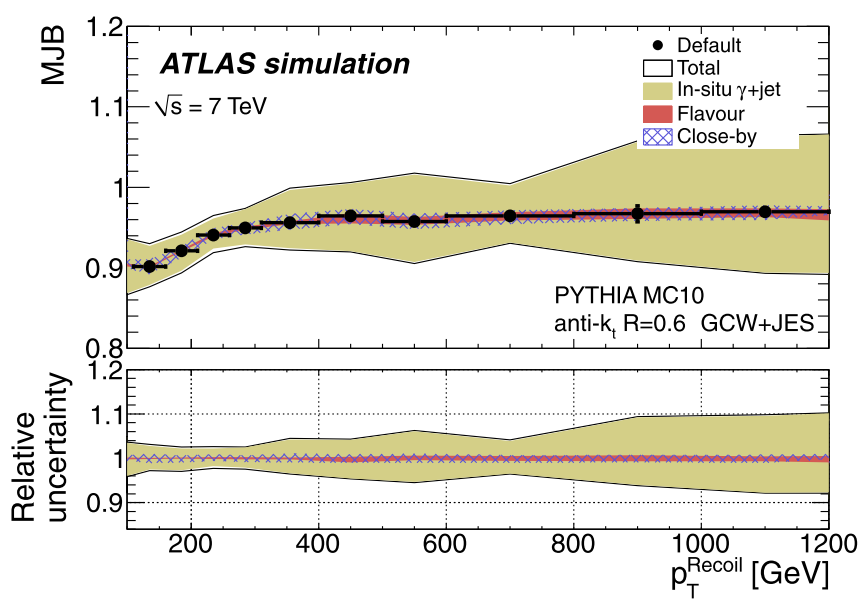

(a) GCW+JES

Fig. 67 The multijet balance $\mathrm{MJB}_{\mathrm{MC}}$ as a function of the recoil system $p_{\mathrm{T}}$ (full dots) for anti- $k_{t}$ jets with $R=0.6$ using the GCW+JES (a) and LCW+JES (b) calibration schemes. The three bands are defined by the maximum shift of MJB when the jets that compose the recoil system are shifted up and down by the JES uncertainty determined from the $\gamma$-jet balance, close-by jet and flavour uncertainties. The black

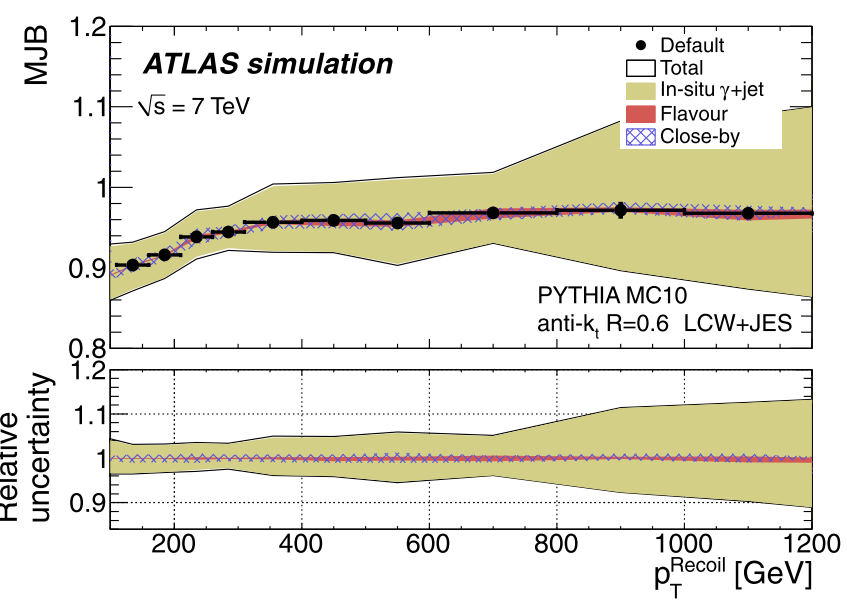

(b) LCW+JES

lines show the total uncertainty obtained by adding in quadrature the individual uncertainties. The lower part of the figure shows the relative uncertainty due to the scale uncertainty of the jets that compose the recoil system, defined as the maximum relative shift with respect to the nominal value, as a function of $p_{\mathrm{T}}^{\text {Recoil }}$ 


\subsubsection{Cell weighting JES uncertainty from combination of in situ techniques}

Figure 69 shows the jet response ratio of data to Monte Carlo simulation for the various in situ techniques as a function of the jet transverse momentum for the GCW+JES (a) and the LCW+JES (b) calibration schemes. Statistical and systematic uncertainties are displayed. The average from the combination of all in situ techniques is overlaid.

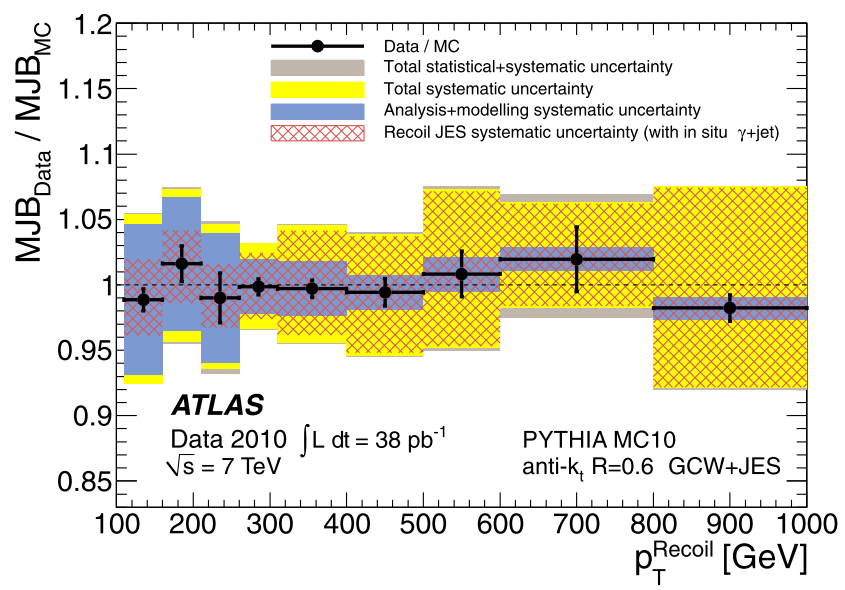

(a) GCW+JES

Fig. 68 Ratio of the data to MC for the multijet balance as a function of the recoil system $p_{\mathrm{T}}$ for anti- $k_{t}$ jets with $R=0.6$ using the GCW+JES (a) and LCW+JES (b) calibration schemes. The various shaded regions show the total uncertainty (dark band) obtained as the squared sum of the total systematic uncertainty (light band) and of the

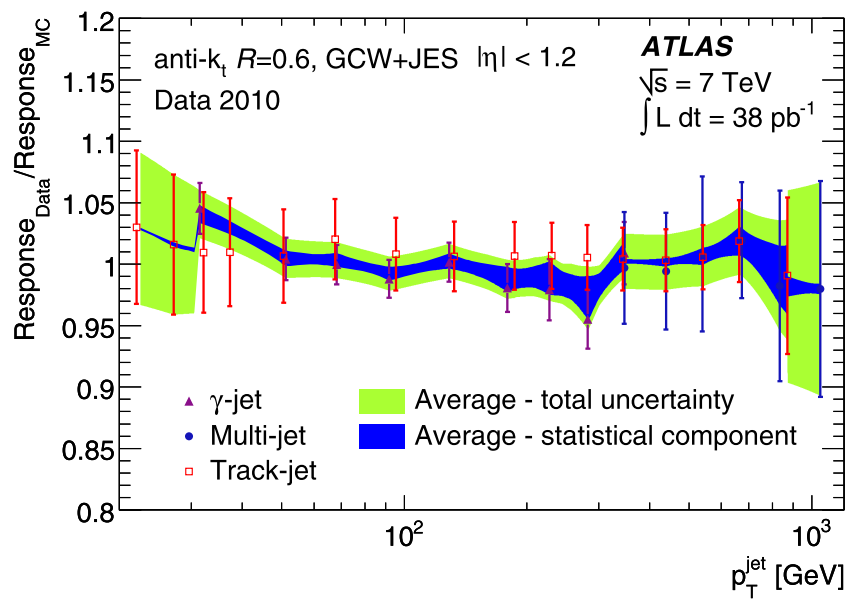

(a) GCW+JES

Fig. 69 Jet response ratio of the data to the Monte Carlo simulation as a function of $p_{\mathrm{T}}^{\text {jet }}$ for three in situ techniques using as reference objects: photons $(\gamma$-jet), a system of low energetic jets (multijet) or the transverse momentum of all tracks associated to jets (tracks in jets). The error bar displays the statistical and systematic uncertainties added in
The weight of each in situ technique contributing to the average is similar to the one for the EM+JES calibration scheme shown in Fig. 44. The contributions are also similar for the LCW+JES and the GCW+JES calibration schemes.

Figure 70 shows (as solid line) the final JES uncertainty for the GCW+JES (a) and the LCW+JES (b) calibration schemes for $|\eta|<1.2$. At the lowest $p_{\mathrm{T}}$ the JES uncertainty is about $9 \%$ to $10 \%$ and decreases for increasing $p_{\mathrm{T}}^{\text {jet }}$. For $p_{\mathrm{T}}^{\text {jet }}>50 \mathrm{GeV}$ it is about $2 \%$ and at $p_{\mathrm{T}}^{\text {jet }}=250 \mathrm{GeV}$ it is

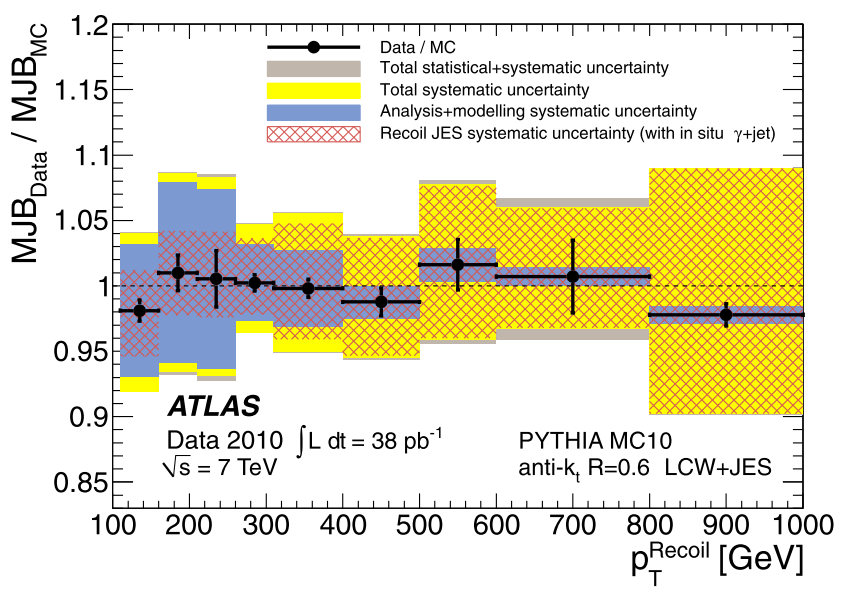

\section{(b) LCW+JES}

statistical uncertainty (error bars). Also displayed are the contributions to the systematic uncertainty due to analysis cuts and event modelling (darkest band) and to the jet energy scale for jets in the recoil system (hatched band)

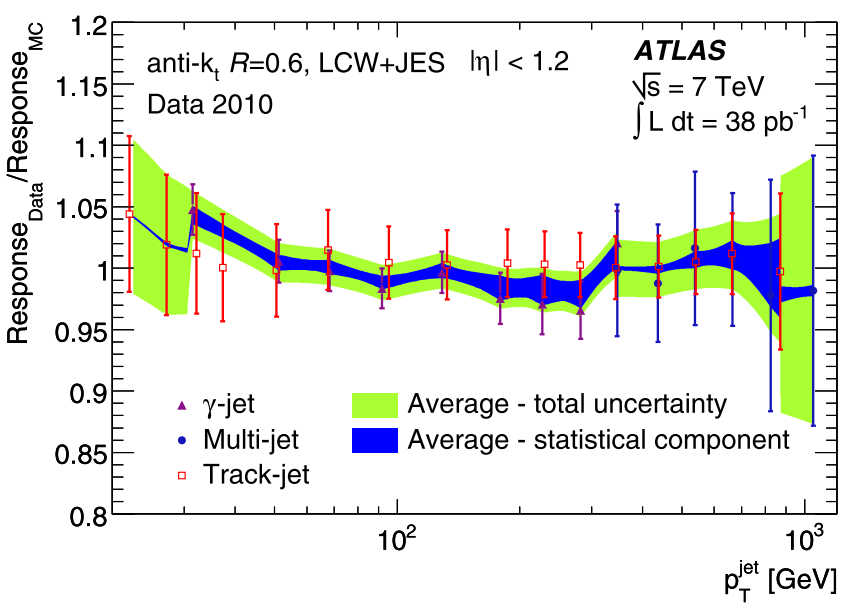

(b) LCW+JES

quadrature. Shown are the results for anti- $k_{t}$ jets with $R=0.6$ calibrated with the GCW+JES (a) and LCW+JES (b) calibration schemes. The light band indicates the combination of the in situ techniques. The inner dark band shows the fraction due to the statistical uncertainty 


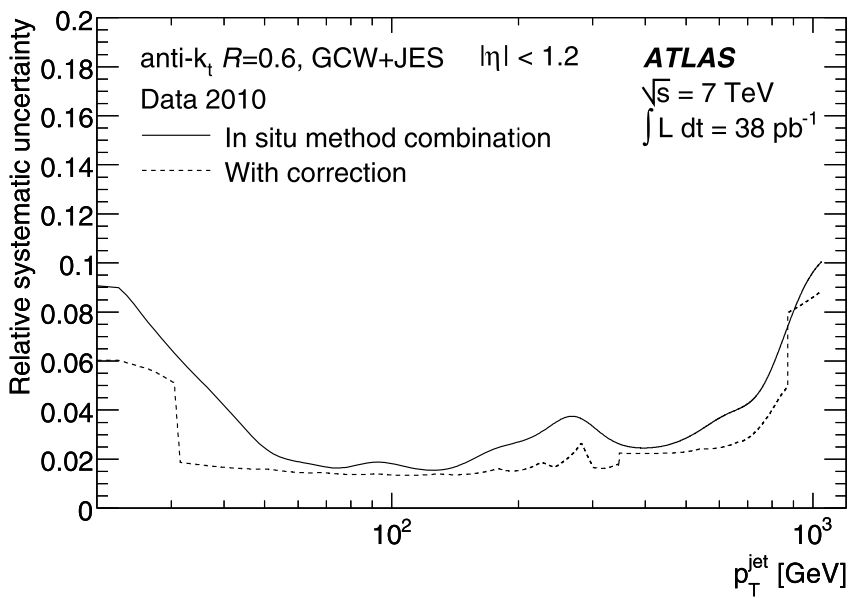

(a) GCW+JES

Fig. 70 Jet energy scale uncertainty (solid line) as a function of $p_{\mathrm{T}}^{\text {jet }}$ for anti- $k_{t}$ jets with $R=0.6$ for $|\eta|<1.2$ calibrated with the GCW+JES (a) and the LCW+JES (b) calibration scheme. The dashed

about 3 to $4 \%$. For jets in the TeV-regime the JES uncertainty is 10 to $12 \%$.

Figure 70 also shows (as dashed line) the JES uncertainty attainable, if the in situ techniques had been used to correct the jet energy. Using the in situ techniques for jet calibration would have resulted in an improved JES uncertainty for both jet calibration schemes based on cell energy weighting.

The JES uncertainty obtained in the central reference region $(|\eta|<1.2)$ is used to derive the JES uncertainty in the forward region using the dijet balance technique. The central region JES uncertainty is combined with the uncertainties from the dijet balance shown in Fig. 65.

\section{Summary of jet energy scale uncertainties of various calibration schemes}

The EM+JES uncertainties are derived from single hadron response measurements and from systematic variations of the Monte Carlo simulation (see Sect. 9).

The JES uncertainty for the GS jet calibration scheme is given by the sum in quadrature of the EM+JES uncertainty and the uncertainty associated to the GS corrections. The latter, derived in Sect. 12, is conservatively taken to be $0.5 \%$ for $30<p_{\mathrm{T}}<800 \mathrm{GeV}$ and $|\eta|<2.1$ and $1 \%$ for $p_{\mathrm{T}}<$ $30 \mathrm{GeV}$ and $2.1<|\eta|<2.8$. These uncertainties are also supported by in situ techniques.

The JES uncertainties in the LCW+JES and GCW+JES jet calibration schemes are derived from a combination of several in situ techniques.

Figure 71 shows a comparison of the JES uncertainties for the EM+JES, the LCW+JES and the GCW+JES jet cal-

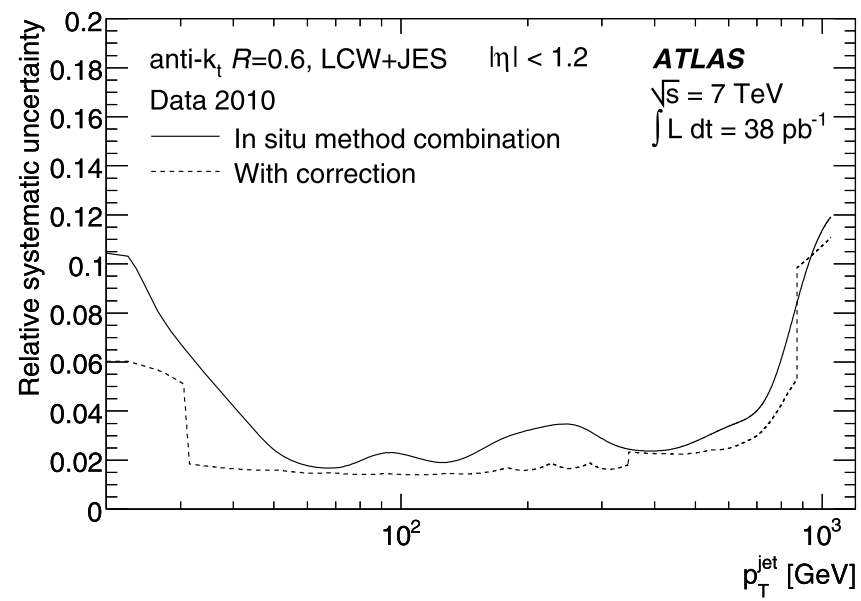

(b) LCW+JES

line shows the JES uncertainty that could have been achieved, if in situ techniques had been used to recalibrate the jets

ibration schemes for various $\eta$-regions. The uncertainties in the LCW+JES and GCW+JES schemes derived in Sect. 14 are similar, but the uncertainty for the GCW+JES calibration scheme is a bit smaller for very low and very large $p_{\mathrm{T}}^{\text {jet }}$.

Over a wide kinematic range, $40 \lesssim p_{\mathrm{T}}^{\text {jet }} \lesssim 600 \mathrm{GeV}$, all calibration schemes show a similar JES uncertainty. At $p_{\mathrm{T}}^{\text {jet }} \approx 250 \mathrm{GeV}$ the uncertainties based on the in situ techniques are about $2 \%$ larger compared to the uncertainty results from the EM+JES calibration scheme.

For $p_{\mathrm{T}}^{\text {jet }}<40 \mathrm{GeV}$ and $p_{\mathrm{T}}^{\text {jet }}>600 \mathrm{GeV}$ the EM+JES calibration scheme has a considerably smaller uncertainty. For the high $p_{\mathrm{T}}$ regions the JES calibration based on in situ suffers from the limited number of events in the data samples. At low $p_{\mathrm{T}}$ the systematic uncertainty on the in situ methods leads to a larger JES uncertainty.

\section{Jet reconstruction efficiency}

A tag-and-probe method is implemented to measure in situ the jet reconstruction efficiency relative to track jets. Because track jets (see Sect. 6.2) and calorimeter jets (see Sect. 6.1) are reconstructed by independent ATLAS subdetectors, a good agreement between data and Monte Carlo simulation for this matching efficiency means that the absolute jet reconstruction efficiency can be determined from the simulation.

\subsection{Efficiency in the Monte Carlo simulation}

The jet reconstruction efficiency is determined in the Monte Carlo simulation by counting in how many cases a calorime- 


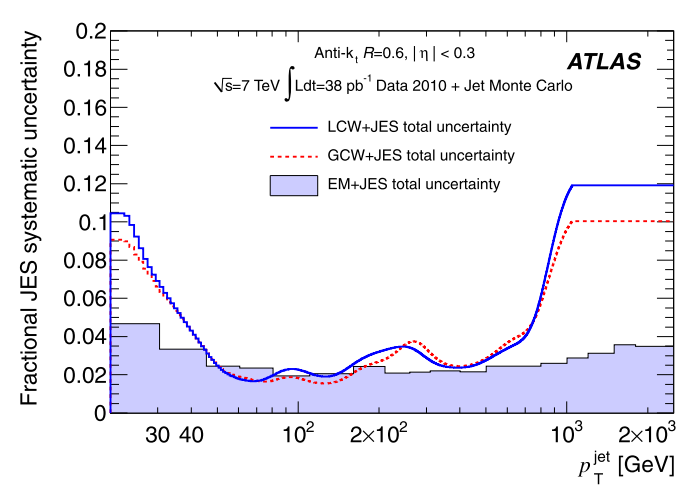

(a) $|\eta|<0.3$

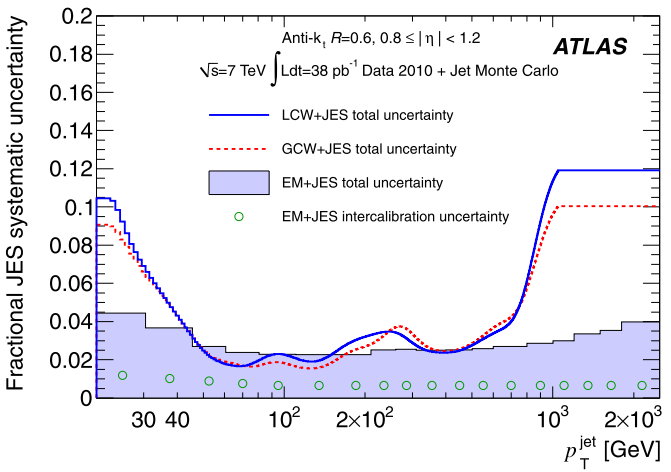

(c) $0.8 \leq|\eta|<1.2$

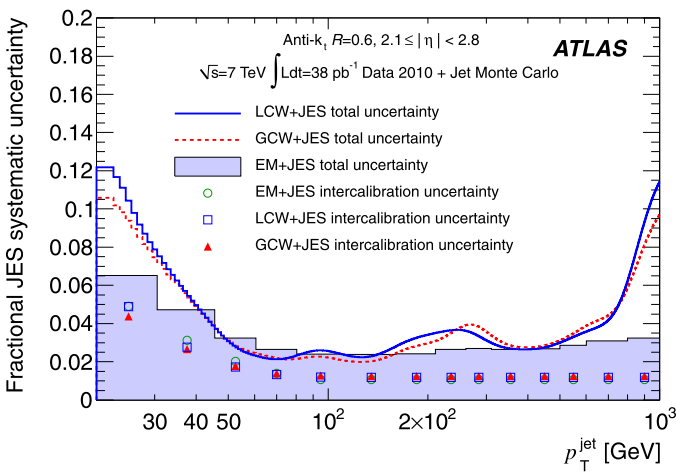

(e) $2.1 \leq|\eta|<2.8$

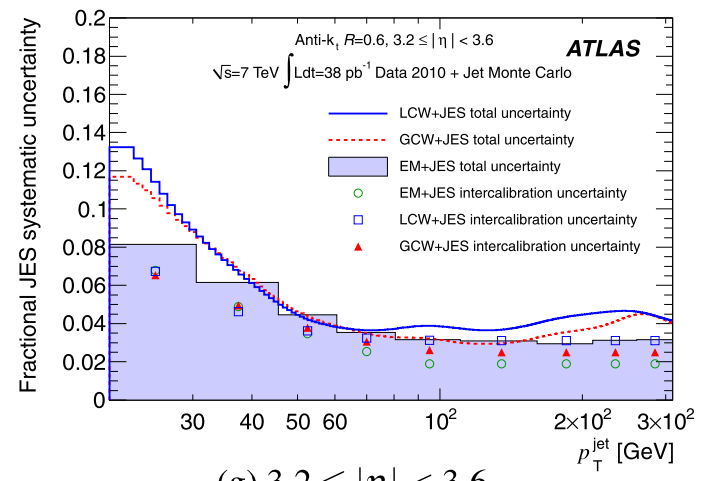

(g) $3.2 \leq|\eta|<3.6$

Fig. 71 Fractional JES uncertainties as a function of $p_{\mathrm{T}}^{\text {jet }}$ for anti- $k_{t}$ jets with $R=0.6$ for the various $\eta$ regions for the LCW+JES (full line) and the GCW+JES (dashed line) schemes. These are derived from a combination of the in situ techniques which are limited in the number of available events at large $p_{\mathrm{T}}^{\text {jet }}$. The fractional JES uncertainty

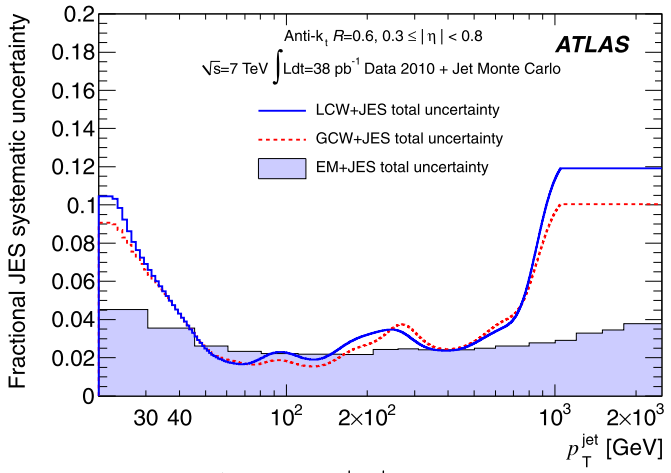

(b) $0.3 \leq|\eta|<0.8$

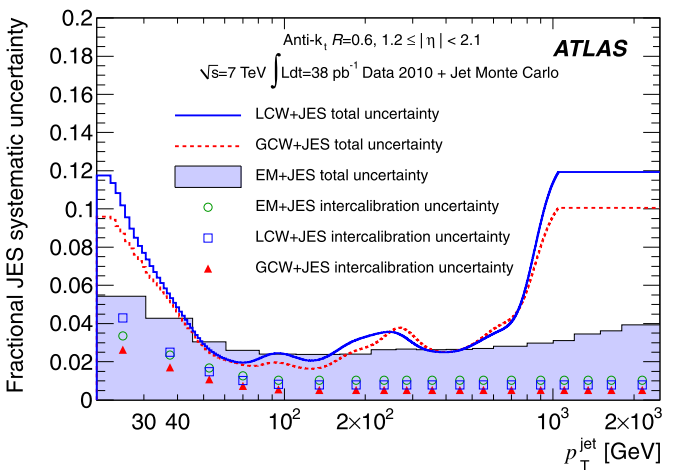

(d) $1.2 \leq|\eta|<2.1$

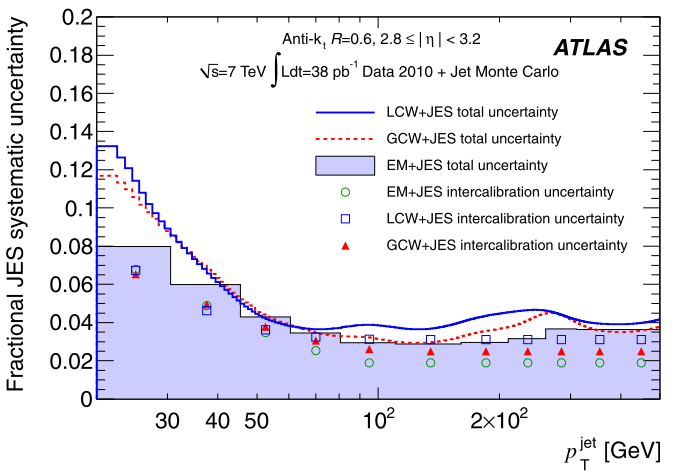

(f) $2.8 \leq|\eta|<3.2$

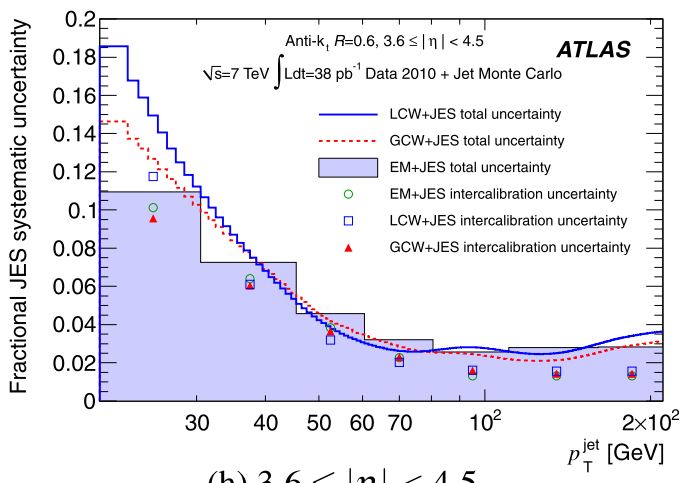

(h) $3.6 \leq|\eta|<4.5$

for EM+JES derived from single hadron response measurements and systematic Monte Carlo simulation variations is overlaid as shaded area for comparison. The $\eta$-intercalibration uncertainty is shown as open symbols for $|\eta|>0.8$ for the EM+JES and for $|\eta|>1.2$ for the LCW+JES and GCW+JES schemes 


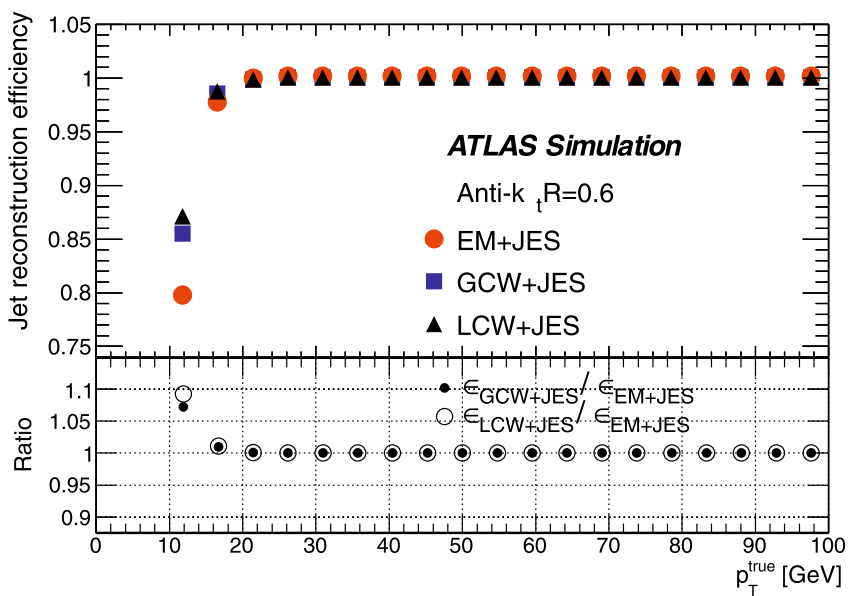

(a) Efficiency from truth jets

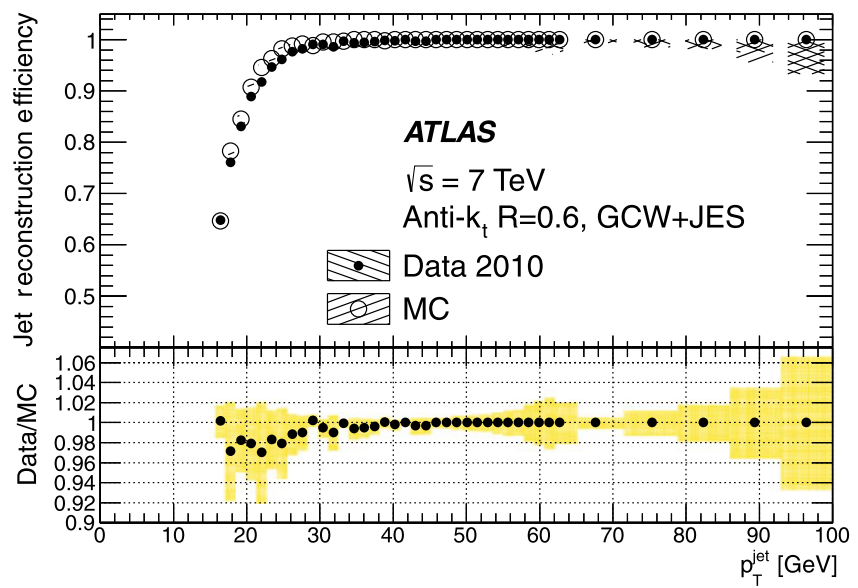

(c) Efficiency from track jets for GCW+JES

Fig. 72 Calorimeter jet reconstruction efficiency with respect to truth jets (a) and track jets $(\mathbf{b}, \mathbf{c}, \mathbf{d})$ as a function of the truth jet (a) or the calorimeter jet $(\mathbf{b}, \mathbf{c}, \mathbf{d}) p_{\mathrm{T}}$ for the three calibration schemes: EM+JES (b), GCW+JES (c) and LCW+JES (d). The lower part of the figure (a) shows ratio of the efficiency of the LCW+JES and the GCW+JES cal-

ter jet can be matched to a truth jet. Reconstructed jets are matched to truth jets, if their jet axes are within $\Delta R<0.4$.

Figure 72a shows the jet reconstruction efficiency for anti- $k_{t}$ jets with $R=0.6$ calibrated with the EM+JES, GCW+JES, and LCW+JES calibration schemes as a function of the transverse momentum of the truth jet. The efficiency stays above a value of $99 \%$ for a truth jet transverse momentum of $20 \mathrm{GeV}$. The lower part of the figure shows the ratio of the efficiency in the GCW+JES and LCW+JES calibration schemes to that obtained from the EM+JES scheme. Similar performance is found for all calibration schemes.

The small differences at low $p_{\mathrm{T}}^{\text {jet }}$ might be caused by the slightly better jet energy resolution obtained with the GCW+JES and the LCW+JES calibration schemes as compared to the EM+JES scheme.

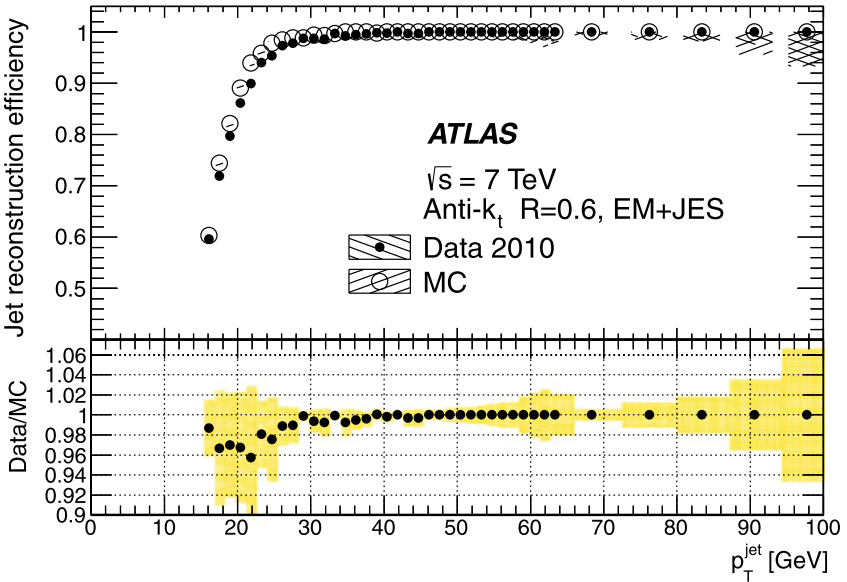

(b) Efficiency from track jets for EM+JES

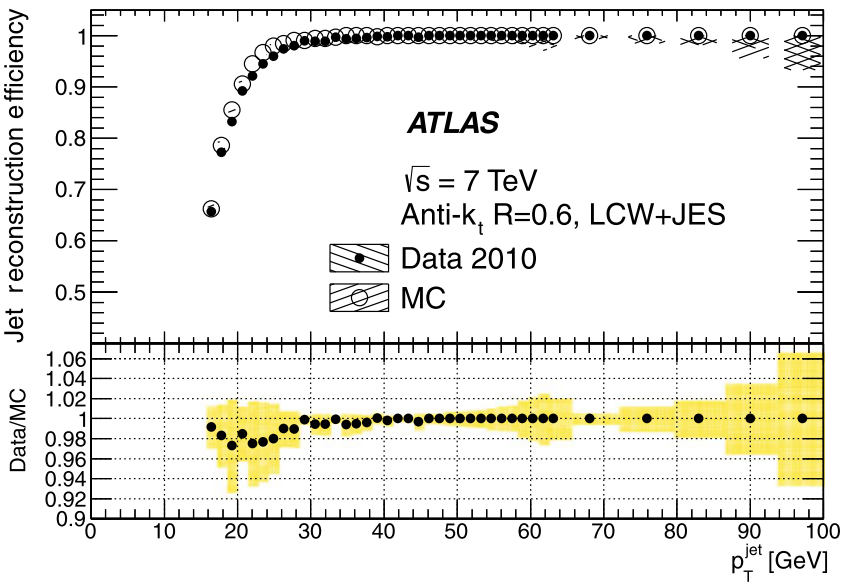

(d) Efficiency from track jets for LCW+JES

ibration schemes to that of the EM+JES calibration scheme. Only jets within $|\eta|<1.9$ are considered. The ratio of data to Monte Carlo simulation is also shown in the lower part of the figure for (b), (c) and (d). The hatched area correspond to the systematic uncertainty obtained by variations in the in situ method

\subsection{Efficiency in situ validation}

The ability of the Monte Carlo simulation to correctly reproduce the jet reconstruction in the data is tested using track jets that provide an independent reference.

A tag-and-probe technique is used as described in the following steps:

1. Only track jets with $p_{\mathrm{T}}>5 \mathrm{GeV}$ and $|\eta|<1.9$ are considered.

2. The track jet with the highest $p_{\mathrm{T}}$ in the event is defined as the reference object.

3. The reference object is required to have $p_{\mathrm{T}}>15 \mathrm{GeV} .{ }^{39}$

\footnotetext{
${ }^{39}$ Reference track jets with $p_{\mathrm{T}}<15 \mathrm{GeV}$ are not used, since they would result in a sample of biased probe track jets. In this case, mostly
} 
4. The reference track jet is matched to a calorimeter jet with $p_{\mathrm{T}}^{\text {jet }}>7 \mathrm{GeV}$, if $\Delta R$ (tag, calo jet $)<0.6{ }^{40}$

5. The probe track jet must be back-to-back to the reference jet in $\phi$ with $|\Delta \phi| \geq 2.8$ radian.

6. Events with additional track jets within $|\Delta \phi| \geq 2.8$ radian are rejected.

7. The calorimeter reconstruction efficiency with respect to track jets is then defined as the fraction of probe track jets matched to a calorimeter jet using $\Delta R$ (probe, calo jet) $<$ $R$ (with $R=0.4$ or $R=0.6$ ) with respect to all probe track jets.

The jet reconstruction efficiency is measured in a sample of minimum bias events and is compared to a minimum bias Monte Carlo simulation. Due to the restriction of $|\eta|<1.9$ on track jets, the measurement is only valid for calorimeter jets with $|\eta|<1.9+R$, where $R=0.4$ or $R=0.6$.

Figures $72 \mathrm{~b}-\mathrm{d}$ show the measured calorimeter reconstruction efficiency with respect to track jets as a function of the calorimeter jet transverse momentum for anti- $k_{t}$ jets with $R=0.6$ calibrated with the EM+JES, GCW+JES, and LCW+JES calibration schemes. ${ }^{41}$ The reconstruction efficiency reaches a plateau close to $100 \%$ at a jet transverse calorimeter momentum of about $25 \mathrm{GeV}$. The matching efficiency in data ( $\left.\varepsilon_{\text {Data }}\right)$ and in Monte Carlo simulation $\left(\varepsilon_{\mathrm{MC}}\right)$ shows a good overall agreement except at low $p_{\mathrm{T}}^{\text {jet }}$ where the efficiency in data is slightly lower than in the Monte Carlo simulation. Similar performance is found for all calibration schemes.

The systematic uncertainties on the jet reconstruction efficiency measured in situ are estimated by varying the following event selection requirements for both data and Monte Carlo simulation: the opening angle $|\Delta \phi|$ between the reference and the probe track jets, the $\Delta R$ requirement between the tag track jet and the calorimeter jet and the probe track jet and the calorimeter jet.

The sensitivity in both data and Monte Carlo simulation to the azimuthal opening angle as well as to the $\Delta R$ (tag, calo jet) variation is small. However, the efficiency shows a sensitivity with respect to the $\Delta R$ (probe jet, calo jet).

events where the probe track jet has fluctuated up in energy (such that it passes the $5 \mathrm{GeV}$ threshold) would be kept. The $15 \mathrm{GeV}$ cut has been determined by measuring the jet reconstruction efficiency relative to track jets as a function of the reference track jet $p_{\mathrm{T}}$. The measured efficiency for low probe track jet $p_{\mathrm{T}}$ was found to be dependent on the reference track jet $p_{\mathrm{T}}$ when the latter is smaller than $15 \mathrm{GeV}$. The jet reconstruction efficiency is stable for a reference track jet $p_{\mathrm{T}}$ greater than $15 \mathrm{GeV}$.

${ }^{40}$ The less restrictive matching criterion with respect to previous sections is motivated by the lower $p_{\mathrm{T}}$.

${ }^{41}$ Technically, the efficiency is first measured as a function of the track jet $p_{\mathrm{T}}$. Using the known relation between the average track jet and the average calorimeter jet $p_{\mathrm{T}}$, the track jet $p_{\mathrm{T}}$ is then converted to the calorimeter jet $p_{\mathrm{T}}^{\text {jet }}$.
The variation of $\varepsilon_{\text {Data }} / \varepsilon_{\mathrm{MC}}$ for these different parameters is shown in Fig. 72. At high $p_{\mathrm{T}}^{\text {jet }}$ the statistical uncertainties after the cut variations lead to an enlarged uncertainty band.

The systematic uncertainty of the in situ determination is larger than the observed shift between data and Monte Carlo simulation. For $p_{\mathrm{T}}^{\text {jet }}<30 \mathrm{GeV}$ a systematic uncertainty of $2 \%$ for jets is assigned.

\subsection{Summary of jet reconstruction efficiency}

The jet reconstruction efficiency is derived using the nominal inclusive jet Monte Carlo simulation sample. The systematic uncertainty is evaluated using a tag-and-probe technique using track jets in both data and Monte Carlo simulation.

The jet reconstruction efficiency is well described by the Monte Carlo simulation and is within the systematic uncertainty of the in situ method. A systematic uncertainty of $2 \%$ for jets with $p_{\mathrm{T}}^{\text {jet }}<30 \mathrm{GeV}$ is assigned and neglected for higher $p_{\mathrm{T}}^{\text {jet }}$.

\section{Response uncertainty of non-isolated jets}

The standard ATLAS jet calibration and associated JES uncertainty is obtained using only isolated jets (see Sect. 8.3). Jets are, however, often produced with nearby jets in a busy environment such as found in multijet topologies or in events where top-quark pairs are produced. Therefore a separate study is needed to determine the additional JES uncertainty for jets with nearby jet activity.

Jets with $p_{\mathrm{T}}^{\text {jet }}>20 \mathrm{GeV}$ and $|y|<2.8$ calibrated with the $\mathrm{EM}+\mathrm{JES}$ scheme are used. The close-by JES uncertainty is evaluated within $|y|<2.0$.

\subsection{Evaluation of close-by jet effects}

The effect due to close-by jets is evaluated in the Monte Carlo simulation by using truth jets as a reference. Similarly, track jets are used as a reference in both data and Monte Carlo simulation (see Sects. 6.2 and 6.3 for comparison). The calorimeter jet response relative to these reference jets is examined for different values of $R_{\min }$, the distance from the calorimeter jet to the closest jet in $\eta-\phi$ space.

The relative calorimeter jet response to the truth jets provides an absolute $p_{\mathrm{T}}$ scale for the calorimeter jets, while the relative response to the track jets allows in situ validation of the calorimeter jet response and the evaluation of the systematic uncertainty. For this purpose, the track jet response in data needs to be established for the non-isolated case and the associated systematic uncertainty has to be understood.

In the relative response measurement in the Monte Carlo simulation, the truth jet is matched to the calorimeter jet or 


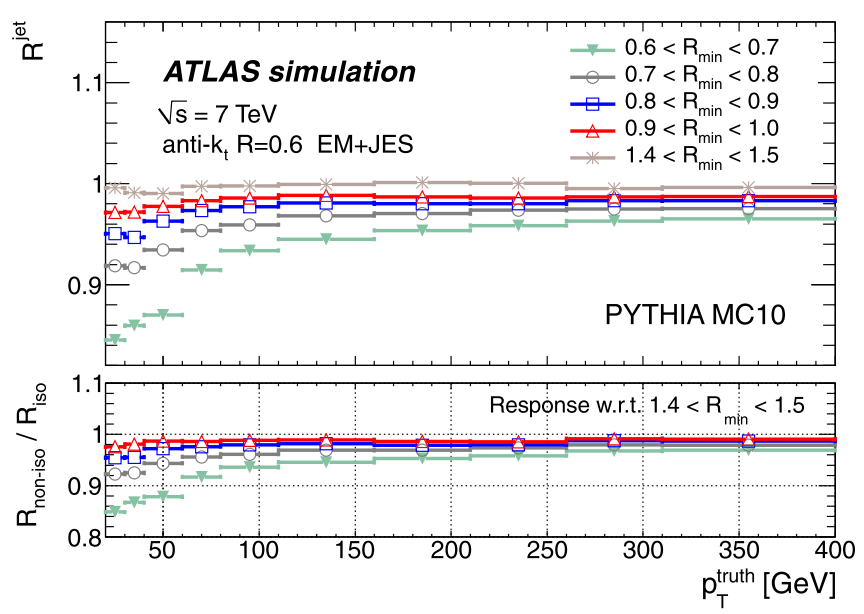

(a) Calorimeter jet

Fig. 73 Average ratio of calorimeter jet (a) and the track jet (b) $p_{\mathrm{T}}$ to the matched truth jet $p_{\mathrm{T}}$ as a function of truth jet $p_{\mathrm{T}}$ for anti- $k_{t}$ jets with $R=0.6$, for different $R_{\min }$ values. The bottom part of the figure shows

track jet in $\eta-\phi$ space by requiring $\Delta R<0.3$. Similarly, the track jet is matched to the calorimeter jet within $\Delta R<0.3$ when the relative response to the track jet is examined. If two or more jets are matched within the $\Delta R$ range, the closest matched jet is taken.

The calorimeter response to the matched track jet is defined as the ratio of the calorimeter jet $p_{\mathrm{T}}^{\mathrm{jet}}$ to the track jet transverse momentum $\left(p_{\mathrm{T}}^{\text {track jet }}\right)$

$r^{\text {calo/track jet }}=p_{\mathrm{T}}^{\text {jet }} / p_{\mathrm{T}}^{\text {track jet }}$.

This response is examined as a function of the jet transverse momentum $p_{\mathrm{T}}^{\text {jet }}$ and for different $R_{\min }$ values measured relative to the closest calorimeter jet with $p_{\mathrm{T}}>7 \mathrm{GeV}$ at the EM energy scale. ${ }^{42}$ The ratio of the calorimeter jet response for non-isolated (i.e. small $R_{\min }$ ) to the response of isolated (large $R_{\min }$ ) jets, is given by

$r_{\text {non-iso/iso }}^{\text {calo/track jet }}=r_{\text {non-iso }}^{\text {calo/track jet }} / r_{\text {iso }}^{\text {calo/track jet }}$.

This ratio is compared between data and Monte Carlo simulations.

$A_{\text {close-by }}=\left[r_{\text {non-iso } / \text { iso }}^{\text {calo } / \text { track jet }}\right]_{\text {Data }} /\left[r_{\text {non-iso } / \text { iso }}^{\text {calo } / \text { track jet }}\right]_{\mathrm{MC}}$.

The deviation of $A_{\text {close-by }}$ is assumed to represent the component of calorimeter JES uncertainty due to close-by jets. This uncertainty, convolved with the systematic uncertainty in the track jet response due to a nearby jet, provides the total JES systematic uncertainty due to the close-by jet effect.

${ }^{42}$ Unless otherwise stated, calorimeter jets (selected as listed below) and nearby jets (selected with $p_{\mathrm{T}}>7 \mathrm{GeV}$ at the EM scale) are both used in the jet response measurement, if a matched track jet is found.

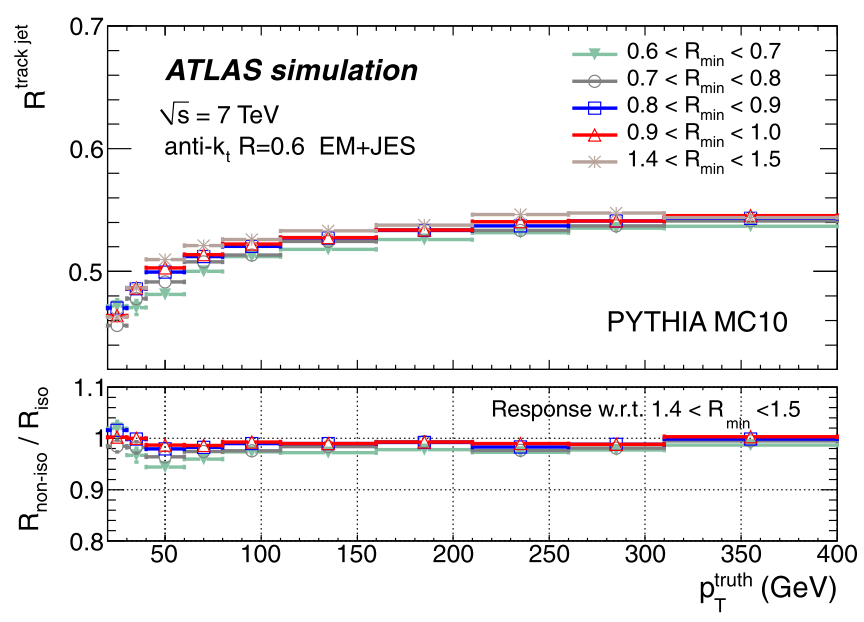

(b) Track jet

the relative response of non-isolated jets with respect to that of isolated jets, obtained as the calorimeter or track jet response for $R_{\min }<1.0$ divided by the jet response for $1.4 \leq R_{\min }<1.5$

\subsection{Non-isolated jet response}

Events that contain at least two jets with $p_{\mathrm{T}}^{\text {jet }}>20 \mathrm{GeV}$ and absolute rapidity $|y|<2.8$ are selected. The response of non-isolated jets is studied in the Monte Carlo simulation using the calorimeter jet response $\mathcal{R}^{\mathrm{jet}}=p_{\mathrm{T}}^{\text {jet }} / p_{\mathrm{T}}^{\text {truth }}$.

Figure 73a shows the calorimeter jet response as a function of $p_{\mathrm{T}}^{\text {truth }}$ for anti- $k_{t}$ jets with $R=0.6$. The jet response was measured for nearby jets in bins of $R_{\min }$ values. The lower part of the figure shows the ratio of the nonisolated jet response for $R_{\min }<1.0$ to the isolated response $1.4 \leq R_{\min }<1.5$,

$\mathcal{R}_{\text {non-iso }}^{\text {jet }} / \mathcal{R}_{\text {iso }}^{\text {jet }}$

The observed behaviour at small $R_{\min }$ values indicates that the non-isolated jet response is lower by up to $15 \%$ relative to the isolated jet response for $p_{\mathrm{T}}^{\text {jet }}>20 \mathrm{GeV}$, if the two jets are close-by. The magnitude of this effect depends on $p_{\mathrm{T}}^{\text {jet }}$ and is largest at low $p_{\mathrm{T}}^{\text {jet }}$.

The track jet response relative to the matched truth jet is defined as

$\mathcal{R}^{\text {track jet }}=p_{\mathrm{T}}^{\text {track jet }} / p_{\mathrm{T}}^{\text {truth }}$.

Figure 73b shows $\mathcal{R}^{\text {track jet }}$ as a function of $p_{\mathrm{T}}^{\text {truth }}$ for anti$k_{t}$ jets with $R=0.6$. The track jet response is more stable against the presence of close-by jets and has a much weaker $R_{\min }$ dependence than the calorimeter jet response. This results from the smaller ambiguity in the matching between the truth and track jets that are both measured from the primary interaction point. Moreover, track jets are less influenced by magnetic field effects than calorimeter jets. 
17.3 Non-isolated jet energy scale uncertainty

The $p_{\mathrm{T}}$ ratio of non-isolated to isolated track jets is defined as:

$r_{\text {non-iso } / \text { iso }}^{\text {track jet }}=p_{\mathrm{T}, \text { non-iso }}^{\text {track jet }} / p_{\mathrm{T}, \text { iso }}^{\text {track jet }}$.

The data to $\mathrm{MC}$ ratio defined as:

$A_{\text {close-by }}^{\text {track jet }}=\left[r_{\text {non-iso } / \text { iso }}^{\text {track jet }}\right]_{\text {Data }} /\left[r_{\text {non-iso } / \text { iso }}^{\text {track jet }}\right]_{\mathrm{MC}}$

is compared between data and Monte Carlo simulations in Fig. 74. This ratio can be used to assess the potential of track jets to test close-by effects in the small $R_{\text {min }}$ range.

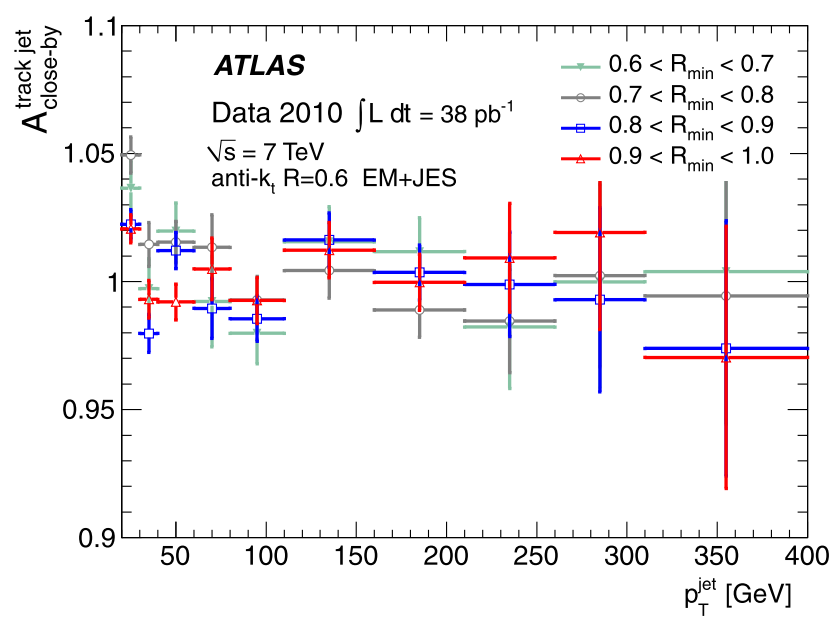

Fig. 74 Ratio of data to Monte Carlo simulation of the track jet $p_{\mathrm{T}}^{\text {track jet }}$ for non-isolated jets divided by the track jet $p_{\mathrm{T}}^{\text {track jet }}$ for isolated jets as a function of the jet $p_{\mathrm{T}}^{\text {jet }}$. Only statistical uncertainties are shown

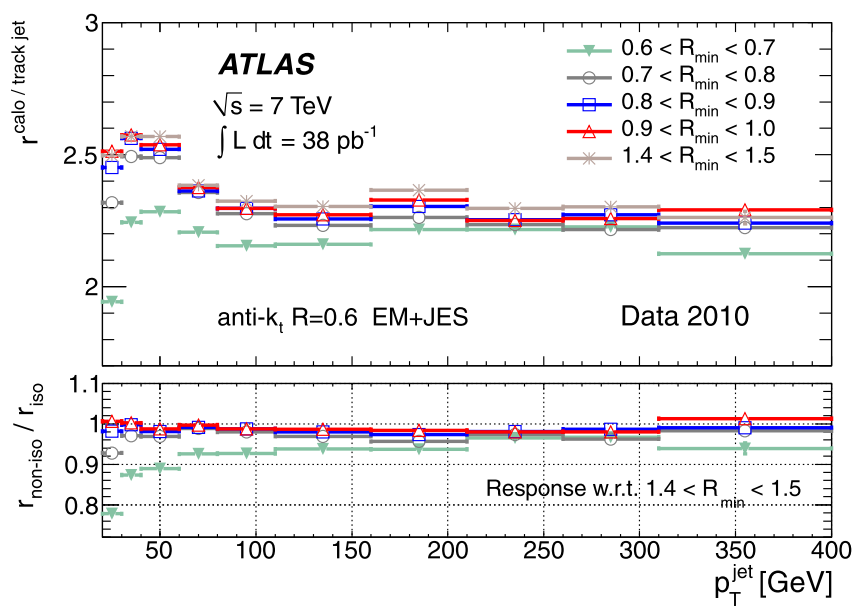

(a) Data

Fig. 75 Ratio of calorimeter jet $p_{\mathrm{T}}^{\text {jet }}$ to the matched track jet $p_{\mathrm{T}}$ as a function of calorimeter jet $p_{\mathrm{T}}^{\text {jet }}$ for anti- $k_{t}$ jets with $R=0.6$ in data (a) and Monte Carlo simulations (b) for different $R_{\min }$ values. The lower
The agreement between data and Monte Carlo simulation is quite satisfactory: within 2 to $3 \%$ for $p_{\mathrm{T}}^{\text {jet }}>30 \mathrm{GeV}$ and slightly worse for $20 \leq p_{\mathrm{T}}^{\text {jet }}<30 \mathrm{GeV}$.

Therefore, the track jet response systematic uncertainty is assigned separately for the two $p_{\mathrm{T}}^{\text {jet }}$ regions: $\left|1-A_{\text {close-by }}^{\text {track jet }}\right|$ is used as the uncertainty for $20 \leq p_{\mathrm{T}}^{\text {jet }}<30 \mathrm{GeV}$, while for $p_{\mathrm{T}}^{\text {jet }}>30 \mathrm{GeV}$ a standard deviation (RMS) of the $A_{\text {close-by }}^{\text {track jet }}$ calculated and assigned as the uncertainty. These uncertainties are typically $1.5 \%(2.0 \%)$ for anti- $k_{t}$ jets with $R=0.6$ $(0.4)$.

The calorimeter jet $p_{\mathrm{T}}^{\text {jet }}$ relative to the matched track jet $p_{\mathrm{T}}^{\text {track jet }}\left(r^{\text {calo/track jet }}\right)$ is shown in Fig. 75 as a function of $p_{\mathrm{T}}^{\text {jet }}$ for anti- $k_{t}$ jets with $R=0.6$ in data and Monte Carlo simulations. The non-isolated jet response relative to the isolated jet response, $r$ non-iso/iso , shown in the bottom part of Fig. 75 reproduces within a few per cent the behaviour in the ratio $\mathcal{R}_{\text {non-iso }}^{\text {jet }} / \mathcal{R}_{\text {iso }}^{\text {jet }}$ for the Monte Carlo simulation response of calorimeter to truth jet $p_{\mathrm{T}}$ in Fig. 73 .

The $r_{\text {non-iso/iso }}^{\text {calotrack jet }}$ data to Monte Carlo ratio $A_{\text {close-by }}$ (see Eq. (52)) is shown in Fig. 76. The $R_{\text {min }}$ dependence of the non-isolated jet response in the data is well described by the Monte Carlo simulation.

Within the statistical uncertainty, $A_{\text {close-by }}$ differs from unity by at most $\sim 3 \%$ depending on the $R_{\text {min }}$ value. No significant $p_{\mathrm{T}}^{\text {jet }}$ dependence is found over the measured $p_{\mathrm{T}}$ range of $20 \leq p_{\mathrm{T}}^{\text {jet }}<400 \mathrm{GeV}$.

The overall JES uncertainty due to nearby jets is taken as the track jet response systematic uncertainty added in quadrature with the deviation from one of the weighted average of $A_{\text {close-by }}$ over the entire $p_{\mathrm{T}}$ range, but added separately for each $R_{\min }$ range. The final uncertainties are sum-

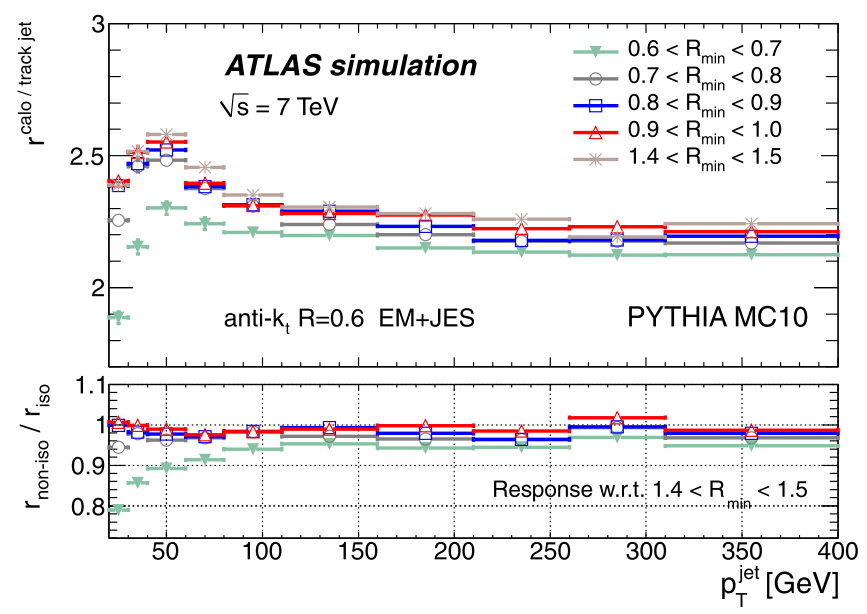

(b) Monte Carlo simulation

part shows the relative response of non-isolated jets with respect to that of isolated jets, obtained as the jet response for $R_{\min }<1.0$ divided by the jet response for $1.4 \leq R_{\min }<1.5$ 


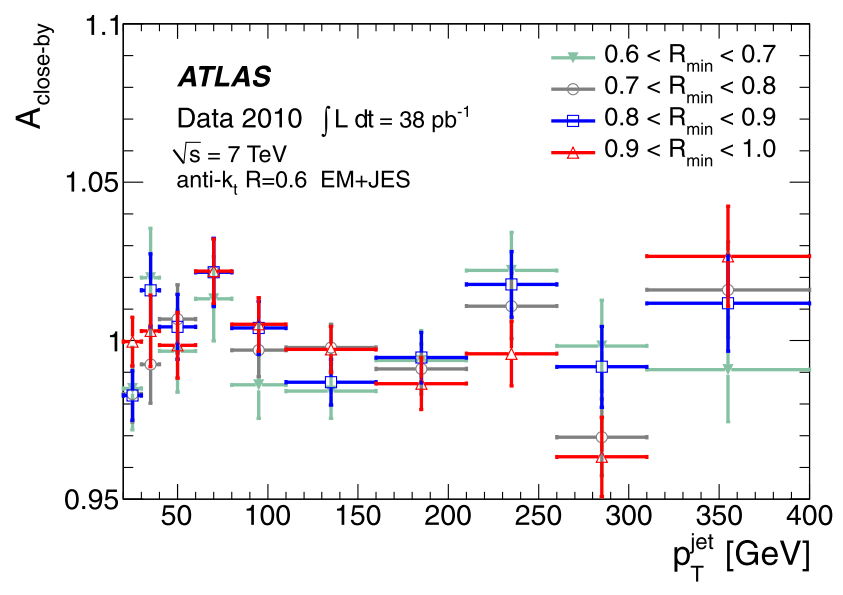

Fig. 76 Data to Monte Carlo simulation ratio of the relative response of non-isolated jets with respect to that of isolated jets for anti- $k_{t}$ jets with $R=0.6$ calibrated with the EM+JES scheme. Only statistical uncertainties are shown

Table 15 Summary of jet energy scale systematic uncertainty assigned for non-isolated jets accompanied by a close-by jet within the denoted $R_{\text {min }}$ ranges. The second row in the table indicates the $p_{\mathrm{T}}$ range of the non-isolated jets. Anti- $k_{t}$ jets with $R=0.6$ and $R=0.4$ are used

\begin{tabular}{llllll}
\hline$p_{\mathrm{T}}^{\text {jet }}[\mathrm{GeV}]$ & \multicolumn{2}{l}{$R=0.6$} & & \multicolumn{2}{l}{$R=0.4$} \\
\cline { 2 - 3 } & $20-30$ & $>30$ & & $20-30$ & $>30$ \\
\hline $0.4 \leq R_{\min }<0.5$ & - & - & $2.7 \%$ & $2.8 \%$ \\
$0.5 \leq R_{\min }<0.6$ & - & - & $1.7 \%$ & $2.3 \%$ \\
$0.6 \leq R_{\min }<0.7$ & $3.9 \%$ & $1.9 \%$ & $2.5 \%$ & $2.7 \%$ \\
$0.7 \leq R_{\min }<0.8$ & $5.1 \%$ & $1.6 \%$ & - & \\
$0.8 \leq R_{\min }<0.9$ & $2.5 \%$ & $1.9 \%$ & - & \\
\hline
\end{tabular}

marised in Table 15 for the two jet distance parameters. The uncertainties are subject to statistical fluctuations.

The $A_{\text {close-by }}$ ratio has been examined for each of the two close-by jets either with the lower or the higher $p_{\mathrm{T}}^{\mathrm{jet}}$, and no apparent difference is observed with respect to the inclusive case shown in Fig. 76. Therefore, both calorimeter jets which are close to each other are subject to this uncertainty.

\subsection{Summary of close-by jet uncertainty}

The uncertainty is estimated by comparing in data and Monte Carlo simulation the track jet response. They are both examined as function of the distance $R_{\min }$ between the jet and the closest jet in the calorimeter.

The close-by jet systematic uncertainty on the jet energy scale is $2.5-5.1 \%(1.7-2.7 \%)$ and $1.6-1.9 \%(2.3-2.8 \%)$ for $R=0.6(R=0.4)$ jets with $20 \leq p_{\mathrm{T}}^{\text {jet }}<30 \mathrm{GeV}$ and $p_{\mathrm{T}}^{\mathrm{jet}}>30 \mathrm{GeV}$, respectively, in the range of $R \leq R_{\min }<$ $R+0.3$ and jet rapidity $|y|<2.0$. When the two jets are separated in distance by $R+0.3$ or more, the jet response becomes similar to that for the isolated jets and hence no additional systematic uncertainty is required. No significant jet $p_{\mathrm{T}}$ dependence is observed at $p_{\mathrm{T}}^{\mathrm{jet}}>30 \mathrm{GeV}$ for the closeby jet systematic uncertainty.

\section{Light quark and gluon jet response and sample characterisation}

In the previous sections the JES uncertainty for inclusive jets was determined. However, details of the jet fragmentation and showering properties can influence the jet response measurement. In this section the JES uncertainties due to jet fragmentation which is correlated to the flavour of the parton initiating that jet (e.g. see Ref. [103]) are investigated. An additional term in the JES uncertainty is derived for event samples that have a different flavour content than the nominal Monte Carlo simulation sample.

The jet energy scale systematic uncertainty due to the difference in response between gluon and light quark initiated jets (henceforth gluon jets and light quark jets) can be reduced by measuring the flavour composition of a sample of jets using template fits to certain jet properties that are sensitive to changes in fragmentation. Although these jet properties may not have sufficient discrimination power to determine the partonic origin of a specific jet, it is possible to determine the average flavour composition of a sufficiently large sample of jets. The average flavour compositions can be determined using jet property templates built in the Monte Carlo simulation for pure samples.

Templates are constructed in dijet events, which are expected to comprise mostly gluon jets at low transverse momentum and central rapidities. They are then applied to events with a high- $p_{\text {T }}$ photon balancing a high- $p_{\mathrm{T}}$ jet $(\gamma$-jet events), which are expected to comprise mostly light quark jets balancing the photon. The application of this technique is further demonstrated with a sample of multijet events, wherein the jets are initiated mostly by gluons from radiation.

\subsection{Data samples for flavour dependence studies}

Two data samples in addition to the inclusive jet sample discussed before are used for the studies of the flavour dependence of the jet response.

1. $\gamma$-jet sample Photons with $p_{\mathrm{T}}>45 \mathrm{GeV}$ are selected in the barrel calorimeter (with pseudorapidity $|\eta|<1.37$ ) and a jet back-to-back ( $\Delta \phi>\pi-0.2$ radians) to the photon is required. The second-leading jet in the event is required to have a $p_{\mathrm{T}}^{\text {jet }}$ below $10 \%$ of the $p_{\mathrm{T}}^{\text {jet }}$ of the leading jet. Anti- $k_{t}$ jets with $R=0.6$ are used.

2. Multijet sample Jets with $p_{\mathrm{T}}^{\text {jet }}>60 \mathrm{GeV}$ and $|\eta|<2.8$ are selected and the number of selected jets defines the sample of at least two, three or four jets. 
18.2 Flavour dependence of the calorimeter response

Jets identified in the Monte Carlo simulation as light quark jets have significantly different response from those identified as gluon jets (see Sect. 6.3 for the definition of the jet flavour).

The flavour-dependence of the jet response is a result of the differences in particle level properties of the two types of jets. For a given jet $p_{\mathrm{T}}$ jets identified as gluon jets tend to have more particles, and those particles tend to be softer than in the case of light quark jets. Additionally, the gluon jets tend to be wider (i.e. with lower energy density in the core of the jet) before interacting with the detector. The magnetic field in the inner detector amplifies the broadness of gluon jets, since their low- $p_{\mathrm{T}}$ charged particles tend to bend more than the higher $p_{\mathrm{T}}$ particles in light quark jets. The harder particles in light quark jets additionally tend to penetrate further into the calorimeter.

The difference in calorimeter response between gluon jets and light quark jets in the Monte Carlo simulation is shown in Fig. 77. Jets in the barrel $(|\eta|<0.8)$ and in the endcap $(2.1 \leq|\eta|<2.8)$ calorimeters are shown separately. For jets calibrated with the EM+JES scheme light quark jets have a 5-6\% higher response than gluon jets at low $p_{\mathrm{T}}^{\text {jet }}$. This difference decreases to about $2 \%$ at high $p_{\mathrm{T}}^{\text {jet }}$.

Since response differences are correlated with differences in the jet properties, more complex jet calibration schemes that are able to account for jet shower properties variations can partially compensate for the flavour dependence. At low $p_{\mathrm{T}}^{\text {jet }}$ the difference in response between light quark jets

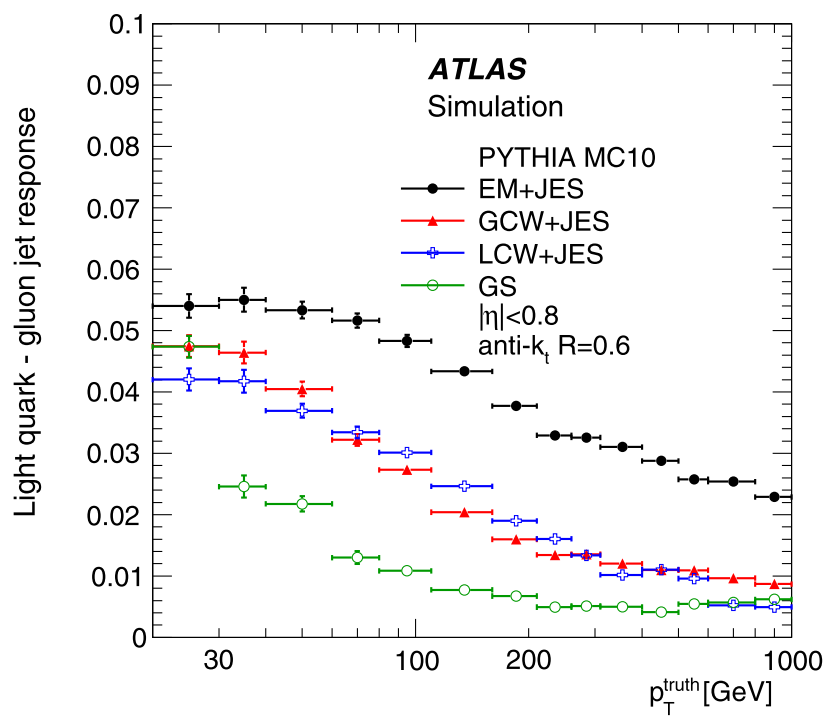

(a) $|\eta|<0.8$

Fig. 77 Difference in average response of gluon and light quark jets as a function of the truth jet $p_{\mathrm{T}}$ for anti- $k_{t}$ jets with $R=0.6$ in the barrel (a) and the endcap (b) calorimeters as determined in Monte Carlo and gluon jets is reduced to 4-5\% for the LCW+JES and GCW+JES schemes and about $3 \%$ for the GS scheme. For $p_{\mathrm{T}}^{\text {jet }}>300 \mathrm{GeV}$ the flavour dependence of the jet response is about $1 \%$ for the LCW+JES and GCW+JES and the GS schemes.

The closer two jets are to one another, the more ambiguous the flavour assignment becomes. The flavour assignment can become particularly problematic when one truth jet is matched to two reconstructed calorimeter jets ("splitting") or two truth jets are matched to one reconstructed calorimeter jet ("merging"). Several different classes of close-by jets are examined for changes in the flavour dependence of the jet response. No significant deviation from the one of isolated jets is found. Therefore, the effects can be treated separately. The jet energy scale uncertainty specific to close-by jets is examined in Sect. 17.

\subsection{Systematic uncertainties due to flavour dependence}

Each jet energy calibration scheme restores the average jet energy to better than $2 \%$ with small uncertainties in a sample of inclusive jets. However, subsamples of jets are not perfectly calibrated, as in the case of light quark jets and gluon jets. The divergence from unity is flavour dependent and may be different in Monte Carlo simulation and data, particularly if the flavour content in the data sample is not well-described by the Monte Carlo simulation. This results in an additional term in the systematic uncertainty for any study using an event or jet selection different from that of the sample in which the jet energy scale was derived.

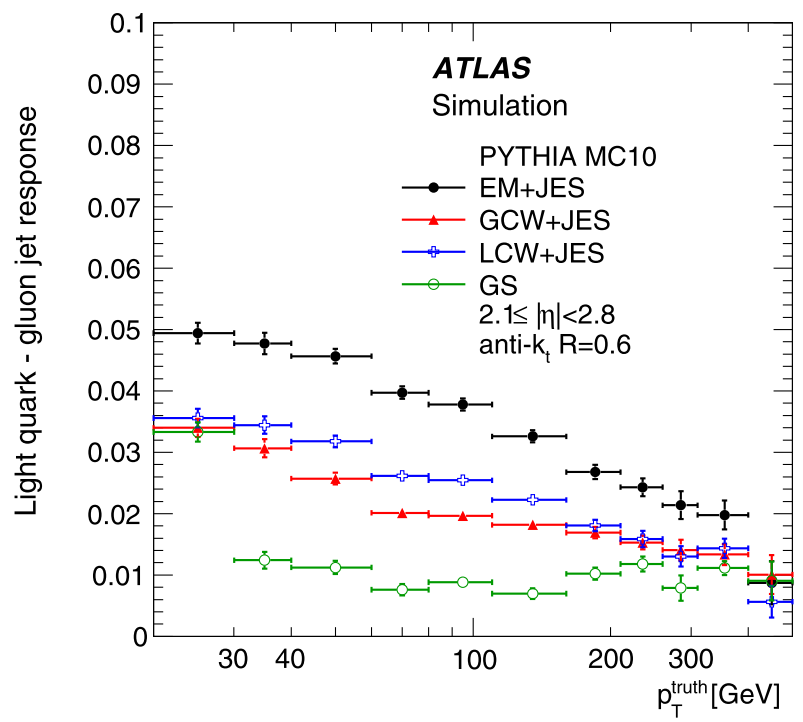

(b) $2.1 \leq|\eta|<2.8$

simulation. Various calibration schemes are shown. The data sample used contains at least two jets with $p_{\mathrm{T}}^{\text {jet }}>60 \mathrm{GeV}$ and $|\eta|<2.8$. Only statistical uncertainties are shown 


\subsubsection{Systematic uncertainty from MC variations}

In order to test the response uncertainties of exclusive samples of either gluon or light quark jets, a large number of systematic variations in the Monte Carlo simulation are investigated (see Ref. [1] for details on the variations). The response difference of quark and gluon jets to that of the inclusive jets is found to be very similar for each of the systematic Monte Carlo variations. Therefore the additional uncertainty on the response of gluon jets is neglected.

These conclusions are in good agreement with the studies which derive the calorimeter jet response using the single hadron response in Ref. [28], where the uncertainties of the calorimeter response to jets initiated by quarks or gluons are similar within $0.5 \%$.

The results are found to be stable under variations of the Monte Carlo simulation samples including soft physics effects like colour reconnections. With more data, a variety of final states may be tested to investigate more details of the light quark and gluon jet response.

\subsubsection{Systematic uncertainty from average flavour content}

The flavour dependent uncertainty term depends on both the average flavour content of the sample and on how well the flavour content is known, e.g. the uncertainty for a generic new physics search with an unknown jet flavour composition is different from the uncertainty on a new physics model in which only light quark jets are produced. The response for any sample of jets, $\mathcal{R}_{s}$, can be written as: ${ }^{43}$

$$
\begin{aligned}
\mathcal{R}_{s}= & f_{g} \times \mathcal{R}_{g}+f_{q} \times \mathcal{R}_{q}+f_{b} \times \mathcal{R}_{b}+f_{c} \times \mathcal{R}_{c} \\
= & 1+f_{g} \times\left(\mathcal{R}_{g}-1\right)+f_{q} \times\left(\mathcal{R}_{q}-1\right) \\
& +f_{b} \times\left(\mathcal{R}_{b}-1\right)+f_{c} \times\left(\mathcal{R}_{c}-1\right),
\end{aligned}
$$

where $R_{x}$ is the detector response to jets and $f_{x}$ is the fraction of jets for $x=g$ (gluon jets), $q$ (light quark jets), $b$ ( $b$ quark jets), and $c$ ( $c$-quark jets) and $f_{g}+f_{q}+f_{b}+f_{c}=1$. For simplicity, the fraction of heavy quark jets is taken to be known. This approximation will be dealt with in the systematic uncertainty analysis for heavy quarks in Sect. 18.4.

Since variations in the flavour fractions and the jet flavour response translate into variations of the jet response for a given sample, the uncertainty on the jet response can be approximately expressed as:

$$
\begin{aligned}
\Delta \mathcal{R}_{s}= & \Delta f_{g} \times\left(\mathcal{R}_{g}-1\right)+\Delta f_{q} \times\left(\mathcal{R}_{q}-1\right) \\
& +f_{g} \times \Delta \mathcal{R}_{g}+f_{q} \times \Delta \mathcal{R}_{q}+f_{b} \times \Delta \mathcal{R}_{b} \\
& +f_{c} \times \Delta \mathcal{R}_{c},
\end{aligned}
$$

\footnotetext{
${ }^{43}$ The following equations are strictly speaking only valid for a given bin in $p_{\mathrm{T}}$ and $\eta$ or in other variables that influence the flavour composition.
}

where $\Delta$ denotes the uncertainty on the individual variables. Since $f_{b}$ and $f_{c}$ are fixed here (i.e. without uncertainty), $\Delta f_{g}=-\Delta f_{q}$. Also, the uncertainties on the response for the exclusive flavour samples (light quark, gluon, $b$, and $c$ quarks) are approximately the same as the inclusive jet response uncertainty $\left(\Delta \mathcal{R}_{j}\right)$. The $b$-jet uncertainty is discussed in Sect. 20 and is neglected here.

The expression can therefore be simplified:

$$
\begin{aligned}
\Delta \mathcal{R}_{s} \approx & -\Delta f_{q} \times\left(\mathcal{R}_{g}-1\right)+\Delta f_{q} \times\left(\mathcal{R}_{q}-1\right) \\
& +f_{g} \times \Delta \mathcal{R}_{j}+f_{q} \times \Delta \mathcal{R}_{j}+f_{b} \times \Delta \mathcal{R}_{j} \\
& \quad+f_{c} \times \Delta \mathcal{R}_{j} \\
= & \Delta f_{q} \times\left(\mathcal{R}_{q}-\mathcal{R}_{g}\right)+\left(f_{g}+f_{q}+f_{b}+f_{c}\right) \times \Delta \mathcal{R}_{j} \\
\approx & \Delta f_{q} \times\left(\mathcal{R}_{q}-\mathcal{R}_{g}\right)+\Delta \mathcal{R}_{j} .
\end{aligned}
$$

The second term is the inclusive jet energy scale systematic uncertainty, and the first term is the additional flavour dependent contribution.

Focusing on the flavor-dependent terms of the jet energy scale uncertainty and rewriting Eq. (59) as a fractional uncertainty, the flavour dependent contribution becomes:

$$
\frac{\Delta \mathcal{R}_{s}}{\mathcal{R}_{s}}=\Delta f_{q} \times\left(\frac{\mathcal{R}_{q}-\mathcal{R}_{g}}{\mathcal{R}_{s}}\right) .
$$

The uncertainty on the flavour content $\left(\Delta f_{q}\right)$ and the inclusive response of the sample $\left(\mathcal{R}_{S}\right)$ depends on the specific analysis. The difference in response between light quark and gluon jets depends on the calibration used, as discussed in Sect. 18.2.

\subsection{Average jet flavour determination}

One way of investigating the flavour composition of a sample is to use different MC generators that cover a reasonable range of flavour compositions. However, these different samples may suffer from under- or overcoverage of the uncertainty or from changes in other sample characteristics, e.g. jet $p_{\mathrm{T}}$ spectra, which may result in a poor estimate of the true uncertainty. Another approach, pursued in this section, is to estimate the flavour composition of the samples by using experimental observables that are sensitive to different jet flavours.

As described in Sect. 18.2, gluon jets tend to have a wider transverse profile and have more particles than light quark jets with the same $p_{\mathrm{T}}$. The jet width, as defined in Eq. (38), and the number of tracks associated to the jet $\left(n_{\text {trk }}\right)$ are thus expected to be sensitive to the difference between light quark jets and gluon jets. The jet width may have contributions from pile-up interactions. In the following discussion 


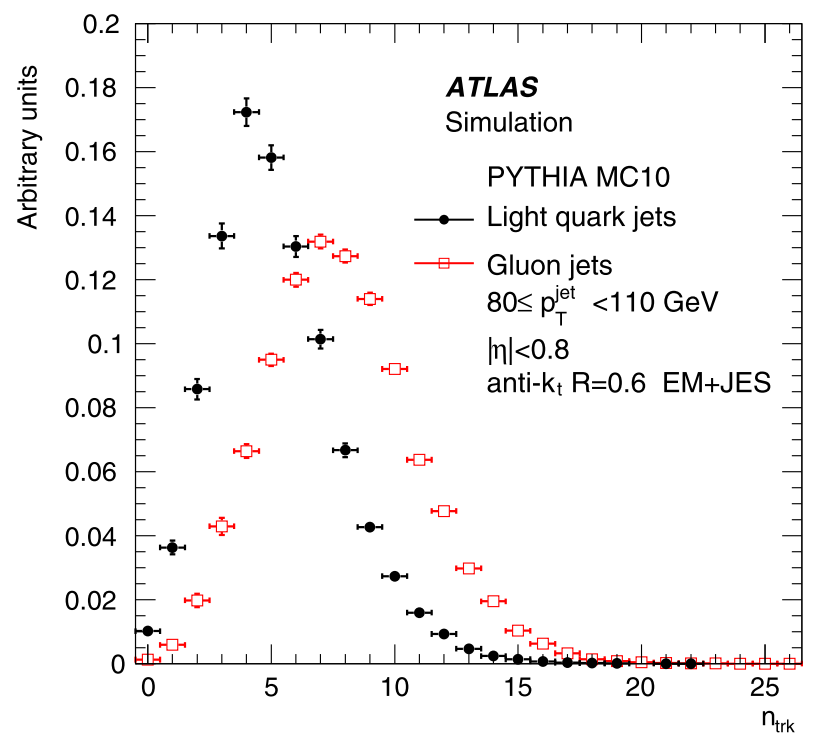

(a) Number of tracks $n_{\text {trk }}$

Fig. 78 Distribution of the number of tracks associated to the jet $n_{\text {trk }}$ (a) and the jet width (b) for isolated anti- $k_{t}$ jets with $R=0.6$ classified as light quark jets (solid circles) and gluon jets (open squares) in the

only events with exactly one reconstructed primary vertex enter the jet width distributions. ${ }^{44}$

The number of tracks associated to a jet is defined by counting the tracks with $p_{\mathrm{T}}>1 \mathrm{GeV}$ coming from the primary hard scattering vertex with an opening angle between the jet and the track momentum direction $\Delta R<0.6$. Figure 78 shows the jet width and $n_{\text {trk }}$ distributions for isolated light quark and gluon jets with $|\eta|<0.8$ and $80 \leq p_{\mathrm{T}}<$ $110 \mathrm{GeV}$ in the inclusive jet Monte Carlo simulation sample. The gluon jets are broader and have more tracks than light quark jets. For this study anti- $k_{t}$ jets with $R=0.6$ calibrated with the EM+JES scheme are used.

Templates are built from the inclusive jet Monte Carlo sample for the jet width and $n_{\text {trk }}$ of light quark and gluon jets separately, ${ }^{45}$ using the flavour tagging algorithm of Sect. 6.3. The templates are constructed in bins of $p_{\mathrm{T}}^{\text {jet }}, \eta$, and isolation ( $\Delta R$ to the nearest jet, $R_{\min }$ ). Fits to the data are performed with these templates to extract the flavour composition.

Comparisons of the inclusive jet width and $n_{\text {trk }}$ distributions in Monte Carlo simulation and data are shown in Fig. 79 for isolated jets with $R=0.6$. The jet width in Monte Carlo simulation is somewhat narrower than in the data for

\footnotetext{
${ }^{44}$ Techniques to correct for these additional interactions are being developed and are discussed in Sect. 9.7.4.

${ }^{45}$ The $n_{\text {trk }}$ and jet width templates are dealt with independently, and the results of their estimates of flavour fraction are not combined.
}

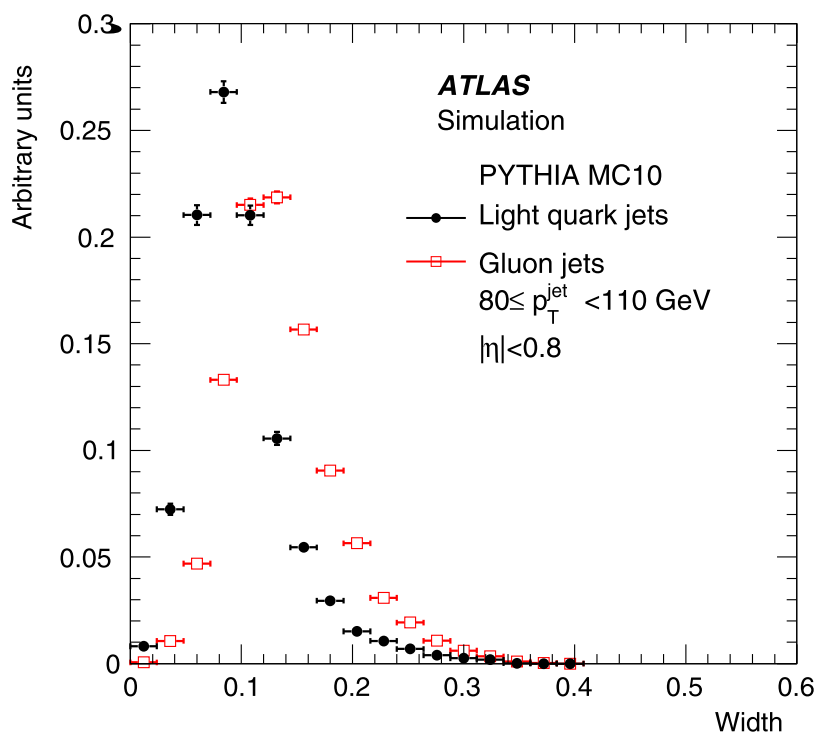

(b) Jet width

Monte Carlo simulation. Jets with $|\eta|<0.8$ and $80 \leq p_{\mathrm{T}}^{\text {jet }}<110 \mathrm{GeV}$ are shown. The distributions are normalised to unit area. Uncertainties are statistical only

the Pythia samples, in agreement with other ATLAS analyses [3].

The inclusive $n_{\text {trk }}$ and jet width Monte Carlo simulation distributions are reweighted bin-by-bin according to the data distribution. This accounts for the differences observed between the data and Monte Carlo simulation. By this reweighting of the inclusive $n_{\text {trk }}$ and jet width distributions, all the different flavours are reweighted in the same way, and the average flavour content of a sample does not change. The reweighted $n_{\text {trk }}$ and jet width distributions for the various Monte Carlo simulation samples are shown in Fig. 80.

After reweighting, the flavour composition of the dijet sample extracted from the data is consistent with that of the Monte Carlo simulation. The extracted values for two representative jet bins are shown in Table 16. This result provides an important validation of the templates.

18.5 Systematic uncertainties of average flavour composition

Uncertainties on the MC-based templates used in fits to the data result in a systematic uncertainty on the extracted flavour composition. Systematic effects from the Monte Carlo modelling of the jet fragmentation, the jet energy scale and resolution as well as the flavour composition of the sample used to extract the templates are discussed in the following. Since there is no single dominant uncertainty, each is individually considered for the extraction of the flavour composition of a sample of jets. 


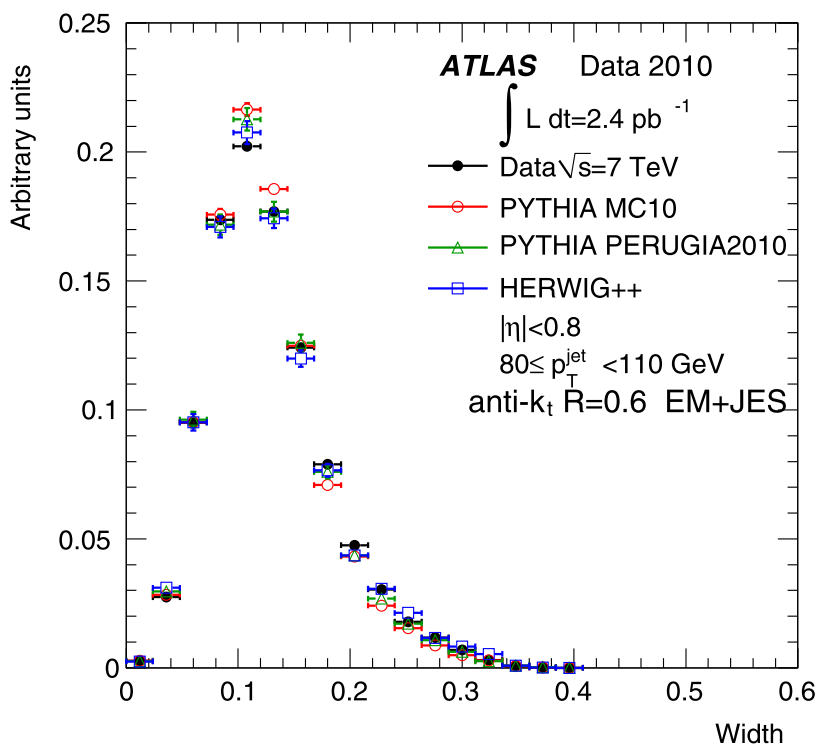

(a) Number of tracks $n_{\text {trk }}$

Fig. 79 Distribution of the number of tracks associated to the jet, $n_{\text {trk }}$ (a) and the jet width (b) for isolated anti- $k_{t}$ jets with $R=0.6$ in data (solid circles) and Monte Carlo simulation. The PythiA MC10 tune (open circles) and PERUGIA2010 tune (open triangles), and HER-

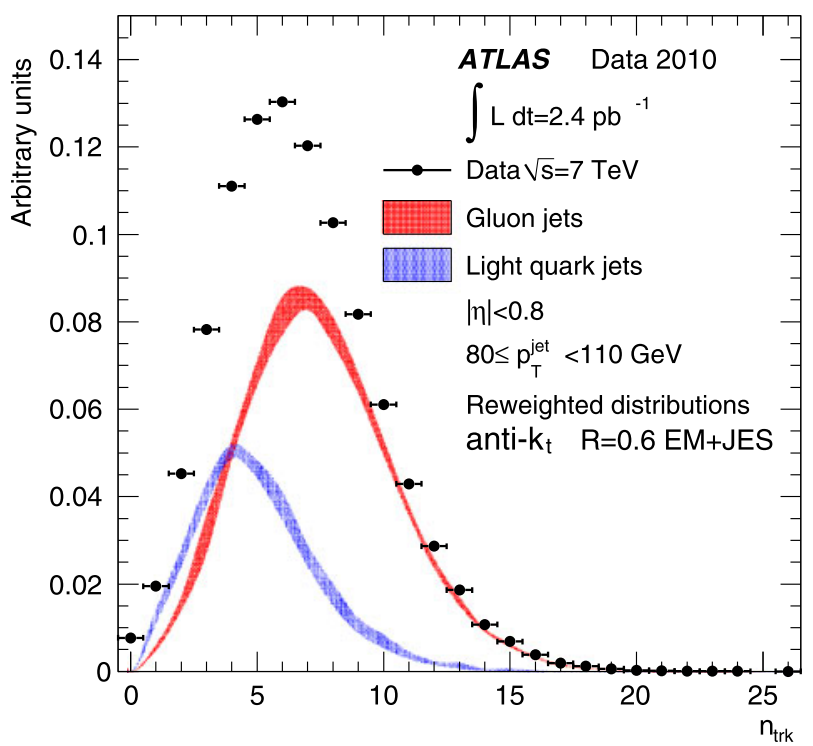

(a) Number of tracks $n_{\text {trk }}$

Fig. 80 Distribution of the number of tracks associated to the jet, $n_{\text {trk }}$ (a) and the jet width (b) for isolated anti- $k_{t}$ jets with $R=0.6$ in data (closed circles) and Monte Carlo simulation (bands). The width of the band represents the maximum variation among the PYTHIA MC10 and PERUgia2010 tunes and the Herwig++ Monte Carlo simulation sam-

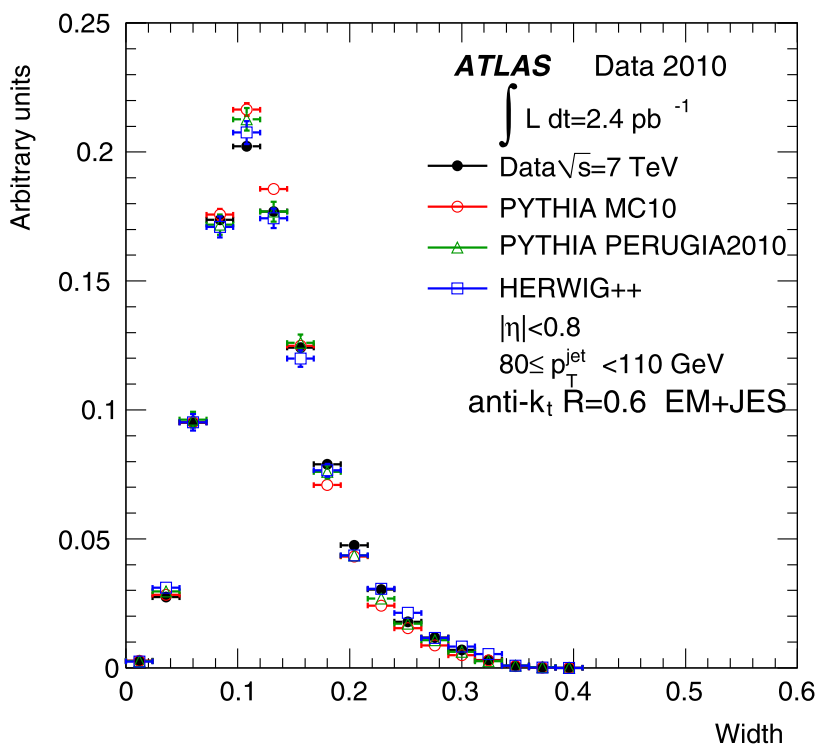

(b) Jet width

WIG++ (open squares) distributions are shown for jets with $|\eta|<0.8$ and $80 \leq p_{\mathrm{T}}^{\text {jet }}<110 \mathrm{GeV}$. The distributions are all normalised to unity. Uncertainties are statistical only

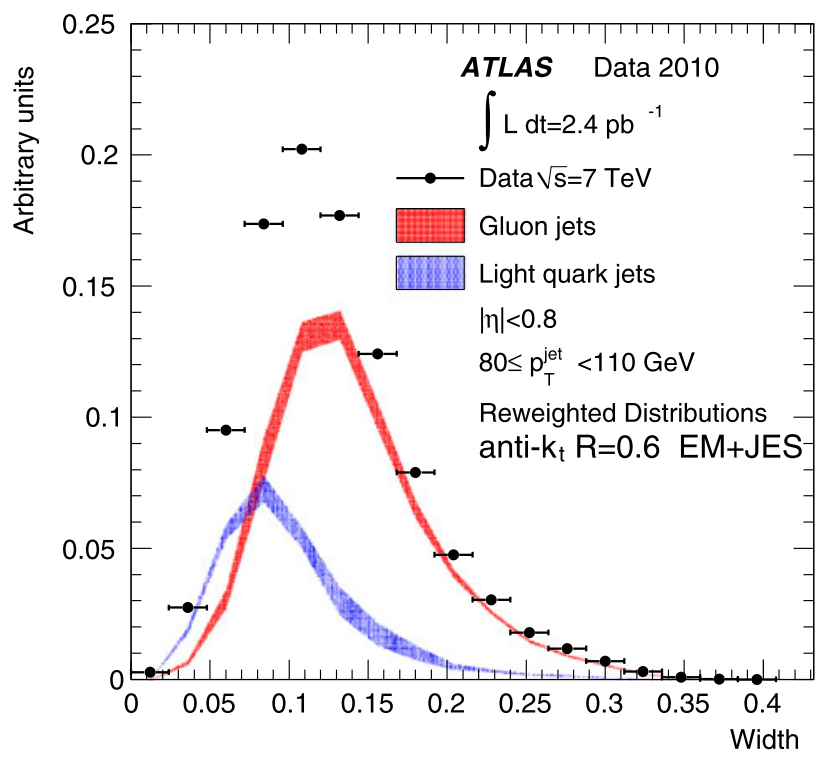

(b) Jet width

ples. Jets with $|\eta|<0.8$ and $80 \leq p_{\mathrm{T}}^{\text {jet }}<110 \mathrm{GeV}$ are included. The inclusive distributions are all normalised to unity. The inclusive Monte Carlo distributions, including the heavy quark jet contributions (not shown), are reweighted to match the inclusive distribution of the data. Uncertainties are statistical only 
Table 16 The results of flavour fits using jet width templates in three data samples: dijet events, $\gamma$-jet events, and multijet events. The Monte Carlo simulation flavour predictions are taken from ALPGEN for the dijet and multijet samples and PYTHIA for the $\gamma$-jet sample. The first uncertainty listed is statistical and the second uncertainty is systematic, and both apply to the measured gluon and light quark jet fractions. The heavy quark jet fractions in the data are constrained to be the same as those in the MC simulation

\begin{tabular}{|c|c|c|c|}
\hline \multirow[t]{2}{*}{ Sample } & \multirow[t]{2}{*}{ Selection } & \multicolumn{2}{|c|}{ Gluon/light/heavy quark jet fraction } \\
\hline & & Data & $\mathrm{MC}$ \\
\hline Dijet & $80 \leq p_{\mathrm{T}}<110 \mathrm{GeV},|\eta|<0.8,1.0 \leq R_{\min }<1.5$ & $\begin{array}{l}73 / 22 / 5 \% \\
\pm 2 \text { (stat.) } \pm 9 \text { (syst.) } \%\end{array}$ & $72 / 23 / 5 \%$ \\
\hline Dijet & $80 \leq p_{\mathrm{T}}<110 \mathrm{GeV}, 2.1 \leq|\eta|<2.8,1.0 \leq R_{\min }<1.5$ & $\begin{array}{l}45 / 52 / 3 \% \\
\pm 3 \text { (stat.) } \pm 12 \text { (syst.) } \%\end{array}$ & $39 / 58 / 3 \%$ \\
\hline$\gamma$-jet & $60 \leq p_{\mathrm{T}}<80 \mathrm{GeV},|\eta|<0.8$, Isolated & $\begin{array}{l}16 / 65 / 19 \% \\
\pm 10 \text { (stat.) } \pm 19 \text { (syst.) } \%\end{array}$ & $6 / 74 / 19 \%$ \\
\hline Multijet & 3-jet, $80 \leq p_{\mathrm{T}}<110 \mathrm{GeV},|\eta|<0.8,0.8 \leq R_{\min }<1.0$ & $\begin{array}{l}83 / 13 / 4 \% \\
\pm 2 \text { (stat.) } \pm 7 \text { (syst.) } \%\end{array}$ & $84 / 12 / 4 \%$ \\
\hline Multijet & 4-jet, $80 \leq p_{\mathrm{T}}<110 \mathrm{GeV},|\eta|<0.8,1.0 \leq \Delta R_{\min }<1.5$ & $\begin{array}{l}89 / 3 / 8 \% \\
\pm 6 \text { (stat.) } \pm 8 \text { (syst.) } \%\end{array}$ & $81 / 11 / 8 \%$ \\
\hline
\end{tabular}

\subsubsection{Monte Carlo modelling of jet width and $n_{\text {trk }}$ distributions}

Monte Carlo simulation samples generated with PYTHIA with the MC10 and the PERUGia2010 tunes and HerWIG++ all show reasonable agreement with data (see Fig. 79). Therefore, two separate fits with templates obtained from the latter two alternative Monte Carlo simulation samples are performed. Reweighting of these alternate samples is performed in the same manner as for the nominal PythiA MC10 sample. The largest of the differences in the flavour fractions with respect to the nominal fits is taken as the uncertainty due to Monte Carlo modelling. This estimate should cover physics effects that may impact light quark and gluon jets differently.

\subsubsection{The jet energy scale uncertainty and finite detector resolution}

The uncertainties in the jet measurement combined with the rapidly falling jet $p_{\mathrm{T}}$ spectrum, lead to $p_{\mathrm{T}}$ bin migrations that affect the templates. Therefore, the templates are rebuilt with all jet momenta scaled up and down according to the inclusive jet energy scale systematic uncertainty. The difference in the flavour content estimated with the modified templates is taken as a systematic uncertainty.

\subsubsection{Flavour composition of the MC simulation}

The fraction of heavy quark jets in the data is assumed to be the same as that predicted by the PYTHIA MC10 Monte Carlo simulation in the template fits. The uncertainty associated with this assumption is estimated by increasing and decreasing this Monte Carlo simulation based fraction of heavy quark jets in the template fits by a factor of two and repeating the fits with the light quark and gluon jet templates. The factor of two is taken in order to be conservative in the $\gamma$-jet and multijet samples, due to the lack of knowledge of gluon splitting fraction to $b \bar{b}$.

The PYTHIA Monte Carlo simulation was produced using the modified LO parton distribution functions, which may not accurately reproduce the true flavour composition. Particularly in the more forward pseudorapidity bins, this could produce some inherent biases in the fits. In order to estimate this uncertainty, the light quark and gluon jet templates from the standard MC sample are combined according to the flavour content of a jet sample generated using ALPGEN. This Monte Carlo generator also uses a leading order PDF, but produces more hard partons via multiparton matrix elements. This new combination is then reweighted to match the inclusive distribution in data, and the reweighted templates are used to extract the flavour composition of the samples. The difference between the flavour composition derived in this manner and the flavour composition derived using the nominal PyтHIA Monte Carlo simulation is taken as a systematic uncertainty.

\subsection{Flavour composition in a photon-jet sample}

The validity of the MC-based templates and fitting method is tested by applying the method to the $\gamma$-jet data sample and comparing the extracted flavour compositions with the $\gamma$-jet Monte Carlo simulation predictions. This sample should contain a considerably higher fraction of light quark jets than the inclusive dijet sample. Figure 81 shows the fit to the jet width in the $\gamma$-jet data for jets with $|\eta|<0.8$ and $60 \leq p_{\mathrm{T}}^{\text {jet }}<80 \mathrm{GeV}$. The heavy quark jet fractions are fixed to those obtained from the $\gamma$-jet Monte Carlo simulation. 


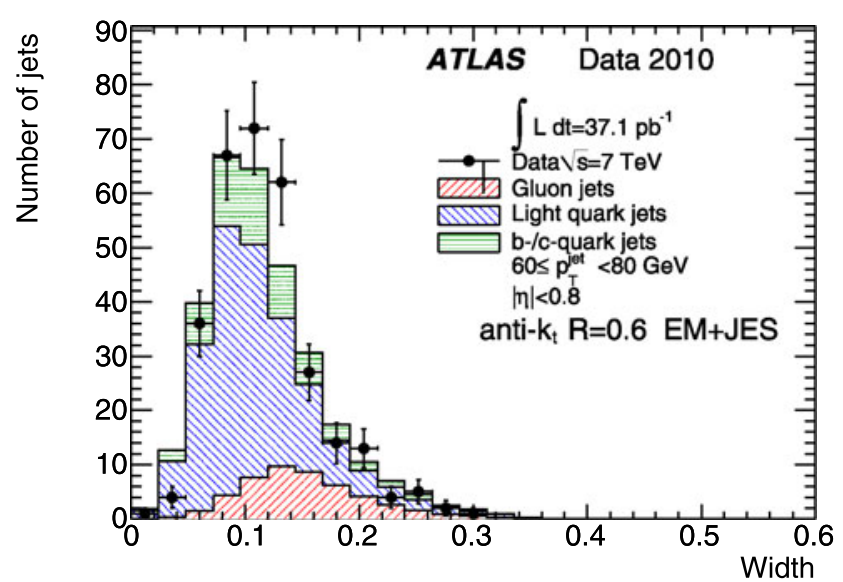

Fig. 81 The jet width template fit in a $\gamma$-jet data sample using templates derived from the inclusive jet Monte Carlo simulation sample created using the PYTHIA MC10 tune. Jets with $|\eta|<0.8$ and $60 \leq p_{\mathrm{T}}^{\text {jet }}<80 \mathrm{GeV}$ are shown. The fraction of heavy quark jets is taken directly from the MC simulation

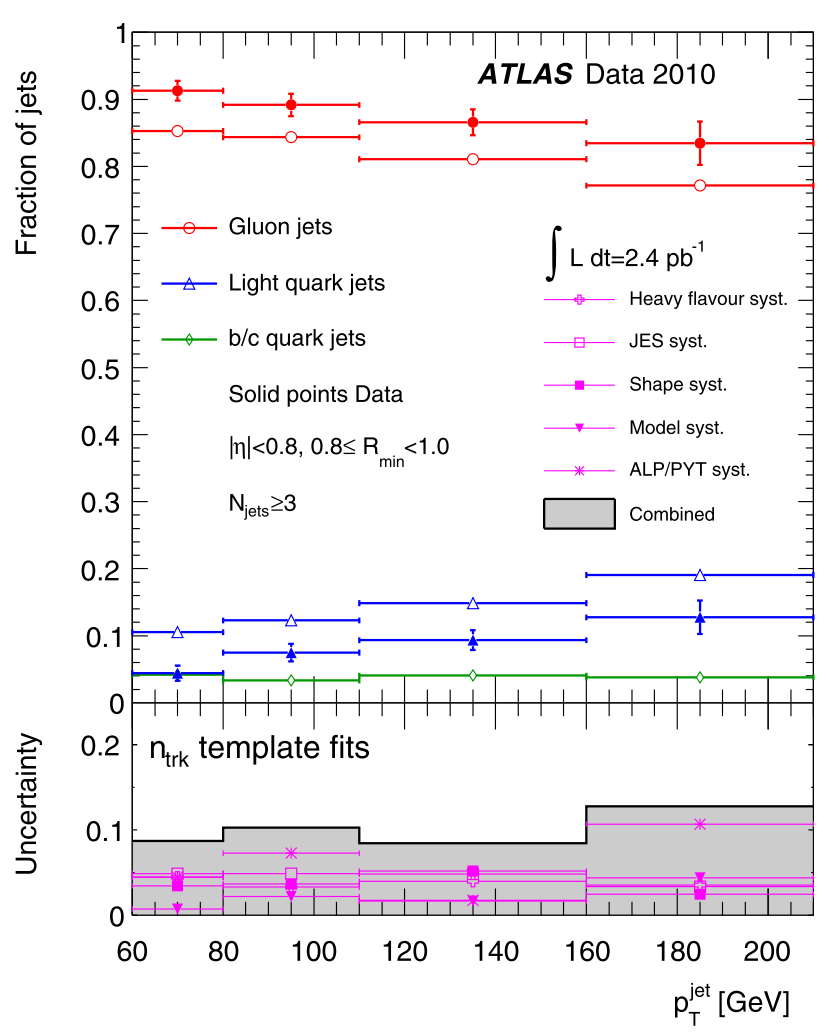

(a) Number of tracks $n_{\text {trk }}$ template fits

Fig. 82 Fitted values of the average light quark and gluon jet fraction in events with three or more jets as a function of $p_{\mathrm{T}}^{\text {jet }}$ calculated using the number of tracks $n_{\text {trk }}$ templates (a) and the jet width templates (b). Non-isolated anti- $k_{t}$ jets $\left(0.8 \leq R_{\min }<1.0\right)$ with $R=0.6$ and with $|\eta|<0.8$ calibrated with the EM+JES scheme are shown. The fraction of heavy quark jets is fixed to that of the Monte Carlo simulation.
The extracted light quark and gluon jet fractions are consistent with the true fractions in Monte Carlo simulation, though with large uncertainties, as shown in Table 16. Using the $n_{\text {trk }}$ variable gives consistent results, but with large systematic uncertainties.

\subsection{Flavour composition in a multijet sample}

The template fit method is also useful for fits to multijet events for various jet multiplicities. These events contain additional jets that mainly result from gluon radiation and hence include a larger fraction of gluon jets than does the $\gamma$-jet sample.

For this particular analysis, the templates built from the inclusive jet sample are used to determine the flavour content for each jet multiplicity bin. However, the $p_{\mathrm{T}}$ spectrum of the sub-leading jets is more steeply falling than the leading jet $p_{\mathrm{T}}$. An additional systematic uncertainty is estimated to account for the difference in $p_{\mathrm{T}}$ spectra. This uncertainty is determined by rederiving templates built with a flat $p_{\mathrm{T}}$ distribution and a significantly steeper $p_{\mathrm{T}}$ distribution than

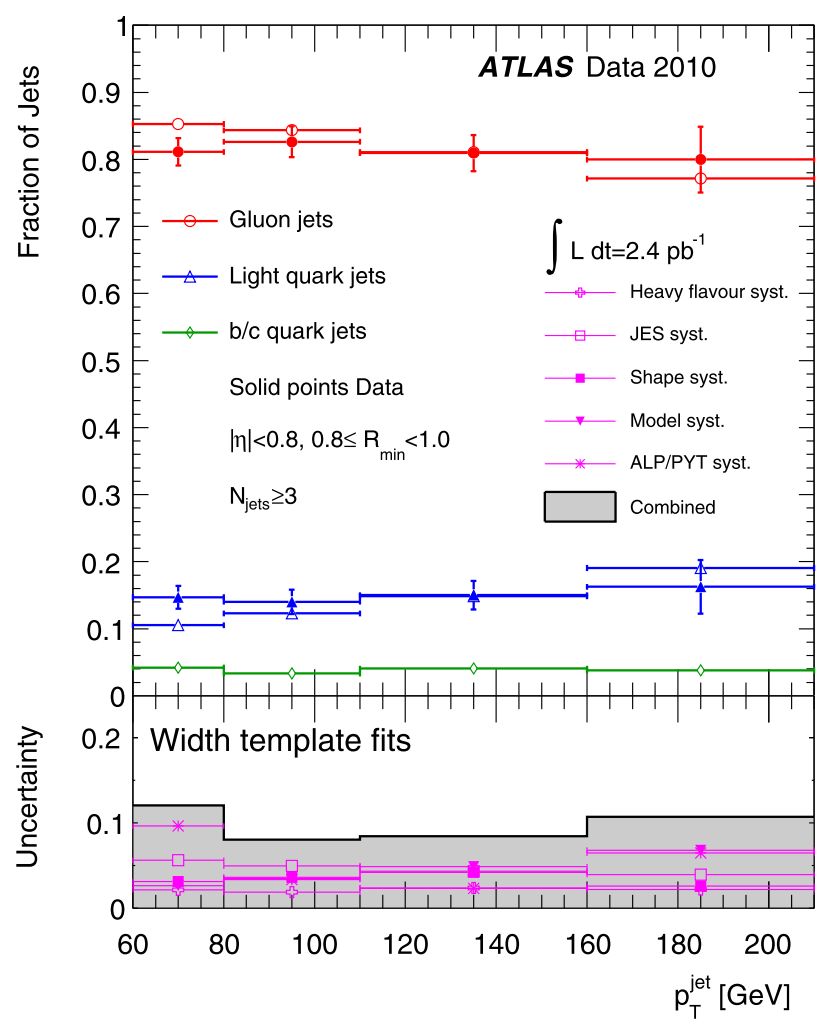

(b) Jet width template fits

The flavour fractions obtained in data are shown with closed markers, while the values obtained from the Monte Carlo simulation are shown with open markers. The error bars indicate the statistical uncertainty of the fit. Below each figure the impact of the different systematic effects is shown with markers and the combined systematic uncertainty is indicated by a shaded band 


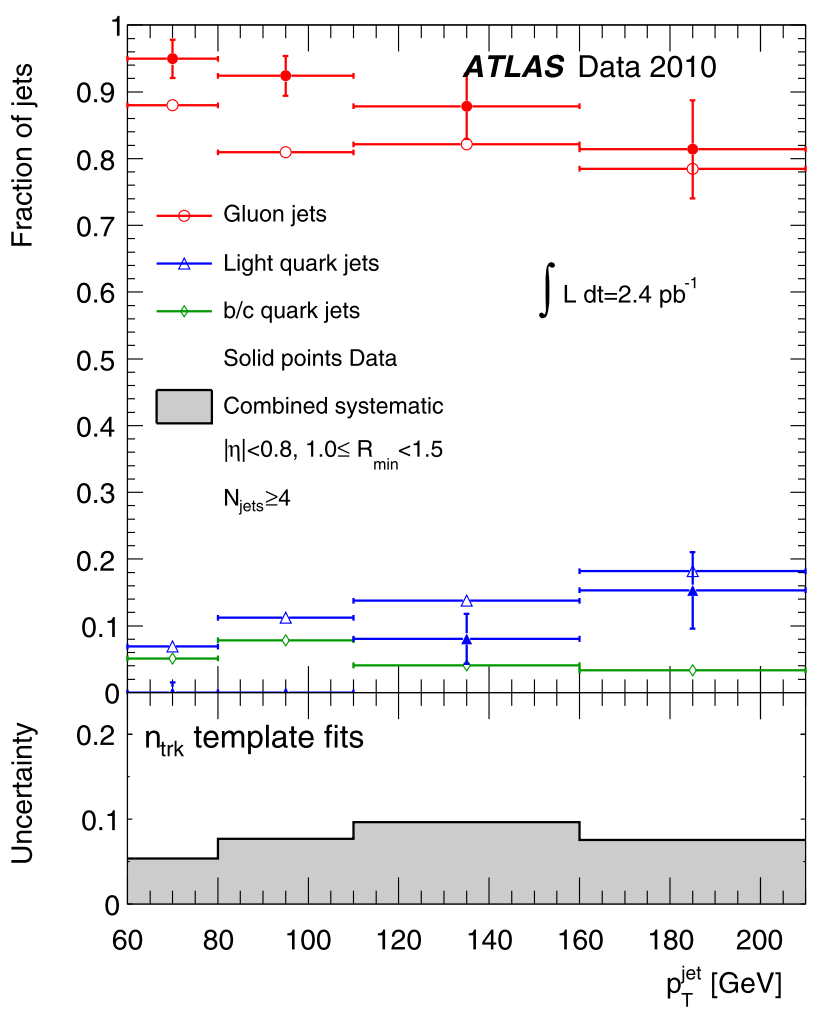

(a) Number of tracks $n_{\text {trk }}$ template fits

Fig. 83 Fitted values of the average light quark and gluon jet fraction in events with four or more jets as a function of $p_{\mathrm{T}}^{\text {jet }}$ for isolated anti$k_{t}$ jets with $R=0.6$ and with $|\eta|<0.8$ calibrated with the EM+JES scheme. The fraction of heavy quark jets is fixed from the Monte Carlo simulation. The number of tracks $n_{\text {trk }}$ (a) and the jet width (b) tem-

that of the dijet sample. The slope of the steeply falling distribution is taken from the $p_{\mathrm{T}}$ of the sixth leading jet in Monte Carlo events with six jets, generated using ALPGEN. The fits are repeated with these modified templates, and the largest difference is assigned as a $p_{\mathrm{T}}^{\text {jet }}$ spectrum shape systematic uncertainty.

Figure 82 compares the fractions of light quark and gluon jets obtained with a fit of the jet width and $n_{\text {trk }}$ distributions in events with three or more jets in data and Monte Carlo simulation as a function of $p_{\mathrm{T}}^{\text {jet }}$ for non-isolated $(0.8 \leq$ $\left.R_{\min }<1.0\right)$ jets with $|\eta|<0.8$. The higher gluon jet fractions predicted by the Monte Carlo simulation are reproduced by the fit, and the data and the Monte Carlo simulation are consistent. The total systematic uncertainty on the measurement is below $10 \%$ over the measured $p_{\mathrm{T}}^{\text {jet }}$ range.

The average flavour fractions obtained from fitting the jet width and $n_{\text {trk }}$ distributions in events with four or more jets are shown in Fig. 83. In both cases, the extracted fractions are consistent with the Monte Carlo predictions within the systematic uncertainties, and the total systematic uncertainty is similar to the one for the three-jet bin.

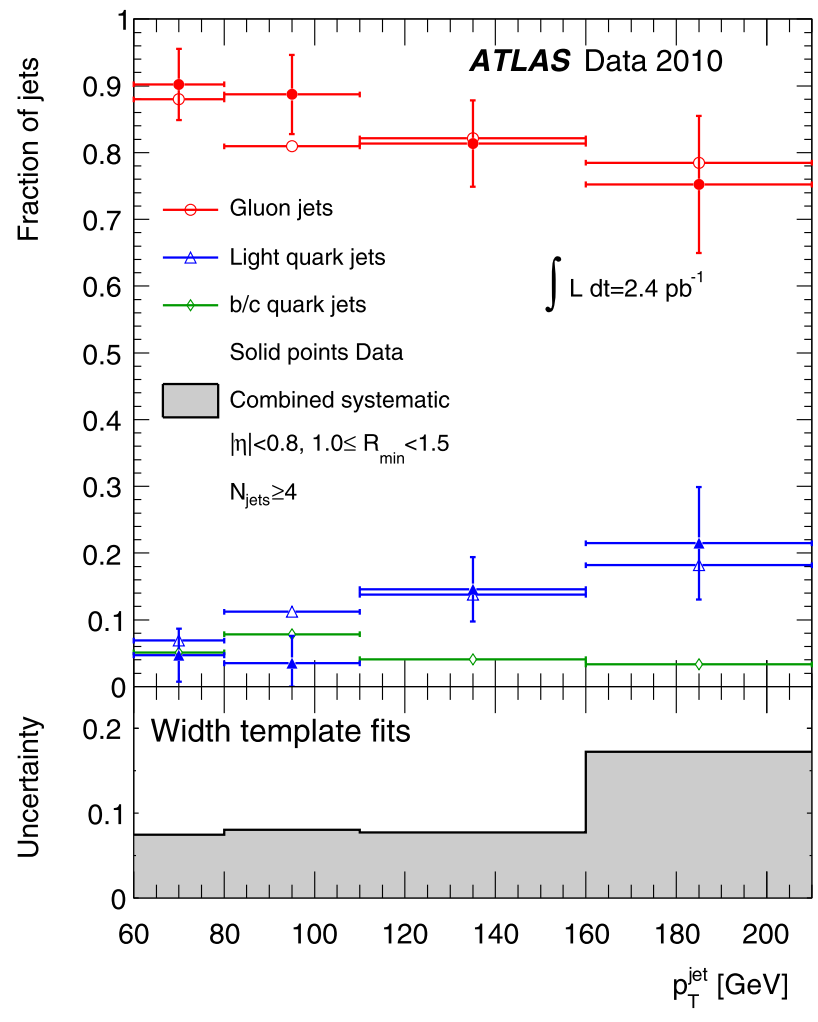

(b) Jet width template fits

plate distributions are used in the fits. The flavour fractions obtained in data are shown with closed markers, while the values obtained from the Monte Carlo simulation are shown with open markers. The error bars indicate the statistical uncertainty of the fit. Below each figure the systematic uncertainty is shown as a shaded band

The extracted light quark and gluon jet fractions, with the total systematic uncertainty from the width and $n_{\text {trk }}$ fits, are summarised in Fig. 84 as a function of inclusive jet multiplicity. The fractions differ by $10 \%$ between the data and the Monte Carlo simulation, but are consistent within uncertainties. The total systematic uncertainty is around $10 \%$ for each multiplicity bin. Thus, for the four-jet bin, the flavour dependent jet energy scale systematic uncertainty can be reduced by a factor of $\sim 10$, from about $6 \%$ obtained assuming a $100 \%$ flavour composition uncertainty to less than $1 \%$ after having determined the flavour composition with a $10 \%$ accuracy. A summary of the flavour fit results using the jet width templates for the different samples is provided in Table 16 .

\subsection{Summary of jet response flavour dependence}

The flavour dependence of the jet response has been studied, and an additional term to the jet energy scale systematic uncertainty has been derived.

A generic template fit method has been developed to reduce this uncertainty significantly for any given sample 


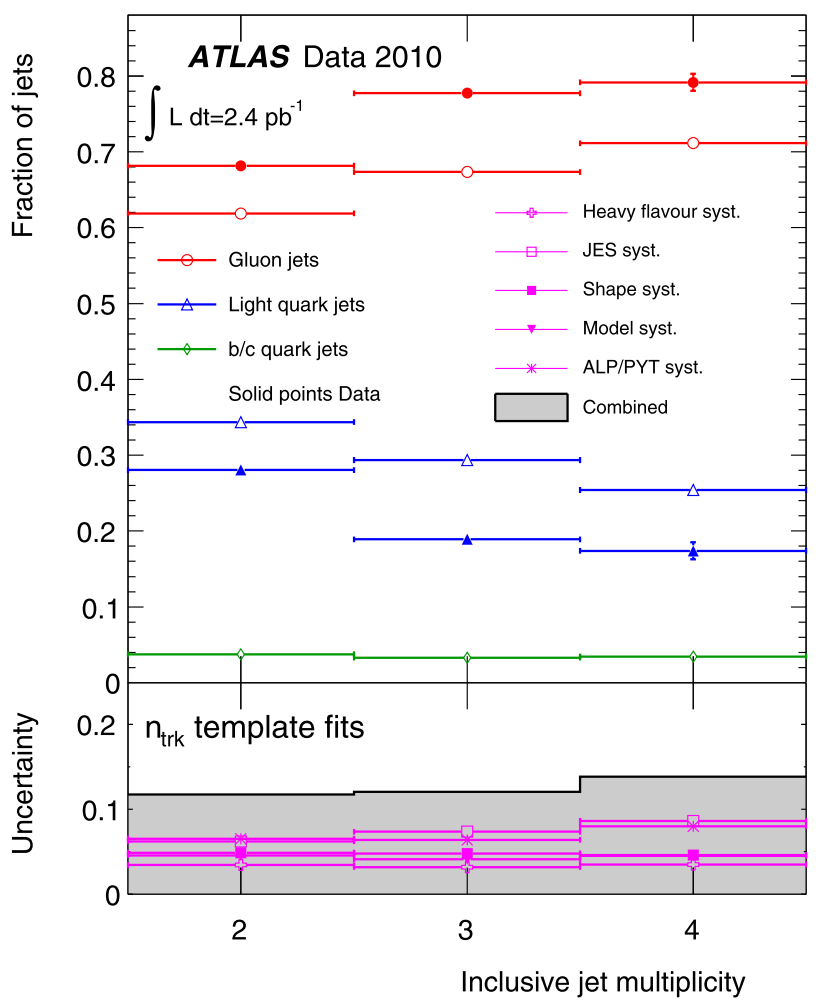

(a) Number of track $n_{\text {trk }}$ template fits

Fig. 84 Fitted values of the average light quark and gluon jet fraction as a function of inclusive jet multiplicity with total uncertainties on the fit as obtained using the number of tracks $n_{\text {trk }}$ (a) and the jet width (b) distributions. The fraction of heavy quark jets is fixed from the Monte Carlo simulation. The flavour fractions obtained in data are shown with closed markers, while the values obtained from the Monte Carlo sim-

of events. Templates derived in dijet events were applied to both $\gamma$-jet and multijet events, demonstrating the potential of the method to reduce the systematic uncertainty. The light-flavour portion of the flavour dependent jet energy scale can be reduced from $\sim 6 \%$ to below $1 \%$.

\section{Global sequential calibrated jet response for a quark sample}

In this section, the performance of the GS calibration (see Sect. 11) is tested for a $\gamma$-jet sample. The jet energy scale after each GS correction can be verified using the in situ techniques such as the direct $p_{\mathrm{T}}$ balance technique in $\gamma$-jet events (see Sect. 10.2), where mainly quark induced jets are tested. The flavour dependence of the GS calibration is tested for jets with $|\eta|<1.2$.

The measurement is first made with jets calibrated with the EM+JES calibration and is repeated after the application of each of the corrections that form the GS calibration. To maximise the available statistics one pseudorapidity bin

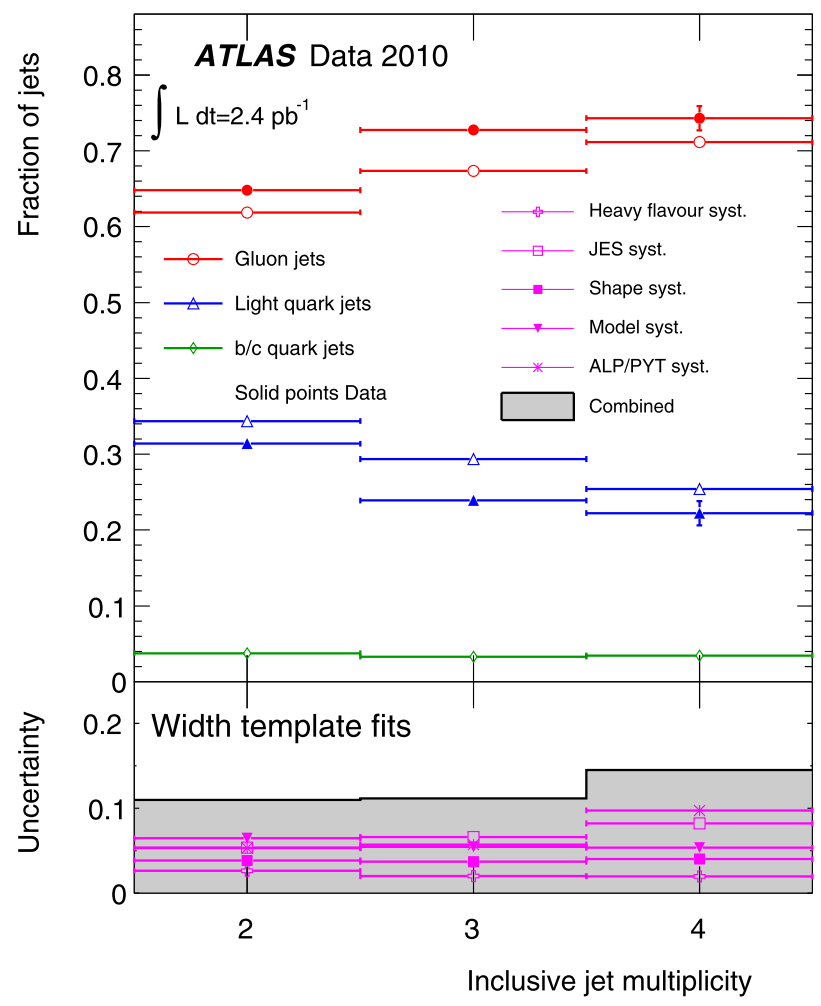

(b) Jet width template fits

ulation are shown with open markers. Anti- $k_{t}$ jets with $R=0.6$ calibrated with the EM+JES scheme are used. The error bars indicate the statistical uncertainty of the fit. Below each figure the impact of the different systematic effects is indicated by markers. and the combined systematic uncertainty is shown at the bottom of the figure as a shaded band

is used $|\eta|<1.2$. The Monte Carlo based GS corrections are applied to both data and Monte Carlo simulation. The systematic uncertainty associated with the GS calibration is evaluated by computing the data to Monte Carlo simulation ratio of the response after the GS calibration relative to that for the $\mathrm{EM}+\mathrm{JES}$ calibration.

For $25 \leq p_{\mathrm{T}}^{\text {jet }}<45 \mathrm{GeV}$, the agreement between the response in data and Monte Carlo simulation is $3.2 \%$ after EM+JES and $4.2 \%$ after GS calibration. For $210 \leq$ $p_{\mathrm{T}}^{\text {jet }}<260 \mathrm{GeV}$, the agreement is $5 \%$ after EM+JES and $2.5 \%$ after GS calibration. Therefore systematic uncertainties derived from the agreement of data and Monte Carlo simulation vary from $1 \%$ at $p_{\mathrm{T}}^{\text {jet }}=25 \mathrm{GeV}$ to $2.5 \%$ for $p_{\mathrm{T}}^{\text {jet }}=260 \mathrm{GeV}$. These results are compatible within the statistical uncertainty with the uncertainty evaluated using inclusive jet events (see Sect. 12.1.3).

The obtained results indicate that the uncertainty in a sample with a high fraction of light quark jets is about the same as in the inclusive jet sample. 


\section{JES uncertainties for jets with identified heavy quark components}

Heavy flavour jets such as jets induced by bottom $(b)$ quarks ( $b$-jets) play an important role in many physics analyses.

The calorimeter jet response uncertainties for $b$-jets is evaluated using single hadron response measurements in samples of inclusive jet and $b \bar{b}$ dijet events. The JES uncertainty arising from the modelling of the $b$-quark production mechanism and the $b$-quark fragmentation can be determined from systematic variations of the Monte Carlo simulation.

Finally, the calorimeter $p_{\mathrm{T}}^{\text {jet }}$ measurement can be compared to the one from tracks associated to the jets for inclusive jets and identified $b$-jets. From the comparison of data to Monte Carlo simulation the $b$-jet energy scale uncertainty relative to the inclusive jet sample is estimated.

\subsection{Selection of identified heavy quark jets}

Jets are reconstructed using the anti- $k_{t}$ jet algorithm with $R=0.4$ and calibrated with the EM+JES scheme. Jets with $p_{\mathrm{T}}^{\text {jet }}>20 \mathrm{GeV}$ and $|\eta|<2.5$ are selected.

A representative sample of identified $b$-jets is selected by a track-based $b$-tagging algorithm, called the SV0 tagger [26, 104, 105]. This algorithm reconstructs a displaced vertex from the decay products of the long-lived $B$-hadron. As input, the SV0 tagging algorithm is given a list of tracks associated to the jet. Secondary vertices are reconstructed starting from two-track vertices which are merged into a common vertex. Tracks giving large $\chi^{2}$ contributions are then iteratively removed until the reconstructed vertex fulfils certain quality criteria. Two-track vertices at a radius consistent with the radius of one of the three pixel detector layers are removed, as these vertices likely originate from material interactions. The decay length significance $L / \sigma(L)>5.72$, where $L$ is the decay length and $\sigma(L)$ its uncertainty, is assigned to each jet as a tagging weight.

A jet is identified as a $b$-jet if this weight exceeds a threshold of 5.85. The b-tagging efficiency and mistag fraction of the SV0 $b$-tagging algorithm have been measured on data as a function of the jet $p_{\mathrm{T}}[104,105]$. The efficiency measurement is based on a sample of jets containing muons and makes use of the transverse momentum of a muon relative to the jet axis. Based on these measurements a dedicated $b$-tagging calibration is applied to the simulation and systematic uncertainties for the calibration are evaluated.

For Monte Carlo studies, a sample of $b$-jets is selected using a geometrical matching of the jet $(\Delta R<0.4)$ to a true $B$-hadron.

\subsection{Calorimeter response uncertainty}

The uncertainty of the calorimeter response to identified $b$-jets has been evaluated using single hadron response mea- surements in situ and in test-beams [28]. The same method as described in Sect. 9.3 is used to estimate the $b$-jet response uncertainty in events with top-quark pairs with respect to the one of inclusive jets.

For jets within $|\eta|<0.8$ and $20 \leq p_{\mathrm{T}}^{\text {jet }}<250 \mathrm{GeV}$ the expected difference in the calorimeter response uncertainty of identified $b$-jets with respect to the one of inclusive jets is less than $0.5 \%$. It is assumed that this uncertainty extends up to $|\eta|<2.5$.

\subsection{Uncertainties due to Monte Carlo modelling}

In Sect. 20.2 the calorimeter $b$-jet response has been estimated from the single hadron response measurement assuming that the Monte Carlo simulation gives a correct description of the $b$-jet fragmentation and the detector geometry. In this section the uncertainties due to these effects are assessed.

The following uncertainties for $b$-jets are studied using systematic variations of the Monte Carlo simulation:

1. Fragmentation and hadronisation modelling uncertainty obtained by comparing the Monte Carlo generators HERWIG vs PYTHIA.

2. Soft physics modelling uncertainty obtained by comparing the PYThia MC10 to the Pythia PerUgia2010 tune.

3. Modelling uncertainty of the detector material in front and in between the calorimeters.

4. Modelling uncertainty of the fragmentation of $b$-quarks.

The event generators PYTHIA and HERWIG++ are used to evaluate the influence of different hadronisation models, different parton showers, as well as differences in the underlying event model (see Sect. 4). Variations in proton parton density functions are also included.

The influence of the soft physics modelling is estimated by replacing the standard PYTHIA MC10 tune by the Pythia Perugia2010 tune. The impact of additional dead material is tested following the prescription detailed in Sect. 9.

The fragmentation function is used to estimate the momentum carried by the $B$-hadron with respect to that of the $b$-quark after quark fragmentation. The contribution of the $b$-quark fragmentation to the JES uncertainty is estimated using Monte Carlo samples generated with different sets of tuning parameters of two fragmentation functions (see Table 17).

The fragmentation function included as default in PYTHIA originates from a detailed study of the $b$-quark fragmentation function in comparison with OPAL [106] and SLD [107] data. The data are better described using the symmetric Bowler fragmentation function with $r_{Q}=0.75$ (PYTHIA PARJ(46)), assuming the same modification for 
Table 17 PyTHIA steering parameters for the considered variations of the $b$-quark fragmentation functions

\begin{tabular}{llll}
\hline Parameter & Nominal & Professor & Bowler-Lund \\
\hline MSTJ(11) & 4 & 5 & 4 \\
MSTJ(22) & 2 & 2 & 2 \\
PARJ(41) & 0.3 & 0.49 & 0.85 \\
PARJ(42) & 0.58 & 1.2 & 1.03 \\
PARJ(46) & 0.75 & 1.0 & 0.85 \\
PARJ(54) & -0.07 & & \\
PARJ(55) & -0.006 & & \\
\hline
\end{tabular}

$b$ - and $c$-quarks. The $a$ (PYTHIA PARJ(41)) and $b$ (PYTHIA PARJ(42)) parameters of the symmetric Lund function were left with the values shown in Table 17. A more detailed discussion of uncertainties in the $b$-quark fragmentation function can be found in Refs. [108-112].

The choice of the fragmentation function for this study is based on comparisons to LEP experimental data, mostly from ALEPH [113] and OPAL [106], as well as from the SLD experiment [107] included in a phenomenological study of the $b$-quark fragmentation in top-quark decay [114].

To assess the impact of the $b$-quark fragmentation, the nominal parameters of the PYTHIA fragmentation function are replaced by the values from a recent tune using the Professor framework [115]. In addition, the nominal fragmentation function is replaced by the modified Bowler-Lund fragmentation function [116].

For each effect listed above the $b$-jet response uncertainty is evaluated from the ratio between the response of $b$-jets in the Monte Carlo samples with systematic variations to the nominal Pythia MC10 $b$-jet sample. The deviation from unity of this ratio is taken as uncertainty:

Uncertainty $=1-\left(\frac{\mathcal{R}_{\text {variation }}^{b \text {-jet }}}{\mathcal{R}_{\text {nominal }}^{b \text {-jet }}}\right)$.

The $b$-jet response obtained with PyTHIA for the MC10 and the Perugia2010 tunes, the Herwig++ Monte Carlo event generator and using a simulation with additional dead material is shown in Fig. 85a. Figure 85b shows the variation with various fragmentation functions, i.e. the standard one in the nominal PYTHIA sample versus the ones in the Pythia Professor tune sample and the Pythia modified Bowler-Lund fragmentation function sample. The response variations are well within about $2 \%$.

\subsection{Final bottom quark JES uncertainty}

The $b$-jet JES uncertainty is obtained adding the calorimeter response uncertainty (see Sect. 20.2) and the uncertainties from the systematic Monte Carlo variations (see Sect. 20.3) in quadrature.

To avoid double counting when combining the $b$-jet uncertainty with the JES uncertainty of inclusive jets the following effects need to be considered:

1. The uncertainty component due to the Perugia2010 tune is not added, since the effect on $b$-jets is similar to the one on inclusive jets where it is already accounted for.

2. The average uncertainty for inclusive jets due to additional dead detector material is subtracted from the corresponding $b$-jet uncertainty component. The JES uncertainty due to dead material is smaller for inclusive jets, since in situ measurements are used.

The resulting additional JES uncertainty for $b$-jets is shown in Fig. 86. It is about $2 \%$ up to $p_{\mathrm{T}}^{\text {jet }} \approx 100 \mathrm{GeV}$ and below $1 \%$ for higher $p_{\mathrm{T}}^{\text {jet }}$. To obtain the overall $b$-jet uncertainty this uncertainty needs to be added in quadrature to the JES uncertainty for inclusive jets described in Sect. 9.

20.5 Validation of the heavy quark energy scale using tracks

The validation of the identified $b$-jet JES uncertainty uses the tracks associated to the $b$-jet as reference object and closely follows the method described in Sect. 10.1. The transverse momentum of a jet is compared to the total transverse momentum measured in tracks associated to the jet (see Eq. (22)).

\subsubsection{Method}

The double ratio of charged-to-total momentum observed in data to that obtained in Monte Carlo simulation defined in

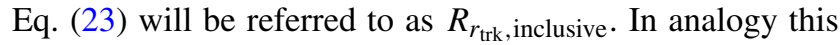
ratio is studied for $b$-tagged jets:

$R_{r_{\text {trk }}, b \text {-jet }} \equiv \frac{\left[\left\langle r_{\text {trk }} b \text {-jet }\right\rangle\right]_{\text {Data }}}{\left[\left\langle r_{\text {trk }} b \text {-jet }\right\rangle\right]_{\mathrm{MC}}}$.

The $r_{\text {trk }}$ distributions for all $p_{\mathrm{T}}$ bins are calculated and the mean values of $r_{\text {trk }}$ for data and Monte Carlo simulation are derived. The relative response to $b$-jets relative to inclusive jets, $R^{\prime}$, is defined as

$R^{\prime} \equiv \frac{R_{r_{\text {trk }}, b \text {-jet }}}{R_{r_{\text {trk }}, \text { inclusive }}}$

\subsubsection{Systematic uncertainties}

The systematic uncertainties arise from the modelling of the $b$-fragmentation, $b$-tagging calibration, jet resolution and 


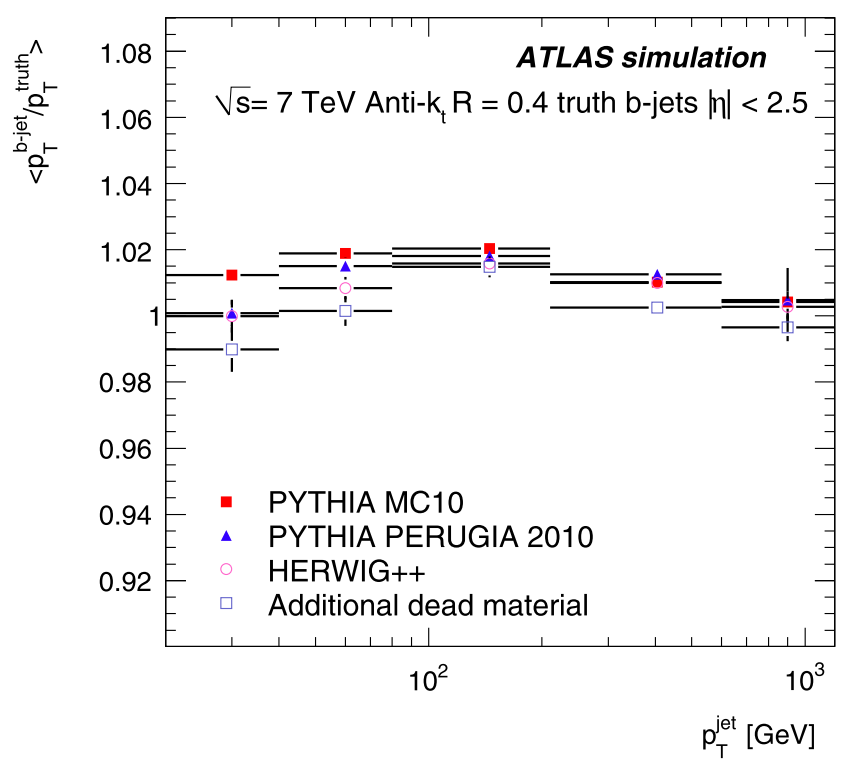

(a) MC generator and detector geometry

Fig. 85 Average response for $b$-jets as a function of $p_{\mathrm{T}}^{\text {jet }}$ obtained with the Monte Carlo event generators PYTHIA with the MC10 and PERUGia2010 tunes and Herwig++ and Pythia simulations with additional dead detector material (a). Average response for $b$-jets us-

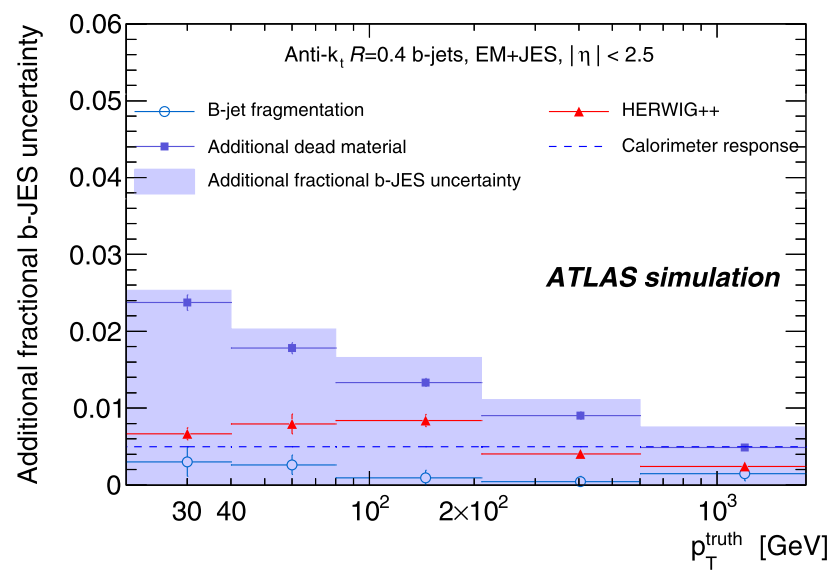

Fig. 86 Additional fractional $b$-jet JES uncertainty as a function of the truth jet transverse momentum for anti- $k_{t}$ jets with $R=0.4$ calibrated with the EM+JES scheme for $|\eta|<2.5$. Shown are systematic Monte Carlo variations using different modelling of the $b$-quark fragmentation and physics effects as well as variations in the detector geometry and the uncertainty in the calorimeter response to $b$-jets as evaluated from single hadron response measurements. Uncertainties on the individual points are statistical only

tracking efficiency. They are assumed to be uncorrelated. The resulting fractional systematic uncertainties are shown on the right part of Fig. 87 and are determined as follows:

1. MC generator: The $r_{\text {trk }}$ distribution is also calculated from HERWIG++ samples. The shift in the distribution is fitted by a constant function. The variations in the data

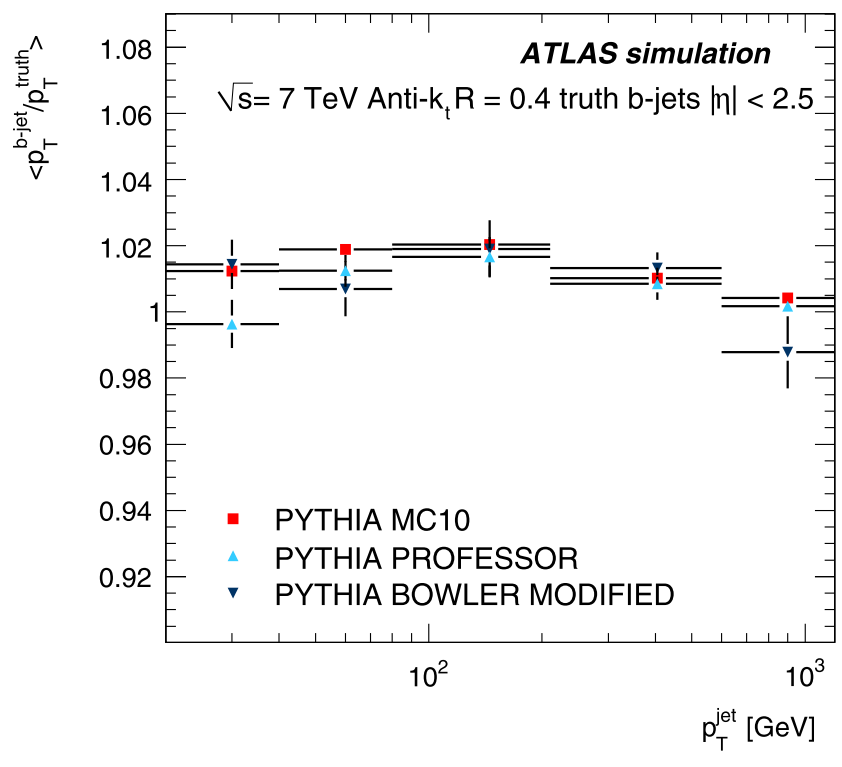

(b) $b$-quark fragmentation

ing the Pythia Professor tune and the PythiA modified Bowler-Lund fragmentation function evaluated with respect to the nominal PYTHIA inclusive jet sample (b). Only statistical uncertainties are shown

to Monte Carlo simulation ratio are taken as a systematic uncertainty.

2. $b$-tagging calibration: The scale factors are varied correlated within their systematic uncertainty in the Monte Carlo simulation and the ratio is re-evaluated. The resulting shifts are added in quadrature to the systematic uncertainty.

3. Material description: The knowledge of the tracking efficiency modelling in Monte Carlo simulation was evaluated in detail in Ref. [93]. The systematic uncertainty on the tracking efficiency for isolated tracks increases from $2 \%\left(\left|\eta^{\text {track }}\right|<1.3\right)$ to $7 \%\left(2.3 \leq\left|\eta^{\text {track }}\right|<2.5\right)$ for tracks with $p_{\mathrm{T}}>500 \mathrm{MeV}$. The resulting effect on $r_{\text {trk }}$ is $2 \%$ for $|y|<1.2,3.1 \%$ for $1.2 \leq|y|<2.1$ and $5.5 \%$ for $2.1 \leq|y|<2.5$.

4. Tracking in jet core: High track densities in the jet core influence the tracking efficiency due to shared hits between tracks, fake tracks and lost tracks. The number of shared hits is well-described in Monte Carlo simulation. The $p_{\mathrm{T}}$ carried by fake tracks is negligible.

A relative systematic uncertainty of $50 \%$ on the loss of efficiency is assigned. The shift of $r_{\text {trk }}$ due to this uncertainty on the loss of efficiency is evaluated in Monte Carlo simulation on generated charged particles. Monte Carlo pseudo-experiments are generated according to the varied inefficiency. For each jet the ratio of the $p_{\mathrm{T}}$ sum of the associated generated particles (truth tracks) with $p_{\mathrm{T}}^{\text {track }}>1 \mathrm{GeV}$ to the $p_{\mathrm{T}}$ sum of those associated truth 

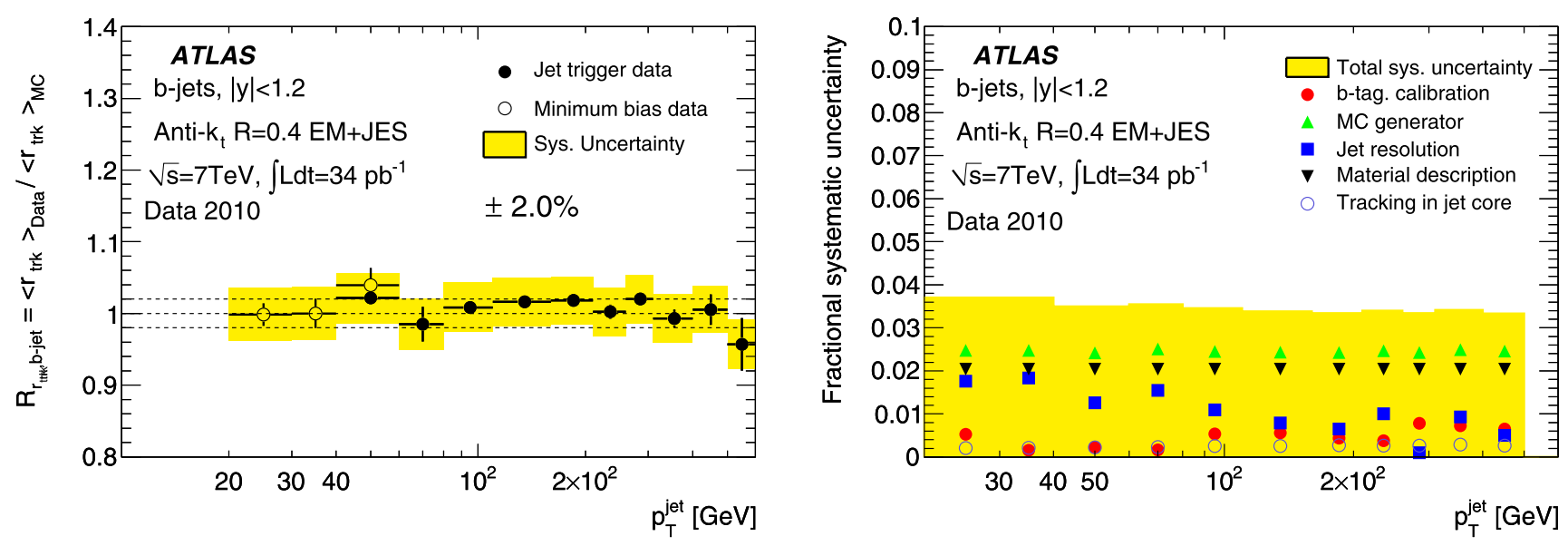

(a) $|y|<1.2$
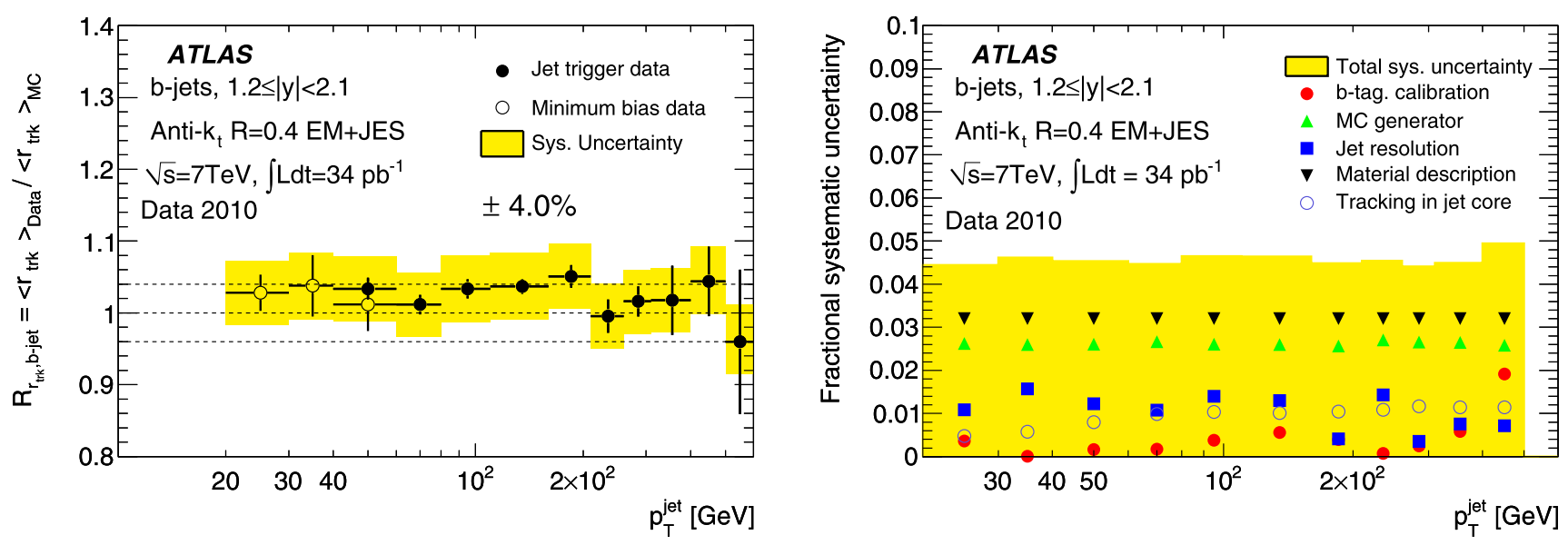

(b) $1.2 \leq|y|<2.1$
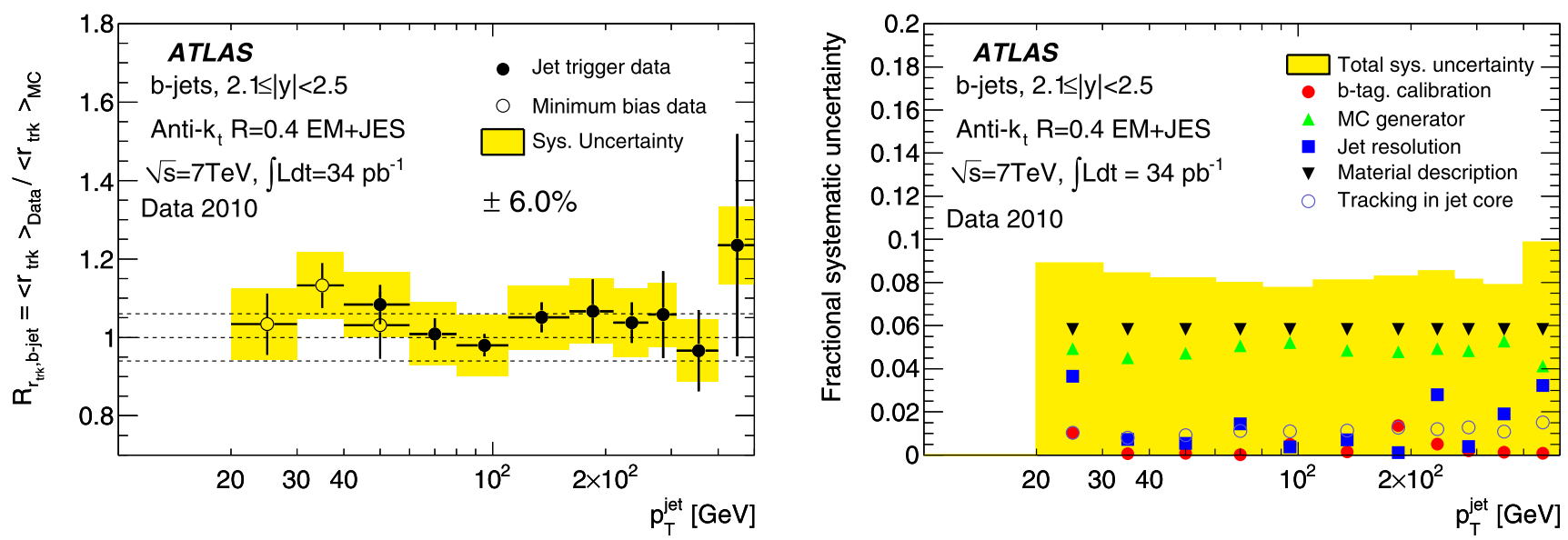

(c) $2.1 \leq|y|<2.5$

Fig. 87 The ratio of the mean value of $r_{\text {trk }}$ in data and Monte Carlo (left) and the fractional systematic uncertainty (right) as a function of $p_{\mathrm{T}}^{\text {jet }}$ for $|y|<1.2(\mathbf{a}), 1.2 \leq|y|<2.1$ (b) and $2.1 \leq|y|<2.5$ (c). Anti- $k_{t}$ jets with $R=0.4$ calibrated with the EM+JES scheme are used. The dashed lines indicate the estimated uncertainty from the data and Monte Carlo simulation agreement. Note the changed axis ranges in (c). Only statistical uncertainties are shown on the data points 

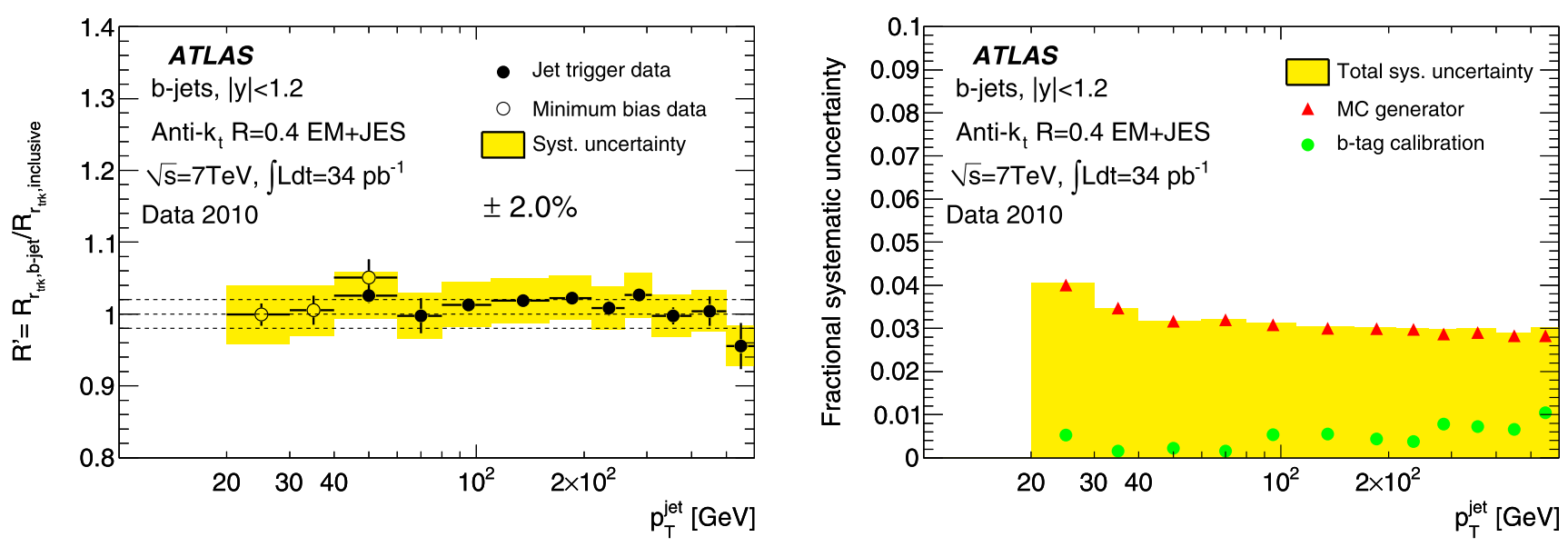

(a) $|y|<1.2$
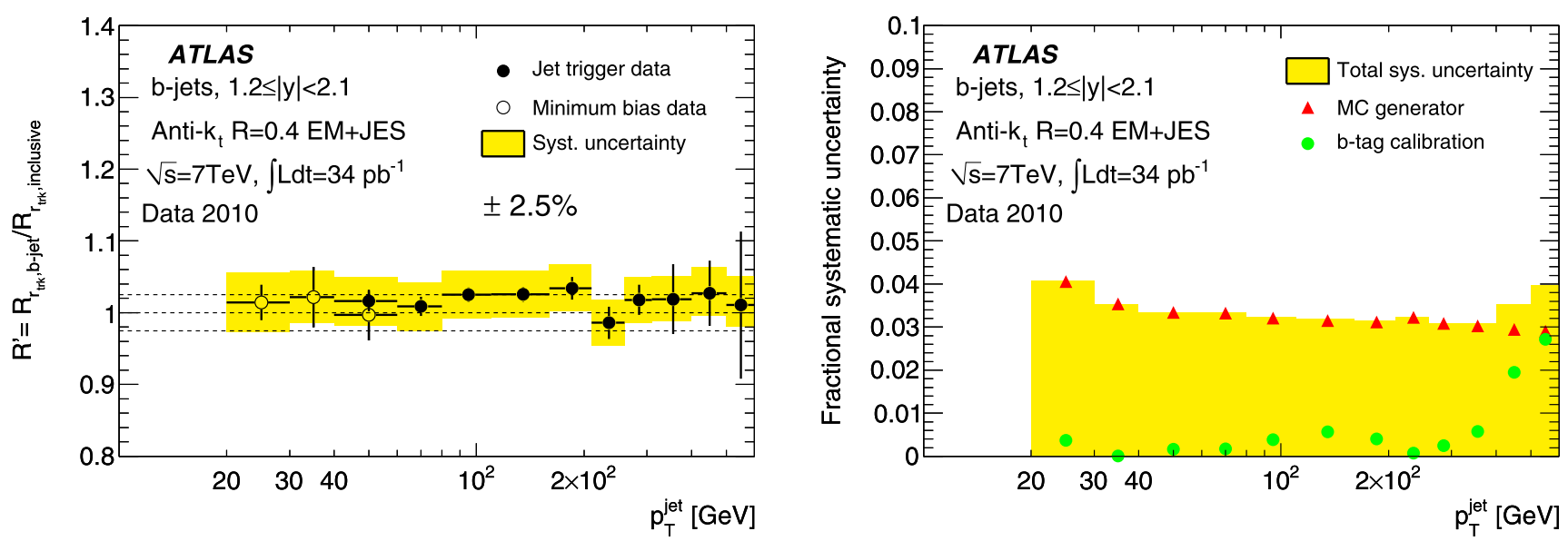

(b) $1.2 \leq|y|<2.1$
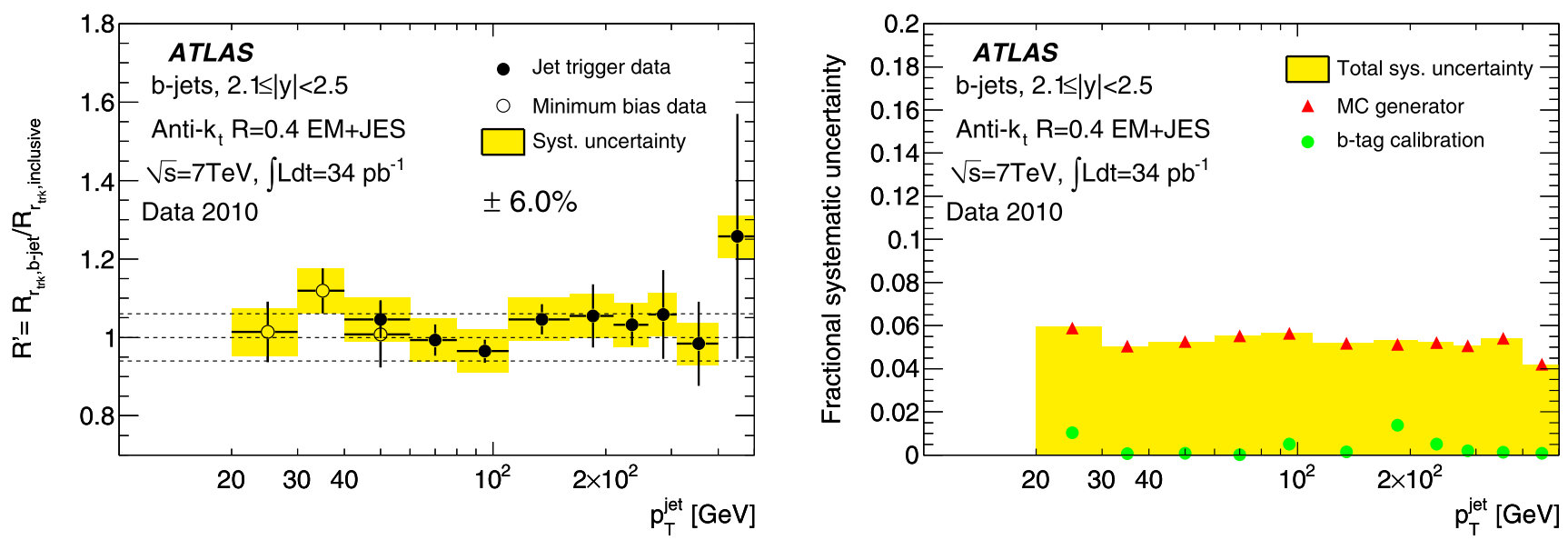

(c) $2.1 \leq|y|<2.5$

Fig. 88 The ratio $R^{\prime}$ (see Eq. (63)) of $R_{r_{\text {trk }}, b \text {-jet }}$ for identified $b$-jets and $R_{r_{\text {trk }} \text {,inclusive }}$ for inclusive jets (left) and the fractional systematic uncertainty (right) as a function of $p_{\mathrm{T}}^{\text {jet }}$ for $|y|<1.2(\mathbf{a}), 1.2 \leq|y|<$ 2.1 (b) and $2.1 \leq|y|<2.5$ (c). Anti- $k_{t}$ jets with $R=0.4$ calibrated with the EM+JES scheme are used. The dashed lines indicate the estimated uncertainty from the data and Monte Carlo simulation agreement. Only statistical uncertainties are shown on the data points. Note the changed axis ranges in (c) 
tracks with $p_{\mathrm{T}}>1 \mathrm{GeV}$ which also have a matched reconstructed track with $p_{\mathrm{T}}^{\text {track }}>1 \mathrm{GeV}$, is calculated. In this latter sample a truth track without or with a reconstructed track with $p_{\mathrm{T}}^{\text {track }}>1 \mathrm{GeV}$ is added or respectively discarded according to the inefficiency uncertainty. The relative shift in the ratio $r_{\text {trk }}$ is added in quadrature to the systematic uncertainty.

5. Jet resolution: The jet energy resolution in Monte Carlo simulation is degraded. A random energy that corresponds to a resolution smearing of $10 \%$ is added to each jet. The resulting shift of the ratio $r_{\text {trk }}$ is evaluated and added in quadrature to the overall systematic uncertainty.

The two biggest contributions to the systematic uncertainty are due to the material description and the difference between the $r_{\text {trk }}$ distribution for HERWIG++ and PYTHIA.

\subsubsection{Results}

Figure 87 (left) shows the ratio of data to Monte Carlo simulation. An agreement of the calorimeter to track jet $p_{\mathrm{T}}$ measurements is found within $2 \%$ in the bin $|y|<1.2$, within $4 \%$ for $1.2 \leq|y|<2.1$ and within $6 \%$ for $2.1 \leq|y|<2.5$.

The relative response $R^{\prime}$ between identified $b$-jets and inclusive jets is shown in Fig. 88 for all $y$-bins indicating the resulting relative $b$-jet energy scale uncertainty with respect to the inclusive jets sample. The uncertainty for $b$-jets is estimated to be $2 \%, 2.5 \%$ and $6 \%$ in the range $|y|<1.2$, $1.2 \leq|y|<2.1$ and $2.1 \leq|y|<2.5$, respectively. For the calculation of the systematic uncertainty in $R^{\prime}$ it is assumed that at first order the uncertainty in the denominator and numerator of $R^{\prime}$ from the tracking, namely tracking efficiency, material description, are fully correlated and cancel. The $p_{\mathrm{T}}^{\text {jet }}$ resolution for inclusive and identified $b$-jets is considered to be similar. Both assumptions are exactly valid for high $p_{\mathrm{T}}$ jets; for low $p_{\mathrm{T}}$ jets the second order deviations are estimated to be about $0.2 \%$.

The most significant systematic uncertainties on $R^{\prime}$ are due to the choice of the Monte Carlo generator and the $b$-tagging calibration. Those independent uncertainties are added in quadrature. The Monte Carlo generator uncertainties from the inclusive sample and from the $b$-tagged sample are also added in quadrature.

\subsubsection{Summary}

The jet energy scale for identified $b$-jets relative to that of inclusive jets is evaluated for anti- $k_{t}$ jets with $R=0.4$ for the EM+JES calibration scheme. The resulting relative $b$-jet energy scale with respect to the inclusive jets sample is derived within $2 \%, 2.5 \%$ and $6 \%$ in the range $|y|<1.2$, $1.2 \leq|y|<2.1$ and $2.1 \leq|y|<2.5$, respectively.

\section{Study of jet punch-through}

For jets at very high transverse momentum it is possible that part of the energy is not deposited in the calorimeter, but leaks out to the detector components beyond the calorimeter. This leads to a systematic reduction in the measured jet energy.

Jets that deposit energy beyond the hadronic Tile calorimeter and in the muon system are called punchthrough jets. A graphical representation of a candidate for a punch-through jet in data is shown in Fig. 89.

In this section the Monte Carlo simulation of energy deposits in the outermost calorimeter layer is tested. Quantitative estimates of the energy lost beyond the calorimeter are obtained using a tag-and-probe technique.

\subsection{Event selection for punch-through analysis}

Anti- $k_{t}$ jets with $R=0.4$ calibrated with the EM+JES scheme are used in this study. Jets with $|\eta|<1.2$ are used. Events with at least two jets are retained, if the highest $p_{\mathrm{T}}$ jet satisfies $p_{\mathrm{T}}^{j 1}>120 \mathrm{GeV}$ and the second highest $p_{\mathrm{T}}$ jet satisfies $p_{\mathrm{T}}^{j 2}>80 \mathrm{GeV}$. The two leading jets are required to be back-to-back requiring $\Delta \phi>170^{\circ}$.

\subsection{Energy depositions in the hadronic calorimeter}

The energy deposits in the outermost layer of the barrel of the Tile calorimeter are a good indicator of the jet en-

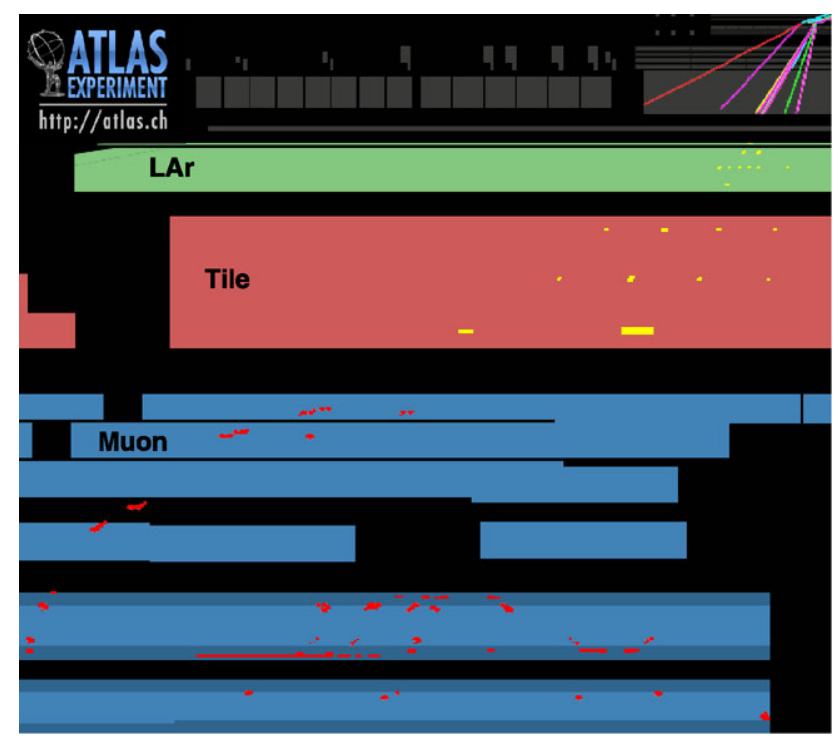

Fig. 89 Graphical representation in a zoomed $x-y$ view of an event candidate with one large transverse momentum jet $\left(p_{\mathrm{T}}^{\text {jet }}=176 \mathrm{GeV}\right)$ having a large activity in the last $\mathrm{Ti}$ le calorimeter layer $(82 \mathrm{GeV}$ at the EM scale) and in the muon detectors. The tracks in the inner detector are shown as lines in the top right, the energy deposits in the LAr and Tile calorimeters are shown as light boxes. The hits in the muon system are shown as points. There are 128 hits measured in the muon system 
ergy depositions beyond the calorimeter. These are shown in Fig. 90 for the leading and the sub-leading jet. Most jets deposit only about 3 to $7 \mathrm{GeV}$ energy in the outermost calorimeter layer. The Monte Carlo simulation gives a good description of the data for $p_{\mathrm{T}}^{\text {jet }}<80 \mathrm{GeV}$. For higher $p_{\mathrm{T}}^{\text {jet }}$ the data distribution is below the Monte Carlo simulation, but the statistical uncertainties are large.

Figure 91 shows the average energy deposition in the outermost layer of the Tile calorimeter measured at the EM scale for the leading and sub-leading jet as a function of the jet $p_{\mathrm{T}}$. The energy deposited in the outermost Tile layer is only a few GeV. This is mainly due to the good calorimeter coverage of the ATLAS detector with a thickness of $\lambda=11$ interaction lengths at $\eta=0$ and the last $\mathrm{Tile}$ layer contributing only $\lambda=1.8$. The energy increases with rising jet $p_{\mathrm{T}}$.

The data are well described by the Monte Carlo simulation in the low $p_{\mathrm{T}}^{\text {jet }}$ region. Starting from about $400 \mathrm{GeV}$ the data tend to be 5-10\% above the Monte Carlo simulation. For high $p_{\mathrm{T}}^{\text {jet }}$ the statistical uncertainties are large.

\subsection{Dijet balance as an indication of punch-through}

The relative calorimeter response between the two jets in a dijet event can be measured using the dijet $p_{\mathrm{T}}$ balance method. In Sect. 9.6 the reference jet is chosen as a wellmeasured object in the central detector region that is used to assess the JES uncertainty of the probe jet in the forward region. However, in the context of punch-through such a distinction cannot be made. Jet punch-through can occur in any detector pseudorapidity region. Fluctuations in the particle composition or in the hadronic shower occur with equal probability for both jets and it is not possible to know a priori which of the jets will be affected.

A different approach is therefore employed. The energy lost beyond the calorimeter will create a component of the missing transverse energy $E_{\mathrm{T}}^{\text {miss }}$ in the direction of the punch-through jet. The punch-through jet can therefore be defined as the one that is closest to the $E_{\mathrm{T}}^{\text {miss }} \phi$-direction. The asymmetry between the transverse momentum of the reference jet ( $p_{\mathrm{T}}^{\text {reference }}$ ) away from the $E_{\mathrm{T}}^{\text {miss }}$ direction and the punch-through jet, acting as probe jet ( $p_{\mathrm{T}}^{\text {punch-through }}$ ), can then be measured as a function of the energy deposition of the jet that is the candidate for punch-through.

Figure 92a show the average ratio of the transverse momentum of the punch-through jet to the one of the reference jet as a function of the energy depositions in the last Tile calorimeter layer measured at the EM-scale with respect to the total jet energy. Figure 92b show the average jet response where $E_{\mathrm{T}}^{\text {miss }}>40 \mathrm{GeV}$ is required. This selection enhances possible punch-through effects. The transverse momentum of the punch-through jet is lower than that of the reference jet as is shown in Fig. 92 for both the inclusive selection and the one with large $E_{\mathrm{T}}^{\text {miss }}$. This is a bias due to the selection of the punch-through jet as the one pointing

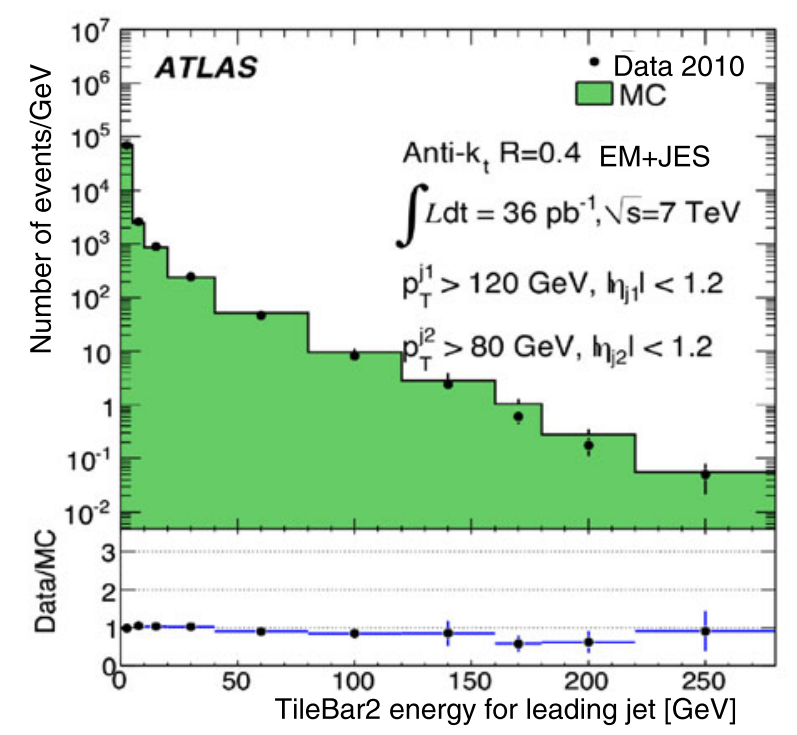

(a) Leading jet

Fig. 90 Distribution of the deposited energy in the outermost layer of the Tile barrel calorimeter measured at the EM-scale for the leading (a) and the sub-leading (b) jet. Anti- $k_{t}$ jets with $R=0.4$ within $|\eta|<1.2$ and calibrated with the EM+JES scheme are used. The lead-

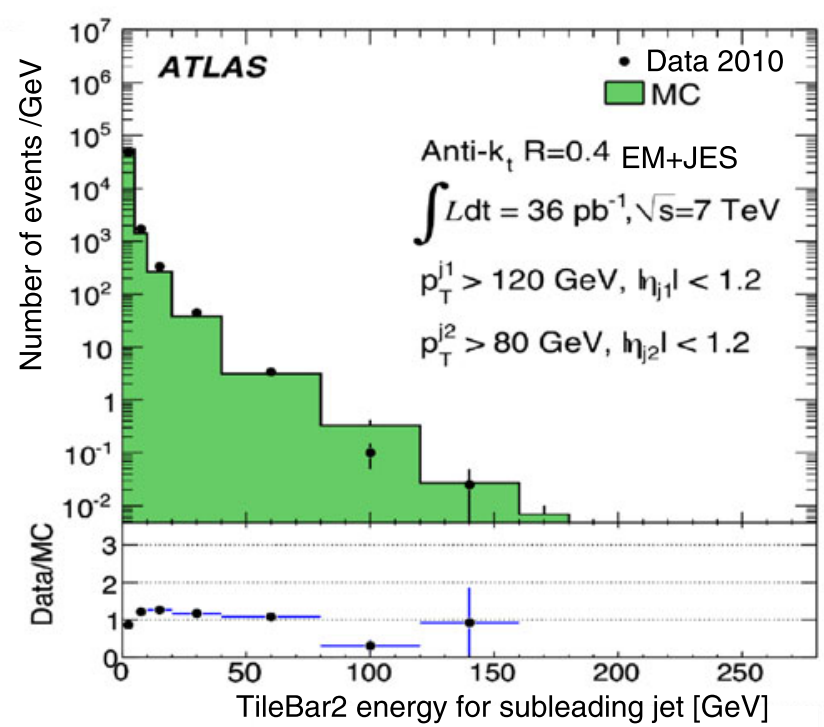

(b) Sub-leading jet

ing jet is required to be above $p_{\mathrm{T}}^{\text {jet }}>120 \mathrm{GeV}$ the sub-leading jet is required to be above $p_{\mathrm{T}}^{\text {jet }}>80 \mathrm{GeV}$. Only statistical uncertainties are shown 


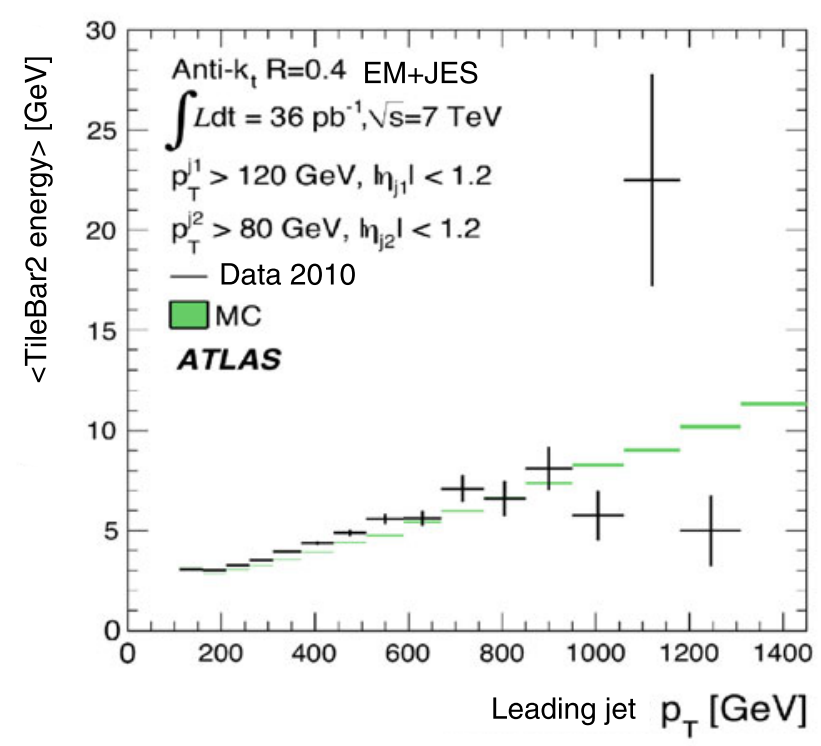

(a) Leading jet

Fig. 91 Average energy deposited in the outermost layer of the Tile barrel calorimeter at the EM-scale for the leading (a) and the sub-leading jet (b) as a function of the jet transverse momentum. Anti- $k_{t}$ jets with $R=0.4$ within $|\eta|<1.2$ and calibrated with

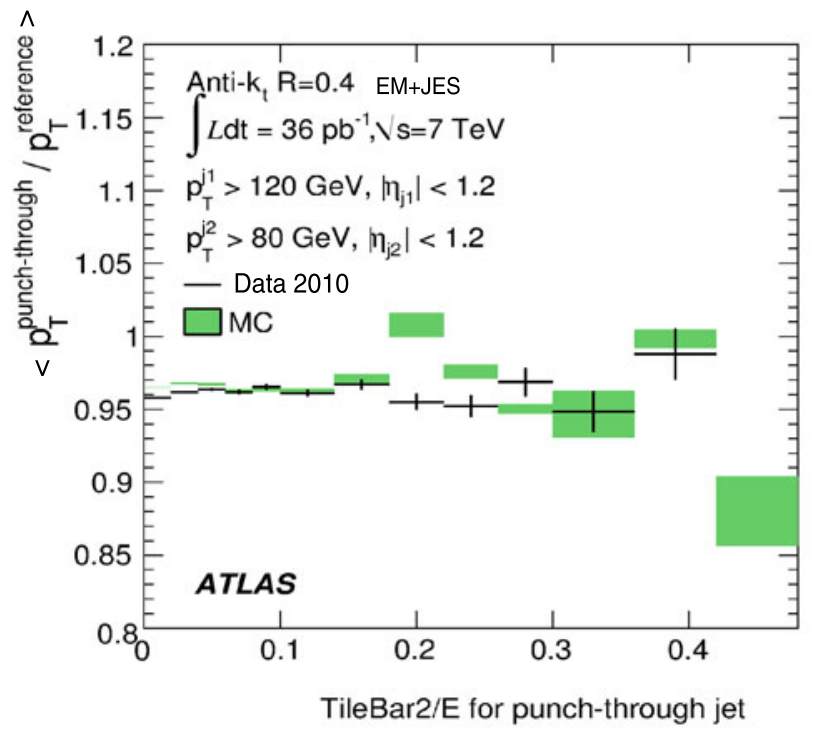

(a) Inclusive events

Fig. 92 Average relative jet response as a function of the energy deposited in the outermost layer of the Tile barrel calorimeter at the EM-scale divided by the total jet energy. In (a) the standard event selection is employed, whilst in (b) an extra requirement is placed upon the missing transverse momentum in the event. Anti- $k_{t}$ jets with $R=0.4$

to the direction of the missing transverse momentum. There is no dependence on the energy fraction in the outermost layer, indicating that energy losses due to punch-through

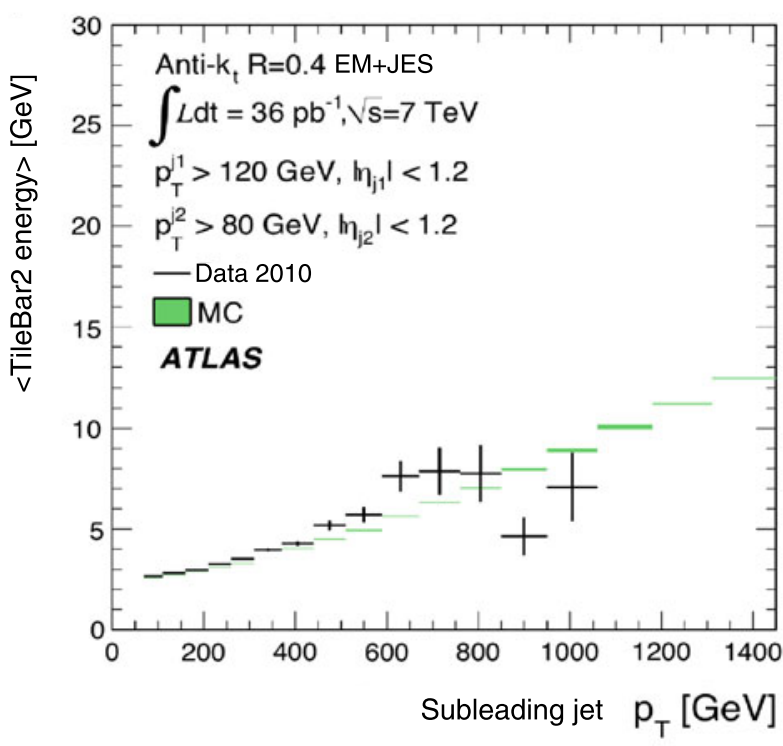

(b) Sub-leading jet

the EM+JES scheme are used. The leading jet is required to be above $p_{\mathrm{T}}^{\text {jet }}>120 \mathrm{GeV}$ the sub-leading jet is required to be above $p_{\mathrm{T}}^{\text {jet }}>80 \mathrm{GeV}$. Only statistical uncertainties are shown

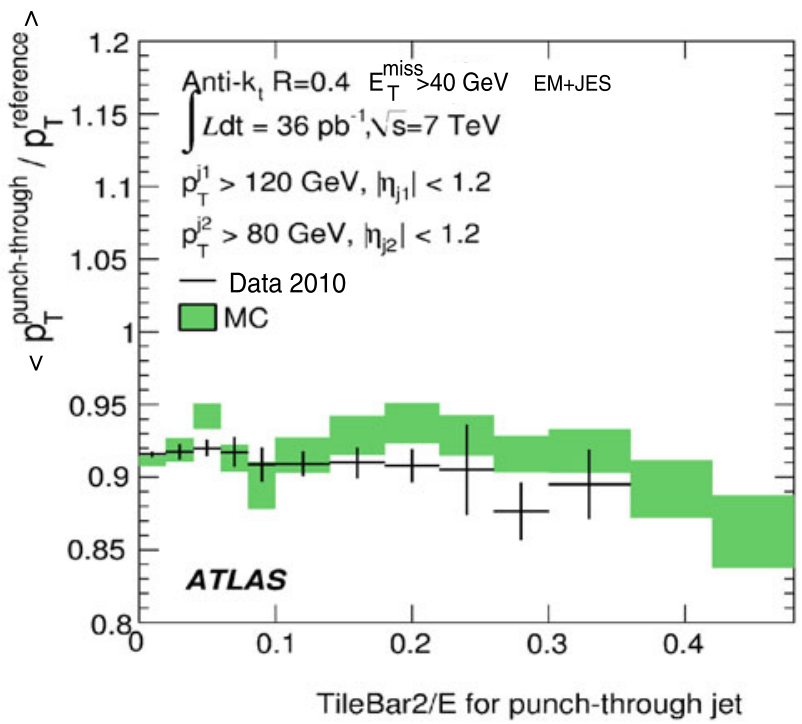

(b) Events with large $E_{\mathrm{T}}^{\text {miss }}$

within $|\eta|<1.2$ and calibrated with the EM+JES scheme are used. The leading jet is required to be above $p_{\mathrm{T}}^{\text {jet }}>120 \mathrm{GeV}$ the sub-leading jet is required to be above $p_{\mathrm{T}}^{\text {jet }}>80 \mathrm{GeV}$. Only statistical uncertainties are shown (errors bars for data, shaded band for Monte Carlo simulation)

are small for the jet $p_{\mathrm{T}}$ range considered in this study. The Monte Carlo simulation describes the data within the statistical uncertainties. 


\subsection{Summary of the jet punch-through study}

The energy deposition in the outer layer of the Tile calorimeter and its dependence on the transverse jet energy is described by the Monte Carlo simulation. This indicates that the Monte Carlo simulation is able to describe energy deposited beyond the calorimeter. For the few jets that are potentially affected by punch-through no additional uncertainty due to punch-through effects is assigned.

\section{Summary}

The jet energy scale (JES) and its systematic uncertainty for various jet calibration schemes are determined for jets measured with the ATLAS detector in the 2010 data set corresponding to an integrated luminosity of $38 \mathrm{pb}^{-1}$. Jets produced in proton-proton collisions at the LHC with a centreof-mass energy of $\sqrt{s}=7 \mathrm{TeV}$ are reconstructed with the anti- $k_{t}$ algorithm with distance parameters $R=0.4$ or $R=$ 0.6. The energy and the direction of the jets are calibrated with simple factors derived from Monte Carlo simulations for transverse jet momenta $p_{\mathrm{T}}^{\text {jet }} \geq 20 \mathrm{GeV}$ and pseudorapidities $|\eta|<4.5$ using various jet calibration schemes.

In the simplest calibration scheme $(\mathrm{EM}+\mathrm{JES})$, where the JES correction factor is directly applied to the calorimeter measurement at the electromagnetic scale, the JES systematic uncertainty is estimated using the single hadron response measured in situ and in test-beams and by studying systematic variations in Monte Carlo simulations. The transverse momentum balance between central and forward jets in dijet events is used to derive the JES uncertainty for forward jets.

In the central region, $|\eta|<0.8$, the EM+JES uncertainty is lower than $4.6 \%$ for all jets with $p_{\mathrm{T}}^{\text {jet }}>20 \mathrm{GeV}$ and less than $2.5 \%$ for jets with $60 \leq p_{\mathrm{T}}^{\text {jet }}<800 \mathrm{GeV}$. Jets with transverse momenta in the TeV-regime have a JES uncertainty of 3 to $4 \%$. Towards the forward region the EM+JES uncertainty increases, taking differences between the Monte Carlo event generators PYTHIA and HERWIG into account when deriving the relative $\eta$-intercalibration uncertainty. The largest JES uncertainty of about $14 \%$ is found for low $p_{\mathrm{T}}$ jets with $20 \leq p_{\mathrm{T}}^{\text {jet }}<30 \mathrm{GeV}$ at $3.6 \leq|\eta|<4.5$. The jet energy scale uncertainty is found to be similar for jets reconstructed with both jet distance parameters studied: $R=0.4$ and $R=0.6$.

The additional energy induced by superimposed events from multiple proton-proton collisions in the same bunch crossing (pile-up) is determined to be $0.5 \mathrm{GeV}$ per additional reconstructed vertex measured on the EM-scale. The JES uncertainty after applying the pile-up correction is estimated as a function of the number of primary vertices. In the case of two primary vertices per event, the uncertainty due to pile-up for jets with $p_{\mathrm{T}}^{\text {jet }} \approx 20 \mathrm{GeV}$ and $0.3 \leq|\eta|<0.8$ is about $1 \%$, while it amounts to about $2 \%$ for jets with $2.1 \leq|\eta|<2.8$. For jets with transverse momentum above $200 \mathrm{GeV}$, the uncertainty due to pile-up is negligible for all jets.

The JES and its uncertainty are validated up to $p_{\mathrm{T}}^{\text {jet }} \lesssim$ $1 \mathrm{TeV}$ to the level of a few per cent using several in situ techniques by comparing the high $p_{\mathrm{T}}$ jet to a well known reference recoiling against it. These reference objects include the sum of the transverse track momenta associated to the jet, a system of low $p_{\mathrm{T}}$ jets or the photon $p_{\mathrm{T}}$. The track-based method covers the full $p_{\mathrm{T}}^{\text {jet }}$ range and has the highest statistical precision. However, the systematic uncertainty of the method is as large as $6 \%$ for very low $p_{\mathrm{T}}$ jets and about 3-4 $\%$ for $40 \leq p_{\mathrm{T}}^{\text {jet }}<800 \mathrm{GeV}$ and somewhat higher for jets outside this $p_{\mathrm{T}}^{\text {jet }}$ range. The $\gamma$-jet method has a systematic uncertainty of about $1 \%$, but is still statistically limited and reaches only $p_{\mathrm{T}}^{\text {jet }} \lesssim 300 \mathrm{GeV}$. Balancing very high $p_{\mathrm{T}}$ jets against a recoil system of lower $p_{\mathrm{T}}$ jets allows the validation of the high $p_{\mathrm{T}}$ jets within $5 \%$ up to $1 \mathrm{TeV}$. In this range the statistical uncertainty is roughly equivalent to, or smaller than, the systematic uncertainty.

The JES uncertainty derived from a combination of in situ techniques is compatible to the one derived from the single hadron response measurements over a wide kinematic range, but it is larger for very low and very large $p_{\mathrm{T}}^{\text {jet }}$, where the uncertainties of the in situ methods are large.

More sophisticated jet energy calibration schemes based on cell energy density weighting or jet properties are studied. These aim at improving the jet energy resolution and reduce the flavour dependence of the jet response.

The JES uncertainty for the GS jet calibration scheme is given by the sum in quadrature of the EM+JES uncertainty and the uncertainty associated to the GS corrections. The latter is conservatively taken to be $0.5 \%$ for $30 \leq p_{\mathrm{T}}<$ $800 \mathrm{GeV}$ and $|\eta|<2.1$ and $1 \%$ for $p_{\mathrm{T}}^{\text {jet }}<30 \mathrm{GeV}$ and $2.1<|\eta|<2$.8. These uncertainties are also supported by in situ measurements with the $\gamma$-jet and the dijet technique.

The global cell weighting scheme (GCW) derives cell weights by optimising the resolution of reconstructed jets relative to their respective truth jets. The local cluster calibration (LCW) derives energy corrections for calorimeter clusters using single hadron Monte Carlo simulations. The JES uncertainty is obtained from in situ techniques. Over a wide kinematic range the JES uncertainties for the various schemes are similar, except at very low and very high $p_{\mathrm{T}}^{\text {jet }}$ where the uncertainty from the in situ techniques are larger. The LCW+JES and the GCW+JES scheme show similar performance.

For all jet calibration methods additional uncertainties are derived for close-by jet topologies and for response differences for jets induced by quarks, gluons or heavy flavour 
quarks. A method to reduce the uncertainty due to uncertainties on the quark and gluon composition in a given event sample is shown. The uncertainty due to close-by jets is largest for low $p_{\mathrm{T}}$ jets, but is at most below 2 to $3 \%$. The additional JES uncertainty of jets containing $B$-hadrons is about $2 \%$ for low- $p_{\mathrm{T}}$ jets and smaller than $1 \%$ for jets with $p_{\mathrm{T}}^{\text {jet }}>200 \mathrm{GeV}$.

The jet reconstruction efficiency is derived using the Monte Carlo simulation and the systematic uncertainty evaluated with a tag-and-probe technique using track jets. The jet reconstruction efficiency is well-described by the Monte Carlo simulation. The associated systematic uncertainty is below $2 \%$ for jets with $p_{\mathrm{T}}^{\text {jet }}<30 \mathrm{GeV}$ and negligible for higher $p_{\mathrm{T}}^{\text {jet }}$.

The Monte Carlo simulation gives a good description of the main aspects of the data. Detailed studies show that the calorimeter cell energy densities in jets, the calorimeter energy topology induced by jets and track related properties are well-described. This includes the amount of energy deposited in the outermost calorimeter layers from which it is inferred that the JES uncertainty due to energy leaking beyond the calorimeter is small and well-described by the Monte Carlo simulation. No additional uncertainty for punch-through effects is assigned for high- $p_{\mathrm{T}}$ jets.

In summary, the precision of the jet energy measurement with the ATLAS detector has been established using various techniques in the first year of proton-proton collisions at the LHC. In the central detector the jet energy can be measured with a precision of about 2 to $3 \%$ over a wide transverse momentum range.

This excellent performance would not have been possible without a very detailed understanding of the detector and sophisticated calorimeter calibration procedures as well as the good description of the ATLAS detector in the Monte Carlo simulation.

Acknowledgements We thank CERN for the very successful operation of the LHC, as well as the support staff from our institutions without whom ATLAS could not be operated efficiently.

We acknowledge the support of ANPCyT, Argentina; YerPhI, Armenia; ARC, Australia; BMWF, Austria; ANAS, Azerbaijan; SSTC, Belarus; CNPq and FAPESP, Brazil; NSERC, NRC and CFI, Canada; CERN; CONICYT, Chile; CAS, MOST and NSFC, China; COLCIENCIAS, Colombia; MSMT CR, MPO CR and VSC CR, Czech Republic; DNRF, DNSRC and Lundbeck Foundation, Denmark; ARTEMIS, European Union; IN2P3-CNRS, CEA-DSM/IRFU, France; GNAS, Georgia; BMBF, DFG, HGF, MPG and AvH Foundation, Germany; GSRT, Greece; ISF, MINERVA, GIF, DIP and Benoziyo Center, Israel; INFN, Italy; MEXT and JSPS, Japan; CNRST, Morocco; FOM and NWO, Netherlands; RCN, Norway; MNiSW, Poland; GRICES and FCT, Portugal; MERYS (MECTS), Romania; MES of Russia and ROSATOM, Russian Federation; JINR; MSTD, Serbia; MSSR, Slovakia; ARRS and MVZT, Slovenia; DST/NRF, South Africa; MICINN, Spain; SRC and Wallenberg Foundation, Sweden; SER, SNSF and Cantons of Bern and Geneva, Switzerland; NSC, Taiwan; TAEK, Turkey; STFC, the Royal Society and Leverhulme Trust, United Kingdom; DOE and NSF, United States of America.
The crucial computing support from all WLCG partners is acknowledged gratefully, in particular from CERN and the ATLAS Tier-1 facilities at TRIUMF (Canada), NDGF (Denmark, Norway, Sweden), CC-IN2P3 (France), KIT/GridKA (Germany), INFN-CNAF (Italy), NL-T1 (Netherlands), PIC (Spain), ASGC (Taiwan), RAL (UK) and BNL (USA) and in the Tier-2 facilities worldwide.

Open Access This article is distributed under the terms of the Creative Commons Attribution License which permits any use, distribution, and reproduction in any medium, provided the original author(s) and the source are credited.

\section{References}

1. ATLAS Collaboration, Measurement of inclusive jet and dijet cross sections in proton-proton collisions at $7 \mathrm{TeV}$ centre-ofmass energy with the ATLAS detector. Eur. Phys. J. C 71, 1512 (2011). arXiv:1009.5908 [hep-ex]

2. ATLAS Collaboration, Measurement of multijet cross sections in proton-proton collisions at a $7 \mathrm{TeV}$ center-of-mass energy. Eur. Phys. J. C 71 (2011). arXiv:1107.2092 [hep-ex]

3. ATLAS Collaboration, Study of jet shapes in inclusive jet production in pp collisions at $\sqrt{s}=7 \mathrm{TeV}$ using the ATLAS detector. Phys. Rev. D 83, 052003 (2011). arXiv:1101.0070 [hep-ex]

4. ATLAS Collaboration, Measurement of dijet azimuthal decorrelations in pp collisions at $\sqrt{s}=7 \mathrm{TeV}$. Phys. Rev. Lett. 106, 172002 (2011). arXiv:1102.2696 [hep-ex]

5. ATLAS Collaboration, Measurement of dijet production with a veto on additional central jet activity in pp collisions at $\sqrt{s}=$ $7 \mathrm{TeV}$ using the ATLAS detector. J. High Energy Phys. 09, 053 (2011). arXiv:1107.1641 [hep-ex]

6. ATLAS Collaboration, Measurement of the production cross section for $W$-bosons in association with jets in pp collisions at $\sqrt{s}=7 \mathrm{TeV}$ with the ATLAS detector. Phys. Lett. B 698, 325345 (2011). arXiv:1012.5382 [hep-ex]

7. ATLAS Collaboration, Measurement of the cross-section for bjets produced in association with a $Z$ boson at $\sqrt{s}=7 \mathrm{TeV}$ with the ATLAS detector. arXiv:1109.1403 [hep-ex]

8. ATLAS Collaboration, Search for quark contact interactions in dijet angular distributions in pp collisions at $\sqrt{s}=7 \mathrm{TeV}$ measured with the ATLAS detector. Phys. Lett. B 694, 327-345 (2011). arXiv:1009.5069 [hep-ex]

9. ATLAS Collaboration, Search for new particles in two-jet final states in $7 \mathrm{TeV}$ proton-proton collisions with the ATLAS detector at the LHC. Phys. Rev. Lett. 105, 161801 (2010). arXiv:1008. 2461 [hep-ex]

10. ATLAS Collaboration, Search for new physics in dijet mass and angular distributions in pp collisions at $\sqrt{s}=7 \mathrm{TeV}$ measured with the ATLAS detector. New J. Phys. 13, 053044 (2011). arXiv:1103.3864 [hep-ex]

11. ATLAS Collaboration, Search for supersymmetry using final states with one lepton, jets, and missing transverse momentum with the ATLAS detector in $\sqrt{s}=7 \mathrm{TeV}$ pp collisions. Phys. Rev. Lett. 106, 131802 (2011). arXiv:1102.2357 [hep-ex]

12. ATLAS Collaboration, Search for squarks and gluinos using final states with jets and missing transverse momentum with the ATLAS detector in $\sqrt{s}=7 \mathrm{TeV}$ proton-proton collisions. Phys. Lett. B 701, 186-203 (2011). arXiv:1102.5290 [hep-ex]

13. ATLAS Collaboration, Search for supersymmetry in pp collisions at $\sqrt{s}=7 \mathrm{TeV}$ in final states with missing transverse momentum and b-jets. Phys. Lett. B 701, 398-416 (2011). arXiv: 1103.4344 [hep-ex]

14. A. Bhatti et al. (CDF Collaboration), Determination of the jet energy scale at the collider detector at Fermilab. Nucl. Instrum. Methods Phys. Res., Sect. A 566, 375-412 (2006). arXiv:hep-ex/ 0510047 
15. B. Abbott et al. (D0 Collaboration), Determination of the absolute jet energy scale in the DZERO calorimeters. Nucl. Instrum. Methods Phys. Res., Sect. A 424, 352-394 (1999)

16. S. Chatrchyan et al. (CMS Collaboration), Determination of jet energy calibration and transverse momentum resolution in CMS. J. Instrum. 6, P11002 (2011). arXiv:1107.4277 [physics.ins-det]

17. V.M. Abazov et al. (D0 Collaboration), Measurement of the inclusive jet cross section in $p \bar{p}$ collisions at $\sqrt{s}=1.96 \mathrm{TeV}$. arXiv: 1110.3771 [hep-ex]

18. T. Aaltonen et al. (CDF Collaboration), Measurement of the inclusive jet cross section at the Fermilab Tevatron $p$ anti-p collider using a cone-based jet algorithm. Phys. Rev. D 78, 052006 (2008). arXiv:0807.2204 [hep-ex]

19. P. Bagnaia et al. (UA2 Collaboration), Measurement of production and properties of jets at the CERN anti-p p collider. Z. Phys. C 20, 117-134 (1983)

20. C. Adloff et al. (H1 Collaboration), Measurement of neutral and charged current cross-sections in positron proton collisions at large momentum transfer. Eur. Phys. J. C 13, 609-639 (2000). arXiv:hep-ex/9908059

21. F. Aaron et al. (H1 Collaboration), A precision measurement of the inclusive ep scattering cross section at HERA. Eur. Phys. J. C 64, 561-587 (2009). arXiv:0904.3513 [hep-ex]

22. S. Chekanov et al. (ZEUS Collaboration), High mass dijet crosssections in photoproduction at HERA. Phys. Lett. B 531, 9-27 (2002). arXiv:hep-ex/0112030

23. S. Chekanov et al. (ZEUS Collaboration), Dijet photoproduction at HERA and the structure of the photon. Eur. Phys. J. C 23, 615-631 (2002). arXiv:hep-ex/0112029

24. M. Wing, Setting the jet energy scale for the ZEUS calorimeter. arXiv:hep-ex/0206036

25. M. Cacciari, G.P. Salam, G. Soyez, The anti- $k_{t}$ jet clustering algorithm. J. High Energy Phys. 04, 063 (2008). arXiv:0802.1189 [hep-ph]

26. ATLAS Collaboration, Expected performance of the ATLAS experiment-detector, trigger and physics, CERN-OPEN-2008020. CERN, Geneva, September 2009. arXiv:0901.0512 [hepex]

27. ATLAS Collaboration, Jet energy resolution in proton-proton collisions at $\sqrt{(s)}=7 \mathrm{TeV}$ recorded in 2010 with the ATLAS detector, CERN-PH-EP-2012-191. Eur. Phys. J. (2012, submitted). arXiv:1210.6210 [hep-ex]

28. ATLAS Collaboration, Single hadron response measurement and calorimeter jet energy scale uncertainty with the ATLAS detector. Eur. Phys. J. (2012, accepted). arXiv:1203.1302v1 [hep-ex]

29. ATLAS Collaboration, The ATLAS experiment at the CERN Large Hadron Collider. J. Instrum. 3, S08003 (2008)

30. ATLAS Collaboration, Performance of the ATLAS trigger system in 2010. Eur. Phys. J. C 72, 1849 (2012). doi:10.1140/epjc/ s10052-011-1849-1. arXiv:1110.1530 [hep-ex]

31. E. Abat et al., Combined performance studies for electrons at the 2004 ATLAS combined test-beam. J. Instrum. 5, P11006 (2010)

32. M. Aharrouche et al., Measurement of the response of the ATLAS liquid argon barrel calorimeter to electrons at the 2004 combined test-beam. Nucl. Instrum. Methods Phys. Res., Sect. A 614, 400-432 (2010)

33. J. Colas et al., Response uniformity of the ATLAS liquid argon electromagnetic calorimeter. Nucl. Instrum. Methods Phys. Res., Sect. A 582, 429-455 (2007). arXiv:0709.1094 [physics.ins-det]

34. M. Aharrouche et al., Energy linearity and resolution of the ATLAS electromagnetic barrel calorimeter in an electron test-beam. Nucl. Instrum. Methods Phys. Res., Sect. A 568, 601-623 (2006)

35. P. Adragna et al., Testbeam studies of production modules of the ATLAS Tile calorimeter. Nucl. Instrum. Methods Phys. Res., Sect. A 606, 362-394 (2009)
36. J. Pinfold et al., Performance of the ATLAS liquid argon endcap calorimeter in the pseudorapidity region $2.5<|\eta|<4.0$ in beam tests. Nucl. Instrum. Methods Phys. Res., Sect. A 593, 324-342 (2008)

37. C. Cojocaru et al., Hadronic calibration of the ATLAS liquid argon end-cap calorimeter in the pseudorapidity region $1.6<|\eta|<$ 1.8 in beam tests. Nucl. Instrum. Methods Phys. Res., Sect. A 531, 481-514 (2004)

38. ATLAS Collaboration, Readiness of the ATLAS liquid argon calorimeter for LHC collisions. Eur. Phys. J. C 70, 723-753 (2010). arXiv:0912.2642 [physics.ins-det]

39. J. Archambault et al., Energy calibration of the ATLAS liquid argon forward calorimeter. J. Instrum. 3, P02002 (2008)

40. M. Aharrouche et al., Study of the response of ATLAS electromagnetic liquid argon calorimeters to muons. Nucl. Instrum. Methods Phys. Res., Sect. A 606, 419-431 (2009)

41. ATLAS Collaboration, Readiness of the ATLAS Tile calorimeter for LHC collisions. Eur. Phys. J. C 70, 1193-1236 (2010). arXiv: 1007.5423 [physics.ins-det]

42. ATLAS Collaboration, Electron performance measurements with the ATLAS detector using the 2010 LHC proton-proton collision data. Eur. Phys. J. C 72, 1909 (2012). arXiv:1110.3174 [hep-ex]

43. A. Buckley et al., General-purpose event generators for LHC physics. Phys. Rep. 504, 145-233 (2011). arXiv:1101.2599 [hep$\mathrm{ph}]$

44. T. Sjostrand, S. Mrenna, P.Z. Skands, PYTHIA 6.4 physics and manual. J. High Energy Phys. 0605, 026 (2006). arXiv:hep-ph/ 0603175

45. R. Corke, T. Sjostrand, Improved parton showers at large transverse momenta. Eur. Phys. J. C 69, 1-18 (2010). arXiv:1003. 2384 [hep-ph]

46. T. Sjostrand, P.Z. Skands, Transverse-momentum-ordered showers and interleaved multiple interactions. Eur. Phys. J. C 39, 129 154 (2005). arXiv:hep-ph/0408302

47. B. Andersson et al., Parton fragmentation and string dynamics. Phys. Rep. 97, 31-145 (1983)

48. A. Sherstnev, R.S. Thorne, Parton distributions for LO generators. Eur. Phys. J. C 55, 553-575 (2008). arXiv:0711.2473 [hep$\mathrm{ph}]$

49. ATLAS Collaboration, Charged particle multiplicities in pp interactions at $\sqrt{s}=0.9$ and $7 \mathrm{TeV}$ in a diffractive limited phasespace measured with the ATLAS detector at the LHC and new PYTHIA6 tune, ATLAS-CONF-2010-031, May 2010

50. ATLAS Collaboration, Charged particle multiplicities in p-p interactions for track $p_{\mathrm{T}}>100 \mathrm{MeV}$ at $\sqrt{s}=0.9$ and $7 \mathrm{TeV}$ measured with the ATLAS detector at the LHC, ATLAS-CONF2010-046, July 2010

51. P.Z. Skands, Tuning Monte Carlo generators: the Perugia tunes. Phys. Rev. D 82, 074018 (2010). arXiv:1005.3457 [hep-ph]

52. G. Marchesini et al., Monte Carlo simulation of general hard processes with coherent QCD radiation. Nucl. Phys. B 310, 461 (1988)

53. G. Marchesini et al., A Monte Carlo event generator for simulating hadron emission reactions with interfering gluons. Comput. Phys. Commun. 67, 465-508 (1991)

54. G. Corcella et al., HERWIG 6.5 release note. arXiv:hep-ph/ 0210213

55. B.R. Webber, A QCD model for jet fragmentation including soft gluon interference. Nucl. Phys. B 238, 492 (1984)

56. J.M. Butterworth, J.R. Forshaw, M.H. Seymour, Multiparton interactions in photoproduction at HERA. Z. Phys. C 72, 637-646 (1996). arXiv:hep-ph/9601371

57. ATLAS Collaboration, First tuning of HERWIG/JIMMY to ATLAS data, ATL-PHYS-PUB-2010-014, October 2010

58. M. Bahr et al., Herwig++ physics and manual. Eur. Phys. J. C 58, 639-707 (2008). arXiv:0803.0883 [hep-ph] 
59. M. Bahr, S. Gieseke, M.H. Seymour, Simulation of multiple partonic interactions in Herwig++. J. High Energy Phys. 07, 076 (2008). arXiv:0803.3633 [hep-ph]

60. M.L. Mangano, M. Moretti, F. Piccinini, R. Pittau, A.D. Polosa, ALPGEN, a generator for hard multiparton processes in hadronic collisions. J. High Energy Phys. 07, 001 (2003). arXiv:hep-ph/ 0206293

61. M.L. Mangano, M. Moretti, R. Pittau, Multijet matrix elements and shower evolution in hadronic collisions: $W b \bar{b}+n$ jets as a case study. Nucl. Phys. B 632, 343-362 (2002). arXiv:hep-ph/ 0108069

62. ATLAS Collaboration, ATLAS MC tunes for MC09, ATLASPHYS-PUB-2010-002, March 2010

63. J. Pumplin et al., New generation of parton distributions with uncertainties from global QCD analysis. J. High Energy Phys. 07, 012 (2002). arXiv:hep-ph/0201195

64. S. Agostinelli et al. (GEANT4 Collaboration), GEANT4: a simulation toolkit. Nucl. Instrum. Methods Phys. Res., Sect. A 506, 250-303 (2003)

65. ATLAS Collaboration, The ATLAS simulation infrastructure. Eur. Phys. J. C 70, 823-874 (2010). arXiv:1005.4568 [physics.ins-det]

66. A. Ribon et al., Status of Geant 4 hadronic physics for the simulation of LHC experiments at the start of the LHC physics program, CERN-LCGAPP-2010-02, May 2010

67. G. Folger, J. Wellisch, String parton models in GEANT4. arXiv: nucl-th/0306007

68. N.S. Amelin et al., Transverse flow and collectivity in ultrarelativistic heavy ion collisions. Phys. Rev. Lett. 67, 1523-1526 (1991)

69. N.S. Amelin, L.P. Csernai, E.F. Staubo, D. Strottman, Collectivity in ultrarelativistic heavy ion collisions. Nucl. Phys. A 544, 463-466 (1992)

70. L.V. Bravina, L.P. Csernai, P. Levai, N.S. Amelin, D. Strottman, Fluid dynamics and quark gluon string model: what we can expect for $\mathrm{Au}+\mathrm{Au}$ collisions at 11.6-A/GeV/c. Nucl. Phys. A 566, 461-464 (1994)

71. L.V. Bravina, Scaling violation of transverse flow in heavy ion collisions at AGS energies. Phys. Lett. B 344, 49-54 (1995)

72. H.W. Bertini, Intranuclear-cascade calculation of the secondary nucleon spectra from nucleon-nucleus interactions in the energy range 340 to $2900 \mathrm{MeV}$ and comparisons with experiment. Phys. Rev. A 188, 1711-1730 (1969)

73. M.P. Guthrie, R.G. Alsmiller, H.W. Bertini, Calculation of the capture of negative pions in light elements and comparison with experiments pertaining to cancer radiotherapy. Nucl. Instrum. Methods Phys. Res. 66, 29-36 (1968)

74. M.P. Guthrie, H.W. Bertini, News item results from mediumenergy intranuclear-cascade calculation. Nucl. Phys. A 169, 670$672(1971)$

75. N.V. Stepanov, Statistical modeling of fission of excited atomic nuclei. 2. Calculation and comparison with experiment, in Russian, ITEP, Moscow, 1988. Preprint:ITEP-88-55

76. P. Adragna et al., Measurement of pion and proton response and longitudinal shower profiles up to 20 nuclear interaction lengths with the ATLAS Tile calorimeter. Nucl. Instrum. Methods Phys. Res., Sect. A 615, 158-181 (2010)

77. E. Abat et al., Response and shower topology of 2 to $180 \mathrm{GeV}$ pions measured with the ATLAS barrel calorimeter at the CERN test-beam and comparison to Monte Carlo simulations, ATLCAL-PUB-2010-001, May 2010

78. E. Abat et al., Study of energy response and resolution of the ATLAS barrel calorimeter to hadrons of energies from $20 \mathrm{GeV}$ to $350 \mathrm{GeV}$. Nucl. Instrum. Methods Phys. Res., Sect. A 621, 134-150 (2010)
79. E. Abat et al., Study of the response of the ATLAS central calorimeter to pions of energies from 3 to $9 \mathrm{GeV}$. Nucl. Instrum. Methods Phys. Res., Sect. A 607, 372-386 (2009)

80. A.E. Kiryunin, H. Oberlack, D. Salihagic, P. Schacht, P. Strizenec, GEANT4 physics evaluation with testbeam data of the ATLAS hadronic end-cap calorimeter. Nucl. Instrum. Methods Phys. Res., Sect. A 560, 278-290 (2006)

81. ATLAS Collaboration, A measurement of the material in the ATLAS inner detector using secondary hadronic interactions. arXiv: 1110.6191 [hep-ex]

82. ATLAS Collaboration, Photon conversions at $\sqrt{s}=900 \mathrm{GeV}$ measured with the ATLAS detector, ATLAS-CONF-2010-007, June 2010

83. ATLAS Collaboration, Probing the material in front of the ATLAS electromagnetic calorimeter with energy flow from $\sqrt{s}=$ $7 \mathrm{TeV}$ minimum bias events. ATLAS-CONF-2010-037, July 2010

84. ATLAS Collaboration, Luminosity determination in p-p collisions at $\sqrt{s}=7 \mathrm{TeV}$ using the ATLAS detector at the LHC. Eur. Phys. J. C 71, 1630 (2011). arXiv:1101.2185 [hep-ex]

85. R. Achenbach et al., The ATLAS Level-1 calorimeter trigger, ATL-DAQ-PUB-2008-001, January 2008

86. M. Cacciari, G.P. Salam, Dispelling the $N^{3}$ myth for the $k_{t}$ jetfinder. Phys. Lett. B 641, 57-61 (2006)

87. M. Cacciari, G.P. Salam, G. Soyez. http://fastjet.fr/

88. W. Lampl et al., Calorimeter clustering algorithms: description and performance, ATL-LARG-PUB-2008-002, April 2008

89. ATLAS Collaboration, Study of the material budget in the ATLAS inner detector with $K_{S}^{0}$ decays in collision data at $\sqrt{s}=$ $900 \mathrm{GeV}$, ATLAS-CONF-2010-019, July 2010

90. ATLAS Collaboration, Mapping the material in the ATLAS inner detector using secondary hadronic interactions in $7 \mathrm{TeV}$ collisions. ATLAS-CONF-2010-058, July 2010

91. ATLAS Collaboration, Measurement of the jet fragmentation function and transverse profile in proton-proton collisions at a center-of-mass energy of $7 \mathrm{TeV}$ with the ATLAS detector. Eur. Phys. J. C 71, 1795 (2011). arXiv:1109.5816 [hep-ex]

92. P. Abreu et al. (DELPHI Collaboration), Tuning and test of fragmentation models based on identified particles and precision event shape data. Z. Phys. C 73, 11-60 (1996)

93. ATLAS Collaboration, Charged-particle multiplicities in p-p interactions measured with the ATLAS detector at the LHC. New J. Phys. 13, 053033 (2011). arXiv:1012.5104 [hep-ex]

94. ATLAS Collaboration, Measurement of the inclusive isolated prompt photon cross section in pp collisions at $\sqrt{s}=7 \mathrm{TeV}$ with the ATLAS detector. Phys. Rev. D 83, 052005 (2010). arXiv: 1012.4389 [hep-ex]

95. M. Davier, A. Hoecker, B. Malaescu, C.Z. Yuan, Z. Zhang, Reevaluation of the hadronic contribution to the muon magnetic anomaly using new $e^{+} e^{-} \rightarrow \pi^{+} \pi^{-}$cross section data from BABAR. Eur. Phys. J. C 66, 1 (2010). arXiv:0908.4300 [hep-ex]

96. M. Holder et al., Performance of a magnetized total absorption calorimeter between $15 \mathrm{GeV}$ and $140 \mathrm{GeV}$. Nucl. Instrum. Methods Phys. Res. 151, 69 (1978)

97. H. Abramowicz et al., The response and resolution of an iron scintillator calorimeter for hadronic and electromagnetic showers between $10 \mathrm{GeV}$ and $140 \mathrm{GeV}$. Nucl. Instrum. Methods Phys. Res. 180, 429 (1981)

98. B. Andrieu et al. (H1 Calorimeter Collaboration), Results from pion calibration runs for the H1 liquid argon calorimeter and comparisons with simulations. Nucl. Instrum. Methods Phys. Res., Sect. A 336, 499 (1993)

99. I. Abt et al. (H1 Collaboration), The tracking, calorimeter and muon detectors of the H1 experiment at HERA. Nucl. Instrum. Methods Phys. Res., Sect. A 386, 348-396 (1997) 
100. C. Issever, K. Borras, D. Wegener, An improved weighting algorithm to achieve software compensation in a fine grained LAr calorimeter. Nucl. Instrum. Methods Phys. Res., Sect. A 545, 803 (2005). arXiv:physics/0408129

101. D. Lincoln, G. Morrow, P. Kasper, A hidden bias in a common calorimeter calibration scheme. Nucl. Instrum. Methods Phys. Res., Sect. A 345, 449-452 (1994)

102. T. Barillari et al., Local hadronic calibration, ATL-LARG-PUB2009-001, December 2008

103. G. Abbiendi et al. (OPAL Collaboration), Experimental properties of gluon and quark jets from a point source. Eur. Phys. J. C 11, 217-238 (1999). arXiv:hep-ex/9903027v1

104. ATLAS Collaboration, Calibrating the b-tag efficiency and mistag rate in $35 \mathrm{pb}^{-1}$ of data with the ATLAS detector. ATLASCONF-2011-089, June 2011

105. ATLAS Collaboration, Measurement of the top quark-pair production cross section with ATLAS in pp collisions at $\sqrt{s}=$ 7 TeV. Eur. Phys. J. C 71, 1577 (2011). arXiv:1012.1792 [hepex]

106. G. Abbiendi et al. (OPAL Collaboration), Inclusive analysis of the b-quark fragmentation function in $Z$ decays at LEP. Eur. Phys. J. C 29, 463-478 (2003). arXiv:hep-ex/0210031

107. K. Abe et al. (SLD Collaboration), Measurement of the b-quark fragmentation function in $Z^{0}$ decays. Phys. Rev. D 65, 092006 (2002). arXiv:hep-ex/0202031
108. T. Carli, V. Chiochia, K. Klimek, Definition and calculation of bottom quark cross-sections in deep inelastic scattering at HERA and determination of their uncertainties. J. High Energy Phys. 09, 070 (2003). arXiv:hep-ph/0305103

109. M. Cacciari, M. Greco, P. Nason, The $p_{\mathrm{T}}$ spectrum in heavy flavour hadroproduction. J. High Energy Phys. 05, 007 (1998). arXiv:hep-ph/9803400

110. A. Banfi, G.P. Salam, G. Zanderighi, Accurate QCD predictions for heavy quark jets at the Tevatron and LHC. J. High Energy Phys. 07, 026 (2007). arXiv:0704.2999 [hep-ph]

111. R.D. Ball, R.K. Ellis, Heavy quark production at high energy. J. High Energy Phys. 05, 053 (2001). arXiv:hep-ph/0101199

112. M. Cacciari, P. Nason, Is there a significant excess in bottom hadroproduction at the Tevatron? Phys. Rev. Lett. 89, 122003 (2002). arXiv:hep-ph/0204025

113. A. Heister et al. (ALEPH Collaboration), Study of the fragmentation of b-quarks into $B$-mesons at the $Z$ peak. Phys. Lett. B 512, 30-48 (2001)

114. G. Corcella, F. Mescia, A phenomenological study of bottom quark fragmentation in top quark decay. Eur. Phys. J. C 65, 171180 (2010). arXiv:0907.5158 [hep-ph]

115. A. Buckley, H. Hoeth, H. Lacker, H. Schulz, J.E. von Seggern, Systematic event generator tuning for the LHC. Eur. Phys. J. C 65, 331-357 (2010). arXiv:0907.2973 [hep-ph]

116. M. Bowler, $e^{+} e^{-}$production of heavy quarks in the string model. Z. Phys. C 11, 169 (1981)

\section{The ATLAS Collaboration}

G. Aad $^{48}$, B. Abbott ${ }^{111}$, J. Abdallah ${ }^{11}$, A.A. Abdelalim ${ }^{49}$, A. Abdesselam ${ }^{118}$, O. Abdinov ${ }^{10}$, B. Abi ${ }^{112}$, M. Abolins ${ }^{88}$, H. Abramowicz ${ }^{153}$, H. Abreu ${ }^{115}$, E. Acerbi ${ }^{89 a, 89 b}$, B.S. Acharya ${ }^{164 a, 164 b}$, D.L. Adams ${ }^{24}$, T.N. Addy ${ }^{56}$, J. Adel$\operatorname{man}^{175}$, M. Aderholz ${ }^{99}$, S. Adomeit ${ }^{98}$, P. Adragna ${ }^{75}$, T. Adye ${ }^{129}$, S. Aefsky ${ }^{22}$, J.A. Aguilar-Saavedra ${ }^{124 b, a}$, M. Aharrouche $^{81}$, S.P. Ahlen $^{21}$, F. Ahles ${ }^{48}$, A. Ahmad ${ }^{148}$, M. Ahsan ${ }^{40}$, G. Aielli133a,133b, T. Akdogan ${ }^{18 a}$, T.P.A. Åkesson ${ }^{79}$, G. Akimoto ${ }^{155}$, A.V. Akimov ${ }^{94}$, A. Akiyama ${ }^{67}$, A. Aktas $^{48}$, M.S. Alam ${ }^{1}$, M.A. Alam ${ }^{76}$, J. Albert ${ }^{169}$, S. Albrand ${ }^{55}$, M. Aleksa ${ }^{29}$, I.N. Aleksandrov ${ }^{65}$, F. Alessandria ${ }^{89 a}$, C. Alexa ${ }^{25 a}$, G. Alexander ${ }^{153}$, G. Alexandre ${ }^{49}$, T. Alexopoulos ${ }^{9}$, M. Alhroob ${ }^{20}$, M. Aliev ${ }^{15}$, G. Alimonti ${ }^{89 a}$, J. Alison ${ }^{120}$, M. Aliyev ${ }^{10}$, P.P. Allport ${ }^{73}$, S.E. Allwood-Spiers ${ }^{53}$, J. Almond ${ }^{82}$, A. Aloisio ${ }^{102 a, 102 b}$, R. Alon ${ }^{171}$, A. Alonso ${ }^{79}$, M.G. Alviggi ${ }^{102 a, 102 b}$, K. Amako ${ }^{66}$, P. Amaral $^{29}$, C. Amelung ${ }^{22}$, V.V. Ammosov $^{128}$, A. Amorim ${ }^{124 a, b}$, G. Amorós ${ }^{167}$, N. Amram ${ }^{153}$, C. Anastopoulos ${ }^{29}$, L.S. Ancu ${ }^{16}$, N. Andari ${ }^{115}$, T. Andeen ${ }^{34}$, C.F. Anders $^{20}$, G. Anders ${ }^{58 a}$, K.J. Anderson ${ }^{30}$, A. Andreazza ${ }^{89 a, 89 b}$, V. Andrei ${ }^{58 a}$, M-L. Andrieux ${ }^{55}$, X.S. Anduaga ${ }^{70}$, A. Angerami ${ }^{34}$, F. Anghinolfi ${ }^{29}$, N. Anjos ${ }^{124 a}$, A. Annovi ${ }^{47}$, A. Antonaki ${ }^{8}$, M. Antonelli ${ }^{47}$, A. Antonov ${ }^{96}$, J. Antos ${ }^{144 b}$, F. Anulli ${ }^{132 a}$, S. Aoun ${ }^{83}$, L. Aperio Bella ${ }^{4}$, R. Apolle ${ }^{118, c}$, G. Arabidze ${ }^{88}$, I. Aracena ${ }^{143}$, Y. Arai ${ }^{66}$, A.T.H. Arce ${ }^{44}$, J.P. Archambault $^{28}$, S. Arfaoui ${ }^{29, d}$, J-F. Arguin ${ }^{14}$, E. Arik ${ }^{18 a, *}$, M. Arik ${ }^{18 a}$, A.J. Armbruster ${ }^{87}$, O. Arnaez ${ }^{81}$, C. Arnault ${ }^{115}$, A. Artamonov ${ }^{95}$, G. Artoni ${ }^{132 a, 132 b}$, D. Arutinov ${ }^{20}$, S. Asai ${ }^{155}$, R. Asfandiyarov ${ }^{172}$, S. Ask ${ }^{27}$, B. Åsman ${ }^{146 a, 146 b}$, D. Asner $^{28}$, L. Asquith ${ }^{5}$, K. Assamagan ${ }^{24}$, A. Astbury ${ }^{169}$, A. Astvatsatourov ${ }^{52}$, G. Atoian ${ }^{175}$, B. Aubert ${ }^{4}$, E. Auge ${ }^{115}$, K. Aug$\operatorname{sten}^{127}$, M. Aurousseau ${ }^{145 a}$, N. Austin ${ }^{73}$, G. Avolio ${ }^{163}$, R. Avramidou ${ }^{9}$, D. Axen ${ }^{168}$, C. Ay ${ }^{54}$, G. Azuelos ${ }^{93, e}$, Y. Azuma ${ }^{155}$, M.A. Baak ${ }^{29}$, G. Baccaglioni ${ }^{89 a}$, C. Bacci ${ }^{134 a, 134 b}$, A.M. Bach ${ }^{14}$, H. Bachacou ${ }^{136}$, K. Bachas ${ }^{29}$, G. Bachy ${ }^{29}$, M. Backes ${ }^{49}$, M. Backhaus $^{20}$, E. Badescu25a, P. Bagnaia ${ }^{132 a, 132 b}$, S. Bahinipati ${ }^{2}$, Y. Bai ${ }^{32 a}$, D.C. Bailey ${ }^{158}$, T. Bain ${ }^{158}$, J.T. Baines ${ }^{129}$, O.K. Baker ${ }^{175}$, M.D. Baker ${ }^{24}$, S. Baker ${ }^{77}$, E. Banas $^{38}$, P. Banerjee ${ }^{93}$, Sw. Banerjee ${ }^{172}$, D. Banfi ${ }^{29}$, A. Bangert ${ }^{137}$, V. Bansal ${ }^{169}$, H.S. Bansil ${ }^{17}$, L. Barak ${ }^{171}$, S.P. Baranov ${ }^{94}$, A. Barashkou ${ }^{65}$, A. Barbaro Galtieri ${ }^{14}$, T. Barber ${ }^{48}$, E.L. Barberio $^{86}$, D. Barberis ${ }^{50 a, 50 b}$, M. Barbero ${ }^{20}$, D.Y. Bardin ${ }^{65}$, T. Barillari ${ }^{99}$, M. Barisonzi ${ }^{174}$, T. Barklow ${ }^{143}$, N. Barlow ${ }^{27}$, B.M. Barnett $^{129}$, R.M. Barnett ${ }^{14}$, A. Baroncelli ${ }^{134 a}$, G. Barone ${ }^{49}$, A.J. Barr ${ }^{18}$, F. Barreiro ${ }^{80}$, J. Barreiro Guimarães da Costa ${ }^{57}$, P. Barrillon ${ }^{115}$, R. Bartoldus ${ }^{143}$, A.E. Barton ${ }^{71}$, D. Bartsch ${ }^{20}$, V. Bartsch ${ }^{149}$, R.L. Bates ${ }^{53}$, L. Batkova ${ }^{144 a}$, J.R. Batley ${ }^{27}$, A. Battaglia ${ }^{16}$, M. Battistin ${ }^{29}$, G. Battistoni ${ }^{89 a}$, F. Bauer ${ }^{136}$, H.S. Bawa ${ }^{143, f}$, B. Beare ${ }^{158}$, T. Beau ${ }^{78}$, P.H. Beauchemin ${ }^{118}$, 
R. Beccherle ${ }^{50 a}$, P. Bechtle ${ }^{41}$, H.P. Beck ${ }^{16}$, G.A. Beck ${ }^{75}$, M. Beckingham ${ }^{138}$, K.H. Becks ${ }^{174}$, A.J. Beddall ${ }^{18 c}$, A. Beddall $^{18 c}$, S. Bedikian ${ }^{175}$, V.A. Bednyakov ${ }^{65}$, C.P. Bee ${ }^{83}$, M. Begel ${ }^{24}$, S. Behar Harpaz ${ }^{152}$, P.K. Behera ${ }^{63}$, M. Beimforde ${ }^{99}$, C. Belanger-Champagne ${ }^{85}$, P.J. Bell ${ }^{49}$, W.H. Bell ${ }^{49}$, G. Bella ${ }^{153}$, L. Bellagamba ${ }^{19 a}$, F. Bellina ${ }^{29}$, M. Bellomo ${ }^{29}$, A. Belloni $^{57}$, O. Beloborodova ${ }^{107}$, K. Belotskiy ${ }^{96}$, O. Beltramello ${ }^{29}$, S. Ben Ami ${ }^{152}$, O. Benary ${ }^{153}$, D. Benchekroun ${ }^{135 a}$, C. Benchouk $^{83}$, M. Bendel ${ }^{81}$, N. Benekos ${ }^{165}$, Y. Benhammou ${ }^{153}$, D.P. Benjamin ${ }^{44}$, M. Benoit ${ }^{115}$, J.R. Bensinger ${ }^{22}$, K. Benslama $^{130}$, S. Bentvelsen ${ }^{105}$, M. Beretta ${ }^{47}$, D. Berge ${ }^{29}$, E. Bergeaas Kuutmann ${ }^{41}$, N. Berger ${ }^{4}$, F. Berghaus ${ }^{169}$, E. Berglund ${ }^{49}$, J. Beringer ${ }^{14}$, K. Bernardet ${ }^{83}$, P. Bernat ${ }^{77}$, R. Bernhard ${ }^{48}$, C. Bernius ${ }^{24}$, T. Berry ${ }^{76}$, A. Bertin ${ }^{19 a, 19 b}$, F. Bertinelli ${ }^{29}$, F. Bertolucci ${ }^{122 a, 122 b}$, M.I. Besana ${ }^{89 a, 89 b}$, N. Besson ${ }^{136}$, S. Bethke ${ }^{99}$, W. Bhimjii ${ }^{45}$, R.M. Bianchi ${ }^{29}$, M. Bianco ${ }^{72 a, 72 b}$, O. Biebel ${ }^{98}$, S.P. Bieniek ${ }^{77}$, K. Bierwagen ${ }^{54}$, J. Biesiada ${ }^{14}$, M. Biglietti ${ }^{134 a, 134 b}$, H. Bilokon ${ }^{47}$, M. Bindi ${ }^{19 a, 19 b}$, S. Binet $^{115}$, A. Bingul ${ }^{18 c}$, C. Bini ${ }^{132 a, 132 b}$, C. Biscarat ${ }^{177}$, U. Bitenc ${ }^{48}$, K.M. Black ${ }^{21}$, R.E. Blair ${ }^{5}$, J.-B. Blanchard ${ }^{115}$, G. Blanchot $^{29}$, T. Blazek ${ }^{144 a}$, C. Blocker ${ }^{22}$, J. Blocki ${ }^{38}$, A. Blondel ${ }^{49}$, W. Blum ${ }^{81}$, U. Blumenschein ${ }^{54}$, G.J. Bobbink ${ }^{105}$, V.B. Bobrovnikov $^{107}$, S.S. Bocchetta ${ }^{79}$, A. Bocci ${ }^{44}$, C.R. Boddy ${ }^{118}$, M. Boehler ${ }^{41}$, J. Boek ${ }^{174}$, N. Boelaert ${ }^{35}$, S. Böser ${ }^{77}$, J.A. Bogaerts $^{29}$, A. Bogdanchikov ${ }^{107}$, A. Bogouch ${ }^{90, *}$, C. Bohm ${ }^{146 a}$, V. Boisvert ${ }^{76}$, T. Bold ${ }^{37}$, V. Boldea ${ }^{25 a}$, N.M. Bolnet ${ }^{136}$, M. Bona ${ }^{75}$, V.G. Bondarenko ${ }^{96}$, M. Bondioli ${ }^{163}$, M. Boonekamp ${ }^{136}$, G. Boorman ${ }^{76}$, C.N. Booth ${ }^{139}$, S. Bordoni $^{78}$, C. Borer $^{16}$, A. Borisov ${ }^{128}$, G. Borissov ${ }^{71}$, I. Borjanovic ${ }^{12 a}$, S. Borroni $^{87}$, K. Bos ${ }^{105}$, D. Boscherini ${ }^{19 a}$, M. Bosman $^{11}$, H. Boterenbrood ${ }^{105}$, D. Botterill $^{129}$, J. Bouchami ${ }^{93}$, J. Boudreau ${ }^{123}$, E.V. Bouhova-Thacker ${ }^{71}$, C. Bourdarios ${ }^{115}$, N. Bousson ${ }^{83}$, A. Boveia ${ }^{30}$, J. Boyd ${ }^{29}$, I.R. Boyko ${ }^{65}$, N.I. Bozhko ${ }^{128}$, I. Bozovic-Jelisavcic ${ }^{12 b}$, J. Bracinik ${ }^{17}$, A. Braem ${ }^{29}$, P. Branchini ${ }^{134 a}$, G.W. Brandenburg ${ }^{57}$, A. Brandt ${ }^{7}$, G. Brandt ${ }^{15}$, O. Brandt ${ }^{54}$, U. Bratzler ${ }^{156}$, B. Brau ${ }^{84}$, J.E. Brau ${ }^{114}$, H.M. Braun ${ }^{174}$, B. Brelier ${ }^{158}$, J. Bremer ${ }^{29}$, R. Brenner ${ }^{166}$, S. Bressler ${ }^{171}$, D. Breton ${ }^{115}$, D. Britton ${ }^{53}$, F.M. Brochu ${ }^{27}$, I. Brock ${ }^{20}$, R. Brock ${ }^{88}$, T.J. Brodbeck $^{71}$, E. Brodet ${ }^{153}$, F. Broggi ${ }^{89 a}$, C. Bromberg ${ }^{88}$, G. Brooijmans ${ }^{34}$, W.K. Brooks ${ }^{31 b}$, G. Brown ${ }^{82}$, H. Brown ${ }^{7}$, P.A. Bruckman de Renstrom ${ }^{38}$, D. Bruncko ${ }^{144 b}$, R. Bruneliere ${ }^{48}$, S. Brunet ${ }^{61}$, A. Bruni ${ }^{19 a}$, G. Bruni ${ }^{19 a}$, M. Bruschi ${ }^{19 a}$, T. Buanes ${ }^{13}$, F. Bucci ${ }^{49}$, J. Buchanan ${ }^{118}$, N.J. Buchanan ${ }^{2}$, P. Buchholz ${ }^{141}$, R.M. Buckingham ${ }^{118}$, A.G. Buckley ${ }^{45}$, S.I. Buda ${ }^{25 a}$, I.A. Budagov ${ }^{65}$, B. Budick ${ }^{108}$, V. Büscher ${ }^{81}$, L. Bugge ${ }^{117}$, D. Buira-Clark ${ }^{118}$, O. Bulekov ${ }^{96}$, M. Bunse ${ }^{42}$, T. Buran ${ }^{117}$, H. Burckhart ${ }^{29}$, S. Burdin ${ }^{73}$, T. Burgess ${ }^{13}$, S. Burke ${ }^{129}$, E. Busato ${ }^{33}$, P. Bussey ${ }^{53}$, C.P. Buszello ${ }^{166}$, F. Butin ${ }^{29}$, B. Butler ${ }^{143}$, J.M. Butler ${ }^{21}$, C.M. Buttar ${ }^{53}$, J.M. Butterworth ${ }^{77}$, W. Buttinger ${ }^{27}$, J. Caballero ${ }^{24}$, S. Cabrera Urbán ${ }^{167}$, D. Caforio ${ }^{19 a, 19 b}$, O. Cakir $^{3 a}$, P. Calafiura ${ }^{14}$, G. Calderini ${ }^{78}$, P. Calfayan ${ }^{98}$, R. Calkins ${ }^{106}$, L.P. Caloba ${ }^{23 a}$, R. Caloi ${ }^{132 a, 132 b}$, D. Calvet ${ }^{33}$, S. Calvet ${ }^{33}$, R. Camacho Toro ${ }^{33}$, P. Camarri ${ }^{133 a, 133 b}$, M. Cambiaghi ${ }^{119 a, 119 b}$, D. Cameron ${ }^{117}$, S. Campana ${ }^{29}$, M. Campanelli $^{77}$, V. Canale ${ }^{102 a, 102 b}$, F. Canelli ${ }^{30, g}$, A. Canepa ${ }^{159 a}$, J. Cantero ${ }^{80}$, L. Capasso ${ }^{102 a, 102 b}$, M.D.M. Capeans Garrido ${ }^{29}$, I. Caprini ${ }^{25 a}$, M. Caprini ${ }^{25 a}$, D. Capriotti ${ }^{99}$, M. Capua ${ }^{36 a, 36 b}$, R. Caputo ${ }^{148}$, C. Caramarcu ${ }^{24}$, R. Cardarelli ${ }^{133 a}$, T. Carli ${ }^{29}$, G. Carlino ${ }^{102 a}$, L. Carminati ${ }^{89 a}, 89 b$, B. Caron ${ }^{159 a}$, S. Caron ${ }^{48}$, G.D. Carrillo Montoya ${ }^{172}$, A.A. Carter ${ }^{75}$, J.R. Carter ${ }^{27}$, J. Carvalho ${ }^{124 a, h}$, D. Casadei ${ }^{108}$, M.P. Casado ${ }^{11}$, M. Cascella ${ }^{122 a, 122 b}$, C. Caso ${ }^{50 a, 50 b, *}$, A.M. Castaneda Hernandez ${ }^{172}$, E. Castaneda-Miranda ${ }^{172}$, V. Castillo Gimenez ${ }^{167}$, N.F. Castro ${ }^{124 a}$, G. Cataldi ${ }^{72 a}$, F. Cataneo ${ }^{29}$, A. Catinaccio ${ }^{29}$, J.R. Catmore $^{71}$, A. Cattai ${ }^{29}$, G. Cattani ${ }^{133 a, 133 b}$, S. Caughron ${ }^{88}$, D. Cauz ${ }^{164 a, 164 c}$, P. Cavalleri ${ }^{78}$, D. Cavalli ${ }^{89 a}$, M. CavalliSforza $^{11}$, V. Cavasinni ${ }^{122 a, 122 b}$, F. Ceradini ${ }^{134 a, 134 b}$, A.S. Cerqueira ${ }^{23 a}$, A. Cerri ${ }^{29}$, L. Cerrito ${ }^{75}$, F. Cerutti ${ }^{47}$, S.A. Cetin ${ }^{18 b}$, F. Cevenini 102a,102b, A. Chafaq ${ }^{135 a}$, D. Chakraborty ${ }^{106}$, K. Chan ${ }^{2}$, B. Chapleau ${ }^{85}$, J.D. Chapman ${ }^{27}$, J.W. Chapman ${ }^{87}$, E. Chareyre $^{78}$, D.G. Charlton ${ }^{17}$, V. Chavda ${ }^{82}$, C.A. Chavez Barajas ${ }^{29}$, S. Cheatham ${ }^{85}$, S. Chekanov ${ }^{5}$, S.V. Chekulaev ${ }^{159 a}$, G.A. Chelkov ${ }^{65}$, M.A. Chelstowska ${ }^{104}$, C. Chen ${ }^{64}$, H. Chen ${ }^{24}$, S. Chen ${ }^{32 \mathrm{c}}$, T. Chen ${ }^{32 \mathrm{c}}$, X. Chen ${ }^{172}$, S. Cheng ${ }^{32 \mathrm{a}}$, A. Cheplakov $^{65}$, V.F. Chepurnov ${ }^{65}$, R. Cherkaoui El Moursli ${ }^{135 e}$, V. Chernyatin ${ }^{24}$, E. Cheu ${ }^{6}$, S.L. Cheung ${ }^{158}$, L. Chevalier ${ }^{136}$, G. Chiefari ${ }^{102 a, 102 b}$, L. Chikovani ${ }^{51 a}$, J.T. Childers ${ }^{58 a}$, A. Chilingarov ${ }^{71}$, G. Chiodini ${ }^{72 a}$, M.V. Chizhov ${ }^{65}$, G. Choudalakis $^{30}$, S. Chouridou ${ }^{137}$, I.A. Christidi ${ }^{77}$, A. Christov ${ }^{48}$, D. Chromek-Burckhart ${ }^{29}$, M.L. Chu ${ }^{151}$, J. Chudoba ${ }^{125}$, G. Ciapetti ${ }^{132 a, 132 b}$, K. Ciba ${ }^{37}$, A.K. Ciftci ${ }^{3 a}$, R. Ciftci ${ }^{3 a}$, D. Cinca ${ }^{33}$, V. Cindro ${ }^{74}$, M.D. Ciobotaru ${ }^{163}$, C. Ciocca ${ }^{19 a}$, A. Ciocio ${ }^{14}$, M. Cirilli ${ }^{87}$, M. Citterio ${ }^{89 a}$, M. Ciubancan ${ }^{25 a}$, A. Clark ${ }^{49}$, P.J. Clark ${ }^{45}$, W. Cleland ${ }^{123}$, J.C. Clemens ${ }^{83}$, B. Clement ${ }^{55}$, C. Clement ${ }^{146 a, 146 b}$, R.W. Clifft ${ }^{129}$, Y. Coadou ${ }^{83}$, M. Cobal ${ }^{164 a, 164 c}$, A. Coccaro ${ }^{50 a, 50 b}$, J. Cochran ${ }^{64}$, P. Coe ${ }^{118}$, J.G. Cogan ${ }^{143}$, J. Coggeshall $^{165}$, E. Cogneras ${ }^{177}$, C.D. Cojocaru ${ }^{28}$, J. Colas ${ }^{4}$, A.P. Colijn ${ }^{105}$, C. Collard ${ }^{115}$, N.J. Collins ${ }^{17}$, C. Collins-Tooth ${ }^{53}$, J. Collot ${ }^{55}$, G. Colon ${ }^{84}$, P. Conde Muiño ${ }^{124 a}$, E. Coniavitis ${ }^{118}$, M.C. Conidi ${ }^{11}$, M. Consonni ${ }^{104}$, V. Consorti ${ }^{48}$, S. Constantinescu $^{25 a}$, C. Conta ${ }^{119 a, 119 b}$, F. Conventi ${ }^{102 a, i}$, J. Cook $^{29}$, M. Cooke ${ }^{14}$, B.D. Cooper ${ }^{77}$, A.M. Cooper-Sarkar ${ }^{118}$, K. Copic ${ }^{34}$, T. Cornelissen ${ }^{174}$, M. Corradi ${ }^{19 a}$, F. Corriveau ${ }^{85, j}$, A. Corso-Radu ${ }^{163}$, A. Cortes-Gonzalez ${ }^{165}$, G. Cortiana ${ }^{99}$, G. Costa ${ }^{89 a}$, M.J. Costa ${ }^{167}$, D. Costanzo ${ }^{139}$, T. Costin $^{30}$, D. Côté ${ }^{29}$, R. Coura Torres ${ }^{23 a}$, L. Courneyea ${ }^{169}$, G. Cowan ${ }^{76}$, C. Cowden $^{27}$, B.E. Cox $^{82}$, K. Cranmer ${ }^{108}$, J. Cranshaw ${ }^{5}$, F. Crescioli ${ }^{122 a, 122 b}$, M. Cristinziani ${ }^{20}$, G. Crosetti ${ }^{36 a, 36 b}$, R. Crupi ${ }^{72 a, 72 b}$, S. Crépé-Renaudin ${ }^{55}$, C.-M. Cuciuc ${ }^{25 a}$, C. Cuenca Almenar ${ }^{175}$, T. Cuhadar Donszelmann ${ }^{139}$, M. Curatolo ${ }^{47}$, C.J. Curtis ${ }^{17}$, P. Cwetanski ${ }^{61}$, H. Czirr ${ }^{141}$, Z. Czyczula ${ }^{175}$, S. D’Auria ${ }^{53}$, M. D’Onofrio ${ }^{73}$, A. D'Orazio ${ }^{132 a, 132 b}$, P.V.M. Da Silva ${ }^{23 a}$, C. Da Via ${ }^{82}$, W. Dabrowski ${ }^{37}$, T. Dai ${ }^{87}$, C. Dallapiccola ${ }^{84}$, C.H. Daly ${ }^{138}$, M. Dam ${ }^{35}$, M. Dameri ${ }^{50,50 b}$, D.S. Damiani $^{137}$, H.O. Danielsson ${ }^{29}$, D. Dannheim ${ }^{99}$, V. Dao ${ }^{49}$, G. Darbo ${ }^{50 a}$, G.L. Darlea ${ }^{25 b}$, C. Daum ${ }^{105}$, J.P. Dauvergne ${ }^{29}$, 
W. Davey ${ }^{20}$, T. Davidek ${ }^{126}$, N. Davidson ${ }^{86}$, R. Davidson ${ }^{71}$, E. Davies ${ }^{118, c}$, M. Davies ${ }^{93}$, A.R. Davison ${ }^{77}$, Y. Davygora ${ }^{58 a}$, E. Dawe ${ }^{142}$, I. Dawson ${ }^{139}$, J.W. Dawson ${ }^{5, *}$, R.K. Daya-Ishmukhametova ${ }^{39}$, K. De $^{7}$, R. de Asmundis ${ }^{102 a}$, S. De Castro $^{19 a, 19 b}$, P.E. De Castro Faria Salgado ${ }^{24}$, S. De Cecco ${ }^{78}$, J. de Graat ${ }^{98}$, N. De Groot ${ }^{104}$, P. de Jong ${ }^{105}$, C. De La Taille ${ }^{115}$, H. De la Torre ${ }^{80}$, B. De Lotto ${ }^{164 a, 164 c}$, L. de Mora $^{71}$, L. De Nooij ${ }^{105}$, D. De Pedis ${ }^{132 a}$, A. De Salvo ${ }^{132 a}$, U. De Sanctis $^{164 a, 164 c}$, A. De Santo ${ }^{149}$, J.B. De Vivie De Regie ${ }^{115}$, S. Dean ${ }^{77}$, R. Debbe ${ }^{24}$, D.V. Dedovich ${ }^{65}$, J. Degenhardt ${ }^{120}$, M. Dehchar $^{118}$, C. Del Papa ${ }^{164 a, 164 c}$, J. Del Peso ${ }^{80}$, T. Del Prete ${ }^{122 a, 122 b}$, M. Deliyergiyev ${ }^{74}$, A. Dell'Acqua ${ }^{29}$, L. Dell' Asta ${ }^{89 a, 89 b}$, M. Della Pietra ${ }^{102 a, i}$, D. della Volpe ${ }^{102 a, 102 b}$, M. Delmastro ${ }^{29}$, P. Delpierre ${ }^{83}$, N. Delruelle ${ }^{29}$, P.A. Delsart ${ }^{55}$, C. Deluca ${ }^{148}$, S. Demers ${ }^{175}$, M. Demichev ${ }^{65}$, B. Demirkoz ${ }^{11, k}$, J. Deng ${ }^{163}$, W. Deng ${ }^{24}$, S.P. Denisov ${ }^{128}$, D. Derendarz ${ }^{38}$, J.E. Derkaoui ${ }^{135 d}$, F. Derue ${ }^{78}$, P. Dervan ${ }^{73}$, K. Desch ${ }^{20}$, E. Devetak ${ }^{148}$, P.O. Deviveiros ${ }^{158}$, A. Dewhurst ${ }^{129}$, B. DeWilde ${ }^{148}$, S. Dhaliwal $^{158}$, R. Dhullipudi ${ }^{24,1}$, A. Di Ciaccio ${ }^{133 a, 133 b}$, L. Di Ciaccio ${ }^{4}$, A. Di Girolamo ${ }^{29}$, B. Di Girolamo ${ }^{29}$, S. Di Luise ${ }^{134 a, 134 b}$, A. Di Mattia ${ }^{172}$, B. Di Micco ${ }^{29}$, R. Di Nardo ${ }^{133 a, 133 b}$, A. Di Simone ${ }^{133 a, 133 b}$, R. Di Sipio ${ }^{19 a, 19 b}$, M.A. Diaz ${ }^{31 a}$, F. Diblen $^{18 c}$, E.B. Diehl ${ }^{87}$, J. Dietrich ${ }^{41}$, T.A. Dietzsch ${ }^{58 a}$, S. Diglio ${ }^{115}$, K. Dindar Yagci ${ }^{39}$, J. Dingfelder ${ }^{20}$, C. Dionisi ${ }^{132 a, 132 b}$, P. Dita ${ }^{25 a}$, S. Dita ${ }^{25 a}$, F. Dittus ${ }^{29}$, F. Djama ${ }^{83}$, T. Djobava ${ }^{51 b}$, M.A.B. do Vale ${ }^{23 c}$, A. Do Valle Wemans ${ }^{124 a}$, T.K.O. Doan ${ }^{4}$, M. Dobbs ${ }^{85}$, R. Dobinson ${ }^{29, *}$, D. Dobos ${ }^{29}$, E. Dobson ${ }^{29}$, M. Dobson ${ }^{163}$, J. Dodd ${ }^{34}$, C. Doglioni ${ }^{118}$, T. Doherty ${ }^{53}$, Y. Doi $^{66, *}$, J. Dolejsi ${ }^{126}$, I. Dolenc ${ }^{74}$, Z. Dolezal ${ }^{126}$, B.A. Dolgoshein ${ }^{96, *}$, T. Dohmae ${ }^{155}$, M. Donadelli ${ }^{23 d}$, M. Donega ${ }^{120}$, J. Donini ${ }^{55}$, J. Dopke ${ }^{29}$, A. Doria ${ }^{102 a}$, A. Dos Anjos ${ }^{172}$, M. Dosil ${ }^{11}$, A. Dotti ${ }^{122 a, 122 b}$, M.T. Dova ${ }^{70}$, J.D. Dowell ${ }^{17}$, A.D. Doxiadis ${ }^{105}$, A.T. Doyle ${ }^{53}$, Z. Drasal ${ }^{126}$, J. Drees ${ }^{174}$, N. Dressnandt ${ }^{120}$, H. Drevermann ${ }^{29}$, C. Driouichi ${ }^{35}$, M. Dris ${ }^{9}$, J. Dubbert ${ }^{99}$, T. Dubbs ${ }^{137}$, S. Dube ${ }^{14}$, E. Duchovni ${ }^{171}$, G. Duckeck ${ }^{98}$, A. Dudarev ${ }^{29}$, F. Dudziak ${ }^{64}$, M. Dührssen ${ }^{29}$, I.P. Duerdoth ${ }^{82}$, L. Duflot ${ }^{115}$, M.-A. Dufour ${ }^{85}$, M. Dunford ${ }^{29}$, H. Duran Yildiz $^{3 b}$, R. Duxfield ${ }^{139}$, M. Dwuznik ${ }^{37}$, F. Dydak ${ }^{29}$, M. Düren ${ }^{52}$, W.L. Ebenstein ${ }^{44}$, J. Ebke ${ }^{98}$, S. Eckert ${ }^{48}$, S. Eckweiler ${ }^{81}$, K. Edmonds ${ }^{81}$, C.A. Edwards ${ }^{76}$, N.C. Edwards ${ }^{53}$, W. Ehrenfeld $^{41}$, T. Ehrich ${ }^{99}$, T. Eifert ${ }^{29}$, G. Eigen ${ }^{13}$, K. Einsweiler ${ }^{14}$, E. Eisenhandler ${ }^{75}$, T. Ekelof ${ }^{166}$, M. El Kacimi ${ }^{135 c}$, M. Ellert ${ }^{166}$, S. Elles ${ }^{4}$, F. Ellinghaus ${ }^{81}$, K. Ellis ${ }^{75}$, N. Ellis ${ }^{29}$, J. Elmsheuser ${ }^{98}$, M. Elsing ${ }^{29}$, D. Emeliyanov ${ }^{129}$, R. Engelmann ${ }^{148}$, A. Engl ${ }^{98}$, B. Epp ${ }^{62}$, A. Eppig ${ }^{87}$, J. Erdmann ${ }^{54}$, A. Ereditato ${ }^{16}$, D. Eriksson ${ }^{146 a}$, J. Ernst ${ }^{1}$, M. Ernst ${ }^{24}$, J. Ernwein ${ }^{136}$, D. Errede ${ }^{165}$, S. Errede ${ }^{165}$, E. Ertel ${ }^{81}$, M. Escalier ${ }^{115}$, C. Escobar ${ }^{123}$, X. Espinal Curull ${ }^{11}$, B. Esposito ${ }^{47}$, F. Etienne ${ }^{83}$, A.I. Etienvre ${ }^{136}$, E. Etzion ${ }^{153}$, D. Evangelakou ${ }^{54}$, H. Evans ${ }^{61}$, L. Fabbri19a,19b ${ }^{13}$ C. Fabre ${ }^{29}$, R.M. Fakhrutdinov ${ }^{128}$, S. Falciano $^{132 a}$, Y. Fang ${ }^{172}$, M. Fanti ${ }^{89 a, 89 b}$, A. Farbin ${ }^{7}$, A. Farilla ${ }^{134 a}$, J. Farley ${ }^{148}$, T. Farooque ${ }^{158}$, S.M. Farrington ${ }^{118}$, P. Farthouat $^{29}$, P. Fassnacht ${ }^{29}$, D. Fassouliotis ${ }^{8}$, B. Fatholahzadeh ${ }^{158}$, A. Favareto ${ }^{89 a, 89 b}$, L. Fayard ${ }^{115}$, S. Fazio ${ }^{36 a, 36 b}$, R. Febbraro $^{33}$, P. Federic ${ }^{144 a}$, O.L. Fedin ${ }^{121}$, W. Fedorko ${ }^{88}$, M. Fehling-Kaschek ${ }^{48}$, L. Feligioni ${ }^{83}$, D. Fellmann ${ }^{5}$, C.U. Felzmann $^{86}$, C. Feng ${ }^{32 d}$, E.J. Feng ${ }^{30}$, A.B. Fenyuk ${ }^{128}$, J. Ferencei ${ }^{144 b}$, J. Ferland ${ }^{93}$, W. Fernando ${ }^{109}$, S. Ferrag ${ }^{53}$ J. Ferrando $^{53}$, V. Ferrara ${ }^{41}$, A. Ferrari ${ }^{166}$, P. Ferrari ${ }^{105}$, R. Ferrari ${ }^{119 a}$, A. Ferrer ${ }^{167}$, M.L. Ferrer ${ }^{47}$, D. Ferrere ${ }^{49}$, C. Ferretti ${ }^{87}$, A. Ferretto Parodi $^{50 a, 50 b}$, M. Fiascaris ${ }^{30}$, F. Fiedler ${ }^{81}$, A. Filipčic ${ }^{74}$, A. Filippas ${ }^{9}$, F. Filthaut ${ }^{104}$, M. Fincke-Keeler ${ }^{169}$, M.C.N. Fiolhais $^{124 a, h}$, L. Fiorini ${ }^{167}$, A. Firan ${ }^{39}$, G. Fischer ${ }^{41}$, P. Fischer ${ }^{20}$, M.J. Fisher ${ }^{109}$, S.M. Fisher ${ }^{129}$, M. Flechl ${ }^{48}$, I. Fleck ${ }^{141}$, J. Fleckner ${ }^{81}$, P. Fleischmann ${ }^{173}$, S. Fleischmann ${ }^{174}$, T. Flick ${ }^{174}$, L.R. Flores Castillo ${ }^{172}$, M.J. Flowerdew ${ }^{99}$, M. Fokitis ${ }^{9}$, T. Fonseca Martin ${ }^{16}$, J. Fopma ${ }^{118}$, D.A. Forbush ${ }^{138}$, A. Formica ${ }^{136}$, A. Forti ${ }^{82}$, D. Fortin ${ }^{159 a}$, J.M. Foster ${ }^{82}$, D. Fournier ${ }^{115}$, A. Foussat ${ }^{29}$, A.J. Fowler ${ }^{44}$, K. Fowler ${ }^{137}$, H. Fox $^{71}$, P. Francavilla ${ }^{122 a, 122 b}$, S. Franchino ${ }^{119 a, 119 b}$, D. Francis ${ }^{29}$, T. Frank $^{171}$, M. Franklin ${ }^{57}$, S. Franz ${ }^{29}$, M. Fraternali119a,119b S. Fratina ${ }^{120}$, J. Freestone ${ }^{82}$, S.T. French ${ }^{27}$, F. Friedrich ${ }^{43}$, R. Froeschl ${ }^{29}$, D. Froidevaux ${ }^{29}$, J.A. Frost ${ }^{27}$, C. Fukunaga ${ }^{156}$, E. Fullana Torregrosa ${ }^{29}$, J. Fuster ${ }^{167}$, C. Gabaldon ${ }^{29}$, O. Gabizon ${ }^{171}$, T. Gadfort ${ }^{24}$, S. Gadomski ${ }^{49}$, G. Gagliardi ${ }^{50 a, 50 b}$, P. Gagnon ${ }^{61}$, C. Galea ${ }^{98}$, E.J. Gallas ${ }^{118}$, V. Gallo ${ }^{16}$, B.J. Gallop ${ }^{129}$, P. Gallus ${ }^{125}$, E. Galyaev ${ }^{40}$, K.K. Gan ${ }^{109}$, Y.S. Gao ${ }^{143, f}$, V.A. Gapienko ${ }^{128}$, A. Gaponenko ${ }^{14}$, F. Garberson ${ }^{175}$, M. GarciaSciveres $^{14}$, C. García ${ }^{167}$, J.E. García Navarro ${ }^{49}$, R.W. Gardner ${ }^{30}$, N. Garelli ${ }^{29}$, H. Garitaonandia ${ }^{105}$, V. Garonne ${ }^{29}$, J. Garvey $^{17}$, C. Gatti $^{47}$, G. Gaudio ${ }^{119 a}$, O. Gaumer ${ }^{49}$, B. Gaur ${ }^{141}$, L. Gauthier ${ }^{136}$, P. Gauzzi ${ }^{132 a}$, I.L. Gavrilenko ${ }^{94}$, C. Gay ${ }^{168}$, G. Gaycken $^{20}$, J-C. Gayde ${ }^{29}$, E.N. Gazis ${ }^{9}$, P. Ge ${ }^{32 d}$, C.N.P. Gee ${ }^{129}$, D.A.A. Geerts ${ }^{105}$, Ch. Geich-Gimbel ${ }^{20}$, K. Gellerst-

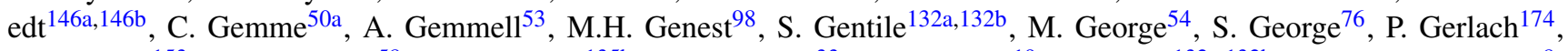
A. Gershon ${ }^{153}$, C. Geweniger ${ }^{58 a}$, H. Ghazlane ${ }^{135 b}$, N. Ghodbane ${ }^{33}$, B. Giacobbe ${ }^{19 a}$, S. Giagu $^{132 a, 132 b}$, V. Giakoumopoulou ${ }^{8}$, V. Giangiobbe ${ }^{122 a, 122 b}$, F. Gianotti ${ }^{29}$, B. Gibbard ${ }^{24}$, A. Gibson ${ }^{158}$, S.M. Gibson ${ }^{29}$, L.M. Gilbert ${ }^{118}$, M. Gilchriese ${ }^{14}$, V. Gilewsky ${ }^{91}$, D. Gillberg ${ }^{28}$, A.R. Gillman ${ }^{129}$, D.M. Gingrich ${ }^{2, \mathrm{e}}$, J. Ginzburg ${ }^{153}$, N. Giokaris ${ }^{8}$, M.P. Giordani ${ }^{164 c}$, R. Giordano $^{102 a, 102 b}$, F.M. Giorgi ${ }^{15}$, P. Giovannini99 ${ }^{99}$, P.F. Giraud ${ }^{136}$, D. Giugni ${ }^{89 a}$, M. Giunta ${ }^{93}$, P. Giusti ${ }^{19 a}$, B.K. Gjelsten ${ }^{117}$, L.K. Gladilin ${ }^{97}$, C. Glasman ${ }^{80}$, J. Glatzer ${ }^{48}$, A. Glazov ${ }^{41}$, K.W. Glitza ${ }^{174}$, G.L. Glonti ${ }^{65}$, J. Godfrey ${ }^{142}$, J. Godlewski ${ }^{29}$, M. Goebel ${ }^{41}$, T. Göpfert ${ }^{43}$, C. Goeringer ${ }^{81}$, C. Gössling ${ }^{42}$, T. Göttfert ${ }^{99}$, S. Goldfarb ${ }^{87}$, T. Golling ${ }^{175}$, S.N. Golovnia ${ }^{128}$, A. Gomes ${ }^{124 a, b}$, L.S. Gomez Fajardo ${ }^{41}$, R. Gonçalo ${ }^{76}$, J. Goncalves Pinto Firmino Da Costa ${ }^{41}$, L. Gonella ${ }^{20}$, A. Gonidec ${ }^{29}$, S. Gonzalez ${ }^{172}$, S. González de la Hoz ${ }^{167}$, M.L. Gonzalez Silva ${ }^{26}$, S. Gonzalez-Sevilla ${ }^{49}$, J.J. Goodson ${ }^{148}$, L. Goossens ${ }^{29}$, P.A. Gorbounov ${ }^{95}$, H.A. Gordon ${ }^{24}$, I. Gorelov ${ }^{103}$, G. Gorfine ${ }^{174}$, B. Gorini ${ }^{29}$, E. Gorini ${ }^{72 a, 72 b}$, A. Gorišek ${ }^{74}$, E. Gornicki $^{38}$, S.A. Gorokhov ${ }^{128}$, V.N. Goryachev ${ }^{128}$, B. Gosdzik ${ }^{41}$, M. Gosselink ${ }^{105}$, M.I. Gostkin ${ }^{65}$, I. Gough Eschrich ${ }^{163}$, 
M. Gouighri1 ${ }^{135 a}$, D. Goujdami ${ }^{135 c}$, M.P. Goulette ${ }^{49}$, A.G. Goussiou ${ }^{138}$, C. Goy ${ }^{4}$, I. Grabowska-Bold ${ }^{163, m}$, P. Grafström²9, C. Grah ${ }^{174}$, K-J. Grahn ${ }^{41}$, F. Grancagnolo ${ }^{72 a}$, S. Grancagnolo ${ }^{15}$, V. Grassi ${ }^{148}$, V. Gratchev ${ }^{121}$, N. Grau ${ }^{34}$, H.M. Gray ${ }^{29}$, J.A. Gray ${ }^{148}$, E. Graziani ${ }^{134 a}$, O.G. Grebenyuk ${ }^{121}$, B. Green ${ }^{76}$, D. Greenfield ${ }^{129}$, T. Greenshaw ${ }^{73}$, Z.D. Greenwood ${ }^{24,1}$, K. Gregersen ${ }^{35}$, I.M. Gregor ${ }^{41}$, P. Grenier ${ }^{143}$, J. Griffiths ${ }^{138}$, N. Grigalashvilii ${ }^{65}$, A.A. Grillo ${ }^{137}$, S. Grinstein ${ }^{11}$, Y.V. Grishkevich $^{97}$, J.-F. Grivaz ${ }^{115}$, M. Groh ${ }^{99}$, E. Gross ${ }^{171}$, J. Grosse-Knetter ${ }^{54}$, J. Groth-Jensen ${ }^{171}$, K. Grybel ${ }^{141}$, V.J. Guarino $^{5}$, D. Guest ${ }^{175}$, C. Guicheney ${ }^{33}$, A. Guida ${ }^{72 a, 72 b}$, S. Guindon ${ }^{54}$, H. Guler ${ }^{85, n}$, J. Gunther ${ }^{125}$, B. Guo ${ }^{158}$, J. Guo ${ }^{34}$, A. Gupta ${ }^{30}$, Y. Gusakov ${ }^{65}$, V.N. Gushchin ${ }^{128}$, A. Gutierrez ${ }^{93}$, P. Gutierrez ${ }^{111}$, N. Guttman ${ }^{153}$, O. Gutzwiller ${ }^{172}$, C. Guyot ${ }^{136}$, C. Gwenlan ${ }^{118}$, C.B. Gwilliam ${ }^{73}$, A. Haas ${ }^{143}$, S. Haas $^{29}$, C. Haber ${ }^{14}$, R. Hackenburg ${ }^{24}$, H.K. Hadavand ${ }^{39}$, D.R. Hadley ${ }^{17}$,

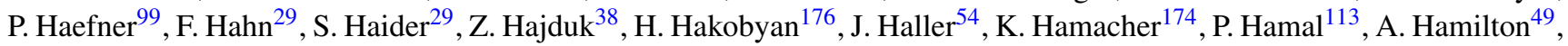

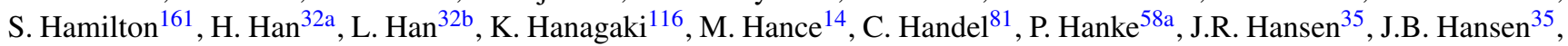
J.D. Hansen ${ }^{35}$, P.H. Hansen ${ }^{35}$, P. Hansson ${ }^{143}$, K. Hara ${ }^{160}$, G.A. Hare ${ }^{137}$, T. Harenberg ${ }^{174}$, S. Harkusha ${ }^{90}$, D. Harper ${ }^{87}$,

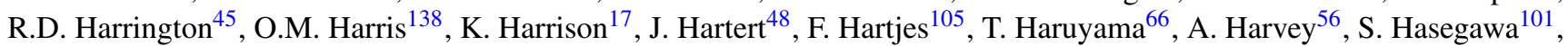
Y. Hasegawa ${ }^{140}$, S. Hassani ${ }^{136}$, M. Hatch ${ }^{29}$, D. Hauff ${ }^{99}$, S. Haug ${ }^{16}$, M. Hauschild ${ }^{29}$, R. Hauser ${ }^{88}$, M. Havranek ${ }^{20}$, B.M. Hawes ${ }^{118}$, C.M. Hawkes ${ }^{17}$, R.J. Hawkings ${ }^{29}$, D. Hawkins ${ }^{163}$, T. Hayakawa ${ }^{67}$, T. Hayashi ${ }^{160}$, D. Hayden ${ }^{76}$, H.S. Hay-

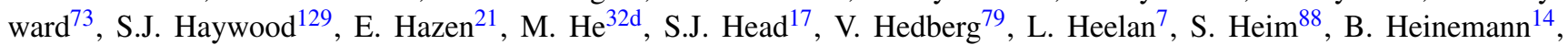
S. Heisterkamp ${ }^{35}$, L. Helary ${ }^{4}$, M. Heller ${ }^{29}$, S. Hellman ${ }^{146 a, 146 b}$, D. Hellmich ${ }^{20}$, C. Helsens ${ }^{11}$, T. Hemperek ${ }^{20}$, R.C.W. Henderson $^{71}$, M. Henke ${ }^{58 a}$, A. Henrichs ${ }^{54}$, A.M. Henriques Correia ${ }^{29}$, S. Henrot-Versille ${ }^{115}$, F. Henry-Couannier ${ }^{83}$, C. Hensel ${ }^{54}$, T. Hen $\beta^{174}$, C.M. Hernandez ${ }^{7}$, Y. Hernández Jiménez ${ }^{167}$, R. Herrberg ${ }^{15}$, A.D. Hershenhorn ${ }^{152}$, G. Herten ${ }^{48}$, R. Hertenberger $^{98}$, L. Hervas ${ }^{29}$, N.P. Hessey ${ }^{105}$, A. Hidvegi ${ }^{146 a}$, E. Higón-Rodriguez ${ }^{167}$, D. Hill ${ }^{5,}$, J.C. Hill ${ }^{27}$, N. Hill ${ }^{5}$, K.H. Hiller ${ }^{41}$, S. Hillert ${ }^{20}$, S.J. Hillier ${ }^{17}$, I. Hinchliffe ${ }^{14}$, E. Hines ${ }^{120}$, M. Hirose ${ }^{116}$, F. Hirsch ${ }^{42}$, D. Hirschbuehl ${ }^{174}$, J. Hobbs ${ }^{148}$, N. Hod ${ }^{153}$, M.C. Hodgkinson ${ }^{139}$, P. Hodgson ${ }^{139}$, A. Hoecker ${ }^{29}$, M.R. Hoeferkamp ${ }^{103}$, J. Hoffman ${ }^{39}$, D. Hoffmann ${ }^{83}$, M. Hohlfeld ${ }^{81}$, M. Holder ${ }^{141}$, S.O. Holmgren ${ }^{146 a}$, T. Holy ${ }^{127}$, J.L. Holzbauer ${ }^{88}$, Y. Homma ${ }^{67}$, T.M. Hong ${ }^{120}$, L. Hooft van Huysduynen $^{108}$, T. Horazdovsky ${ }^{127}$, C. Horn ${ }^{143}$, S. Horner ${ }^{48}$, K. Horton ${ }^{118}$, J-Y. Hostachy ${ }^{55}$, S. Hou ${ }^{151}$, M.A. Houlden ${ }^{73}$, A. Houm$\operatorname{mada}^{135 a}$, J. Howarth ${ }^{82}$, D.F. Howell ${ }^{118}$, I. Hristova ${ }^{15}$, J. Hrivnac ${ }^{115}$, I. Hruska ${ }^{125}$, T. Hryn'ova ${ }^{4}$, P.J. Hsu ${ }^{175}$, S.-C. Hsu ${ }^{14}$, G.S. Huang ${ }^{111}$, Z. Hubacek ${ }^{127}$, F. Hubaut ${ }^{83}$, F. Huegging ${ }^{20}$, T.B. Huffman ${ }^{118}$, E.W. Hughes ${ }^{34}$, G. Hughes ${ }^{71}$, R.E. Hughes-

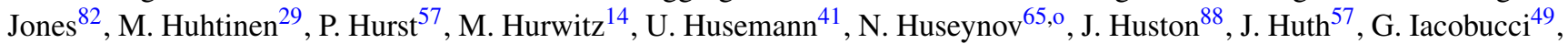
G. Iakovidis ${ }^{9}$, M. Ibbotson ${ }^{82}$, I. Ibragimov ${ }^{141}$, R. Ichimiya ${ }^{67}$, L. Iconomidou-Fayard ${ }^{115}$, J. Idarraga ${ }^{115}$, P. Iengo ${ }^{102 a, 102 b}$, O. Igonkina ${ }^{105}$, Y. Ikegami ${ }^{66}$, M. Ikeno ${ }^{66}$, Y. Ilchenko ${ }^{39}$, D. Iliadis ${ }^{154}$, D. Imbault ${ }^{78}$, M. Imori ${ }^{155}$, T. Ince In $^{20}$, J. InigoGolfin $^{29}$, P. Ioannou ${ }^{8}$, M. Iodice ${ }^{134 a}$, A. Irles Quiles ${ }^{167}$, A. Ishikawa ${ }^{67}$, M. Ishino ${ }^{68}$, R. Ishmukhametov ${ }^{39}$, C. Issever ${ }^{118}$, S. Istin ${ }^{18 a}$, A.V. Ivashin ${ }^{128}$, W. Iwanski ${ }^{38}$, H. Iwasaki ${ }^{66}$, J.M. Izen $^{40}$, V. Izzo ${ }^{102 a}$, B. Jackson ${ }^{120}$, J.N. Jackson ${ }^{73}$, P. Jackson ${ }^{143}$, M.R. Jaekel ${ }^{29}$, V. Jain ${ }^{61}$, K. Jakobs ${ }^{48}$, S. Jakobsen ${ }^{35}$, J. Jakubek ${ }^{127}$, D.K. Jana ${ }^{111}$, E. Jankowski ${ }^{158}$, E. Jansen ${ }^{77}$, A. Jantsch ${ }^{99}$, M. Janus ${ }^{20}$, G. Jarlskog ${ }^{79}$, L. Jeanty ${ }^{57}$, K. Jelen ${ }^{37}$, I. Jen-La Plante ${ }^{30}$, P. Jenni ${ }^{29}$, A. Jeremie ${ }^{4}$, P. Jež ${ }^{35}$, S. Jézéquel ${ }^{4}$, M.K. Jha ${ }^{19 a}$, H. Ji ${ }^{172}$, W. Ji ${ }^{81}$, J. Jia ${ }^{148}$, Y. Jiang ${ }^{32 b}$, M. Jimenez Belenguer ${ }^{41}$, G. Jin ${ }^{32 b}$, S. Jin ${ }^{32 a}$, O. Jinnouchi ${ }^{157}$, M.D. Joergensen $^{35}$, D. Joffe ${ }^{39}$, L.G. Johansen ${ }^{13}$, M. Johansen ${ }^{146 a, 146 b}$, K.E. Johansson ${ }^{146 a}$, P. Johansson ${ }^{139}$, S. Johnert ${ }^{41}$, K.A. Johns ${ }^{6}$, K. Jon-And ${ }^{146 a, 146 b}$, G. Jones ${ }^{82}$, R.W.L. Jones ${ }^{71}$, T.W. Jones ${ }^{77}$, T.J. Jones ${ }^{73}$, O. Jonsson ${ }^{29}$, C. Joram ${ }^{29}$, P.M. Jorge ${ }^{124 a}$, J. Joseph ${ }^{14}$, T. Jovin ${ }^{12 b}$, X. Ju ${ }^{130}$, C.A. Jung ${ }^{42}$, V. Juranek ${ }^{125}$, P. Jussel ${ }^{62}$, A. Juste Rozas ${ }^{11}$, V.V. Kabachenko ${ }^{128}$, S. Kabana $^{16}$, M. Kaci ${ }^{167}$, A. Kaczmarska ${ }^{38}$, P. Kadlecik ${ }^{35}$, M. Kado ${ }^{115}$, H. Kagan ${ }^{109}$, M. Kagan ${ }^{57}$, S. Kaiser ${ }^{99}$, E. Kajomovitz $^{152}$, S. Kalinin ${ }^{174}$, L.V. Kalinovskaya ${ }^{65}$, S. Kama ${ }^{39}$, N. Kanaya ${ }^{155}$, M. Kaneda ${ }^{29}$, T. Kanno ${ }^{157}$, V.A. Kantserov ${ }^{96}$, J. Kanzaki ${ }^{66}$, B. Kaplan ${ }^{175}$, A. Kapliy ${ }^{30}$, J. Kaplon ${ }^{29}$, D. Kar ${ }^{43}$, M. Karagounis ${ }^{20}$, M. Karagoz ${ }^{118}$, M. Karnevskiy ${ }^{41}$, K. Karr ${ }^{5}$, V. Kartvelishvili71 ${ }^{71}$, A.N. Karyukhin ${ }^{128}$, L. Kashif ${ }^{172}$, A. Kasmi ${ }^{39}$, R.D. Kass ${ }^{109}$, A. Kastanas ${ }^{13}$, M. Kataoka ${ }^{4}$, Y. Kataoka ${ }^{155}$, E. Katsoufis ${ }^{9}$, J. Katzy ${ }^{41}$, V. Kaushik ${ }^{6}$, K. Kawagoe ${ }^{67}$, T. Kawamoto ${ }^{155}$, G. Kawamura ${ }^{81}$, M.S. Kayl ${ }^{105}$, V.A. Kazanin ${ }^{107}$, M.Y. Kazarinov ${ }^{65}$, J.R. Keates ${ }^{82}$, R. Keeler ${ }^{169}$, R. Kehoe ${ }^{39}$, M. Keil ${ }^{54}$, G.D. Kekelidze ${ }^{65}$, M. Kelly ${ }^{82}$, J. Kennedy ${ }^{98}$, C.J. Kenney ${ }^{143}$, M. Kenyon ${ }^{53}$, O. Kepka ${ }^{125}$, N. Kerschen ${ }^{29}$, B.P. Kerševan ${ }^{74}$, S. Kersten ${ }^{174}$, K. Kessoku ${ }^{155}$, C. Ketterer ${ }^{48}$, J. Keung ${ }^{158}$, M. Khakzad ${ }^{28}$, F. Khalil-zada ${ }^{10}$, H. Khandanyan ${ }^{165}$, A. Khanov ${ }^{112}$, D. Kharchenko ${ }^{65}$, A. Khodinov $^{96}$, A.G. Kholodenko ${ }^{128}$, A. Khomich ${ }^{58 a}$, T.J. Khoo $^{27}$, G. Khoriauli ${ }^{20}$, A. Khoroshilov ${ }^{174}$, N. Khovanskiy ${ }^{65}$, V. Khovan-

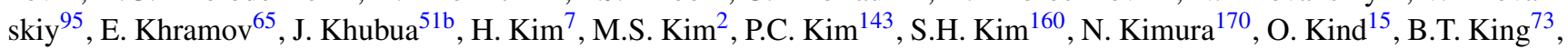
M. King ${ }^{67}$, R.S.B. King ${ }^{118}$, J. Kirk ${ }^{129}$, L.E. Kirsch ${ }^{22}$, A.E. Kiryunin ${ }^{99}$, T. Kishimoto ${ }^{67}$, D. Kisielewska ${ }^{37}$, T. Kittelmann $^{123}$, A.M. Kiver ${ }^{128}$, E. Kladiva ${ }^{144 b}$, J. Klaiber-Lodewigs ${ }^{42}$, M. Klein ${ }^{73}$, U. Klein ${ }^{73}$, K. Kleinknecht ${ }^{81}$, M. Klemetti ${ }^{85}$, A. Klier ${ }^{171}$, A. Klimentov ${ }^{24}$, R. Klingenberg ${ }^{42}$, E.B. Klinkby ${ }^{35}$, T. Klioutchnikova ${ }^{29}$, P.F. Klok ${ }^{104}$, S. Klous ${ }^{105}$, E.E. Kluge ${ }^{58 a}$, T. Kluge ${ }^{73}$, P. Kluit ${ }^{105}$, S. Kluth ${ }^{99}$, N.S. Knecht ${ }^{158}$, E. Kneringer ${ }^{62}$, J. Knobloch ${ }^{29}$, E.B.F.G. Knoops ${ }^{83}$, A. Knue ${ }^{54}$, B.R. Ko ${ }^{44}$, T. Kobayashi ${ }^{155}$, M. Kobel ${ }^{43}$, M. Kocian ${ }^{143}$, A. Kocnar ${ }^{113}$, P. Kodys ${ }^{126}$, K. Köneke ${ }^{29}$, A.C. König ${ }^{104}$, S. Koenig ${ }^{81}$, L. Köpke ${ }^{81}$, F. Koetsveld ${ }^{104}$, P. Koevesarki ${ }^{20}$, T. Koffas ${ }^{28}$, E. Koffeman ${ }^{105}$, F. Kohn ${ }^{54}$, Z. Kohout ${ }^{127}$, T. Kohriki ${ }^{66}$, T. Koi ${ }^{143}$, T. $\operatorname{Kokott}^{20}$, G.M. Kolachev ${ }^{107}$, H. Kolanoski ${ }^{15}$, V. Kolesnikov ${ }^{65}$, I. Koletsou ${ }^{89 a}$, J. Koll ${ }^{88}$, D. Kol- 
$\operatorname{lar}^{29}$, M. Kollefrath ${ }^{48}$, S.D. Kolya ${ }^{82}$, A.A. Komar ${ }^{94}$, Y. Komori ${ }^{155}$, T. Kondo ${ }^{66}$, T. Kono ${ }^{41, p}$, A.I. Kononov ${ }^{48}$, R. Konoplich $^{108, q}$, N. Konstantinidis ${ }^{77}$, A. Kootz ${ }^{174}$, S. Koperny ${ }^{37}$, S.V. Kopikov ${ }^{128}$, K. Korcyl ${ }^{38}$, K. Kordas ${ }^{154}$, V. Koreshev ${ }^{128}$, A. Korn ${ }^{118}$, A. Korol ${ }^{107}$, I. Korolkov ${ }^{11}$, E.V. Korolkova ${ }^{139}$, V.A. Korotkov ${ }^{128}$, O. Kortner ${ }^{99}$, S. Kortner ${ }^{99}$, V.V. Kostyukhin ${ }^{20}$, M.J. Kotamäki ${ }^{29}$, S. Kotov ${ }^{99}$, V.M. Kotov ${ }^{65}$, A. Kotwal ${ }^{44}$, C. Kourkoumelis ${ }^{8}$, V. Kouskoura ${ }^{154}$, A. Koutsman ${ }^{105}$, R. Kowalewski ${ }^{169}$, T.Z. Kowalski ${ }^{37}$, W. Kozanecki ${ }^{136}$, A.S. Kozhin ${ }^{128}$, V. Kral ${ }^{127}$, V.A. Kramarenko ${ }^{97}$, G. Kramberger ${ }^{74}$, M.W. Krasny ${ }^{78}$, A. Krasznahorkay ${ }^{108}$, J. Kraus ${ }^{88}$, J.K. Kraus ${ }^{20}$, A. Kreisel ${ }^{153}$, F. Krejci ${ }^{127}$, J. Kretzschmar ${ }^{73}$, N. Krieger ${ }^{54}$, P. Krieger ${ }^{158}$, K. Kroeninger ${ }^{54}$, H. Kroha ${ }^{99}$, J. Kroll ${ }^{120}$, J. Kroseberg ${ }^{20}$, J. Krstic ${ }^{12 a}$, U. Kruchonak ${ }^{65}$, H. Krüger ${ }^{20}$, T. Kruker ${ }^{16}$, Z.V. Krumshteyn ${ }^{65}$, A. Kruth ${ }^{20}$, T. Kubota ${ }^{86}$, S. Kuehn ${ }^{48}$, A. Kugel ${ }^{58 c}$, T. Kuhl ${ }^{41}$, D. Kuhn ${ }^{62}$, V. Kukhtin ${ }^{65}$, Y. Kulchitsky ${ }^{90}$, S. Kuleshov ${ }^{31 b}$, C. Kummer ${ }^{98}$, M. Kuna ${ }^{78}$, N. Kundu ${ }^{118}$, J. Kunkle ${ }^{120}$, A. Kupco ${ }^{125}$, H. Kurashige ${ }^{67}$, M. Ku-

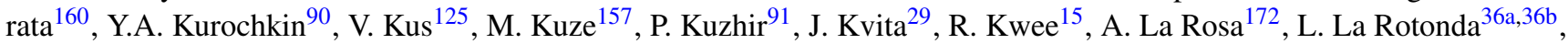
L. Labarga ${ }^{80}$, J. Labbe ${ }^{4}$, S. Lablak ${ }^{135 a}$, C. Lacasta ${ }^{167}$, F. Lacava132a,132b, H. Lacker ${ }^{15}$, D. Lacour ${ }^{78}$, V.R. Lacuesta ${ }^{167}$, E. Ladygin ${ }^{65}$, R. Lafaye ${ }^{4}$, B. Laforge ${ }^{78}$, T. Lagouri ${ }^{80}$, S. Lai ${ }^{48}$, E. Laisne ${ }^{55}$, M. Lamanna ${ }^{29}$, C.L. Lampen ${ }^{6}$, W. Lampl ${ }^{6}$, E. Lancon ${ }^{136}$, U. Landgraf ${ }^{48}$, M.P.J. Landon ${ }^{75}$, H. Landsman ${ }^{152}$, J.L. Lane ${ }^{82}$, C. Lange ${ }^{41}$, A.J. Lankford ${ }^{163}$, F. Lanni ${ }^{24}$, K. Lantzsch ${ }^{174}$, A. Lanza ${ }^{119}$, S. Laplace ${ }^{78}$, C. Lapoire ${ }^{20}$, J.F. Laporte ${ }^{136}$, T. Lari ${ }^{89 a}$, A.V. Larionov ${ }^{128}$, A. Larner ${ }^{118}$, C. Lasseur $^{29}$, M. Lassnig ${ }^{29}$, P. Laurelli ${ }^{47}$, W. Lavrijsen ${ }^{14}$, P. Laycock ${ }^{73}$, A.B. Lazarev ${ }^{65}$, O. Le Dortz ${ }^{78}$, E. Le Guirriec ${ }^{83}$, C. Le Maner ${ }^{158}$, E. Le Menedeu ${ }^{136}$, C. Lebel ${ }^{93}$, T. LeCompte ${ }^{5}$, F. Ledroit-Guillon ${ }^{55}$, H. Lee ${ }^{105}$, J.S.H. Lee ${ }^{116}$, S.C. Lee ${ }^{151}$, L. Lee ${ }^{175}$, M. Lefebvre ${ }^{169}$, M. Legendre ${ }^{136}$, A. Leger ${ }^{49}$, B.C. LeGeyt ${ }^{120}$, F. Legger ${ }^{98}$, C. Leggett ${ }^{14}$, M. Lehmacher ${ }^{20}$, G. Lehmann Miotto ${ }^{29}$, X. Lei ${ }^{6}$, M.A.L. Leite ${ }^{23 d}$, R. Leitner ${ }^{126}$, D. Lellouch ${ }^{171}$, M. Leltchouk ${ }^{34}$, B. Lemmer ${ }^{54}$, V. Lendermann $^{58 a}$, K.J.C. Leney ${ }^{145 b}$, T. Lenz ${ }^{105}$, G. Lenzen ${ }^{174}$, B. Lenzi ${ }^{29}$, K. Leonhardt ${ }^{43}$, S. Leontsinis ${ }^{9}$, C. Leroy ${ }^{93}$, JR. Lessard ${ }^{169}$, J. Lesser ${ }^{146 a}$, C.G. Lester ${ }^{27}$, A. Leung Fook Cheong ${ }^{172}$, J. Levêque ${ }^{4}$, D. Levin ${ }^{87}$, L.J. Levinson ${ }^{171}$, M.S. Levitski $^{128}$, M. Lewandowska ${ }^{21}$, A. Lewis ${ }^{118}$, G.H. Lewis ${ }^{108}$, A.M. Leyko ${ }^{20}$, M. Leyton ${ }^{15}$, B. Li ${ }^{83}, \mathrm{H}_{\text {. Li }}{ }^{172}, \mathrm{~S}_{\text {. Li }}{ }^{32 \mathrm{~b}, \mathrm{~d}}, \mathrm{X} . \mathrm{Li}^{87}$, Z. Liang ${ }^{39}$, Z. Liang ${ }^{118, \mathrm{r}}$, H. Liao ${ }^{33}$, B. Liberti ${ }^{133 a}$, P. Lichard ${ }^{29}$, M. Lichtnecker ${ }^{98}$, K. Lie ${ }^{165}$, W. Liebig ${ }^{13}$, R. Lifshitz ${ }^{152}$,

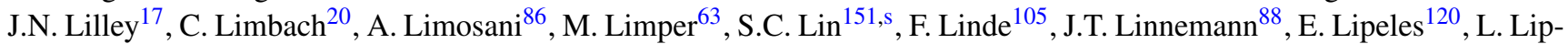

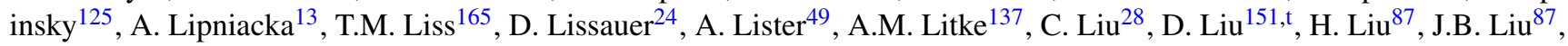
M. Liu ${ }^{32 b}$, S. Liu $^{2}$, Y. Liu ${ }^{32 b}$, M. Livan ${ }^{119 a, 119 b}$, S.S.A. Livermore ${ }^{118}$, A. Lleres ${ }^{55}$, J. Llorente Merino ${ }^{80}$, S.L. Lloyd ${ }^{75}$, E. Lobodzinska ${ }^{41}$, P. Loch $^{6}$, W.S. Lockman ${ }^{137}$, T. Loddenkoetter ${ }^{20}$, F.K. Loebinger ${ }^{82}$, A. Loginov ${ }^{175}$, C.W. Loh ${ }^{168}$, T. Lohse ${ }^{15}$, K. Lohwasser ${ }^{48}$, M. Lokajicek ${ }^{125}$, J. Loken ${ }^{118}$, V.P. Lombardo ${ }^{4}$, R.E. Long ${ }^{71}$, L. Lopes ${ }^{124 a, b}$, D. Lopez Mateos ${ }^{57}$, M. Losada ${ }^{162}$, P. Loscutoff ${ }^{14}$, F. Lo Sterzo ${ }^{132 a, 132 b}$, M.J. Losty ${ }^{159 a}$, X. Lou ${ }^{40}$, A. Lounis ${ }^{115}$, K.F. Loureiro ${ }^{162}$, J. Love ${ }^{21}$, P.A. Love ${ }^{71}$, A.J. Lowe ${ }^{143, f}$, F. Lu ${ }^{32 a}$, H.J. Lubatti ${ }^{138}$, C. Luci ${ }^{132 a, 132 b}$, A. Lucotte ${ }^{55}$, A. Ludwig ${ }^{43}$, D. Ludwig ${ }^{41}$, I. Ludwig $^{48}$, J. Ludwig ${ }^{48}$, F. Luehring ${ }^{61}$, G. Luijckx ${ }^{105}$, D. Lumb ${ }^{48}$, L. Luminari ${ }^{132 a}$, E. Lund ${ }^{117}$, B. Lund-Jensen ${ }^{147}$, B. Lundberg $^{79}$, J. Lundberg ${ }^{146 a, 146 b}$, J. Lundquist ${ }^{35}$, M. Lungwitz ${ }^{81}$, A. Lupi ${ }^{122 a, 122 b}$, G. Lutz ${ }^{99}$, D. Lynn ${ }^{24}$, J. Lys ${ }^{14}$, E. Lytken ${ }^{79}$, H. $\mathrm{Ma}^{24}$, L.L. Ma ${ }^{172}$, J.A. Macana Goia ${ }^{93}$, G. Maccarrone ${ }^{47}$, A. Macchiolo ${ }^{99}$, B. Maček ${ }^{74}$, J. Machado Miguens ${ }^{124 a}$, R. Mackeprang ${ }^{35}$, R.J. Madaras ${ }^{14}$, W.F. Mader ${ }^{43}$, R. Maenner ${ }^{58 c}$, T. Maeno $^{24}$, P. Mättig ${ }^{174}$, S. Mättig ${ }^{41}$, L. Magnoni ${ }^{29}$, E. Magradze ${ }^{54}$, Y. Mahalalel ${ }^{153}$, K. Mahboubi ${ }^{48}$, G. Mahout $^{17}$, C. Maiani ${ }^{132 a, 132 b}$, C. Maidantchik ${ }^{23 a}$, A. Maio ${ }^{124 a, b}$, S. Majewski ${ }^{24}$, Y. Makida ${ }^{66}$, N. Makovec ${ }^{115}$, P. Mal ${ }^{6}$, B. Malaescu ${ }^{29}$, Pa. Malecki ${ }^{38}$, P. Malecki ${ }^{38}$, V.P. Maleev ${ }^{121}$, F. Malek ${ }^{55}$, U. Mallik ${ }^{63}$, D. Malon ${ }^{5}$, C. Malone ${ }^{143}$, S. Maltezos ${ }^{9}$, V. Malyshev ${ }^{107}$, S. Malyukov ${ }^{29}$, R. Mameghani ${ }^{98}$, J. Mamuzic $^{12 b}$, A. Manabe ${ }^{66}$, L. Mandelli ${ }^{89 a}$, I. Mandić ${ }^{74}$, R. Mandrysch ${ }^{15}$, J. Maneira ${ }^{124 a}$, P.S. Mangeard ${ }^{88}$, I.D. Manjavidze ${ }^{65}$, A. Mann ${ }^{54}$, P.M. Manning ${ }^{137}$, A. Manousakis-Katsikakis ${ }^{8}$, B. Mansoulie ${ }^{136}$, A. Manz ${ }^{99}$, A. Mapelli29, L. Mapelli ${ }^{29}$, L. March $^{80}$, J.F. Marchand ${ }^{29}$, F. Marchese ${ }^{133 a, 133 b}$, G. Marchiori ${ }^{78}$, M. Marcisovsky ${ }^{125}$, A. Marin ${ }^{21, *}$, C.P. Marino ${ }^{169}$, F. Marroquim ${ }^{23 a}$, R. Marshall ${ }^{82}$, Z. Marshall ${ }^{29}$, F.K. Martens ${ }^{158}$, S. Marti-Garcia ${ }^{167}$, A.J. Martin ${ }^{175}$, B. Martin ${ }^{29}$, B. Mar$\operatorname{tin}^{88}$, F.F. Martin ${ }^{120}$, J.P. Martin ${ }^{93}$, Ph. Martin55, T.A. Martin ${ }^{17}$, V.J. Martin ${ }^{45}$, B. Martin dit Latour ${ }^{49}$, S. MartinHaugh $^{149}$, M. Martinez ${ }^{11}$, V. Martinez Outschoorn ${ }^{57}$, A.C. Martyniuk ${ }^{82}$, M. Marx ${ }^{82}$, F. Marzano ${ }^{132 a}$, A. Marzin ${ }^{111}$, L. Masetti ${ }^{81}$, T. Mashimo ${ }^{155}$, R. Mashinistov ${ }^{94}$, J. Masik ${ }^{82}$, A.L. Maslennikov ${ }^{107}$, I. Massa ${ }^{19 a, 19 b}$, G. Massaro ${ }^{105}$, N. Mas$\mathrm{sol}^{4}$, P. Mastrandrea ${ }^{132 \mathrm{a}, 132 \mathrm{~b}}$, A. Mastroberardino ${ }^{36 \mathrm{a}, 36 \mathrm{~b}}$, T. Masubuchi ${ }^{155}$, M. Mathes ${ }^{20}$, P. Matricon ${ }^{115}$, H. Matsumoto ${ }^{155}$, H. Matsunaga ${ }^{155}$, T. Matsushita ${ }^{67}$, C. Mattravers ${ }^{118, c}$, J.M. Maugain ${ }^{29}$, S.J. Maxfield ${ }^{73}$, D.A. Maximov ${ }^{107}$, E.N. May ${ }^{5}$, A. Mayne ${ }^{139}$, R. Mazini ${ }^{151}$, M. Mazur ${ }^{20}$, M. Mazzanti ${ }^{89 a}$, E. Mazzoni ${ }^{122 a, 122 b}$, S.P. Mc Kee ${ }^{87}$, A. McCarn ${ }^{165}$, R.L. McCarthy $^{148}$, T.G. McCarthy ${ }^{28}$, N.A. McCubbin ${ }^{129}$, K.W. McFarlane ${ }^{56}$, J.A. Mcfayden ${ }^{139}$, H. McGlone ${ }^{53}$, G. Mchedlidze ${ }^{51 b}$, R.A. McLaren ${ }^{29}$, T. Mclaughlan ${ }^{17}$, S.J. McMahon ${ }^{129}$, R.A. McPherson ${ }^{169, j}$, A. Meade ${ }^{84}$, J. Mechnich ${ }^{105}$, M. Mechtel ${ }^{174}$, M. Medinnis ${ }^{41}$, R. Meera-Lebbai ${ }^{111}$, T. Meguro ${ }^{116}$, R. Mehdiyev ${ }^{93}$, S. Mehlhase ${ }^{35}$, A. Mehta ${ }^{73}$, K. Meier ${ }^{58 a}$, J. Meinhardt $^{48}$, B. Meirose ${ }^{79}$, C. Melachrinos ${ }^{30}$, B.R. Mellado Garcia ${ }^{172}$, L. Mendoza Navas ${ }^{162}$, Z. Meng ${ }^{151, t}$, A. Mengarelli ${ }^{19 a, 19 b}$, S. Menke ${ }^{99}$, C. Menot $^{29}$, E. Meoni ${ }^{11}$, K.M. Mercurio ${ }^{57}$, P. Mermod ${ }^{118}$, L. Merola ${ }^{102 a, 102 b}$, C. Meroni ${ }^{89 a}$, F.S. Merritt $^{30}$, A. Messina ${ }^{29}$, J. Metcalfe ${ }^{103}$, A.S. Mete ${ }^{64}$, C. Meyer $^{81}$, J-P. Meyer ${ }^{136}$, J. Meyer ${ }^{173}$, J. Meyer ${ }^{54}$, T.C. Meyer ${ }^{29}$, W.T. Meyer ${ }^{64}$, J. Miao ${ }^{32 d}$, S. Michal ${ }^{29}$, L. Micu ${ }^{25 a}$, R.P. Middleton ${ }^{129}$, P. Miele ${ }^{29}$, S. Migas ${ }^{73}$, L. Mijović ${ }^{41}$, G. Miken- 
berg $^{171}$, M. Mikestikova ${ }^{125}$, M. Mikuž ${ }^{74}$, D.W. Miller ${ }^{30}$, R.J. Miller ${ }^{88}$, W.J. Mills ${ }^{168}$, C. Mills ${ }^{57}$, A. Milov ${ }^{171}$, D.A. Milstead $^{146 a, 146 b}$, D. Milstein ${ }^{171}$, A.A. Minaenko ${ }^{128}$, M. Miñano Moya ${ }^{167}$, I.A. Minashvili ${ }^{65}$, A.I. Mincer ${ }^{108}$, B. Mindur ${ }^{37}$, M. Mineev ${ }^{65}$, Y. Ming ${ }^{130}$, L.M. Mir ${ }^{11}$, G. Mirabelli ${ }^{132 a}$, L. Miralles Verge ${ }^{11}$, S. Misawa ${ }^{24}$, A. Misiejuk ${ }^{76}$, J. Mitrevski ${ }^{137}$, G.Y. Mitrofanov ${ }^{128}$, V.A. Mitsou ${ }^{167}$, S. Mitsui ${ }^{66}$, P.S. Miyagawa ${ }^{139}$, K. Miyazaki ${ }^{67}$, J.U. Mjörnmark ${ }^{79}$, T. Moa ${ }^{146 a, 146 b}$, P. Mockett ${ }^{138}$, S. Moed ${ }^{57}$, V. Moeller ${ }^{27}$, K. Mönig ${ }^{41}$, N. Möser ${ }^{20}$, S. Mohapatra ${ }^{148}$, W. Mohr ${ }^{48}$, S. Mohrdieck-Möck ${ }^{99}$, A.M. Moisseev ${ }^{128, *}$, R. Moles-Valls ${ }^{167}$, J. Molina-Perez ${ }^{29}$, J. Monk ${ }^{77}$, E. Monnier ${ }^{83}$, S. Montesano ${ }^{89 a, 89 b}$, F. Monticelli $^{70}$, S. Monzani ${ }^{19 a, 19 b}$, R.W. Moore ${ }^{2}$, G.F. Moorhead ${ }^{86}$, C. Mora Herrera ${ }^{49}$, A. Moraes ${ }^{53}$, N. Morange ${ }^{136}$, J. Morel ${ }^{54}$, G. Morello ${ }^{36 a, 36 b}$, D. Moreno ${ }^{81}$, M. Moreno Llácer ${ }^{167}$, P. Morettini ${ }^{50 a}$, M. Morii ${ }^{57}$, J. Morin ${ }^{75}$, A.K. Morley ${ }^{29}$, G. Mornacchi $^{29}$, S.V. Morozov ${ }^{96}$, J.D. Morris ${ }^{75}$, L. Morvaj ${ }^{101}$, H.G. Moser ${ }^{99}$, M. Mosidze ${ }^{51 b}$, J. Moss ${ }^{109}$, R. Mount ${ }^{143}$, E. Mountricha $^{136}$, S.V. Mouraviev ${ }^{94}$, E.J.W. Moyse ${ }^{84}$, M. Mudrinic ${ }^{12 b}$, F. Mueller ${ }^{58 a}$, J. Mueller ${ }^{123}$, K. Mueller ${ }^{20}$, T.A. Müller ${ }^{98}$, D. Muenstermann ${ }^{29}$, A. Muir ${ }^{168}$, Y. Munwes ${ }^{153}$, W.J. Murray ${ }^{129}$, I. Mussche ${ }^{105}$, E. Musto ${ }^{102 a, 102 b}$, A.G. Myagkov ${ }^{128}$, M. Myska ${ }^{125}$, J. Nadal ${ }^{11}$, K. Nagai ${ }^{160}$, K. Nagano ${ }^{66}$, Y. Nagasaka ${ }^{60}$, A.M. Nairz ${ }^{29}$, Y. Nakahama ${ }^{29}$, K. Nakamura ${ }^{155}$, T. Nakamura ${ }^{155}$, I. Nakano ${ }^{110}$, G. Nanava ${ }^{20}$, A. Napier ${ }^{161}$, M. Nash ${ }^{77, c}$, N.R. Nation ${ }^{21}$, T. Nattermann ${ }^{20}$, T. Naumann ${ }^{41}$, G. Navarro ${ }^{162}$, H.A. Neal ${ }^{87}$, E. Nebot ${ }^{80}$, P.Yu. Nechaeva ${ }^{94}$, A. Negri1 ${ }^{19 a, 119 b}$, G. Negri ${ }^{29}$, S. Nektarijevic ${ }^{49}$, A. Nelson $^{163}$, S. Nelson ${ }^{143}$, T.K. Nelson ${ }^{143}$, S. Nemecek ${ }^{125}$, P. Nemethy ${ }^{108}$, A.A. Nepomuceno ${ }^{23 a}$, M. Nessi ${ }^{29, u}$, S.Y. Nesterov ${ }^{121}$, M.S. Neubauer ${ }^{165}$, A. Neusiedl ${ }^{81}$, R.M. Neves ${ }^{108}$, P. Nevski ${ }^{24}$, P.R. Newman ${ }^{17}$, V. Nguyen Thi Hong ${ }^{136}$, R.B. Nicker$\operatorname{son}^{118}$, R. Nicolaidou ${ }^{136}$, L. Nicolas ${ }^{139}$, G. Nicoletti ${ }^{47}$, B. Nicquevert ${ }^{29}$, F. Niedercorn ${ }^{115}$, J. Nielsen ${ }^{137}$, T. Niinikoski ${ }^{29}$, N. Nikiforou ${ }^{34}$, A. Nikiforov ${ }^{15}$, V. Nikolaenko ${ }^{128}$, K. Nikolaev ${ }^{65}$, I. Nikolic-Audit ${ }^{78}$, K. Nikolics ${ }^{49}$, K. Nikolopoulos ${ }^{24}$, H. Nilsen ${ }^{48}$, P. Nilsson ${ }^{7}$, Y. Ninomiya ${ }^{155}$, A. Nisati ${ }^{132 a}$, T. Nishiyama ${ }^{67}$, R. Nisius ${ }^{99}$, L. Nodulman ${ }^{5}$, M. Nomachi ${ }^{116}$, I. No$\operatorname{midis}^{154}$, M. Nordberg ${ }^{29}$, B. Nordkvist ${ }^{146 a, 146 b}$, P.R. Norton ${ }^{129}$, D. Notz ${ }^{41}$, J. Novakova ${ }^{126}$, M. Nozaki ${ }^{66}$, L. Nozka ${ }^{113}$, I.M. Nugent ${ }^{159}$ a , A.-E. Nuncio-Quiroz ${ }^{20}$, G. Nunes Hanninger ${ }^{86}$, T. Nunnemann ${ }^{98}$, E. Nurse ${ }^{77}$, T. Nyman ${ }^{29}$, B.J. O'Brien ${ }^{45}$, S.W. O’Neale ${ }^{17, *}$, D.C. O’Neil ${ }^{142}$, V. O’Shea ${ }^{53}$, F.G. Oakham ${ }^{28, e}$, H. Oberlack ${ }^{99}$, J. Ocariz ${ }^{78}$, A. Ochi ${ }^{67}$, S. Oda ${ }^{155}$, S. Odaka ${ }^{66}$, J. Odier ${ }^{83}$, H. Ogren ${ }^{61}$, A. $\mathrm{Oh}^{82}$, S.H. Oh ${ }^{44}$, C.C. Ohm ${ }^{146 a, 146 b}$, T. Ohshima ${ }^{101}$, H. Ohshita ${ }^{140}$, T. Ohsugi ${ }^{59}$, S. Okada ${ }^{67}$, H. Okawa ${ }^{163}$, Y. Okumura ${ }^{101}$, T. Okuyama ${ }^{155}$, M. Olcese ${ }^{50 a}$, A.G. Olchevski ${ }^{65}$, M. Oliveira ${ }^{124 a, h}$, D. Oliveira Damazio $^{24}$, E. Oliver Garcia ${ }^{167}$, D. Olivito ${ }^{120}$, A. Olszewski ${ }^{38}$, J. Olszowska ${ }^{38}$, C. Omachi ${ }^{67}$, A. Onofre ${ }^{124 a, v}$, P.U.E. Onyisi $^{30}$, C.J. Oram ${ }^{159 a}$, M.J. Oreglia ${ }^{30}$, Y. Oren ${ }^{153}$, D. Orestano ${ }^{134 a, 134 b}$, I. Orlov ${ }^{107}$, C. Oropeza Barrera ${ }^{53}$, R.S. Orr ${ }^{158}$, B. Osculati ${ }^{50 a, 50 b}$, R. Ospanov ${ }^{120}$, C. Osuna ${ }^{11}$, G. Otero y Garzon $^{26}$, J.P. Ottersbach ${ }^{105}$, M. Ouchrif ${ }^{135 d}$, F. Ould-Saada ${ }^{117}$, A. Ouraou ${ }^{136}$, Q. Ouyang ${ }^{32 a}$, M. Owen ${ }^{82}$, S. Owen ${ }^{139}$, V.E. Ozcan ${ }^{18 a}$, N. Ozturk ${ }^{7}$, A. Pacheco Pages ${ }^{11}$, C. Padilla Aranda ${ }^{11}$, S. Pagan $\mathrm{Griso}^{14}$, E. Paganis ${ }^{139}$, F. Paige ${ }^{24}$, K. Pajchel ${ }^{117}$, G. Palacino ${ }^{159 b}$, C.P. Paleari ${ }^{6}$, S. Palestini ${ }^{29}$, D. Pallin ${ }^{33}$, A. Palma ${ }^{124 a}$, J.D. Palmer ${ }^{17}$, Y.B. Pan ${ }^{172}$, E. Panagiotopoulou ${ }^{9}$, B. Panes ${ }^{31 a}$, N. Panikashvili ${ }^{87}$, S. Panitkin ${ }^{24}$, D. Pantea ${ }^{25 a}$, M. Panuskova ${ }^{125}$, V. Paolone ${ }^{123}$, A. Papadelis ${ }^{146 a}$, Th.D. Papadopoulou' ${ }^{9}$ A. Paramonov ${ }^{5}$, W. Park ${ }^{24, w}$, M.A. Parker ${ }^{27}$, F. Parodi ${ }^{50 a, 50 b}$, J.A. Parsons ${ }^{34}$, U. Parzefall ${ }^{48}$, E. Pasqualucci ${ }^{132 a}$, A. Passeri ${ }^{134 a}$, F. Pastore ${ }^{134 a, 134 b}$, Fr. Pastore ${ }^{76}$, G. Pásztor $^{49, x}$, S. Pataraia ${ }^{174}$, N. Patel ${ }^{150}$, J.R. Pater ${ }^{82}$, S. Patricellii ${ }^{102 a, 102 b}$, T. Pauly ${ }^{29}$, M. Pecsy ${ }^{144 a}$, M.I. Pedraza Morales ${ }^{172}$, S.V. Peleganchuk ${ }^{107}$, H. Peng ${ }^{32 b}$, R. Pengo ${ }^{29}$, A. Penson ${ }^{34}$, J. Penwell ${ }^{61}$, M. Perantoni ${ }^{23 a}$, K. Perez ${ }^{34, y}$, T. Perez Cavalcanti $^{41}$, E. Perez Codina ${ }^{11}$, M.T. Pérez García-Estañ ${ }^{167}$, V. Perez Reale ${ }^{34}$, L. Perini ${ }^{89 a, 89 b}$, H. Pernegger ${ }^{29}$, R. Perrino $^{72 a}$, P. Perrodo ${ }^{4}$, S. Persembe ${ }^{3 a}$, A. Perus ${ }^{115}$, V.D. Peshekhonov ${ }^{65}$, B.A. Petersen ${ }^{29}$, J. Petersen ${ }^{29}$, T.C. Petersen ${ }^{35}$, E. Petit ${ }^{83}$, A. Petridis ${ }^{154}$, C. Petridou ${ }^{154}$, E. Petrolo ${ }^{132 a}$, F. Petrucci ${ }^{134 a, 134 b}$, D. Petschull ${ }^{41}$, M. Petteni ${ }^{142}$, R. Pezoa ${ }^{31 b}$, B. Pfeifer ${ }^{48}$, A. Phan ${ }^{86}$, A.W. Phillips ${ }^{27}$, P.W. Phillips ${ }^{129}$, G. Piacquadio ${ }^{29}$, E. Piccaro ${ }^{75}$, M. Piccinini ${ }^{19 a, 19 b}$, A. Pickford ${ }^{53}$, S.M. Piec ${ }^{41}$, R. Piegaia ${ }^{26}$, J.E. Pilcher ${ }^{30}$, A.D. Pilkington ${ }^{82}$, J. Pina ${ }^{124 a, b}$, M. Pinamonti ${ }^{164 a, 164 c}$, A. Pinder ${ }^{118}$, J.L. Pinfold ${ }^{2}$, J. Ping ${ }^{32 c}$, B. Pinto ${ }^{124 a, b}$, O. Pirotte ${ }^{29}$, C. Pizio ${ }^{89 a, 89 b}$, R. Placakyte ${ }^{41}$, M. Plamondon ${ }^{169}$, M.-A. Pleier ${ }^{24}$, A.V. Pleskach ${ }^{128}$, A. Poblaguev ${ }^{24}$, S. Poddar ${ }^{58 a}$, F. Podlyski ${ }^{33}$, L. Poggioli ${ }^{115}$, T. Poghosyan ${ }^{20}$, M. Pohl ${ }^{49}$, F. Polci ${ }^{55}$, G. Polesello ${ }^{119 a}$, A. Policicchio $^{138}$, A. Polini ${ }^{19 a}$, J. Poll ${ }^{75}$, V. Polychronakos ${ }^{24}$, D.M. Pomarede ${ }^{136}$, D. Pomeroy ${ }^{22}$, K. Pommès ${ }^{29}$, L. Pontecorvo ${ }^{132 a}$, B.G. Pope ${ }^{88}$, G.A. Popeneciu ${ }^{25 a}$, D.S. Popovic ${ }^{12 a}$, A. Poppleton ${ }^{29}$, X. Portell Bueso ${ }^{29}$, C. Posch ${ }^{21}$, G.E. Pospelov ${ }^{99}$, S. Pospisil ${ }^{127}$, M. Potekhin ${ }^{24}$, I.N. Potrap ${ }^{99}$, C.J. Potter ${ }^{149}$, K.P. Potter $^{82}$, C.T. Potter ${ }^{114}$, G. Poulard ${ }^{29}$, J. Poveda ${ }^{172}$,

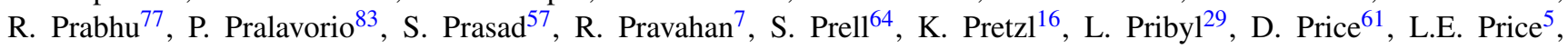
M.J. Price ${ }^{29}$, P.M. Prichard ${ }^{73}$, D. Prieur ${ }^{123}$, M. Primavera ${ }^{72 a}$, K. Prokofiev ${ }^{108}$, F. Prokoshin ${ }^{31 b}$, S. Protopopescu ${ }^{24}$, J. Proudfoot $^{5}$, X. Prudent ${ }^{43}$, H. Przysiezniak ${ }^{4}$, S. Psoroulas ${ }^{20}$, E. Ptacek ${ }^{114}$, E. Pueschel ${ }^{84}$, J. Purdham ${ }^{87}$, M. Purohit ${ }^{24, w}$, P. Puzo ${ }^{115}$,

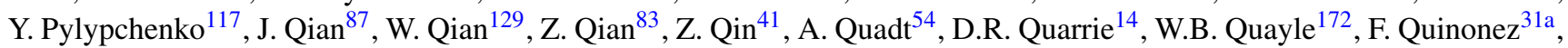
M. Raas ${ }^{104}$, V. Radeka ${ }^{24}$, V. Radescu ${ }^{58 b}$, B. Radics ${ }^{20}$, T. Rador ${ }^{18 a}$, F. Ragusa ${ }^{89 a, 89 b}$, G. Rahal ${ }^{177}$, A.M. Rahimi ${ }^{109}$, D. Rahm $^{24}$, S. Rajagopalan ${ }^{24}$, M. Rammensee ${ }^{48}$, M. Rammes ${ }^{141}$, M. Ramstedt ${ }^{146 a, 146 b}$, A.S. Randle-Conde ${ }^{39}$, K. Randrianarivony ${ }^{28}$, P.N. Ratoff ${ }^{71}$, F. Rauscher ${ }^{98}$, E. Rauter ${ }^{99}$, M. Raymond ${ }^{29}$, A.L. Read ${ }^{117}$, D.M. Rebuzzi ${ }^{119 a, 119 b}$, A. Redelbach $^{173}$, G. Redlinger ${ }^{24}$, R. Reece ${ }^{120}$, K. Reeves ${ }^{40}$, A. Reichold ${ }^{105}$, E. Reinherz-Aronis ${ }^{153}$, A. Reinsch ${ }^{114}$, I. Reisinger ${ }^{42}$, D. Reljic ${ }^{12 a}$, C. Rembser ${ }^{29}$, Z.L. Ren ${ }^{151}$, A. Renaud ${ }^{115}$, P. Renkel ${ }^{39}$, S. Rescia ${ }^{24}$, M. Rescigno ${ }^{132 a}$, S. Resconi ${ }^{89 a}$, B. Re- 
sende $^{136}$, P. Reznicek ${ }^{98}$, R. Rezvani ${ }^{158}$, A. Richards ${ }^{77}$, R. Richter ${ }^{99}$, E. Richter-Was ${ }^{4, z}$, M. Ridel ${ }^{78}$, S. Rieke $^{81}$, M. Ri- $^{2}$ jpstra $^{105}$, M. Rijssenbeek ${ }^{148}$, A. Rimoldi ${ }^{119 a, 119 b}$, L. Rinaldi ${ }^{19 a}$, R.R. Rios ${ }^{39}$, I. Riu ${ }^{11}$, G. Rivoltella ${ }^{89 a, 89 b}$, F. Rizatdinova $^{112}$, E. Rizvi ${ }^{75}$, S.H. Robertson ${ }^{85, j}$, A. Robichaud-Veronneau ${ }^{118}$, D. Robinson ${ }^{27}$, J.E.M. Robinson ${ }^{77}$, M. Robinson ${ }^{114}$, A. Robson ${ }^{53}$, J.G. Rocha de Lima ${ }^{106}$, C. Roda ${ }^{122 a, 122 b}$, D. Roda Dos Santos ${ }^{29}$, S. Rodier ${ }^{80}$, D. Rodriguez ${ }^{162}$, Y. Rodriguez Garcia ${ }^{162}$, A. Roe ${ }^{54}$, S. Roe ${ }^{29}$, O. Røhne ${ }^{117}$, V. Rojo ${ }^{1}$, S. Rolli ${ }^{161}$, A. Romaniouk ${ }^{96}$, M. Romano ${ }^{19 a, 19 b}$, V.M. Romanov $^{65}$, G. Romeo ${ }^{26}$, L. Roos $^{78}$, E. Ros ${ }^{167}$, S. Rosati ${ }^{132 a, 132 b}$, K. Rosbach ${ }^{49}$, A. Rose ${ }^{149}$, M. Rose ${ }^{76}$, G.A. Rosenbaum $^{158}$, E.I. Rosenberg ${ }^{64}$, P.L. Rosendahl ${ }^{13}$, O. Rosenthal ${ }^{141}$, L. Rosselet ${ }^{49}$, V. Rossetti ${ }^{11}$, E. Rossi ${ }^{132 a, 132 b}$, L.P. Rossi ${ }^{50 a}$, L. Rossi ${ }^{89 a, 89 b}$, M. Rotaru ${ }^{25 a}$, I. Roth ${ }^{171}$, J. Rothberg ${ }^{138}$, D. Rousseau ${ }^{115}$, C.R. Royon ${ }^{136}$, A. Rozanov ${ }^{83}$, Y. Rozen ${ }^{152}$, X. Ruan ${ }^{115}$, I. Rubinskiy ${ }^{41}$, B. Ruckert ${ }^{98}$, N. Ruckstuhl ${ }^{105}$, V.I. Rud ${ }^{97}$, C. Rudolph ${ }^{43}$, G. Rudolph ${ }^{62}$, F. Rühr ${ }^{6}$, F. Ruggieri $^{134 a, 134 b}$, A. Ruiz-Martinez ${ }^{64}$, E. Rulikowska-Zarebska ${ }^{37}$, V. Rumiantsev ${ }^{91, *}$, L. Rumyantsev ${ }^{65}$, K. Runge ${ }^{48}$, O. Runolfsson $^{20}$, Z. Rurikova ${ }^{48}$, N.A. Rusakovich ${ }^{65}$, D.R. Rust ${ }^{61}$, J.P. Rutherfoord ${ }^{6}$, C. Ruwiedel ${ }^{14}$, P. Ruzicka ${ }^{125}$, Y.F. Ryabov ${ }^{121}$, V. Ryadovikov ${ }^{128}$, P. Ryan ${ }^{88}$, M. Rybar ${ }^{126}$, G. Rybkin ${ }^{115}$, N.C. Ryder ${ }^{118}$, S. Rzaeva ${ }^{10}$, A.F. Saavedra ${ }^{150}$, I. Sadeh ${ }^{153}$, H.FW. Sadrozinski ${ }^{137}$, R. Sadykov ${ }^{65}$, F. Safai Tehrani ${ }^{132 a, 132 b}$, H. Sakamoto ${ }^{155}$, G. Salamanna ${ }^{75}$, A. Salamon ${ }^{133 a}$, M. Saleem ${ }^{111}$, D. Salihagic ${ }^{99}$, A. Salnikov ${ }^{143}$, J. Salt ${ }^{167}$, B.M. Salvachua Ferrando ${ }^{5}$, D. Salvatore ${ }^{36 a, 36 b}$, F. Salvatore ${ }^{149}$, A. Salvucci ${ }^{104}$, A. Salzburger ${ }^{29}$, D. Sampsonidis ${ }^{154}$, B.H. Samset ${ }^{117}$, A. Sanchez ${ }^{102 a, 102 b}$, H. Sandaker ${ }^{13}$, H.G. Sander ${ }^{81}$, M.P. Sanders ${ }^{98}$, M. Sandhoff ${ }^{174}$, T. Sandoval ${ }^{27}$, C. Sandoval ${ }^{162}$, R. Sandstroem ${ }^{99}$, S. Sandvoss ${ }^{174}$, D.P.C. Sankey ${ }^{129}$, A. Sansoni ${ }^{47}$, C. Santamarina Rios $^{85}$, C. Santoni ${ }^{33}$, R. Santonico ${ }^{133 a, 133 b}$, H. Santos ${ }^{124 a}$, J.G. Saraiva ${ }^{124 a}$, T. Sarangi ${ }^{172}$, E. Sarkisyan-Grinbaum ${ }^{7}$, F. Sarri ${ }^{122 a, 122 b}$, G. Sartisohn ${ }^{174}$, O. Sasaki ${ }^{66}$, T. Sasaki ${ }^{66}$, N. Sasao ${ }^{68}$, I. Satsounkevitch ${ }^{90}$, G. Sauvage ${ }^{4}$, E. Sauvan ${ }^{4}$, J.B. Sauvan ${ }^{115}$, P. Savard ${ }^{158, \text { e }}$, A.Y. Savine ${ }^{6}$, V. Savinov ${ }^{123}$, D.O. Savu ${ }^{29}$, P. Savva ${ }^{9}$, L. Sawyer ${ }^{24,1}$, D.H. Saxon ${ }^{53}$, L.P. Says ${ }^{33}$, C. Sbarra ${ }^{19 a}$, A. Sbrizzi ${ }^{19 a, 19 b}$, O. Scallon ${ }^{93}$, D.A. Scannicchio ${ }^{163}$, J. Schaarschmidt ${ }^{115}$, P. Schacht ${ }^{99}$, U. Schäfer ${ }^{81}$, S. Schaepe ${ }^{20}$, S. Schaetzel ${ }^{58 b}$, A.C. Schaffer ${ }^{115}$, D. Schaile ${ }^{98}$, R.D. Schamberger ${ }^{148}$, A.G. Schamov ${ }^{107}$, V. Scharf ${ }^{58 a}$, V.A. Schegelsky ${ }^{121}$, D. Scheirich ${ }^{87}$, M. Schernau ${ }^{163}$, M.I. Scherzer ${ }^{14}$, C. Schiavi ${ }^{50 a, 50 b}$, J. Schieck ${ }^{98}$, M. Schioppa ${ }^{36 a, 36 b}$, S. Schlenker ${ }^{29}$, J.L. Schlereth ${ }^{5}$, E. Schmidt ${ }^{48}$, K. Schmieden ${ }^{20}$, C. Schmitt ${ }^{81}$, S. Schmitt ${ }^{58 b}$, M. Schmitz ${ }^{20}$, A. Schöning $^{58 b}$, M. Schott ${ }^{29}$, D. Schouten ${ }^{159 a}$, J. Schovancova ${ }^{125}$, M. Schram ${ }^{85}$, C. Schroeder ${ }^{81}$, N. Schroer ${ }^{58 c}$, S. Schuh ${ }^{29}$, G. Schuler ${ }^{29}$, J. Schultes ${ }^{174}$, H.-C. Schultz-Coulon ${ }^{58 a}$, H. Schulz ${ }^{15}$, J.W. Schumacher ${ }^{20}$, M. Schumacher ${ }^{48}$, B.A. Schumm ${ }^{137}$, $\mathrm{Ph}$. Schune ${ }^{136}$, C. Schwanenberger ${ }^{82}$, A. Schwartzman ${ }^{143}$, Ph. Schwemling ${ }^{78}$, R. Schwienhorst ${ }^{88}$, R. Schwierz ${ }^{43}$, J. Schwindling $^{136}$, T. Schwindt ${ }^{20}$, W.G. Scott ${ }^{129}$, J. Searcy ${ }^{114}$, G. Sedov $^{41}$, E. Sedykh ${ }^{121}$, E. Segura ${ }^{11}$, S.C. Seidel ${ }^{103}$, A. Seiden $^{137}$, F. Seifert ${ }^{43}$, J.M. Seixas ${ }^{23 a}$, G. Sekhniaidze ${ }^{102 a}$, D.M. Seliverstov ${ }^{121}$, B. Sellden ${ }^{146 a}$, G. Sellers ${ }^{73}$, M. Seman ${ }^{144 b}$, N. Semprini-Cesari ${ }^{19 a, 19 b}$, C. Serfon ${ }^{98}$, L. Serin ${ }^{115}$, R. Seuster ${ }^{99}$, H. Severini ${ }^{111}$, M.E. Sevior ${ }^{86}$, A. Sfyrla ${ }^{29}$, E. Shabalina ${ }^{54}$, M. Shamim ${ }^{114}$, L.Y. Shan ${ }^{32 a}$, J.T. Shank ${ }^{21}$, Q.T. Shao ${ }^{86}$, M. Shapiro ${ }^{14}$, P.B. Shatalov ${ }^{95}$, L. Shaver ${ }^{6}$, K. Shaw ${ }^{164 a, 164 c}$, D. Sherman ${ }^{175}$, P. Sherwood ${ }^{77}$, A. Shibata ${ }^{108}$, H. Shichi ${ }^{101}$, S. Shimizu ${ }^{29}$, M. Shimojima ${ }^{100}$, T. Shin ${ }^{56}$, A. Shmeleva ${ }^{94}$, M.J. Shochet ${ }^{30}$, D. Short ${ }^{118}$, M.A. Shupe ${ }^{6}$, P. Sicho ${ }^{125}$, A. Sidoti ${ }^{132 a, 132 b}$, A. Siebel ${ }^{174}$, F. Siegert ${ }^{48}$, J. Siegrist ${ }^{14}$, Dj. Sijacki $^{12 a}$, O. Silbert ${ }^{171}$, J. Silva ${ }^{124 a, b}$, Y. Silver ${ }^{153}$, D. Silverstein ${ }^{143}$, S.B. Silverstein ${ }^{146 a}$, V. Simak ${ }^{127}$, O. Simard ${ }^{136}$, Lj. Simic ${ }^{12 a}$, S. Simion ${ }^{115}$, B. Simmons ${ }^{77}$, M. Simonyan ${ }^{35}$, P. Sinervo ${ }^{158}$, N.B. Sinev ${ }^{114}$, V. Sipica ${ }^{141}$, G. Siragusa ${ }^{173}$, A. $\operatorname{Sircar}^{24}$, A.N. Sisakyan ${ }^{65}$, S.Yu. Sivoklokov ${ }^{97}$, J. Sjölin ${ }^{146 a, 146 b}$, T.B. Sjursen ${ }^{13}$, L.A. Skinnari ${ }^{14}$, H.P. Skottowe ${ }^{57}$, K. Skovpen ${ }^{107}$, P. Skubic ${ }^{111}$, N. Skvorodnev ${ }^{22}$, M. Slater ${ }^{17}$, T. Slavicek ${ }^{127}$, K. Sliwa ${ }^{161}$, J. Sloper ${ }^{29}$, V. Smakhtin ${ }^{171}$, S.Yu. Smirnov ${ }^{96}$, Y. Smirnov ${ }^{24}$, L.N. Smirnova ${ }^{97}$, O. Smirnova ${ }^{79}$, B.C. Smith ${ }^{57}$, D. Smith ${ }^{143}$, K.M. Smith ${ }^{53}$, M. Smizanska $^{71}$, K. Smolek ${ }^{127}$, A.A. Snesarev ${ }^{94}$, S.W. Snow ${ }^{82}$, J. Snow ${ }^{111}$, J. Snuverink ${ }^{105}$, S. Snyder ${ }^{24}$, M. Soares ${ }^{124 a}$, R. Sobie ${ }^{169, j}$, J. Sodomka ${ }^{127}$, A. Soffer ${ }^{153}$, C.A. Solans ${ }^{167}$, M. Solar ${ }^{127}$, J. Solc ${ }^{127}$, E. Soldatov ${ }^{96}$, U. Soldevila ${ }^{167}$, E. Solfaroli Camillocci $^{132 a, 132 b}$, A.A. Solodkov ${ }^{128}$, O.V. Solovyanov ${ }^{128}$, J. Sondericker ${ }^{24}$, N. Soni ${ }^{2}$, V. Sopko ${ }^{127}$, B. Sopko ${ }^{127}$, M. Sorbi ${ }^{89 a, 89 b}$, M. Sosebee ${ }^{7}$, R. Soualah ${ }^{164 a, 164 c}$, A. Soukharev ${ }^{107}$, S. Spagnolo ${ }^{72 a, 72 b}$, F. Spanò ${ }^{76}$, R. Spighi ${ }^{19 a}$, G. Spigo ${ }^{29}$, F. Spila ${ }^{132 a, 132 b}$, E. Spiriti ${ }^{134 a}$, R. Spiwoks ${ }^{29}$, M. Spousta ${ }^{126}$, T. Spreitzer ${ }^{158}$, B. Spurlock ${ }^{7}$, R.D. St. Denis ${ }^{53}$, T. Stahl ${ }^{141}$, J. Stahlman ${ }^{120}$, R. Stamen ${ }^{58 a}$, E. Stanecka ${ }^{29}$, R.W. Stanek ${ }^{5}$, C. Stanescu ${ }^{134 a}$, S. Stapnes ${ }^{117}$, E.A. Starchenko ${ }^{128}$, J. Stark ${ }^{55}$, P. Staroba ${ }^{125}$, P. Starovoitov ${ }^{91}$, A. Staude ${ }^{98}$, P. Stavina ${ }^{144 a}$, G. Stavropoulos ${ }^{14}$, G. Steele ${ }^{53}$, P. Steinbach ${ }^{43}$, P. Steinberg ${ }^{24}$, I. Stekl ${ }^{127}$, B. Stelzer ${ }^{142}$, H.J. Stelzer ${ }^{88}$, O. Stelzer-Chilton ${ }^{159 a}$, H. Stenzel ${ }^{52}$, K. Stevenson ${ }^{75}$, G.A. Stewart ${ }^{29}$, J.A. Stillings ${ }^{20}$, T. Stockmanns $^{20}$, M.C. Stockton ${ }^{29}$, K. Stoerig ${ }^{48}$, G. Stoicea ${ }^{25 a}$, S. Stonjek ${ }^{99}$, P. Strachota ${ }^{126}$, A.R. Stradling ${ }^{7}$, A. Straessner ${ }^{43}$, J. Strandberg ${ }^{147}$, S. Strandberg ${ }^{146 a, 146 b}$, A. Strandlie ${ }^{117}$, M. Strang ${ }^{109}$, E. Strauss ${ }^{143}$, M. Strauss ${ }^{111}$, P. Strizenec ${ }^{144 b}$, R. Ströhmer $^{173}$, D.M. Strom ${ }^{114}$, J.A. Strong ${ }^{76, *}$, R. Stroynowski ${ }^{39}$, J. Strube ${ }^{129}$, B. Stugu ${ }^{13}$, I. Stumer ${ }^{24, *}$, J. Stupak ${ }^{148}$, P. Sturm ${ }^{174}$, D.A. Soh ${ }^{151, \mathrm{r}}$, D. Su ${ }^{143}$, HS. Subramania ${ }^{2}$, A. Succurro ${ }^{11}$, Y. Sugaya ${ }^{116}$, T. Sugimoto ${ }^{101}$, C. Suhr ${ }^{106}$, K. Suita ${ }^{67}$, M. Suk ${ }^{126}$, V.V. Sulin ${ }^{94}$, S. Sultansoy ${ }^{3 d}$, T. Sumida ${ }^{29}$, X. Sun ${ }^{55}$, J.E. Sundermann ${ }^{48}$, K. Suruliz ${ }^{139}$, S. Sushkov ${ }^{11}$, G. Susinno ${ }^{36 a, 36 b}$, M.R. Sutton ${ }^{149}$, Y. Suzuki ${ }^{66}$, Y. Suzuki ${ }^{67}$, M. Svatos ${ }^{125}$, Yu.M. Sviridov ${ }^{128}$, S. Swedish ${ }^{168}$, I. Sykora ${ }^{144 a}$, T. Sykora ${ }^{126}$, B. Szeless ${ }^{29}$, J. Sánchez ${ }^{167}$, D. Ta ${ }^{105}$, K. Tackmann ${ }^{41}$, A. Taffard ${ }^{163}$, R. Tafirout ${ }^{159 a}$, N. Taiblum ${ }^{153}$, Y. Takahashi ${ }^{101}$, H. Takai ${ }^{24}$, R. Takashima ${ }^{69}$, H. Takeda ${ }^{67}$, T. Takeshita ${ }^{140}$, M. Talby ${ }^{83}$, A. Talyshev ${ }^{107}$, M.C. Tamsett ${ }^{24}$, J. Tanaka ${ }^{155}$, 
R. Tanaka ${ }^{115}$, S. Tanaka ${ }^{131}$, S. Tanaka ${ }^{66}$, Y. Tanaka ${ }^{100}$, K. Tani ${ }^{67}$, N. Tannoury ${ }^{83}$, G.P. Tappern ${ }^{29}$, S. Tapprogge ${ }^{81}$, D. Tardif ${ }^{158}$, S. Tarem ${ }^{152}$, F. Tarrade ${ }^{28}$, G.F. Tartarelli ${ }^{89 a}$, P. Tas ${ }^{126}$, M. Tasevsky ${ }^{125}$, E. Tassi ${ }^{36 a, 36 b}$, M. Tatarkhanov ${ }^{14}$, Y. Tayalati ${ }^{135 d}$, C. Taylor $^{77}$, F.E. Taylor ${ }^{92}$, G.N. Taylor ${ }^{86}$, W. Taylor ${ }^{159 b}$, M. Teinturier ${ }^{115}$, M. Teixeira Dias Castanheira $^{75}$, P. Teixeira-Dias ${ }^{76}$, K.K. Temming ${ }^{48}$, H. Ten Kate ${ }^{29}$, P.K. Teng ${ }^{151}$, S. Terada ${ }^{66}$, K. Terashi $^{155}$, J. Terron $^{80}$, M. Terwort $^{41, \mathrm{p}}$, M. Testa ${ }^{47}$, R.J. Teuscher ${ }^{158, j}$, J. Thadome ${ }^{174}$, J. Therhaag ${ }^{20}$, T. Theveneaux-Pelzer ${ }^{78}$, M. Thioye ${ }^{175}$, S. Thoma ${ }^{48}$, J.P. Thomas ${ }^{17}$, E.N. Thompson ${ }^{34}$, P.D. Thompson ${ }^{17}$, P.D. Thompson ${ }^{158}$, A.S. Thompson ${ }^{53}$, E. Thomson ${ }^{120}$, M. Thom$\operatorname{son}^{27}$, R.J. Thompson ${ }^{82}$, R.P. Thun ${ }^{87}$, F. Tian ${ }^{34}$, T. Tic ${ }^{125}$, V.O. Tikhomirov ${ }^{94}$, Y.A. Tikhonov ${ }^{107}$, C.J.W.P. Timmermans $^{104}$, P. Tipton ${ }^{175}$, F.J. Tique Aires Viegas ${ }^{29}$, S. Tisserant ${ }^{83}$, J. Tobias ${ }^{48}$, B. Toczek ${ }^{37}$, T. Todorov ${ }^{4}$, S. TodorovaNova $^{161}$, B. Toggerson ${ }^{163}$, J. Tojo ${ }^{66}$, S. Tokár ${ }^{144 a}$, K. Tokunaga ${ }^{67}$, K. Tokushuku ${ }^{66}$, K. Tollefson ${ }^{88}$, M. Tomoto ${ }^{101}$, L. Tompkins $^{14}$, K. Toms ${ }^{103}$, G. Tong ${ }^{32 a}$, A. Tonoyan ${ }^{13}$, C. Topfel ${ }^{16}$, N.D. Topilin ${ }^{65}$, I. Torchiani ${ }^{29}$, E. Torrence ${ }^{114}$, H. Torres ${ }^{78}$, E. Torró Pastor ${ }^{167}$, J. Toth ${ }^{83, \mathrm{x}}$, F. Touchard ${ }^{83}$, D.R. Tovey ${ }^{139}$, D. Traynor ${ }^{75}$, T. Trefzger ${ }^{173}$, L. Tremblet ${ }^{29}$, A. Tricoli ${ }^{29}$, I.M. Trigger ${ }^{159}$ a , S. Trincaz-Duvoid ${ }^{78}$, T.N. Trinh ${ }^{78}$, M.F. Tripiana ${ }^{70}$, W. Trischuk ${ }^{158}$, A. Trivedi ${ }^{24, w}$, B. Trocmé ${ }^{55}$, C. Tron$\operatorname{con}^{89 a}$, M. Trottier-McDonald ${ }^{142}$, A. Trzupek ${ }^{38}$, C. Tsarouchas ${ }^{29}$, J.C-L. Tseng ${ }^{118}$, M. Tsiakiris ${ }^{105}$, P.V. Tsiareshka ${ }^{90}$, D. Tsionou ${ }^{4}$, G. Tsipolitis ${ }^{9}$, V. Tsiskaridze ${ }^{48}$, E.G. Tskhadadze ${ }^{51 \text { a }}$, I.I. Tsukerman ${ }^{95}$, V. Tsulaia ${ }^{14}$, J.-W. Tsung ${ }^{20}$, S. Tsuno ${ }^{66}$, D. Tsybychev ${ }^{148}$, A. Tua ${ }^{139}$, A. Tudorache ${ }^{25 a}$, V. Tudorache ${ }^{25 a}$, J.M. Tuggle ${ }^{30}$, M. Turala ${ }^{38}$, D. Turecek ${ }^{127}$, I. Turk Cakir $^{3 \mathrm{e}}$, E. Turlay ${ }^{105}$, R. Turra ${ }^{89 a, 89 b}$, P.M. Tuts ${ }^{34}$, M.S. Twomey ${ }^{138}$, A. Tykhonov ${ }^{74}$, M. Tylmad ${ }^{146 a, 146 b}$, M. Tyndel ${ }^{129}$, H. Tyrvainen ${ }^{29}$, G. Tzanakos ${ }^{8}$, K. Uchida ${ }^{20}$, I. Ueda ${ }^{155}$, R. Ueno ${ }^{28}$, M. Ugland ${ }^{13}$, M. Uhlenbrock ${ }^{20}$, M. Uhrmacher ${ }^{54}$, F. Ukegawa ${ }^{160}$, G. Unal ${ }^{29}$, D.G. Underwood ${ }^{5}$, A. Undrus ${ }^{24}$, G. Unel ${ }^{163}$, Y. Unno ${ }^{66}$, D. Urbaniec ${ }^{34}$, E. Urkovsky ${ }^{153}$, P. Urrejola $^{31 a}$, G. Usai ${ }^{7}$, M. Uslenghi ${ }^{19 a, 119 b}$, L. Vacavant ${ }^{83}$, V. Vacek ${ }^{127}$, B. Vachon ${ }^{85}$, S. Vahsen ${ }^{14}$, J. Valenta ${ }^{125}$, P. Valente ${ }^{132 a}$, S. Valentinetti ${ }^{19 a, 19 b}$, S. Valkar ${ }^{126}$, E. Valladolid Gallego ${ }^{167}$, S. Vallecorsa ${ }^{152}$, J.A. Valls Ferrer ${ }^{167}$, H. van der Graaf ${ }^{105}$, E. van der Kraaij ${ }^{105}$, R. Van Der Leeuw ${ }^{105}$, E. van der Poel $^{105}$, D. van der Ster $^{29}$, N. van Eldik ${ }^{84}$, P. van Gemmeren ${ }^{5}$, Z. van Kesteren ${ }^{105}$, I. van Vulpen ${ }^{105}$, M. Vanadia ${ }^{99}$, W. Vandelli ${ }^{29}$, G. Vandoni ${ }^{29}$, A. Vaniachine ${ }^{5}$, P. Vankov ${ }^{41}$, F. Vannucci ${ }^{78}$, F. Varela Rodriguez ${ }^{29}$, R. Vari ${ }^{132 a}$, E.W. Varnes ${ }^{6}$, D. Varouchas ${ }^{14}$, A. Vartapetian ${ }^{7}$, K.E. Varvell ${ }^{150}$, V.I. Vassilakopoulos ${ }^{56}$, F. Vazeille ${ }^{33}$, G. Vegni ${ }^{89 a, 89 b}$, J.J. Veillet ${ }^{115}$, C. Vellidis ${ }^{8}$, F. Veloso ${ }^{124 a}$, R. Veness ${ }^{29}$, S. Veneziano ${ }^{132 a}$, A. Ventura ${ }^{72 a, 72 b}$, D. Ventura ${ }^{138}$, M. Venturi ${ }^{48}$, N. Venturi ${ }^{16}$, V. Vercesi ${ }^{119 a}$, M. Verducci ${ }^{138}$, W. Verkerke ${ }^{105}$, J.C. Vermeulen ${ }^{105}$, A. Vest ${ }^{43}$, M.C. Vetterli ${ }^{142, e}$, I. Vichou ${ }^{165}$, T. Vickey ${ }^{145 b \text {,aa }}$, O.E. Vickey Boeriu ${ }^{145 b}$, G.H.A. Viehhauser ${ }^{118}$, S. Viel ${ }^{168}$, M. Villa ${ }^{19 a, 19 b}$, E.G. Villani ${ }^{129}$, M. Villaplana Perez ${ }^{167}$, E. Vilucchi ${ }^{47}$, M.G. Vincter ${ }^{28}$, E. Vinek ${ }^{29}$, V.B. Vinogradov ${ }^{65}$, M. Virchaux ${ }^{136, *}$, J. Virzi ${ }^{14}$, O. Vitells ${ }^{171}$, M. Viti ${ }^{41}$, I. Vivarellii ${ }^{48}$, F. Vives Vaque ${ }^{2}$, S. Vlachos ${ }^{9}$, D. Vladoiu ${ }^{98}$, M. Vlasak ${ }^{127}$, N. Vlasov ${ }^{20}$, A. Vogel ${ }^{20}$, P. $\operatorname{Vokac}^{127}$, G. Volpi ${ }^{47}$, M. Volpi ${ }^{86}$, G. Volpini ${ }^{89 a}$, H. von der Schmitt ${ }^{99}$, J. von Loeben ${ }^{99}$, H. von Radziewski ${ }^{48}$, E. von Toerne ${ }^{20}$, V. Vorobel ${ }^{126}$, A.P. Vorobiev ${ }^{128}$, V. Vorwerk ${ }^{11}$, M. $\operatorname{Vos}^{167}$, R. Voss ${ }^{29}$, T.T. Voss ${ }^{174}$, J.H. Vossebeld ${ }^{73}$, N. Vranjes ${ }^{12 a}$, M. Vranjes Milosavljevic ${ }^{105}$, V. Vrba ${ }^{125}$, M. Vreeswijk ${ }^{105}$, T. Vu Anh ${ }^{81}$, R. Vuillermet ${ }^{29}$, M. Vujicic ${ }^{28}$, I. Vukotic ${ }^{115}$, W. Wagner ${ }^{174}$, P. Wagner ${ }^{120}$, H. Wahlen ${ }^{174}$, J. Wakabayashi ${ }^{101}$, J. Walbersloh ${ }^{42}$, S. Walch ${ }^{87}$, J. Walder ${ }^{71}$, R. Walker ${ }^{98}$, W. Walkowiak ${ }^{141}$, R. Wall ${ }^{175}$, P. Waller ${ }^{73}$, C. Wang ${ }^{44}$, H. Wang ${ }^{172}$, H. Wang ${ }^{32 b, a b}$, J. Wang ${ }^{151}$, J. Wang ${ }^{32 d}$, J.C. Wang ${ }^{138}$, R. Wang ${ }^{103}$, S.M. Wang ${ }^{151}$, A. Warburton ${ }^{85}$, C.P. Ward ${ }^{27}$, M. Warsinsky ${ }^{48}$, R. Wastie ${ }^{118}$, P.M. Watkins ${ }^{17}$, A.T. Watson ${ }^{17}$, M.F. Watson ${ }^{17}$, G. Watts ${ }^{138}$, S. Watts ${ }^{82}$, A.T. Waugh ${ }^{150}$, B.M. Waugh ${ }^{77}$, J. Weber ${ }^{42}$, M. Weber ${ }^{129}$, M.S. Weber $^{16}$, P. Weber ${ }^{54}$, A.R. Weidberg ${ }^{118}$, P. Weigell ${ }^{99}$, J. Weingarten ${ }^{54}$, C. Weiser ${ }^{48}$, H. Wellenstein ${ }^{22}$, P.S. Wells ${ }^{29}$, M. Wen $^{47}$, T. Wenaus ${ }^{24}$, S. Wendler ${ }^{123}$, Z. Weng ${ }^{151, \mathrm{r}}$, T. Wengler ${ }^{29}$, S. Wenig $^{29}$, N. Wermes ${ }^{20}$, M. Werner ${ }^{48}$, P. Werner $^{29}$, M. Werth $^{163}$, M. Wessels ${ }^{58 a}$, C. Weydert ${ }^{55}$, K. Whalen ${ }^{28}$, S.J. Wheeler-Ellis ${ }^{163}$, S.P. Whitaker ${ }^{21}$, A. White ${ }^{7}$, M.J. White ${ }^{86}$, S. White ${ }^{24}$, S.R. Whitehead ${ }^{118}$, D. Whiteson ${ }^{163}$, D. Whittington ${ }^{61}$, F. Wicek ${ }^{115}$, D. Wicke ${ }^{174}$, F.J. Wickens ${ }^{129}$, W. Wiedenmann ${ }^{172}$, M. Wielers ${ }^{129}$, P. Wienemann ${ }^{20}$, C. Wiglesworth ${ }^{75}$, L.A.M. Wiik-Fuchs ${ }^{48}$, P.A. Wijeratne ${ }^{77}$, A. Wildauer ${ }^{167}$, M.A. Wildt ${ }^{41, p}$, I. Wilhelm ${ }^{126}$, H.G. Wilkens ${ }^{29}$, J.Z. Will ${ }^{98}$, E. Williams ${ }^{34}$, H.H. Williams ${ }^{120}$, W. Willis ${ }^{34}$, S. Willocq ${ }^{84}$, J.A. Wilson ${ }^{17}$, M.G. Wilson ${ }^{143}$, A. Wilson ${ }^{87}$, I. Wingerter-Seez ${ }^{4}$, S. Winkelmann ${ }^{48}$, F. Winklmeier ${ }^{29}$, M. Wittgen ${ }^{143}$, M.W. Wolter ${ }^{38}$, H. Wolters ${ }^{124 a, h}$, W.C. Wong ${ }^{40}$, G. Wooden ${ }^{87}$, B.K. Wosiek ${ }^{38}$, J. Wotschack ${ }^{29}$, M.J. Woudstra ${ }^{84}$, K. Wraight ${ }^{53}$, C. Wright ${ }^{53}$, M. Wright ${ }^{53}$, D. Wright ${ }^{143}$, B. Wrona ${ }^{73}$, S.L. Wu ${ }^{172}, X$ Wu $^{49}$, Y. Wu ${ }^{32 b, a c}$, E. Wulf ${ }^{34}$, R. Wunstorf ${ }^{42}$, B.M. Wynne ${ }^{45}$, L. Xaplanteris ${ }^{9}$, S. Xella ${ }^{35}$, S. Xie ${ }^{48}$, Y. Xie ${ }^{32 a}$, C. Xu ${ }^{32 b, a d}$, D. Xu ${ }^{139}$, G. Xu $^{32 a}$, B. Yabsley ${ }^{150}$, S. Yacoob ${ }^{145 b}$, M. Ya$\operatorname{mada}^{66}$, H. Yamaguchi ${ }^{155}$, A. Yamamoto ${ }^{66}$, K. Yamamoto ${ }^{64}$, S. Yamamoto ${ }^{155}$, T. Yamamura ${ }^{155}$, T. Yamanaka ${ }^{155}$, J. Ya-

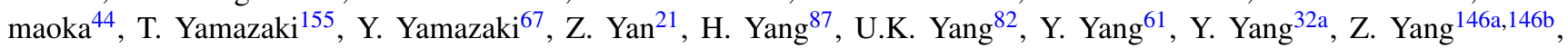
S. Yanush ${ }^{91}$, Y. Yao $^{14}$, Y. Yasu ${ }^{66}$, G.V. Ybeles Smit ${ }^{130}$, J. Ye ${ }^{39}$, S. Ye ${ }^{24}$, M. Yilmaz ${ }^{3 c}$, R. Yoosoofmiya ${ }^{123}$, K. Yorita ${ }^{170}$, R. Yoshida ${ }^{5}$, C. Young ${ }^{143}$, S. Youssef ${ }^{21}$, D. Yu ${ }^{24}$, J. Yu ${ }^{7}$, J. Yu ${ }^{112}$, L. Yuan ${ }^{32 a, a e}$, A. Yurkewicz ${ }^{148}$, V.G. Zaets ${ }^{128}$, R. Zaidan ${ }^{63}$, A.M. Zaitsev ${ }^{128}$, Z. Zajacova ${ }^{29}$, Yo.K. Zalite ${ }^{121}$, L. Zanello ${ }^{132 a, 132 b}$, P. Zarzhitsky ${ }^{39}$, A. Zaytsev ${ }^{107}$, C. Zeitnitz ${ }^{174}$, M. Zeller ${ }^{175}$, M. Zeman ${ }^{125}$, A. Zemla ${ }^{38}$, C. Zendler ${ }^{20}$, O. Zenin ${ }^{128}$, T. Ženišs ${ }^{144 a}$, Z. Zenonos ${ }^{122 a, 122 b}$, S. Zenz ${ }^{14}$, D. Zerwas ${ }^{115}$, G. Zevi della Porta ${ }^{57}$, Z. Zhan ${ }^{32 d}$, D. Zhang ${ }^{32 b, a b}$, H. Zhang ${ }^{88}$, J. Zhang ${ }^{5}$, X. Zhang ${ }^{32 d}$, Z. Zhang ${ }^{115}$, Q. Zhang ${ }^{5}$, L. Zhao ${ }^{108}$, T. Zhao ${ }^{138}$, Z. Zhao ${ }^{32 b}$, A. Zhemchugov ${ }^{65}$, S. Zheng ${ }^{32 a}$, J. Zhong ${ }^{151, a f}$, B. Zhou ${ }^{87}$, N. Zhou ${ }^{163}$, 
Y. Zhou ${ }^{151}$, C.G. Zhu ${ }^{32 d}$, H. Zhu ${ }^{41}$, J. Zhu ${ }^{87}$, Y. Zhu ${ }^{32 b}$, X. Zhuang ${ }^{98}$, V. Zhuravlov ${ }^{99}$, D. Zieminska ${ }^{61}$, R. Zimmermann ${ }^{20}$, S. Zimmermann ${ }^{20}$, S. Zimmermann ${ }^{48}$, Z. Zinonos ${ }^{122 a}$, M. Ziolkowski ${ }^{141}$, R. Zitoun ${ }^{4}$, L. Živković ${ }^{34}$, V.V. Zmouchko ${ }^{128, *}$, G. Zobernig ${ }^{172}$, A. Zoccoli ${ }^{19 a, 19 b}$, Y. Zolnierowski ${ }^{4}$, A. Zsenei ${ }^{29}$, M. zur Nedden ${ }^{15}$, V. Zutshi ${ }^{106}$, L. Zwalinski ${ }^{29}$

${ }^{1}$ University at Albany, Albany NY, United States of America

${ }^{2}$ Department of Physics, University of Alberta, Edmonton AB, Canada

${ }^{3(a)}$ Department of Physics, Ankara University, Ankara; ${ }^{(b)}$ Department of Physics, Dumlupinar University, Kutahya;

${ }^{(c)}$ Department of Physics, Gazi University, Ankara; ${ }^{(d)}$ Division of Physics, TOBB University of Economics and

Technology, Ankara; ${ }^{(\mathrm{e})}$ Turkish Atomic Energy Authority, Ankara, Turkey

${ }^{4}$ LAPP, CNRS/IN2P3 and Université de Savoie, Annecy-le-Vieux, France

${ }^{5}$ High Energy Physics Division, Argonne National Laboratory, Argonne IL, United States of America

${ }^{6}$ Department of Physics, University of Arizona, Tucson AZ, United States of America

${ }^{7}$ Department of Physics, The University of Texas at Arlington, Arlington TX, United States of America

${ }^{8}$ Physics Department, University of Athens, Athens, Greece

${ }^{9}$ Physics Department, National Technical University of Athens, Zografou, Greece

${ }^{10}$ Institute of Physics, Azerbaijan Academy of Sciences, Baku, Azerbaijan

${ }^{11}$ Institut de Física d'Altes Energies and Departament de Física de la Universitat Autònoma de Barcelona and ICREA,

Barcelona, Spain

12(a) Institute of Physics, University of Belgrade, Belgrade; ${ }^{(b)}$ Vinca Institute of Nuclear Sciences, Belgrade, Serbia

${ }^{13}$ Department for Physics and Technology, University of Bergen, Bergen, Norway

${ }^{14}$ Physics Division, Lawrence Berkeley National Laboratory and University of California, Berkeley CA, United States of America

${ }^{15}$ Department of Physics, Humboldt University, Berlin, Germany

${ }^{16}$ Albert Einstein Center for Fundamental Physics and Laboratory for High Energy Physics, University of Bern, Bern, Switzerland

${ }^{17}$ School of Physics and Astronomy, University of Birmingham, Birmingham, United Kingdom

${ }^{18(a)}$ Department of Physics, Bogazici University, Istanbul; ${ }^{(b)}$ Division of Physics, Dogus University, Istanbul;

${ }^{(c)}$ Department of Physics Engineering, Gaziantep University, Gaziantep; ${ }^{(d)}$ Department of Physics, Istanbul Technical University, Istanbul, Turkey

19(a) INFN Sezione di Bologna; ${ }^{(b)}$ Dipartimento di Fisica, Università di Bologna, Bologna, Italy

${ }^{20}$ Physikalisches Institut, University of Bonn, Bonn, Germany

${ }^{21}$ Department of Physics, Boston University, Boston MA, United States of America

${ }^{22}$ Department of Physics, Brandeis University, Waltham MA, United States of America

23(a) Universidade Federal do Rio De Janeiro COPPE/EE/IF, Rio de Janeiro; ${ }^{(b)}$ Federal University of Juiz de Fora (UFJF), Juiz de Fora; ${ }^{\left({ }^{c}\right)}$ Federal University of Sao Joao del Rei (UFSJ), Sao Joao del Rei; ${ }^{(d)}$ Instituto de Fisica, Universidade de Sao Paulo, Sao Paulo, Brazil

${ }^{24}$ Physics Department, Brookhaven National Laboratory, Upton NY, United States of America

${ }^{25(a)}$ National Institute of Physics and Nuclear Engineering, Bucharest; ${ }^{(b)}$ University Politehnica Bucharest, Bucharest;

${ }^{(c)}$ West University in Timisoara, Timisoara, Romania

${ }^{26}$ Departamento de Física, Universidad de Buenos Aires, Buenos Aires, Argentina

${ }^{27}$ Cavendish Laboratory, University of Cambridge, Cambridge, United Kingdom

${ }^{28}$ Department of Physics, Carleton University, Ottawa ON, Canada

${ }^{29}$ CERN, Geneva, Switzerland

${ }^{30}$ Enrico Fermi Institute, University of Chicago, Chicago IL, United States of America

31(a) Departamento de Fisica, Pontificia Universidad Católica de Chile, Santiago; ${ }^{(b)}$ Departamento de Física, Universidad Técnica Federico Santa María, Valparaíso, Chile

32 (a) Institute of High Energy Physics, Chinese Academy of Sciences, Beijing; ${ }^{(b)}$ Department of Modern Physics, University of Science and Technology of China, Anhui; ${ }^{(c)}$ Department of Physics, Nanjing University, Jiangsu; ${ }^{\left({ }^{(d)}\right.}$ High Energy Physics Group, Shandong University, Shandong, China

${ }^{33}$ Laboratoire de Physique Corpusculaire, Clermont Université and Université Blaise Pascal and CNRS/IN2P3, Aubiere Cedex, France

${ }^{34}$ Nevis Laboratory, Columbia University, Irvington NY, United States of America

${ }^{35}$ Niels Bohr Institute, University of Copenhagen, Kobenhavn, Denmark

${ }^{36(a)}$ INFN Gruppo Collegato di Cosenza; ${ }^{(b)}$ Dipartimento di Fisica, Università della Calabria, Arcavata di Rende, Italy 
${ }^{37}$ Faculty of Physics and Applied Computer Science, AGH-University of Science and Technology, Krakow, Poland

${ }^{38}$ The Henryk Niewodniczanski Institute of Nuclear Physics, Polish Academy of Sciences, Krakow, Poland

${ }^{39}$ Physics Department, Southern Methodist University, Dallas TX, United States of America

${ }^{40}$ Physics Department, University of Texas at Dallas, Richardson TX, United States of America

${ }^{41}$ DESY, Hamburg and Zeuthen, Germany

${ }^{42}$ Institut für Experimentelle Physik IV, Technische Universität Dortmund, Dortmund, Germany

${ }^{43}$ Institut für Kern- und Teilchenphysik, Technical University Dresden, Dresden, Germany

${ }^{44}$ Department of Physics, Duke University, Durham NC, United States of America

${ }^{45}$ SUPA - School of Physics and Astronomy, University of Edinburgh, Edinburgh, United Kingdom

${ }^{46}$ Fachhochschule Wiener Neustadt, Johannes Gutenbergstrasse 3, 2700 Wiener Neustadt, Austria

${ }^{47}$ INFN Laboratori Nazionali di Frascati, Frascati, Italy

${ }^{48}$ Fakultät für Mathematik und Physik, Albert-Ludwigs-Universität, Freiburg i.Br., Germany

${ }^{49}$ Section de Physique, Université de Genève, Geneva, Switzerland

50(a) INFN Sezione di Genova; ${ }^{(b)}$ Dipartimento di Fisica, Università di Genova, Genova, Italy

${ }^{51(a)}$ E.Andronikashvili Institute of Physics, Georgian Academy of Sciences, Tbilisi; ${ }^{(b)}$ High Energy Physics Institute,

Tbilisi State University, Tbilisi, Georgia

${ }^{52}$ II Physikalisches Institut, Justus-Liebig-Universität Giessen, Giessen, Germany

${ }^{53}$ SUPA - School of Physics and Astronomy, University of Glasgow, Glasgow, United Kingdom

${ }^{54}$ II Physikalisches Institut, Georg-August-Universität, Göttingen, Germany

${ }^{55}$ Laboratoire de Physique Subatomique et de Cosmologie, Université Joseph Fourier and CNRS/IN2P3 and Institut

National Polytechnique de Grenoble, Grenoble, France

${ }^{56}$ Department of Physics, Hampton University, Hampton VA, United States of America

${ }^{57}$ Laboratory for Particle Physics and Cosmology, Harvard University, Cambridge MA, United States of America

${ }^{58(a)}$ Kirchhoff-Institut für Physik, Ruprecht-Karls-Universität Heidelberg, Heidelberg; ${ }^{(b)}$ Physikalisches Institut,

Ruprecht-Karls-Universität Heidelberg, Heidelberg; ${ }^{\text {c) }}$ ZITI Institut für technische Informatik,

Ruprecht-Karls-Universität Heidelberg, Mannheim, Germany

${ }^{59}$ Faculty of Science, Hiroshima University, Hiroshima, Japan

${ }^{60}$ Faculty of Applied Information Science, Hiroshima Institute of Technology, Hiroshima, Japan

${ }^{61}$ Department of Physics, Indiana University, Bloomington IN, United States of America

${ }^{62}$ Institut für Astro- und Teilchenphysik, Leopold-Franzens-Universität, Innsbruck, Austria

${ }^{63}$ University of Iowa, Iowa City IA, United States of America

${ }^{64}$ Department of Physics and Astronomy, Iowa State University, Ames IA, United States of America

${ }^{65}$ Joint Institute for Nuclear Research, JINR Dubna, Dubna, Russia

${ }^{66}$ KEK, High Energy Accelerator Research Organization, Tsukuba, Japan

${ }^{67}$ Graduate School of Science, Kobe University, Kobe, Japan

${ }^{68}$ Faculty of Science, Kyoto University, Kyoto, Japan

${ }^{69}$ Kyoto University of Education, Kyoto, Japan

${ }^{70}$ Instituto de Física La Plata, Universidad Nacional de La Plata and CONICET, La Plata, Argentina

${ }^{71}$ Physics Department, Lancaster University, Lancaster, United Kingdom

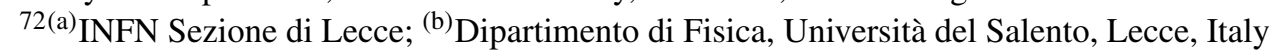

${ }^{73}$ Oliver Lodge Laboratory, University of Liverpool, Liverpool, United Kingdom

${ }^{74}$ Department of Physics, Jožef Stefan Institute and University of Ljubljana, Ljubljana, Slovenia

${ }^{75}$ School of Physics and Astronomy, Queen Mary University of London, London, United Kingdom

${ }^{76}$ Department of Physics, Royal Holloway University of London, Surrey, United Kingdom

${ }^{77}$ Department of Physics and Astronomy, University College London, London, United Kingdom

${ }^{78}$ Laboratoire de Physique Nucléaire et de Hautes Energies, UPMC and Université Paris-Diderot and CNRS/IN2P3, Paris, France

${ }^{79}$ Fysiska institutionen, Lunds universitet, Lund, Sweden

${ }^{80}$ Departamento de Fisica Teorica C-15, Universidad Autonoma de Madrid, Madrid, Spain

${ }^{81}$ Institut für Physik, Universität Mainz, Mainz, Germany

${ }^{82}$ School of Physics and Astronomy, University of Manchester, Manchester, United Kingdom

${ }^{83}$ CPPM, Aix-Marseille Université and CNRS/IN2P3, Marseille, France

${ }^{84}$ Department of Physics, University of Massachusetts, Amherst MA, United States of America 
${ }^{85}$ Department of Physics, McGill University, Montreal QC, Canada

${ }^{86}$ School of Physics, University of Melbourne, Victoria, Australia

${ }^{87}$ Department of Physics, The University of Michigan, Ann Arbor MI, United States of America

${ }^{88}$ Department of Physics and Astronomy, Michigan State University, East Lansing MI, United States of America

${ }^{89}$ (a) INFN Sezione di Milano; ${ }^{(b)}$ Dipartimento di Fisica, Università di Milano, Milano, Italy

${ }^{90}$ B.I. Stepanov Institute of Physics, National Academy of Sciences of Belarus, Minsk, Republic of Belarus

${ }^{91}$ National Scientific and Educational Centre for Particle and High Energy Physics, Minsk, Republic of Belarus

${ }^{92}$ Department of Physics, Massachusetts Institute of Technology, Cambridge MA, United States of America

${ }^{93}$ Group of Particle Physics, University of Montreal, Montreal QC, Canada

${ }^{94}$ P.N. Lebedev Institute of Physics, Academy of Sciences, Moscow, Russia

${ }^{95}$ Institute for Theoretical and Experimental Physics (ITEP), Moscow, Russia

${ }^{96}$ Moscow Engineering and Physics Institute (MEPhI), Moscow, Russia

${ }^{97}$ Skobeltsyn Institute of Nuclear Physics, Lomonosov Moscow State University, Moscow, Russia

${ }^{98}$ Fakultät für Physik, Ludwig-Maximilians-Universität München, München, Germany

${ }^{99}$ Max-Planck-Institut für Physik (Werner-Heisenberg-Institut), München, Germany

${ }^{100}$ Nagasaki Institute of Applied Science, Nagasaki, Japan

${ }^{101}$ Graduate School of Science, Nagoya University, Nagoya, Japan

102(a) INFN Sezione di Napoli; ${ }^{\left({ }^{b}\right)}$ Dipartimento di Scienze Fisiche, Università di Napoli, Napoli, Italy

${ }^{103}$ Department of Physics and Astronomy, University of New Mexico, Albuquerque NM, United States of America

${ }^{104}$ Institute for Mathematics, Astrophysics and Particle Physics, Radboud University Nijmegen/Nikhef, Nijmegen,

Netherlands

${ }^{105}$ Nikhef National Institute for Subatomic Physics and University of Amsterdam, Amsterdam, Netherlands

${ }^{106}$ Department of Physics, Northern Illinois University, DeKalb IL, United States of America

${ }^{107}$ Budker Institute of Nuclear Physics (BINP), Novosibirsk, Russia

${ }^{108}$ Department of Physics, New York University, New York NY, United States of America

${ }^{109}$ Ohio State University, Columbus OH, United States of America

${ }^{110}$ Faculty of Science, Okayama University, Okayama, Japan

${ }^{111}$ Homer L. Dodge Department of Physics and Astronomy, University of Oklahoma, Norman OK, United States of America

${ }^{112}$ Department of Physics, Oklahoma State University, Stillwater OK, United States of America

${ }^{113}$ Palacký University, RCPTM, Olomouc, Czech Republic

${ }^{114}$ Center for High Energy Physics, University of Oregon, Eugene OR, United States of America

${ }^{115}$ LAL, Univ. Paris-Sud and CNRS/IN2P3, Orsay, France

${ }^{116}$ Graduate School of Science, Osaka University, Osaka, Japan

${ }^{117}$ Department of Physics, University of Oslo, Oslo, Norway

${ }^{118}$ Department of Physics, Oxford University, Oxford, United Kingdom

119(a) INFN Sezione di Pavia; ${ }^{(b)}$ Dipartimento di Fisica Nucleare e Teorica, Università di Pavia, Pavia, Italy

${ }^{120}$ Department of Physics, University of Pennsylvania, Philadelphia PA, United States of America

${ }^{121}$ Petersburg Nuclear Physics Institute, Gatchina, Russia

122(a) INFN Sezione di Pisa; ${ }^{(b)}$ Dipartimento di Fisica E. Fermi, Università di Pisa, Pisa, Italy

${ }^{123}$ Department of Physics and Astronomy, University of Pittsburgh, Pittsburgh PA, United States of America

${ }^{124(a)}$ Laboratorio de Instrumentacao e Fisica Experimental de Particulas - LIP, Lisboa, Portugal; (b) Departamento de Fisica

Teorica y del Cosmos and CAFPE, Universidad de Granada, Granada, Spain

${ }^{125}$ Institute of Physics, Academy of Sciences of the Czech Republic, Praha, Czech Republic

${ }^{126}$ Faculty of Mathematics and Physics, Charles University in Prague, Praha, Czech Republic

${ }^{127}$ Czech Technical University in Prague, Praha, Czech Republic

${ }^{128}$ State Research Center Institute for High Energy Physics, Protvino, Russia

${ }^{129}$ Particle Physics Department, Rutherford Appleton Laboratory, Didcot, United Kingdom

${ }^{130}$ Physics Department, University of Regina, Regina SK, Canada

${ }^{131}$ Ritsumeikan University, Kusatsu, Shiga, Japan

132(a) INFN Sezione di Roma I; ${ }^{(b)}$ Dipartimento di Fisica, Università La Sapienza, Roma, Italy

133(a) INFN Sezione di Roma Tor Vergata; ${ }^{(b)}$ Dipartimento di Fisica, Università di Roma Tor Vergata, Roma, Italy

134(a) INFN Sezione di Roma Tre; ${ }^{(b)}$ Dipartimento di Fisica, Università Roma Tre, Roma, Italy 
${ }^{135(a)}$ Faculté des Sciences Ain Chock, Réseau Universitaire de Physique des Hautes Energies - Université Hassan II, Casablanca; ${ }^{(b)}$ Centre National de l'Energie des Sciences Techniques Nucleaires, Rabat; ${ }^{(c)}$ Université Cadi Ayyad, Faculté des sciences Semlalia Département de Physique, B.P. 2390 Marrakech 40000; ${ }^{(d)}$ Faculté des Sciences, Université Mohamed Premier and LPTPM, Oujda; ${ }^{(e)}$ Faculté des Sciences, Université Mohammed V, Rabat, Morocco

${ }^{136}$ DSM/IRFU (Institut de Recherches sur les Lois Fondamentales de l'Univers), CEA Saclay (Commissariat a l'Energie Atomique), Gif-sur-Yvette, France

${ }^{137}$ Santa Cruz Institute for Particle Physics, University of California Santa Cruz, Santa Cruz CA, United States of America

${ }^{138}$ Department of Physics, University of Washington, Seattle WA, United States of America

${ }^{139}$ Department of Physics and Astronomy, University of Sheffield, Sheffield, United Kingdom

${ }^{140}$ Department of Physics, Shinshu University, Nagano, Japan

${ }^{141}$ Fachbereich Physik, Universität Siegen, Siegen, Germany

${ }^{142}$ Department of Physics, Simon Fraser University, Burnaby BC, Canada

${ }^{143}$ SLAC National Accelerator Laboratory, Stanford CA, United States of America

${ }^{144(a)}$ Faculty of Mathematics, Physics \& Informatics, Comenius University, Bratislava; ${ }^{(b)}$ Department of Subnuclear

Physics, Institute of Experimental Physics of the Slovak Academy of Sciences, Kosice, Slovak Republic

${ }^{145(a)}$ Department of Physics, University of Johannesburg, Johannesburg; ${ }^{\left({ }^{b}\right)}$ School of Physics, University of the

Witwatersrand, Johannesburg, South Africa

146(a) Department of Physics, Stockholm University; ${ }^{(b)}$ The Oskar Klein Centre, Stockholm, Sweden

${ }^{147}$ Physics Department, Royal Institute of Technology, Stockholm, Sweden

${ }^{148}$ Department of Physics and Astronomy, Stony Brook University, Stony Brook NY, United States of America

${ }^{149}$ Department of Physics and Astronomy, University of Sussex, Brighton, United Kingdom

${ }^{150}$ School of Physics, University of Sydney, Sydney, Australia

${ }^{151}$ Institute of Physics, Academia Sinica, Taipei, Taiwan

${ }^{152}$ Department of Physics, Technion: Israel Inst. of Technology, Haifa, Israel

${ }^{153}$ Raymond and Beverly Sackler School of Physics and Astronomy, Tel Aviv University, Tel Aviv, Israel

${ }^{154}$ Department of Physics, Aristotle University of Thessaloniki, Thessaloniki, Greece

${ }^{155}$ International Center for Elementary Particle Physics and Department of Physics, The University of Tokyo, Tokyo, Japan

${ }^{156}$ Graduate School of Science and Technology, Tokyo Metropolitan University, Tokyo, Japan

${ }^{157}$ Department of Physics, Tokyo Institute of Technology, Tokyo, Japan

${ }^{158}$ Department of Physics, University of Toronto, Toronto ON, Canada

159(a) TRIUMF, Vancouver BC; ${ }^{(b)}$ Department of Physics and Astronomy, York University, Toronto ON, Canada

${ }^{160}$ Institute of Pure and Applied Sciences, University of Tsukuba, 1-1-1 Tennodai, Tsukuba, Ibaraki 305-8571, Japan

${ }^{161}$ Science and Technology Center, Tufts University, Medford MA, United States of America

${ }^{162}$ Centro de Investigaciones, Universidad Antonio Narino, Bogota, Colombia

${ }^{163}$ Department of Physics and Astronomy, University of California Irvine, Irvine CA, United States of America

${ }^{164(a)}$ INFN Gruppo Collegato di Udine, Udine; ${ }^{(b)}$ ICTP, Trieste; ${ }^{(c)}$ Dipartimento di Chimica, Fisica e Ambiente, Università di Udine, Udine, Italy

${ }^{165}$ Department of Physics, University of Illinois, Urbana IL, United States of America

${ }^{166}$ Department of Physics and Astronomy, University of Uppsala, Uppsala, Sweden

${ }^{167}$ Instituto de Física Corpuscular (IFIC) and Departamento de Física Atómica, Molecular y Nuclear and Departamento de Ingenierá Electrónica and Instituto de Microelectrónica de Barcelona (IMB-CNM), University of Valencia and CSIC, Valencia, Spain

${ }^{168}$ Department of Physics, University of British Columbia, Vancouver BC, Canada

${ }^{169}$ Department of Physics and Astronomy, University of Victoria, Victoria BC, Canada

${ }^{170}$ Waseda University, Tokyo, Japan

${ }^{171}$ Department of Particle Physics, The Weizmann Institute of Science, Rehovot, Israel

${ }^{172}$ Department of Physics, University of Wisconsin, Madison WI, United States of America

${ }^{173}$ Fakultät für Physik und Astronomie, Julius-Maximilians-Universität, Würzburg, Germany

${ }^{174}$ Fachbereich C Physik, Bergische Universität Wuppertal, Wuppertal, Germany

${ }^{175}$ Department of Physics, Yale University, New Haven CT, United States of America

${ }^{176}$ Yerevan Physics Institute, Yerevan, Armenia

${ }^{177}$ Domaine scientifique de la Doua, Centre de Calcul CNRS/IN2P3, Villeurbanne Cedex, France

${ }^{a}$ Also at Laboratorio de Instrumentacao e Fisica Experimental de Particulas - LIP, Lisboa, Portugal 
${ }^{\mathrm{b}}$ Also at Faculdade de Ciencias and CFNUL, Universidade de Lisboa, Lisboa, Portugal

${ }^{\mathrm{c}}$ Also at Particle Physics Department, Rutherford Appleton Laboratory, Didcot, United Kingdom

${ }^{\mathrm{d}}$ Also at CPPM, Aix-Marseille Université and CNRS/IN2P3, Marseille, France

${ }^{\mathrm{e}}$ Also at TRIUMF, Vancouver BC, Canada

${ }^{\mathrm{f}}$ Also at Department of Physics, California State University, Fresno CA, United States of America

${ }^{g}$ Also at Fermilab, Batavia IL, United States of America

${ }^{\mathrm{h}}$ Also at Department of Physics, University of Coimbra, Coimbra, Portugal

${ }^{i}$ Also at Università di Napoli Parthenope, Napoli, Italy

${ }^{\mathrm{j}}$ Also at Institute of Particle Physics (IPP), Canada

${ }^{\mathrm{k}}$ Also at Department of Physics, Middle East Technical University, Ankara, Turkey

${ }^{1}$ Also at Louisiana Tech University, Ruston LA, United States of America

mAlso at Faculty of Physics and Applied Computer Science, AGH-University of Science and Technology, Krakow, Poland

${ }^{\mathrm{n}}$ Also at Group of Particle Physics, University of Montreal, Montreal QC, Canada

${ }^{\circ}$ Also at Institute of Physics, Azerbaijan Academy of Sciences, Baku, Azerbaijan

pAlso at Institut für Experimentalphysik, Universität Hamburg, Hamburg, Germany

${ }^{\mathrm{q}}$ Also at Manhattan College, New York NY, United States of America

${ }^{\mathrm{r}}$ Also at School of Physics and Engineering, Sun Yat-sen University, Guanzhou, China

${ }^{s}$ Also at Academia Sinica Grid Computing, Institute of Physics, Academia Sinica, Taipei, Taiwan

${ }^{t}$ Also at High Energy Physics Group, Shandong University, Shandong, China

"Also at Section de Physique, Université de Genève, Geneva, Switzerland

${ }^{v}$ Also at Departamento de Fisica, Universidade de Minho, Braga, Portugal

${ }^{w}$ Also at Department of Physics and Astronomy, University of South Carolina, Columbia SC, United States of America

${ }^{x}$ Also at KFKI Research Institute for Particle and Nuclear Physics, Budapest, Hungary

${ }^{y}$ Also at California Institute of Technology, Pasadena CA, United States of America

${ }^{\mathrm{z}}$ Also at Institute of Physics, Jagiellonian University, Krakow, Poland

${ }^{a}$ Also at Department of Physics, Oxford University, Oxford, United Kingdom

${ }^{a b}$ Also at Institute of Physics, Academia Sinica, Taipei, Taiwan

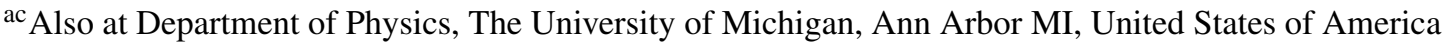

${ }^{a d}$ Also at DSM/IRFU (Institut de Recherches sur les Lois Fondamentales de l'Univers), CEA Saclay (Commissariat a l'Energie Atomique), Gif-sur-Yvette, France

${ }^{a e}$ Also at Laboratoire de Physique Nucléaire et de Hautes Energies, UPMC and Université Paris-Diderot and CNRS/IN2P3, Paris, France

af Also at Department of Physics, Nanjing University, Jiangsu, China

*Deceased 